RECEI: 83

JAN 30 IYY6

WHC-EP-0888

OSTI

\title{
1995 Solid Waste 30-Year Characteristics Volume Summary
}

Prepared for the U.S. Department of Energy Office of Environmental Restoration and Waste Management

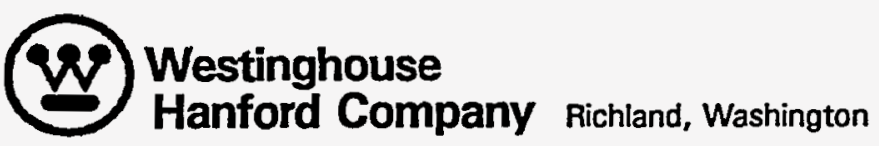

Management and Operations Contractor for the

U.S. Department of Energy under Contract DE-ACO6-87RL10930

Approved for Public Release 


\section{LECAL DISCLAMER}

This report was prepared as an account of work sponsored by an egency of the United States Government. Neither the United States Government nor any agency thereof, nor any of their employees, nor any of their contractors, subcontractors or their employees, makes any warranty, express or implied, or assumes any legal liability or responsibility for the accuracy, completeness, or any third party's use or the results of such use of any information, apparatus, product, or process disclosed, or represents that its use would not infringe privately owned rights. Reference herein to any specific commercial product, process, or service by trade name, trademark, manufacturer, or otherwise, does not necessarily constitute or imply its endorsement, recommendation, or favoring by the United States Government or any agency thereof or its contractors or subcontractors. The views and opinions of authors expressed herein do not necessarily state or reflect those of the United States Government or any agency thereof.

This report has been reproduced from the best available copy. Available in paper copy and microfiche.

Available to the U.S. Department of Energy and its contractors from

Office of Scientific and Technical Information

P.O. Box 62

Oak Ridge, TN 37831

(615) $576-8401$

Available to the public from the U.S. Department of Commerce National Technical Information Service 5285 Port Royal Road

Springfield, VA 22161

(703) $487-4650$

Printed in the United Statee of America 


\title{
1995 Solid Waste 30-Year Characteristics Volume Summary
}

\author{
K. J. Templeton \\ T. J. DeForest \\ G. I. Rice \\ Pacific Northwest National Laboratory \\ Date Published \\ October 1995
}

Prepared for the U.S. Department of Energy Office of Environmental Restoration and Waste Management

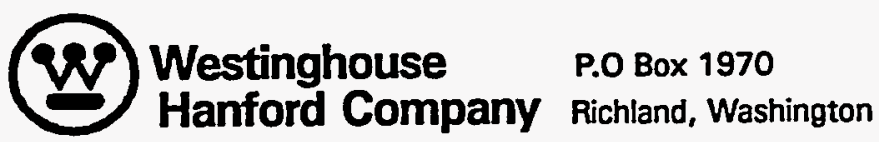

Management and Operations Contractor for the

U.S. Department of Energy under Contract DE-AC06-87RL10930

Approved for Public Release 


\section{RELEASE AUTHORIZATION}

Document Number: WHC-EP-0888, Rev. 0

Document Title: $\quad 1995$ Solid Waste 30-Year Characteristics Volume Summary

Release Date: $\quad 11 / 10 / 95$

This document was reviewed following the procedures described in WHC-CM-3-4 and is:

APPROVED FOR PUBLIC RELEASE

WHC Information Release Administration Specialist:

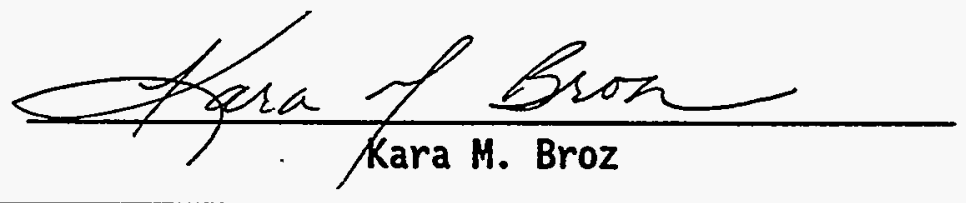

November 10,1995 


\section{EXECUTIVE SUMMARY}

This document, prepared by Pacific Northwest Laboratory (PNL) under the direction of Westinghouse Hanford Company (WHC), describes the characteristics of the waste to be managed at Hanford's Solid Waste Operations Complex (SWOC) over the next 30 years. The characteristics discussed are the physical waste forms and the hazardous waste constituents for low-level mixed waste (LLMW) and transuranic/transuranic-mixed waste (TRU_TRUM). Low-level waste (LLW) is described by physical waste forms in Appendi $\bar{x} A$, since the majority of LLW wil1 not require treatment.

The data presented in this report will change as waste generator planning and missions change and/or become better defined. However, the information still provides useful insights into Hanford's solid waste management needs, both for the present and the future. Waste characteristic information is provided to assist in planning Hanford's treatment, storage, and disposal (TSD) activities over the next several decades.

A baseline volume of approximately $235,560 \mathrm{~m}^{3}$ of LLMW and TRU_TRUM waste is expected to be managed at Hanford's SWOC over the next 30 years. Based on range information provided by the individual waste generators, this baseline volume could fluctuate between a maximum of $260,500 \mathrm{~m}^{3}$ and a minimum of $203,680 \mathrm{~m}^{3}$.

The main physical waste form that wil. be present in the baseline volume is steel shielding, which will make up $30 \%$ of the LLMW and TRU TRUM waste. Retrieval of long-length equipment from the single-shell tank farms is the primary source of steel shielding. The decontamination and decomissioning of the Plutonium Finishing Plant will generate a large portion of the metal debris, which is a major physical waste form that will make up $27 \%$ of the waste. The other major physical waste form that will comprise $23 \%$ of the LLMW and TRU TRUM waste is contaminated soil, generated primarily by Past Practice Remediation.

The main hazardous waste constituents of the LLMW and TRUM waste are organics mixed with metals without mercury, which are expected to be present in $43 \%$ of the mixed waste. The main generator of this hazardous constituent is Single-Shell Tank Long-Length Equipment. Metals without mercury will contaminate $17 \%$ of the waste, which is generated primarily by Past Practice Remediation. Corrosive hazardous constituents will be present in $13 \%$ of the LLMW and TRU_TRUM waste as a result of Past Practice Remediation. 
WHC-EP-0888

Intentionally Left Blank 


\section{CONTENTS}

EXECUTIVE SUMMARY .................... i

ACRONYMS AND ABBREVIATIONS ...................... xi

1.0 INTRODUCTION ........................... 1-1

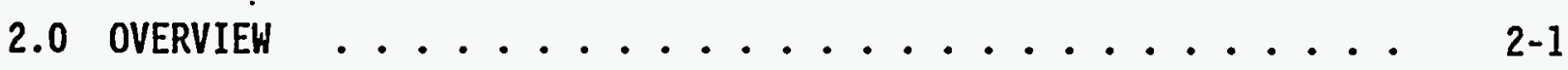

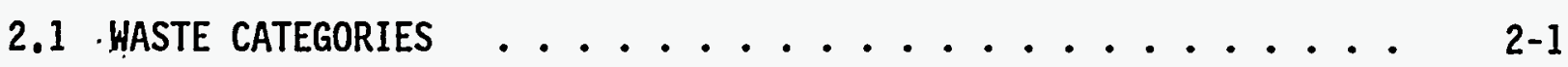

2.2 PHYSICAL WASTE CHARACTERISTICS .......... $2-2$

2.3 HAZARDOUS WASTE CHARACTERISTICS ........... 2-... 2-...

2.4 FORECASTED CHARACTERISTICS AND WASTE CATEGORIES ..... 2-3

2.4.1 Volumes by Physical Waste Form ........ 2-. 2-

2.4.2 LLMH and TRU_TRUM Haste Volumes by Hazardous Waste Constituents ............ 2-. 2-5

2.4.3 LLMW and TRU_TRUM Waste VoTumes by Waste Category ................ 2-5

2.4.4 Major LLMH and TRU_TRUM Waste Generators . . . 2-6

2.5 WASTE CHARACTERISTIC ASSUMPTIONS ......... 2-. 2-.

3.0 LOH-LEVEL MIXED WASTE ..................... 3-1

3.1 LOW-LEVEL MIXED WASTE VOLUME ............. 3-. . . . .

3.2 LOH-LEVEL MIXED WASTE CATEGORIES BY PHYSICAL WASTE FORM - . 3-2

3.2.1 Contact-Handled Low-Level Mixed Waste ...... 3-3

3.2.2 Remote-Handled Low-Level Mixed Waste ..... 3-6

3.3 LOH-LEVEL MIXED WASTE CATEGORIES BY HAZARDOUS WASTE

CONSTITUENTS ................... 3-7

3.3.1 Contact-Handled Low-Level Mixed Haste ...... 3-7

3.3.2 Remote-Handled Low-Level Mixed Waste ...... 3-9

4.0 TRANSURANIC ANid TRANSURANIC MIXED WASTE ......... 4- . . 
4.1 TRU_TRUM WASTE VOLUME .................. 4-1

4.2 TRU_TRUM WASTE CATEGORIES BY PHYSICAL WASTE FORM . . . . 4-2

4.2.1 Contact-Handled Transuranic and Transuranic Mixed Waste ............ 4-. 4

4.2.2 Remote-Handled Transuranic and Transuranic Mixed Waste ............... 4-4

4.3 TRU TRUM WASTE CATEGORIES BY HAZARDOUS WASTE CONSTITUENTS ................... 4-6

4.3.1 Contact-Handled Transuranic Mixed Waste . . . . . 4-6

4.3.2 Remote-Handled Transuranic Mixed Waste ..... 4-7

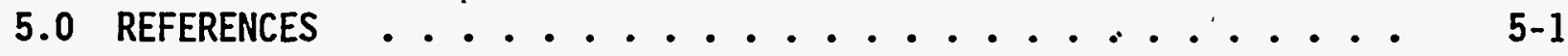

APPENDIX A - LOH-LEVEL HASTE ............... A-1

APPENDIX B - HASTE FORM AND HAZARDOUS CONSTITUENTS FORECAST REQUEST FORM ............... B- B

APPENDIX C - ANNUAL WASTE CATEGORY VOLUMES BY PHYSICAL WASTE FORM AND HAZARDOUS WASTE CONSTITUENT ......... C-1

APPENDIX D - ANNUAL VOLUMES OF PHYSICAL WASTE FORMS AND HAZARDOUS CONSTITUENTS BY WASTE GENERATOR ......... D-1

APPENDIX E - WASTE GENERATOR ABBREVIATIONS ......... E-1 


\section{FIGURES}

2-1 LLMH and TRU_TRUM Waste Volumes by Physical Haste Form . . . . 2-4.

2-2 Annual Baseline Volume of LLMH and TRU_TRUM Waste by

Physical Waste Form ................ . . 2-5

2-3 LLMH and TRUM Waste Volumes by Hazardous Haste Constituent . . 2-6

2-4 LLMW and TRU_TRUM Haste Volumes by Waste Category . . . . . 2-7

2-5 LLMW and TRU TRUM Haste Generators . . . . . . . . . . . 2-7

3-1 LLMW Volumes by Waste Category . . . . . . . . . . . 3-1

3-2 Annual Baseline Volume of LLMW by Waste Category . . . . . . 3-2

3-3 Major LLMW Generators ................... 3-2

3-4 CH_LLMW Volumes by Physical Waste Form . . . . . . . . . 3-3

3-5 Annual CH_LLMH Baseline Volume by Physical Waste Form . . . . 3-4

3-6 RH_LLMH Volumes by Physical Haste Form . . . . . . . . . 3-6

3-7 Annual RH_LLMW Baseline Volume by Physical Waste Form . . . . 3-7

3-8 CH_LLMW Volumes by Hazardous Waste Constituent . . . . . . 3-8

3-9 Annual CH_LLMW Baseline Volumes by Hazardous Haste Category . . 3-9

3-10 RH_LLMW Volumes by Hazardous Waste Category ........ . . 3-11

3-11 RH_LLMW Baseline Volumes by Hazardous Haste Category . . . . 3-12

4-1 TRU_TRUM Waste Volumes by Waste Category . . . . . . . . 4-1

4-2 Annual Baseline Volumes of TRU_TRUM Waste by Waste Category . . 4-2

4-3 Major TRU_TRUM Waste Generators ............... 4-3

4-4 CH_TRU_TRUM Volumes by Physical Waste Form .......... 4-3

4-5 Annual CH_TRU_TRUM Baseline Volumes by Physical Waste Form . . 4-4

4-6 RH_TRU_TRUM BaseTine Votumes by Physical Waste Form . . . . . 4-5

4-7 Annual RH_TRU_TRUM Baseline Volume by Physical Waste Form . . . 4-6 
4-8 CH_TRUM Waste Volumes by Hazardous Haste Category . . . . . . . 4-8

4-9 CH_TRUM Baseline Waste Volumes by Hazardous Waste Category - . 4-8

4-10 RH_TRU< Waste Volumes by Hazardous Waste Category . . . . . . 4-9

4-11 Annual RH_TRUM Baseline Waste Volumes by Hazardous

Waste Catégory ................... . 4-10

A-1 Total LLW Volume by Waste Category . . . . . . . . . . A-2

A-2 Annual LLW Baseline Volume by Waste Category . . . . . . . A-2

A-3 Major LLW Generators . . . . . . . . . . . . A-3

A-4 CH LLW Volumes by Physical Waste Form . . . . . . . . . . A-4

A-5 CH_LLH Volumes by Physical Waste Form . . . . . . . . . . A-4

A-6 RH_LLW Volumes by Physical Waste Form . . . . . . . . . . A-7

A-7 RH_LLH Annual Baseline Volumes by Physical Waste Form . . . . A-7 


\section{TABLES}

2-1 Past Practịce Remediation Characteristic Assumptions . . . 2-9

3-1 CH_LLMW Generators by Physical Waste Form . . . . . . . . 3-4

3-2 RH_LLMW Generators by Physical Haste Form . . . . . . . . 3-8

3-3 CH_LLMW Generators by Hazardous Waste Constituents ..... 3-10

3-4 . RH_LLMW Generators by Hazardous Waste Constituents . . . . 3-13

4-1 CH_TRU_TRUM Waste Generators by Physicat Haste Form . . . . 4 4-5

4-2 RH_TRU_TRUM Waste Generators by Physical Waste Form ..... 4-7

4-3 CH_TRUM Waste Generators by Hazardous Waste Constituents . . 4-9

4-4 RH_TRUM Waste Generators by Hazardous Waste Constituents . . . 4-11

A-1 CH_LLW Generators by Physical Waste Form ......... A-5

A-2 RH_LLW Generators by Physical Waste Form ......... A-8

E-1 Waste Generator List by Hanford Program Area ....... E-1 
WHC-EP-0888

Intentional1y Left Blank 


\section{ACRONYMS AND ABBREVIATIONS}

$\begin{array}{ll}\text { CH } & \text { contact-handled (waste) } \\ \text { ER } & \text { environmental restoration } \\ \text { ERD } & \text { Environmental Restoration Division } \\ \text { LLMH } & \text { low-level mixed waste } \\ \text { LLW } & \text { low-level waste } \\ \text { PCB } & \text { polychlorinated biphenyl } \\ \text { PFP } & \text { Plutonium Finishing Plant } \\ \text { PNL } & \text { Pacific Northwest Laboratory } \\ \text { PUREX } & \text { Plutonium uranium extraction } \\ \text { RH } & \text { remote-handled (waste) } \\ \text { SHOC } & \text { Solid Haste Operations Complex } \\ \text { TRU } & \text { transuranic (waste) } \\ \text { TRUM } & \text { transuranic mixed (waste) } \\ \text { TSD } & \text { treatment, storage, and disposal } \\ \text { WHC } & \text { Hestinghouse Hanford Company } \\ \text { WIDS } & \text { Haste Information Database System }\end{array}$


WHC-EP-0888

Intentionally Left Blank 


\subsection{INTRODUCTION}

The Hanford Site has been designated by the U.S. Department of Energy (DOE) to store, treat, and dispose of solid waste received from both onsite and offsite generators. This waste is currently or planned to be generated from ongoing operations, maintenance and deactivation activities, decontamination and decommissioning (D\&D) of facilities, and environmental restoration (ER) activities.

For the past five years, each onsite and offsite generator has provided an annual forecast of the waste volume that it plans to ship to Hanford's Solid Waste Operations Complex (SWOC) over the next 30 years. Each generator's forecast summarizes the waste that will be sent to the Hanford Site including the waste volume, container data, physical waste forms, hazardous constituents, and radionuclide data if available. After analyzing the 1995 forecasts, it was determined that additional waste was expected from onsite D\&D and remedial action (RA) activities. Special studies and interviews were conducted to obtain these volumes. These special study volumes and the 1994 30-year forecast waste volumes are outlined in the 1995 Solid Waste 30-Year Volume Summary (VaTero et a]. 1995). This document describes the waste characteristics (physical waste forms and hazardous constituents) for the solid waste volumes reported in that document.

This document, prepared by Pacific Northwest Laboratory (PNL) under the direction of Hestinghouse Hanford Company (WHC), describes the characteristics of the waste to be shipped to Hanford's SWOC. The physical waste forms and hazardous constituents are described for the low-level mixed waste (LLMH) and the transuranic_transuranic mixed waste (TRU_TRUM). Low-7evel waste (LLH) characteristics are provided in Appendix $A$ of this report.

An overall LLMH and TRU_TRUM waste baseline volume of $235,560 \mathrm{~m}^{3}$ is expected to be managed at the SHOC. Based on ranges provided by the waste generators, this baseline volume could fluctuate between a minimum of $203,680 \mathrm{~m}^{3}$ and a maximum of $260,500 \mathrm{~m}^{3}$. The largest portion of the base 7 ine waste will be CH_LLMW, which is expected to account for $77 \%\left(182,160 \mathrm{~m}^{3}\right)$ of the total baseline volume. RH LLMW accounts for another $3 \%\left(6,670 \mathrm{~m}^{3}\right)$, CH_TRU_TRUM for $15 \%\left(35,100 \mathrm{~m}^{3}\right)^{-}$, and RH_TRU_TRUM for $5 \%\left(11,630 \mathrm{~m}^{3}\right)$.

Section 2.0 provides an overview of the waste volumes described by waste categories, which are contact-handled (CH) LLMH, remote-handled (RH) LLMH, CH_TRU_TRUM, and RH_TRU_TRUM. The waste characteristics are also defined in Section 2.0. Sections $\overline{3} .0$ and 4.0 describe the physical waste forms and hazardous constituents of the LLMW and TRU_TRUM waste, respectively.

Low-level waste (LLW) characteristics are provided in Appendix A. Appendix B contains a table from the 1994 forecast that was sent to each generator to complete. This table provides the possible physical waste forms and hazardous constituents of the waste contributed by the generators. Appendix C contains a database report summarizing the 1994 Forecast annual 
waste category volumes by physical waste form and hazardous waste constituent. Appendix $D$ contains a database report of the volumes of the physical waste forms and hazardous constituents for each waste generator. A list of waste generator abbreviations is provided in Appendix $E$. 


\subsection{OVERVIEW}

This section provides a high-level description of the LLMW and TRU TRUM solid waste volumes by waste category that are expected to be generated by onsite and offsite waste generators in the next 30 years. Brief descriptions of the waste categories, physical waste characteristics, and hazardous constituents are provided as well. The major waste generators of the LLMW and TRU_TRUM. waste volumes are identified and discussed. The purposes of the information presented in this section are to provide a general description of the volumes that will be managed within Hanford's solid waste management facilities and to identify the terms that will be used throughout this report.

\subsection{WASTE CATEGORIES}

The waste volumes and waste characteristics are described in this document by waste category. The waste is divided into four broad categories based on concentrations of radionuclides and surface dose rates of the waste package. For the purposes of this document, the contact-handled $(\mathrm{CH})$ and remote-handled (RH) categories will be combined for each waste classification to form the basis of each section. The categories are briefly described below:

- Contact-handled low-level mixed waste (CH_LLMW): This waste has a dose rate less than or equal to $200 \mathrm{mrem} / \overline{\mathrm{h}}$. The waste contains radioactivity that is not classified as transuranic waste or spent nuclear fuel; however, the waste does contain dangerous constituents as identified by Washington Administrative Code (WAC) 173-303 (Ecology 1993). The concentration of transuranic radionuclides is less than or equal to $100 \mathrm{nCi} / \mathrm{g}$ of the waste matrix.

- Remote-handled low-level mixed waste (RH_LLMH): This waste has a dose rate greater than $200 \mathrm{mrem} / \mathrm{h}$. The waste contains radioactivity that is not classified as transuranic waste or spent nuclear fue?; however, the waste does contain dangerous constituents as identified by WAC 173-303. The concentration of transuranic radionuclides is less than or equal to $100 \mathrm{nCi} / \mathrm{g}$ of the waste matrix.

- Contact-handled transuranic/transuranic mixed waste (CH_TRU/TRUM) : This waste has a dose rate less than or equal to $200 \mathrm{mrem} / \mathrm{h}$ at contact with the waste. At the time of assay, this waste contains more than $100 \mathrm{nCi} / \mathrm{g}$ of alpha-emitting isotopes with atomic numbers greater than 92 and half-lives greater than 20 years. TRUM waste is TRU waste that is also classified as dangerous waste as defined in WAC 173-303.

- Remote-handled transuranic/transuranic mixed waste (RH_TRU/TRUM): This waste has a dose rate greater than $200 \mathrm{mrem} / \mathrm{h}$ at contact with 
the waste. At the time of assay, this waste contains more than $100 \mathrm{nCi} / \mathrm{g}$ of alpha-emitting isotopes with atomic numbers greater than 92 and half-lives greater than 20 years. TRUM waste is TRU waste that is also classified as dangerous waste as defined in WAC 173-303.

\subsection{PHYSICAL WASTE CHARACTERISTICS}

In the 1994 Solid Haste Forecasts, waste volumes are further defined by the specific physical characteristics that will be generated. The main physical characteristics of most of the waste volume can be delineated into these eight main categories, which are based on the DOE final Treatability Group Guidance document:-

- Inorganics - material that has an inorganic matrix or content.

- Organics - chemical compounds based on carbon, hydrogen, and oxygen, usually derived from living matter.

- Debris - wastes that meet the U.S. Environmental Protection Agency (EPA) criteria for "debris." Debris materials are divided into four groups as either metal (inorganic), inorganic non-metal, combustible (organic), or mixtures of materials (heterogeneous). If the waste is dominated by one type of material it should be classed as that material; otherwise, it is classified as heterogenous.

- Labpacks/Containerized Liquids - various quantities of compatible waste within the same DOT hazard class, packaged in vessels such as cans or bottles.

- Soils - soil contaminated with hazardous and/or radioactive materials.

- Shielding - the three types of shielding include steel, lead, and concrete. It is assumed that, if shielding is required, then the shielding material is handled as solid waste.

- Void Space - space within a container that is not occupied by waste.

- Unknown - in several cases, physical waste form information is not known; therefore, "unknown" has been used to denote these areas of uncertainty. 


\subsection{HAZARDOUS WASTE CHARACTERISTICS}

In the 1994 Solid Haste Forecasts, hazardous characteristics are identified for radioactive and hazardous mixed waste and hazardous waste. These hazardous characteristics can be divided into eight main categories that may be individual or mixed. Several hazardous constituents might be present in a particular waste form and require definition by waste volume percentage as mixed hazardous constituents (e.g., if a physical waste form in a 55-gallon drum contains ignitables and corrosives, then the package is $100 \%$ ignitables and corrosives). A brief definition of the nine main hazardous characteristics that were used for this report follows:

- Ignitabies (D001) - term used by Ecology (WAC 173-303-090) for a waste that can cause a fire through friction, absorption of moisture, or spontaneous chemical reaction.

- Corrosives (D002) - any liquid or solid that causes destruction of human skin tissue or that has a severe corrosion rate on steel.

- Reactive Metals (D003) - typically sodium metal or alkali metal alloys but can also be particulate fines of aluminum, uranium, zirconium, or other pyrophoric materials, and may be mixed with stabilizing materials.

- Metals Without Mercury (D004-D011) - toxic metals that are not contaminated with mercury.

- Metals with Mercury (D009, P065, P092, U15i) - toxic metals that are specifically contaminated with mercury.

- Toxic Organics (D018-D043) - toxic organics.

- State Regulated - waste that is defined as hazardous only under Washington State regulations.

- PCBs - polychlorinated biphenyl-contaminated materials (designated in 40 CFR 761 or HAC 173-303-071). where PCB concentration is further divided into two categories: less than 50 parts per million (ppm) and greater than $50 \mathrm{ppm}$.

- Unknown - in some cases, hazardous constituent data are not known; "Unknown" has been used to denote these areas of uncertainty.

\subsection{FORECASTED CHARACTERISTICS AND WASTE CATEGORIES}

This section provides a brief summary of the waste volumes that will be generated by physical waste forms, hazardous constituents, and waste category. This information provides an overview of the quantities of waste that will be 
sent to the Hanford Site for future treatment, storage, and disposal and provides a profile of each of the waste generators planning on shipping large quantities of waste to the SWOC.

\subsubsection{Volumes by Physical Waste Form}

The baseline, minimum, and maximum volumes of LLMW and TRU_TRUM waste are represented by physical waste form in Figure 2-1. As shown in the figure, the expected baseline volume is $235,560 \mathrm{~m}^{3}$, the minimum is $203,680 \mathrm{~m}^{3}$, and the maximum is $260,500 \mathrm{~m}^{3}$. The maximum and minimum ranges were provided by the individual waste generators; however, the baseline volume is the best estimate for future generated waste. The main physical waste form is steel shielding from Single-She11 Tank Long-Length Equipment, which comprises $30 \%$ of the baseline volume:- Metal debris wi.7 comprise $27 \%$ of the baseline, $23 \%$ will be contaminated soil, and the remaining $20 \%$ will be made up of other physical waste forms.

Figure 2-2 displays the LLMW and TRU TRUM annual baseline waste volumes by physical waste form. The figure shows that contaminated soils is the major waste form from 1997 to 2010; after that, steel shielding and then metal debris are the predominant forms. The peak from 2013 through 2017 represents the assumed schedule for D\&D of the Plutonium Finishing Plant (PFP) and retrieval of long-length equipment. The dates for shipment of D\&D waste from

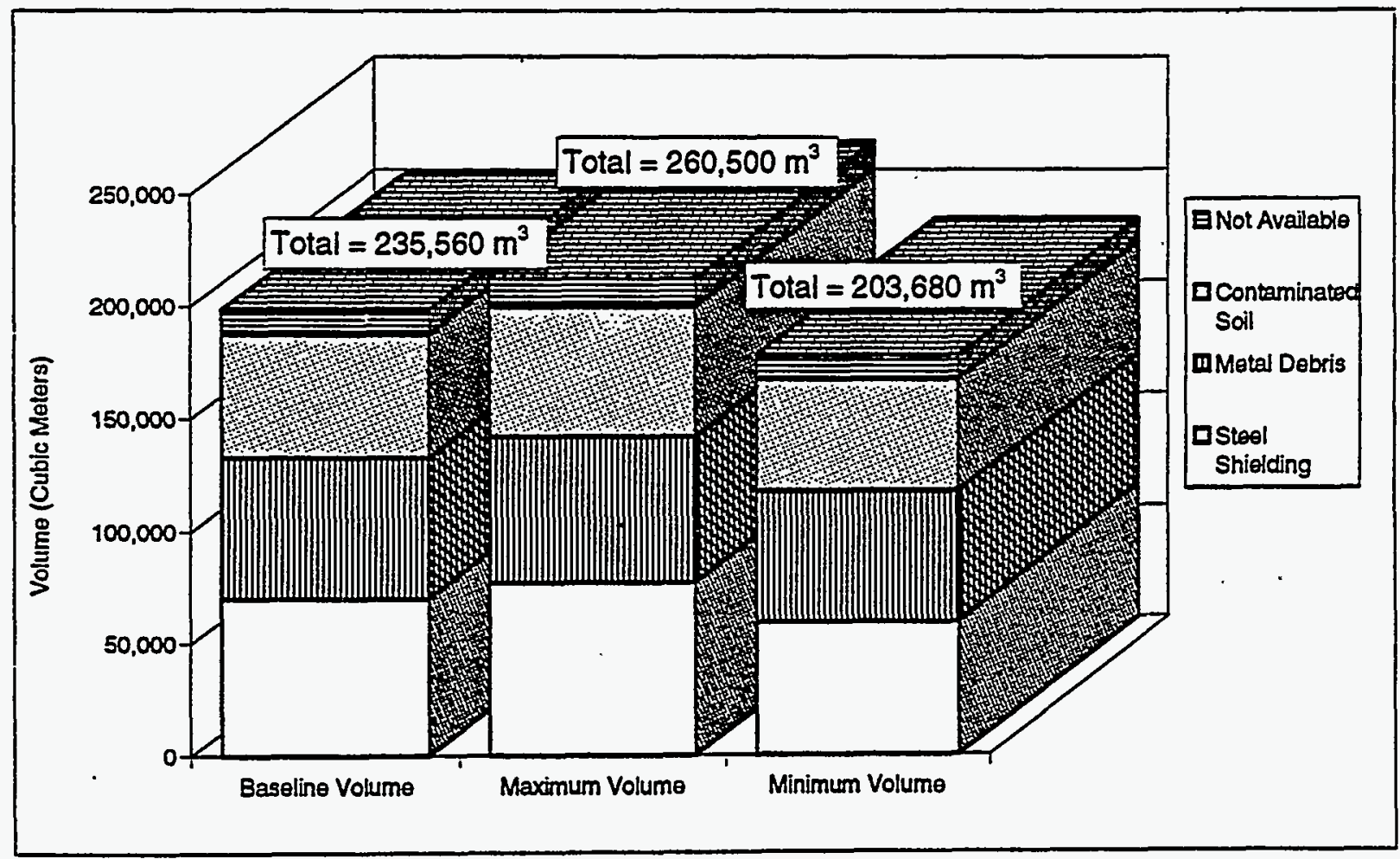

Figure 2-1. LLMW and TRU_TRUM Haste Volumes by Physical Haste Form 


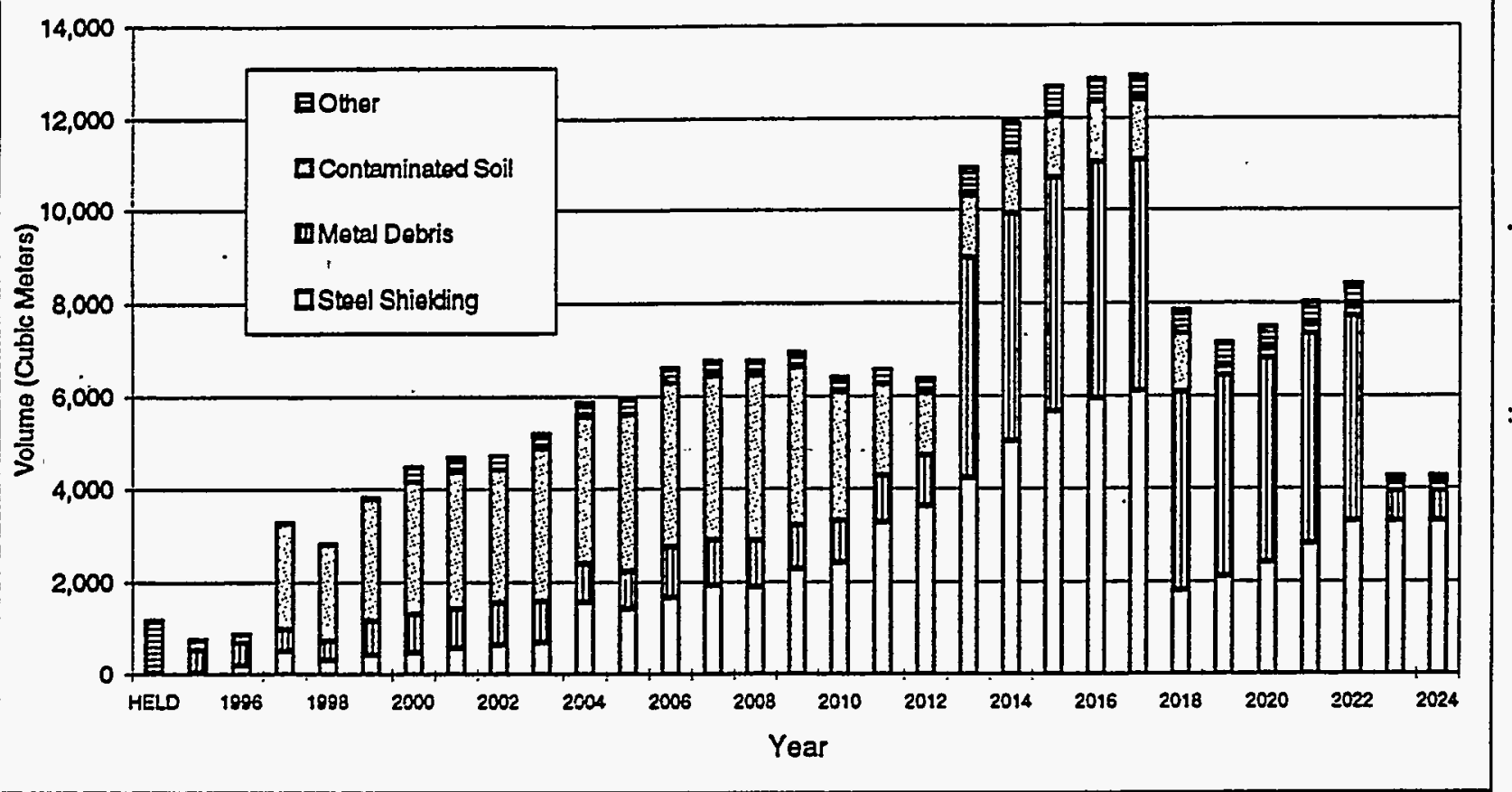

Figure 2-2. Annual Baseline Volume of LLMW and TRU_TRUM Waste by Physical Waste Form

the PFP are not now known; however, an assumption was made that until further planning has been completed, the waste will be shipped from 2013 through 2022. Detailed information on the assumptions made for D\&D waste generators is contained in Templeton et a1. (1995).

\subsubsection{LLMW and TRU_TRUM Waste Volumes by Hazardous Waste Constituents}

Figure 2-3 displays the baseline, maximum, and minimum volumes by hazardous constituents. OnTy LLMW and TRUM waste is represented since only these waste categories contain hazardous constituents. Organics mixed with metais without mercury comprise $43 \%$ of the LLMW and TRUM waste volume. Metals without mercury comprise $17 \%$, corrosives are expected to be $13 \%$, and the remaining $27 \%$ is other hazardous constituents.

\section{2:4.3 LLMW and TRU_TRUM Waste Volumes by Waste Category}

The baseline, maximum, and minimum volumes by waste category are displayed in Figure 2-4. The majority of the waste over the next 30 years is expected to be CH_LLMW, which comprises $77 \%$ of the total baseline volume. The main generator of the CH_LLMW is Single-Shell Tank Long-Length Equipment, which is described in Section 2.4.4. CH_TRU_TRUM will comprise 15\%, while RH_LLMW and RH_TRU_TRUM will make up the remaining $8 \%$ of the baseline volume. 


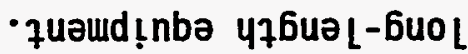

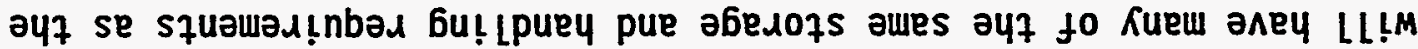

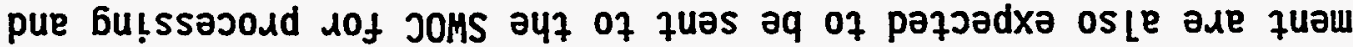

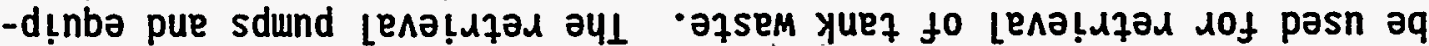

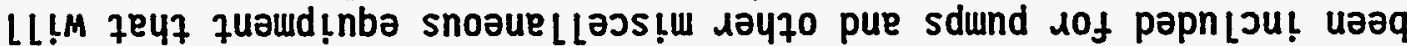

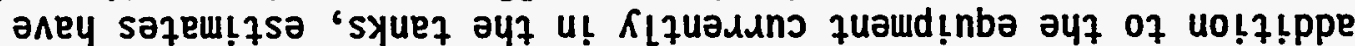

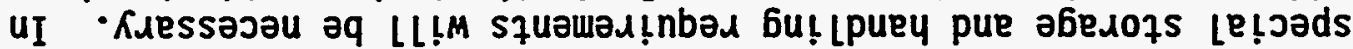
'sә7sem әsә47 to Uo!feanf!fuos Lensnun ayf to asnejag $\cdot$ (7f 09)

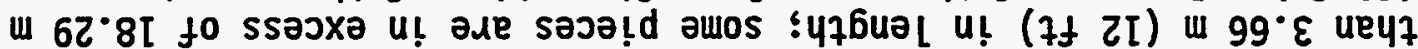

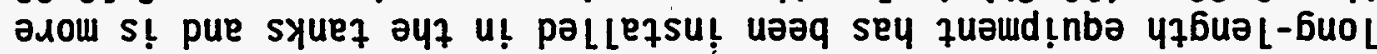

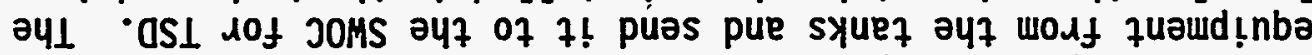

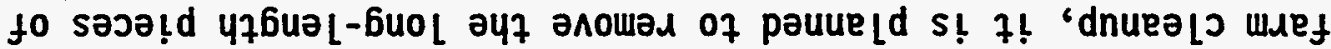

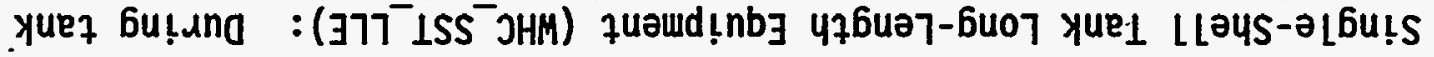

$\cdot(966 \mathrm{I})$

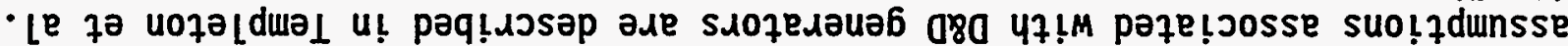

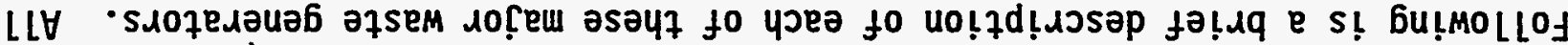

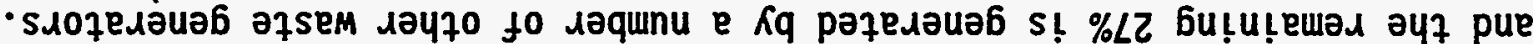

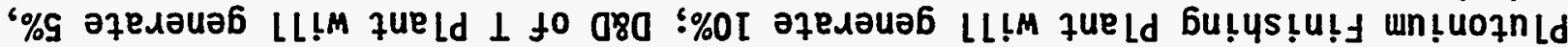

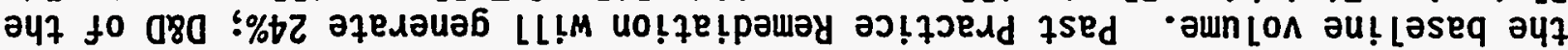

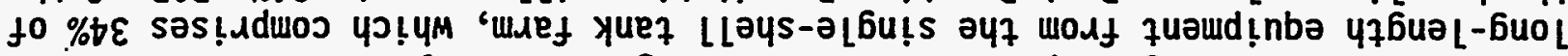

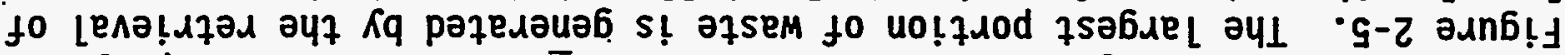

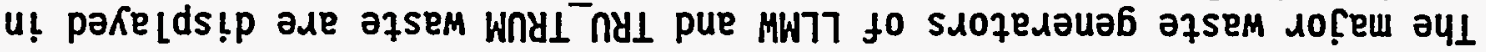

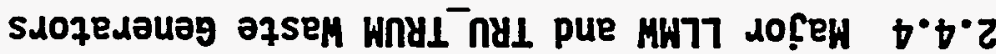

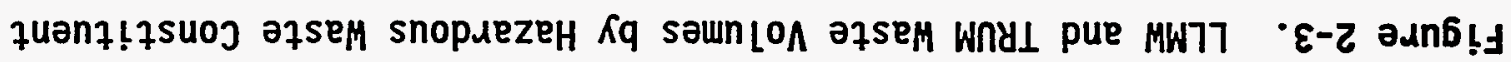

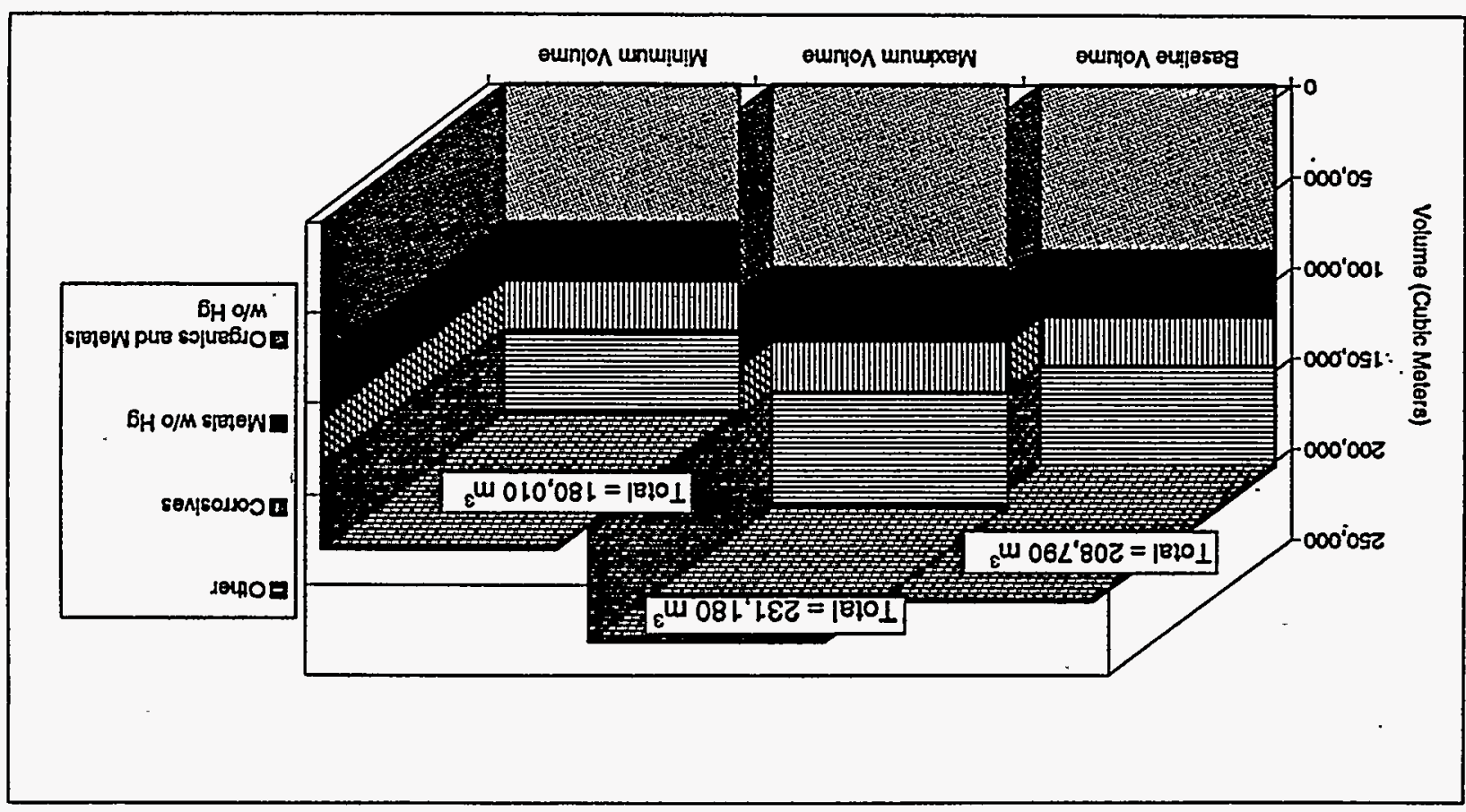




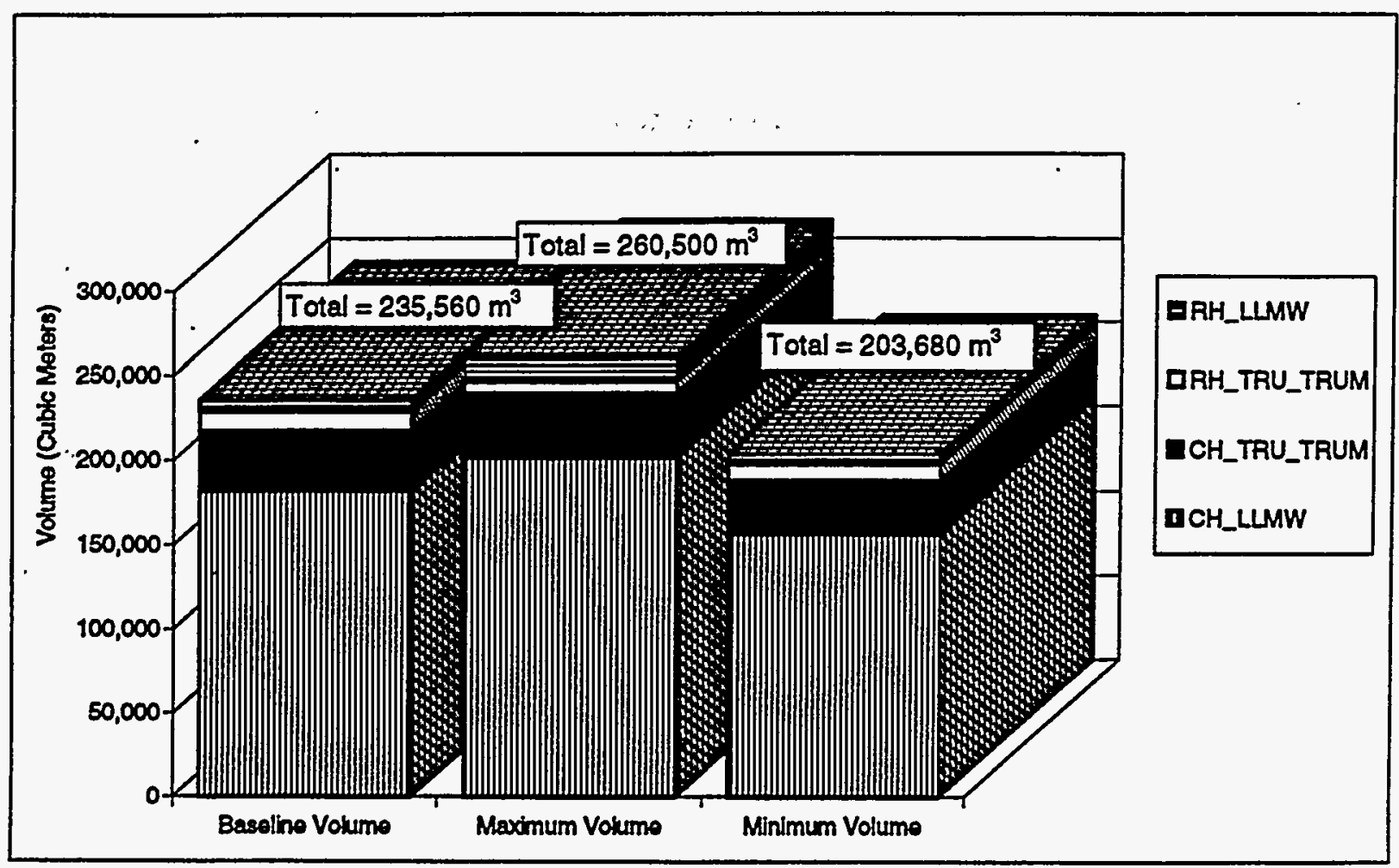

Figure 2-4: LLMH and TRU_TRUM Waste. Volumes by Waste Category

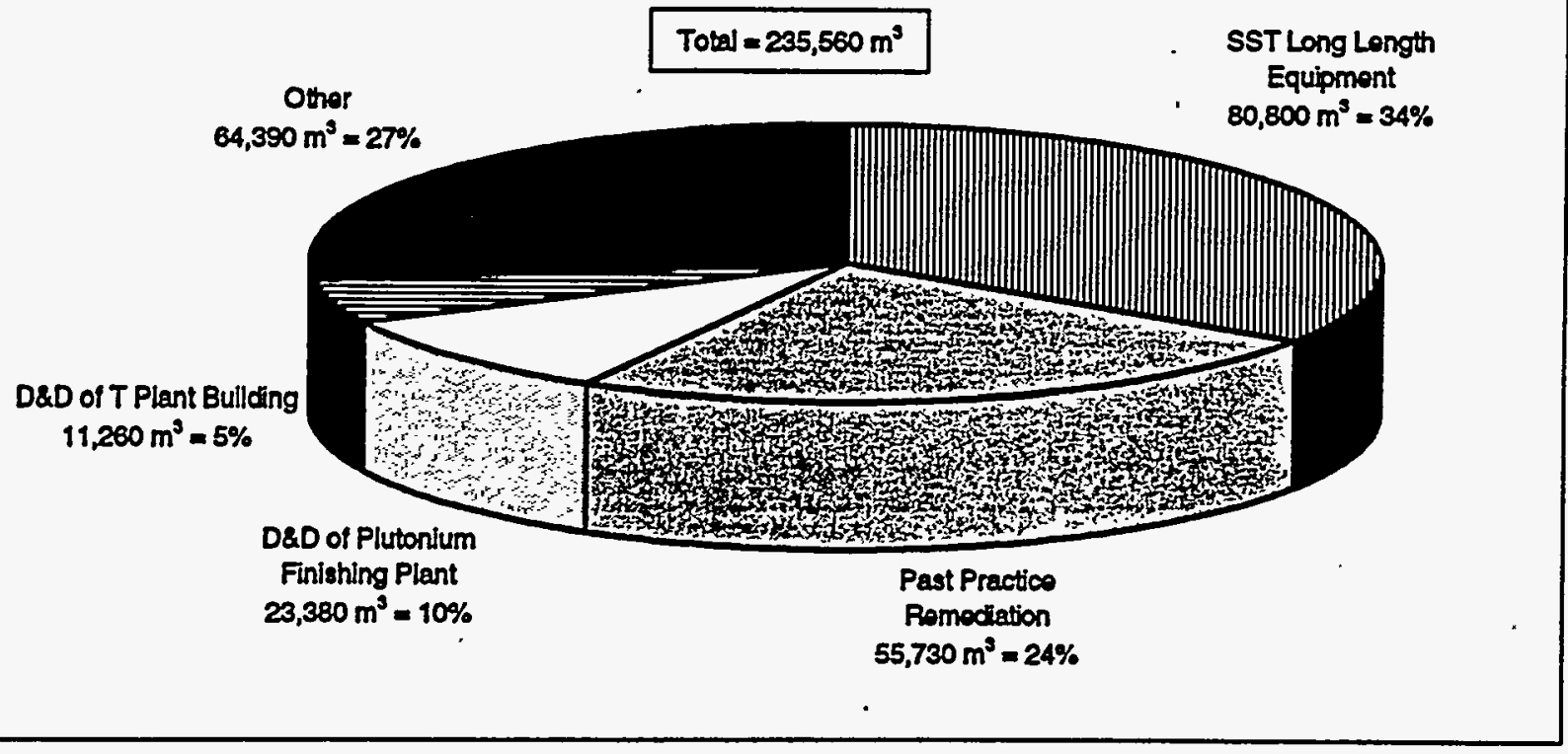

Figure 2-5. LLMW and TRU_TRUM Waste Generators 
This generator forecasted all of its waste to be CH LLMH III. There is a high possibility, however, that much or all of the waste will be remote-handled. In addition, some of this waste may also be TRUM, depending on the degree of decontamination at the time of removal from the waste tank. Both of these issues could significantly affect the TSD options being considered for the long-length contaminated equipment. In addition, there is a possibility that the long-length contaminated equipment might not be sent to the SWOC for processing; however, this scenario was not an option when the forecast data were submitted. This new scenario would dramatically reduce the expected volume of waste at the SHOC.

- Past Practice Remediation Projects (PAST_PRAC_REM): It is assumed that the SWD Program will" be responsible for the TRU TRUM waste and a portion of the LLMW from past practice sites, because they will have treatment technologies for this waste. The Environmental Restoration Division (ERD) is responsible for other remediation wastes. The wastes included for this generator are $10 \%$ of the tota1 LLMW generated and a11 expected TRU_TRUM waste and greaterthan-Class-3 waste; all other waste will be handled by ERD. Estimation of PUREX Equipment and Materials That are Candidates for Removal and Waste Processing During PUREX Plant Closure (LATA 1994) did not distinguish between LLW and LLMW for greater-than-Class-3 waste; for the purposes of this document, this waste was assumed to be LLMW.

- D\&D of Plutonium Finishing P1ant (THHC PFP): In operational mode, the PFP processed plutonium-based chemical solutions and converted them to metals and oxides. The D\&D volume estimate includes the canyon building $2345-Z$ and its associated buildings $241-Z, 242-Z$, and $236-Z$. Contaminated materials included in the volume estimate are glove boxes, pedestal storage, ventilation systems, pencil tanks, and ventilation fans.

- D\&D of T-Plant (THHC TPLANT): T P7ant, a canyon building, is used to decontaminate equipment and sort and package waste from other facilities. Solid waste from the D\&D of T Plant includes predominately contaminated equipment such as pumps, tanks, jumpers, and silver reactors.

\subsection{WASTE CHARACTERISTIC ASSUMPTIONS}

Forecast data were not provided for waste expected from past practice sites (Past Practice Remediation waste generator). Thus physical waste form characteristics were based on data from 100 and 300 Area Remedial Action sites (200 Area sites have not been included but would not change the physical characteristics split). Hazardous waste characteristics for soil are based on hazardous chemical data for 25 inactive 1 iquid waste discharge sites in the 100 Areas, as reported in the Waste Information Database System (WIDS). It is 
assumed that 300 Area liquid discharge sites would also be contaminated with metals without mercury and corrosives. Metal debris waste would come mainly from burial grounds and buried pipelines; these sites are assumed to be contaminated with metals without mercury as well. Table 2-1 displays the assumptions used for Past Practice Remediation.

Haste characteristics data were not available for several waste generators, or the available data were inconsistent. In cases where no information was available, the physical waste form or hazardous waste constituent was simply listed as unknown. The total volume of waste for which physical waste form data were unavailable is $8,623 \mathrm{~m}^{3}$. The volume of waste for which no hazardous waste constituent data were avajlable amounts to $23,279 \mathrm{~m}^{3}$. In all cases, waste without physical waste forms had no hazardous waste constituents; however, several generators provided physical waste form information but were unable to provide hazardous waste constituent data.

Table 2-1. Past Practice Remediation Characteristic Assumptions

\begin{tabular}{|c|c|c|c|}
\hline $\begin{array}{c}\text { Waste } \\
\text { Generator }\end{array}$ & Waste Class & Physical Waste Form Assumption & $\begin{array}{c}\text { Hazardous Waste } \\
\text { Constituent Assumption }\end{array}$ \\
\hline \multirow[t]{2}{*}{$\begin{array}{l}\text { Past Practice } \\
\text { Remediation }\end{array}$} & CH_LLMH & $\begin{array}{l}92 \% \text { contaminated soils } \\
7 \% \text { debris contaminated soils } \\
1 \% \text { metal debris }\end{array}$ & \multirow[t]{2}{*}{$\begin{array}{l}50 \% \text { corrosives } \\
50 \% \text { metals without mercury }\end{array}$} \\
\hline & CH_TRU_TRU: & $\begin{array}{l}9 \% \text { contaminated soils } \\
1 \% \text { debris contaminated soils } \\
45 \% \text { metal debris } \\
45 \% \text { inorganic non-metal debris }\end{array}$ & \\
\hline
\end{tabular}


WHC-EP-0888

Intentionally Left Blank 


\subsection{LOW-LEVEL MIXED WASTE}

This section provides LLMW volumes by waste category, physical waste form, and hazardous constituents. The major waste generators of LLMW are also 7isted. Valero et al. (1995) provides more detailed information on the individual waste generators and their missions. Templeton et al. (1995) also provides a comparison of the 1994 reported LLMW volumes with the LLMH volumes reported in .1995.

\section{1 - LOW-LEVEL MIXED WASTE VOLUME}

Approximately $188,830 \mathrm{~m}^{3}$ of LLMW wiTl be shipped to the SWOC in the next 30 years; however, the maximum amount expected is $209,080 \mathrm{~m}^{3}$, and the minimum is $161,600 \mathrm{~m}^{3}$. As depicted in Figure $3-1$, the majority (96\%) of the LLMW is CH_LLMH, and $4 \%$ of the baseline volume is RH_LLMH.

Figure 3-2 displays the annual baseline waste volumes for the next 30 years by waste category. The volume of LLMW gradually increases until it peaks in 2017 at approximately $10,000 \mathrm{~m}^{3}$. This gradual increase corresponds to the expected retrieval of the long-length equipment from the single-shel1 tank farms.

The major waste generators of LLMW are depicted in Figure 3-3. As shown, 43\% is generated by Single-She11 Tank Long-Length Equipment. Other primary contributors of LLMH include Past Practice Remediation (26\%), Buried Equipment (4\%), and other waste generators (27\%).

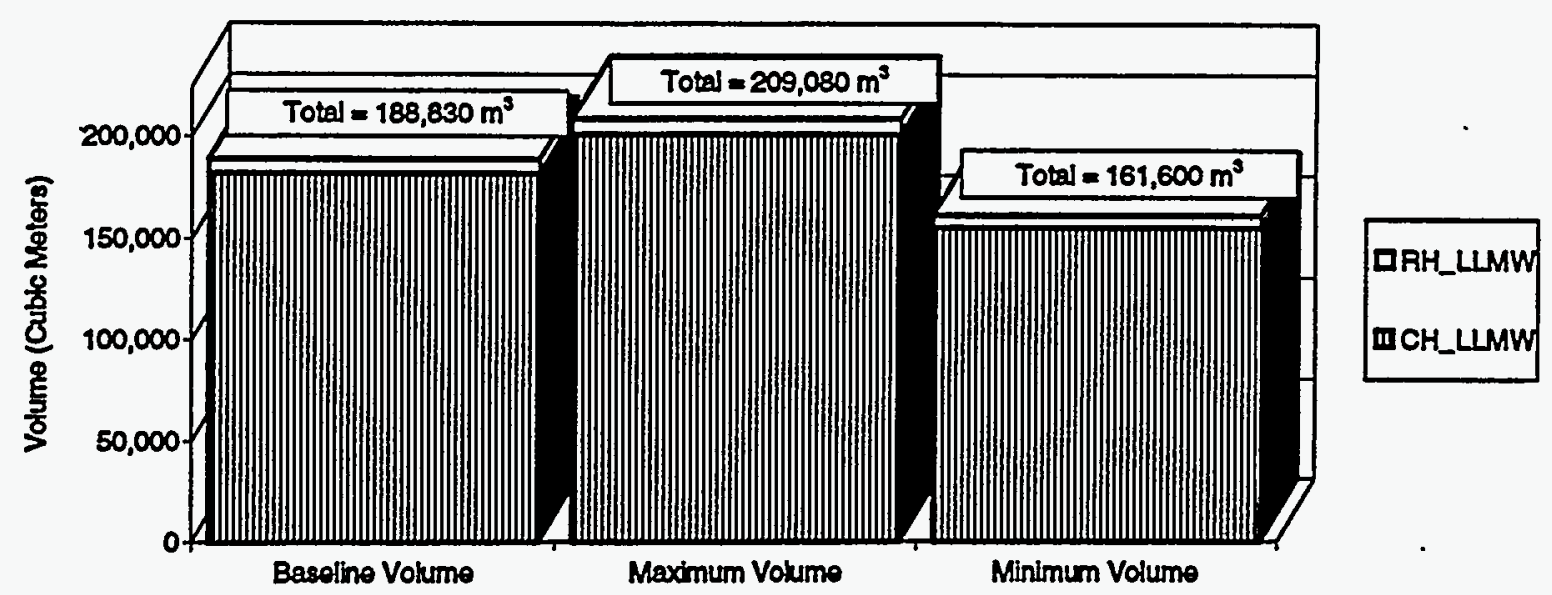

Figure 3-1. LLMW Volumes by Waste Category 


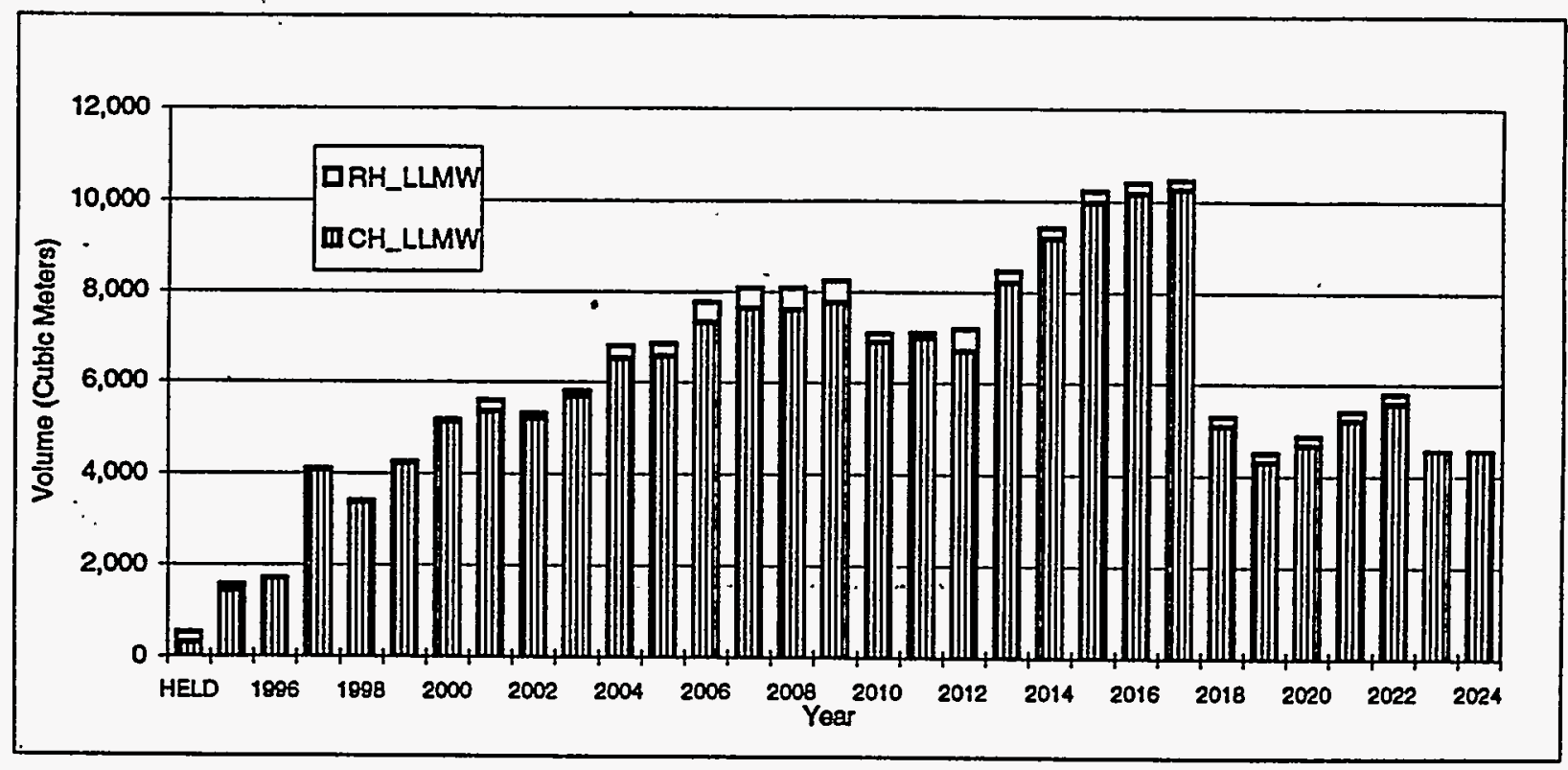

Figure 3-2. Annual Baseline Volume of LLMW by Waste Category

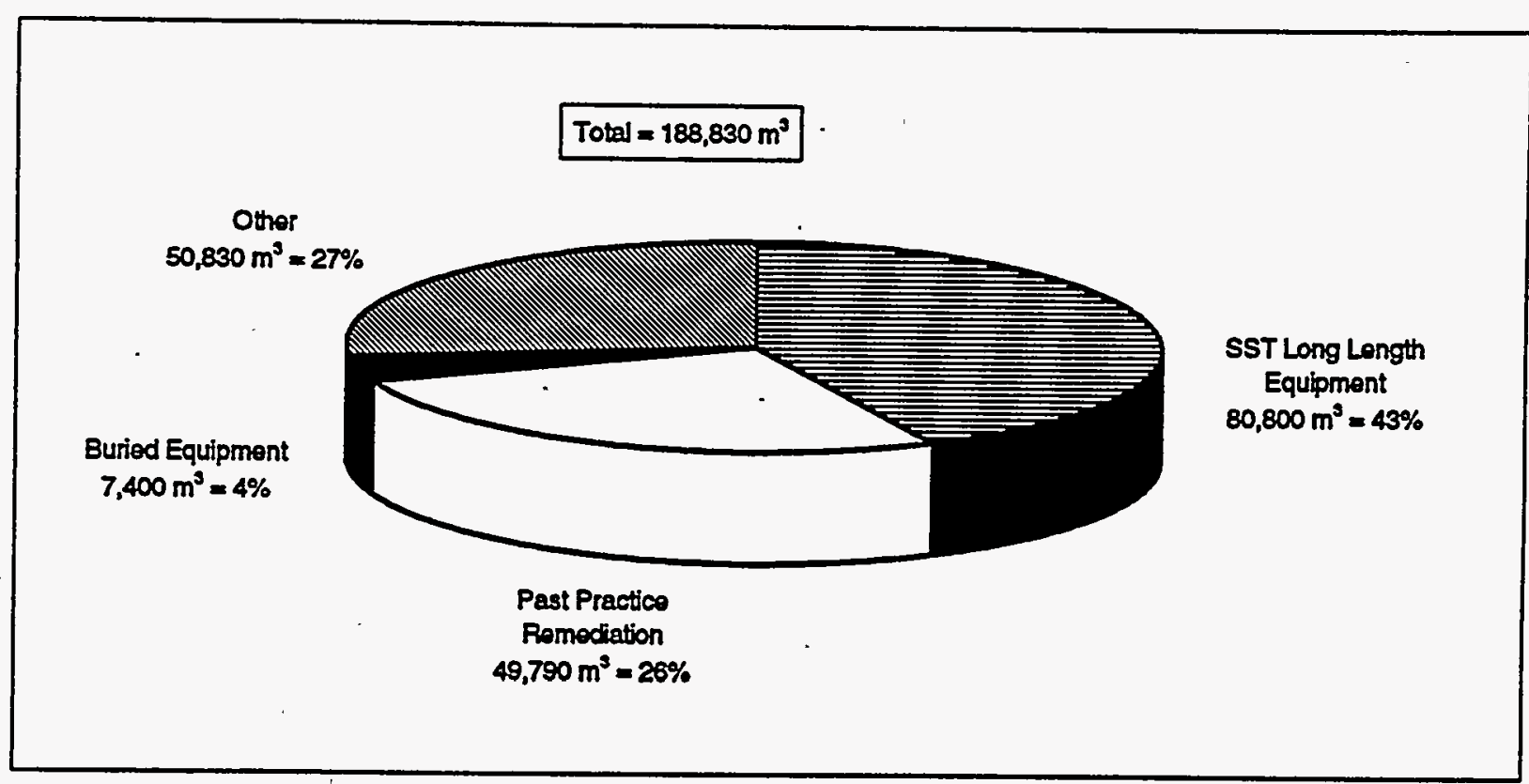

Figure 3-3. Major LLMW Generators

\subsection{LOW-LEVEL MIXED WASTE CATEgORIES BY PHYSICAL WASTE FORM}

CH_LLMW and RH_LLMW volumes by physical waste form are described in the following sections. The annual volumes by physical waste form are included, as are the waste generators. 


\subsubsection{Contact-Handled Low-Level Mixed Waste}

The total baseline volume of CH LLMW to be shipped to the SHOC in the next 30 years is approximately $182,1 \overline{60} \mathrm{~m}^{3}$, while the expected maximum and minimum volumes are $201,520 \mathrm{~m}^{3}$ and $155,720 \mathrm{~m}^{3}$, respectively. Figure $3-4$ provides the breakout of the physical waste forms for $\mathrm{CH}$ LLMH. As shown in the figure, $39 \%$ of the CH_LLMH is expected to be steel shiēiding. Contaminated soil will account for another 30\%, metal debris for 16\%, and other physical waste forms approximately $15 \%$.

Figure 3-5 displays the annual CH_LLMW baseline volumes by physical waste form. As shown in the figure, waste volumes gradually increase until 2017 due to the increase in steel shielding generated by Single-Shell Tank Long-Length Equipment. From 1997 through 2010, the volume of contaminated soil from Past Practice Remediation remains at approximately 2,500 $\mathrm{m}^{3}$ annually. In 2018, the annual generated volumes decrease to approximately $4,000 \mathrm{~m}^{3}$ due to the near-completion of long-length equipment retrieval and to the operations of Past Practice Remediation.

Table 3-1 provides the volumes forecasted by each waste generator arranged by primary physical waste forms. The only waste generator of the CH_LLMW steel shielding is Single-She11 Tank Long-Length Equipment. As shown in the table, Past Practice Remediation is the primary contributor of

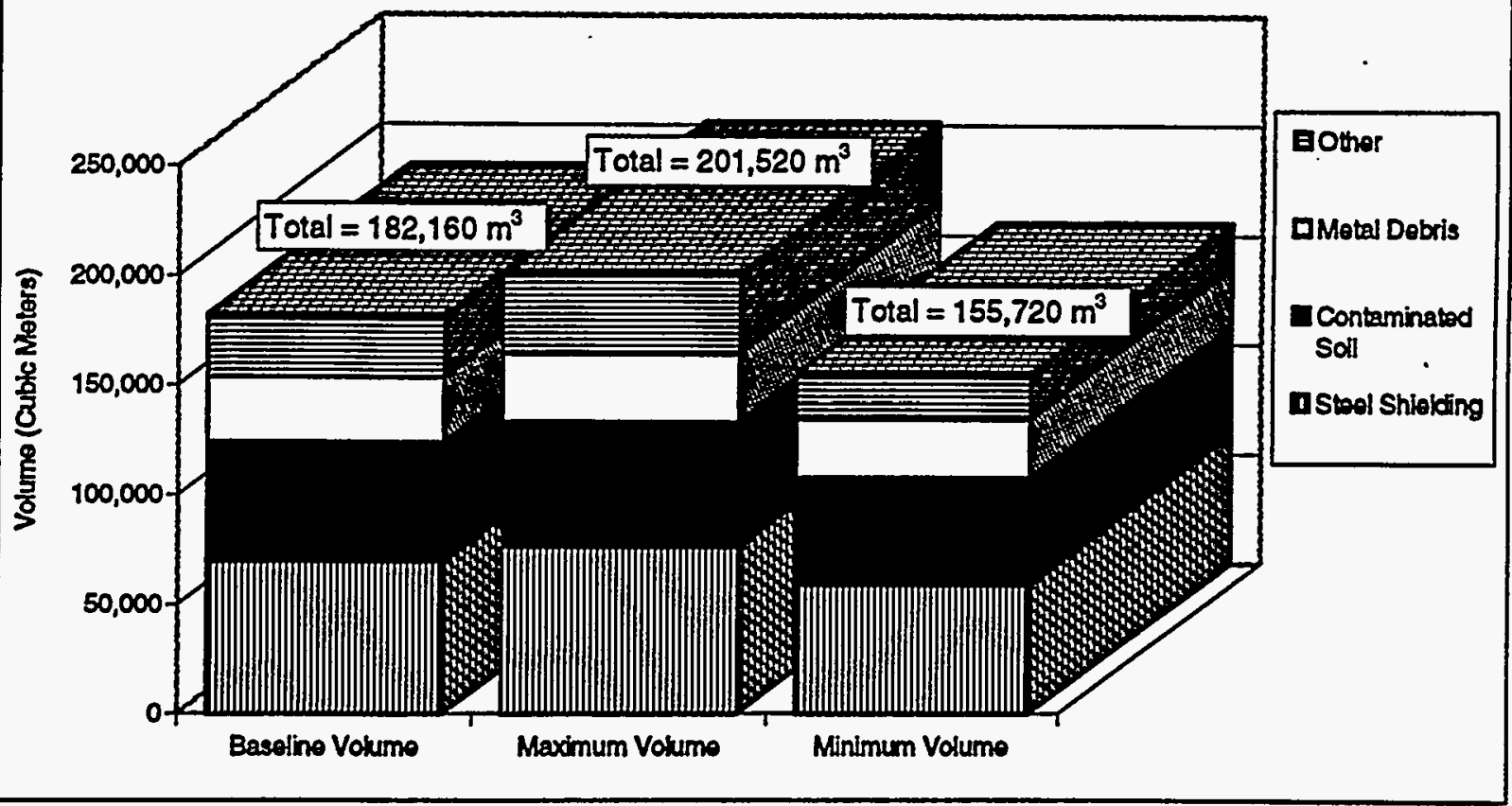

Figure 3-4. CH_LLMH VoTumes by Physical Waste Form 


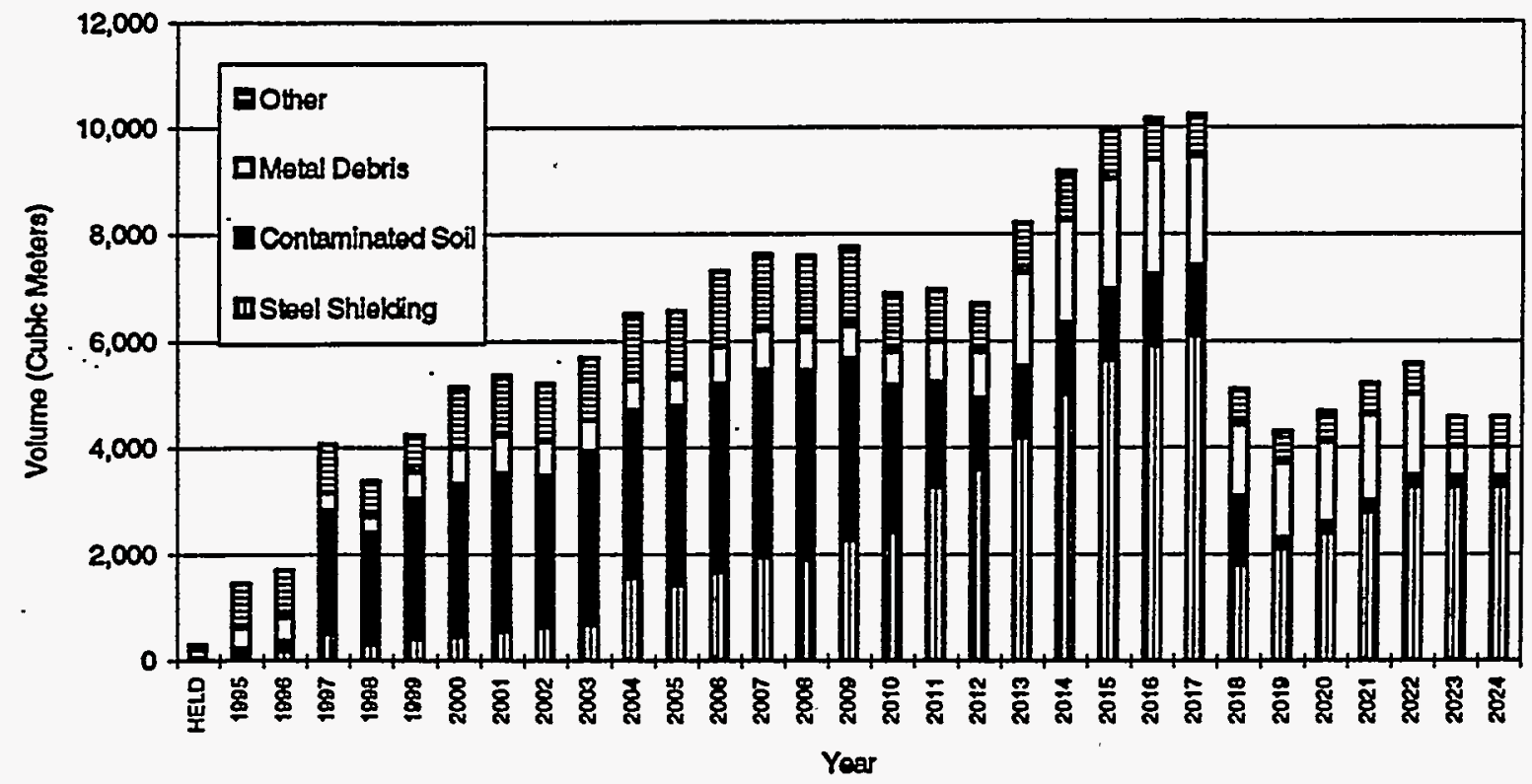

Figure 3-5. Annual CH_LLMW Baseline Volume by Physical Waste Form

Table 3-1. CH_LLMW Generators by Physical Waste Form ${ }^{(a, b)}$

\begin{tabular}{||l|r|r|r|r|r|}
\hline \multicolumn{1}{|c|}{ Waste Generator } & $\begin{array}{r}\text { Steel } \\
\text { Shielding }\end{array}$ & $\begin{array}{c}\text { Contaminated } \\
\text { Soil }\end{array}$ & $\begin{array}{c}\text { Metal } \\
\text { Debris }\end{array}$ & Other & Total \\
\hline SST Long-Length Equipment & 69,828 & 0 & 10,976 & 0 & 80,804 \\
\hline Past Practice Remediation & 0 & 45,749 & 498 & 3,546 & 49,793 \\
\hline Buried Equipment & 0 & 0 & 7,403 & 0 & 7,403 \\
\hline Portsmouth - Energy Systems & 0 & 0 & 0 & 6,448 & 6,448 \\
\hline Tank Farm Operations & 0 & 308 & 1,652 & 3,633 & 5,593 \\
\hline D\&D of T Plant & 0 & 0 & 5,438 & 0 & 5,438 \\
\hline Paducah - Energy Systems & 0 & 4,619 & 0 & 513 & 5,132 \\
\hline Tank Farm Restoration & 0 & 3,184 & 782 & 521 & 4,487 \\
\hline Surplus Facilities & 0 & 396 & 71 & 2,379 & 2,846 \\
\hline 200 Area Effluent Treatment Facility & 0 & 0 & 0 & 2,384 & 2,384 \\
\hline Plutonium Finishing Plant & 0 & 0 & 0 & 2,187 & 2,187 \\
\hline D\&D of 200 Area Effluent Treatment Facility & 0 & 0 & 1,569 & 30 & 1,599 \\
\hline Argonne National Laboratory - East & 0 & 9 & 0 & 1,153 & 1,162 \\
\hline SST Retrieval (149 tanks) & 0 & 97 & 119 & 864 & 1,081 \\
\hline 222-S Laboratory & 0 & 0 & 0 & 885 & 885 \\
\hline Pacific Northwest Laboratory & 0 & 0 & 0 & 737 & 737 \\
\hline High-Level Vitrification Project & 0 & 0 & 69 & 619 & 688 \\
\hline D\&D of 242-A Evaporator. & 0 & 0 & 326 & 0 & 326 \\
\hline D\&D of 242-S Evaporator & 0 & 0 & 326 & 0 & 326 \\
\hline Tank 106-C Sluicing & 0 & 0 & 29 & 265 & 294 \\
\hline
\end{tabular}


Table 3-1. (contd)

\begin{tabular}{|c|c|c|c|c|c|}
\hline Waste Generator & $\begin{array}{c}\text { Steel } \\
\text { Shielding }\end{array}$ & $\begin{array}{c}\text { Contaminated } \\
\text { Soil }\end{array}$ & $\begin{array}{c}\text { Hetal } \\
\text { Debris }\end{array}$ & Other & Total \\
\hline D\&D of B Plant & 이 & 0) & 277 & 0] & 277 \\
\hline D\&D of PUREX & 요 & 0 & 0 & 236 & 236 \\
\hline Laurence Berkeley Laboratory & 0 & 0 & 0 & 233 & 233 \\
\hline Portsmouth - Utility Services & 0 & 0 & 0) & 214 & 214 \\
\hline T Plant & 0 & 6 & 49 & 158 & 213 \\
\hline Bettis Atomic Power Laboratory & 이 & 5 & 0 & 190 & 195 \\
\hline Knol ls Atomic Power Laboratory & 0] & 42 & 15 & 69 & 126 \\
\hline 300 Area Treated Effluent Disposal Facility & 이 & 0 & 0) & 119 & 119 \\
\hline FERMI Mational Accelerator Laboratory & 요 & 0) & 0] & 78 & 78 \\
\hline PUREX & o & 2 & 0) & 65 & 67 \\
\hline D\&D of 242-T Evaporator & 0 & 0 & 64 & 0 & 64 \\
\hline 100 Area Transition & 요 & 0 & 10 & 471 & 57 \\
\hline Brookhaven National Laboratory & 0 & 0 & 6 & 50 & 55 \\
\hline Princetón Plasma Physics Laboratory & 0 & 0 & 이 & 53 & 53 \\
\hline LoH-Level Vitrification Project & 0 & 0 & 5 & 47) & 52 \\
\hline Battelle Columbus Laboratories & 0 & 0 & 요 & 50 & 50 \\
\hline B Plant & 요 & 0 & 0 & 50 & 50 \\
\hline K Basin Operations & 이 & 0 & 13 & 29 & 42 \\
\hline D\&D of 222-S Laboratory & 0 & D & 41 & 0 & 41 \\
\hline Waste Heutralization Facility & of & 0 & 4 & 35 & 39 \\
\hline General Atomics & 요 & 3 & 0 & 30 & 33 \\
\hline Low-Level Waste Burial Ground & 0 & 0 & o & 31 & 31 \\
\hline PUREX SUrveillance and Maintenance & 0) & 1. & 이 & 27 & 28 \\
\hline Tank Waste Pretreatment Facility & 요 & 0 & 3 & 25 & 27 \\
\hline Paducah - Utility Services & 0 & 0 & 의 & 25 & 25 \\
\hline Well Drilling & 요 & 19 & 1 & 4 & 24 \\
\hline D\&D of PHL 326 Building & o) & 0 & 9 & 9 & 18 \\
\hline Rockuell - Canoga Park & 여 & 이 & 이 & 16 & 16 \\
\hline 300 Area Fuel Supply & 인 & 1 & of & 13 & 14 \\
\hline DED Of TRUSAF & 0 & o. & 6 & 7 & 13 \\
\hline Kaiser Construction Services & 0 & 11 & 이 & 1) & 11 \\
\hline University of California - Davis & 0. & 0 & 1 & 10) & 11 \\
\hline Stanford Linear Accelerator Center & o & 요 & of & 11 & 11 \\
\hline University of Utah & 0 & 0 & 0 & 9 & 9 \\
\hline Geotechnical Engineering Laboratory & 0 & 0 & 0] & 9 & 9 \\
\hline DED of PNL 324 Building & 0 & 0] & of & 3 & 3 \\
\hline TRUSAF & o) & of & 요 & 3 & 3 \\
\hline Ames Laboratory & 0 & 이 & 0] & 1 & 1 \\
\hline Large Sodiun Fire Facility & 이 & 이 & 이 & 1 & 1 \\
\hline Total & 69,828 & 54,453 & 29,762 & 28,919 & 182,163 \\
\hline \multicolumn{6}{|c|}{$\begin{array}{l}\text { (a) Sum of individual values may not match totals due to rounding. } \\
\text { (b) Amounts reported in cubic meters. }\end{array}$} \\
\hline
\end{tabular}


CH_LLMH-contaminated soils. Metal debris is generated primarily by SingleShê11 Tank Long-Length Equipment, Buried Equipment, and D\&D of T P Tant.

\subsubsection{Remote-Handled Low-Level Hixed Waste}

Approximately $6,670 \mathrm{~m}^{3}$ of RH_LLMW will be shipped to the SWOC over the next 30 years. The maximum and minimum volumes expected are $7,560 \mathrm{~m}^{3}$ and $5,890 \mathrm{~m}^{3}$, respectively. Figure 3-6 displays the total $\mathrm{RH}$ LLMW volume by physical waste form. Almost 38\% of the RH_LLMH forecasted to be shipped to SWOC is metal debris. Twenty-nine percent of the RH_LLMW will be organic particulates, $13 \%$ will be void space in the containers, and the remaining $20 \%$ will. be other physical waste forms.

Annual RH_LLMW baseline volumes are depicted in Figure 3-7. The $242 \mathrm{~m}^{3}$ of "HELD" waste is forecasted by the PUREX tunnels. Annual volumes peak in 2006 through 2009 at approximately $500 \mathrm{~m}^{3}$ due to the large amounts of organic particulates expected to be generated by Surplus Facilities. In 2012, the volume also peaks at $500 \mathrm{~m}^{3}$ due to the amount of void space forecasted by Double-She17 Tank Retrieval. From 2012 through 2022, the waste will be primarily metal debris, due to the predicted schedule for D\&D of T Plant.

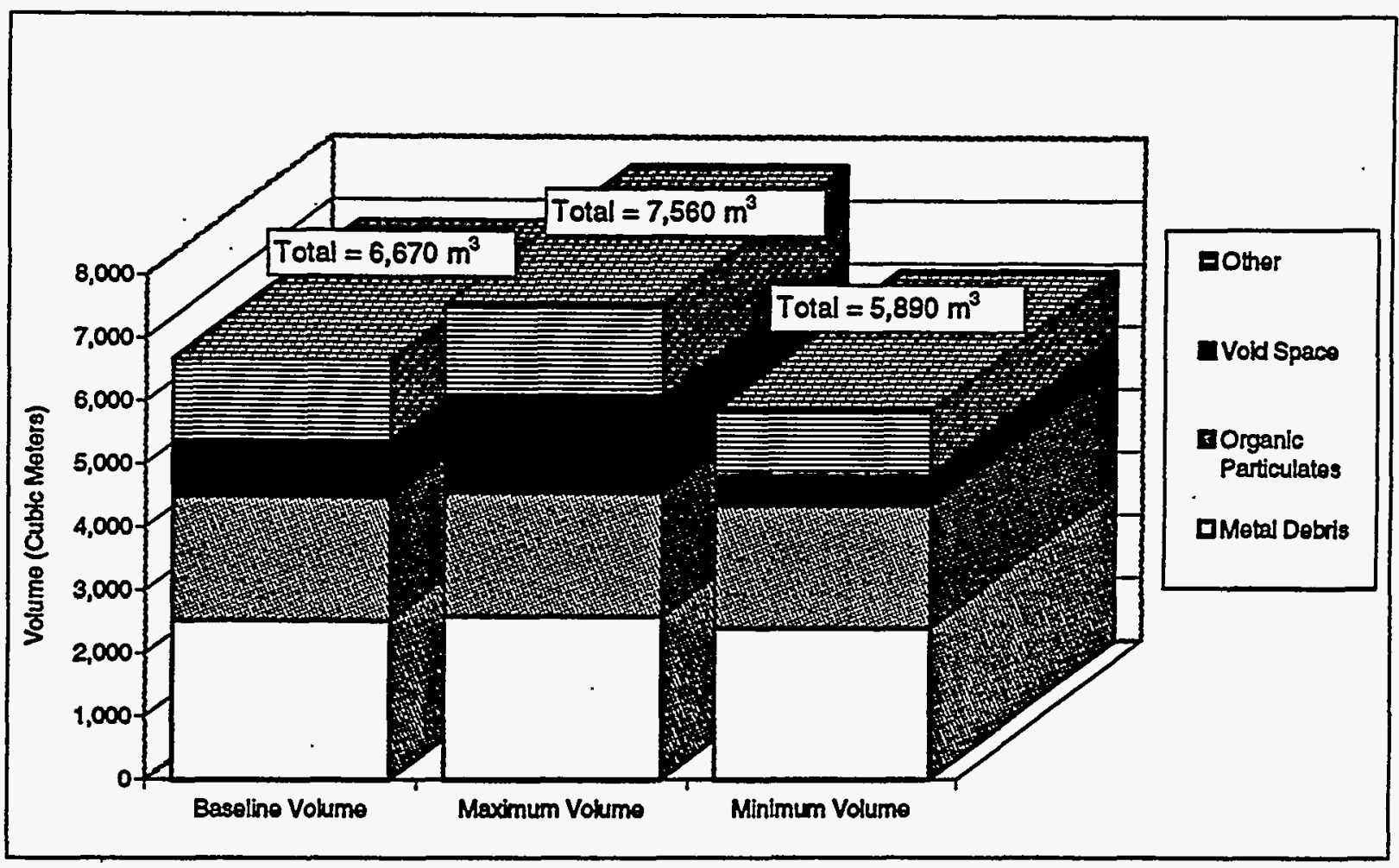

Figure 3-6. RH_LLMW Votumes by Physical Waste Form 


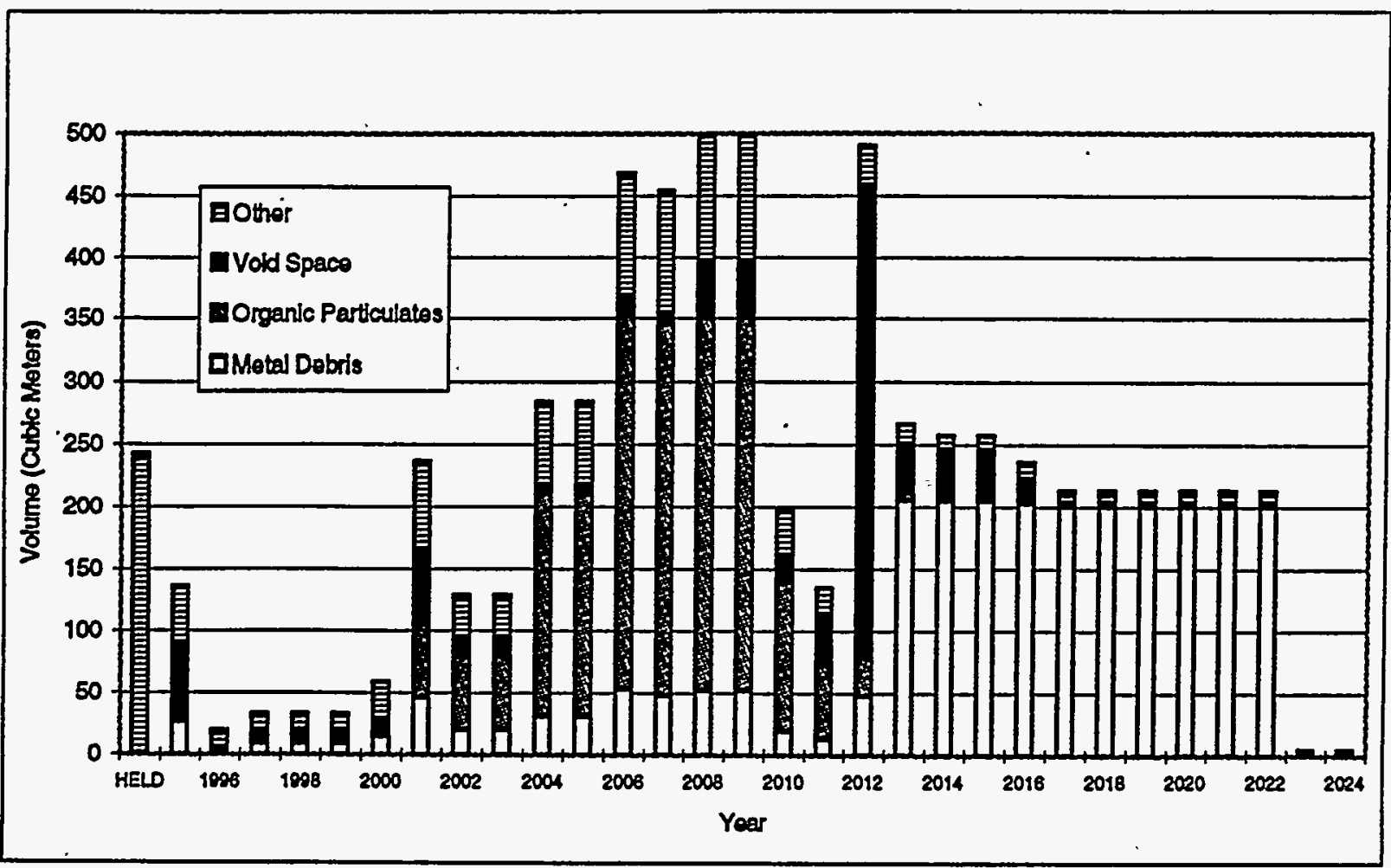

Figure 3-7. Annual RH_LLMH Baseline Volume by Physical Waste Form

As shown in Table 3-2, Surplus Facilities is the primary generator of the RH LLMW organic particulates. D\&D of T Plant is the main contributor of metal debris, while Double-She11 Tank Retrieval generates the largest portion of void space.

\subsection{LOW-LEVEL MIXED WASTE CATEGORIES BY HAZARDOUS HASTE CONSTITUENTS}

A description of the CH_LLMW and RH_LLMW by the hazardous constituents that contaminate the waste is included below. The total volume and the waste generators for each waste category are also provided.

\subsubsection{Contact-Handled Low-Level Mixed Waste}

The total baseline volume of CH LLMH to be shipped to the SWOC in the next 30 years is approximately $182,1 \overline{6} 0 \mathrm{~m}^{3}$, while the maximum and minimum volume ranges are $201,520 \mathrm{~m}^{3}$ and $155,720 \mathrm{~m}^{3}$, respectively. The primary hazardous constituents that will contaminate the CH_LLMW are depicted in Figure 3-8. As shown in the figure, $50 \%$ of the waste is mixed with organics and metal without mercury; $18 \%$ contains metals without mercury; $14 \%$ will contain corrosives; organics and other hazardous constituents will each contain $9 \%$ of the baseline volume. 
Tabie 3-2. RH_LLMW Generators by Physical Waste Form ${ }^{(a, b)}$

\begin{tabular}{|l|r|r|r|r|r|}
\hline \multicolumn{1}{|c|}{ Uaste Generator } & $\begin{array}{r}\text { Metal } \\
\text { Debris }\end{array}$ & $\begin{array}{c}\text { Organic } \\
\text { Particulates }\end{array}$ & $\begin{array}{c}\text { Void } \\
\text { Space }\end{array}$ & other & Total \\
\hline \hline Surplus Facilities & 27 & 1,939 & 0 & 554 & 2,770 \\
\hline D\&D of T Plant & 1,360 & 0 & 0 & 0 & 1,360 \\
\hline DST Retrieval (17 tanks) & 76 & 0 & 642 & 38 & 755 \\
\hline D\&D of PHL 324 Bui lding & 424 & 0 & 0 & 0 & 424 \\
\hline DST Retrieval Systems (10 tanks) & 102 & 0 & 128 & 26 & 255 \\
\hline PUREX Turnel Waste & 0 & 0 & 0 & 242 & 242 \\
\hline 101-AZ Retrieval (one tank) & 50 & 0 & 105 & 68 & 224 \\
\hline Pacific Northwest Laboratory & 0 & 18 & 0 & 173 & 191 \\
\hline D\&D of 222-S Laboratory & 96 & 0 & 0 & 11 & 107 \\
\hline T Plant & 20 & 1 & 0 & 65 & 85 \\
\hline D\&D of B Plant & 69 & 0 & 0 & 0 & 69 \\
\hline D\&D of PUREX & 0 & 0 & 0 & 59 & 59 \\
\hline 222-S Laboratory & 0 & 0 & 0 & 56 & 56 \\
\hline D\&D of 242-A Evaporator & 22 & 0 & 0 & 0 & 22 \\
\hline D\&D of 242-S Evaporator & 22 & 0 & 0 & 0 & 22 \\
\hline D\&D of 242-T Evaporator & 12 & 0 & 0 & 0 & 12 \\
\hline D\&D of PHL 326 Building & 0 & 0 & 0 & 7 & 7 \\
\hline B Plant & 1 & 0 & 0 & 5 & 6 \\
\hline \hline Total & 2,530 & 1,958 & 875 & 1,303 & 6,666 \\
\hline \hline (a) Sum of individual values may not match totals due to rounding. & \\
(b) Amounts reported in cubic meters. & & & & \\
\hline
\end{tabular}

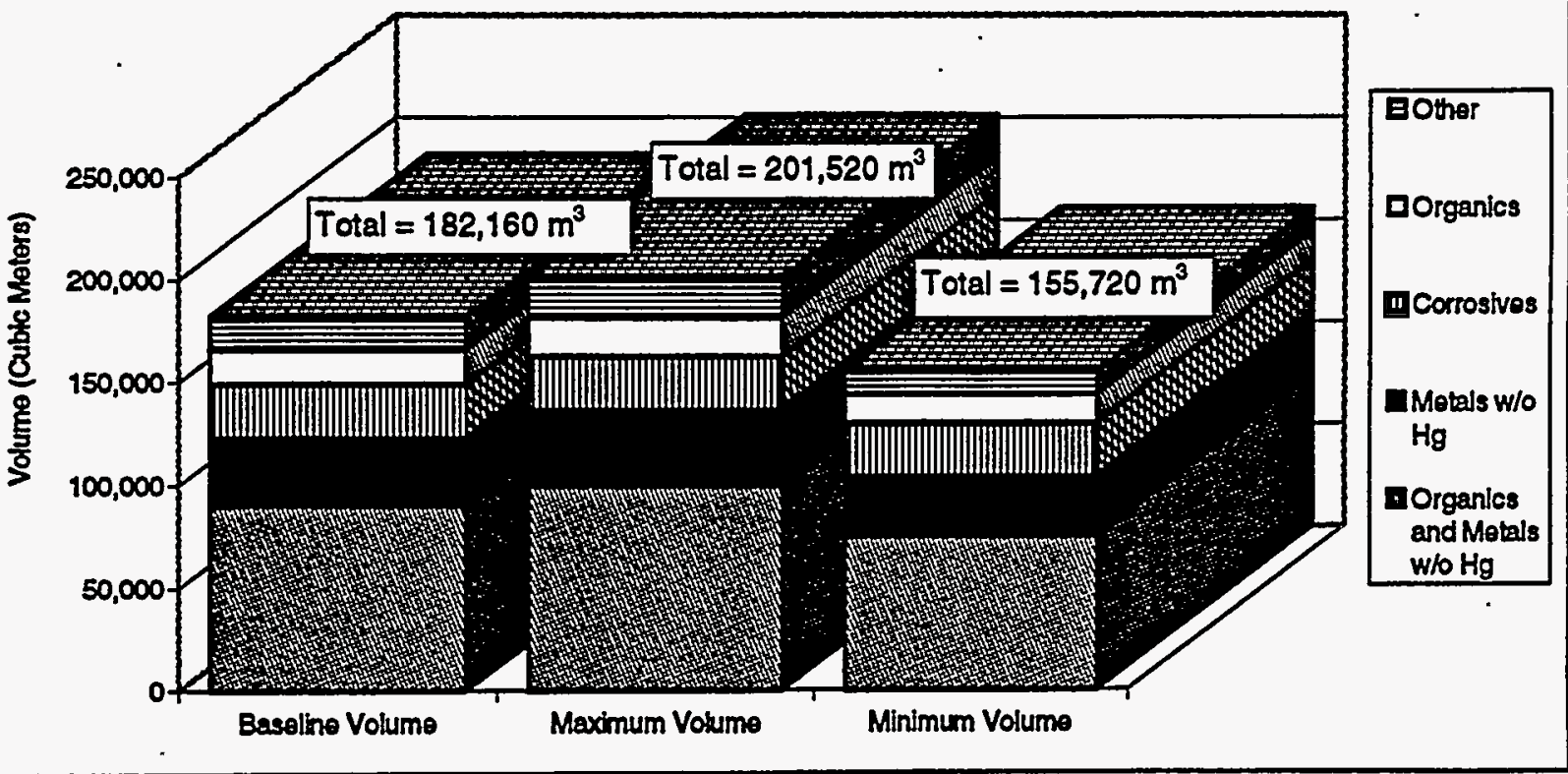

Figure 3-8. CH_LLMH Volumes by Hazardous Waste Constituent 
Figure 3-9 displays the annual CH_LLMW baseline volumes by hazardous waste constituents. Metals without mercury mixed with organics gradually increases through 2017, which corresponds to the retrieval of the long-length equipment from the single-she11 tank farms. After 2017, metals without mercury mixed with organics decreases significantly due to the near-completion of the long-length equipment retrieval. In addition, metals without mercury and corrosives are predominant from 1997 through 2010 due to Past Practice Remediation's expected shipping schedule.

The major generators that forecast the above hazardous constituents are provided in Tabie 3-3. Single-Shell Tank Long-Length Equipment is the primary producer of metals without mercury mixed with organics, forecasting approximately $89 \%$ of the volume contaminated with this hazardous constituent. Past Practice Remediation is the primary generator of waste containing metals without mercury and waste containing corrosives. This generator forecasted $76 \%$ of the CH_LLMW containing metals without mercury and $94 \%$ of the waste contaminated with corrosives.

\subsubsection{Remote-Handled Low-Level Mixed Waste}

Approximateiy $6,670 \mathrm{~m}^{3}$ of RH LLMW will be shipped to the SWOC over the next 30 years. The maximum and minimum expected volumes are $7,560 \mathrm{~m}^{3}$ and $5,890 \mathrm{~m}^{3}$, respectively. Figure 3-10 displays the RH_LLMW baseline volume

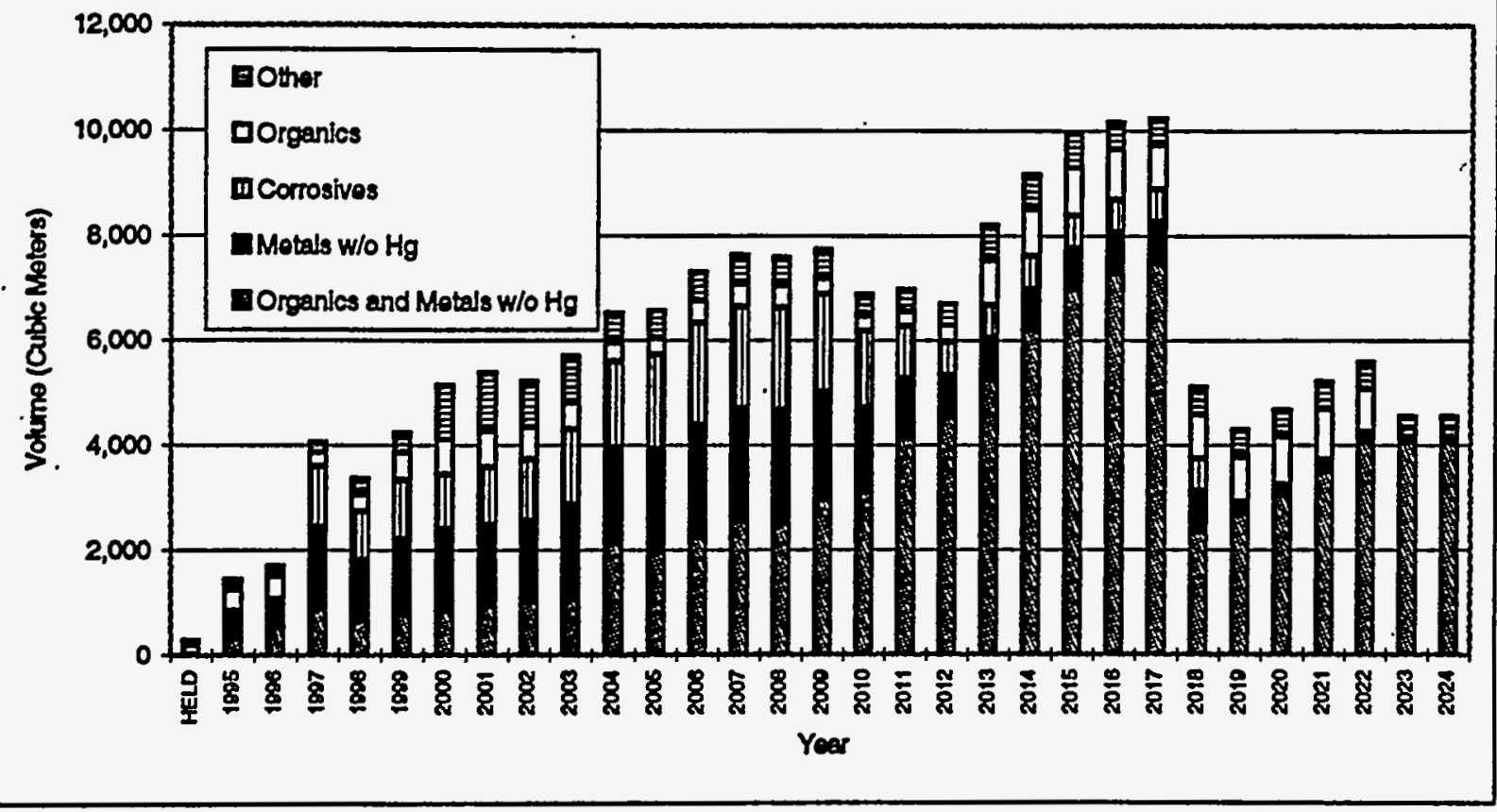

Figure 3-9. Annual CH_LLMH Baseline Volumes by Hazardous Waste Category 
Table 3-3. CH_LLMW Generators by Hazardous Waste Constituents ${ }^{(a, b)}$

\begin{tabular}{|c|c|c|c|c|c|c|}
\hline Waste Generator & $\begin{array}{c}\text { Organics and } \\
\text { Metals Without } \\
\text { Mercury }\end{array}$ & \begin{tabular}{|l|} 
Metals \\
Without \\
Mercury \\
\end{tabular} & Corrosives & Organics & Other & Total \\
\hline SST Long-Length Equipment & 80,804 & 0 & 0 & 0) & 0 & 80,804 \\
\hline Past Practice Remediation & 0 & 25,146 & 24,648 & 0 & 0 & 49,793 \\
\hline Buried Equipment & 0) & 1,160 & 19 & 6,219 & 4 & 7,402 \\
\hline Portsmouth - Energy' Systems & 0 & 0 & 0 & 0 & 6,448 & 6,448 \\
\hline Tank Farm Operations & 5,423 & 0 & 0 & 0 & 170 & 5,593 \\
\hline D\&D of $T$ Plant & 0] & 0 & 0 & 5,438 & 0 & 5,438 \\
\hline Paducah - Energy Systems & 2,566 & 0 & 0 & 2,566 & 0 & 5,132 \\
\hline Tank Farm Restoration & 0] & 1,083 & 0 & 1,083 & 2,322 & 4,487 \\
\hline Surplus Facilities & 0] & 1,178 & 996 & 171 & 502 & 2,846 \\
\hline 200 Area Effluent Treatment Facility & 263 & 1,595 & 0 & 526 & 0 & 2,384 \\
\hline Plutonium Finishing Plant & 237 & 244 & 0 & 0) & 1,706 & 2,187 \\
\hline $\begin{array}{l}\text { D\&D of } 200 \text { Area Effluent Treatment } \\
\text { Facility }\end{array}$ & 0] & 0 & 0 & 0 & 1,599 & 1,599 \\
\hline Argonne National Laboratory - East & 0 & 1,076 & 5 & 6 & 75 & 1,162 \\
\hline SST Retrieval (149 Tanks) & 1,081 & 0 & 0 & 0 & 0 & 1,081 \\
\hline 222-S Laboratory & 89 & 43 & 0 & 242 & 511 & 885 \\
\hline Pacific Northwest Laboratory & 0 & 272 & 92 & 82 & 291 & 737 \\
\hline High-Level Vitrification Project & 0 & 99 & 151 & 120 & 318 & 688 \\
\hline D\&D of 242-A Evaporator & 0] & 0) & .0 & of & 326 & 326 \\
\hline D\&D of 242-S Evaporator & 0 & 0 & 0 & 0 & 326 & 326 \\
\hline Tank 106-C Sluicing & 0 & 294 & 0 & 0] & 0. & 294 \\
\hline D\&D of B Plant & 0) & 277 & 0 & 0] & 0] & 277 \\
\hline D\&D of PUREX & 0 & 0 & 0 & 0) & 236 & 236 \\
\hline Lawrence Berkeley Laboratory & 0] & 30 & 14 & 55 & 134 & 233 \\
\hline Portsmouth - Utility Services & 0) & ㅁ) & 0 & 이 & 214 & 214 \\
\hline T Plant & 0 & 24 & 7 & 126 & 56 & 213 \\
\hline Bettis Atomic Power Laboratory & 0] & 150 & 0 & 1 & 44 & 195 \\
\hline Knolls Atomic Power Laboratory & 0 & 83 & 2 & 16 & 25 & 126 \\
\hline $\begin{array}{l}300 \text { Area Treated Effluent Disposal } \\
\text { Facility }\end{array}$ & 0 & 0) & 0 & 0| & 119 & 119 \\
\hline FERHI National Accelerator Laboratory & 0] & 23 & 0 & 0 & 55 & 78 \\
\hline PUREX & 0 & 11 & 1 & 0] & 55 & 67 \\
\hline D\&D of 242-T Evaporator & 0] & 0 & 0 & 0] & 64 & 64 \\
\hline 100 Area Transition & 0) & 23 & 7 & 13 & 14 & 57 \\
\hline Brookhaven National Laboratory & 0] & 3 & 11 & 0 & 41 & 55 \\
\hline Princeton Plasma Physics Laboratory & 0] & 30 & 0 & 0] & 23 & 53 \\
\hline Low-Level Vitrification Project & 0 & 7 & 13 & 9 & 22 & 52 \\
\hline Battelle Columbus Laboratories & 0] & 17 & 0 & 4 & 30 & 50 \\
\hline B Plant & 0) & 8 & 6 & 15 & 21 & 50 \\
\hline K Basin Operations & 0) & 13 & 0) & 0] & 29 & 42. \\
\hline D\&D of 222-S Laboratory & 0) & 1 & 0 & 10) & 30 & 41 \\
\hline Waste Neutralization Facility & 이 & D] & 0) & 35 & 4 & 39 \\
\hline General Atomics & 0) & 15 & 0) & 13 & 4 & 33 \\
\hline Low-Level Waste Burial Ground & 이 & 0 & 0 & 0 & 31 & 31 \\
\hline PUREX Surveillance and Maintenance & 0 & 6 & 1 & 0] & 21 & 28 \\
\hline Tank Waste Pretreatment Facility & 0 & 4 & 7 & 5) & 12] & 27 \\
\hline
\end{tabular}


Table 3-3. (contd)

\begin{tabular}{|c|c|c|c|c|c|c|}
\hline Waste Generator & $\begin{array}{c}\text { Organics and } \\
\text { Metals Without } \\
\text { Mercury }\end{array}$ & \begin{tabular}{|l|} 
Metals \\
Without \\
Hercury \\
\end{tabular} & Corrosives & Organics & Other & Total \\
\hline Paducah - Utility Services & $\begin{array}{ll} & 0 \\
\end{array}$ & 이 & 0 & 0 & 25 & 25 \\
\hline Well Drilling & 0 & 0 & 0 & 24 & 0 & 24 \\
\hline D\&D of PNL 326 Building & 0 & 0 & 0) & 0 & 18 & 18 \\
\hline Rockwell - Canoga Park & 이 & 0) & 0 & 0 & 16 & 16 \\
\hline 300 Area Fuel Supply & 0 & 8 & 3 & 0 & 3 & 14 \\
\hline DED of TRUSAF & 0 & 0 & 0) & 0 & 13 & 13 \\
\hline Kaiser Construction Services & o] & 11 & 0 & 0] & 0 & 11 \\
\hline University of California - Davis & 0) & 0 & 0) & 0 & 11 & 11 \\
\hline Stanford Linear Accelerator Center. & 0 & 8 & 0) & 0 & 3 & 11 \\
\hline University of Utah & 0 & 0 & 0 & 9 & 0 & 9 \\
\hline Geotechnical Engineering Laboratory & 0] & 요 & 9 & 0 & 0 & 9 \\
\hline D\&D of PNL 324 Building & 0 & 3 & 0) & 0 & o & 3 \\
\hline TRUSAF & 0] & 0] & 0 & 0 & 3 & 3 \\
\hline Ames Laboratory & 0 & 0 & 0 & 0] & 0 & 1 \\
\hline Large Sodium Fire Facility & 0 & of & of & 0 & 1 & 1 \\
\hline Total. & 90,463 & 32,944 & 25,991 & 16,788 & 15,976 & 182,162 \\
\hline $\begin{array}{l}\text { (a) Sum of individual values may } \\
\text { (b) Amounts reported in cubic met }\end{array}$ & & & & & & \\
\hline
\end{tabular}

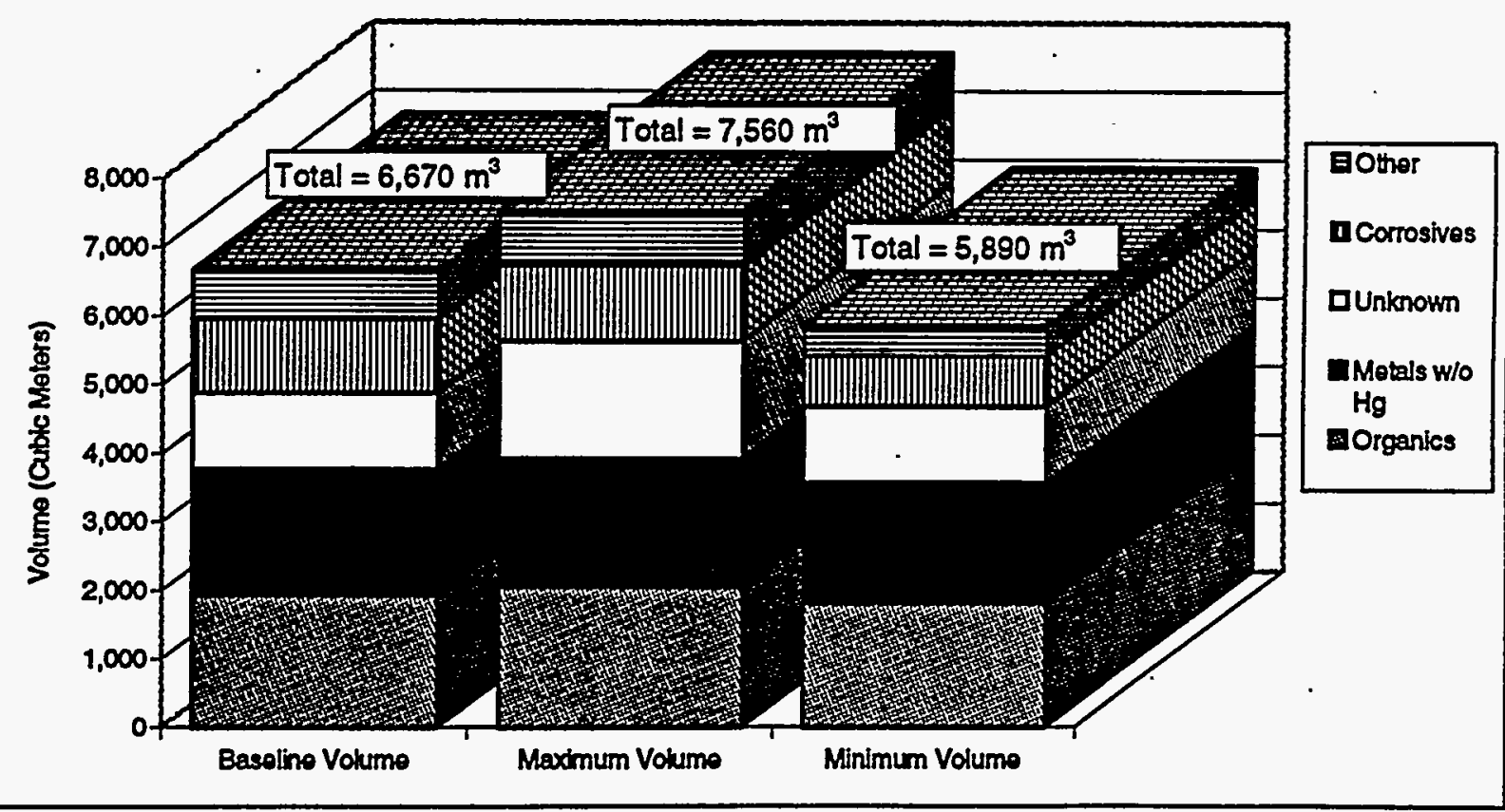

Figure 3-10. RH_LLMW Volumes by Hazardous Waste Category 
according to the hazardous constituents that contaminate the waste. As shown, $29 \%$ of the forecasted RH LLMW wi11 contain organics; $27 \%$ will contain metals without mercury; $17 \%$ wi $\overline{1}$ contain corrosives; and $16 \%$ of the waste has no hazardous constituent data available.

Figure 3-11 displays the annual RH_LLMW baseline volumes by hazardous constituents. The held volume is forecasted by the PUREX tunnels, which does not have hazardous constituent data available. In 2012, waste generation by Double-Shel1. Tank Retrieval also generates waste with unknown hazardous constituents. The peak from 2004 through 2009 is due to large amounts of metals without mercury and corrosives being generated by Surplus Facilities. The organic waste is constant from 2013 through 2022 since this waste will be generated during D\&D of T Plant. The schedule for the waste from D\&D of $t$ Plant was assumed to be uniformly distributed from 2013 to 2022.

The major waste generators of the RH LLMW contaminated with the above hazardous waste constituents are listed in Table 3-4. Surplus Facilities is the primary generator of both metals without mercury and corrosives. D\&D of $T$ Plant will generate most of the organic contaminated waste; in fact, this is the only waste generated by D\&D of T Plant.

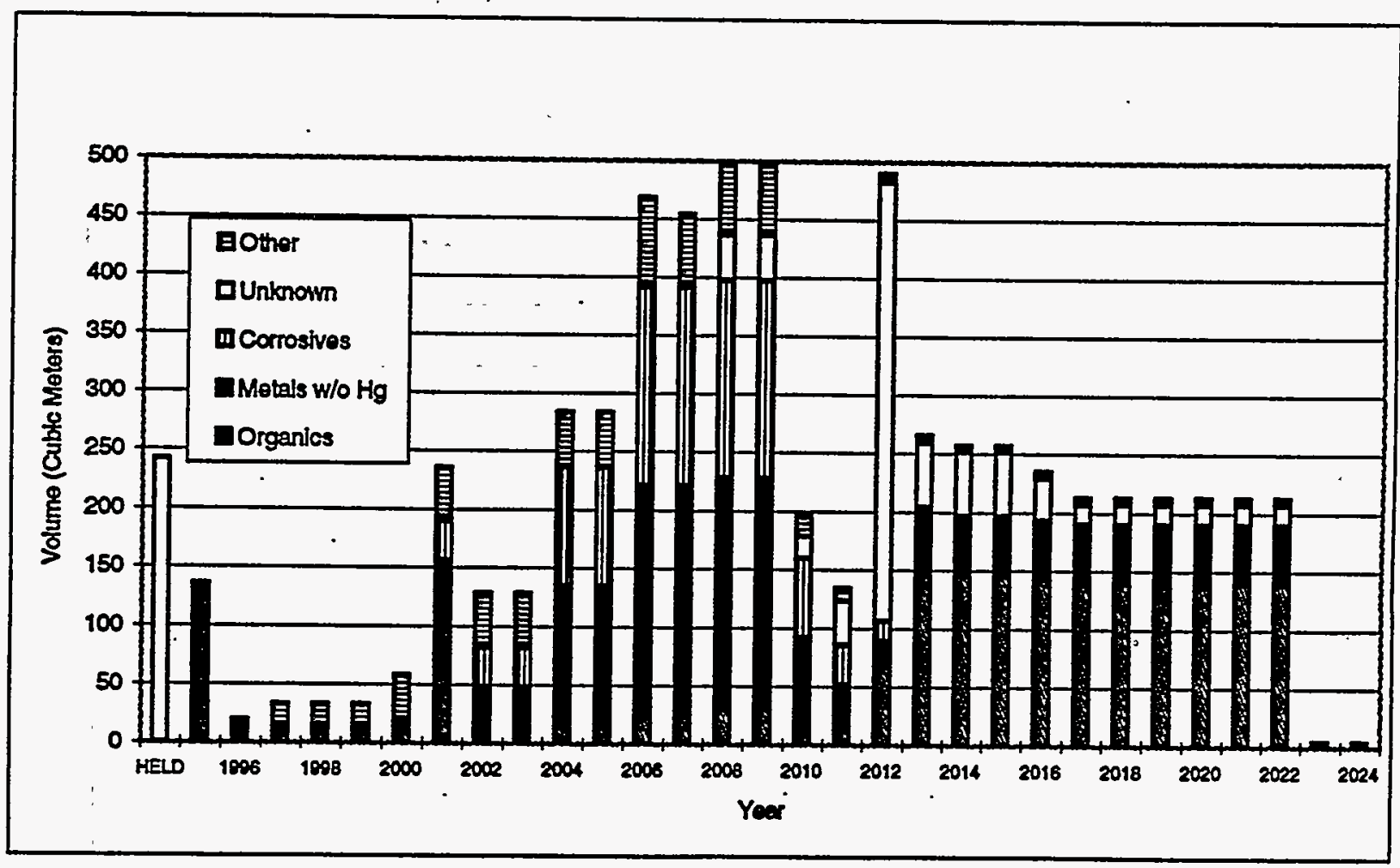

Figure 3-11. RH_LLMH Baseline Volumes by hazardous Waste Category 
Table 3-4. RH_LLMW Generators by Hazardous Waste Constituents $(a, b)$

\begin{tabular}{|c|c|c|c|c|c|c|}
\hline Waste Generator & Organics & $\begin{array}{c}\text { Metals wi thout } \\
\text { Mercury }\end{array}$ & Corrosives & Unknown & Other & Total \\
\hline Surplus Facilities & 152 & 1,260 & 1,108 & $\mathbf{0}$ & 249 & 2,770 \\
\hline D\&D of $T$ Plant & 1,360 & $\mathbf{0}$ & $\mathbf{0}$ & 0 & 0 & 1,360 \\
\hline DST Retrieval (17 tanks) & 113 & $\mathbf{0}$ & 0 & 642 & 0 & 755 \\
\hline D\&D of PNL 324 Building & 0 & 424 & 0 & 0 & 0 & 424 \\
\hline DST Retrieval Systems ( 10 tanks) & 0 & 0 & $\mathbf{0}$ & 0 & 255 & 255 \\
\hline PUREX Tunnel Waste & 0 & $\mathbf{0}$ & 0 & 242 & $\mathbf{0}$ & 242 \\
\hline 101-Az Retrieval (one tank) & 224 & 0 & 0 & 0 & $\mathbf{0}$ & 224 \\
\hline Pacific Northwest Laboratory & 0 & 54 & 0 & 9 & 128 & 191 \\
\hline D\&D of 222-S Laboratory & 26 & 3 & 0 & 50 & 28 & 107 \\
\hline$I$ Plant & 50 & 10 & 3 & 13 & 9 & 85 \\
\hline DED of B Plant & 0 & 69 & 0 & 0 & 0 & 69 \\
\hline DED OF PUREX & $\mathbf{0}$ & 0 & $\mathbf{0}$ & 59 & 0 & 59 \\
\hline 222-s Laboratory & 18 & 4 & 0 & 0 & 34 & 56 \\
\hline D\&D of 242-A Evaporator & 0 & 0 & 0 & 22 & 0 & 22 \\
\hline D\&D of 242-S Evaporator & 0 & 0 & 0 & 22 & 0 & 22 \\
\hline D\&d of 242-T Evaporator & 0 & 0 & 0 & 12 & 0 & 12 \\
\hline D\&D of PNL 326 Building & 0 & 0 & 0 & 7 & 0 & 7 \\
\hline B Plant & 0 & 1 & 0 & 0 & 4. & 6 \\
\hline Total & 1,943 & 1,826 & 1,111 & 1,079 & 708 & 6,666 \\
\hline
\end{tabular}




\section{WHC-EP-0888}

Intentionally Left Blank 


\subsection{TRANSURANIC AND TRANSURANIC MIXED MASTE}

This section provides waste volumes by physical waste form and hazardous constituents for TRU_TRUM waste. The major waste generators of TRU TRUM waste are also described. The 1995 Solid Haste 30-Year Volume Summary (Vālero et al. 1995) provides more detailed information about these waste generators and their missions; the 1995 Solid Waste 30-Year Container Volume Summary (Templeton et a1. 1995) provides a comparison of the 1994 and 1995 reported TRU_TRUM waste volumes.

\subsection{TRU_TRUM WASTE VOLUME}

The baseline amount of TRU TRUM waste to be shipped to the SHOC over the next 30 years is approximately $\overline{46}, 730 \mathrm{~m}^{3}$. The maximum reported volume is $51,420 \mathrm{~m}^{3}$, while the minimum reported volume is $42,070 \mathrm{~m}^{3}$. As shown in Figure 4-1, 75\% of the TRU_TRUM baseline waste is CH_TRU_TRUM, while the remaining 25\% is RH_TRU_TRUM. Figure 4-2 displays the annuāi baseline volume of TRU TRUM waste by waste category. As shown in the figure, the volumes remain at approximately $600 \mathrm{~m}^{3}$ from 1995 through 2012. From 2013 through 2022, the TRU_TRUM annual volume increases to approximately $3,100 \mathrm{~m}^{3}$, which reflects the

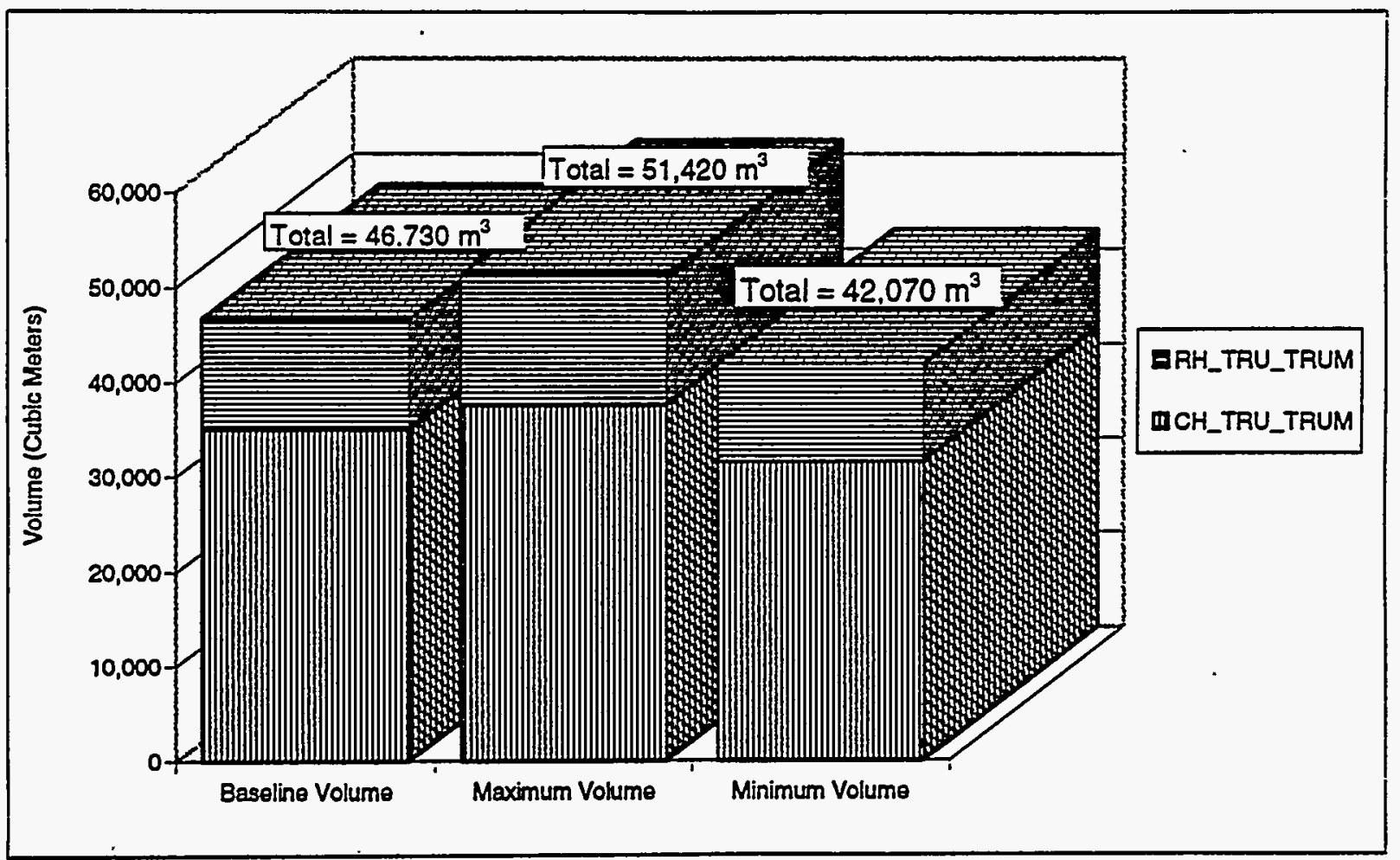

Figure 4-1. TRU_TRUM Waste Volumes by Waste Category 


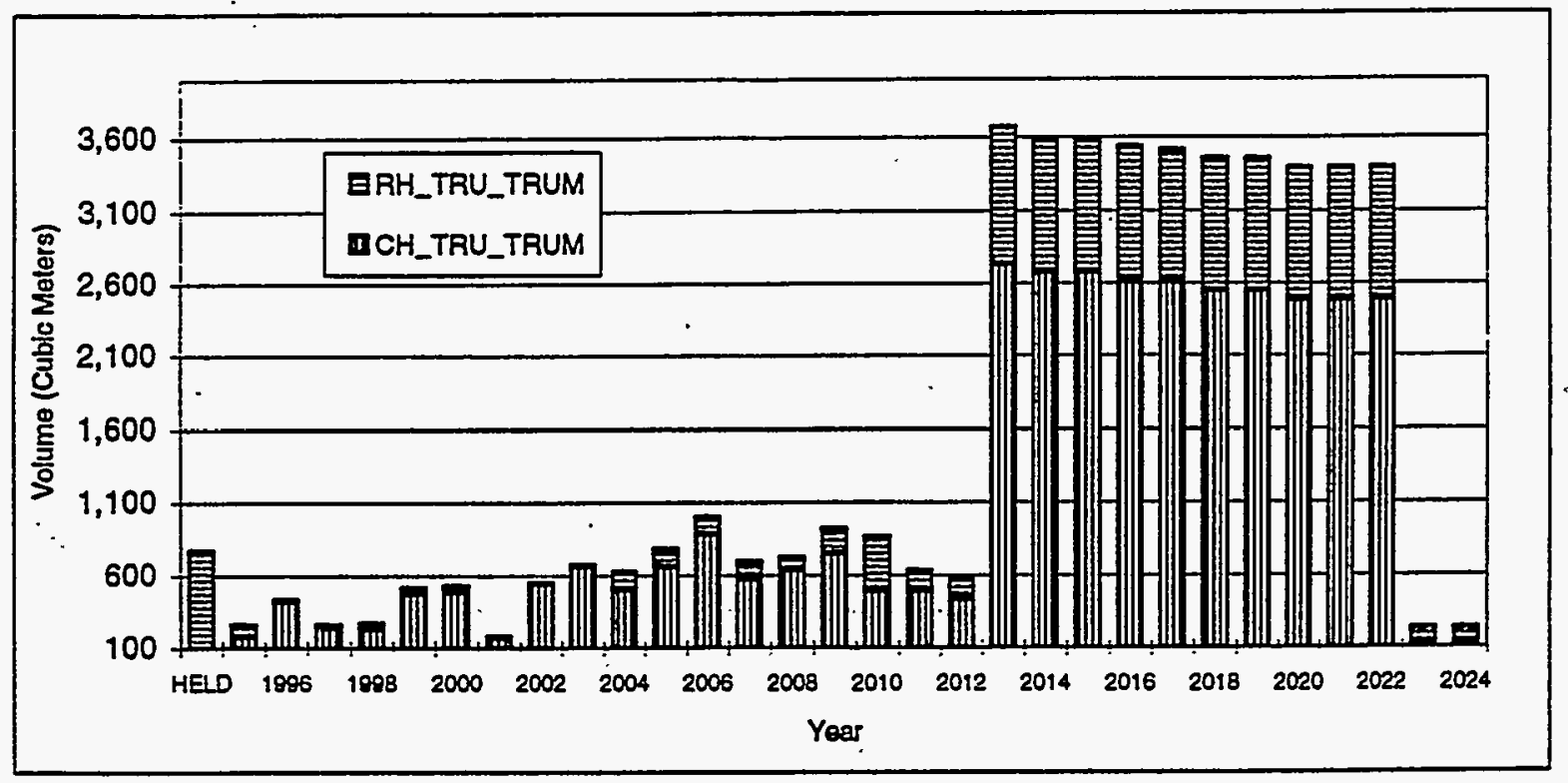

Figure 4-2. Annual Baseline Volumes of TRU_TRUM Wàste by Waste Category

anticipated schedule for D\&D of the Plutonium Finishing Plant (PFP) and other non-surplus facilities. The schedule for these non-surplus facilities was assumed to be from 2013 through 2022.

The major waste generators of TRU TRUM waste are depicted in Figure 4-3. As shown, $49 \%$ will be generated by D\&D of the PFP; Past Practice Remediation comprises 13\%; D\&D of T-Plant will generate 10\%; and the remaining $28 \%$ will be generated by other waste generators.

\subsection{TRU_TRUM. HASTE CATEGORIES BY PHYSICAL MASTE FORM}

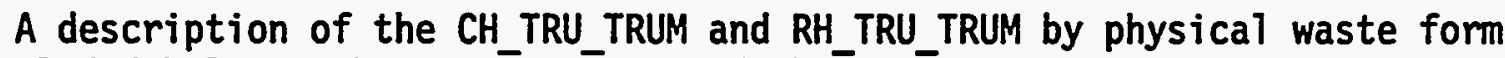
is included below. The tota $\bar{\top}$ vōume and the was $\bar{t} e$ generators for each waste category are also described.

\subsubsection{Contact-Handled Transuranic and Transuranic Mixed Waste}

The baseline volume of CH TRU TRUM to be shipped to the SHOC in the next 30 years is approximately $35,100 \mathrm{~m}^{3}$. The minimum and maximum ranges are $31,720 \mathrm{~m}^{3}$ and $37,690 \mathrm{~m}^{3}$, respectively. Figure 4-4 displays the baseline, minimum, and maximum volumes of CH_TRU_TRUM by physical waste forms. As displayed, 68\% of the CH_TRU_TRUM base7 ine waste is metal debris. The primary generator of the CH TRU TRUM metal debris is D\&D of the PFP. Figure 4-5 depicts the annual $\bar{C}$ _TRU_TRUM baseline waste volumes by physïcal waste form. From 1995 through $201 \overline{2}$ volumes remain relatively low, at approximately $500 \mathrm{~m}^{3}$ and are composed primarily of metal debris and inorganic non-metal debris. From 2013 through 2022, the annual volume increases to approximately $2,500 \mathrm{~m}^{3}$, 


\section{Total $=46,730 \mathrm{~m}^{5}$}

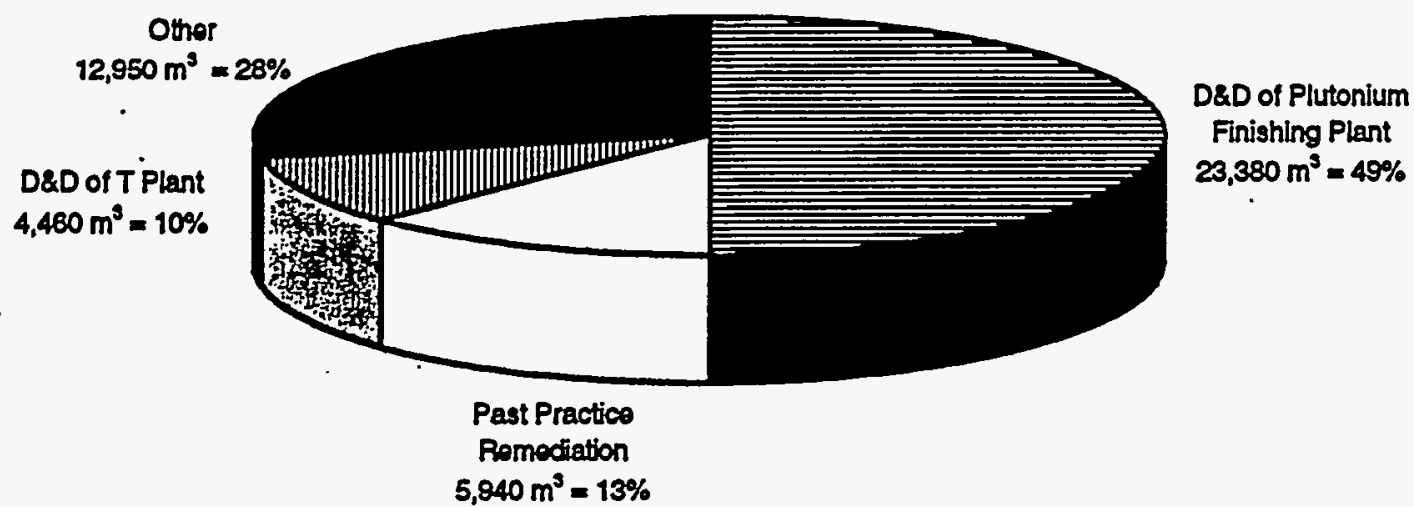

Figure 4-3. Major TRU_TRUM Waste Generators

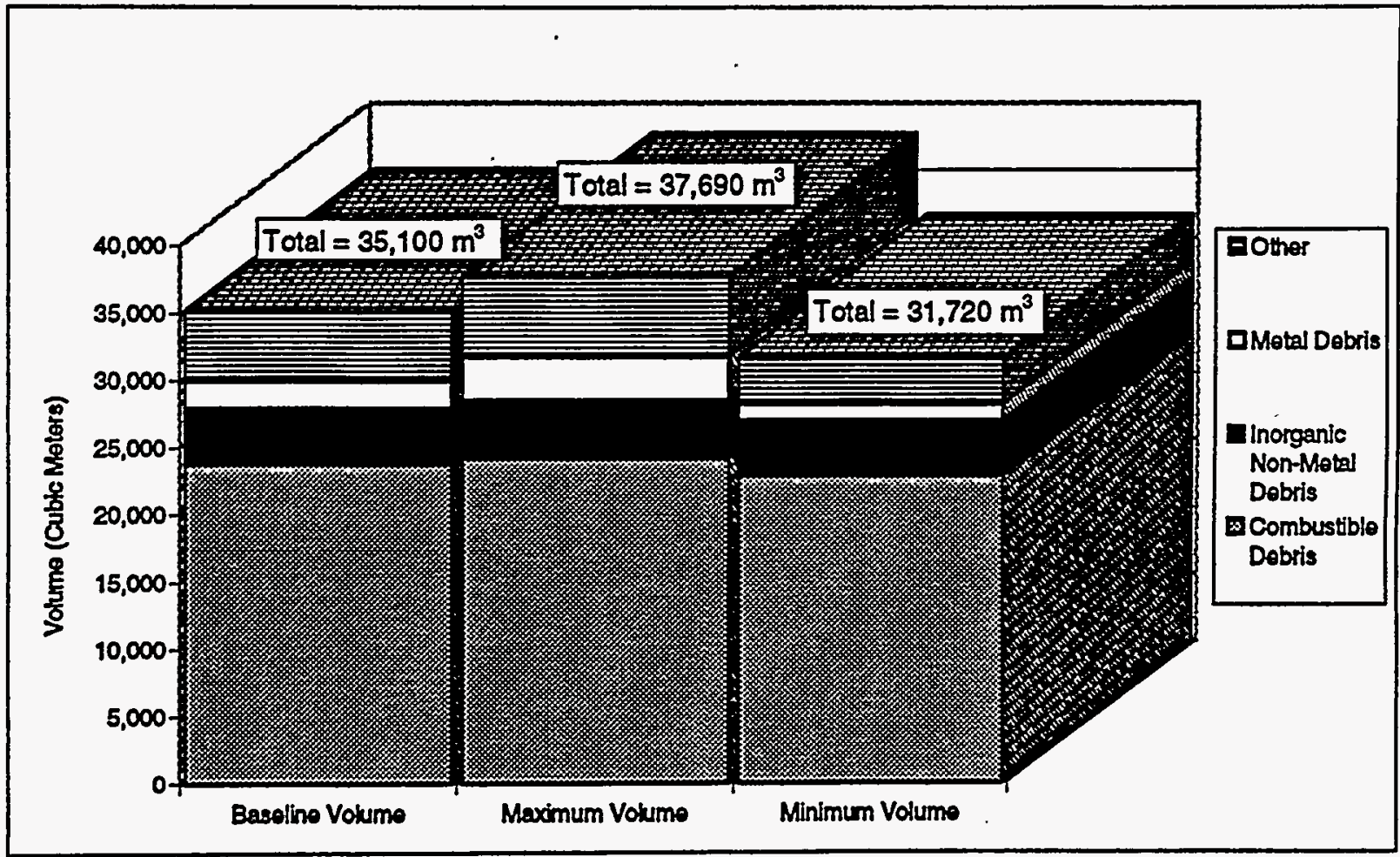

Figure 4-4. CH_TRU_TRUM Volumes by Physical Waste Form 


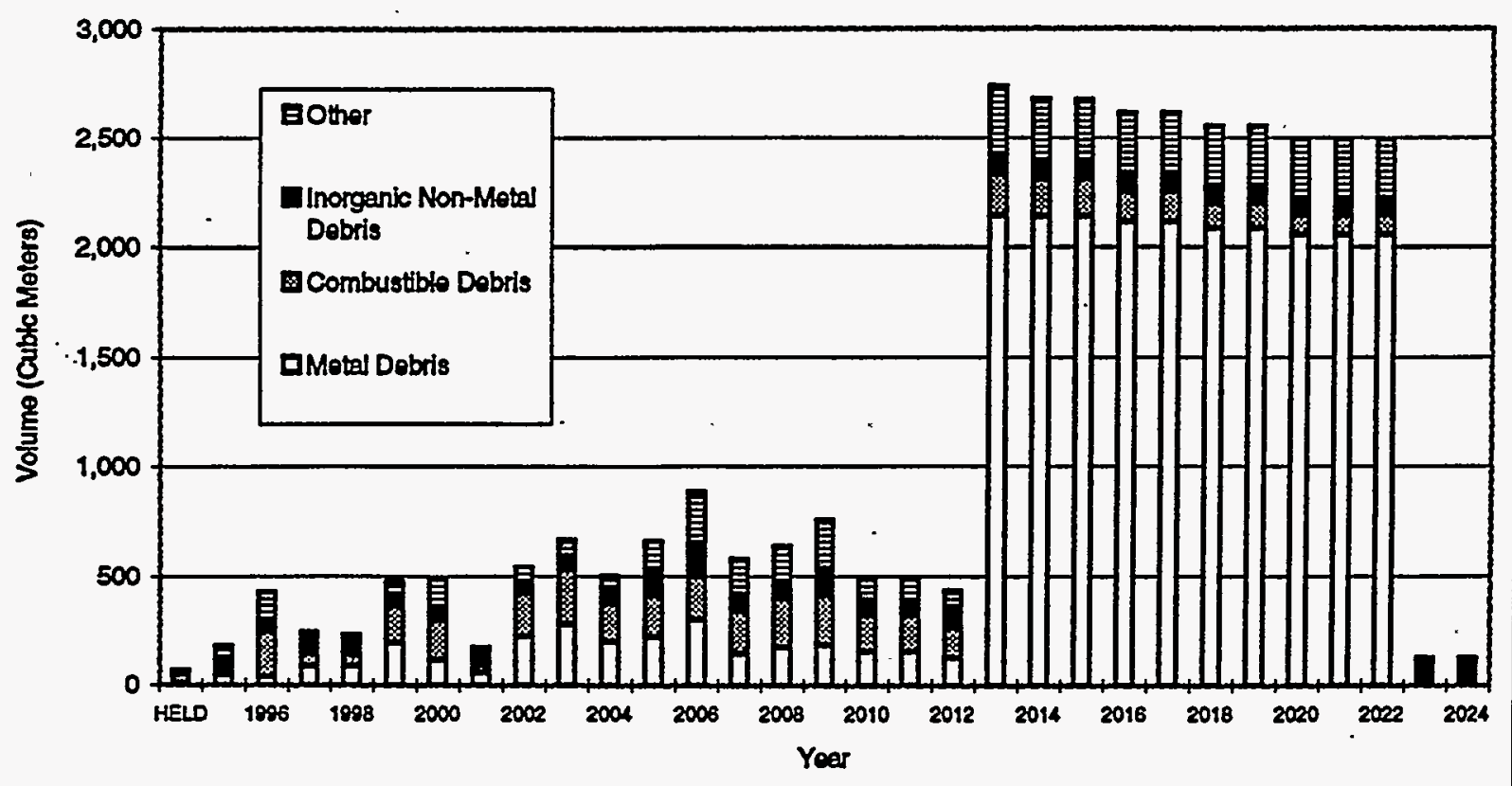

Figure 4-5. Annual CH_TRU_TRUM Baseline Volumes by Physical Haste Form

which corresponds to the expected schedule for D\&D of the PFP. During these years, the waste is primarily metal debris.

As shown in Table 4-1, D\&D of PFP forecasts $72 \%$ of the metal debris. Past Practice Remediation is the primary generator of inorganic non-metal debris, while the PFP and the High-Leve Vitrification Project are the main producers of combustible debris.

\subsubsection{Remote-Handled Transuranic and Transuranic Mixed Waste}

Approximately $11,630 \mathrm{~m}^{3}$ of RH_TRU_TRUM waste will be shipped to the SHOC over the next 30 years. The minimum and maximum amounts that could be shipped are $13,730 \mathrm{~m}^{3}$ and $10,350 \mathrm{~m}^{3}$, respectively. The primary RH_TRU_TRUM physica? waste forms are displayed in Figure 4-6. As depicted, 56\% of the RH_TRU_TRUM basel ine waste will be metal debris. Another $20 \%$ of the waste does not have physical waste form data available; $4 \%$ is expected to be combustible debris; and the remaining $20 \%$ will be other physical waste forms.

Figure 4-7 displays the baseline volume annually by physical waste forms. The large amount of held waste is forecasted by the PUREX tunnels, which currently does not have a shipping schedule for this waste. From 1996 through 2003, very little RH_TRU_TRUM is expected. Then from 2004 through 
Table 4-1. CH_TRU_TRUM Waste Generators by Physical Waste Form ${ }^{(a, b)}$

\begin{tabular}{|c|c|c|c|c|c|}
\hline Waste Generator & $\begin{array}{c}\text { Metal } \\
\text { Debris }\end{array}$ & $\begin{array}{c}\text { Inorganic } \\
\text { Non-Metal Debris }\end{array}$ & $\begin{array}{c}\text { Combust ible } \\
\text { Debris }\end{array}$ & Other & Total \\
\hline D\&D of PFP & 17,199 & 0 & 요 & 1,505 & 18,704 \\
\hline Past Practice Renediation & 2,671 & 2,671 & 0] & 594 & 5,935 \\
\hline DED of $T$ Plant & 2,720 & 0 & 0 & 850 & 3,570 \\
\hline Plutoniun Finishing Plant & 795 & 이 & 847 & 466 & 2,027 \\
\hline High-Level Vitrification Project & 188 & 94 & 984 & 609 & 1,875 \\
\hline Surplus Facilities & 34 & 841 & 84 & 868 & 1,827 \\
\hline DED of PUREX & 76 & 525 & 0 & 3 & 604 \\
\hline Argonne Hational Laboratory - East & 57 & 21 & 21 & 92 & 192 \\
\hline Pacific Morthwest Laboratory & 0 & 0 & 81 & 67 & 149 \\
\hline D\&D of Fuels Development Laboratory & 85 & 0] & 1 & 5 & 90 \\
\hline Mixed Waste Storage Facility & 0 & 요 & o & 38 & 38 \\
\hline D\&D of PHL 231-2 Building & 29 & 0 & 0 & 5 & 34 \\
\hline D8d of PHL 327 Building & 27 & 0 & 0] & 0 & 27 \\
\hline PUREX & 13 & o & 9 & 이 & 22 \\
\hline Fuels Development Laboratory & 1 & 요 & 요 & 이 & 1 \\
\hline Ames Laboratory & 요 & 0 & 0 & 이 & 0 \\
\hline Total & 23,812 & 4,152 & 2,028 & 5,103 & 35,095 \\
\hline
\end{tabular}

(a) Sum of individual values may not match totals due to rounding.

(b) Anounts reported in cubic meters.

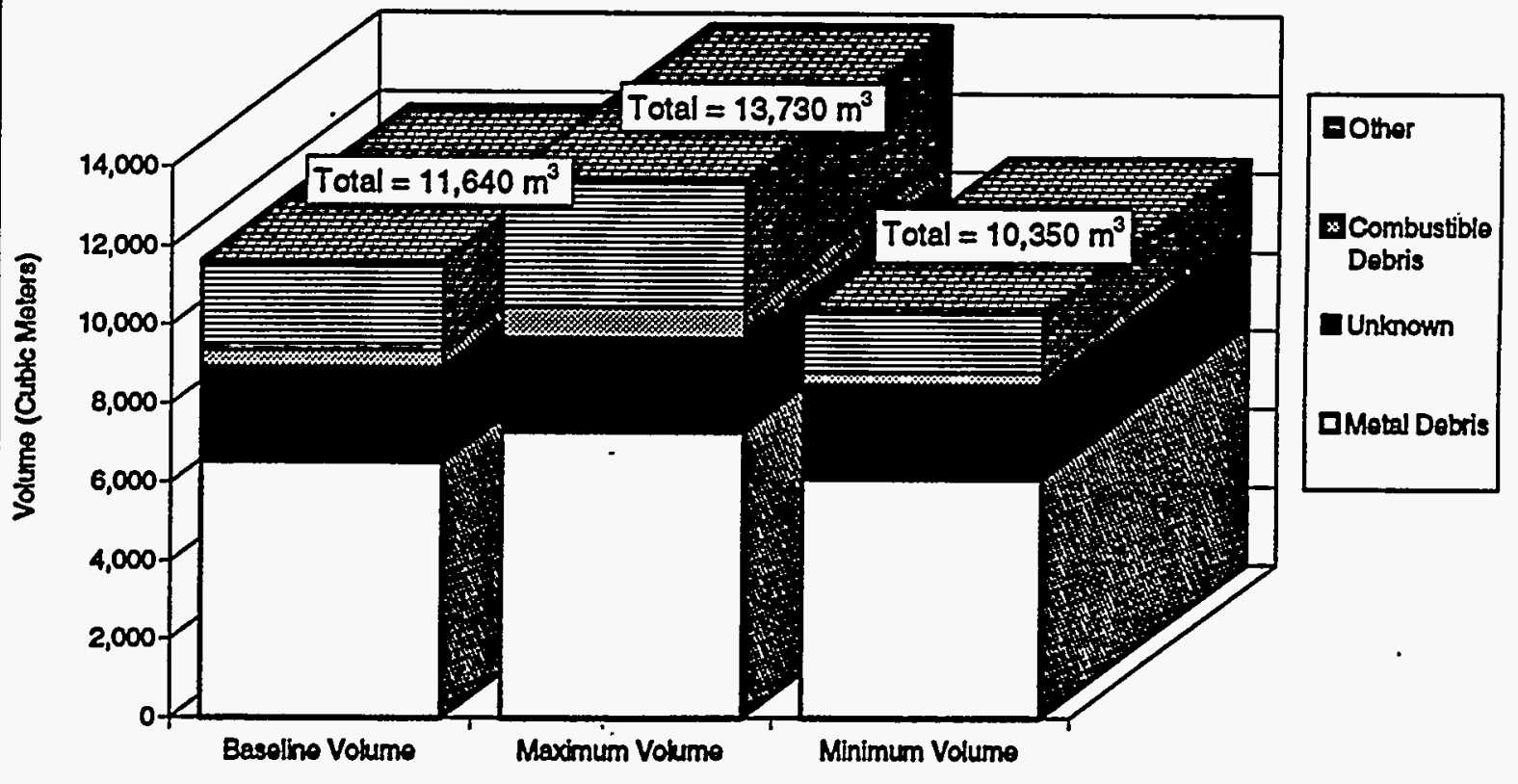

Figure. 4-6. RH_TRU_TRUM Baseline Volumes by Physical Waste Form 


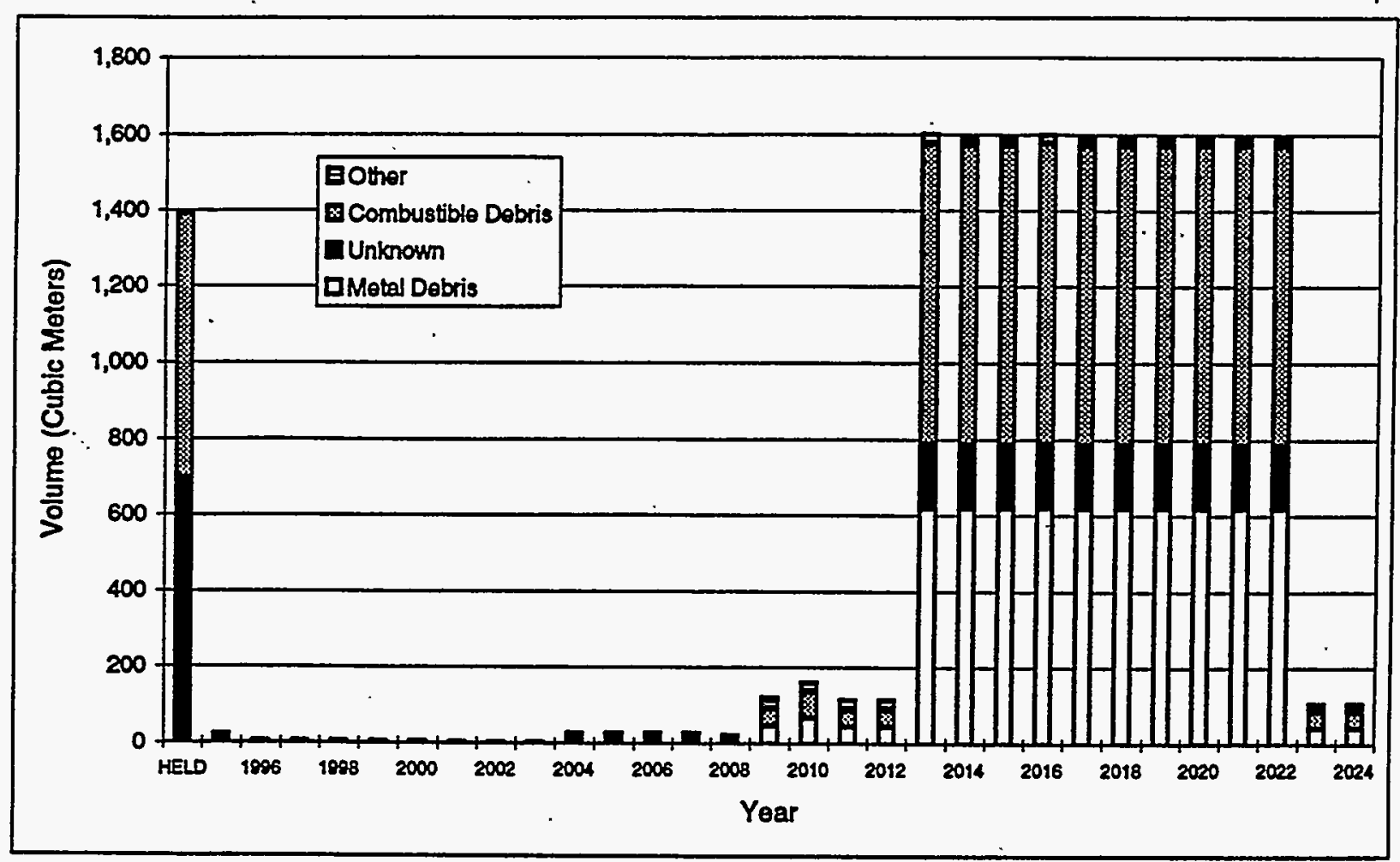

Figure 4-7. Annual RH_TRU_TRUM Baseline Volume by Physical Waste Form

2012, smal1 amounts (approximately $100 \mathrm{~m}^{3}$ ) will be generated. From 2013 through 2022, large amounts of metal debris will be generated due to the expected schedule for D\&D of the PFP.

The waste generators for RH_TRU_TRUM that forecast the primary physical waste forms are shown in Table 4-2. The D\&D of PFP will generate $66 \%$ of the metal debris. The only generator that was unable to provide physical waste form data for their RH_TRU_TRUM waste was the PUREX tunnels. The principal generator of combustibīe debris is the High-Level Vitrification Plant.

\subsection{TRU_TRUM WASTE CATEGORIES BY HAZARDOUS WASTE CONSTITUENTS}

A description of the CH_TRU_TRUM waste and RH_TRU_TRUM waste by the hazardous constituents that contaminate the waste $\overline{i s}$ included below. Although TRU_TRUM waste is combined as one waste category, the hazardous constituents describe only the volumes of TRUM waste, since hazardous constituent data are not applicable to mixed TRU waste.

\subsubsection{Contact-Handled Transuranic Mixed Haste}

The baseline volume of $\mathrm{CH}$ TRUM to be shipped to the SWOC in the next 30 years is approximately $14,760 \mathrm{~m}^{3}$, with the minimum and maximum ranges reported 
Table 4-2. RH_TRU_TRUM Waste Generators by Physical Waste Form ${ }^{(a, b)}$

\begin{tabular}{|l|r|r|r|r|r|}
\hline \multicolumn{1}{|c|}{ Waste Generator } & $\begin{array}{r}\text { Metal } \\
\text { Debris }\end{array}$ & Unknokn & $\begin{array}{c}\text { Combustible } \\
\text { Debris }\end{array}$ & other & Total \\
\hline \hline D\&D of Plutonium Finishing Plant & 4,300 & 314 & 0 & 62 & 4,676 \\
\hline High-Level Vitrification Project & 678 & 0 & 339 & 678 & 1,696 \\
\hline D\&D of H Reactor & 0 & 1,186 & 0 & 0 & 1,186 \\
\hline D\&D of PHL 327 Building & 729 & 171 & 0 & 123 & 1,023 \\
\hline D\&D of T Plant & 680 & 0 & 0 & 212 & 892 \\
\hline Surplus Facilities & 35 & 0 & 89 & 587 & 711 \\
\hline PUREX Tunnel Waste & 0 & 697 & 0 & 0 & 697 \\
\hline DST Retrieval (17 tanks) & 25 & 0 & 0 & 228 & 253 \\
\hline D\&D of PUREX & 25 & 0 & 10 & 116 & 151 \\
\hline Pacific Horthwest Laboratory & 8 & 0 & 7 & 79 & 93 \\
\hline Battelle Columbus Laboratories & 0 & 0 & 0 & 71 & 71 \\
\hline K Basin Operations & 10 & 0 & 0 & 58 & 68 \\
\hline Argonne Hational Laboratory - East & 31 & 0 & 10 & 10 & 51 \\
\hline T Plant & 0 & 0 & 0 & 28 & 28 \\
\hline D\&D of Waste Neutralization Facility & 23 & 0 & 0 & 0 & 23 \\
\hline 222-S Laboratory & 0 & 0 & 6 & 9 & 15 \\
\hline \hline Total & 6,544 & 2,367 & 460 & 2,263 & 19,634 \\
\hline \hline (a) Sum of individual values may not match totals due to rounding- & \\
(b) Amounts reported in cubic meters. & & & & \\
\hline
\end{tabular}

as $13,890 \mathrm{~m}^{3}$ and $15,710 \mathrm{~m}^{3}$, respectively. Figure 4-8 displays the baseline, minimum, and maximum waste volumes by hazardous waste constituents. As shown in the figure, a majority (68\%) of the CH_TRUM baseline waste has no hazardous constituent data available. Nineteen percent of the waste is expected to be organics; $5 \%$ will be metals without mercury; and the remaining $7 \%$ will be made up of other hazardous waste constituents.

Figure 4-9 displays the annual baseline $\mathrm{CH}_{-}$TRUM volume by hazardous waste constituents. From 1995 through 2005, the annual volume remains below $50 \mathrm{~m}^{3}$. From 2006 through 2012, the volume increases to approximately $100 \mathrm{~m}^{3}$. The annual volume increases to $1,300 \mathrm{~m}^{3}$ from 2013 through 2022, an increase which corresponds to the expected schedule of the D\&D of PFP. Since most of the hazardous constituent data for the D\&D of PFP are not available, "unknown" predominates from 2013 through 2022.

Table 4-3 provides the main waste generators for each of the primary hazardous constituents that make up the $\mathrm{CH}_{-}$TRUM waste. D\&D of the PFP is the only generator with unavailable data. The D\&D of T Plant is the major generator of the $\mathrm{CH}_{-}$TRUM waste containing organics.

\subsubsection{Remote-Handled Transuranic Mixed Waste}

Approximately $5,200 \mathrm{~m}^{3}$ of RH_TRUM waste will be shipped to the SHOC over the next 30 years. The maximum range is $6,390 \mathrm{~m}^{3}$, and the minimum range is 4,520 $\mathrm{m}^{3}$. Figure 4-10 displays the baseline, maximum, and minimum volumes for RH_TRUM waste by the hazardous constituents that contaminate the waste. As 


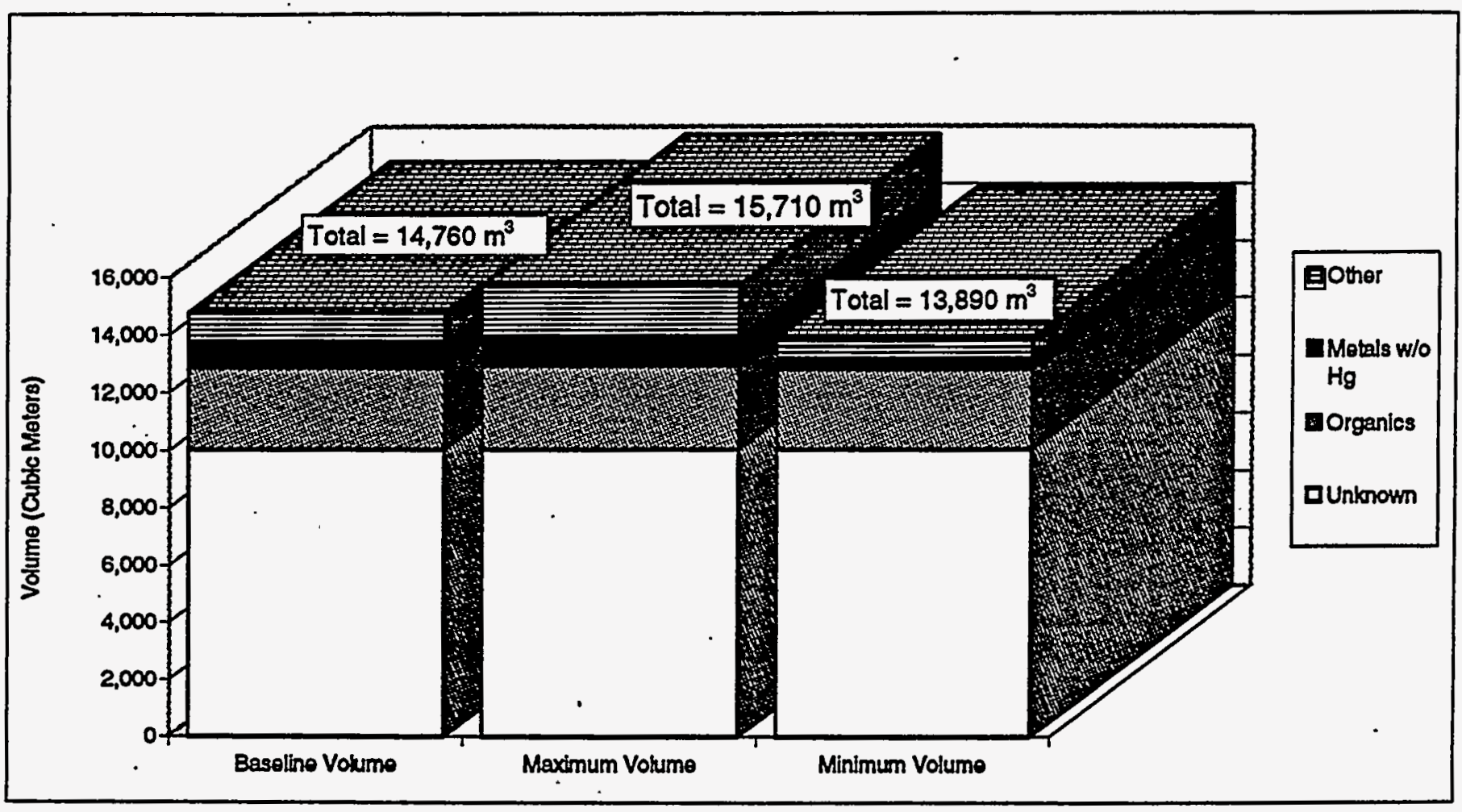

Figure 4-8. CH_TRUM Waste Volumes by Hazardous Waste Category

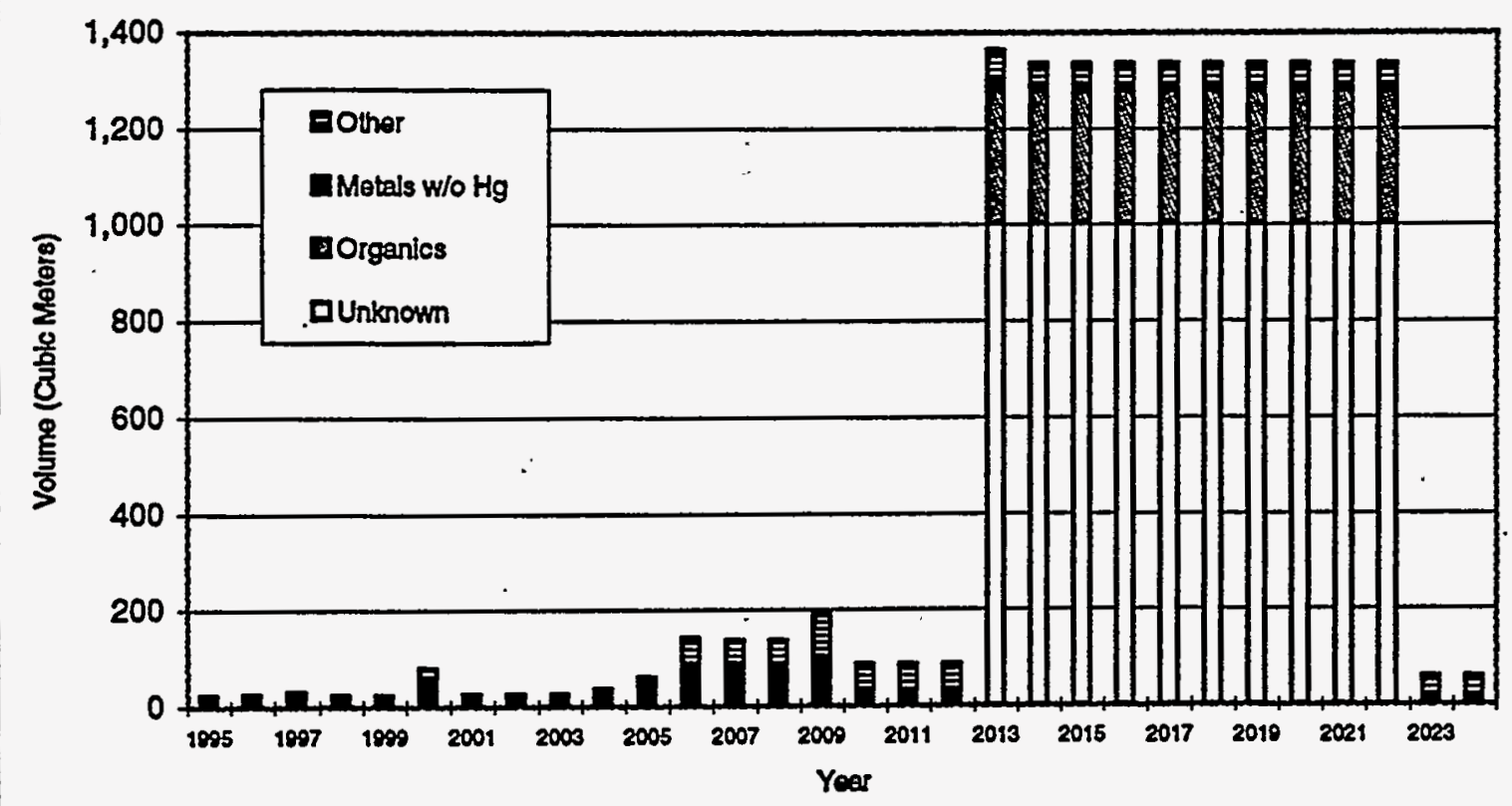

Figure 4-9. CH_TRUM Baseline Waste Volumes by Hazardous Waste Category 
Table 4-3:. CH_TRUM Waste Generators by Hazardous Waste Constituents ${ }^{(a, b)}$

\begin{tabular}{|c|c|c|c|c|c|}
\hline Waste Generator & Unknown & Organics & $\begin{array}{c}\text { Metals Without } \\
\text { Mercury }\end{array}$ & Other & Total \\
\hline D\&D of Plutonium Finishing Plant & 10,041 & $\begin{array}{r}0 \\
\end{array}$ & 0 & 0 & 10,041 \\
\hline DED of $T$ Plant & 0 & 2,720 & 0 & 0 & 2,720 \\
\hline High-Level Vitrification Project & 0 & 70 & 109 & 758 & 938 \\
\hline Surplus Facilities & 0 & 50 & 289 & 334 & 674 \\
\hline Plutonium Finishing Plant & 0 & o) & 366 & 0 & 366 \\
\hline D\&D of PUREX & $\mathbf{0}$ & 0 & 9 & 0 & 9 \\
\hline Argonne Mational Laboratory - East & 0 & 0 & 6 & 2 & 8 \\
\hline Pacific Northwest Laboratory & 0 & 0 & 3 & 0 & 3 \\
\hline DED of PNL 231-2 Building & 0 & 0 & 0 & 2 & 2 \\
\hline PUREX & 0 & 0 & 1 & 0 & 1 \\
\hline Ames Laboratory & 0 & 0 & 0 & 0 & 0 \\
\hline Total & 10,041 & 2,840 & 782 & 1,097 & 14,760 \\
\hline $\begin{array}{l}\text { (a) Sum of individual values may } \\
\text { (b) Amounts reported in cubic mete }\end{array}$ & $\begin{array}{l}t \text { match } \\
5 \text {. }\end{array}$ & & & & \\
\hline
\end{tabular}

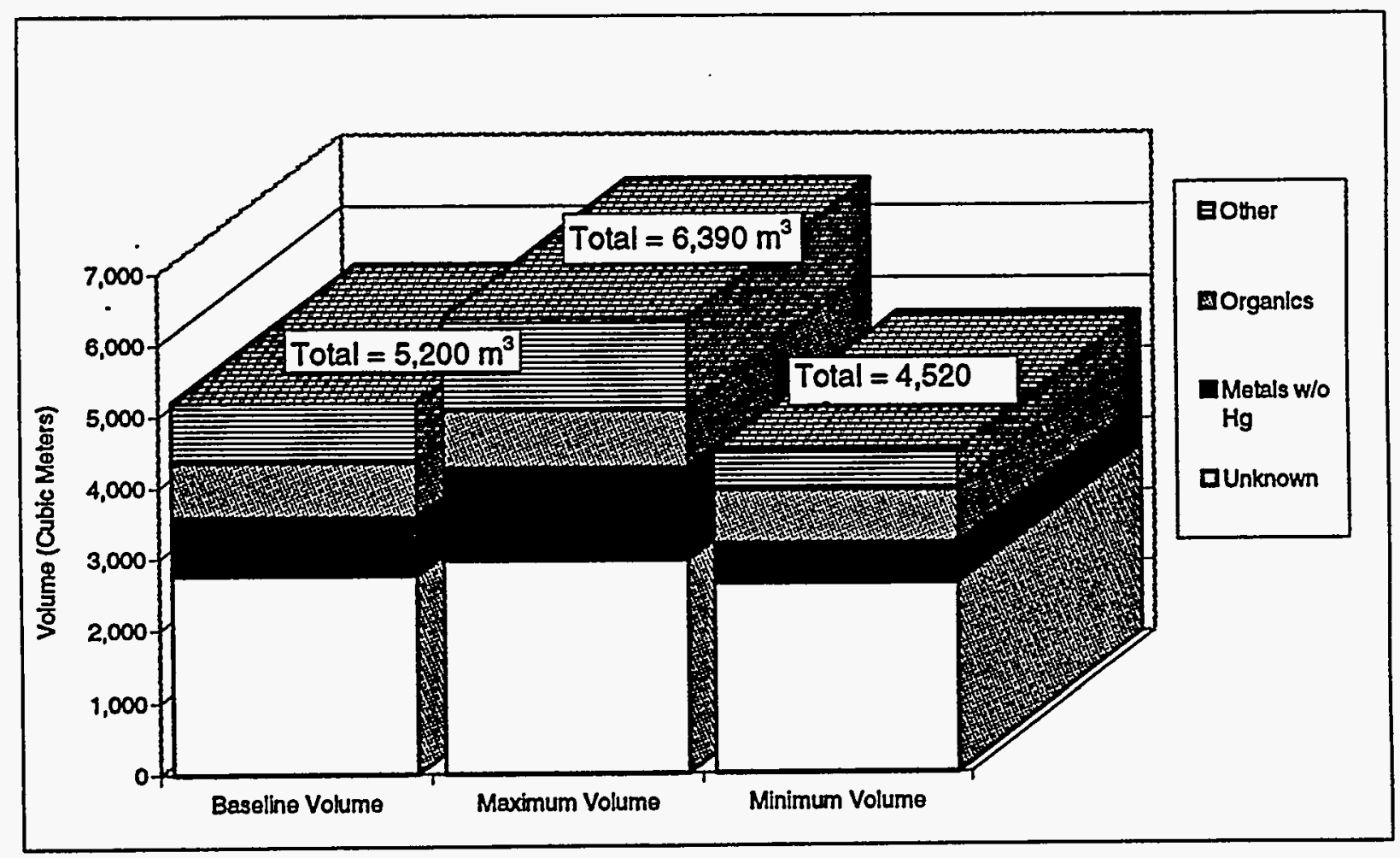

Figure 4-10. RH_TRUM Waste Volumes by Hazardous Waste Category 
depicted, $53 \%$ of the RH TRUM baseline waste does not have hazardous constituent data available. Metals without mercury account for $16 \%$, organics for $15 \%$, and other hazardous components, $16 \%$.

Figure 4-11 displays the annual RH_TRUM volumes by hazardous waste constituents. Less than $50 \mathrm{~m}^{3}$ of RH_TRUM is expected annuality from 1995 through 2003. From 2004 through 2009, there is an increase in waste volume that comprises mainly metals without mercury and other hazardous waste constituents. that are generated by the High-Level Vitrification Project. In 2010, hazardous constituents that were not described will be generated by the Double-She11 Tank Retrieva1. From 2013 through 2022, large amounts of waste with unknown hazardous constituents will be generated by the D\&D of PFP.

Table 4-4 displays the waste generators of RH. TRUM waste by the major hazardous constituents that contaminate the waste. D\&D of PFP has the largest volume of RH TRUM waste that has unavailable hazardous constituent data. Metals without mercury are generated primarily by the High-Level Vitrification Project and Surplus Facilities. Organics are expected to be generated by the D\&D of T Plant.

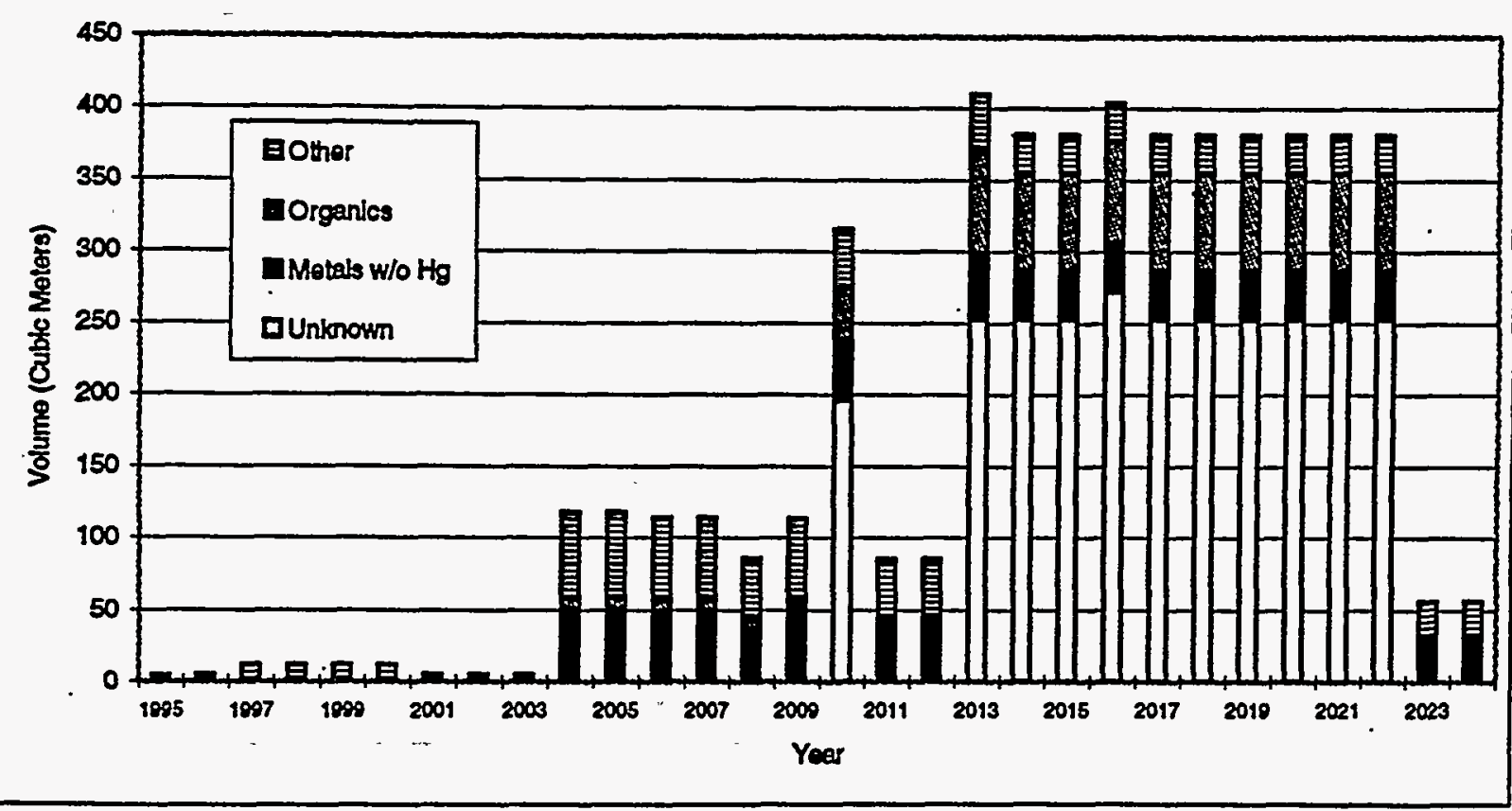

Figure 4-11. Annual RH_TRUM Baseline Waste Volumes by Hazardous Haste Category 
Table 4-4.' RH_TRUM Waste Generators by Hazardous Waste Constituents ${ }^{(a, b)}$

\begin{tabular}{|l|r|r|r|r|r|}
\hline \multicolumn{1}{|c|}{ Waste Generator } & Unknown & $\begin{array}{r}\text { Metals Without } \\
\text { Mercury }\end{array}$ & Organics & Other & $\begin{array}{r}\text { Grand } \\
\text { Total }\end{array}$ \\
\hline \hline D\&D of PFP & 2.510 & 0 & 0 & 0 & 2.510 \\
\hline High-Level Vitrification Project & 0 & 478 & 0 & 419 & 898 \\
\hline Surplus Facilities & 0 & 304 & 53 & 351 & 708 \\
\hline D\&D of T Plant & 0 & 0 & 680 & 0 & 680 \\
\hline DS.T Retrieval (17 tanks) & 215 & 0 & 38 & 0 & 253 \\
\hline Pacific Northwest Laboratory & 7 & 12 & 0 & 28 & 46 \\
\hline D\&D of PUREX & 0 & 32 & 0 & 0 & 32 \\
\hline T Plant & 0 & 0 & 0 & 28 & 28 \\
\hline D\&D of Waste Neutralization Plant & 23 & 0 & 0 & 0 & 23 \\
\hline $222-S$ Laboratory & 0 & 1 & 5 & 9 & 15 \\
\hline D\&D of PNL 327 Building & 0 & 6 & 2 & 0 & 8 \\
\hline \hline Total & 2.755 & 833 & 778 & 836 & 5,201 \\
\hline \hline
\end{tabular}

(a) Sum of individual values may not match totals due to rounding. (b) Amounts reported in cubic meters. 
WHC-EP-0888

Intentionally Left Blank 


\subsection{REFERENCES}

40-CFR-761. 1993. U.S. Environmental Protection Agency. Polychlorinated Biphenyls (PCBs) Manufacturing, Processing, Distribution in Commerce, and Use Prohibitions. U.S. Code of Federal Regulations.

Los A1 amos Technical Associates, Inc. 1994. Estimation of PUREX Equipment and Material.s That are Candidates for Removal and Waste Processing During PUREX Plant Closure. WHC-IP-0977, Westinghouse Hanford Company, Richland, Washington.

Tempieton, K. J., T. J. Deforest, and M. D. Patridge. 1995. 1995 Solid Waste 30-Year Container Volume Summary. WHC-EP-0871, Hestinghouse Hanford Company, Richland, Washington.

VaTero, 0. J., K. J. Templeton, and T. J. DeForest. 1995. 1995 Solid Waste 30-Year Volume Summary. WHC-EP-0865, Hestinghouse Hanford Company, Richland, Hashington.

Washington State Department of Ecology. 1993. Dangerous Waste Regulations. Washington State Administrative Code, 173-303. 


\section{WHC-EP-0888}

\section{Intentionally Left Blank}


WHC-EP-0888

APPERDIX A

\section{LOW-LEVEL WASTE}

A- $\mathbf{i}$ 
WHC-EP-0888

Intentionally Left Blank

A- $i i$ 
WHC-EP-0888

\title{
APPENDIX A
}

\author{
LOW-LEVEL WASTE
}

This appendix provides Tow-level waste (LLW) volumes by physical waste form for both contact-handled (CH) and remote-handled (RH) categories of LLW, which are described below:

- Contact-handled low-level waste (CH_LLH): This waste has a dose rate less than or equal to $200 \mathrm{mrem} / \mathrm{h}$ at contact with the waste container. The waste contains radioactivity that is not classified as transuranic waste; radioactive mixed waste, or spent nuclear fuel. The concentration of transuranic radionuclides is less than or equal to $100 \mathrm{nCi} / \mathrm{g}$ of the waste matrix.

- Remote-handled low-level waste (RH_LLW): This waste has a dose rate greater than $200 \mathrm{mrem} / \mathrm{h}$ at contact with the waste container. The waste contains radioactivity that is not classified as transuranic waste, radioactive mixed waste, or spent nuclear fuel. The concentration of transuranic radionuclides is less than or equal to $100 \mathrm{nCi} / \mathrm{g}$ of the waste matrix.

Hazardous waste constituent data will not be described, since LLW does not have hazardous constituents; only low-level mixed waste, which is described in Section 3.0 of the main report, contains hazardous constituents. The major generators of LLW will also be provided in this appendix; however, a detailed discussion of the associated waste generator missions is contained in Valero et a1. (1995). LLW will not be handled by the SWOC except for selective sampling. Current plans are to send the majority of the LLW directly to the Hanford LLW burial grounds.

\section{A.1 LOW-LEVEL WASTE VOLUME}

Approximately $1,461,580 \mathrm{~m}^{3}$ of $\mathrm{LLW}$ is expected at the Hanford Site over the next 30 years. Of this waste, $96 \%$ is $\mathrm{CH}$ LLH, and the remaining $4 \%$ is RH_LLW, as depicted in Figure $A-1$. Figure $A-1$ also displays the expected maximum and minimum waste volumes, $1,594,180 \mathrm{~m}^{3}$ and $1,381,860 \mathrm{~m}^{3}$, respectively. Figure A-2 displays the annual baseline volume of $L L H$ by waste category. As depicted in the figure, waste volumes gradually increase from 2002 through 2007 , when they peak at approximately $210,000 \mathrm{~m}^{3}$. This peak corresponds to the anticipated shipping schedule of Surplus Facilities.

Figure A-3 displays the major waste generators of LLW. Seventy-six percent will be generated by Surplus Facilities; the Formerly Utilized Sites Remedial Action Program (FUSRAP) will generate 4\%; D\&D of B Plant will generate $3 \%$; and the remaining $17 \%$ will be generated by other miscellaneous waste generators. 
WHC-EP-0888

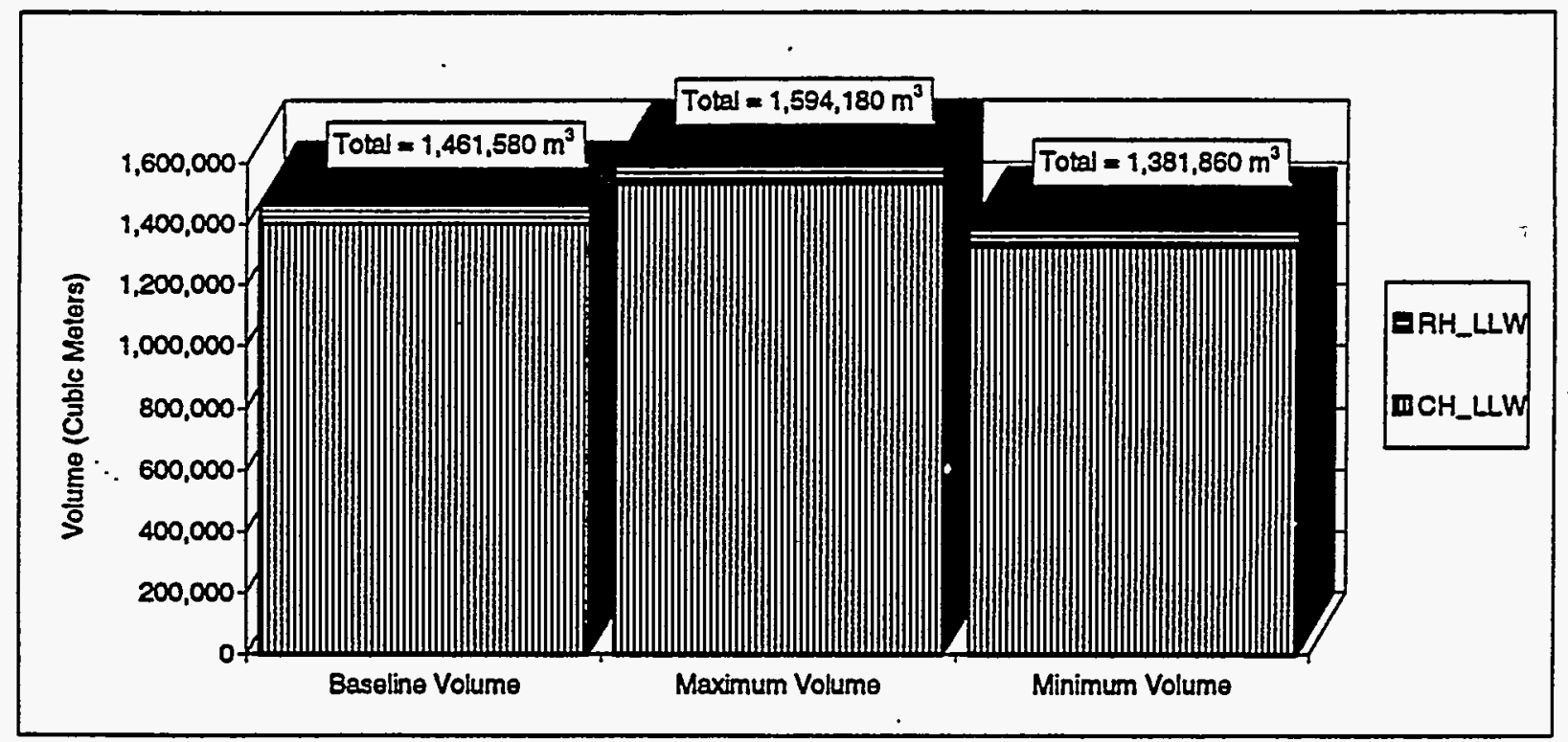

Figure A-1. Total LLW Volume by Waste Category

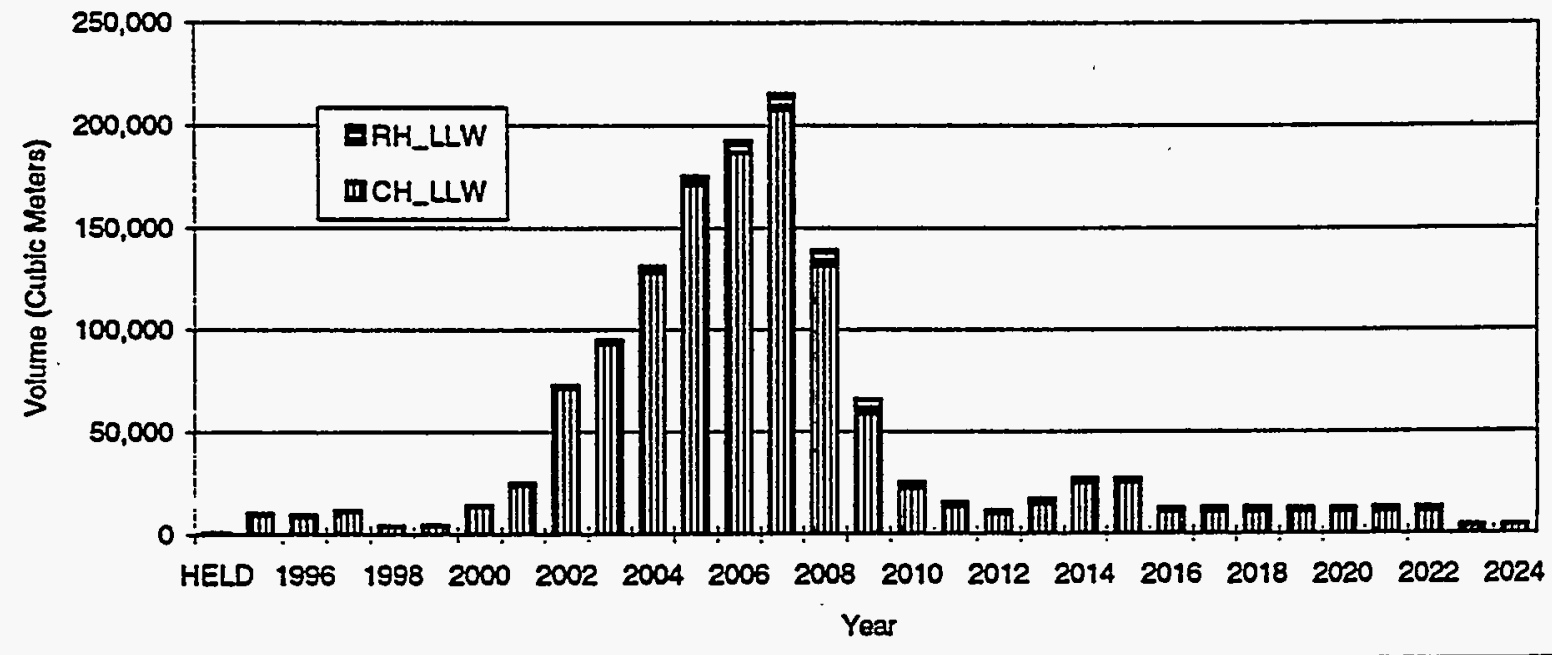

Figure A-2. Annual LLW Baseline Volume by Haste Category

\section{A.2 LOW-LEVEL WASTE CATEGORIES BY PHYSICAL WASTE FORM}

This section describes the LLW volumes by waste category and physical waste form. The total volume and the annual volume by physical waste form are discussed. The major waste generators for each waste category are also provided. 


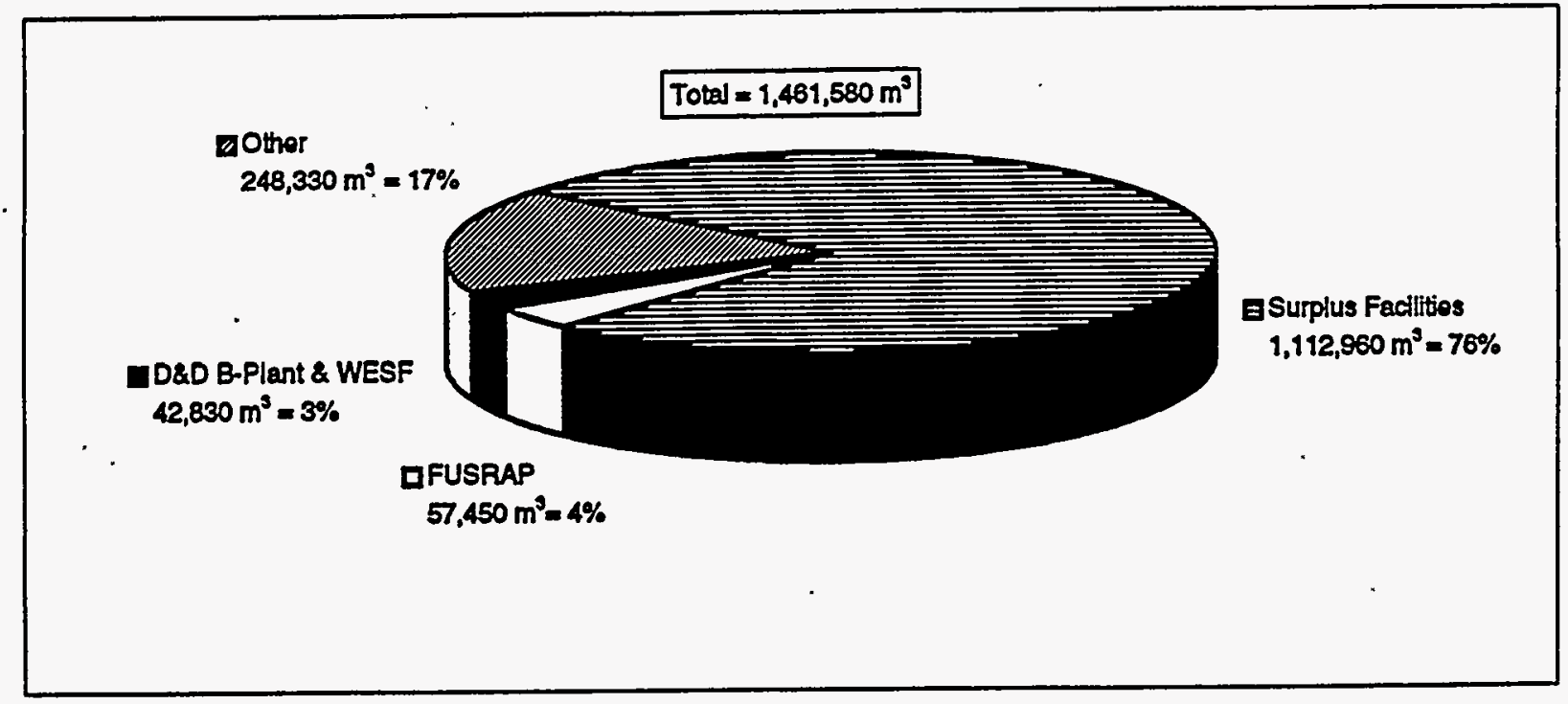

Figure A-3. Major LLW Generators

\section{A.2.1 Contact-Handled Low-Level Waste}

The total baseline amount of CH LLW to be shipped to the Hanford Site over the next 30 years is $1,403,200 \mathrm{~m}^{3}$. Figure A-4 displays the baseline, maximum, and minimum volumes of CH_LLW by physical waste form. The maximum and minimum expected volumes are $1,532,370 \mathrm{~m}^{3}$ and $1,325,770 \mathrm{~m}^{3}$, respectively. As depicted in the figure, inorganic non-metal debris will make up the largest (57\%) portion of $\mathrm{CH}$ LLW; contaminated soil will be $13 \%$; metal debris and inorganic absorbed Tiquid/sludges will each be $7 \%$; and other physical waste forms will constitute $15 \%$ of the CH_LLW.

Figure A- 5 displays the annual $\mathrm{CH}_{-}$LLW baseline volumes by physical waste form. As shown in the figure, there is a gradual increase in waste from 2002 through 2007. This peak represents the shipping schedule of inorganic nonmetal debris by Surplus Facilities.

The waste generators for CH_LLW by major physical waste form are displayed in Table A-1. Surplus Facilities is the major waste generator of CH LLW and the primary generator of all major CH_LLW physical waste forms except metal debris. FUSRAP generates primarily inorganic non-metal debris, and the D\&D of B PTant will mainly generate metal debris.

\section{A.2.2 Remote-Handled Low-Level Waste}

The total base7ine amount of RH_LLW to be shipped to the Hanford Site over the next 30 years is $58,390 \mathrm{~m}^{3}$. The maximum and minimum expected volumes are $61,800 \mathrm{~m}^{3}$ and $56,090 \mathrm{~m}^{3}$, respectively. Figure 4-6 displays the baseline, maximum, and minimum volumes by physical waste forms. As depicted, inorganic 


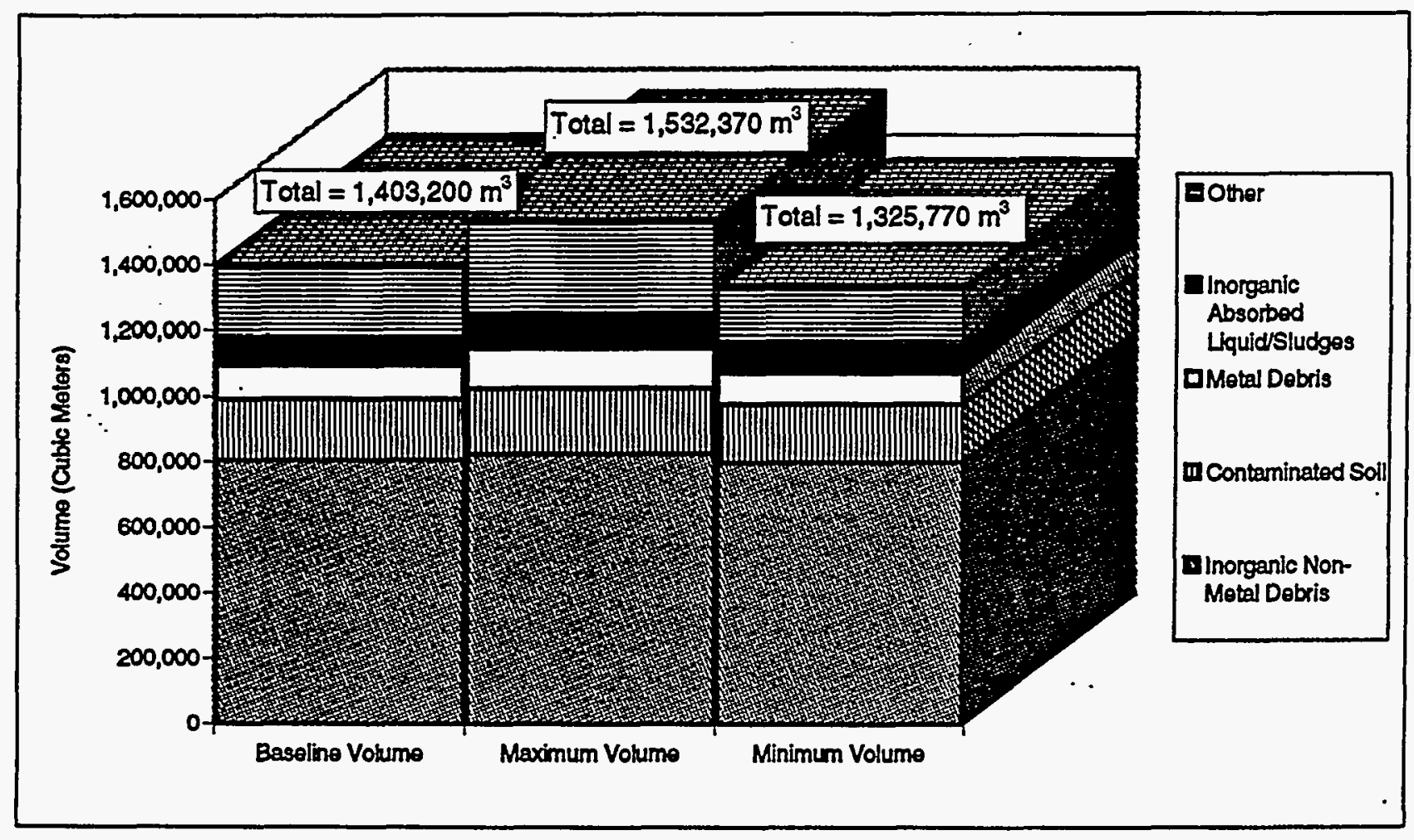

Figure A-4. Annual LLW Baseline Volume by Waste Category

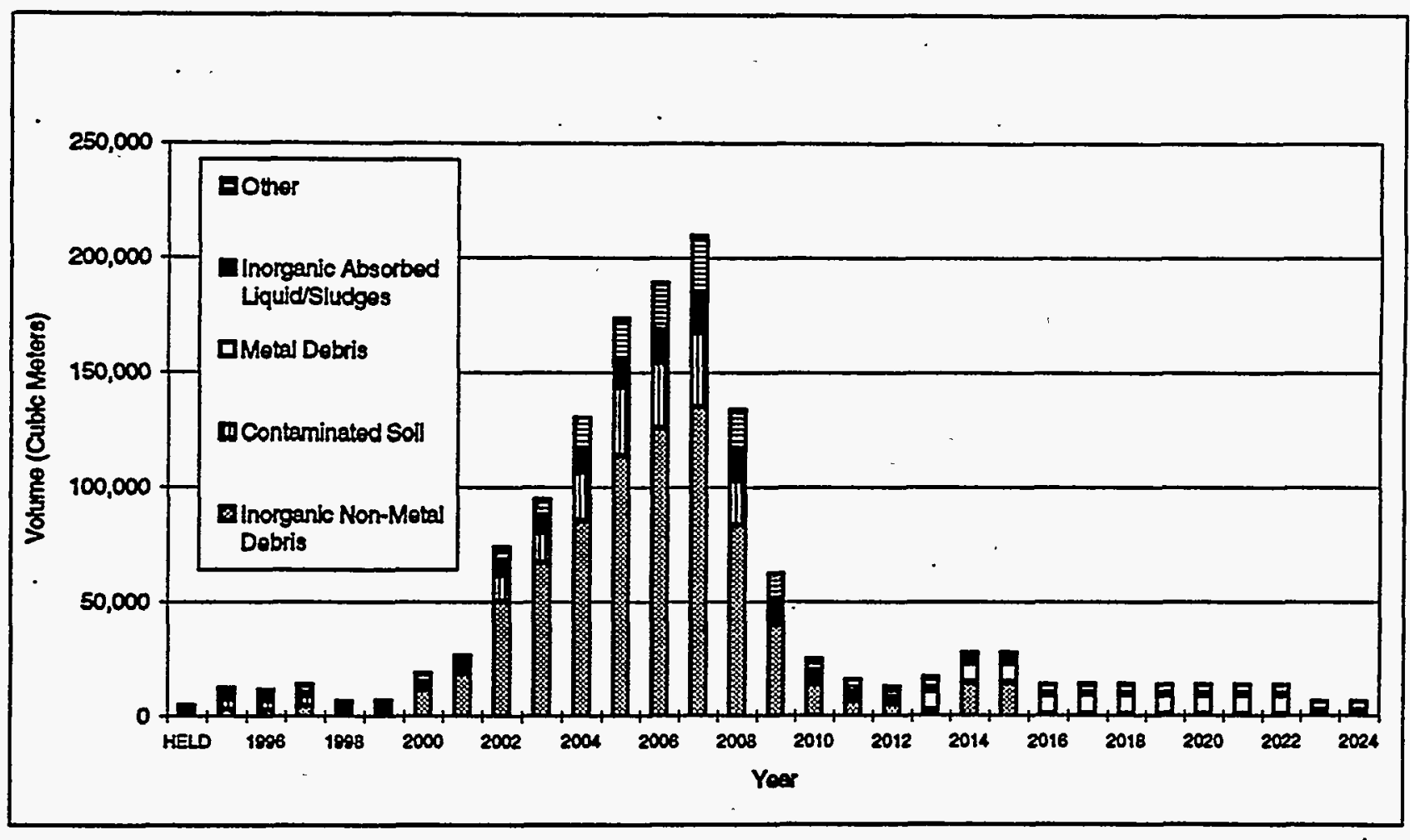

Figure A-5. RH_LLW Volumes by Physical Waste Form 
Table A-1. CH_LLW Generators by Physical Waste Form ${ }^{(a, b)}$

\begin{tabular}{|c|c|c|c|c|c|c|}
\hline Waste Generator & $\begin{array}{c}\text { Inorganic } \\
\text { Non-Metá] } \\
\text { Debris }\end{array}$ & $\begin{array}{c}\text { Contaiminated } \\
\text { Soil }\end{array}$ & $\begin{array}{l}\text { Metal } \\
\text { Debris }\end{array}$ & $\begin{array}{c}\text { Inorganic } \\
\text { Absorbed } \\
\text { Liquid/Sludges }\end{array}$ & Other & Total \\
\hline Surplus Facilities & 736,313 & 146,919 & $\overline{0}$ & 83,501 & 106,288 & $1,073,020$ \\
\hline FUSRAP & 40,727 & 15,401 & $\overline{0}$ & 0 & 1,321 & 57,450 \\
\hline D\&D of B Plant & 0 & 0 & 34,267 & 0 & 0 & 34,267 \\
\hline High-Level Vitrification -Project & 2,016 & 0 & 2,016 & $\mathbf{0}$ & 16,127 & 20,158 \\
\hline Brookhaven National Laboratory & 1,566 & 1,044 & 7,973 & 664 & 7,736 & 18,983 \\
\hline Pacific Northwest Laboratory & 0 & 37 & 0 & $\mathbf{0}$ & 18,307 & 18,344 \\
\hline Lawrence Berkeley Laboratory & 13,943 & 0 & 1,073 & $\mathbf{0}$ & 760 & 15,776 \\
\hline Portsmouth - Energy Systems & 216 & 10,140 & 17 & 4,850 & 256 & 15,480 \\
\hline D\&D of 222-S Laboratory & 0 & 0 & 11,838 & 0 & 965 & 12,803 \\
\hline Low-Level Vitrification Project & 1,134 & 0 & 1,134 & 0 & 9,075 & 11,344 \\
\hline 300 Area Treated Effluent Disposal Facility & 0 & 0 & 0 & 9,944 & 1,105 & 11,049 \\
\hline $222-5$ Laboratory & 882 & 110 & 3,866 & 0 & 5,365 & 10,222 \\
\hline Argonne National Laboratory - East & 1,102 & 3,157 & 1,004 & 129 & 4,365 & 9,758 \\
\hline Tank Farm Operations & 1,558 & 0 & 2,266 & 71 & 5,046 & 8,940 \\
\hline D\&D of $N$ Reactor & 0 & of & 5,183 & 0 & 3,144 & 8,326 \\
\hline Princeton Plasma Physics Laboratory & 728 & 0 & 4,528 & 0 & 2,903 & 8,159 \\
\hline D\&D of T Plant & 0 & 0 & 7.144 & 0 & 0 & 7.144 \\
\hline Paducah - Energy Systems & 1,091 & 4,509 & 0 & 0 & 1,091 & 6,690 \\
\hline Plutonium Finishing Plant & 599 & 139 & 774 & 0 & 4,660 & 6,171 \\
\hline Tank Farm Pretreatment Facility & 596 & 0 & 596 & 0 & 4,764 & 5,956 \\
\hline D\&D of PUREX & 0 & 0 & 4,768 & 0 & 0 & 4,768 \\
\hline B Plant & 368 & 351 & 376 & 159 & 3,174 & 4,428 \\
\hline K Basin Operations & 0 & 0 & 1,434 & 0 & 2,992 & 4,426 \\
\hline Laundry/Equipment Decontamination & 0 & 0 & 0 & 0 & 3,399 & 3,399 \\
\hline D\&D of PNL 314 Building & 0 & 0 & 3,178 & 0 & 0 & 3,178 \\
\hline DST Retrieval Systems (10 tanks) & 0 & 1,785 & 198 & 0 & 0 & 1,983 \\
\hline D\&D of PNL 331 Building & 0 & 0 & 1,717 & 0 & 191 & 1,908 \\
\hline University of California - Davis & 83 & 1,552 & 49 & 0 & 152 & 1,836 \\
\hline Portsmouth - Utility Services & 0 & 0 & 355 & 0 & 1,421 & 1,776 \\
\hline Battelle Columbus Laboratories & 601 & 195 & 360 & 0 & 345 & 1,501 \\
\hline 100 Area Transition & 647 & 0 & 0 & 0 & 733 & 1,380 \\
\hline FERMI National Accelerator Laboratory & 27 & 16 & 1,083 & 32 & 197 & 1,356 \\
\hline Rockwell - Canoga Park & 191 & 255 & 255 & 13 & 561 & 1,274 \\
\hline D\&D of PNL 327 Building & 0 & 0 & 907 & 0 & 242 & 1,149 \\
\hline Stanford Línear Accelerator Center & 1 & 0] & 942 & $\mathbf{0}$ & 14 & 958 \\
\hline Paducah - Utility Services & 0 & 0 & 371 & 0 & 556 & 926 \\
\hline General Atomics & of & 101 & 0 & 0 & 609 & 710 \\
\hline DST Retrieval (17 tanks) & 0) & 605 & 67 & 0 & 0 & 673 \\
\hline Tank 106-C Manipulator & 0 & 0 & 49 & 0 & 570 & 620 \\
\hline \begin{tabular}{|l|} 
PUREX \\
\end{tabular} & 39 & 8 & 192 & 0 & 325 & 564 \\
\hline SST Retrieval (149 tanks) & 119 & 0 & 173 & 5 & 243 & 540 \\
\hline T Plant & 21 & 21 & 64 & 4 & 315 & 426 \\
\hline Cross-Site Transfer System & of & 365 & 4 & 0 & 0 & 368 \\
\hline DED of PNL 324 Building & 0 & 0 & 292 & 0 & 68 & 360 \\
\hline PUREX Surveilliance and Maintenance & 21 & 4 & 107 & 0 & 186 & 318 \\
\hline Knolls Atomic Power Shipyards & 0 & 0 & 297 & 0 & 0 & 297 \\
\hline EG\&G Rocky Flats Plant & 16 & 0 & 2 & 0 & 273 & 291 \\
\hline 300 Area Fuel Supply & 65 & 23 & 88 & 0 & 44 & 220 \\
\hline
\end{tabular}


Table A-1. (contd)

\begin{tabular}{|c|c|c|c|c|c|c|}
\hline Waste Generator & $\begin{array}{c}\text { Inorganic } \\
\text { Non-Hetai } \\
\text { Debris }\end{array}$ & $\begin{array}{c}\text { Contaninated } \\
\text { Soil }\end{array}$ & $\begin{array}{l}\text { Metal } \\
\text { Debris } \\
\end{array}$ & $\begin{array}{c}\text { Inorganic } \\
\text { Absorbed } \\
\text { Liquid/Sludges } \\
\end{array}$ & Other & Total \\
\hline D\&D of Waste Neutralization Facility & 0 & 0 & 125 & 0 & 74 & 198 \\
\hline Tank 106-C Sluicing & 70 & 12 & 70 & 0 & 40 & 192 \\
\hline Low-Level Waste Burial Grounds & 0 & 13 & 5 & 0 & 171 & 189 \\
\hline Bettis Atomic Power Laboratory & 6 & 0 & 126 & 0 & 21 & 153 \\
\hline D\&D of Fuels Developinent Laboratory & 5 & 0 & 54 & 0 & 69 & 129 \\
\hline FFTF & 0 & 0 & 28 & 0 & 73 & 101 \\
\hline Ames National Laboratory & 68 & 0 & 0 & 0 & 29 & 97 \\
\hline D\&D of PHL 242 Building & 0 & 0 & 62 & 0 & 34 & 96 \\
\hline Kaiser Construction Services & 13 & 64 & 0 & 0 & 0 & 77 \\
\hline Tank Farm Ventilation Upgrades ... & 26 & 10 & 14 & 0 & 6 & 57 \\
\hline D\&D of PNL 306W Building & 0 & 0 & 44 & 0 & 7 & 51 \\
\hline Well Drilling & 4 & 31 & 2 & 0 & 2 & 39 \\
\hline Geotechnical Engineering Laboratory & 0 & 0 & 16 & 0 & 16 & 31 \\
\hline 101-AZ Retrieval (1 tank) & 0 & 3 & 0 & 0 & 16 & 19 \\
\hline Fueis Development Laboratory & 2 & 0 & 5 & 0 & 12 & 19 \\
\hline D\&D of PNL 326 Building & 0 & 0 & 12 & 0 & 5 & 16 \\
\hline TRUSAF & 0 & 0 & 1 & 0 & 10 & 11 \\
\hline University of Utah & 0 & 0 & 0 & 0 & 9 & 9 \\
\hline Tank 106-C Manipulator & 0 & 8 & 0 & 0) & 0 & 8 \\
\hline Aging Waste Transfer Lines & 0 & 6 & 1 & 0 & 0] & 7 \\
\hline Bettis Atomic Power Shipyards & 0 & 0 & 7 & 0 & 0 & 7 \\
\hline $\begin{array}{l}\text { D\&D of Development/Fabrication Test } \\
\text { Laboratory }\end{array}$ & 0 & 0 & 2 & 0 & 4 & 5 \\
\hline D\&D of PML 2312 Building & 0 & 0 & 0 & 0 & 4 & 4 \\
\hline 384 Power House & 1 & 1 & 0 & 0 & 1 & 3 \\
\hline Bates Accelerator, MA & 0 & 0 & 0] & 0 & 2 & 2 \\
\hline Large Sodium Fire Facility & 0 & 0 & 0 & 0 & 2 & 2 \\
\hline Pilot-Scale Retrieval & 0 & 0 & 1 & 01 & 이 & 1 \\
\hline Total & 804,863 & 186,885 & 101,584 & 99,373 & 210,492 & $1,403,197$ \\
\hline \multicolumn{7}{|c|}{$\begin{array}{l}\text { (a) Sum of individual values may not match totals due to rounding. } \\
\text { (b) Amounts reported in cubic meters. }\end{array}$} \\
\hline
\end{tabular}

and organic absorbed 1iquid/sludges each will comprise $27 \%$ of the RH LLW. Metal debris will be $22 \%$, inorganic non-metal debris will make up $16 \overline{\%}$, and other physical waste forms will be $8 \%$ of the RH_LLW.

Figure A-7 displays the annual RH LLH baseline volume by physical waste form. The figure shows a peak from $20 \overline{2} 2$ through 2009 , which corresponds to the shipping schedule of absorbed liquid/sludges from the Surplus Facilities waste generator.

The waste generators for RH LLW by major physical waste forms are provided in Table A-2. Surplus Facilities will be the only producer of inorganic and organic absorbed liquid/sludges. Metal debris will be generated primarily by D\&D of B Plant, and inorganic non-metal debris will be primarily generated by Surpius Facilities. 


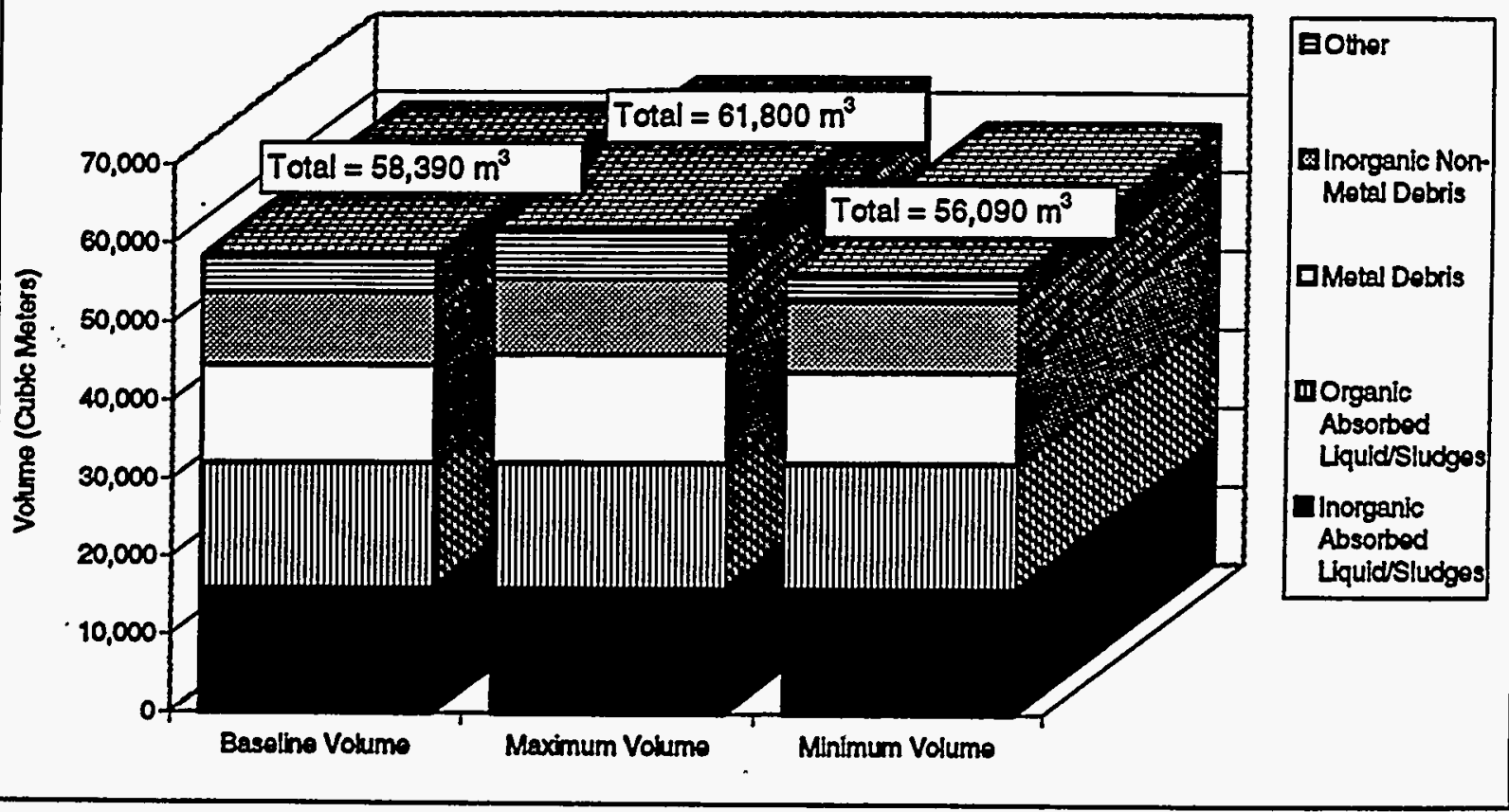

Figure A-6. RH_LLW Volumes by Physical Waste Form

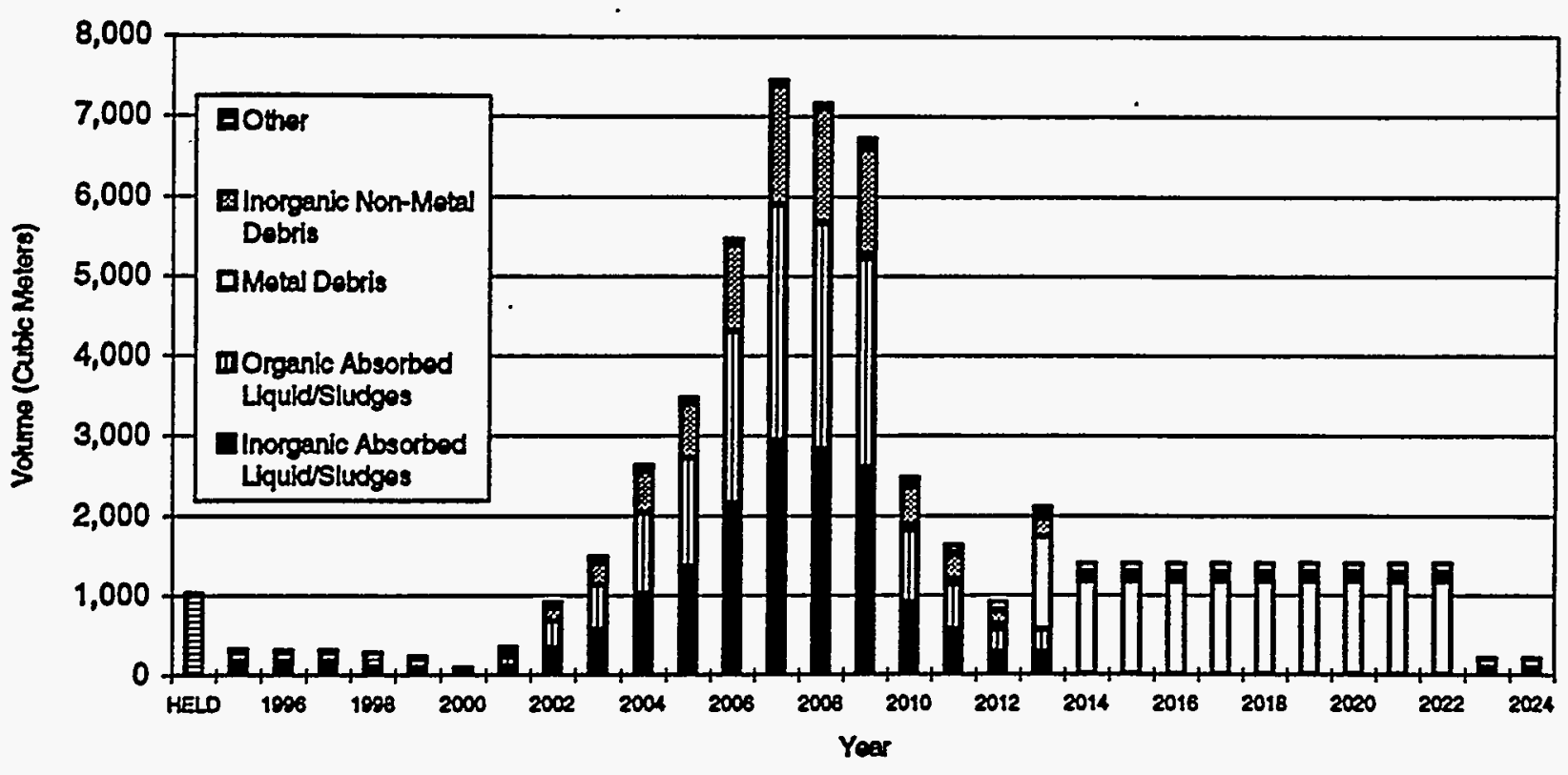

Figure A-7. RH_LLW Annual Baseline Volumes by Physical Waste Form 
Table A-2. RH_LLW Generators by Physical Waste Form ${ }^{(a, b)}$

\begin{tabular}{|c|c|c|c|c|c|c|}
\hline Haste Generator & \begin{tabular}{|c|} 
Inorganic \\
Absorbed \\
Liquid/Sludges
\end{tabular} & $\begin{array}{c}\text { Organic } \\
\text { Absorbed } \\
\text { Liquid/sludges }\end{array}$ & $\begin{array}{l}\text { Metal } \\
\text { Debris }\end{array}$ & $\begin{array}{c}\text { Inorganic } \\
\text { Non-Metal } \\
\text { Debris }\end{array}$ & lother & Total \\
\hline Surplus Facilites & 15,977 & 15,977 & 0 & 7,989 & 0 & 39,943 \\
\hline D\&D of B Plant & 0 & $\mathbf{0}$ & 8,567 & 0 & 0 & 8,567 \\
\hline High-Level Vitrification Project & 0 & 0 & 914 & 203 & 914 & 2,030 \\
\hline D\&D of T Plant & 0 & $\mathbf{0}$ & 1,786 & 0 & 0 & 1,786 \\
\hline Pacific Northwest Laboratory & 0 & 0 & 0 & 1 & 1,728 & 1,729 \\
\hline D\&D of PUREX & 0 & 0 & 400 & 792 & 0 & 1,192 \\
\hline PUREX Tunnel Waste & 0 & 0 & 0 & 0 & 1,022 & 1,022 \\
\hline B Plant & 0 & $\mathbf{0}$ & 157 & 157 & 298 & 612 \\
\hline Low-Level Vitrification Project. & 0 & 0 & 196 & 44 & 196 & 436 \\
\hline D\&D of PNL 327 Building & 0 & 0 & 214 & 0 & 101 & 315 \\
\hline Tank Waste Pretreatment Facility & 0 & 0 & 137 & 30 & 137 & 305 \\
\hline General Atomics & 0 & 0 & 99 & 33 & 0 & 132 \\
\hline$T$ Plant & 0 & 0 & 43 & 4 & 38 & 85 \\
\hline 100 Area Transition & 0 & 0 & $\mathbf{0}$ & 49 & 10 & 59 \\
\hline FFTF & 0 & 0 & 3 & 0 & 45 & 48 \\
\hline K Basin Operations & 0 & 0 & 17 & $\mathbf{0}$ & 29 & 47 \\
\hline Argonne National Laboratory - East & 0 & $\mathbf{0}$ & 32 & 4 & 0 & 36 \\
\hline D\&D of PNL 324 Building & 0) & 0 & 26 & $\overline{0}$ & 4 & 30 \\
\hline Brookhaven National Laboratory & of & 0 & 4 & 0 & 5 & 10 \\
\hline Tank 106-C Manipulator & 0) & $\mathbf{0}$ & $\mathbf{0}$ & 6 & 0 & 6 \\
\hline Total & 15,977 & 15,977 & 12,594 & 9,312 & 4,527 & 58,387 \\
\hline
\end{tabular}


WHC-EP-0888

\section{APPENDIX B}

\section{WASTE FORH AND HAZARDOUS CONSTITUENT}

FORECAST REQUEST FORM

B- $\mathbf{i}$ 
WHC-EP-0888

Intentionally Left Blank

$B-i i$ 


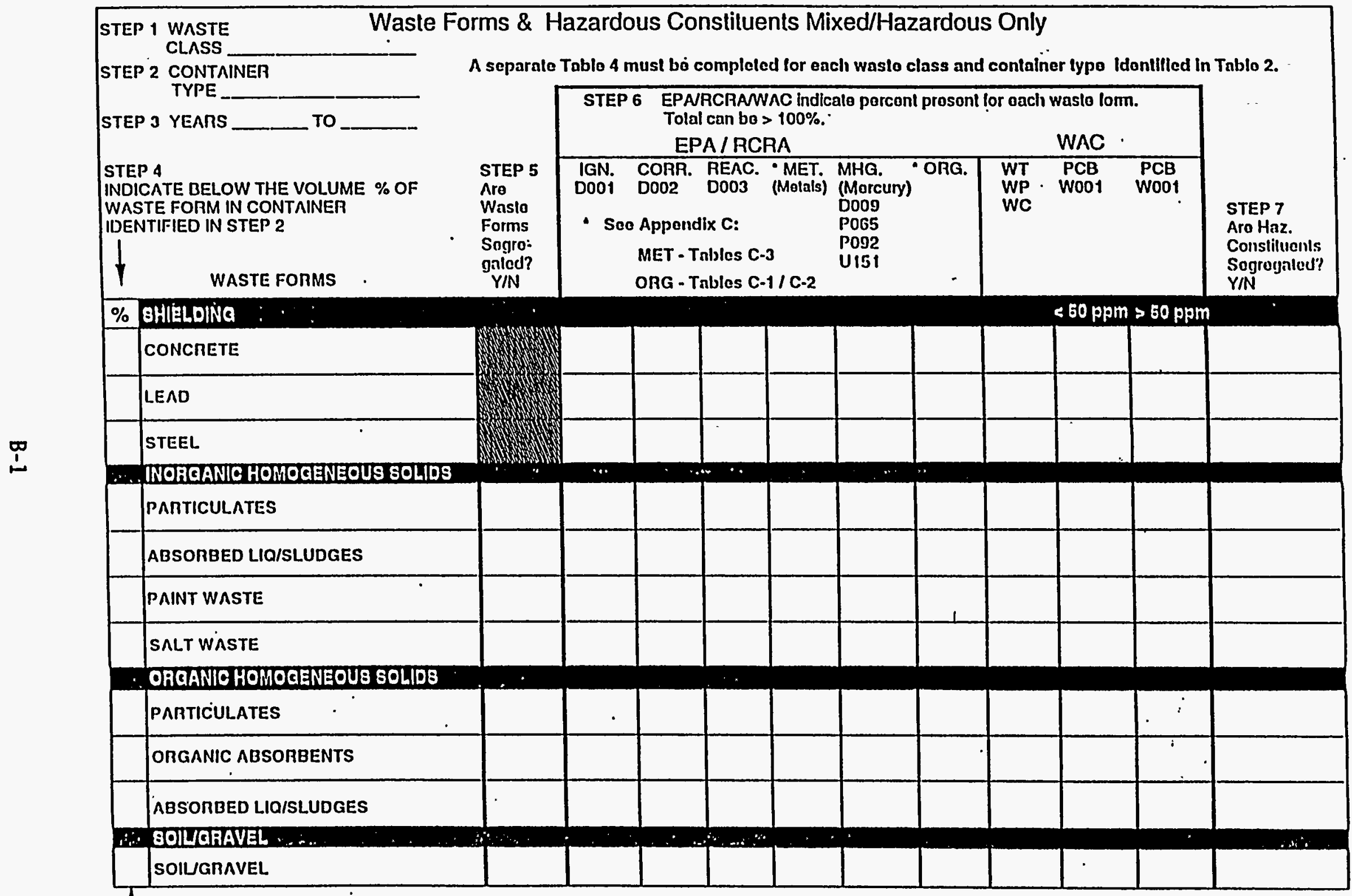

NOTE: Waste Form \% should be totaled on Table 4 Page 2 of 2 and MUST equal 100\%. 


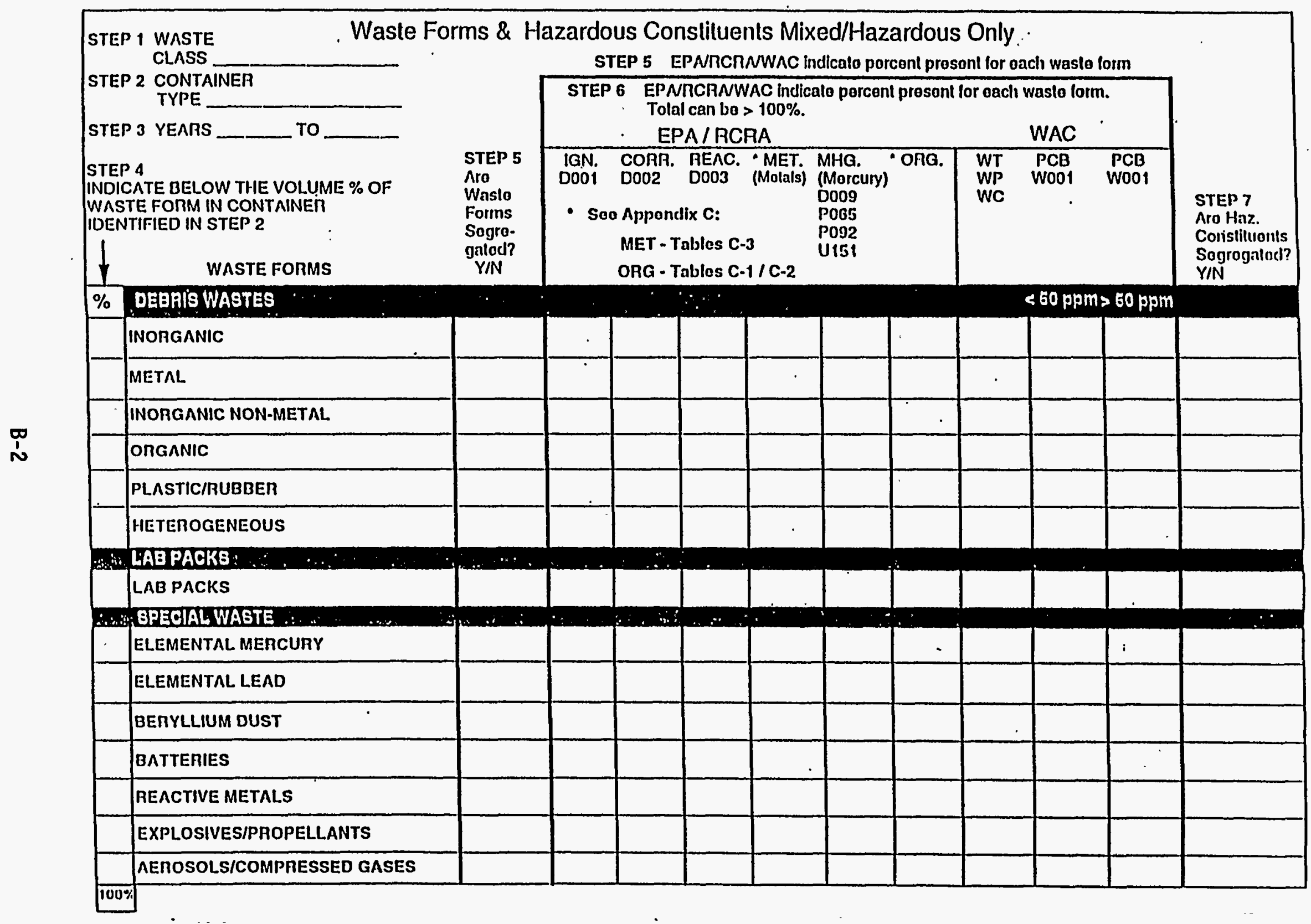


WHC-EP-0888

APPENDIX C

ANNUAL MASTE CATEGORY VOLUMÉS BY PHYSICAL WASTE FORM AND HAZARDOUS WASTE CONSTITUENT 
WHC-EP-0888

Intentional1y Left $\mathrm{B}$ lank

$C-i j$ 
: $\quad$ oे

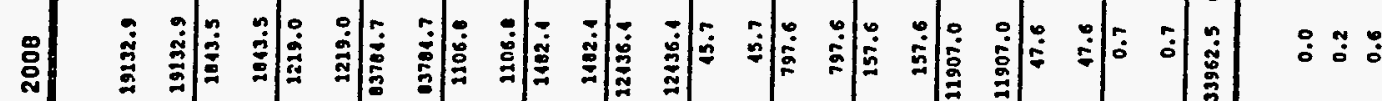

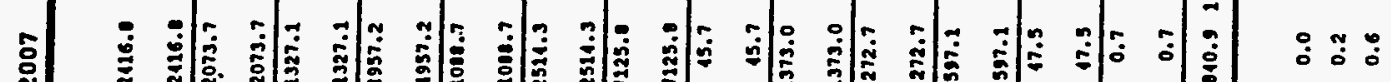

N

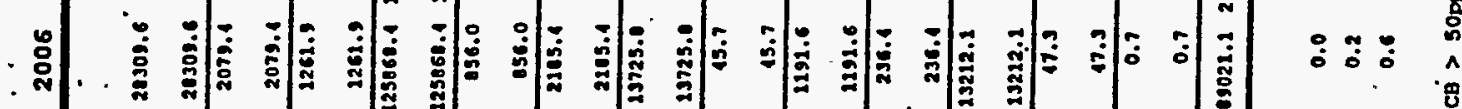

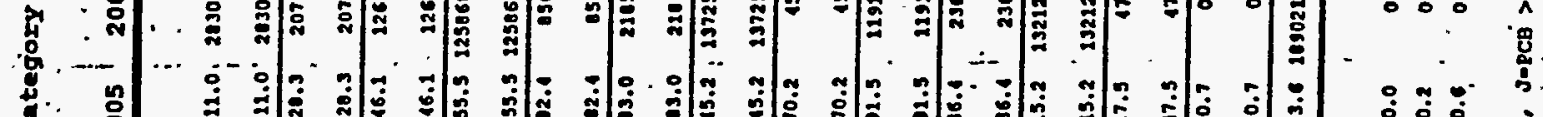

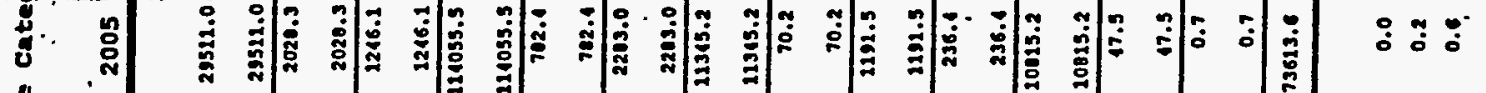

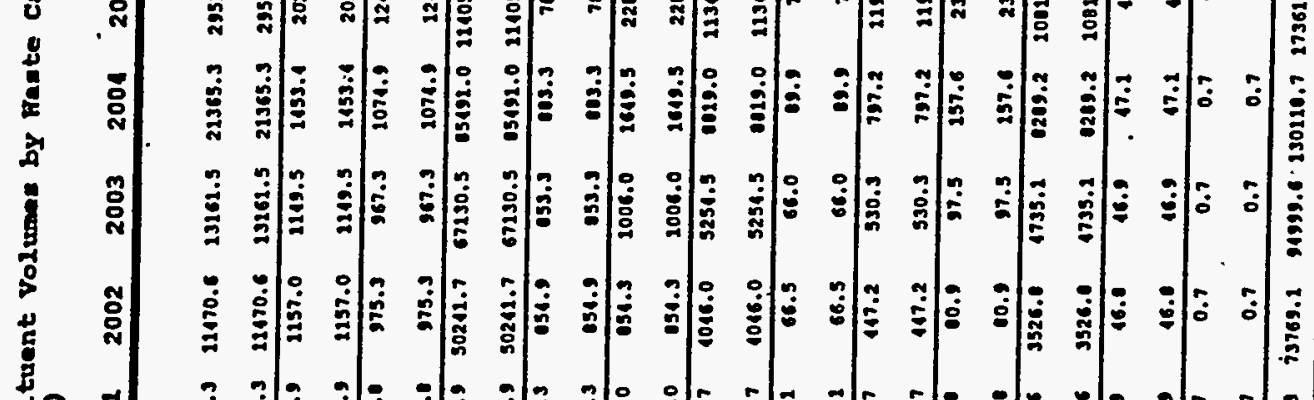

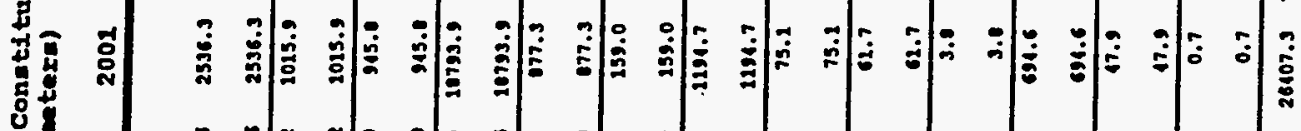

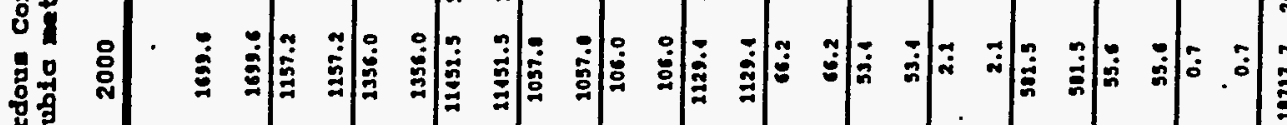

要

照

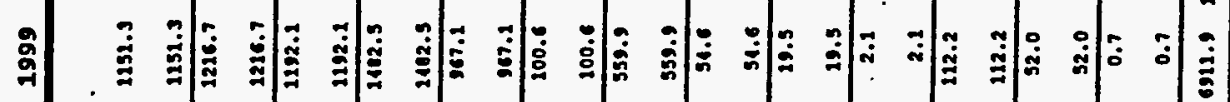

:웅

$\frac{1}{x}$

:ํํㅇ

:ํㅗㅇํำ

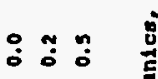

:: : :

$\div:$

(2)

$\stackrel{0}{0}: \stackrel{0}{\circ}$

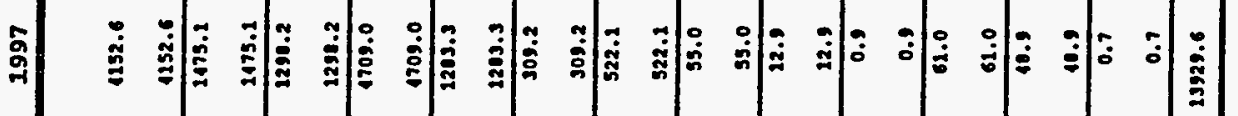

뭉ํํ.

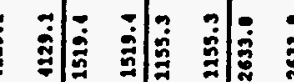

วิे

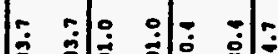

: :

$\therefore$ :

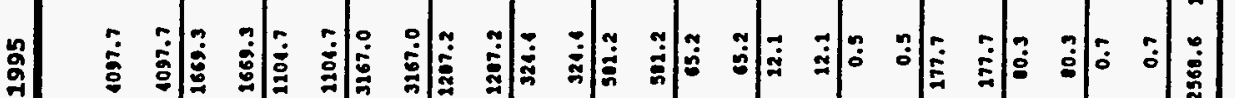

ํํำ

ㅇํㅇ

บับ

恖

$\stackrel{\circ}{\circ}: \stackrel{\circ}{\circ}$

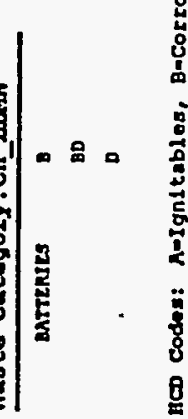


Annual Physical Waste Form and Hazaxdous Constituent Volumes by Waste Category (in cubio meters)

\begin{tabular}{|c|c|c|c|c|c|c|c|c|c|c|c|c|c|c|c|c|c|}
\hline FWF & $\mathrm{HCD}$ & Held & 1995 & 1996 & 1997 & 1998 & 1999 & 2000 & 2001 & 2002 & 2003 & 2004 & 2005 & 2006 & 2007 & 2008 & 2009 \\
\hline TOtal BATTERIEs & & 0.0 & 2.6 & 3.5 & 2.1 & 2.1 & 1.2 & 0.1 & 0.7 & 0.1 & 0.7 & 0.7 & 0.0 & 0.0 & 0.0 & 0.8 & 1.7 \\
\hline BERYLLIUYH & D & 0.0 & 0.2 & 0.9 & 3.9 & 2.2 & 0.5 & 0.2 & 0.2 & 0.2 & 0.2 & 0.2 & 0.2 & 0.5 & 3.9 & 3.9 & $\overline{0.5}$ \\
\hline TOLAI BERYLLAUM & & 0.0 & 0.2 & 0.9 & 3.9 & 2.2 & 0.5 & 0.2 & 0.2 & 0.2 & 0.2 & 0.2 & 0.2 & 0.5 & 3.9 & 3.9 & 0.5 \\
\hline CONT soll & $\bar{n}$ & 0.0 & 0.1 & 0.0 & 0.0 & 0.0 & 0.1 & 0.0 & 1.1 & 2.1 & 1.1 & 3.5 & 3.5 & 5.9 & 5.9 & 3.9 & $\overline{5.9}$ \\
\hline \multirow{10}{*}{. } & AC & 0.0 & 2.8 & 3.0 & 3.0 & 3.0 & 3.0 & 3.0 & 3.0 & 3.0 & 3.0 & 3.0 & 3.0 & 3.0 & 3.0 & 3.0 & 3.0 \\
\hline & AEFG & 0.0 & 0.5 & 0.0 & 0.1 & 0.4 & 0.0 & 0.0 & 0.0 & 0.0 & 0.0 & 0.0 & 0.0 & 0.0 & 0.0 & 0.0 & 0.0 \\
\hline & : & 0.0 & 1.7 & 0.3 & 1061.6 & 043.4 & 2031.4 & 969.9 & 985.0 & 2058.8 & 1308.3 & 1437.2 & 1581.3 & 1660.1 & 1668.1 & 1675.1 & 1597.8 \\
\hline & D & 0.0 & 15.7 & 1.8 & 1063.7 & 899.3 & 1125.3 & 1154.0 & 1190.6 & 1192.6 & 1423.2 & 1458.3 & 2584.1 & 1672.3 & 1672.3 & 1679.3 & 1602.0 \\
\hline & DE & 24.5 & 03.0 & 83.7 & 84.3 & 03.7 & 03.7 & 83.7 & 83.7 & 03.7 & 83.7 & 05.6 & 85.6 & 86.3 & 86.9 & 86.9 & 08.2 \\
\hline & $\varepsilon$ & .0 .0 & 0.0 & 0.0 & 0.0 & 0.0 & 0.0 & 0.0 & 0.0 & 0.0 & 0.0 & 0.0 & 0.0 & 0.0 & 0.0 & 0.0 & 0.0 \\
\hline & $\mathbf{r}$ & 14.5 & 77.7 & 77.6 & 77.6 & 131.6 & 170.1 & 260.6 & 201.7 & 209.9 & 191.2 & $97.3^{\circ}$ & 79.3 & 80.4 & 80.1 & 80.1 & 80.1 \\
\hline & FG & 0.0 & 0.2 & 0.9 & 0.7 & 0.5 & 0.5 & 0.5 & 0.0 & 0.0 & 0.0 & 0.0 & 0.0 & 0.0 & 0.0 & 0.0 & 0.0 \\
\hline & 0 & 0.0 & 0.0 & 0.0 & 0.0 & 108.3 & 184.8 & 365.3 & 107.5 & 263.7 & 226.0 & 36.1 & 0.0 & 0.0 & 0.0 & 0.0 & 0.0 \\
\hline & $\mathbf{J}$ & 0.0 & 0.0 & 0.0 & 0.0 & 0.0 & 0.0 & 0.2 & 0.0 & 0.0 & 0.0 & 0.0 & 0.0 & 0.0 & 0.0 & 0.0 & 0.0 \\
\hline TOL.n1 CONT SOIL & & 29.1 & 202.5 & 168.6 & 2291.9 & 20170.6 & $2 ! 990.0$ & 28.37 .6 & 2953.3 & 2.813 .4 & 3237.0 & 3121.8 & 3337.6 & 3516.5 & 3517.2 & 3531.3 & 3378.0 \\
\hline D-cotitust & $\Lambda$ & 0.0 & 1.1 & 0.8 & 2.1 & 3.8 & 1.6 & 0.4 & 1.5 & 1.5 & 1.5 & 3.8 & 9.1 & 6.7 & 7.6 & 7.6 & 11.0 \\
\hline \multirow[t]{16}{*}{-. } & $A B G$ & 0.0 & 0.0 & 0.0 & 0.0 & 0.0 & 0.0 & 0.0 & 0.0 & 0.0 & 0.0 & 0.0 & 0.0 & 0.0 & 0.0 & 0.0 & 0.0 \\
\hline & $\wedge C$ & 0.0 & 0.2 & 0.2 & 0.2 & 0.2 & 0.2 & 0.2 & 0.2 & 0.2 & 0.2 & 0.2 & 0.2 & 0.2 & 0.2 & 0.2 & 0.2 \\
\hline & AEFG & 0.0 & 1.1 & 1.7 & 0.9 & 0.9 & 0.1 & 0.1 & 0.1 & 0.1 & 0.1 & 0.1 & 0.1 & 0.1 & 0.1 & 0.1 & 0.1 \\
\hline & AFG & 0.0 & 0.1 & 0.1 & 0.1 & 0.1 & 0.1 & 0.1 & 0.1 & 0.1 & 0.1 & 0.1 & 0.1 & 0.1 & 0.1 & 0.1 & 0.1 \\
\hline & $A 6$ & 0.0 & 0.1 & 0.4 & $n .3$ & 0.3 & 0.3 & 0.3 & 0.3 & 0.3 & 0.3 & 0.3 & 0.3 & 0.3 & 0.3 & 0.3 & 0.3 \\
\hline & B & $0.0:$ & 1.9. & 0.7 & 0.7 & 0.4 & 2.1 & 0.1 & 4.5 & 4.5 & 4.5 & 13.3 & 23.3 & 22.1 & 22.2 & 22.1 & 22.1 \\
\hline & c & 0.0 & 0.0 & 0.0 & 0.0 & 0.0 & 0.0 & 0.0 & 0.0 & 0.0 & 0.0 & 0.0 & 0.0 & 0.0 & 0.0 & 0.0 & 0.0 \\
\hline & 0 & 0.0 & 14.8 & 16.2 & 67.5 & 14.4 & 9.2 & 7.1 & 12.5 & 12.5 & 12.5 & 23.6 & 23.6 & 34.6 & 34.6 & 34.6 & 34.6 \\
\hline & Dr & 0.0 & 69.1 & 76.1 & 80.9 & 75.9 & 75.9 & 75.9 & 75.9 & 73.9 & 75.9 & 90.7 & 90.7 & 93.6 & 100.6 & 100.6 & 110.4 \\
\hline & Dro & 0.0 & 0.4 & 0.4 & 0.3 & 0.3 & 0.3 & 0.3 & 0.3 & 0.3 & 0.3 & 0.3 & 0.3 & 0.3 & 0.3 & 0.3 & 0.3 \\
\hline & DG. & $0 . n$ & 2.7 & 2.2 & 1.7 & 1.7 & 1.7 & 1.7 & 2.7 & 1.7 & 1.7 & 1.7 & 1.7 & 1.7 & 1.7 & 1.7 & 1.7 \\
\hline & & 0.0 & 0.0 & $n .0$ & 0.0 & 0.0 & 0.0 & 0.0 & 0.0 & 0.0 & 0.0 & 0.0 & 0.0 & 0.0 & 0.0 & 0.0 & 0.0 \\
\hline & $\mathbf{r}$ & 0.0 & 5.9 & 17.5 & 16.9 & 16.9 & 16.9 & 16.6 & 16.6 & 16.6 & 16.6 & 16.6 & 26.6 & 16.6 & 16.6 & 16.6 & 16.6 \\
\hline & 86 & 0.0 & 0.3 & 0.3 & 0.2 & 0.2 & 0.2 & 0.2 & 0.2 & 0.2 & 0.2 & 0.2 & 0.2 & 0.2 & 0.2 & 0.2 & 0.2 \\
\hline & G & 0.0 & 0.6 & a.e & 0.6 & 0.4 & 0.1 & 0.1 & 0.4 & 0.4 & 0.4 & 0.1 & 0.5 & 0.5 & 0.5 & 0.5 & 0.5 \\
\hline & UNKNOWN & 25.1 & 0.0 & 0.0 & 0.0 & 0.0 & 0.0 & 0.0 & 0.0 & 0.0 & 0.0 & 0.0 & 0.0 & 0.0 & 0.0 & 0.0 & 0.0 \\
\hline Total D-Congust & & 25.1 & 99.0 & 110.1 & 173.1 & 116.1 & 109.8 & 104.2 & 115.0 & 115.0 & 115.0 & 152.1 & 152.3 & 179.0 & $185 . \dot{6}$ & 205.6 & 198.9 \\
\hline \multirow[t]{4}{*}{ D-HET } & $n$ & 0.0 & 0.0 & 0.0 & 0.0 & 0.0 & 0.0 & 0.0 & 0.0 & 0.0 & 0.0 & 0.0 & 0.0 & 0.0 & 0.0 & 0.0 & 1.4 \\
\hline & $A B G$ & 0.0 & 0.0 & 0.0 & 0.0 & 0.0 & $0 . n$ & $n . n$ & 0.0 & D. n & 0.0 & 0.0 & 0.0 & 0.0 & 0.0 & 0.0 & 0.0 \\
\hline & AEFG & 0.0 & 0.5 & 0.8 & 0.4 & 0.4 & $0 . n$ & 0.0 & 0.0 & 0.0 & 0.0 & 0.0 & 0.0 & 0.0 & 0.0 & 0.0 & 0.0 \\
\hline & AFG & 0.0 & 0.0 & 0.0 & 0.0 & 0.0 & 0.0 & 0.0 & 0.0 & 0.0 & 0.0 & 0.0 & 0.0 & 0.0 & 0.0 & 0.0 & 0.0 \\
\hline
\end{tabular}

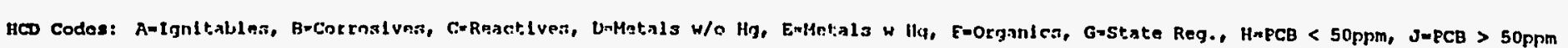


Annual Physical Waste Form and Bazardous Constituent Volumes by Waste Category (in cubic meters)

\begin{tabular}{|c|c|c|c|c|c|c|c|c|c|c|c|c|c|c|c|c|c|}
\hline PWE & HCD & Held & 1995 & 1996 & 1997 & 1998 & 1999 & 2000 & 2001 & 2002 & 2003 & 2004 & 2005 & 2006 & 2007 & 2008 & 2009 \\
\hline & $A G$ & 0.0 & 0.0 & 0.0 & 0.0 & 0.0 & 0.0 & 0.0 & 0.0 & 0.0 & 0.0 & 0.0 & 0.0 & 0.0 & 0.0 & 0.0 & 0.0 \\
\hline & B & 0.0 & 0.0 & 0.0 & 0.0 & 0.0 & 0.0 & 0.0 & 0.0 & 0.0 & 0.0 & 0.2 & 0.2 & 0.2 & 0.2 & 0.2 & 0.2 \\
\hline & c & $0 . n$ & 0.0 & 0.0 & 0.0 & 0.0 & 0.0 & 0.0 & 0.0 & 0.0 & 0.0 & 0.0 & 0.1 & 0.1 & 0.1 & 0.1 & 1.5 \\
\hline & D & 0.0 & 31.7 & 24.7 & 13.0 & 8.0 & 9.3 & 53.7 & 24.1 & 23.1 & 26.9 & 15.1 & 12.6 & 23.4 & 13.4 & 13.1 & 13.4 \\
\hline & or & 0.0 & 18.6 & 28.1 & 28.3 & 28.0 & 20.0 & 28.0 & 28.0 & 28.0 & 28.0 & 28.7 & 20.7 & 28.9 & 29.1 & 29.1 & 29.6 \\
\hline & Dro & 0.0 & 5.8 & 5.0 & 5.0 & 0.0 & 0.0 & 0.0 & 0.0 & 0.0 & 0.0 & 0.0 & 0.0 & 0.0 & 0.0 & 0.0 & 0.0 \\
\hline & $D G$ & 0.0 & 20.0 & 28.0 & 28.8 & 28.0 & 28.8 & 28.0 & 28.8 & 20.0 & 20.0 & 20.8 & 28.8 & 20.8 & 20.8 & 20.8 & 28.0 \\
\hline & $\varepsilon$ & 0.0 & 0.0 & 0.0 & 0.0 & 0.0 & 0.0 & 0.0 & 0.0 & 0.0 & 0.0 & 0.0 & 0.0 & 0.0 & 0.0 & 0.0 & 0.0 \\
\hline & $5 G$ & 0.0 & 0.0 & 0.0 & 0.0 & 0.0 & 0.0 & 0.0 & 0.0 & 0.0 & 0.0 & 0.0 & 0.0 & 0.0 & 0.0 & 0.0 & 0.0 \\
\hline & $F$ & 0.0 & 6.3 & 2.9 & 2.0 & 3.9 & 1.4 & 20.1 & 19.9 & 13.0 & 17.5 & 1.9 & 2.5 & 2.3 & 2.5 & 2.5 & 2.5 \\
\hline & FG & 0.0 & 3.5 & 1.9 & 3.8. & 2.6 & 2.6 & 2.6 & 0.3 & 0.3 & 0.3 & 0.3 & 0.3 & 0.3 & 0.3 & 0.3 & 0.3 \\
\hline & G & 15.7 & 6.8 & 6.2 & 12.3 & 8.2 & 9.8 & 10.7 & 10.9 & 20.5 & 39.4 & 11.7 & 13.0 & 14.6 & 6.0 & 6.0 & 12.1 \\
\hline & Unknown & $0 . n$ & 0.0 & 0.0 & 0.0 & 0.0 & 0.0 & 0.0 & 0.0 & 0.0 & 0.0 & 0.0 & 0.0 & 0.0 & 0.0 & 0.0 & 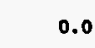 \\
\hline TOt.n1 D-HET & & 15.7 & 102.6 & 92.7 & $96.5^{\circ}$ & 00.5 & 03.5 & 172.5 & 112.6 & 123.1 & 111.5 & 90.1 & 07.5 & 09.1 & 81.0 & 01.0 & \\
\hline \multirow[t]{16}{*}{ D-IN HON-MTL } & $\bar{A}$ & 1.3 & 0.1 & 0.2 & 0.1 & 0.1 & 0.5 & 0.1 & 1.2 & 1.4 & 1.2 & 3.9 & 3.0 & 6.5 & 6.3 & 6.5 & $\overline{6.3}$ \\
\hline & ABG & 0.0 & 0.0 & 0.0 & 0.0 & n.o & $n . n$ & 0.0 & 0.0 & 0.0 & 0.0 & 0.0 & 0.0 & 0.0 & 0.0 & 0.0 & 0.0 \\
\hline & $\wedge c$ & $0 . n$ & 2.3 & 2.5 & 2.5 & 2.5 & 2.5 & 2.5 & 2.5 & 2.5 & 2.5 & 2.5 & 2.5 & 2.5. & 2.3 & 2.5 & 2.5 \\
\hline & Ars & 0.0 & 0.1 & 0.1 & 0.0 & 0.0 & 0.0 & 0.0 & 0.0 & 0.0 & 0.0 & 0.0 & 0.0 & 0.0 & 0.0 & 0.0 & 0.0 \\
\hline & $\Lambda a$ & 0.0 & 0.3 & 0.2 & 0.2 & 0.2 & 0.2 & 0.2 & 0.2 & 0.2 & 0.2 & 0.2 & 0.2 & 0.2 & 0.2 & 0.2 & 0.2 \\
\hline & B & 0.0 & 2.1 & 1.1 & $1 . n$ & 0.6 & 2.5 & 0.6 & 5.7 & 5.7 & 5.7 & 15.9 & 15.9 & 26.1 & 26.1 & 26.1 & 27.1 \\
\hline & c & 0.0 & 0.0 & 0.0 & 0.0 & 0.0 & 0.0 & 0.0 & 0.0 & 0.0 & 0.0 & 0.0 & 0.0 & 0.0 & 0.0 & 0.0 & 0.5 \\
\hline & D & 0.0 & 9.0 & 6.2 & 23.3 & 5.8 & 6.0 & 9.7 & 16.0 & 13.5 & 25.1 & 21.5 & 20.3 & 32.0 & 32.0 & 32.0 & 32.3 \\
\hline & DF & 1.6 & 11.0 & 14.1 & 41.5 & 41.0 & 14.0 & 14.0 & 44.0 & 14.0 & 14.0 & 45.5 & 45.5 & 16.0 & 16.5 & 16.5 & 47.5 \\
\hline & DFG & 0.0 & 0.3 & 0.2 & 0.2 & 0.2 & 0.2 & 0.2 & 0.2 & 0.2 & 0.2 & 0.2 & 0.2 & 0.2 & 0.2 & 0.2 & 0.2 \\
\hline & DG & 0.0 & 1.0 & 1.5 & 1.2 & 1.2 & 1.2 & 1.2 & 1.2 & 1.2 & 1.2 & 1.2 & 1.2 & 1.2 & 1.2 & 1.2 & 1.2 \\
\hline & $E$ & 0.0 & 0.0 & .0 .0 & 0.0 & 0.0 & 0.0 & 0.0 & 0.0 & 0.0 & 0.0 & 0.0 & 0.0 & 0.0 & 0.0 & 0.0 & 0.0 \\
\hline & $\mathbf{r}$ & 1.6 & 11.2 & 10.7 & 10.3 & 10.7 & 11.3 & 16.6 & 18.0 & 15.5 & 17.0 & 13.2 & 12.3 & 13.5 & 23.5 & 13.5 & 13.5 \\
\hline & EG & 0.0 & 0.2 & 0.2 & 0.1 & 0.1 & 0.1 & 0.1 & 0.1 & 0.1 & 0.1 & 0.1 & 0.1 & 0.1 & 0.1 & 0.1 & 0.1 \\
\hline & $G$ & 0.0 & 2.1 & 2.0 & 2.0 & 5.8 & 8.5 & 52.9 & 30.7 & 39.0 & 51.1 & 10.6 & 2.9 & 2.9 & 2.9 & 2.9 & 3.1 \\
\hline & ल1 & 0.0 & 0.0 & 0.0 & 0.0 & 0.0 & 0.0 & $0 . n$ & 0.0 & 0.0 & $0.0 .^{\circ}$ & 0.0 & 0.0 & 0.0 & 0.0 & 0.0 & 0.0 \\
\hline \multirow{8}{*}{ D-METAL } & & 1.6 & 71.9 & 69.7 & 85.0 & 71.8 & 77.6 & 128.7 & 148.4 & 123.0 & 130.8 & 115.1 & 105.8 & 131.0 & 132.2 & 132.1 & 135.4 \\
\hline & $\lambda$ & 0.0 & 0.5 & 0.3 & 0.2 & 0.2 & 0.2 & 0.2 & 0.2 & 0.2 & 0.2 & 0.2 & 0.2 & 0.2 & 0.2 & 0.2 & 0.2 \\
\hline & $B$ & 0.0 & 1.1 & 0.9 & 0.1 & 0.1 & 1.2 & 0.8 & 1.7 & 1.7 & 1.4 & 3.1 & 3.1 & 5.0 & 5.0 & 5.0 & 1.6 \\
\hline & c & 0.0 & 0.0 & 0.0 & 0.0 & 0.0 & 0.0 & 0.0 & 0.0 & 0.0 & 0.0 & 0.0 & 0.0 & 0.0 & 0.0 & 0.0 & 0.0 \\
\hline & D & 0.2 & 50.3 & 63.5 & 72.7 & 53.9 & 07.1 & 124.2 & 130.9 & 114.0 & 111.0 & 77.8 & 73.9 & 95.4 & 95.4 & 95.6 & 71.5 \\
\hline & $\mathrm{DE}$ & $n . n$ & 66.9 & 85.5 & 137.2 & 105.3 & 122.3 & 129.1 & 142.4 & 156.1 & 263.4 & 3113.9 & 281.7 & 320.9 & 364.5 & 358.5 & $\$ 18.1$ \\
\hline & $D G$ & 0.0 & 1.1 & 0.1 & 0.0 & 0.0 & 0.0 & 0.4 & 0.0 & 0.0 & 0.0 & 0.0 & 0.0 & 0.0 & 0.0 & 0.0 & 0.0 \\
\hline & $\varepsilon$ & $n . n$ & 0.0 & 0.0 & 0.0 & 0.0 & $0 . n$ & 0.0 & 0.0 & 0.0 & 0.0 & 0.0 & 0.0 & 0.0 & 0.0 & 0.0 & \\
\hline
\end{tabular}

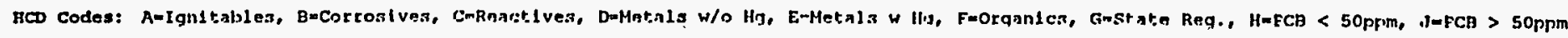


WHC-EP-0888

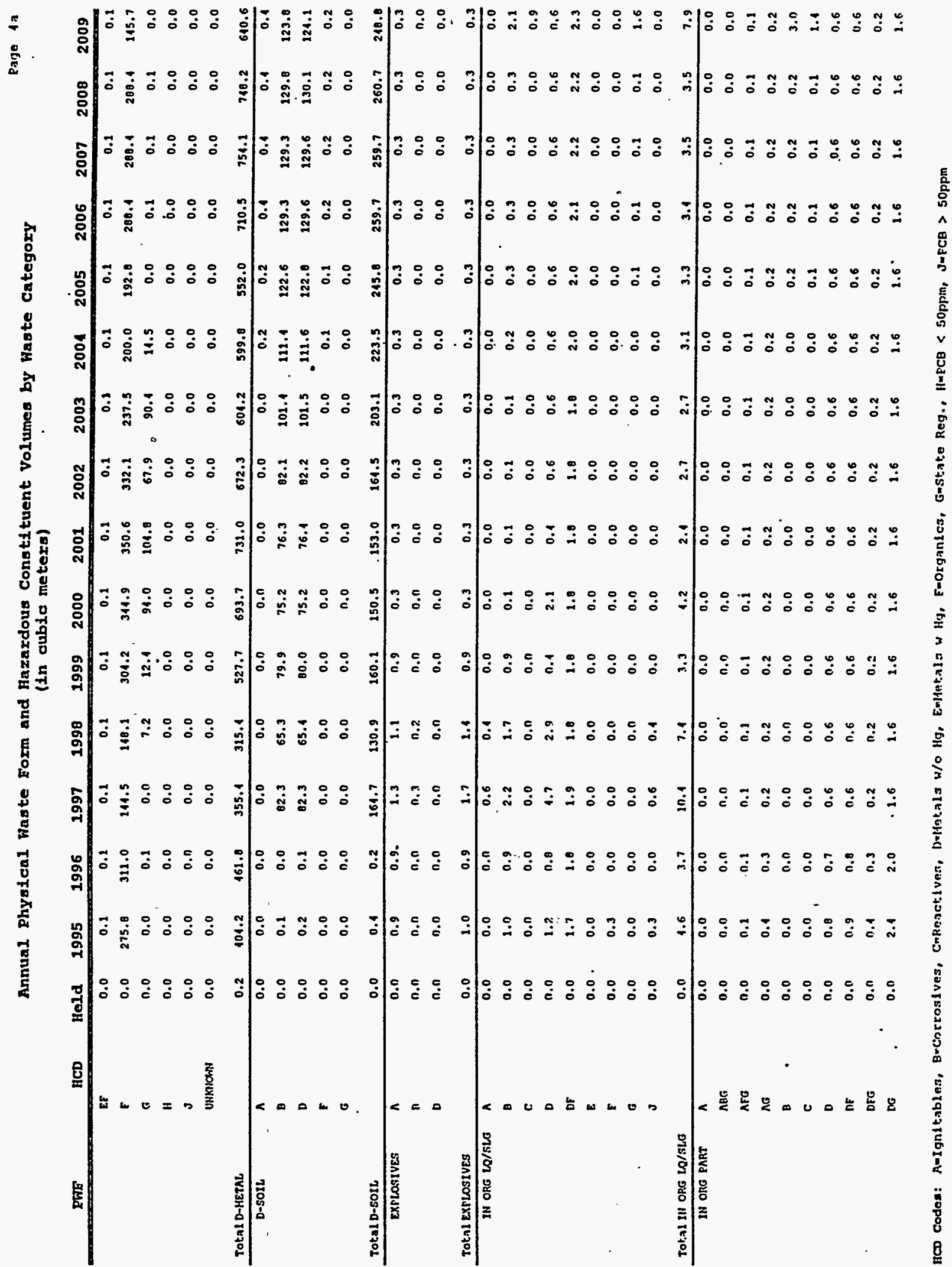




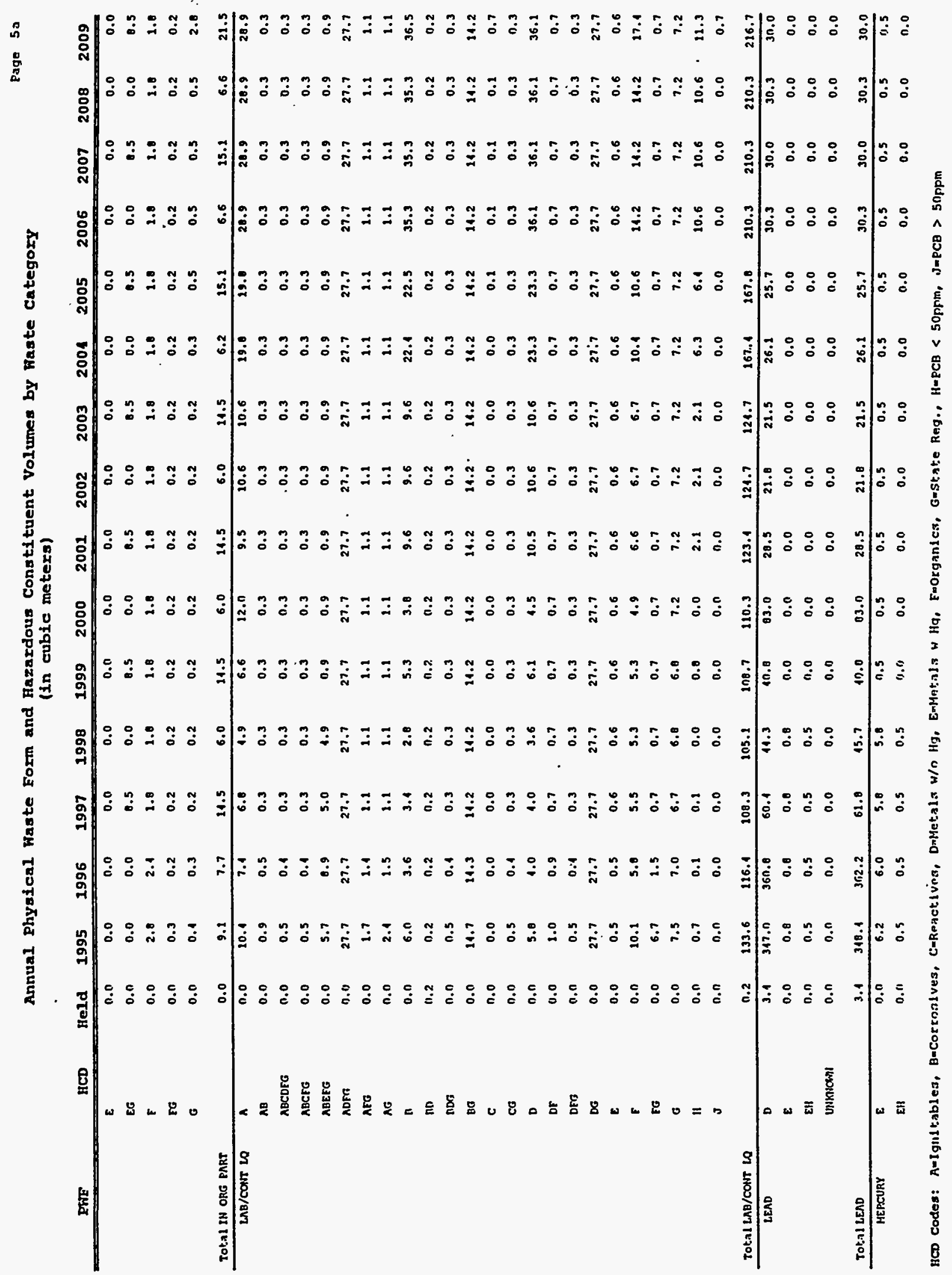


Annual Physical Waste Form and Hazardous Constituent Volumes by Waste Category (in cubic meters)

DFE

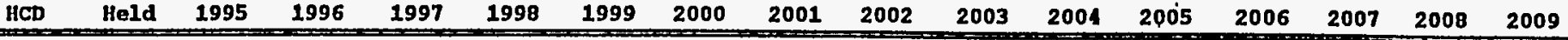

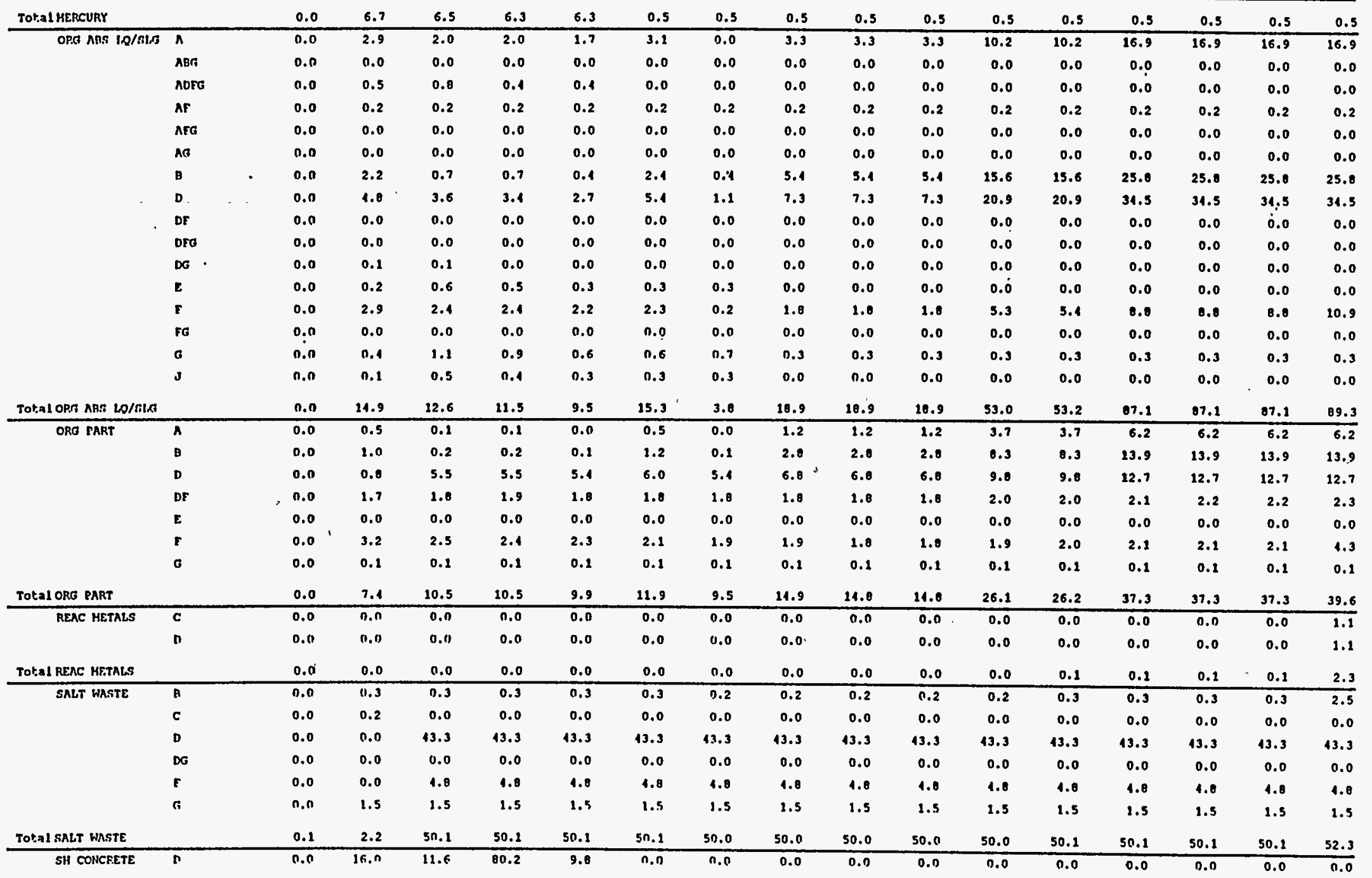

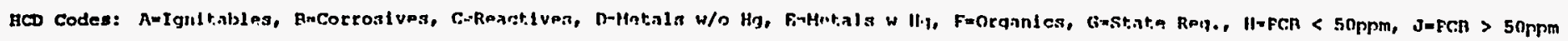




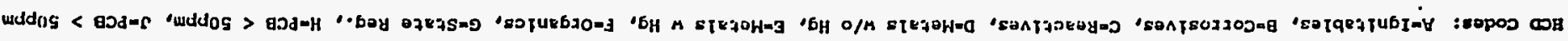

\begin{tabular}{|c|c|c|c|c|c|c|c|c|c|c|c|c|c|c|c|c|c|}
\hline$i \cdot z$ & $1 \cdot 2$ & $1 \cdot 2$ & $\tau \cdot 2$ & $\tau \cdot 2$ & $i \cdot z$ & $I \cdot z$ & $x \cdot z$ & T.2 & $t \cdot 2$ & $r \cdot 2$ & 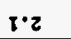 & $x \cdot 2$ & $\tau \cdot \mathrm{L}$ & $i \cdot z$ & 0.0 & 3NOH & D7s/OT DY0 $\mathrm{NI}$ \\
\hline $2 \cdot z$ & $2 \cdot 2$ & $\iota \cdot 7$ & 6.1 & $2 \cdot 1$ & $9 \cdot z$ & $6 \cdot \varepsilon$ & $0 \cdot \varepsilon$ & 1.0 & $E \cdot 1$ & $9 \cdot 2$ & $8 \cdot 0$ & $0 \cdot 0$ & 0.0 & $6 \cdot 2$ & 0.0 & & TIOS-a IE'T01 \\
\hline $2 \cdot 2$ & $z \cdot z$ & $\angle \cdot 1$ & L.t & $\boldsymbol{L} \cdot \boldsymbol{I}$ & 9.2 & $6 \cdot \varepsilon$ & $0 . \varepsilon$ & .0 & c.t & $9 \cdot 2$ & 0.0 & 0.0 & 0.0 & L.2 & 0.0 & $3110 \mathrm{~N}$ & $7105-0$ \\
\hline$L \cdot V L T$ & 6.895 & $E^{\prime} t \boldsymbol{t}$ & 0.562 & 6.012 & $E \cdot 66 I$ & 0.642 & $6 \cdot \mathrm{EzZ}$ & L'as & $\theta \cdot c t t$ & 1.265 & $c \cdot 90$ & $\theta \cdot 2 \theta$ & $5 \cdot 9 \varepsilon$ & 0.20 & 0.0 & & TV13H-Q IR;OOL \\
\hline$\angle \cdot 6<1$ & 6.091 & E'trI & e.562 & 6.812 & $c \cdot 661$ & 0.612 & $6.82 z$ & $L \cdot 05$ & o.git & .961 & $c \cdot 98$ & 0.20 & S.9E & 0.21 & 0.0 & $31 \mathrm{kOA1}$ & TW13k-a \\
\hline 5.02z & $9 \cdot 412$ & 8.062 & $1.06 t$ & I.06โ & L'ELT & $\cdot \cdot z s z$ & E. $\angle 6 \mathrm{I}$ & I.0E & E'zoI & Q.59t & L.SS & l'ss & $9 \cdot 012$ & 9.9 & 0.0 & & TLH-HON N1-Q TY:TOL \\
\hline s.0zz & $9 \cdot 4 \pi z$ & $r \cdot 06 \tau$ & $\tau \cdot 06 \pi$ & $\tau .06 t$ & $\mathcal{L} \cdot \mathcal{E} L I$ & $1 \cdot z s z$ & {$[.26 \pi$} & $\tau \cdot 0 \varepsilon$ & $c \cdot 2 \theta t$ & 0.591 & L.SS & L'ss & 9.012 & 9.9 & 0.0 & 31NㅇN & TLH-NOH NI-O \\
\hline $9 \cdot \angle \varepsilon$ & $0 \cdot \varepsilon z$ & $0 \cdot \varepsilon z$ & 1.60 & $9 \cdot 25$ & $\varepsilon \cdot 1$ & s.t & $s \cdot 1$ & 6.0 & $2 \cdot 0 z$ & $\varepsilon \cdot 0$ & c'0 & $6 \cdot 0$ & $2 \cdot 09$ & $i \cdot b$ & O.5s & & $1311-01=701$ \\
\hline $9 \cdot 2 \varepsilon$ & $0 \cdot \varepsilon \imath$ & $0 \cdot \mathfrak{\varepsilon} \varepsilon$ & $1 \cdot 68$ & $9 \cdot 25$ & E. & $s \cdot 8$ & $s \cdot 1$ & 6.0 & $L \cdot \theta z$ & $\varepsilon \cdot 0$ & c.o & E.o & L.09 & $L \cdot 1$ & 0.55 & 3MON & $23 H-a$ \\
\hline $5 \cdot 20$ & 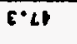 & $\varepsilon \cdot \omega$ & $2 \cdot 621$ & 1.06 & E.61 & s.9r & s.ar & s'or & 2.65 & 2.61 & L.61 & $z \cdot t s$ & $0 \cdot 25$ & $2 \cdot \theta S$ & C.ct & & 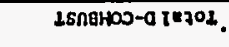 \\
\hline s.zo & $E \cdot 4$ & E.L & L.62I & 1.06 & E.61 & 5.06 & $s \cdot a r$ & $5 \cdot 96$ & L.61 & L.6r & L.61 & $2 \cdot 15$ & 0.25 & $2 \cdot 85$ & $\varepsilon \cdot \varepsilon$ & 3NON & $15 n e+100-a$ \\
\hline$\varepsilon \cdot \theta z$ & $\varepsilon \cdot a z$ & ¿.2z & $L \cdot 2 z$ & $6 \cdot 2 z$ & $0 . \mathrm{OE}$ & I. Is & ८.6E & 9.5 & $0 . \angle \pi$ & $0 \cdot \mathrm{BE}$ & $E \cdot I t$ & $\varepsilon \cdot t$ & 0.0 & S.SE & 0.0 & & TIOS LNOOS T\&7OL \\
\hline
\end{tabular}

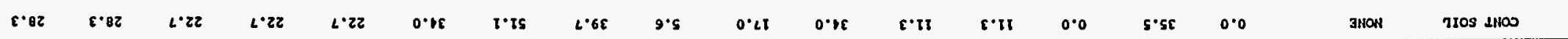

\begin{tabular}{|c|c|c|c|c|c|c|c|c|c|c|c|c|c|c|c|c|c|}
\hline \multirow[b]{2}{*}{$2 \cdot \operatorname{sill}$} & \multirow[b]{2}{*}{$2.065 L$} & \multirow[b]{2}{*}{ G.L29L } & \multicolumn{3}{|c|}{ - } & \multirow[b]{2}{*}{0.2695} & \multirow[b]{2}{*}{0.6025} & \multirow[b]{2}{*}{ B०น9ES } & \multirow[b]{2}{*}{ o.iris } & \multirow[b]{2}{*}{$6 \cdot 8 \varepsilon z 6$} & \multirow[b]{2}{*}{$9 \cdot 09 \varepsilon \varepsilon$} & \multirow[b]{2}{*}{$z \cdot z L 01$} & \multirow[b]{2}{*}{$0.00<5$} & \multirow[b]{2}{*}{$0.61+1$} & \multirow[b]{2}{*}{$1 \cdot 206$} & \multicolumn{2}{|c|}{ 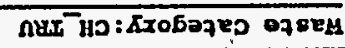 } \\
\hline & & & c'roel & $\tau .9959$ & S.6ts9 & & & & & & & & & & & & 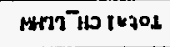 \\
\hline E'0IE & E.0TE & E.otc & E'otc & c'ote & E'DTE & c.ots & E'OtE & Cote & E'otc & $9 \cdot 1$ & $9 \cdot 1$ & $2 \cdot 1$ & $9 \cdot 1$ & $9 \cdot 1$ & s.โ12 & & 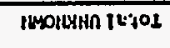 \\
\hline E'ote & c’ole & c·ote & E.0te & $\varepsilon \cdot$ ore & $\varepsilon \cdot 01 \varepsilon$ & c.ots & $\varepsilon \cdot 0 t \varepsilon$ & 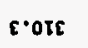 & \&'otc & $I \cdot T$ & $\boldsymbol{t} \cdot \boldsymbol{t}$ & $\boldsymbol{\tau} \cdot \boldsymbol{\tau}$ & $\boldsymbol{I} \cdot \boldsymbol{t}$ & เ. & s.extz & myonisn & \\
\hline 0.0 & 0.0 & 0.0 & 0.0 & 0.0 & 0.0 & 0.0 & 0.0 & 0.0 & $0 \cdot 0$ & $0 \cdot 0$ & 0.0 & 0.0 & $0 \cdot 0$ & 0.0 & u.u & F & \\
\hline 0.0 & 0.0 & 0.0 & 0.0 & 0.0 & 0.0 & 0.0 & 0.0 & 0.0 & $0 \cdot 0$ & $5 \cdot 0$ & s.o & $5 \cdot 0$ & $5 \cdot 0$ & s.0 & 0.0 & 4 & MUONXNก \\
\hline $1 \cdot 9<22$ & 8.9061 & $9 \cdot 1167$ & $6 . T L 9 T$ & $2 \cdot 2212$ & 8.0951 & $5 \cdot 609$ & s'zrg & L'Sss & B.0L4 & I'Lit & $2 \cdot 618$ & V.LTS & $2 \cdot 261$ & $9 \cdot 62$ & 0.0 & & T331S UIS I<701 \\
\hline$\tau \cdot 9 L 2 z$ & c.905t & $9 \cdot 6068$ & 6.7491 & $\tau \cdot L z+\pi$ & $0 \cdot 0952$ & 7.609 & $5 \cdot 289$ & L.sss & P.0Lr & $t \cdot 2 z t$ & 2.618 & $\because$ LIS & L.26I & 9.62 & 0.0 & so & T3318 H3 \\
\hline $2 \cdot \mathbf{E}$ & $2 \cdot \varepsilon$ & $2: \varepsilon$ & $z \cdot \varepsilon$ & $z \cdot \varepsilon$ & $2 \cdot 5$ & $\tau \cdot \varepsilon$ & $2 \cdot 8$ & $2 \cdot 8$ & $2 \cdot \varepsilon$ & $2 \cdot 8$ & 9.5 & $2 \cdot 52$ & 6.9 & $L \cdot 9$ & 0.0 & & aV37 HS t $t=301$ \\
\hline
\end{tabular}

\begin{tabular}{|c|c|c|c|c|c|c|c|c|c|c|c|c|c|c|c|c|}
\hline$\tau \cdot 0$ & t.0 & $1 \cdot 0$ & I.0 & 100 & $1 \cdot 0$ & $1 \cdot 0$ & $1 \cdot 0$ & $1 \cdot 0$ & I.0 & $r^{\circ} 0$ & $1 \cdot 0$ & 1.0 & $2 \cdot 0$ & 0.0 & 2 & \\
\hline t・o & $t \cdot 0$ & $r \cdot 0$ & 1.0 & 1.0 & $\tau \cdot 0$ & r.o & $r \cdot 0$ & 1.0 & I.0 & $r \cdot 0$ & $t \cdot 0$ & $1 \cdot 0$ & $1 \cdot 0$ & 0.0 & 23 & \\
\hline 6.0 & 6.0 & 6.0 & 6.0 & 6.0 & 6.0 & 6.0 & 6.0 & 6.0 & 6.0 & 6.0 & 6.0 & $\tau \cdot \tau$ & 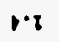 & 0.0 & $\mathbf{3}$ & \\
\hline 0.0 & 8.0 & 0.0 & $0 \cdot 0$ & $9 \cdot 0$ & $9 \cdot 0$ & 00 & 0.0 & 0.0 & 0.0 & 0.0 & 8.0 & $0 . \tau$ & $\tau \cdot \pi$ & 0.0 & $\boldsymbol{x a}$ & \\
\hline I.0 & I०0 & $1 \cdot 0$ & $1 \cdot 0$ & $1 \cdot 0$ & $t \cdot 0$ & $\because \because 0$ & $\tau=0$ & r.0 & 80 & $1 \% 0$ & 8.0 & I.0 & $z \cdot 0$ & 0.0 & 230 & \\
\hline$\varepsilon \cdot 0$ & $\varepsilon \cdot 0$ & $\varepsilon \cdot 0$ & $\varepsilon \cdot 0$ & $8 \cdot 0$ & $\varepsilon \cdot 0$ & E.0 & $\varepsilon \cdot 0$ & $\varepsilon \cdot 0$ & $\varepsilon \cdot 0$ & $\varepsilon \cdot 0$ & $\varepsilon \cdot 0$ & $1 \cdot 0$ & .0 & 0.0 & sa & \\
\hline s.0 & S.0 & s:0 & s.0 & s.o & $s=0$ & s.o & s.o & s.0 & $5 \%$ & $0 \cdot \varepsilon$ & $9 \cdot 0 z$ & $s \cdot \varepsilon$ & 9.1 & 0.0 & a & \\
\hline I.0 & $r \cdot 0$ & $1=0$ & $x \cdot 0$ & $r \cdot 0$ & $\tau \cdot 0$ & 2.0 & $r \cdot 0$ & 1.0 & $1 \cdot 0$ & 1.0 & $1 \cdot 0$ & $t \cdot 0$ & $2 \cdot 0$ & 0.0 & 9v & \\
\hline 0.0 & 0.0 & 0.0 & 0.0 & 0.0 & 0.0 & 0.0 & 0.0 & 0.0 & 0.0 & 0.0 & 0.0 & 0.0 & 0.0 & 0.0 & odv & \\
\hline 0.0 & 0.0 & 0.0 & 0.0 & 0.0 & 0.0 & 0.0 & 0.0 & 0.0 & 0.0 & 0.0 & 0.0 & 0.0 & 0.0 & 0.0 & อ8v & gvan us \\
\hline 0.0 & 0.0 & 0.0 & 0.0 & 0.0 & 0.0 & 0.0 & 0.0 & 0.0 & 0.0 & 8.6 & $2 \cdot 08$ & $9 \cdot \mathrm{Tt}$ & 0.91 & 0.0 & & З \\
\hline
\end{tabular}

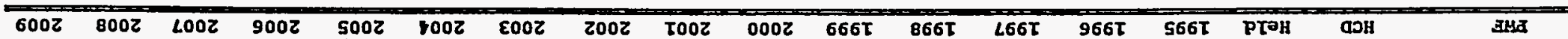

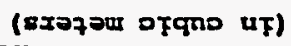

$\angle 2$ abts

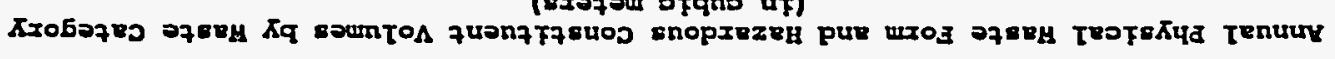




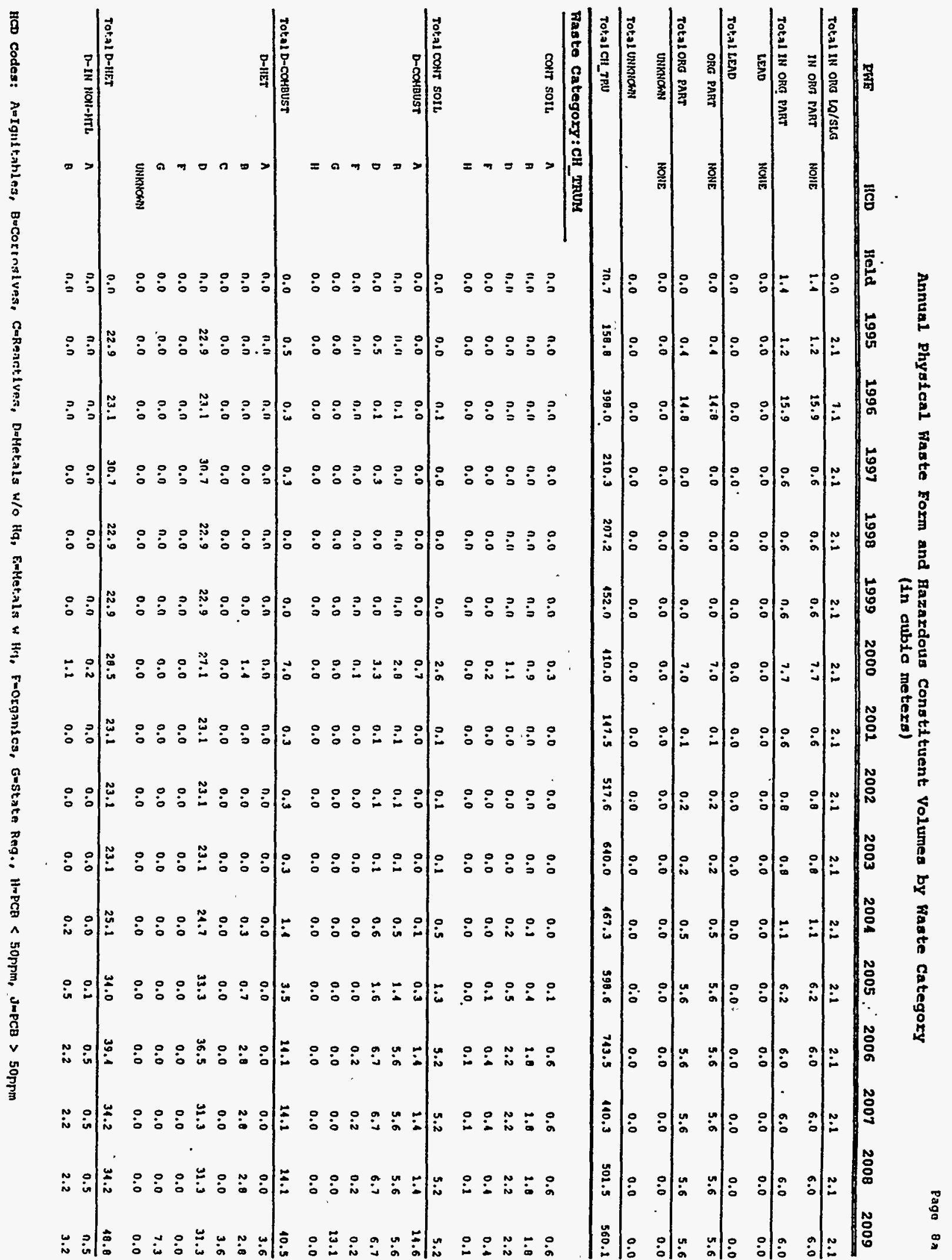




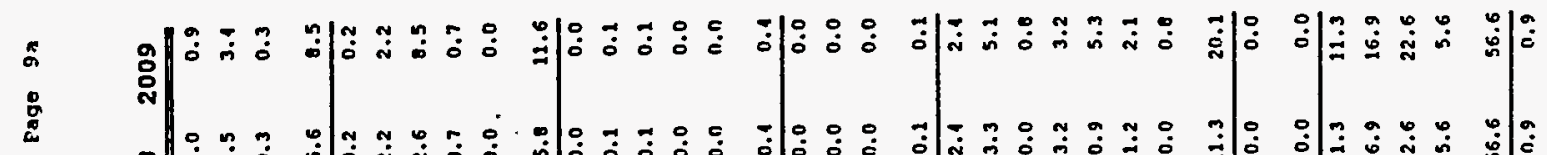

a

a

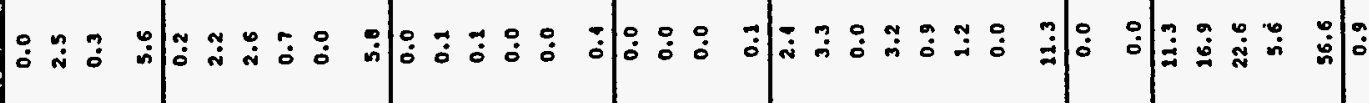

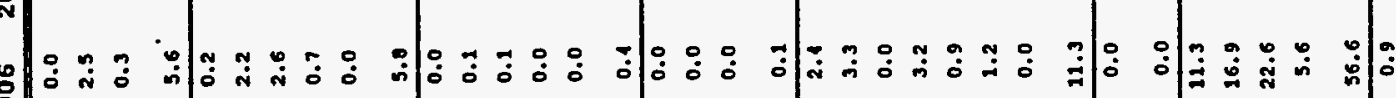

in

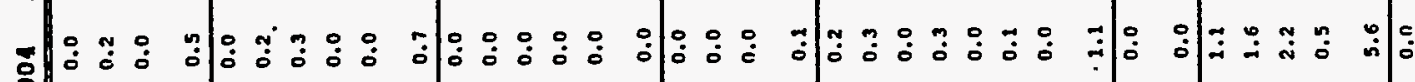

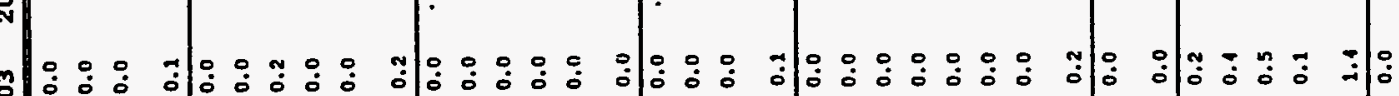

过

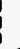

.

要势

.

悬

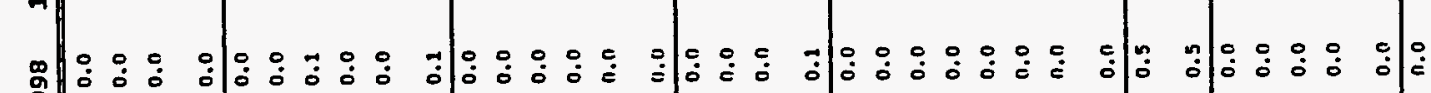

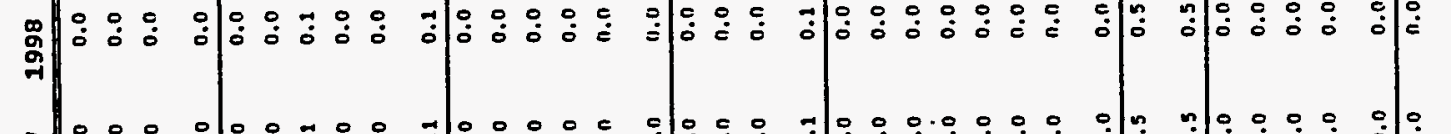

L

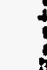

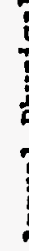

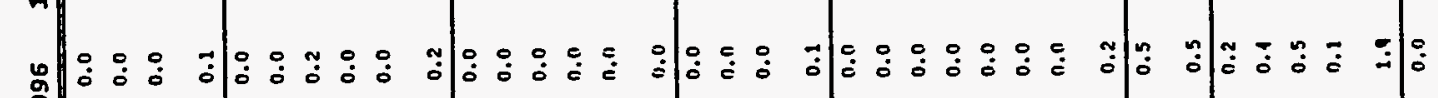

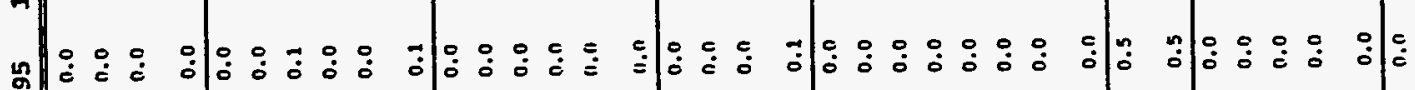

竎

(2)

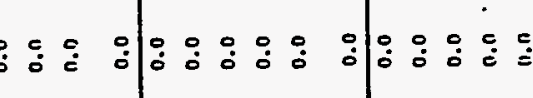

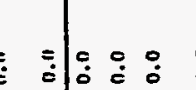



Annual Physical Waste Form and Hazardous Constituent Volumes by faste Category
(In cublo metera)

\begin{tabular}{|c|c|c|c|c|c|c|c|c|c|c|c|c|c|c|c|c|c|c|}
\hline & PWF & HCD & Held & 1995 & 1996 & 1997 & 1998 & 1999 & 2000 & 2001 & 2002 & 2003 & 2004 & 2005 & 2006 & 2007 & 2008 & 2009 \\
\hline & & $B$ & 0.0 & 0.0 & 0.0 & 0.0 & 0.0 & 0.0 & 0.8 & 0.0 & 0.0 & 0.0 & 0.1 & 0.1 & 1.6 & 1.6 & 1.6 & 1.6 \\
\hline & & 0 & 0.0 & 0.0 & 0.0 & 0.0 & 0.0 & 0.0 & 0.0 & 0.0 & 0.0 & 0.0 & 0.0 & 0.0 & 0.1 & 0.2 & 0.1 & 0.1 \\
\hline & Totnl ORG PART & & 0.0 & 0.0 & 0.0 & 0.0 & 0.0 & 0.0 & 1.4 & 0.0 & 0.0 & 0.0 & 0.2 & 0.7 & 2.0 & 2.0 & 2.0 & 2.8 \\
\hline & Tatal CH_TRUM & & 0.0 & 24.2 & 26.5 & 31.0 & 23.7 & 23.7 & 79.9 & 26.0 & 26.0 & 26.0 & 35.6 & 59.8 & 111.7 & 136.3 & 236.5 & 195.1 \\
\hline & Faste Catego & $\operatorname{Anz}$ & & & & & & & & & & & & & & & & \\
\hline & BATTERIES & $A B$ & 0.0 & 0.0 & 0.0 & 0.0 & 0.0 & 0.0 & 0.0 & 0.0 & 0.0 & 0.0 & 0.0 & 0.0 & 0.0 & 0.0 & 0.0 & 0.0 \\
\hline & & $B$ & 0.0 & 5.9 & 6.0 & 5.9 & 5.7 & 5.6 & 2.3 & 2.5 & 2.1 & 2.4 & 2.2 & 2.4 & 2.4 & 2.4 & 2.1 & 2.6 \\
\hline & & BC & 0.0 & 0.2 & 0.2 & 0.2 & 0.2 & 0.2 & 0.2 & 0.2 & 0.2 & 0.2 & 0.2 & 0.2 & 0.1 & 0.1 & 0.1 & 0.1 \\
\hline & & 80 & 0.0 & 0.1 & 0.1 & 0.1 & 0.1 & 0.0 & 0.0 & 0.0 & 0.0 & 0.0 & 0.0 & 0.0 & 0.0 & 0.0 & 0.0 & 0.0 \\
\hline & & RDF & 0.0 & 0.6 & 0.7 & 0.7 & 0.7 & 0.7 & 0.7 & 0.7 & 0.7 & 0.7 & 0.7 & 0.7 & 0.7 & 0.7 & 0.7 & 0.1 \\
\hline & & $B D G$ & 0.0 & 0.0 & 0.8 & 0.8 & 0.8 & 0.8 & 0.0 & 0.8 & 0.8 & 0.8 & 0.0 & 0.0 & 0.0 & 0.8 & 0.8 & 0.8 \\
\hline . & & RE & 0.0 & 0.1 & 0.1 & 0.1 & 0.1 & 0.1 & 0.1 & 0.1 & 0.2 & 0.1 & 0.1 & 0.1 & 0.1 & 0.1 & 0.1 & 0.1 \\
\hline & & BEO & 0.0 & 0.6 & 0.6 & 0.6 & 0.6 & 0.6 & 0.6 & 0.6 & 0.6 & 0.6 & 0.6 & 0.6 & 0.6 & 0.6 & 0.6 & 0.6 \\
\hline & & $\ln$ & $n: n$ & 0.1 & 0.1 & 0.2 & 0.2 & 0.2 & 0.2 & 0.3 & 0.3 & 0.1 & 0.4 & 0.5 & 0.6 & 0.0 & 1.0 & 1.2 \\
\hline & & 0 & $0.1^{\circ}$ & 2.2 & 2.2 & 2.2 & 2.2 & 2.2 & 0.0 & 0.0 & 0.0 & 0.0 & 0.1 & 0.3 & 0.3 & 0.3 & 0.3 & 0.5 \\
\hline & & $D$ & 0.0 & 0.1 & 0.4 & 0.4 & 0.1 & 0.4 & 0.1 & 0.1 & 0.1 & 0.1 & 0.4 & 0.1 & 0.4 & 0.1 & 0.4 & 0.4 \\
\hline & & $\mathbf{E}$ & $0 . n$ & 1.5 & 1.5 & 1.5 & 1.5 & 1.5 & 1.5 & 1.5 & 1.5 & 1.5 & 1.5 & 1.5 & 1.5 & 1.5 & 1.5 & 1.5 \\
\hline & TOtaL BATTERLES & & 0.0 & 13.1 & 13.2 & 13.1 & 12.9 & 12.9 & 7.4 & 7.7 & 7.6 & 7.7 & 7.6 & 0.0 & 0.0 & 0.1 & 0.3 & 9.0 \\
\hline & contr sotl & $n$ & 0.0 & 0.2 & 0.3 & 0.3 & 0.3 & 0.1 & 1.2 & 5.2 & 6.9 & 10.8 & 19.7 & 19.7 & 29.7 & 14.7 & 9.8 & 7.9 \\
\hline & & AEFG & 0.0 & 0.1 & 0.1 & 0.1 & 0.1 . & 0.0 & 0.0 & 0.0 & 0.0 & 0.0 & 0.0 & 0.0 & 0.0 & 0.0 & 0.0 & 0.0 \\
\hline & & B & 0.0 & 0.9 & 1.1 & 1.0 & 0.9 & 1.1 & 2.7 & 10.7 & 11,0 & 21.9 & 39.4 & 39.4 & 39.4 & 29.5 & 19.7 & 9.8 \\
\hline & & c & 0.0 & 0.0 & 0.0 & 0.0 & 0.0 & 0.0 & 0.0 & 0.0 & 0.0 & 0.0 & 0.0 & 0.0 & 0.0 & 0.0 & 0.0 & 0.0 \\
\hline & & D & 0.0 & 0.8 & 1.0 & 1.0 & 1.0 & 1.3 & -3.4 & 13.9 & 28.4 & 28.9 & 52.6 & 52.6 & 52.6 & 39.4 & 26.3 & 13.1 \\
\hline & & $\mathbf{F}$ & 0.0 & 5.1 & 5.5 & 6.2 & 3.4 & 5.5 & 6.0 & 8.7 & 9.8 & 12.4 & 10.3 & 18.3 & 20.3 & 15.0 & 11.7 & 0.4 \\
\hline & & G & 0.0 & 0.1 & 0.1 & 0.4 & 0.4 & 0.1 & 0.1 & 0.4 & 0.4 & 0.4 & 0.4 & 0.1 & 0.2 & 0.2 & 0.2 & 0.2 \\
\hline & & H & 0.0 & 0.0 & 0.1 & 0.1 & 0.1 & 0.1 & 0.4 & 1.7 & 2.3 & 3.6 & 6.5 & 6.5 & 6.5 & 4.9 & 3.2 & 1.6 \\
\hline & Tot:al rowt soll & & $n . n$ & 8.2 & 0.9 & 9.5 & 8.6 & 9.1 & 14.4 & 40.8 & 51.9 & 78.2 & 237.1 & 237.1 & 136.9 & 204.0 & 71.2 & 38.3 \\
\hline & D-cotbust & 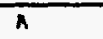 & 0.0 & 1.0 & 4.3 & 4.2 & 3.6 & 3.7 & 1.0 & 5.6 & 6.0 & 7.2 & 0.1 & 9.3 & 9.9 & 10.0 & 10.4 & 11.3 \\
\hline & & ABCG & $n . n$ & 0.0 & 0.0 & 0.0 & 0.0 & 0.0 & 0.0 & 0.0 & 0.0 & 0.0 & 0.0 & 0.0 & 0.0 & 0.0 & 0.0 & 0.0 \\
\hline & & MEnF & 0.0 & 6.8 & 7.5 & 7.5 & 7.5 & 7.5 & 1.5 & 7.5 & 7.5 & 7.5 & 7.5 & 7.3 & 7.5 & 7.5 & 7.5 & 7.5 \\
\hline & & ABEFG & $n .0$ & 0.3 & 0.3 & 0.3 & 0.3 & n.o & 0.0 & 0.0 & 0.0 & 0.0 & 0.0 & 0.0 & 0.0 & 0.0 & 0.0 & 0.0 \\
\hline & & $M B G$ & 0.0 & $0 . n$ & 0.0 & 0.0 & 0.0 & n.o & 0.0 & 0.0 & 0.0 & 0.0 & 0.0 & 0.0 & 0.0 & 0.0 & 0.0 & 0.0 \\
\hline & & $A C$ & 0.0 & 0.7 & 0.7 & 0.5 & 0.5 & 0.3 & 0.3 & 0.3 & 0.3 & 0.3 & 0.3 & 0.3 & 0.3 & 0.3 & 0.3 & 0.3 \\
\hline & & $A D G$ & 0.0 & n.0 & 0.0 & 0.0 & $0 . n$ & 0.0 & 0.0 & 0.0 & 0.0 & 0.0 & 0.0 & 0.0 & 0.0 & 0.0 & 0.0 & 0.0 \\
\hline & & AETG & $n, 0$ & n.o & 0.0 & 0.0 & 0.0 & $0, n$ & 0.0 & 0.0 & $0 . n$ & 0.0 & 0.0 & 0.0 & 0.0 & 0.0 & 0.0 & 0.0 \\
\hline & & $A F$ & 0.0 & 5.1 & 1.5 & 1.5 & 1.5 & 1.5 & 1.5 & 1.3 & 2.5 & 1.5 & 1.5 & 1.5 & 1.5 & 1.5 & 1.5 & 1.5 \\
\hline & & AFG & $n . n$ & 0.2 & 0.3 & 0.1 & $0.5^{\prime}$ & 0.6 & 0.8 & 1.0 & 1.3 & 1.6 & 2.1 & 2.6 & 3.3 & 4.1 & 5.1 & 6.4 \\
\hline
\end{tabular}

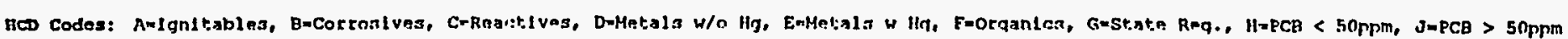




\section{WHC-EP-0888}

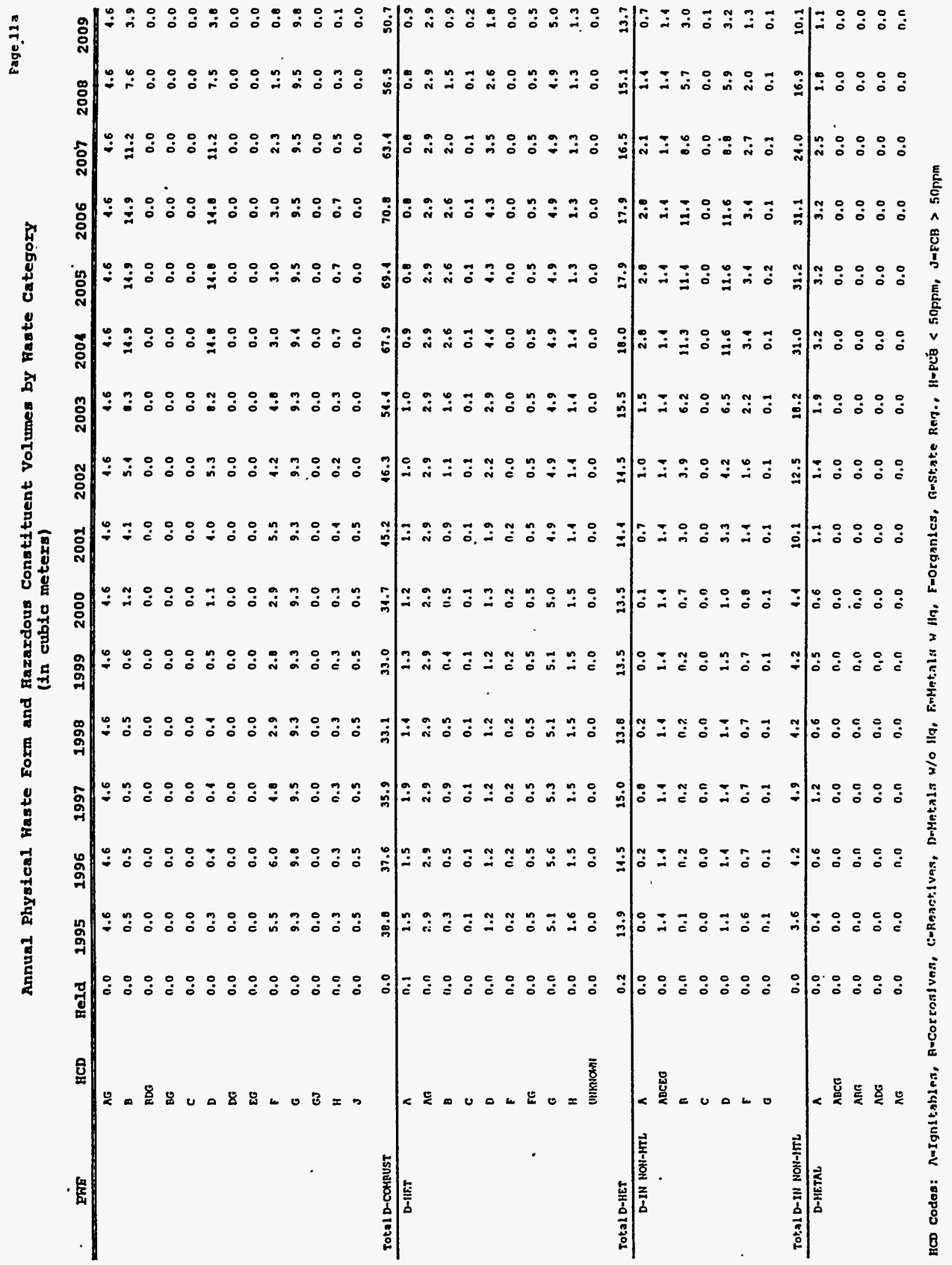

C-11 


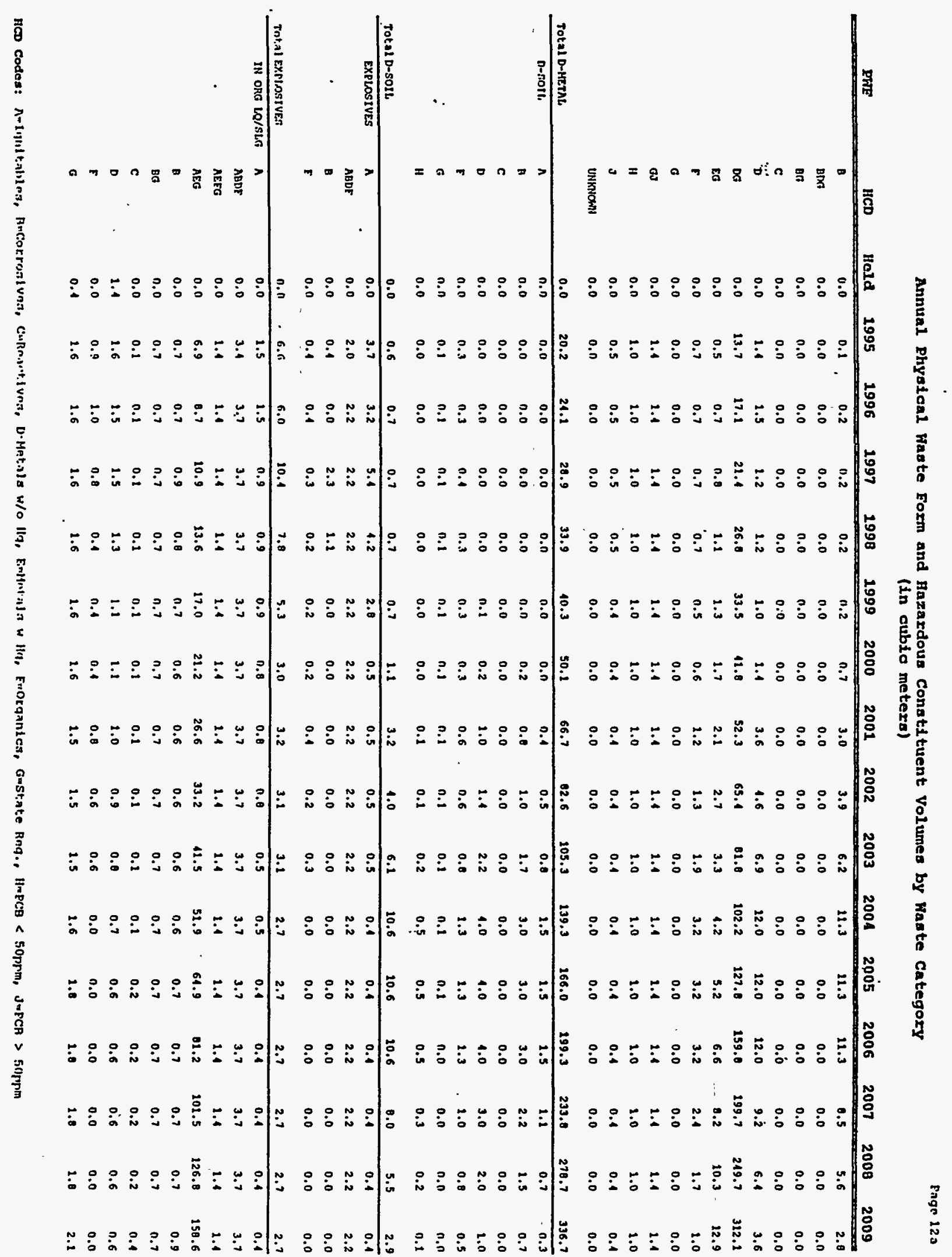




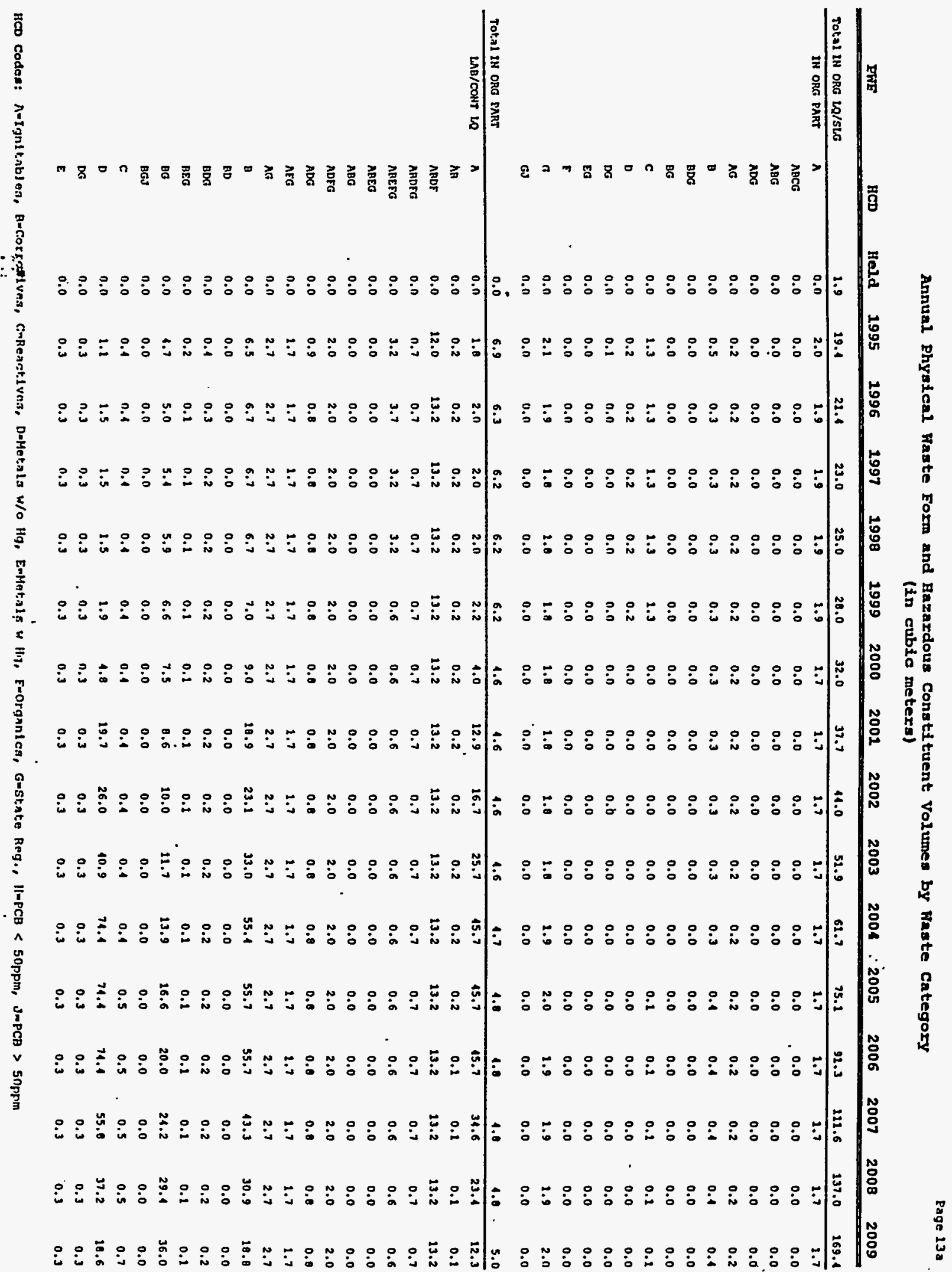


Annual Physical Faste Form and Hazardous Constltuent Volumes by Waate Category (in cubio metern)

\begin{tabular}{|c|c|c|c|c|c|c|c|c|c|c|c|c|c|c|c|c|c|}
\hline PWE & HCD & Held & 1995 & 1996 & 1997 & 1998 & 1999 & 2000 & 2001 & 2002 & 2003 & 2004 & 2005 & 2006 & 2007 & 2008 & 2009 \\
\hline & 8.6 & 11.0 & 0.1 & 0.1 & 0.1 & 0.1 & 0.1 & 0.1 & 0.1 & 0.1 & 0.1 & 0.1 & 0.1 & 0.1 & 0.1 & 0.1 & 0.1 \\
\hline & $F$ & 0.0 & 1.0 & 1.1 & 1.1 & 1.1 & 1.2 & 2.0 & 5.9 & 7.6 & 11.6 & 20.8 & 22.4 & 21.4 & 16.4 & 11.5 & 7.3 \\
\hline & FG & 0.0 & 0.1 & 0.5 & 0.6 & 0.7 & 0.9 & 1.1 & 2.4 & 1.7 & 2.2 & 2.7 & 3.3 & 4.1 & 3.1 & 6.1 & 8.0 \\
\hline & $G$ & 0.0 & 18.0 & 17.9 & 17.8 & 17.8 & 17.8 & 27.0 & 17.8 & 17.8 & 17.8 & 17.0 & 17.0 & $17.0^{\circ}$ & 17.0 & 17.8 & 17.8 \\
\hline & H & 0.0 & 0.1 & 0.1 & 0.1 & 0.1 & 0.2 & 0.6 & 2.6 & 3.4 & 5.4 & 9.9 & $10.0:$ & 10.0 & 7.6 & 5.1 & 2.0 \\
\hline & J & 0.0 & 0.0 & 0.0 & 0.0 & 0.0 & 0.0 & 0.0 & 0.0 & 0.0 & 0.0 & 0.0 & .0 .1 & 0.1 & 0.1 & 0.2 & 0.3 \\
\hline TOtA1 LAB/CONT LQ & & 0.0 & 59.9 & 62.0 & 62.5 & 63.2 & 62.5 & 71.5 & 112.5 & 131.1 & 172.9 & 265.4 . & 269.9 & 273.9 & 229.6 & 286.5 & 146.8 \\
\hline \multirow{6}{*}{ LEAD } & $A D F$ & 0.0 & 0.3 & 0.3 & 0.3 & 0.3 & 0.3 & 0.3 & 0.3 & 0.3 & 0.3 & 0.3 & 0.3 & 0.3 & 0.3 & 0.3 & $\overline{0.3}$ \\
\hline & BDG & 0.0 & 0.3 & 0.1 & 0.5 & $0 . \dot{6}$ & 0.7 & 0.9 & 1.2 & 1.5 & 1.9 & 2.3 & 2.9 & 3.7. & 4.6 & 5.8 & 7.3 \\
\hline & D & 0.0 & 0.5 & 0.6 & 0.6 & 0.6 & 0.7 & 1.2 & 4.1 & 4.9 & 7.8 & 14.1 & 14.2 & 24.1 & 10.6 & 7.0 & 3.5 \\
\hline & $\varepsilon$ & 0.0 & 0.5 & 0.5 & 0.5 & 0.5 & 0.5 & 0.5 & 0.5 & 0.5 & 0.5 & 0.5 & 0.5 & 0.5 & 0.5 & 0.5 & 0.5 \\
\hline & c & 0.0 & 0.1 & 0.1 & 0.1 & 0.1 & 0.1 & 0.1 & 0.1 & 0.1 & 0.1 & 0.1 & 0.1 & 0.1 & 0.1 & 0.1 & 0.1 \\
\hline & Uninatomn & 0.0 & 0.0 & $0.0^{\circ}$ & 0.0 & 0.0 & 0.0 & 0.0 & 0.0 & 0.0 & 0.0 & 0.0 & 0.0 & 0.0 & 0.0 & 0.0 & 0.0 \\
\hline TOt.AL LEAD & & 0.0 & 1.9 & 2.1 & 2.2 & 2.3 & 2.5 & 3.3 & 6.1 & 7.5 & 10.7 & 17.6 & 10.2 & 18.9 & 16.3 & 13.9 & 11.9 \\
\hline \multirow[t]{3}{*}{ MEISTYURY } & c & $n .1$ & 0.1 & $n .1$ & 0.1 & 0.1 & 0.1 & 0.1 & 0.1 & $n, n$ & $n, n$ & 0.0 & 0.0 & 0.0 & 0.0 & 0.0 & 0.0 \\
\hline & $D$ & 0.0 & 0.5 & 0.5 & 0.5 & 0.5 & 0.5 & 0.5 & 0.5 & 0.5 & 0.5 & 0.5 & 0.5 & 0.5 & 0.5 & 0.5 & 0.5 \\
\hline & $\varepsilon$ & 0.0 & 0.5 & 0.5 & 0.5 & 0.5 & 0.5 & 0.5 & 0.5 & 0.5 & 0.5 & 0.5 & 0.5 & 0.1 & 0.4 & 0.4 & 0.4 \\
\hline Tota I MERCURY & & 0.0 & 1.1 & 1.2 & 1.2 & 1.2 & 1.2 & 1.2 & 1.1 & 1.1 & 1.1 & 1.1 & 1.1 & 1.0 & 1.0 & 1.0 & 1.0 \\
\hline \multirow[t]{14}{*}{ ORG ABS LQ/SLG } & $\Lambda$ & 0.0 & 0.2 & 0.2 & 0.2 & 0.2 & 0.2 & 0.2 & 0.2 & 0.2 & 0.2 & 0.2 & 0.2 & 0.2 & 0.2 & 0.2 & 0.2 \\
\hline & ABDE & $n .0$ & 6.0 & 7.5 & 7.5 & 7.5 & 1.5 & 7.5 & 7.5 & 7.5 & 7.5 & 1.5 & 7.5 & 1.5 & 7.5 & 7.5 & 7.5 \\
\hline & AREG & $0 . n$ & 12.5 & 15.6 & 29.6 & 24.5 & 30.6 & 38.3 & 47.9 & 59.8 & 24.0 & 93.5 & 216.9 & 146.1 & 182.7 & 228.4 & 285.5 \\
\hline & AF & 0.0 & 0.4 & 0.1 & 0.3 & 0.3 & 0.2 & 0.2 & 0.2 & 0.2 & 0.2 & 0.2 & 0.2 & 0.2 & 0.2 & 0.2 & 0.2 \\
\hline & AFG & 0.0 & $\cdot 0.3$ & 0.3 & 0.3 & 0.3 & 0.3 & 0.3 & 0.3 & 0.3 & 0.3 & 0.3 & 0.3 & 0.3 & 0.3 & 0.3 & 0.3 \\
\hline & $\Lambda G$ & 0.0 & 0.0 & 0.0 & 0.0 & 0.0 & 0.0 & 0.0 & 0.0 & 0.0 & 0.0 & 0.0 & 0.0 & 0.0 & 0.0 & 0.0 & 0.0 \\
\hline & B & 0.0 & 0.1 & 0.2 & 0.1 & 0.1 & 0.1 & 0.1 & 0.1 & 0.1 & 0.1 & 0.1 & 0.1 & 0.1 & 0.1 & 0.1 & 0.1 \\
\hline & c & 0.0 & 0.0 & 0.0 & 0.0 & 0.0 & 0.0 & 0.0 & 0.0 & 0.0 & 0.0 & 0.0 & 0.0 & 0.0 & 0.0 & 0.0 & 0.0 \\
\hline & $E$ & 0.0 & 0.1 & 0.1 & 0.0 & 0.0 & 0.0 & 0.0 & 0.0 & 0.0 & 0.0 & 0.0 & 0.0 & 0.0 & 0.0 & 0.0 & 0.0 \\
\hline & EG & 0.0 & 0.2 & 0.2 & 0.2 & 0.2 & 0.2 & 0.2 & 0.2 & 0.2 & 0.2 & 0.2 & 0.2 & 0.2 & 0.2 & 0.2 & 0.2 \\
\hline & $F$ & 0.4 & 5.1 & 5.0 & 4.5 & 3.9 & 3.0 & 3.2 & 3.1 & 3.1 & 2.9 & 2.1 & 2.1 & 2.1 & 2.1 & 2.1 & 2.3 \\
\hline & 80 & 0.0 & 5.0 & 5.0 & 5.1 & 5.2 & 5.4 & 3.6 & 5.8 & 6.0 & 6.1 & 6.8 & 7.3 & 0.0 & 0.0 & 9.9 & 11.2 \\
\hline & 6 & 0.0 & 1.9 & 1.9 & 1.9 & 1.9 & 1.9 & 1.9 & 1.9 & 1.9 & 1.9 & 1.9 & 1.9 & 1.6 & 1.6 & 1.6 & 1.6 \\
\hline & s & 0.0 & 0.6 & 0.6 & 0.6 & 0.6 & 0.6 & 0.6 & 0.6 & $0: 0$ & 0.0 & 0.0 & 0.0 & 0.0 & 0.0 & 0.0 & 0.0 \\
\hline TOLAL ORG ABS LQ/SLG & & 0.1 & 33.6 & 37.1 & 10.9 & 45.3 & 51.3 & 58.6 & 68.6 & 79.8 & 94.9 & 113.3 & 137.2 & 266.9 & 204.2 & 251.0 & 309.6 \\
\hline \multirow[t]{4}{*}{ OPG PART } & $\pi$ & 0.0 & 0.2 & 0.3 & 0.3 & 0.3 & 0.4 & 1.1 & 4.5 & 5.9 & 9.3 & 17.0 & 17.0 & 17.0 & 12.7 & 8.5 & $\overline{1.2}$ \\
\hline & AEDF & 0.11 & 1.7 & 1.8 & 1.8 & 1.8 & 1.0 & 1.8 & 1.8 & .1 .0 & 1.0 & 1.8 & 1.8 & 1.0 & 1.0 & 1.8 & 1.0 \\
\hline & B & 0.0 & 0.5 & 0.6 & 0.6 & 0.6 & 0.8 & 2.2 & 9.0 & 11.8 & 18.6 & 33.9 & 33.9 & 33.9 & 25.4 & 16.9 & 0.4 \\
\hline & c & 0.0 & 0.1 & 0.7 & 0.7 & 0.7 & 0.7 & 0.0 & 0.0 & 0.0 & 0.0 & 0.0 & 0.0 & 0.0 & 0.0 & 0.0 & 0.0 \\
\hline
\end{tabular}

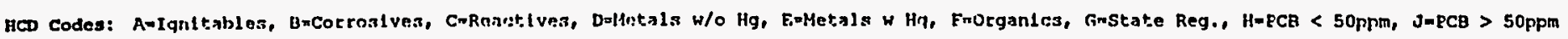


(in cubic meters)

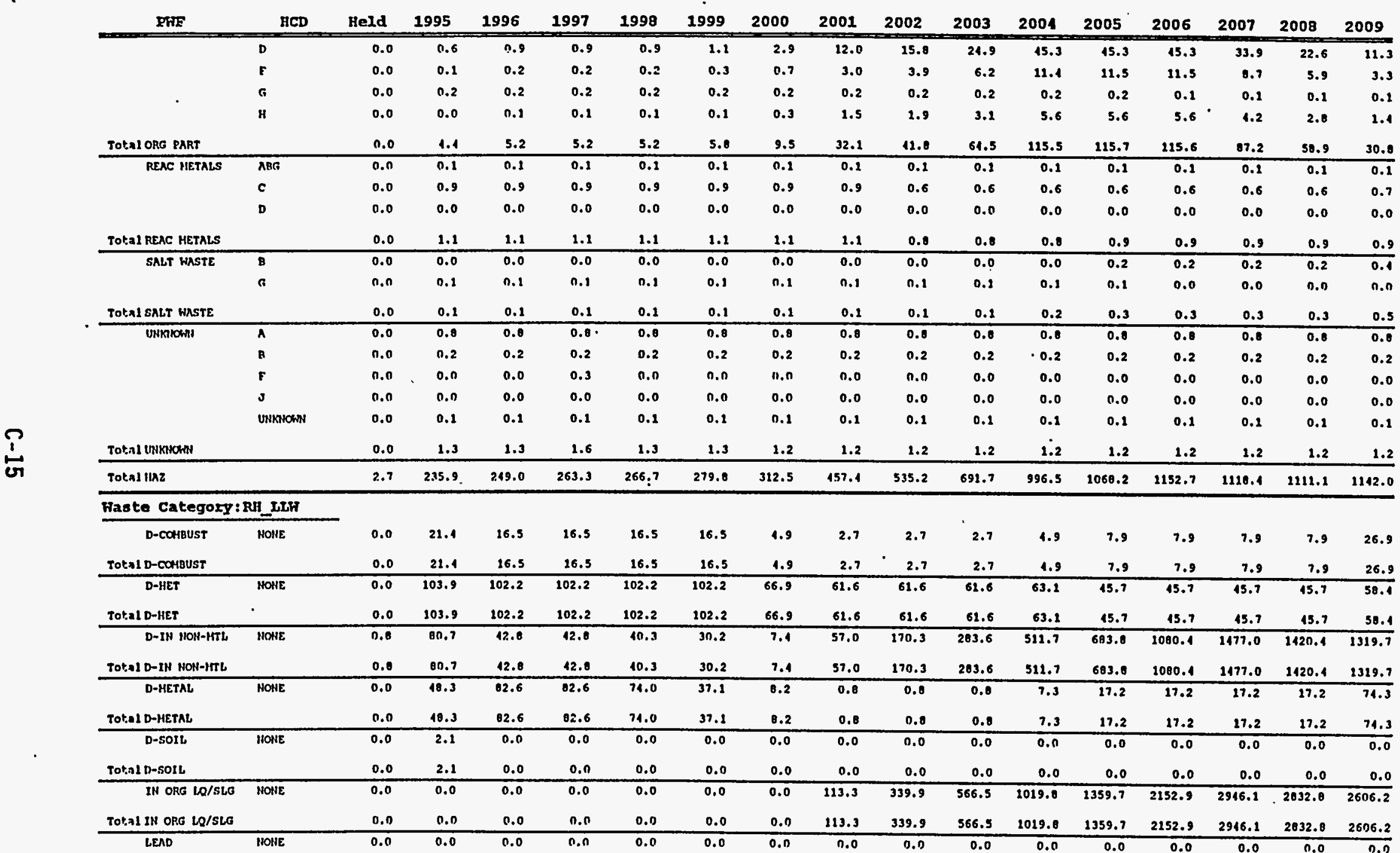

gCD Codes: A=1gnit.ables, Bucotrosives, CmRenetlves, DeMatals w/O Hg, E-Mntals w Hg, Fmorganics, Gestate Reg., H-PCB < 50ppm, J-PCB > 50ppm 


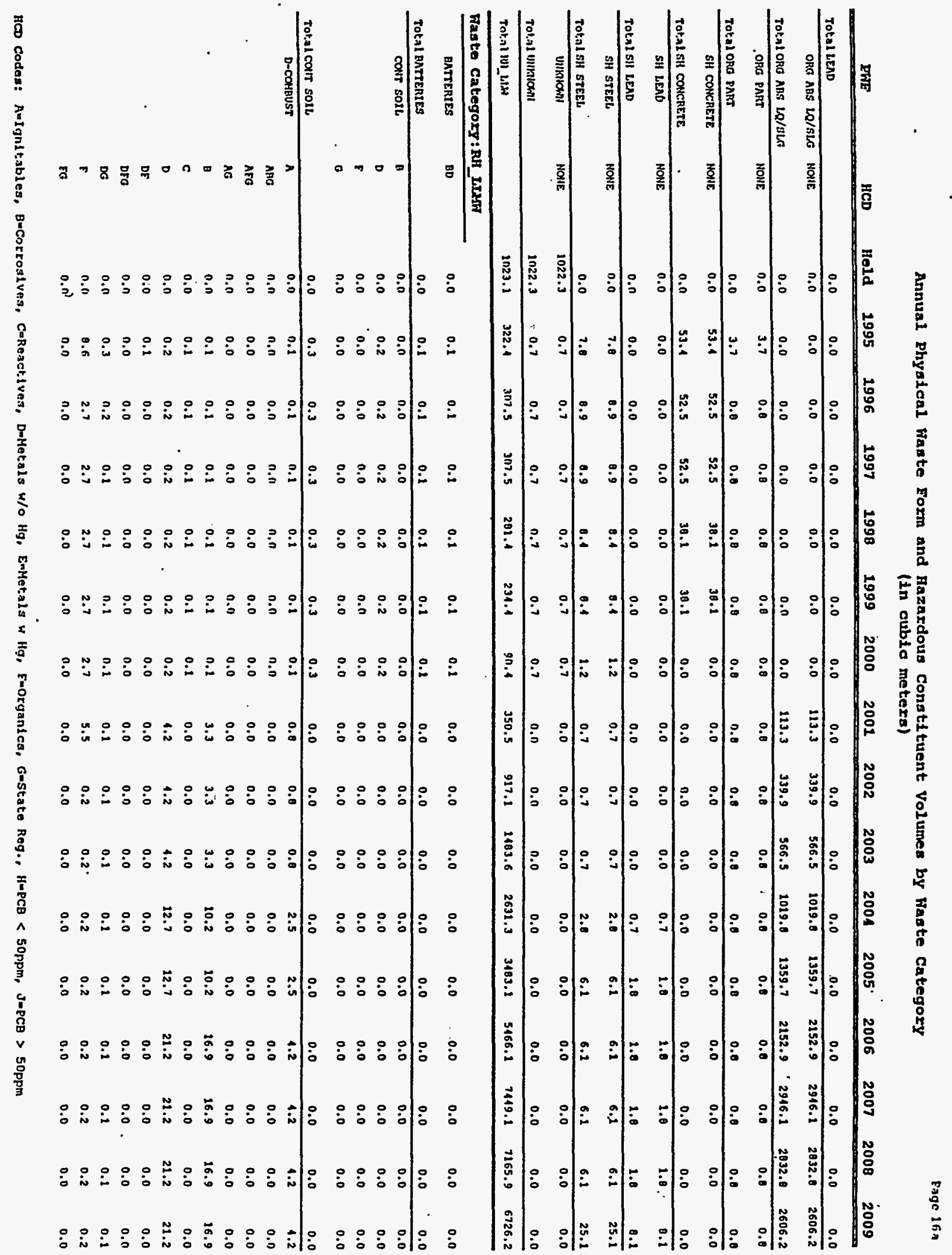




\section{LI-J}

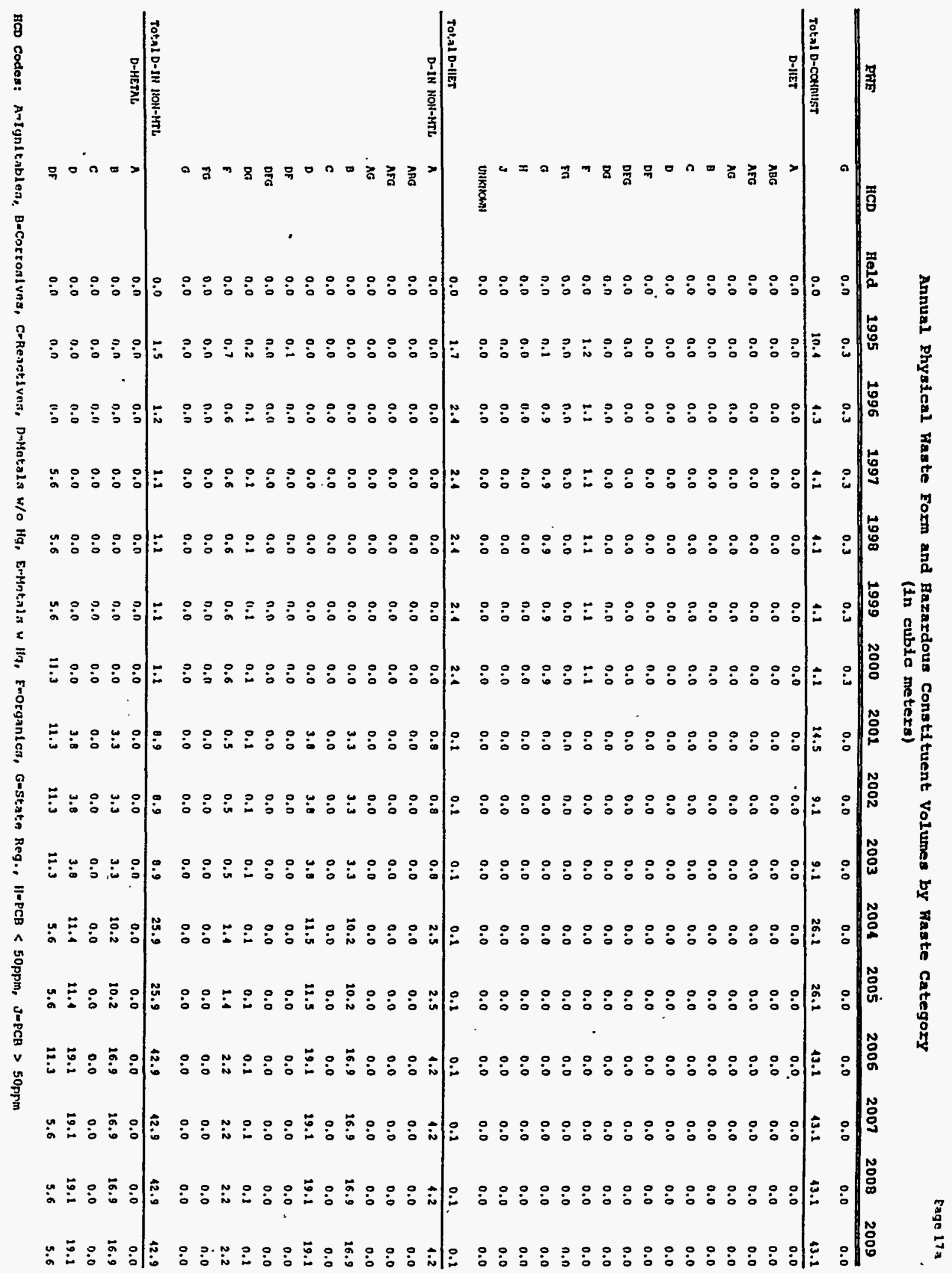




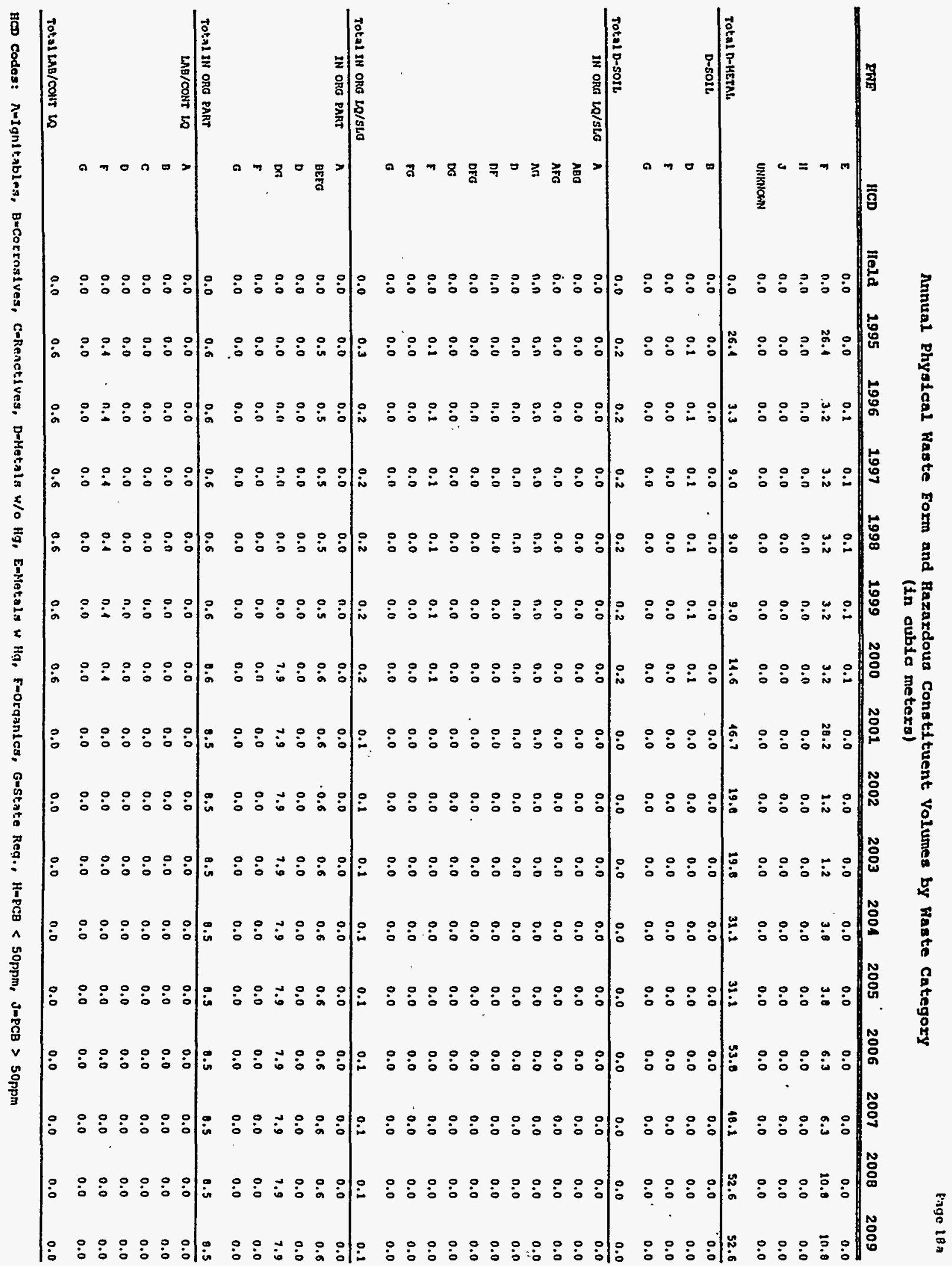




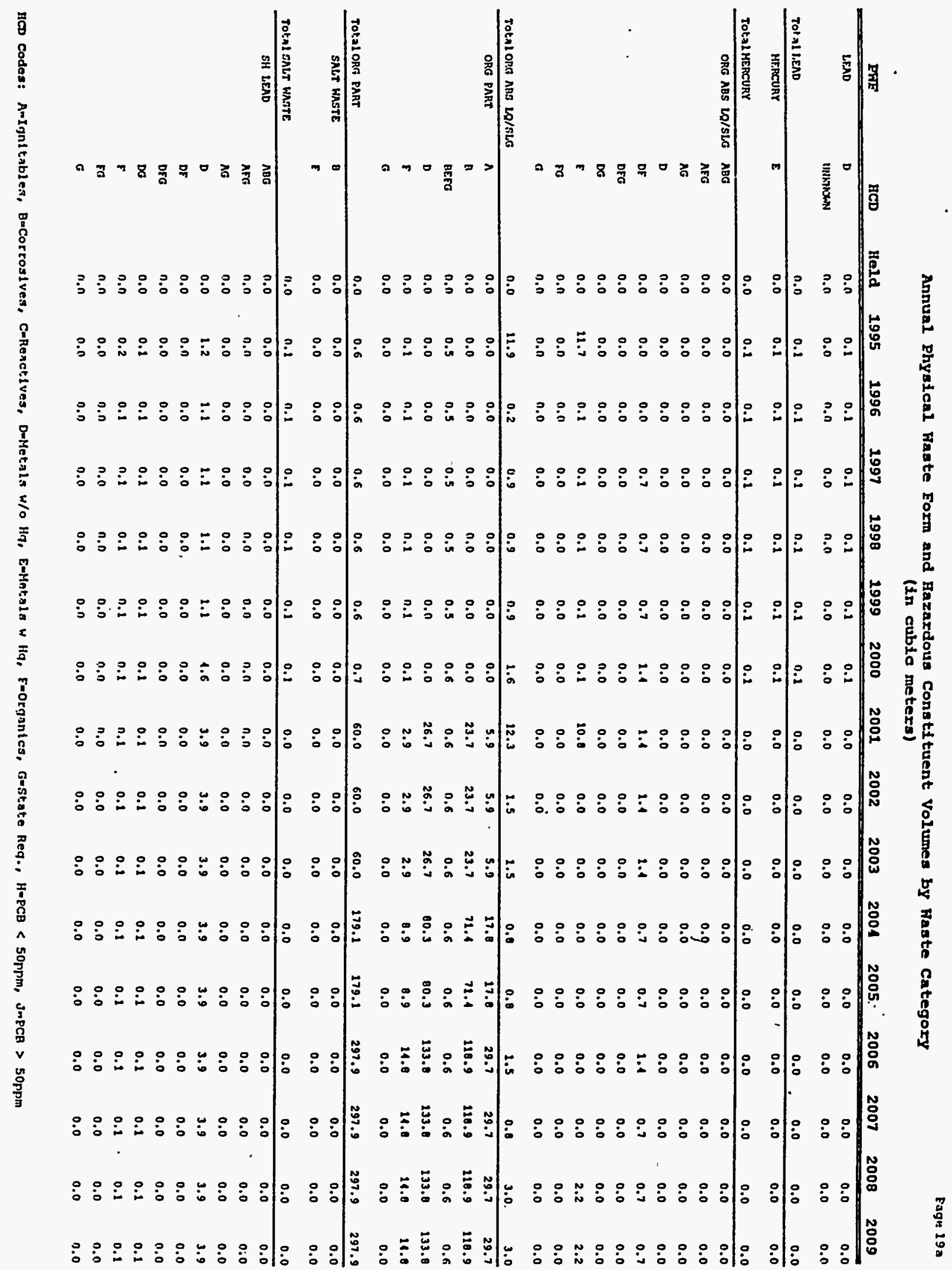


Annual Physical Faste Form and Hazardous Constituent Volumes by Haste Category (in cubic meters)

\begin{tabular}{|c|c|c|c|c|c|c|c|c|c|c|c|c|c|c|c|c|c|}
\hline FWE & HCD & Held & 1995 & 1996 & 1997 & 1998 & 1999 & 2000 & 2001 & 2002 & 2003 & 2004 & 2005 & 2006 & 2007 & 2008 & 2009 \\
\hline TOtA1 BU LEAD & & 0.0 & 1.0 & 1.6 & 1.5 & 1.5 & 1.5 & 4.9 & 1.2 & 4.2 & 4.2 & 1.2 & 4.2 & 4.2 & 4.2 & 1.2 & 1.2 \\
\hline \multirow[t]{4}{*}{ SH STEEL } & D & 0.0 & 0.0 & 0.2 & 0.2 & 0.2 & 0.2 & 0.2 & 0.0 & 0.0 & 0.0 & 0.0 & 0.0 & 0.0 & 0.0 & 0.0 & $\overline{0.0}$ \\
\hline & DF & 0.0 & 0.0 & 0.0 & 0.7 & 0.7 & 0.7 & 1.4 & 1.4 & 1.4 & 1.4 & 0.7 & 0.7 & 2.4. & 0.7 & 0.7 & 0.7 \\
\hline & $r$ & 0.0 & 13.0 & 0.0 & 0.0 & 0.0 & 0.0 & 0.0 & 21.5 & 0.0 & 0.0 & 0.0 & 0.0 & 0.0 & 0.0 & 0.0 & 0.0 \\
\hline & UHKNOWM & 0.0 & 0.2 & 0.2 & 0.2 & 0.2 & 0.2 & 0.3 & 0.3 & 0.3 & 0.3 & 0.3 & 0.3 & 0.3 & 0.3 & 0.3 & 0.3 \\
\hline TOt:A1 SH STEEC & & 0.0 & 13.3 & 0.1 & 1.2 & 1.2 & 1.2 & 1.9 & 23.2 & 1.7 & 1.7 & 1.0 & 1.0 & 1.7 & 1.0 & 1.0 & 1.0 \\
\hline \multirow[t]{3}{*}{ SH VoId } & DF & 0.0 & 0.0 & 0.0 & 7.1 & 7.1 & 7.1 & 11.1 & 11.1 & 14.1 & 14.1 & 7.1 & 7.1 & 14.1 & 7.1 & 7.1 & $\overline{7.1}$ \\
\hline & $\mathbf{F}$ & 0.0 & 62.3 & 0.0 & 0.0 & 0.0 & 0.0 & 0.0 & 13.1 & 0.0 & 0.0 & 0.0 & 0.0 & 0.0 & 0.0 & 0.0 & 0.0 \\
\hline & UNKNoWns & 0.0 & 0.0 & 0.0 & 0.0 & 0.0 & 0.0 & 0.0 & 0.0 & 0.0 & 0.0 & 0.0 & 0.0 & 0.0 & 0.0 & 37.9 & 37.9 \\
\hline TOt:a1 SH VoID & & 0.0 & 62.3 & 0.0 & 7.1 & 7.1 & 7.1 & 14.1 & 57.2 & 14.1 & 24.2 & 7.1 & 7.1 & 14.1 & 7.1 & 15.0 & 45.0 \\
\hline UNKNOWN & UHentoms & 212.2 & 2.2 & 2.2 & 2.2 & 2.2 & 2.2 & 2.2 & 0.0 & 0.0 & 0.0 & 0.0 & 0.0 & 0.0 & 0.0 & 0.0 & 0.0 \\
\hline Total unhrown & & 242.2 & 2.2 & 2.2 & 2.2 & 2.2 & 2.2 & 2.2 & 0.0 & 0.0 & 0.0 & 0.0 & 0.0 & 0.0 & 0.0 & 0.0 & 0.0 \\
\hline Tot:11 NI_L:LAN & & 242.2 & 135.4 & 19.3 & 33.1 & 33.1 & 33.1 & 58.8 & 236.2 & 128.4 & 120.4 & 204.4 & 284.4 & .168 .3 & 454.2 & 498.9 & 198.9 \\
\hline \multicolumn{2}{|c|}{ Faste Category:Rn_IRU } & & & & & & & & & & & & & & & & \\
\hline D-contust & NONE & 0.0 & 1.0 & 0.7 & 0.7 & 0.7 & 0.7 & 0.7 & 0.7 & 0.7 & 0.7 & 0.7 & 0.7 & 0.7 & 0.7 & 0.7 & 10.7 \\
\hline Tot:a1 D-CoMaUST & & 0.0 & 1.0 & 0.7 & 0.7 & 0.7 & 0.7 & 0.7 & 0.7 & 0.7 & 0.7 & 0.7 & 0.7 & 0.1 & 0.7 & 0.7 & 10.7 \\
\hline D-HET & MONE & 1.0 & 51.1 & 0.2 & 0.0 & 0.0 & 0.0 & 0.0 & 0.0 & 0.0 & 0.0 & 0.0 & 0.0 & 0.0 & 0.0 & 0.0 & $\overline{4.9}$ \\
\hline TOEA1 D-IIET & & 1.0 & 51.1 & 0.2 & 0.0 & 0.0 & 0.0 & 0.0 & 0.0 & 0.0 & 0.0 & 0.0 & 0.0 & 0.0 & 0.0 & 0.0 & 1.9 \\
\hline D-IN MON-MTL & HONE & 4.0 & 0.3 & 2.8 & .0 .3 & 15.1 & 14.2 & 11.2 & 0.3 & 0.3 & 0.3 & 0.3 & 0.3 & 0.3 & 0.3 & 0.3 & 5.3 \\
\hline Totn1 D-IN NON-HTL & & 1.0 & 0.3 & 2.0 & 0.3 & 25.4 & 14.2 & 14.2 & 0.3 & 0.3 & 0.3 & 0.3 & 0.3 & 0.3 & 0.3 & 0.3 & 5.3 \\
\hline D-METAL & MONE & 0.0 & 11.7 & 2.0 & 2.0 & 2.0 & 2.0 & 2.0 & 1.2 & 1.2 & 2.2 & 1.2 & 1.2 & 1.2 & 1.2 & 1.2 & $\overline{21.1}$ \\
\hline Tot:Al D-HETAL & & 0.0 & 11.7 & 2.0 & 2.0 & 2.0 & 2.0 & 2.0 & 1.2 & 1.2 & 1.2 & 1.2 & 1.2 & 1.2 & 1.2 & 1.2 & 21.1 \\
\hline IN ORG PART & NONE & 0.0 & $0 . n$ & 0.0 & 0.0 & 0.0 & 0.0 & 8.0 & 0.0 & 0.0 & 0.0 & 0.0 & 0.0 & 0.0 & 0.0 & 0.0 & 0.0 \\
\hline TOtn1 IN ORG BART & & 0.0 & 0.0 & 0.0 & 0.0 & 8.8 & 0.0 & 8.0 & 0.0 & 0.0 & 0.0 & 0.0 & 0.0 & 0.0 & 0.0 & 0.0 & 0.0 \\
\hline LEND & NONE & 0.0 & 0.0 & 0.0 & 0.0 & 0.0 & 0.0 & 0.0 & 0.0 & 0.0 & 0.0 & 0.0 & 0.0 & 0.0 & 0.0 & 0.0 & 0.0 \\
\hline Tota1 LEAD & & 0.0 & 0.0 & 0.0 & 0.0 & 0.0 & 0.0 & 0.0 & 0.0 & 0.0 & 0.0 & 0.0 & 0.0 & 0.0 & 0.0 & 0.0 & 0.0 \\
\hline ORG PART & NONE & 0.0 & 6.8 & 0.0 & 0.0 & 0.0 & 0.0 & 0.0 & 0.0 & 0.0 & 0.0 & 0.0 & 0.0 & 0.0 & 0.0 & 0.0 & 0.0 \\
\hline Total ORG PART & & 0.0 & 6.8 & 0.0 & 0.0 & 0.0 & 0.0 & 0.0 & 0.0 & 0.0 & 0.0 & 0.0 & 0.0 & 0.0 & 0.0 & 0.0 & 0.0 \\
\hline SH LEAD & NONE & 0.0 & 2.9 & 2.1 & 2.1 & 3.3 & 3.2 & 3.2 & 1.1 & 1.4 & 1.4 & 1.4 & 1.4 & 1.4 & 1.4 & 1.1 & 6.4 \\
\hline TOt.21 SH LEAD & & 0.0 & 2.9 & 2.1 & 2.1 & 3.3 & 3.2 & 3.2 & 1.4 & 1.1 & 1.1 & 1.4 & 1.4 & 2.1 & 1.4 & 1.1 & 6.1 \\
\hline SH STEEL & MONE & 0.0 & 0.1 & 0.1 & 0.1 & $n .1$ & 0.1 & 0.1 & 0.0 & 0.0 & 0.0 & 0.0 & 0.0 & 0.0 & 0.0 & 0.0 & 4.9 \\
\hline TOt:A1 SI STEEL & & 0.0 & 0.1 & 0.1 & 0.1 & 0.1 & 0.1 & 0.1 & 0.0 & 0.0 & 0.0 & 0.0 & 0.0 & 0.0 & 0.0 & 0.0 & 4.9 \\
\hline
\end{tabular}

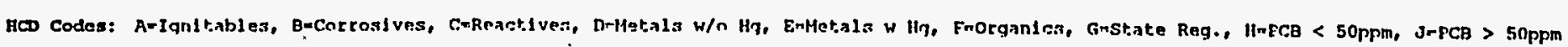




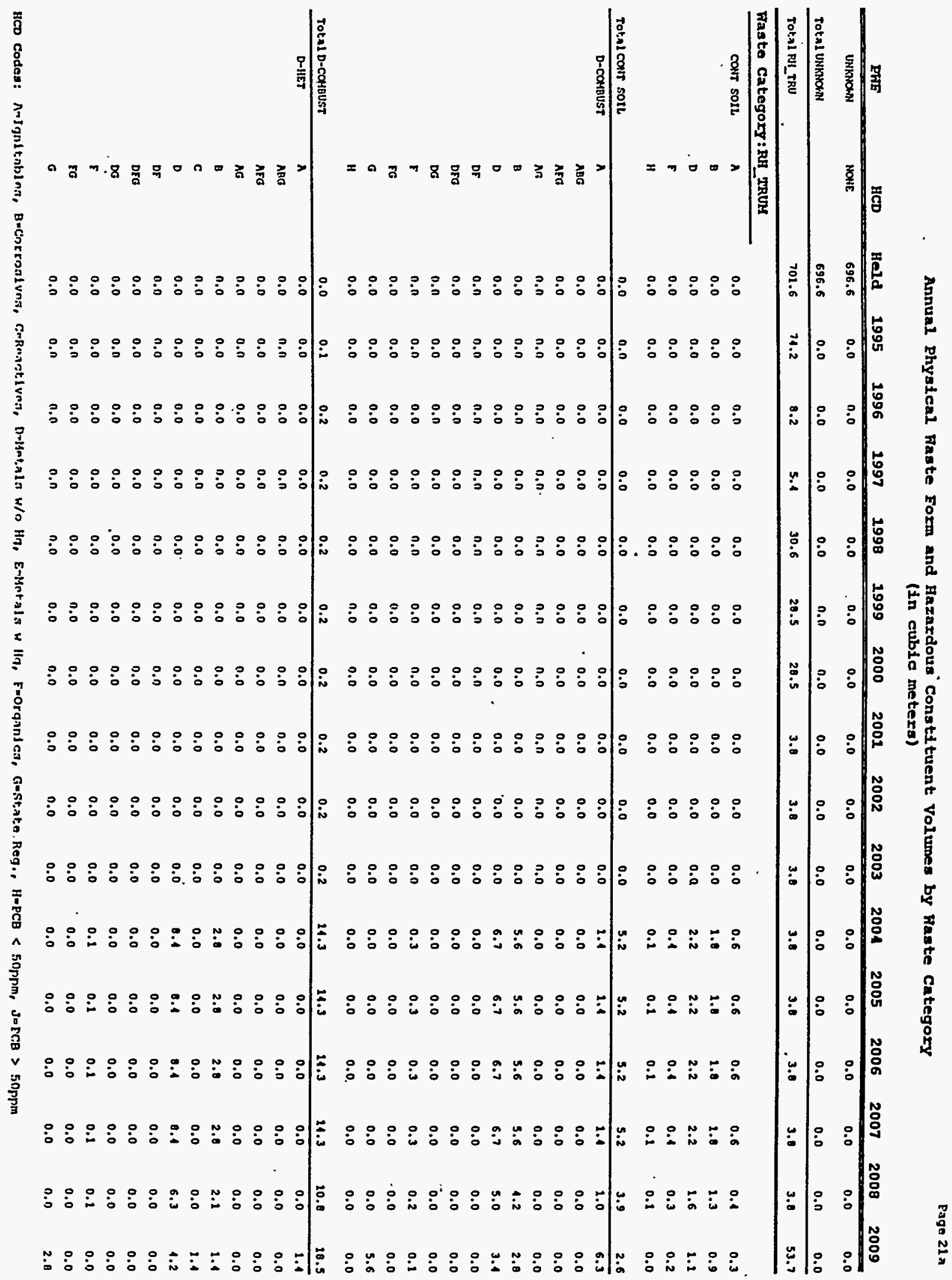




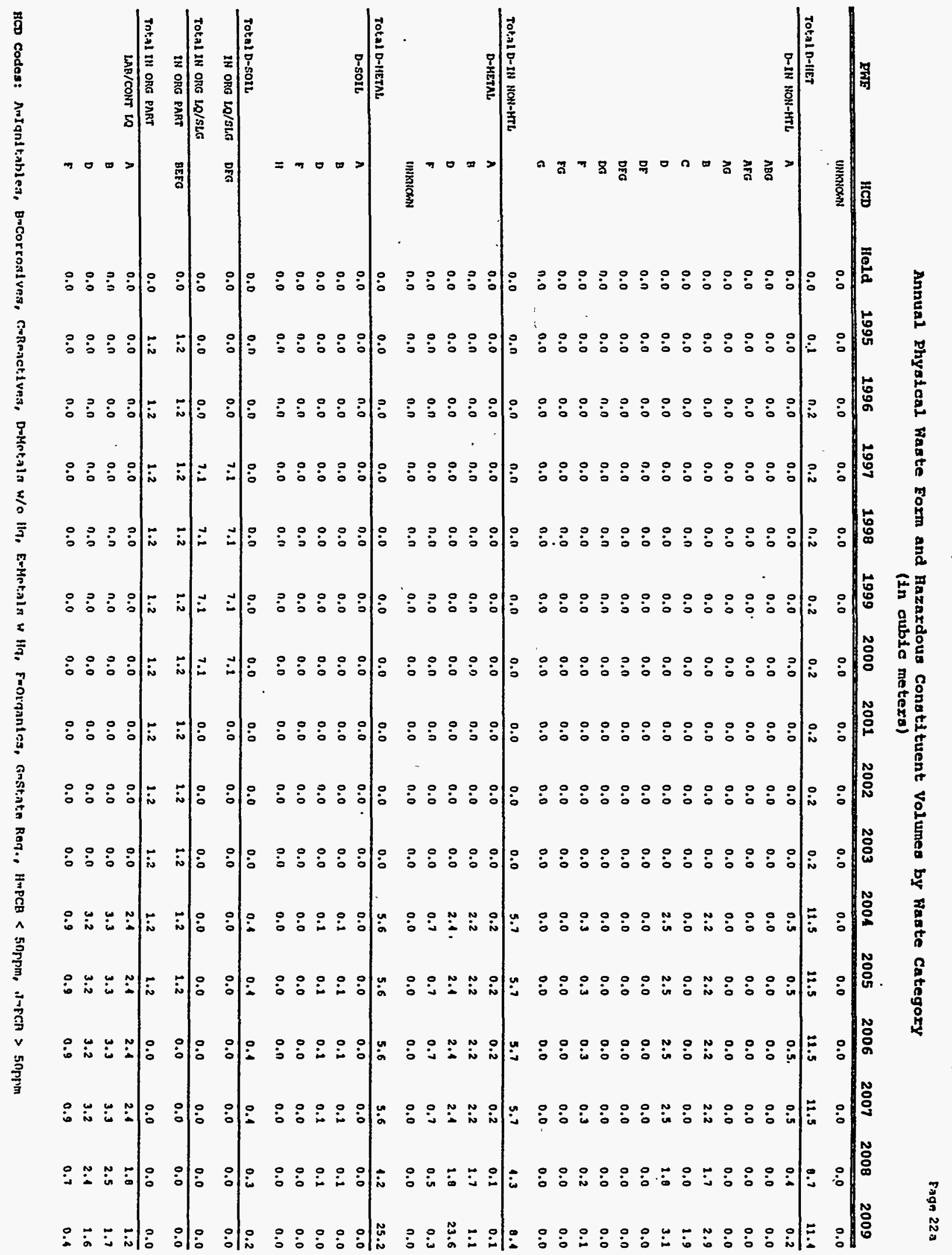




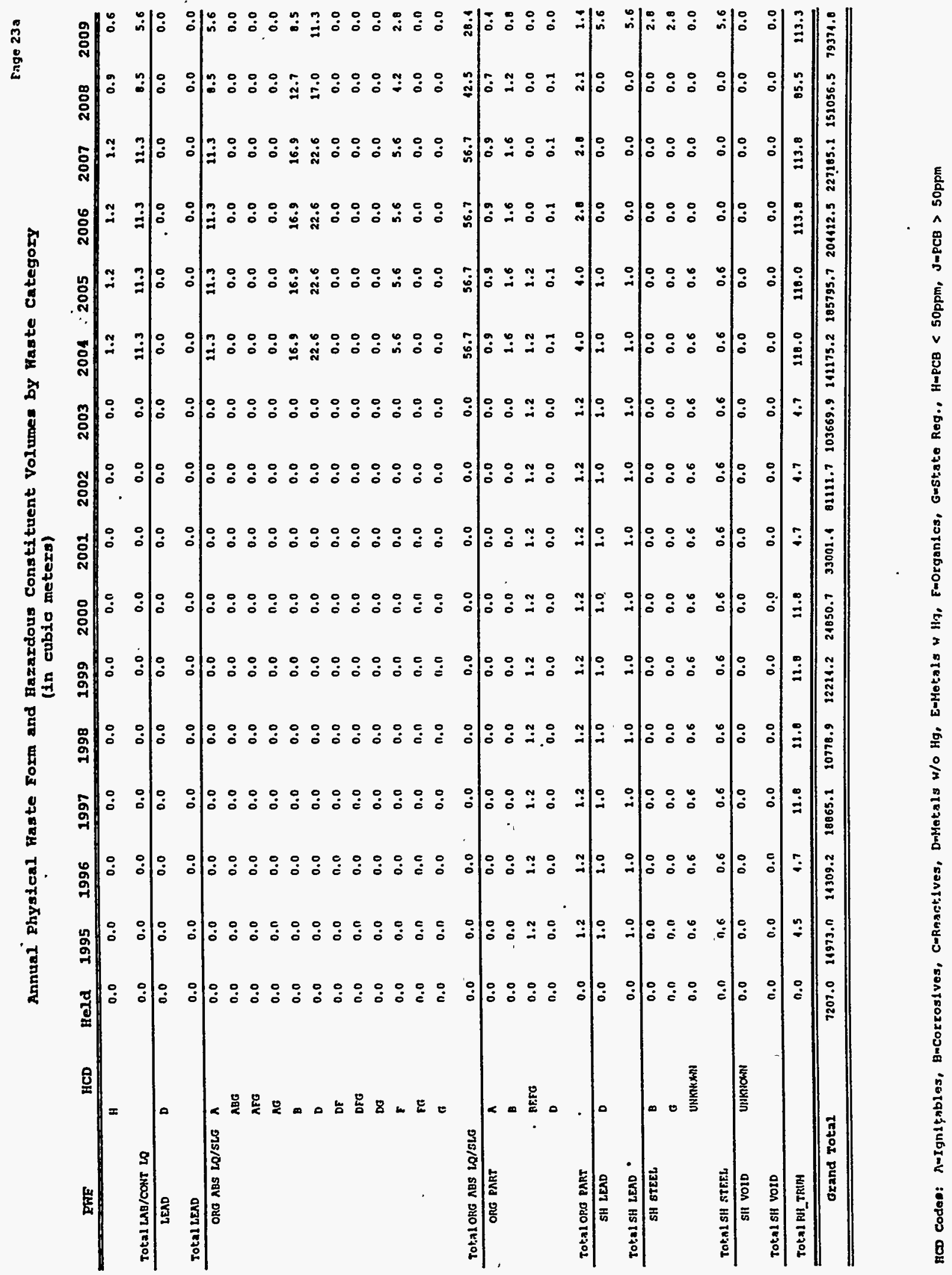


Annual Physical waste Form and Hazardous Constituent Volumes by Waste Category

\begin{tabular}{|c|c|c|c|c|c|c|c|c|c|c|c|c|c|c|c|c|c|}
\hline PWE & HCD & 2010 & 2011 & 2012 & 2013 & 2014 & 2015 & 2016 & 2017 & 2018 & 2019 & 2020 & 2021 & 2022 & 2023 & 2024 & Total Pot \\
\hline \multicolumn{18}{|c|}{ Waste Category: CH_LWW } \\
\hline cont sorl & NoNE & 1249.1 & 813.6 & 803.2 & 795.5 & 761.0 & 763.4 & 186.0 & 150.4 & 152.7 & 455.1 & 157.6 & 460.3 & 463.0 & 166.0 & 169.0 & 186004.6100 .0 \\
\hline TOtAICONT SOLL & & 1249.1 & 013.6 & 003.2 & 795.5 & 761.0 & 763.4 & 186.0 & 150.1 & 452.7 & 455.1 & 157.6 & 460.3 & 463.0 & 166.0 & 469.0 & 106804.6100 .0 \\
\hline D-Corbust & NONE & 2164.6 & 2161.1 & 2163.8 & 2161.6 & 2165.7 & 2169.9 & 2192.6 & 2192.6 & 2160.7 & 2160.7 & 2130.0 & 2138.0 & 2130.0 & 2130.3 & 2130.3 & 38164.6100 .0 \\
\hline Tota1D-congust & & 2164.6 & 2161.4 & 2163.0 & 2161.6 & 2165.7 & 2169.9 & 2192.6 & 2192.6 & 2160.7 & 2160.7 & 2138.0 & 2136.0 & 2138.0 & 2130.3 & 2130.3 & 58160.6100 .0 \\
\hline D-HET & HONE & 1107.2 & 1192.0 & 1200.1 & 1535.5 & 1511.4 & 1540.0 & 1560.7 & 1609.2 & 1619.9 & 1602.0 & 1604.8 & 1617.9 & 1631.6 & 2319.5 & 1334.6 & 39749.0100 .0 \\
\hline Tota1 D-HIET & & 1107.2 & 1192.0 & 1200.1 & 1535.5 & 1514.4 & 1548.0 & 1568.7 & 1609.2 & 1619.9 & 1602.0 & 1604.8 & 1627.9 & 1631.6 & 1319.5 & 2334.6 & 39749.0100 .0 \\
\hline D-IN NON-HTL & MONE & 13553.1 & 6543.7 & 5025.6 & 1933.7 & 14222.0 & 14222.0 & 467.0 & 500.0 & 487.1 & 161.3 & 155.4 & 159.4 & 163.5 & 167.4 & 412.0 & 804862.6100 .0 \\
\hline TOL:A1 D-IN NON-MTL & & 13553.4 & 6543.7 & 5025.6 & 1933.7 & 14222.0 & 21222.0 & 167.0 & 500.0 & 187.1 & 461.3 & 155.4 & 159.4 & 163.5 & 167.4 & 172.0 & 801862.6100 .0 \\
\hline D-METAL & MONE & 923.7 & 931.1 & 952.3 & 7916.1 & 7933.9 & 7947.3 & 0013.6 & 8264.0 & $025 \theta .2$ & 0038.5 & 8003.3 & 0023.5 & 8041.6 & 1107.6 & 1130.9 & 101584.4100 .0 \\
\hline Tot:AID-METAL & & 923.7 & 931.1 & 952.3 & 7916.1 & 7933.9 & 7947.3 & 8013.6 & 0261.0 & 0230.2 & 0030.5 & 8003.3 & 0023.5 & 6044.6 & 1107.6 & 2130.9 & 101584.1100 .0 \\
\hline D-sorL & MONE & 106.1 & 80.0 & 81.4 & 85.3 & 06.7 & 91.0 & 95.5 & 100.2 & 105.2 & 110.4 & 115.9 & 121.6 & 127.7 & 134.0 & 140.7 & 15121.2100 .0 \\
\hline Tot.n10-501L & & 2n6.1 & 80.8 & 01.1 & 85.3 & 86.7 & 91.0 & 95.5 & 100.2 & 105.2 & 110.4 & 115.9 & 121.6 & 127.7 & 134.0 & 140.7 & 15121.2100 .0 \\
\hline IN ORG LR/ALG & MOHE & 3155.0 & 2282.9 & 1425.5 & 2126.7 & 570.5 & 571.9 & 165.6 & 166.9 & 167.7 & 169.2 & 470.8 & 172.5 & 174.2 & 176.1 & 478.0 & 99372.6100 .0 \\
\hline TOt:Al IN ORG LQ/SLG & & 3155.0 & 2282.9 & 1425.3 & 1426.7 & 570.5 & 511.9 & 465.6 & 166.9 & 167.7 & 169.2 & 470.8 & 172.5 & 474.2 & 176.1 & 178.0 & 99372.8100 .0 \\
\hline IN ORG PART & NONE & 16.0 & 16.0 & 46.0 & 16.0 & 16.0 & 16.0 & 16.0 & 16.0 & 45.9 & 15.9 & 15.9 & 15.9 & 15.9 & 15.9 & 15.9 & 1686.9100 .0 \\
\hline Total IN ORO TMRT & & 46.0 & 46.0 & 16.0 & 16.0 & 46.0 & 16.0 & 16.0 & 16.0 & 15.9 & 15.9 & 45.9 & 15.9 & 15.9 & 45.9 & 15.9 & 1686.9100 .0 \\
\hline LAB/CONT LQ & MONE & 29.0 & 13.2 & 11.6 & 11.7 & 20.3 & 20.5 & 10.7 & 10.9 & 11.1 & 11.3 & 11.6 & 11.0 & 12.1 & 12.3 & 12.6 & 6767.4100 .0 \\
\hline TOt:al LAB/CONT LQ & & 29.0 & 13.2 & 11.6 & 11.7 & 10.3 & 10.5 & 10.7 & 10.9 & 11.1 & 11.3 & 11.6 & 11.0 & 12.1 & 12.3 & 12.6 & 6767.4100 .0 \\
\hline LEND & NONE & 3.8 & 0.6 & 0.3 & 4.3 & 4.0 & 1.0 & 1.0 & 1.0 & 1.0 & 4.0 & 4.0 & 4.0 & 4.0 & 0.0 & 0.0 & 1306.6100 .0 \\
\hline TotalLEAD & & 3.8 & 0.6 & 0.3 & 4.3 & 1.0 & 1.0 & 1.0 & 4.0 & 4.0 & 4.0 & 4.0 & 1.0 & 4.0 & 0.0 & 0.0 & 1306.6100 .0 \\
\hline ORG ABS LQ/SLG & MONE & 2620.9 & 1747.9 & 009.5 & 089.7 & 32.4 & 32.5 & 49.7 & 35.8 & 36.0 & 50.3 & 33.6 & 33.0 & 34.1 & 34.3 & 34.6 & 84735.7100 .0 \\
\hline Tota1ORG ABS LQ/SLG & & 2620.9 & 1747.9 & 889.5 & 889.7 & 32.4 & 32.5 & 19.7 & 35.0 & 36.0 & 50.3 & 33.6 & 33.0 & 34.1 & 34.3 & 34.6 & 89735.7100 .0 \\
\hline ORG EART & NONE & 18.3 & 18.2 & 18.5 & 18.7 & 49.0 & 19.6 & 49.1 & 49.6 & 19.1 & 19.3 & 19.9 & 19.8 & 50.1 & 50.3 & 50.6 & 1513.6100 .0 \\
\hline Tot.Al ORG PART & & 18.3 & 40.2 & 18.5 & 48.7 & 19.0 & 19.6 & 19.1 & 49.6 & 19.1 & 19.3 & 19.9 & 19.8 & 50.1 & 50.3 & 50.6 & 1513.6100 .0 \\
\hline UAKNoWn & MONE & 0.7 & 0.7 & 0.7 & 142.8 & 142.0 & 112.8 & 312.8 & 342.8 & 112.0 & 112.8 & 142.0 & 142.8 & 212.0 & 0.7 & 0.7 & 1142.9100 .0 \\
\hline Total UNKNOWN & & 0.7 & 0.7 & 0.7 & 142.8 & 142.0 & $142 . \theta$ & 112.0 & 112.0 & 212.8 & 142.8 & 112.0 & 142.0 & 142.0 & 0.7 & 0.7 & 1412.9100 .0 \\
\hline TOt:नICH_Ltw & & $2508 \mathrm{~B} .3$ & 15062.7 & 12649.0 & 16998.2 & 27370.1 & $276 n n .3$ & 13592.2 & 13873.1 & 13841.2 & 13601.5 & 13534.1 & 13581.0 . & .13632 .2 & 6241.9 & 6300.11 & 1403196.9 \\
\hline Waste Category: & CH_LSMH & & & & & & & & & & & & & & & & \\
\hline PATTERIES & a & 0.5 & 0.5 & 0.5 & 0.5 & 0.5 & 0.5 & 0.5 & 0.5 & 0.5 & 0.5 & 0.5 & 0.5 & 0.5 & 0.5 & 0.5 & 9.320 .8 \\
\hline & BD & 0.2 & 0.2 & 0.2 & 0.2 & 0.2 & 0.2 & 0.2 & 0.2 & 0.2 & 0.2 & 0.2 & 0.2 & 0.2 & 0.2 & 0.2 & $6.1 \quad 13.6$ \\
\hline & D & 1.0 & 1.0 & 1.0 & 0.7 & 0.7 & 0.7 & 0.7 & 0.7 & 0.7 & 0.7 & 0.7 & 0.7 & 0.7 & 0.7 & 0.7 & $29.2 \quad 65.1$ \\
\hline
\end{tabular}

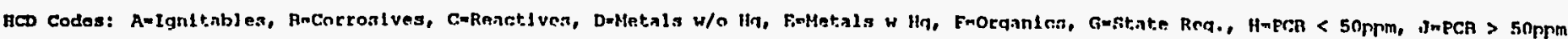




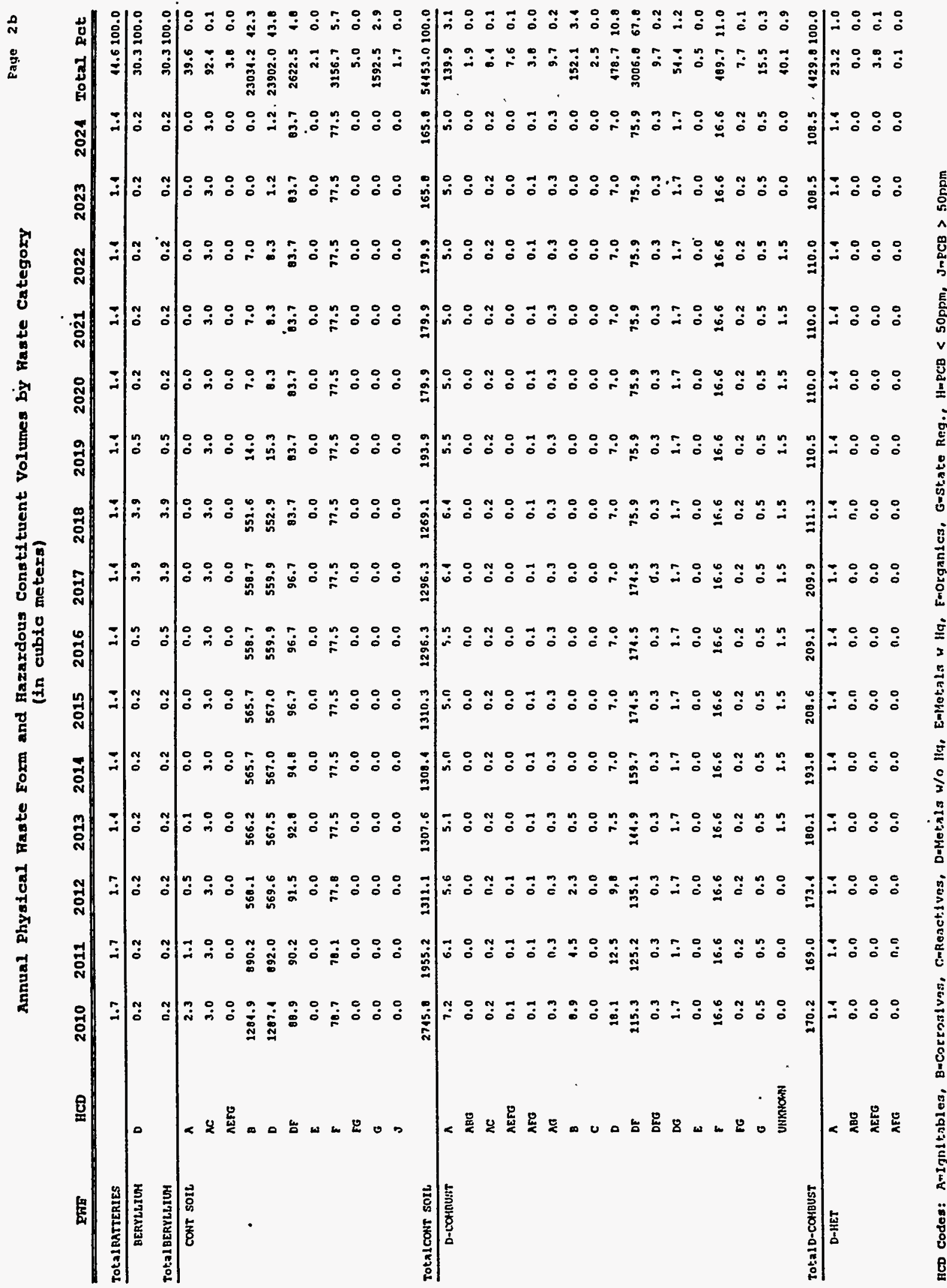


Ainual Phyoical Faste Form and Hazardous Constituent Volumes by Faste Category (In cubic meters)

\begin{tabular}{|c|c|c|c|c|c|c|c|c|c|c|c|c|c|c|c|c|c|c|}
\hline PWE & HCD & 2010 & 2011 & 2012 & 2013 & 2014 & 2015 & 2016 & 2017 & 2018 & 2019 & 2020 & 2021 & 2022 & 2023 & 2024 & Total P & Rot \\
\hline & $A B$ & 0.0 & 0.0 & 0.0 & 0.0 & 0.0 & 0.0 & 0.0 & 0.0 & 0.0 & 0.0 & 0.0 & 0.0 & 0.0 & 0.0 & 0.0 & 0.1 & 0.0 \\
\hline & B & 0.1 & 0.1 & 0.1 & 0.1 & 0.1 & 0.1 & 0.1 & 0.1 & 0.1 & 0.1 & 0.1 & 0.1 & 0.1 & 0.1 & 0.1 & 1.2 & 0.1 \\
\hline & c & 1.5 & 1.5 & 1.5 & . 1.5 & 1.5 & 1.5 & 1.5 & 1.5 & 1.5 & 1.5 & 1.5 & 1.5 & 1.5 & .1 .5 & 1.5 & 25.6 & 1.1 \\
\hline & D & 12.2 & 11.8 & 11.6 & 11.3 & 11.1 & 11.4 & 11.4 & 11.4 & 11.1 & 11.4 & 11.4 & 21.4 & 21.4 & 21.1 & 11.4 & 161.1 & 20.2 \\
\hline & DF & 17.1 & 18.2 & 10.6 & 19.0 & 19.7 & 19.6 & 19.6 & 19.6 & 15.3 & 15.3 & 15.3 & 15.3 & 25.3 & 25.3 & 15.3 & 678.0 & 29.7 \\
\hline & DFG & 0.0 & 0.0 & 0.0 & 0.0 & 0.0 & 0.0 & 0.0 & 0.0 & 0.0 & 0.0 & 0.0 & 0.0 & 0.0 & 0.0 & 0.0 & 17.9 & 0.7 \\
\hline & DG & 4.3 & 4.3 & 4.3 & 1.3 & 1.3 & 2.9 & 2.9 & 2.9 & 2.9 & 2.9 & 2.9 & 2,9 & 2.9 & 2.9 & 2.9 & 403.5 & 21.2 \\
\hline & $\mathbf{E}$ & 0.0 & 0.0 & 0.0 & 0.0 & 0.0 & 0.0 & 0.0 & 0.0 & 0.0 & 0.0 & 0.0 & 0.0 & 0.0 & 0.0 & 0.0 & 0.8 & 0.0 \\
\hline & EG & 0.0 & 0.0 & 0.0 & 0.0 & 0.0 & 0.0 & 0.0 & 0.0 & 0.0 & 0.0 & 0.0 & 0.0 & 0.0 & 0.0 & 0.0 & 0.2 & 0.0 \\
\hline & F & 2.5 & 2.5 & 2.5 & 2.5 & 2.5 & 2.5 & 2.5 & 2.5 & 2.5 & 2.5 & 2.5 & 2.5 & 2.5 & 2.5 & 2.5 & i45.1 & 6.3 \\
\hline & EG & 0.3 & 0.3 & 0.3 & 0.3 & 0.3 & 0.3 & 0.3 & 0.3 & 0.3 & 0.3 & 0.3 & 0.3 & 0.3 & 0.3 & 0.3 & 28.7 & 1.2 \\
\hline & G & 6.3 & 6.3 & 9.7 & 6.3 & 6.3 & 9.5 & 6.1 & 6.1 & 9.5 & 6.1 & 6.1 & 9.5 & 6.1 & 6.1 & 6.1 & 381.0 & 16.7 \\
\hline & UNANOONA & 0.0 & 0.0 & 0.0 & 2.5 & 2.5 & 2.5 & 2.5 & 2.5 & 2.5 & 2.5 & 2.5 & 2.3 & 2.5 & 0.0 & 0.0 & 25.9 & 1.2 \\
\hline Totn1D-HET & & 47.0 & 17.0 & 30.6 & 50.0 & 50.6 & 52.3 & 48.9 & 18.9 & 18.0 & 14.6 & 41.6 & 18.0 & 14.6 & 12.0 & 12.0 & 2280.71 & 100.0 \\
\hline D-IN MOt-MTI, & $\wedge$ & 2.6 & 1.2 & 0.1 & 0.1 & 0.1 & 0.0 & 0.1 & 0.0 & 0.1 & 0.0 & 0.1 & 0.0 & 0.1 & 0.0 & 0.1 & 16.3 & 1.6 \\
\hline & Anis: & 0.0 & 0.0 & $0 . n$ & 0.0 & 0.0 & 0.0 & n.0 & 0.0 & 0.0 & 0.0 & 0.0 & 0.0 & 0.0 & 0.0 & 0.0 & 1.3 & 0.0 \\
\hline & ns. & 2.5 & 2.5 & 2.5 & 2.5 & 2.5 & 2.5 & 2.5 & 2.5 & 2.5 & 2.5 & 2.5 & 2.5 & 2.5 & 2.5 & 2.5 & 77.5 & 2.7 \\
\hline & AFs & 0.0 & 0.0 & 0.0 & 0.0 & 0.0 & 0.0 & 0.0 & 0.0 & 0.0 & 0.0 & 0.0 & 0.0 & 0.0 & 0.0 & 0.0 & 2.6 & 0.0 \\
\hline & AG & 0.2 & 0.2 & 0.2 & 0.2 & 0.2 & 0.2 & 0.2 & 0.2 & 0.2 & 0.2 & 0.2 & 0.2 & 0.2 & 0.2 & 0.2 & 6.6 & 0.2 \\
\hline & B & 11.0 & 6.7 & 4.1 & 2.0 & 1.5 & 1.5 & 1.5 & 1.5 & 1.5 & 1.5 & 1.5 & 1.5 & 1.5 & 1.5 & 1.5 & 205.2 & 7.3 \\
\hline & c & 0.5 & 0.5 & 0.5 & 0.5 & 0.5 & 0.5 & 0.5 & 0.5 & 0.5 & 0.5 & 0.5 & 0.5 & 0.5 & 0.5 & 0.5 & 8.5 & 0.3 \\
\hline & D & 15.1 & 9.4 & 6.5 & 1.2 & 3.6 & 3.6 & 3.6 & 3.6 & 3.6 & 3.6 & 3.6 & 3.6 & 3.6 & 3.6 & 3.6 & 351.1 & 12.5 \\
\hline & DE & 16.0 & 19.0 & 50.0 & 51.1 & 52.6 & 58.1 & 54.1 & 54.1 & 44.0 & 41.0 & 14.0 & 14.0 & 14.0 & 16.0 & 14.0 & 1394.6 & 19.7 \\
\hline & DEG _ & 0.2 & 0.2 & 0.2 & 0.2 & 0.2 & 0.2 & 0.2 . & 0.2 & 0.2 & 0.2 & 0.2 & 0.2 & 0.2 & 0.2 & 0.2 & 6.6 & 0.2 \\
\hline & DG & 1.2 & 1.2 & 1.2 & 1.2 & 1.2 & 1.2 & 1.2 & 1.2 & 1.2 & 1.2 & 1.2 & 1.2 & 1.2 & 1.2 & 1.2 & 37.1 & 1.3 \\
\hline & E & 0.0 & 0.0 & 0.0 & 0.0 & 0.0 & 0.0 & 0.0 & 0.0 & 0.0 & 0.0 & 0.0 & 0.0 & 0.0 & 0.0 & 0.0 & 1.4 & 0.0 \\
\hline & r & 11.6 & 11.0 & 10.7 & 10.4 & 10.3 & 20.3 & 10.3 & 10.3 & 10.3 & 10.3 & 20.3. & 10.3 & 10.3 & 10.3 & 10.3 & 361.4 & 12.0 \\
\hline & ro & 0.1 & 0.1 & 0.1 & 0.1 & 0.1 & 0.1 & 0.1 & 0.1 & 0.1 & 0.1 & 0.1 & 0.1 & 0.1 & 0.1 & 0.1 & 5.3 & 0.1 \\
\hline & 6 & 3.4 & 3.4 & 3.4 & 3.4 & 3.1 & 3.4 & 3.4 & 3.1 & 3.4 & 3.4 & 3.1 & 3.4 & 3.4 & 3.4 & 3.4 & 299.4 & 10.6 \\
\hline & 애 & 0.0 & 0.0 & 0.0 & 0.0 & 0.0 & 0.0 & 0.0 & 0.0 & 0.0 & 0.0 & 0.0 & 0.0 & 0.0 & 0.0 & 0.0 & 0.0 & 0.0 \\
\hline Total D-IN NON-HTL & & 97.8 & 85.9 & 80.7 & 76.5 & 76.8 & 78.2 & 78.4 & 78.2 & 68.2 & 68.1 & 68.2 & 68.1 & 69.2 & 68.1 & 68.2 & 2005.41 & 100.0 \\
\hline D-METAL & $A$ & 0.2 & 0.2 & 0.3 & 1.2 & 1.2 & 1.2 & 1.2 & 1.2 & 1.2 & 1.2 & 1.2 & 1.2 & 1.2 & 0.1 & 0.1 & 17.8 & 0.0 \\
\hline • & B & 2.1 & 1.2 & 0.9 & 0.6 & 0.6 & 0.7 & 0.8 & 0.1 & 0.4 & 0.5 & 0.7 & 0.8 & 0.1 & 0.0 & 0.0 & 16.9 & 0.1 \\
\hline & c & 0.0 & 0.0 & 0.0 & 0.0 & 0.0 & 0.0 & 0.0 & 0.0 & 0.0 & 0.0 & 0.0 & 0.0 & 0.0 & 0.0 & 0.0 & 0.4 & 0.0 \\
\hline & D & 62.0 & 52.5 & 53.9 & 01.3 & 85.6 & 90.1 & 98.8 & 76.6 & 76.4 & 69.2 & 77.9 & 86.8 & 60.1 & 5.5 & 5.5 & 2300.8 & 7.7 \\
\hline & nF & 140.8 & 577.1 & 632.7 & 727.7 & 856.6 & 959.9 & 1002.1 & 1030.0 & 330.9 & 385.4 & 432.3 & 195.5 & 570.1 & 570.1 & 570.1 & 12746.5 & 12.0 \\
\hline - & DG & 0.0 & 0.0 & 0.0 & 0.0 & 0.0 & 0.0 & n. 0 & 0.0 & 0.0 & 0.0 & 0.0 & 0.0 & 0.0 & 0.0 & 0.0 & 1.2 & 0.0 \\
\hline & E & 0.0 & 0.0 & 0.0 & 0.0 & 0.0 & 0.0 & $0 . n$ & 0.0 & 0.0 & 0.0 & 0.0 & 0.0 & 0.0 & 0.0 & 0.0 & 0.7 & 0.0 \\
\hline
\end{tabular}

RCD Codes: AnIgnitahles, B-Corrosives, GeReactives, DeMetals w/o Hg, E-Metals w Hg, Emorganics, G-State Reg., H-PCB < 50ppm, J-PCB > 50ppm 

(in cubla meters)

\begin{tabular}{|c|c|c|c|c|c|c|c|c|c|c|c|c|c|c|c|c|c|}
\hline PHE & $\mathrm{HCD}$ & 2010 & 2011 & 2012 & 2013 & 2014 & 2015 & 2016 & 2017 & 2018 & 2019 & 2020 & 2021 & 2022 & 2023 & 2021 & Total Pat \\
\hline & EE & 0.1 & 0.1 & 0.1 & 0.1 & 0.1 & 0.1 & 0.1 & 0.1 & 0.1 & 0.1 & 0.1 & 0.1 & 0.1 & 0.1 & 0.1 & 3.90 .0 \\
\hline & $\bar{F}$ & 145.0 & 141.7 & 192.1 & 236.0 & 760.7 & 784.5 & 032.0 & 713.0 & 713.0 & 736.8 & 784.5 & 832.0 & 689.3 & 1.6 & 1.6 & $11921.5 \quad 10.0$ \\
\hline . & G & 0.0 & 0.0 & 0.0 & 0.0 & 0.0 & 0.0 & 0.1 & 0.0 & 0.0 & 0.0 & 0.0 & 0.1 & 0.0 & 0.0 & 0.0 & $393.1 \quad 1.3$ \\
\hline & H & 0.0 & 0.0 & 0.0 & 0.0 & 0.0 & 0.0 & 0.0 & 0.0 & 0.0 & 0.0 & 0.0 & 0.0 & 0.0 & 0.0 & 0.0 & 0.2 \\
\hline & J & 0.0 & 0.0 & 0.0 & 0.0 & 0.0 & 0.0 & 0.0 & 0.0 & 0.0 & 0.0 & 0.0 & 0.0 & 0.0 & 0.0 & 0.0 & 0.2 \\
\hline & UNBWOWN & 0.0 & 0.0 & 0.0 & 231.9 & 231.9 & 231.9 & 231.9 & 231.9 & 231.9 & 231.9 & 231.9 & 231.9 & 231.9 & 0.0 & 0.0 & 2319.6 \\
\hline TOt:A1 D-HETAL & & 650.4 & 776.0 & 800.3 & 1780.1 & 1937.1 & 2068.7 & 2167.1 & 2053.7 & 1362.5 & 1425.6 & 1529.0 & 1640.0 & 1553.5 & 377.6 & 577.7 & 29761.399 .9 \\
\hline D-soll & $\pi$ & 0.1 & 0.0 & 0.0 & 0.0 & 0.0 & $0.0^{\circ}$ & 0.0 & 0.0 & 0.0 & 0.0 & 0.0 & 0.0 & 0.0 & 0.0 & 0.0 & $3.0 \quad 0.0$ \\
\hline & B & 99.6 & 69.0 & 14.0 & 43.9 & 13.0 & 43.0 & 13.3 & 43.3 & 12.7 & 1.1 & 0.5 & 0.5 & 0.5 & 0.0 & 0.0 & $27306.1 \quad 19.8$ \\
\hline & D & 99.7 & 69.1 & 41.1 & 43.9 & 13.9 & 43.9 & 13.3 & 43.3 & 12.0 & 1.1 & 0.6 & 0.6 & 0.6 & 0.0 & 0.0 & $1789.1 \quad 19.9$ \\
\hline & $\varepsilon$ & 0.1 & 0.0 & 0.0 & 0.0 & 0.0 & 0.0 & 0.0 & 0.0 & 0.0 & 0.0 & 0.0 & 0.0 & 0.0 & 0.0 & 0.0 & $\begin{array}{ll}3.0 & 0.0\end{array}$ \\
\hline & o & 0.0 & 0.0 & 0.0 & 0.0 & 0.0 & 0.0 & 0.0 & 0.0 & 0.0 & 0.0 & 0.0 & 0.0 & 0.0 & 0.0 & 0.0 & 0.30 .0 \\
\hline TotalD-sole & & 199.7 & 138.3 & 88.3 & 07.9 & 07.0 & 87.0 & 86.7 & 86.7 & 05.6 & 2.3 & 1.2 & 1.2 & 1.2 & 0.1 & 0.1 & 3502.1100 .0 \\
\hline EXPLOSIVES & $A$ & 0.3 & 0.3 & 0.3 & 0.3 & 0.3 & 0.3 & 0.3 & 0.3 & 0.3 & 0.3 & 0.3 & 0.3 & 0.3 & 0.3 & 0.3 & 14.295 .2 \\
\hline & A & 0.0 & $0 . n$ & 0.0 & 0.0 & $n . n$ & 0.0 & 0.0 & $n . n$ & 0.0 & 0.0 & 0.0 & 0.0 & 0.0 & 0.0 & 0.0 & $0.5 \quad 3.0$ \\
\hline & D & 0.0 & 0.0 & 0.0 & 0.0 & 0.0 & 0.0 & 0.0 & 0.0 & 0.0 & 0.0 & 0.0 & 0.0 & 0.0 & 0.0 & 0.0 & $\begin{array}{ll}0.1 & 0.9\end{array}$ \\
\hline TOtaL EXPLOSIVES & & 0.3 & 0.3 & 0.3 & 0.3 & 0.3 & 0.3 & 0.3 & 0.3 & 0.3 & 0.3 & 0.3 & 0.3 & 0.3 & 0.3 & 0.3 & 14.9100 .0 \\
\hline IN ORG LQ/SLG & $n$ & 0.0 & 0.0 & 0.0 & 0.0 & 0.0 & 0.0 & 0.0 & 0.0 & 0.0 & 0.0 & 0.0 & 0.0 & 0.0 & 0.0 & 0.0 & 1.40 .7 \\
\hline & B & 2.1 & 2.1 & 2.1 & 2.1 & 2.1 & 2.1 & 2.1 & 2.1 & 2.1 & 2.1 & 2.1 & 2.1 & 2.1 & 2.1 & 2.1 & $13.6 \quad 23.2$ \\
\hline & c & 0.9 & 0.9 & 0.9 & 0.9 & 0.9 & 0.9 & 0.9 & 0.9 & 0.9 & 0.9 & 0.9 & 0.9 & 0.9 & 0.9 & 0.9 & 16.10 .5 \\
\hline & $D$ & 0.6 & 0.6 & 0.6 & 0.6 & 0.6 & 0.6 & 0.6 & 0.6 & 0.6 & 0.6 & 0.6 & 0.6 & 0.6 & 0.6 & 0.6 & $20.5 \quad 15.1$ \\
\hline & DE & 2.4 & 2.5 & 2.7 & 2.8 & 3.1 & 3.3 & 3.3 & 3.3 & 1.8 & 1.0 & 1.8 & 1.8 & 1.0 & 1.8 & 2.8 & $66.7 \quad 35.5$ \\
\hline & $\mathbf{E}$ & 0.0 & 0.0 & 0.0 & 0.0 & 0.0 & 0.0 & 0.0 & 0.0 & 0.0 & 0.0 & 0.0 & 0.0 & 0.0 & 0.0 & 0.0 & 0.10 .1 \\
\hline & $r$ & 0.0 & 0.0 & 0.0 & 0.0 & 0.0 & 0.0 & 0.0 & 0.0 & 0.0 & 0.0 & 0.0 & 0.0 & 0.0 & 0.0 & 0.0 & $1.9 \quad 1.0$ \\
\hline & 0 & 1.6 & 1.6 & 1.6 & 1.6 & 1.6 & 1.6 & 1.6 & 1.6 & 1.6 & 1.6 & 1.6 & 1.6 & 1.6 & 1.6 & 1.6 & $27.7 \quad 14.7$ \\
\hline & 3 & 0.0 & 0.0 & 0.0 & 0.0 & 0.0 & 0.0 & 0.0 & 0.0 & 0.0 & 0.0 & 0.0 & 0.0 & 0.0 & 0.0 & 0.0 & $\begin{array}{ll}1.1 & 0.7\end{array}$ \\
\hline TOtAL IN ORG LO/SLC & & 0.0 & 0.1 & 0.3 & 0.1 & 0.6 & 0.9 & 0.9 & 8.9 & 7.1 & 7.1 & 7.4 & 7.4 & 7.1 & 7.1 & 7.1 & 107.7100 .0 \\
\hline IN ORG PART & 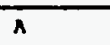 & 0.0 & 0.0 & 0.0 & 0.0 & 0.0 & 0.0 & 0.0 & 0.0 & 0.0 & 0.0 & 0.0 & 0.0 & 0.0 & 0.0 & 0.0 & 0.20 .0 \\
\hline & ABG & 0.0 & 0.0 & 0.0 & 0.0 & 0.0 & 0.0 & 0.0 & 0.0 & 0.0 & 0.0 & 0.0 & 0.0 & 0.0 & 0.0 & 0.0 & $\begin{array}{ll}1.7 & 0.4\end{array}$ \\
\hline & Ars & 0.1 & 0.1 & 0.1 & 0.1 & 0.1 & 0.1 & 0.1 & 0.1 & 0.1 & 0.1 & 0.1 & 0.1 & 0.1 & 0.1 & 0.1 & $\begin{array}{ll}3.5 & 0.8\end{array}$ \\
\hline & $A G$ & 0.2 & 0.2 & 0.2 & 0.2 & 0.2 & 0.2 & 0.2 & 0.2 & 0.2 & 0.2 & 0.2 & 0.2 & 0.2 & 0.2 & 0.2 & 0.02 .1 \\
\hline & B & 3.0 & 3.0 & 3.0 & 3.0 & 3.0 & 3.0 & 3.0 & 3.0 & $3: 0$ & 3.0 & 3.0 & 3.0 & 3.0 & 3.0 & 3.0 & 19.711 .8 \\
\hline & c & 1.1 & 1.1 & 1.4 & 1.1 & 1.4 & 1.4 & 2.4 & 1.4 & 1.4 & 1.4 & 1.4 & 1.4 & 1.1 & 1.1 & 1.4 & $24.1 \quad 5.7$ \\
\hline & $\mathbf{D}$ & 0.6 & 0.6 & 0.6 & 0.6 & 0.6 & 0.6 & 0.6 & 0.6 & 0.6 & 0.6 & 0.6 & 0.6 & 0.6 & 0.6 & 0.6 & 19.0 \\
\hline & $\mathrm{DF}$ & 0.6 & 0.6 & 0.6 & 0.6 & 0.6 & 0.6 & 0.6 & 0.6 & 0.6 & 0.6 & 0.6 & 0.6 & 0.6 & 0.6 & 0.6 & 19.4 \\
\hline & Dro & 0.2 & 0.2 & 0.2 & 0.2 & 0.2 & 0.2 & 0.2 & 0.2 & 0.2 & 0.2 & 0.2 & 0.2 & 0.2 & 0.2 & 0.2 & 8.8 2.1 \\
\hline & $D G$ & 1.6 & 1.6 & 1.6 & 1.6 & 1.6 & 1.6 & 1.6 & 1.6 & 1.6 & 1.6 & 1.6 & 1.6 & 1.6 & 1.6 & 1.6 & 49.511 .7 \\
\hline
\end{tabular}

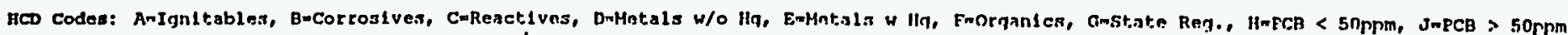


Annual Physical Waste Form and Hazardous Constituent Volumes by Faste Category (in cubia meters)

\begin{tabular}{|c|c|c|c|c|c|c|c|c|c|c|c|c|c|c|c|c|c|}
\hline PWE & HCD & 2010 & 2011 & 2012 & 2013 & 2014 & 2015 & 2016 & 2017 & 2018 & 2019 & 2020 & 2021: & 2022 & 2023 & 2021 & Total Pct \\
\hline & $\varepsilon$ & 0.0 & 0.0 . & 0.0 & 0.0 & 0.0 & 0.0 & 0.0 & 0.0 & 0.0 & 0.0 & 0.0 & 0.0 & 0.0 & 0.0 & 0.0 & 0.10 .0 \\
\hline & $\varepsilon 0$ & 0.0 & 0.5 & 0.0 & 0.5 & 0.0 & 8.5 & 0.0 & 0.5 & 0.0 & 0.5 & 0.0 & 0.5 & 0.0 & 8.5 & 0.0 & $119.0 \quad 28.3$ \\
\hline & E & 1.8 & 1.8 & 1.8 & 1.0 & 1.0 & 1.8 & 1.8 & 1.0 & 1.8 & 1.8 & 1.8 & 1.0 & 1.0 & 1.0 & 1.8 & $58.0 \quad 13.8$ \\
\hline & EG & 0.2 & 0.2 & 0.2 & 0.2 & 0.2 & 0.2 & 0.2 & 0.2 & 0.2 & 0.2 & 0.2 & 0.2 & 0.2 & 0.2 & 0.2 & $\begin{array}{ll}7.0 & 1.6\end{array}$ \\
\hline & a & 2.0 & 2.0 & 2.0 & 2.8 & 2.8 & 2.8 & 2.8 & 2.0 & 2.8 & 2.0 & 2.8 & 2.8 & 2.0 & 2.8 & 2.0 & 50.112 .0 \\
\hline TOtal IN ORG PART & & 13.0 & 21.5 & 13.0 & 21.5 & 13.0 & 21.5 & 13.0 & 21.5 & 13.0 & 21.5 & 13.0 & 21.5 & 23.0 & 21.5 & 13.0 & $\$ 19.9100 .0$ \\
\hline LMB/CONT LQ & $\wedge$ & 15.2 & 10.6 & 8.1 & 6.5 & 6.1 & 6.1 & 6.1 & 6.1 & 6.1 & 6.1 & 6.1 & 6.1 & 6.1 & 6.1 & 6.1 & $343.2 \quad 11.2$ \\
\hline & AB & 0.3 & 0.3 & 0.3 & 0.3 & 0.3 & 0.3 & 0.3 & 0.3 & 0.3 & 0.3 & 0.3 & 0.3 & 0.3 & 0.3 & 0.3 & 11.0 \\
\hline & ABCDEG & 0.3 & 0.3 & 0.3 & 0.3 & 0.3 & 0.3 & 0.3 & 0.3 & 0.3 & 0.3 & 0.3 & 0.3 & 0.3 & 0.3 & 0.3 & 10.9 \\
\hline & AECFG & 0.3 & 0.3 & 0.3 & 0.3 & 0.3 & 0.3 & 0.3 & 0.3 & 0.3 & 0.3 & 0.3 & 0.3 & 0.3 & 0.3 & 0.3 & 10.9 \\
\hline & AgEFG & 0.9 & 0.9 & 0.9 & $0 . n$ & 0.0 & 0.0 & 0.0 & 0.0 & 0.0 & 0.0 & 0.0 & 0.0 & 0.0 & 0.0 & 0.0 & 38.0 \\
\hline & Abro & 4.1 & 4.1 & 4.1 & 4.1 & 4.1 & 2.7 & 2.7 & 2.7 & 2.7 & 2.7 & 2.7 & 2.7 & 2.7 & 2.7 & 2.7 & $\begin{array}{lll}164.6 \quad 15.2\end{array}$ \\
\hline & AFG & 1.1 & 1.1 & 1.1 & 1.1 & 1.2 & 1.1 & 1.1 & 1.1 & 1.1 & 1.1 & 1.1 & 1.1 & 1.1 & 1.1 & 1.1 & 35.6 \\
\hline & $\lambda G$ & 1.1 & 1.1 & 1.1 & 1.1 & 1.1 & 1.1 & 1.1 & 1.1 & 1.1 & 1.1 & 1.1 & 1.1 & 1.1 & 2.2 & 1.1 & 36.3 \\
\hline & B & 17.4 & 11.0 & 7.9 & 5.3 & 1.7 & 4.7 & 1.7 & 1.7 & 1.7 & 4.7 & 1.7 & 4.7 & 4.7 & 4.7 & 4.7 & 335.611 .0 \\
\hline & AD & 0.2 & 0.2 & 0.2 & 0.2 & 0.2 & 0.2 & 0.2 & 0.2 & 0.2 & 0.2 & 0.2 & 0.2 & 0.2 & 0.2 & 0.2 & $\begin{array}{ll}0.0 & 0.2\end{array}$ \\
\hline & ADG & 0.3 & 0.3 & 0.3 & 0.3 & 0.3 & 0.3 & 0.3 & 0.3 & 0.3 & 0.3 & 0.3 & 0.3 & 0.3 & 0.3 & 0.3 & 10.9 \\
\hline & BG & 2.4 & 2.1 & 2.4 & 2.1 & 2.4 & 1.7 & 1.7 & 1.7 & 1.7 & 1.7 & 1.7 & 1.7 & 1.7 & 1.7 & 2.7 & 243.6 \\
\hline & c & 0.7 & 0.7 & 0.7 & 0.7 & 0.7 & 0.7 & 0.7 & 0.7 & 0.7 & 0.7 & 0.7 & 0.7 & 0.7 & 0.7 & 0.7 & 13.8 \\
\hline & 66 & 0.3 & 0.3 & 0.3 & 0.3 & 0.3 & $n, 3$ & 0.3 & 0.3 & 0.3 & 0.3 & 0.3 & 0.3 & 0.3 & 0.3 & 0.3 & 10.9 \\
\hline & D & 17.0 & 20.6 & 7.1 & 1.0 & 1.2 & 1.2 & 4.2 & 1.2 & 4.2 & 4.2 & 4.2 & 4.2 & 1.2 & 4.2 & 1.2 & 338.511 .1 \\
\hline - & DE & 0.7 & 0.7 & 0.7 & 0.7 & 0.7 & 0.7 & 0.7 & 0.7 . & 0.7 & 0.7 & 0.7 & 0.7 & 0.7 & 0.7 & 0.7 & $21.9 \quad 0.7$ \\
\hline & DFG & 0.3 & 0.3 & 0.3 & 0.3 & 0.3 & 0.3 & 0.3 & 0.3 & 0.3 & 0.3 & 0.3 & 0.3 & 0.3 & 0.3 & 0.3 & 10.9 \\
\hline & DG & 4.2 & 1.1 & 4.1 & 1.1 & 1.1 & 2.7 & 2.7 & 2.7 & 2.7 & 2.7 & 2.7 & 2.7 & 2.7 & 2.7 & 2.7 & $161.6 \quad 15.2$ \\
\hline & $\varepsilon$ & 0.6 & 0.6 & 0.6 & 0.6 & 0.6 & 0.6 & 0.6 & 0.6 & 0.6 & 0.6 & 0.6 & 0.6 & 0.6 & 0.6 & 0.6 & 19.40 .6 \\
\hline & $\boldsymbol{\varepsilon}$ & 12.0 & 10.2 & 9.3 & 0.6 & 8.1 & 8.4 & 8.1 & 0.4 & 0.1 & 8.1 & 0.1 & 8.4 & 8.1 & 8.4 & 0.1 & 271.3 \\
\hline & .60 & 0.7 & 0.7 & 0.7 & 0.7 & 0.7 & 0.7 & 0.7 & 0.7 & 0.7 & 0.7 & 0.7 & 0.7 & 0.7 & 0.7 & 0.7 & 20.2 \\
\hline & G & 7.2 & 7.2 & 7.2 & 7.2 & 7.2 & 7.2 & 7.2 & 7.2 & 1.2 & 7.2 & 7.2 & 7.2 & 7.2 & 7.2 & 7.2 & 216.8 \\
\hline - & H & 4.9 & 2.0 & 1.7 & 0.9 & 0.7 & 0.7 & 0.7 & 0.7 & 0.7 & 0.7 & 0.7 & 0.7 & 0.7 & 0.7 & 0.7 & 82.6 \\
\hline & $\mathbf{J}$ & 0.7 & 0.7 & 0.7 & 0.7 & 0.7 & 0.7 & 0.7 & 0.7 & 0.7 & 0.7 & 0.7 & 0.7 & 0.7 & 0.7 & 0.7 & 12.5 \\
\hline TOt:al LAB/CONT LR & & 94.0 & 12.7 & 62.1 & 52.5 & 50.1 & 17.0 & 47.0 & 17.0 & 17.0 & 17.0 & 17.0 & 17.0 & 17.0 & 17.0 & 47.0 & 3041.0100 .0 \\
\hline LEAD & D & 12.1 & 9.7 & 8.9 & 7.9 & 8.0 & 7.0 & 7.3 & 7.0 & 7.3 & 7.0 & 7.3 & 7.0 & 7.3 & 6.7 & 7.0 & $1302.6 \quad 99.4$ \\
\hline & E & 0.0 & 0.0 & 0.0 & 0.0 & 0.0 & 0.0 & 0.0 & 0.0 & 0.0 & 0.0 & 0.0 & 0.0 & 0.0 & 0.0 & 0.0 & 3.4 \\
\hline & 태 & 0.0 & 0.0 & 0.0 & 0.0 & 0.0 & 0.0 & 0.0 & 0.0 & 0.0 & 0.0 & 0.0 & 0.0 & 0.0 & 0.0 & 0.0 & 2.2 \\
\hline & UNKNOWN & 0.0 & 0.0 & 0.0 & 0.2 & 0.2 & 0.2 & 0.2 & 0.2 & 0.2 & 0.2 & 0.2 & 0.2 & 0.2 & 0.0 & 0.0 & 2.1 \\
\hline TOtallEAD & & 12.1 & 9.7 & 8.9 & 0.1 & 8.2 & 7.2 & 7.5 & 7.2 & 7.5 & 1.2 & 7.5 & 7.2 & 1.5 & 6.7 & 7.0 & 1310.1100 .0 \\
\hline MERCURY & E & 0.5 & 0.5 & 0.5 & 0.1 & 0.1 & 0.1 & 0.4 & 0.1 & 0.1 & 0.4 & 0.1 & 0.4 & 0.4 & 0.4 & 0.1 & $37.0 \quad 94.2$ \\
\hline & EH & 0.0 & 0.0 & 0.0 & 0.0 & 0.0 & 0.0 & 0.0 & 0.0 & 0.0 & 0.0 & 0.0 & 0.0 & 0.0 & 0.0 & 0.0 & 2.2 \\
\hline
\end{tabular}

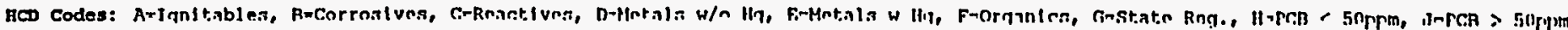




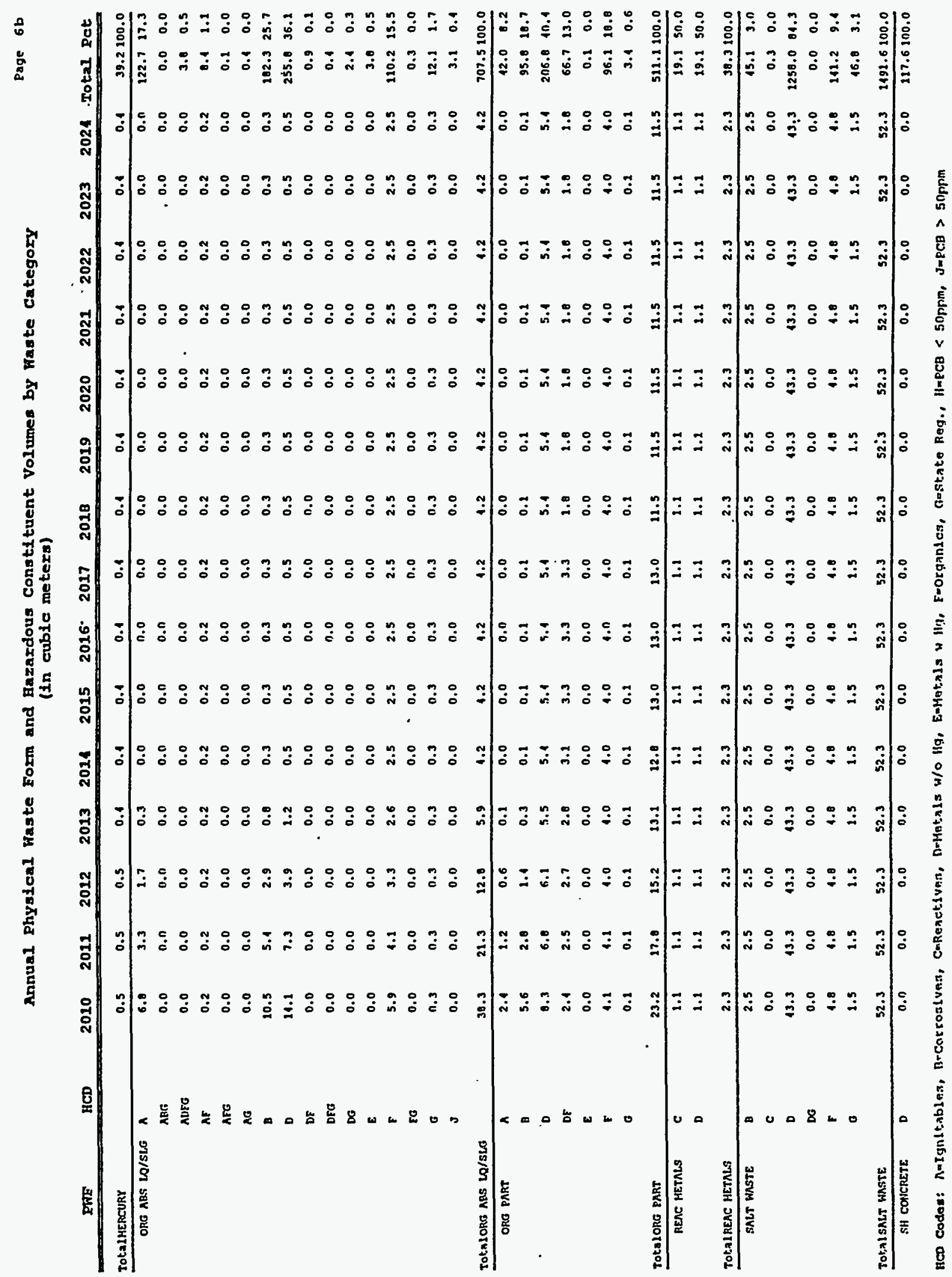




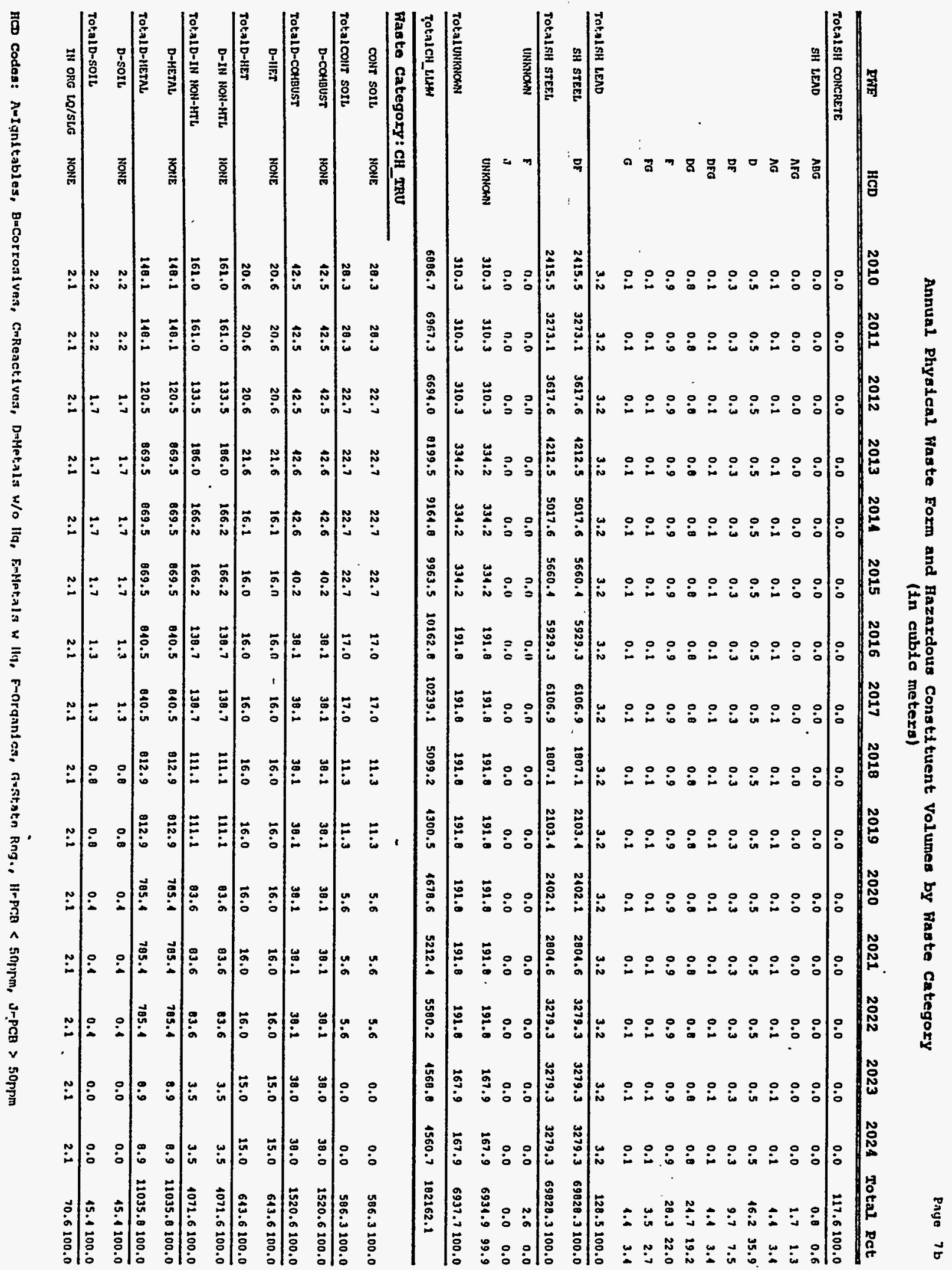




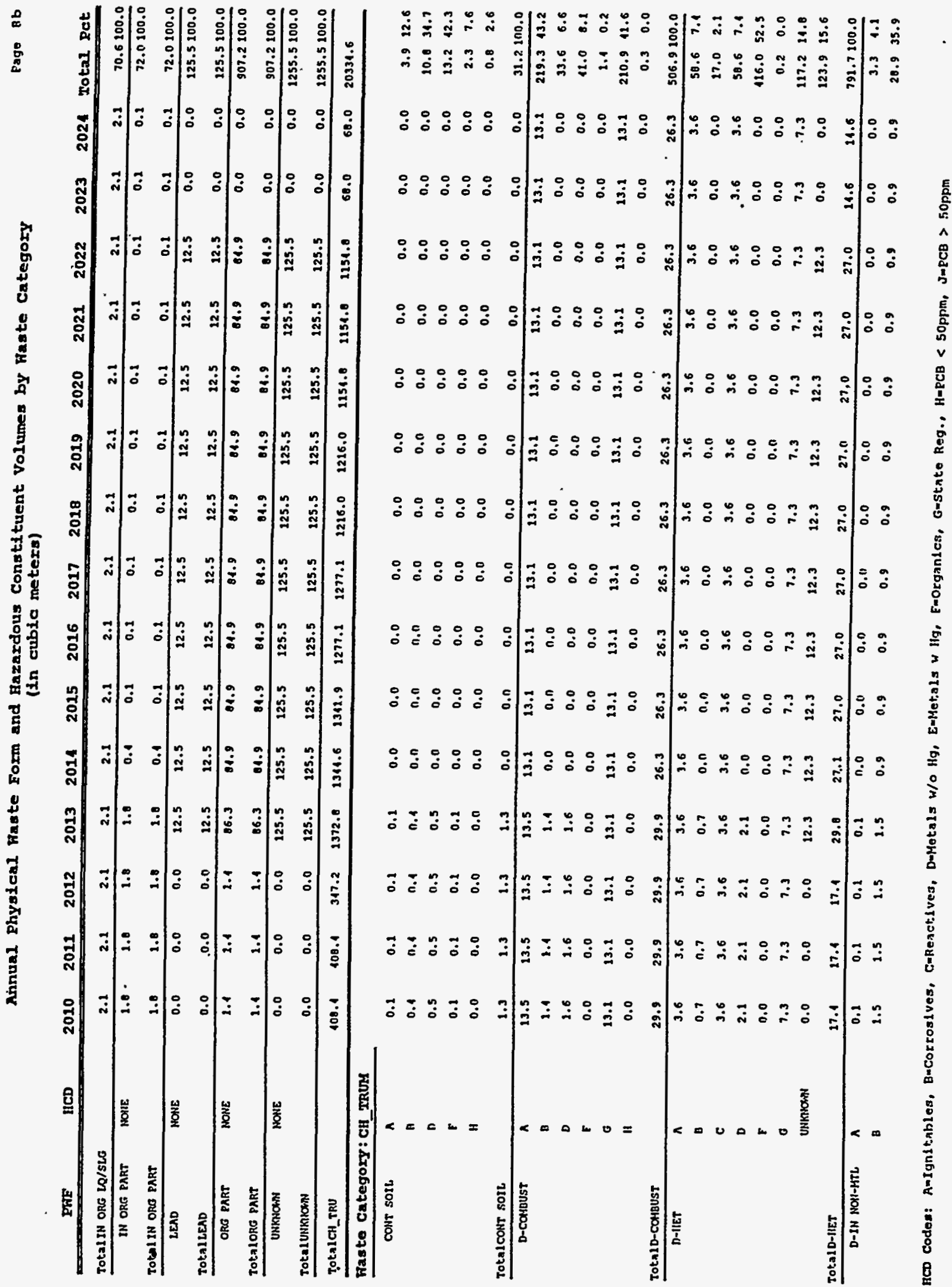




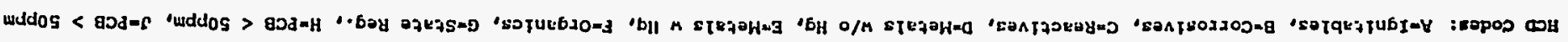

\begin{tabular}{|c|c|c|c|c|c|c|c|c|c|c|c|c|c|c|c|c|c|c|}
\hline $0.2 \varepsilon$ & 0.5 & 0.0 & 0.0 & 0.0 & 0.0 & 0.0 & 0.0 & 0.0 & 0.0 & 0.0 & 0.0 & 0.0 & $z \cdot 0$ & $2 \cdot 0$ & $2 \cdot 0$ & $2 \cdot 0$ & $v$ & LAY3 O40 \\
\hline $0.00 \mathrm{r}$ & $6.98 E$ & 0.0 & 0.0 & 0.0 & 0.0 & 0.0 & 0.0 & 0.0 & 0.0 & 0.0 & 0.0 & 0.0 & $t \cdot n t$ & $t \cdot n$ & $t \cdot \operatorname{tr}$ & $T \cdot \operatorname{tin}$ & & D7S/OT SQV 5YOI E'TO \\
\hline 0.01 & '•रह & 0.0 & 0.0 & 0.0 & 0.0 & 0.0 & 0.0 & 0.0 & 0.0 & 0.0 & 0.0 & 0.0 & $\bullet \mathrm{I}$ & 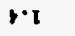 & $\cdot 1$ & $\because 1$ & $\mathbf{d}$ & \\
\hline 0.04 & L'VEt & 0.0 & 0.0 & 0.0 & 0.0 & 0.0 & 0.0 & 0.0 & 0.0 & 0.0 & 0.0 & 0.0 & $9 \cdot 5$ & $9 \cdot 5$ & $9 \cdot 5$ & $9 \cdot \varepsilon$ & a & \\
\hline $0.0 \varepsilon$ & 0.101 & 0.0 & 0.0 & 0.0 & 0.0 & 0.0 & 0.0 & 0.0 & 0.0 & 0.0 & 0.0 & 0.0 & $2 \cdot 1$ & $2 \cdot V$ & $2 \cdot \boldsymbol{r}$ & $2 \cdot 1$ & g & \\
\hline 0.02 & $\varepsilon \cdot\llcorner 9$ & 0.0 & 0.0 & 0.0 & 0.0 & 0.0 & 0.0 & 0.0 & 0.0 & 0.0 & 0.0 & 0.0 & a.z & $a \cdot z$ & $\theta \cdot z$ & $0 \cdot 2$ & $v$ & פ7S/OT SUY 980 \\
\hline 0.001 & $2 \cdot 5$ & 0.0 & 0.0 & $2 \cdot 0$ & $2 \cdot 0$ & $2 \cdot 0$ & $2 \cdot 0$ & $2 \cdot 0$ & $2 \cdot 0$ & $2 \cdot 0$ & $2 \cdot 0$ & $2 \cdot 0$ & $2 \cdot 0$ & 0.0 & 0.0 & 0.0 & & av37tejos \\
\hline $0.00 \mathrm{r}$ & $z \cdot s$ & 0.0 & 0.0 & 2.0 & 200 & 2.0 & 2.0 & $2 \cdot 0$ & $2 \cdot 0$ & 200 & $2 \cdot 0$ & $2 \cdot 0$ & $2 \cdot 0$ & 0.0 & 0.0 & 0.0 & a & qv37 \\
\hline 0.001 & 0.002 & L. & $\iota \cdot 9$ & $4 \cdot 8$ & $6 \cdot 8$ & L.9 & $2 \cdot 8$ & $L \cdot \theta$ & $2 \cdot \theta$ & $2 \cdot 0$ & $L^{\circ} \theta$ & $2 \cdot 8$ & $9 \cdot \pi$ & $9 \cdot \pi$ & $9 \cdot 4$ & $2 \cdot \pi$ & & 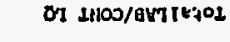 \\
\hline L.9 & $0 . n t$ & 0.0 & 0 & 0.0 & 0.0 & 0.0 & 8.0 & 0.0 & $8 \cdot 0$ & $\theta \cdot 0$ & 0.0 & 0.0 & $8 \cdot 0$ & D.0 & 0.0 & $8 \cdot 0$ & $s$ & \\
\hline r.or & L. $2 z$ & 8.0 & $e \cdot 0$ & $8 \cdot 0$ & 0.0 & 0.0 & 8.0 & 0.0 & 0.0 & 0.0 & $0 \cdot 0$ & 0.0 & $\tau \cdot \mathbf{t}$ & $2 \cdot 1$ & $2 \cdot 1$ & $2 \cdot 1$ & H & \\
\hline $9 \cdot 9 \varepsilon$ & r.9e & c. & $c \cdot 1$ & $E \cdot b$ & $c \cdot 1$ & $\varepsilon \cdot$ & $\varepsilon \cdot V$ & $\varepsilon \cdot r$ & $\varepsilon \cdot \boldsymbol{r}$ & $\varepsilon \cdot \boldsymbol{r}$ & $E \cdot 1$ & $\varepsilon \cdot \boldsymbol{r}$ & $9 \cdot 1$ & $9 \cdot 1$ & 9.8 & $9 \cdot 6$ & 3 & \\
\hline 2.6 & $2.6 \mathrm{I}$ & 0.0 & 0.0 & 0.0 & 0.0 & 0.0 & 0.0 & 0.0 & 0.0 & 0.0 & 0.0 & 0.0 & $0 \cdot 0$ & $9 \cdot 0$ & $8 \cdot 0$ & 0.0 & a & \\
\hline .9. & $0 \cdot n$ & 0.0 & $0 \cdot 0$ & 0.0 & 0.0 & 0.0 & 00 & $8 \cdot 0$ & 0.0 & $\theta \cdot 0$ & 0.0 & $0 \cdot 0$ & $8 \cdot 0$ & $8 \cdot 0$ & 0.0 & 8.0 & o & \\
\hline$z \cdot \varepsilon \tau$ & $\varepsilon \cdot 8 r$ & $L^{\circ} \tau$ & $\iota \cdot \tau$ & $\iota \cdot \tau$ & 4.1 & $L \cdot x$ & $3 \cdot 1$ & 4.5 & $\iota \cdot \tau$ & $L \cdot \tau$ & $L \cdot 1$ & $L \cdot \tau$ & $9 \cdot 2$ & $9 \cdot z$ & $9 \cdot z^{\circ}$ & $9 \cdot 2$ & a & \\
\hline 0.9 & $E \cdot b t$ & 0.0 & 0.0 & 0.0 & 0.0 & 0.0 & 0.0 & 0.0 & 0.0 & 0.0 & 0.0 & 0.0 & $9 \cdot 0$ & $9 \cdot 0$ & 9.0 & $9 \cdot 0$ & $v$ & on 21100/8\%7 \\
\hline $0.00 \tau$ & $\vdash \cdot \mathcal{E}$ & $r \cdot 0$ & $t \cdot 0$ & $1 \cdot 0$ & $\mathfrak{x} \cdot 0$ & $1 \% 0$ & $I \cdot 0$ & $t \cdot 0$ & $1 \cdot 0$ & $t \cdot 0$ & I.0 & $r \cdot 0$ & $1 \cdot 0$ & $1 \cdot 0$ & $1 \cdot 0$ & $1 \cdot 0$ & & 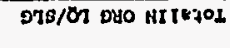 \\
\hline 0.05 & I. & 0.0 & 0.0 & 0.0 & 0.0 & 0.0 & 0.0 & 0.0 & 0.0 & 0.0 & 0.0 & 0.0 & 0.0 & 0.0 & 0.0 & 0.0 & a & \\
\hline 0.02 & $\cdot 0$ & 0.0 & 0.0 & 0.0 & 0.0 & 0.0 & 0.0 & 0.0 & 0.0 & $0 \cdot 0$ & 0.0 & 0.0 & 0.0 & 0.0 & 0.0 & 0.0 & 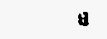 & \\
\hline 0.08 & $\tau \cdot \tau$ & 0.0 & 0.0 & 0.0 & 0.0 & 0.0 & 0.0 & 0.0 & 0.0 & 0.0 & 0.0 & $0 \therefore 0$ & 0.0 & 0.0 & 0.0 & 0.0 & $v$ & 975/07 080 $\mathrm{HI}$ \\
\hline 0.0011 & $\cdot z$ & 0.0 & 0.0 & $0 \cdot 0$ & 0.0 & 0.0 & 0.0 & 0.0 & 0.0 & 0.0 & 0.0 & 0.0 & 1.0 & $1 \cdot 1)$ & $1 \cdot 0$ & $1 ?$ & & $7105-1276401$ \\
\hline $9 \cdot z$ & 0.0 & 0.0 & 0.0 & 0.0 & 0.0 & $0 \cdot 0$ & 0.0 & 0.0 & 0.0 & $0 \cdot 0$ & 0.0 & 0.0 & 0.0 & u.u & 0.0 & $u \cdot u$ & II & \\
\hline 9.2 & 1.0 & 0.0 & 0.0 & 0.0 & 0.0 & 0.0 & 0.0 & 0.0 & $0 \cdot 0$ & 0.0 & 0.0 & 0.0 & 0.0 & 0.0 & 0.0 & 0.0 & 3 & \\
\hline$\varepsilon=21$ & $0 \cdot \tau$ & 0.0 & 0.0 & 0.0 & 0.0 & 0.0 & 0.0 & 0.0 & 0.0 & 0.0 & 0.0 & 0.0 & 0.0 & 0.0 & 0.0 & 0.0 & a & \\
\hline $9 \cdot 1 \varepsilon$ & 8.0 & 0.0 & 0.0 & 0.0 & 0.0 & 0.0 & 0.0 & 0.0 & 0.0 & 0.0 & 0.0 & 0.0 & 0.0 & 0.0 & 0.0 & 0.0 & B & \\
\hline 9.21 & E.0 & 0.0 & 0.0 & 0.0 & 0.0 & 0.0 & 0.0 & 0.0 & 0.0 & 0.0 & 0.0 & 0.0 & 0.0 & 0.0 & 0.0 & 0.0 & $v$ & $710 \mathrm{~s}-\mathrm{a}$ \\
\hline $0.00 t$ & S.9LLZT & 6.5 & 6.5. & $\vdash \cdot 0<2 t$ & $r \cdot 0 L Z T$ & $1 \cdot 0<2 \pi$ & VOLLt & V.0LzI & $r 0<z I$ & $\because 0 L z \tau$ & $\because 0 \angle 2 t$ & $.0 L 2 t$ & $6.7 L 2 t$ & $\varepsilon \cdot L$ & $\varepsilon \cdot L$ & $\varepsilon^{\circ} \cdot L$ & & TVL:SH-dTEjOL \\
\hline $9^{\circ}<\mathrm{L}$ & PCI56 & 0.0 & 0.0 & L. 266 & 2.766 & เ.166 & $L^{\circ} .766$ & '. 166 & $\mathcal{L} \cdot 166$ & $\cdot \cdot 166$ & ¿.166 & L.166 & $\iota^{\circ} \tau 66$ & 0.0 & 0.0 & 0.0 & MHONYMN & \\
\hline$c \cdot 12$ & $t \cdot r z<2$ & 0.0 & 0.0 & $0 . z L z$ & $0 \cdot z<z$ & $0 \cdot z<z$ & $0.2<z$ & $0 \cdot z\llcorner z$ & $0 \cdot z L z$ & $0 \cdot z \iota z$ & $0 \cdot z\llcorner z$ & $0.2 L z$ & $I \cdot z<2$ & 1.0 & $r \cdot 0$ & $1 \cdot 0$ & 4 & \\
\hline 6.02 & $2 \cdot 811$ & $6 \cdot 5$ & 6.5 & s'o & $s \cdot 9$ & $5 \cdot 9$ & $s \cdot 9$ & $\mathbf{s} \cdot 9$ & $s \cdot 9$ & $5 \cdot 9$ & $s \cdot 9$ & $s \cdot 9$ & $r \cdot 2$ & $5 \cdot 9$ & $s \cdot 9$ & $5 \cdot 9$ & a & \\
\hline I.0 & V'ST & 0.0 & 0.0 & $2 \cdot 0$ & $2 \cdot 0$ & $2 \cdot 0$ & $2 \cdot 0$ & $2 \cdot 0$ & 2.0 & 2.0 & $2 \cdot 0$ & $2 \cdot 0$ & $2 \cdot 0$ & $s \cdot 0$ & $s \cdot 0$ & s.o & a & \\
\hline 0.0 & $2 \cdot \tau$ & 0.0 & 0.0 & 0.0 & 0.0 & 0.0 & 0.0 & 0.0 & 0.0 & 0.0 & 0.0 & 0.0 & 0.0 & 0.0 & 0.0 & 0.0 & $v$ & TVLIJH-a \\
\hline 0.008 & 5.00 & 6.2 & $6 \cdot 2$ & 6.2 & $6 \cdot 2$ & 6.2 & $6 \cdot 2$ & 6.2 & 6.2 & $6 \cdot 2$ & 6.2 & 6.2 & E'V & $E \cdot r$ & $\varepsilon \cdot 1$ & $E \cdot 6$ & & 7IH-HOW NI-OTEJOI \\
\hline 1.26 & $6 \cdot r$ & 0.0 & 0.0 & 0.0 & 0.0 & 0.0 & 0.0 & 0.0 & 0.0 & 0.0 & 0.0 & 0.0 & 0.0 & 0.0 & 0.0 & 0.0 & 3 & \\
\hline 9.2E & c.o & $6 \cdot 0$ & 6.0 & $6 \cdot 0$ & 6.0 & $6 \cdot 0$ & 6.0 & $6 \cdot 0$ & 6.0 & 6.0 & 6.0 & 6.0 & $s \cdot r$ & $s \cdot \tau$ & $\boldsymbol{s} \cdot \boldsymbol{t}$ & $s \cdot t$ & a & $\cdot$ \\
\hline 1.61 & 6.51 & 6.0 & 6.0 & 6.0 & 6.0 & 6.0 & 6.0 & 6.0 & 6.0 & 6.0 & 6.0 & 6.0 & 6.0 & 6.0 & 6.0 & 6.0 & $\mathrm{~J}$ & \\
\hline
\end{tabular}

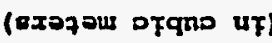

96 obed 

(in cubia meters)

\begin{tabular}{|c|c|c|c|c|c|c|c|c|c|c|c|c|c|c|c|c|c|c|}
\hline FWF & HCD & 2010 & 2011 & 2012 & 2013 & 2014 & 2015 & 2016 & 2017 & 2018 & 2019 & 2020 & 2021 & 2022 & 2023 & 2024 & Total PC & et \\
\hline & 8 & 0.1 & 0.1 & 0.4 & 0.4 & 0.0 & 0.0 & 0.0 & 0.0 & 0.0 & 0.0 & 0.0 & 0.0 & 0.0 & 0.0 & 0.0 & 10.160 & 60.0 \\
\hline & D & 0.0 & 0.0 & 0.0 & 0.0 & 0.0 & 0.0 & 0.0 & 0.0 & 0.0 & 0.0 & 0.0 & 0.0 & 0.0 & 0.0 & 0.0 & 0.8 & 5.0 \\
\hline Tota1ORG PART & & 0.7 & 0.7 & 0.7 & 0.7 & 0.0 & 0.0 & 0.0 & 0.0 & 0.0 & 0.0 & 0.0 & 0.0 & 0.0 & .0 .0 & 0.0 & 16.8100 & 00.0 \\
\hline TPOtalCH_TRIMH & & 07.1 & 87.1 & 07.1 & 1361.3 & 1336.1 & 1336.0 & 1336.0 & 2336.0 & 1336.0 & 2336.0 & 1336.0 & 1336.0 & 1336.0 & 38.8 & 50.0 & 14760.4 & \\
\hline Faste Categ & IY: $\mathrm{knz}$ & & & & & & & & & & & & & & & & & \\
\hline BATTERIES & $A B$ & 0.0 & 0.0 & 0.0 & 0.0 & 0.0 & 0.0 & 0.0 & 0.0 & 0.0 & 0.0 & 0.0 & 0.0 & 0.0 & 0.0 & 0.0 & 0.5 & 0.1 \\
\hline & B & 2.6 & 2.6 & 2.6 & 2.6 & 2.6 & 2.6 & 2.6 & 2.6 & 2.6 & 2.6 & 2.6 & 2.6 & 2.6 & 2.6 & 2.6 & 93.02 & 24.1 \\
\hline & BC & 0.1 & 0.1 & 0.1 & 0.1 & 0.1 & 0.1 & 0.1 & 0.1 & 0.1 & 0.1 & 0.1 & 0.1 & 0.1 & 0.1 & 0.1 & 5.1 & 1.3 \\
\hline & BD & 0.1 & 0.1 & 0.1 & 0.1 & 0.1 & 0.1 & 0.1 & 0.1 & 0.1 & 0.1 & 0.1 & 0.1 & 0.1 & 0.1 & 0.1 & 3.5 & 0.9 \\
\hline & BDF & 0.7 & 0.7 & 0.7 & 0.7 & 0.7 & 0.7 & 0.7 & 0.7 & 0.7 & 0.7 & 0.7 & 0.7 & 0.7 & 0.7 & 0.7 & 22.6 & 5.8 \\
\hline & BDG & 0.1 & 0.1 & 0.1 & 0.1 & 0.1 & 0.2 & 0.1 & 0.1 & 0.1 & 0.1 & 0.1 & 0.1 & 0.1 & 0.1 & 0.2 & 14.6 & 3.8 \\
\hline & BE & 0.1 & 0.1 & 0.1 & 0.1 & 0.1 & 0.1 & 0.1 & 0.1 & 0.1 & 0.1 & 0.1 & 0.1 & 0.1 & 0.1 & 0.1 & 3.8 & 1.5 \\
\hline & BEG & 0.6 & 0.6 & 0.6 & 0.6 & 0.6 & 0.6 & 0.6 & 0.6 & 0.6 & 0.6 & 0.6 & 0.6 & 0.6 & 0.6 & 0.6 & 18.3 & 1.7 \\
\hline & BG & 1.4 & 1.0 & 2.2 & 2.7 & 3.4 & 1.2 & 5.3 & 6.6 & 0.2 & 10.2 & 12.8 & 16.0 & 19.9 & 24.9 & 31.2 & 158.711 & 41.1 \\
\hline & D & 0.5 & 0.5 & 0.5 & 0.5 & 0.5 & 0.5 & 0.5 & 0.5 & 0.5 & 0.5 & 0.5 & 0.5 & 0.5 & 0.5 & $\cdot 0.5$ & 22.0 & 5.7 \\
\hline & no & 0.0 & $0 . n$ & 0.0 & 0.0 & 0.0 & 0.0 & 0.0 & 0.0 & 0.0 & 0.0 & 0.0 & 0.0 & 0.0 & 0.0 & 0.0 & 6.5 & 1.6 \\
\hline & E & 0.7 & 0.7 & 0.7 & 0.7 & 0.7 & 0.7 & 0.7 & 0.7 & 0.7 & 0.7 & 0.7 & 0.7 & 0.7 & 0.7 & 0.7 & 34.5 & 8.9 \\
\hline TOLALARTTERIES & & 7.1 & 7.7 & 0.1 & 0.7 & 9.3 & 10.2 & 11.2 & 12.5 & 14.1 & 16.2 & 10.7 & 21.9 & 23.0 & 30.8 & 31.0 & 305.6100 & 00.0 \\
\hline CONT SOIL & $A$ & 0.9 & 0.9 & 0.9 & 0.9 & 0.0 & 0.0 & 0.0 & 0.0 & 0.0 & 0.0 & 0.0 & 0.0 & 0.0 & 0.0 & 0.0 & 110.912 & 12.3 \\
\hline & AETG & 0.0 & 0.0 & 0.0 & 0.0 & 0.0 & 0.0 & $0 . n$ & 0.0 & 0.0 & 0.0 & 0.0 & 0.0 & 0.0 & 0.0 & 0.0 & 1.0 & 0.1 \\
\hline & B & 1.9 & 1.9 & 1.9 & 1.9 & 0.0 & 0.0 & 0.0 & 0.0 & 0.0 & 0.0 & 0.0 & 0.0 & 0.0 & 0.0 & 0.0 & 210.324 & 24.9 \\
\hline & c & 0.0 & 0.0 & 0.0 & 0.0 & 0.0 & 0.0 & 0,0 & 0.0 & 0.0 & 0.0 & 0.0 & 0.0 & 0.0 & 0.0 & 0.0 & 0.0 & 0.0 \\
\hline & D & 2.6 & 2.6 & 2.6 & 2.6 & 0.0 & 0.0 & 0.0 & 0.0 & 0.0 & 0.0 & 0.0 & 0.0 & 0.0 & 0.0 & 0.0 & 318.233 & 33.0 \\
\hline & $\mathbf{F}$ & 5.0 & 5.0 & 5.8 & 5.8 & 3.2 & 5.2 & 5.2 & 5.2 & 3.2 & 5.2 & 3.2 & 5.2 & 5.2 & 3.2 & 5.2 & 236.524 & 24.5 \\
\hline & G & 0.2 & 0.2 & 0.2 & 0.2 & 0.2 & 0.2 & 0.2 & 0.2 & 0.2 & 0.2 & 0.2 & 0.2 & 0.2 & 0.2 & 0.2 & 0.9 & 0.9 \\
\hline & H & 0.3 & 0.3 & 0.3 & 0.3 & 0.0 & 0.0 & 0.0 & 0.0 & 0.0 & 0.0 & 0.0 & 0.0 & 0.0 & 0.0 & 0.0 & 39.6 & 4.1 \\
\hline Tota1CoNT SOIL & & 12.0 & 12.0 & 12.0 & 12.0 & 3.4 & 5.1 & 5.1 & 5.4 & 5.4 & 5.1 & .5 .1 & 5.4 & 3.1 & 5.1 & 5.4 & 963.7100 & 00.0 \\
\hline D-COMBUST & $\boldsymbol{A}$ & 12.7 & 15.2 & 18.3 & 22.2 & 26.8 & 32.9 & 10.5 & 49.9 & 61.7 & 76.5 & 95.0 & 118.1 & 116.9 & 103.0 & 228.1 & $1231.2 \quad 10$ & 10.5 \\
\hline & ARCG & 0.0 & 0.0 . & 0.0 & 0.0 & 0.0 & 0.0 & 0.0 & 0.0 & 0.0 & 0.0 & 0.0 & 0.0 & 0.0 & 0.0 & 0.0 & 0.2 & 0.0 \\
\hline & AMDE & 7.5 & 7.5 & 7.5 & 7.5 & 7.5 & 7.5 & 1.5 & 7.5 & 7.5 & 7.5 & 7.5 & 7.5 & 7.5 & 7.5 & 7.5 & 226.1 & 7.4 \\
\hline & ABEEG & 0.0 & 0.0 & 0.0 & 0.0 & 0.0 & 0.0 & 0.0 & 0.0 & 0.0 & 0.0 & 0.0 & 0.0 & 0.0 & 0.0 & 0.0 & 2.0 & 0.0 \\
\hline & $A B G$ & 0.0 & 0.0 & 0.0 & 0.0 & 0.0 & 0.0 & 0.0 & 0.0 & 0.0 & 0.0 & 0.0 & 0.0 & 0.0 & 0.0 & 0.0 & 0.9 & 0.0 \\
\hline & $A C$ & 0.3 & 0.3 & 0.3 & 0.3 & 0.3 & 0.3 & 0.3. & 0.3 & 0.3 & 0.3 & 0.3 & 0.3 & 0.3 & 0.3 & 0.3 & 11.5 & 0.3 \\
\hline & ADG & 0.0 & 0.0 & 0.0 & 0.0 & 0.0 & 0.0 & 0.0 & 0.0 & 0.0 & 0.0 & 0.0 & 0.0 & 0.0 & 0.0 & 0.0 & 0.5 & 0.0 \\
\hline & AEFG & 0.0 & 0.0 & 0.0 & 0.0 & 0.0 & 0.0 & 0.0 & 0.0 & 0.0 & 0.0 & 0.0 & 0.0 & 0.0 & 0.0 & 0.0 & 0.1 & 0.0 \\
\hline & AF & 1.5 & 1.5 & 1.5 & 1.5 & 1.5 & 1.5 & 1.5 & 1.5 & 1.5 & 1.5 & 1.5 & 1.5 & 1.5 & 1.5 & 1.5 & 30.0 & 1.6 \\
\hline & ArG & 0.0 & 10.0 & 12.6 & 15.7 & 19.7 & 24.6 & 30.8 & 38.5 & 40.1 & 60.1 & 75.2 & 94.0 & 217.5 & 146.9 & 103.6 & 917.230 & 30.1 \\
\hline
\end{tabular}

HCD Codes: AnIgnitables, B-Corrosives, CeRnactivas, D-Methls w/O Hg, EmMetals w Hg, EoOrganlcs, G-Stante Reg., HmFCB < 50ppm, JmECB > 50ppm 

Annual Physical Waste Form and Hazardous Constituent Volumes by Haste Category
(In cubic meters)

\begin{tabular}{|c|c|c|c|c|c|c|c|c|c|c|c|c|c|c|c|c|c|c|}
\hline PWE & HCD & 2010 & 2011 & 2012 & 2013 & 2014 & 2015 & 2016 & 2017 & 2018 & 2019 & 2020 & 2021 & 2022 & 2023 & 2024 & Total & Pat \\
\hline & AG & 3.0 & 3.8 & 3.8 & 3.0 & 3.0 & 3.0 & 3.0 & 3.8 & 3.8 & 3.8 & 3.8 & 3.8 & 3.0 & 3.0 & 3.0 & 127.8 & 4.2 \\
\hline & B & 1.0 & 1.0 & 1.0 & 1.0 & 0.3 & 0.3 & 0.3 & 0.3 & 0.3 & 0.3 & 0.3 & 0.3 & 0.3 & 0.3 & 0.3 & 97.7 & 3.2 \\
\hline & BDS & 0.0 & 0.0 & 0.0 & 0.0 & $0 . n$ & 0.0 & 0.0 & 0.0 & 0.0 & 0.0 & 0.0 & 0.0 & 0.0 & 0.0 & 0.0 & 0.2 & $0 . n$ \\
\hline & 86 & 0.0 & 0.0 & 0.0 & 0.0 & 0.0 & 0.0 & 0.0 & 0.0 & 0.0 & 0.0 & 0.0 & 0.0 & 0.0 & $0.0^{\circ}$ & 0.0 & 0.2 & 0.0 \\
\hline & $c$ & 0.0 & 0.0 & 0.0 & 0.0 & 0.0 & 0.0 & 0.0 & 0.0 & 0.0 & 0.0 & 0.0 & 0.0 & 0.0 & 0.0 & 0.0 & 1.0 & 0.0 \\
\hline & D & 1.0 & 1.0 & 1.0 & 1.0 & 0.2 & 0.2 & 0.2 & 0.2 & 0.2 & 0.2 & 0.2 & 0.2 & 0.2 & 0.2 & 0.2 & 95.6 & 3.1 \\
\hline & $D G$ & 0.0 & 0.0 & 0.0 & 0.0 & 0.0 & 0.0 & 0.0 & 0.0 & 0.0 & 0.0 & 0.0 & 0.0 & 0.0 & 0.0 & 0.0 & 1.0 & 0.0 \\
\hline & Ec & 0.0 & 0.0 & 0.0 & 0.0 & 0.0 & 0.0 & 0.0 & 0.0 & 0.0 & 0.0 & 0.0 & 0.0 & 0.0 & 0.0 & 0.0 & 0.5 & 0.0 \\
\hline & $\mathbf{r}$ & 0.2 & 0.2 & 0.2 & 0.2 & 0.1 & 0.1 & 0.1 & 0.1 & 0.1 & 0.1 & 0.1 & 0.1 & 0.1 & 0.1 & 0.1 & 55.9 & 1.0 \\
\hline & $G$ & 1.2 & 4.2 & 4.2 & 4.2 & 4.2 & 1.2 & 4.2 & 4.2 & 4.2 & 4.2 & 1.2 & 1.2 & 4.2 & 4.2 & 4.2 & 206.2 & 6.7 \\
\hline & G & 0.0 & 0.0 & 0.0 & 0.0 & 0.0 & 0.0 & 0.0 & 0.0 & 0.0 & 0.0 & 0.0 & 0.0 & 0.0 & 0.0 & 0.0 & 0.5 & 0.0 \\
\hline & $"$ & 0.0 & 0.0 & 0.0 & 0.0 & 0.0 & 0.0 & 0.0 & 0.0 & 0.0 & 0.0 & 0.0 & 0.0 & 0.0 & 0.0 & 0.0 & 6.3 & 0.2 \\
\hline & ง & 0.0 & 0.0 & 0.0 & 0.0 & 0.0 & 0.0 & 0.0 & 0.0 & 0.0 & 0.0 & 0.0 & 0.0 & 0.0 & 0.0 & 0.0 & 4.1 & 0.1 \\
\hline TOt:al D-COHRUST & & 41.1 & 15.6 & 51.2 & $58.2 *$ & 65.1 & 76.1 & 89.0 & 107.0 & 228.4 & 255.2 & 188.7 & 230.6 & 203.0 & 348.5 & 430.3 & 3037.9 & 100.0 \\
\hline D-HET & $\wedge$ & 0.9 & 0.9 & 0.9 & 0.9 & 0.9 & 0.9 & 0.9 & 0.9 & 0.9 & 0.9 & 0.9 & 0.9 & 0.9 & 0.9 & 0.9 & 32.1 & 21.3 \\
\hline & $\Lambda G$ & $n .0$ & 0.0 & 0.0 & 0.0 & 0.0 & 0.0 & $0 . n$ & 0.0 & 0.0 & 0.0 & 0.0 & 0.0 & 0.0 & 0.0 & 0.0 & 14.4. & 15.6 \\
\hline & n & 0.5 & 0.5 & 0.5 & 0.5 & 0.1 & 0.1 & $0.4^{\circ}$ & 0.1 & 0.4 & 0.4 & 0.4 & 0.4 & 0.4 & 0.4 & 0.1 & 26.0 & 9.2 \\
\hline & $c$ & 0.2 & $n .2$ & 0.2 & 0.2 & 0.2 & 0.2 & 0.2 & 0.2 & 0.2 & 0.2 & 0.2 & 0.2 & 0.2 & 0.2 & 0.2 & 5.7 & 2.0 \\
\hline & D & 0.4 & 0.4 & 0.4 & 0.1 & 0.2 & 0.2 & 0.2 & 0.2 & 0.2 & 0.2 & 0.2 & 0.2 & 0.2 & 0.2 & 0.2 & 11.0 & 14.4 \\
\hline & $r$ & 0.0 & 0.0 & 0.0 & 0.0 & 0.0 & 0.0 & 0.0 & 0.0 & 0.0 & 0.0 & 0.0 & 0.0 & 0.0 & 0.0 & 0.0 & 2.1 & 0.8 \\
\hline 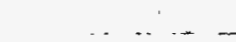 & IG & 0.0 & 0.0 & 0.0 & 0.0 & 0.0 & 0.0 & $\ldots 0.0$ & 0.0 & 0.0 & 0.0 & 0.0 & 0.0 & 0.0 & 0.0 & 0.0 & 7.6 & 2.6 \\
\hline & 6 & 0.2 & 0.2 & 0.2 & 0.2 & 0.2 & 0.2 & 0.2 & 0.2 & 0.2 & 0.2 & 0.2 & 0.2 & 0.2 & 0.2 & 0.2 & 00.6 & 28.4 \\
\hline & $H$ & 1.3 & 1.3 & 1.3 & 1.3 & 1.3 & 1.3 & 1.3 & 2.3 & 1.3 & 1.3 & 1.3 & 1.3 & 2.3 & 1.3 & 1.3 & 12.9 & 15.1 \\
\hline & unknowns & 0.0 & 0.0 & 0.0 & 0.0 & 0.0 & 0.0 & 0.0 & 0.0 & 0.0 & 0.0 & 0.0 & 0.0 & 0.0 & 0.0 & 0.0 & 0.0 & 0.0 \\
\hline TOt:al D-HET & & 3.0 & 3.8 & 3.8 & 3.0 & 3.5 & 3.5 & 3.3 & 3.5 & 3.5 & 3.5 & 3.5 & 3.5 & 3.5 & 3.5 & 3.5 & 283.3 & 99.9 \\
\hline D-IN NON-MTL & $\boldsymbol{A}$ & 0.1 & 0.1 & 0.1 . & 0.1 & 0.0 & 0.0 & 0.0 & 0.0 & 0.0 & 0.0 & 0.0 & 0.0 & 0.0 & 0.0 & 0.0 & 18.5 & 7.0 \\
\hline & ABCEG & 1.1 & 1.4 & 1.4 & 1.4 & 1.4 & 1.4 & 1.4 & 1.4 & 1.1 & 1.1 & 1.1 & 1.4 & 1.4 & 1.4 & 2.4 & 13.9 & 16.6 \\
\hline & B & 0.7 & 0.7 & 0.7 & 0.7 & 0.2 & 0.2 & 0.2 & 0.2 & 0.2 & 0.2 & 0.2 & 0.2 & 0.2 & 0.2 & 0.2 & 72.3 & 27.4 \\
\hline & c & 0.1 & 0.1 & 0.1 & 0.1 & 0.1 & 0.1 & 0.1 & 0.1 & 0.1 & 0.1 & 0.1 & 0.1 & 0.1 & 0.1 & 0.1 & 2.1 & 0.8 \\
\hline & D & 1.0 & 1.0 & 1.0 & 1.0 & 0.4 & 0.1 & 0.4 & 0.1 & 0.1 & 0.1 & 0.4 & 0.1 & 0.1 & 0.1 & 0.1 & 84.6 & 32.1 \\
\hline & $F$ & 0.7 & 0.7 & 0.7 & 0.7 & 0.6 & 0.6 & 0.6 & 0.6 & 0.6 & 0.6 & 0.6 & 0.6 & 0.8 & 0.6 & 0.6 & 36,5 & 13.0 \\
\hline & G & 0.1 & 0.1 & 0.1 & 0.1 & 0.1 & 0.1 & 0.1 & 0.1 & 0.1 & 0.1 & 0.1 & 0.1 & 0.1 & 0.1 & 0.1 & 5.5 & 2.1 \\
\hline TOtalD-IN IONS-HTL & & 1.5 & 1.5 & 1.5 & 4.5 & 3.1 & 3.1 & 3.1 & 3.1 & 3.1 & 3.1 & 3.1 & 3.1 & 3.1 & 3.1 & 3.1 & 263.8 & 100.0 \\
\hline D-METAL & $A$ & 0.5 & 0.5 & 0.5 & 0.5 & 0.4 & 0.4 & 0.4 & 0.1 & 0.4 & 0.1 & 0.4 & 0.1 & 0.4 & 0.4 & 0.1 & 31.3 & 0.0 \\
\hline & ABCG & 0.0 & 0.0 & 0.0 & 0.0 & 0.0 & 0.0 & 0.0 & 0.0 & 0.0 & 0.0 & 0.0 & 0.0 & 0.0 & 0.0 & 0.0 & 0.0 & 0.0 \\
\hline & $A B G$ & 0.0 & 0.0 & $0, n$ & 0.0 & 0.0 & 0.0 & 0.0 & 0.0 & 0.0 & 0.0 & 0.0 & 0.0 & 0.0 & 0.0 & 0.0 & 0.1 & 0.0 \\
\hline & $A D G$ & 0.0 & 0.0 & 0.0 & $0 . n$ & 0.0 & 0.0 & 0.0 & 0.0 & 0.0 & 0.0 & 0.0 & 0.0 & 0.0 & 0.0 & 0.0 & 0.0 & 0.0 \\
\hline & $A G$ & 0.0 & 0.0 & 0.0 & 0.0 & 0.0 & 0.0 & 0.0 & 0.0 & 0.0 & 0.0 & 0.0 & 0.0 & 0.0 & 0.0 & 0.0 & 0.0 & 0.0 \\
\hline
\end{tabular}

HCD Codon: A-Ignltables, B-Corrosives, C=Reactives, D=Metals w/O Hg, E-Metals w Hg, F=Organics, G=State Reg., H-PCB < 50ppm, J-PCB > 50ppm 

(in cubic meters)

\begin{tabular}{|c|c|c|c|c|c|c|c|c|c|c|c|c|c|c|c|c|c|c|}
\hline PFE & HCD & 2010 & 2011 & 2012 & 2013 & 2014 & 2015 & 2016 & 2017 & 2018 & 2019 & 2020 & 2021 & 2022 & 2023 & 2024 & Total & Pet \\
\hline \multirow{13}{*}{. } & a & 0.5 & 0.5 & 0.5 & 0.5 & 0.0 & 0.0 & 0.0 & 0.0 & 0.0 & 0.0 & 0.0 & 0.0 & 0.0 & 0.0 & 0.0 & 68.3 & \\
\hline & anc & 0.0 & 0.0 & 0.0 & 0.0 & 0.0 & 0.0 & 0.0 & 0.0 & 0.0 & 0.0 & 0.0 & 0.0 & 0.0 & 0.0 & 0.0 & 0.0 & \\
\hline & BG & 0.0 & 0.0 & 0.0 & $0 . n$ & 0.0 & 0.0 & 0.0 & 0.0 & 0.0 & 0.0 & 0.0 & 0.0 & 0.0 & 0.0 & 0.0 & 0.0 & \\
\hline & $c$ & 0.0 & 0.0 & 0.0 & 0.0 & 0.0 & 0.0 & 0.0 & 0.0 & 0.0 & 0.0 & 0.0 & 0.0 & 0.0 & 0.0 & 0.0 & 1.4 & \\
\hline & D & 1.4 & 1.4 & 1.4 & 1.4 & 0.8 & 0.8 & 0.0 & 0.0 & 0.8 & 0.8 & 0.8 & 0.0 & 0.0 & 0.8 & 0.0 & 93.8 & \\
\hline & DG & 390.1 & 187.7 & $6 n 9.6$ & 762.0 & 952.5 & 1190.7 & 2488.3 & 1860.4 & 2325.6 & 2906.9 & 3633.7 & 4542.1 & 5677.6 & 7097.1 & 8871.3 & 41302.1 & \\
\hline & EG & 16.1 & 20.1 & 25.2 & 31.5 & 39.1 & 19.3 & 61.6 & 77.0 & 96.3 & 120.3 & 250.4 & 188.0 & 235.1 & 293.0 & 367.3 & 1034.5 & \\
\hline & $F$ & 0.5 & 0.5 & 0.5 & 0.4 & 0.3 & 0.3 & 0.3 & 0.3 & 0.3 & 0.3 & 0.3 & 0.3 & 0.3 & 0.3 & 0.3 & 29.4 & \\
\hline & $G$ & 0.0 & 0.0 & 0.0 & 0.0 & 0.0 & 0.0 & 0.0 & 0.0 & 0.0 & 0.0 & 0.0 & 0.0 & 0.0 & 0.0 & 0.0 & 0.1 & \\
\hline & as & 1.4 & 1.4 & 1.4 & 1.4 & 1.4 & 1.4 & 1.1 & 1.1 & 1.4 & 1.1 & 1.1 & 1.1 & 1.1 & 1.1 & 1.4 & 12.9 & \\
\hline & H & 1.0 & 1.0 & 1.0 & 1.0 & 1.0 & 1.0 & 1.0 & 1.0 & 1.0 & 1.0 & 1.0 & 1.0 & 1.0 & 1.0 & 1.0 & 31.7 & \\
\hline & J & 0.0 & 0.0 & 0.0 & 0.0 & 0.0 & 0.0 & 0.0 & 0.0 & 0.0 & 0.0 & 0.0 & 0.0 & 0.0 & 0.0 & 0.0 & 7.3 & \\
\hline & UnKNOWN & 0.0 & 0.0 & 0.0 & 0.4 & 0.4 & 0.4 & 0.1 & 0.1 & 0.1 & 0.1 & 0.1 & 0.4 & 0.4 & 0.0 & 0.0 & 4.1 & \\
\hline TOt:a1D-METAL & & 112.0 & 513.5 & 640.5 & 799.6 & 996.6 & 1244.6 & 1551.6 & 1912.1 & 2126.5 & 3031.9 & 3786.8 & 4734.8 & 3917.4 & 7395.2 & 9212.9 & 16418.2 & \\
\hline \multirow[t]{7}{*}{ n-moll. } & $\Lambda$ & 0.0 & 0.0 & 0.0 & 0.0 & 0.0 & 0.0 & 0.0 & 0.0 & 0.0 & 0.0 & 0.0 & 0.0 & 0.0 & 0.0 & 0.0 & 9.2 & \\
\hline & B & 0.1 & 0.1 & 0.1 & 0.1 & $0 . n$ & 0.0 & 0.0 & $0 . n$ & $n .0$ & 0.0 & 0.0 & 0.0 & 0.0 & 0.0 & 0.0 & 10.0 & \\
\hline & c & $n .0$ & 0.0 & 0.0 & 0.0 & 0.0 & $n .0$ & 0.0 & 0.0 & 0.0 & 0.0 & 0.0 & 0.0 & 0.0 & 0.0 & 0.0 & 0.0 & \\
\hline & D & 0.2 & 0.2 & 0.2 & 0.2 & 0.0 & 0.0 & 0.0 & 0.0 & 0.0 & 0.0 & 0.0 & 0.0 & 0.0 & 0.0 & 0.0 & 25.3 & \\
\hline & $\mathbf{F}$ & 0.3 & 0.3 & 0.3 & 0.3 & 0.3 & 0.3 & 0.3 & 0.3 & 0.3 & 0.3 & 0.3 & 0.3 & 0.3 & 0.3 & 0.3 & 15.9 & \\
\hline & $G$ & 0.0 & 0.0 & 0.0 & 0.0 & 0.0 & 0.0 & 0.0 & 0.0 & 0.0 & 0.0 & 0.0 & 0.0 & 0.0 & 0.0 & 0.0 & 3.0 & \\
\hline & " & 0.0 & 0.0 & 0.0 & 0.0 & 0.0 & 0.0 & 0.0 & 0.0 & 0.0 & 0.0 & 0.0 & 0.0 & 0.0 & 0.0 & 0.0 & 3.0 & \\
\hline Tot.a1 D-SoIL & & 0.9 & 0.9 & 0.9 & 0.9 & 0.1 & 0.4 & 0.1 & 0.1 & 0.1 & 0.4 & 0.1 & 0.4 & 0.1 & 0.1 & 0.4 & 75.6 & \\
\hline \multirow[t]{4}{*}{ EXPLOSIVES } & $A$ & 0.4 & 0.4 & 0.1 & 0.1 & 0.4 & 0.4 & 0.4 & 0.1 & 0.1 & 0.1 & 0.1 & 0.1 & 0.1 & 0.4 & 0.4 & 31.3 & \\
\hline & ABDE & 2.2 & 2.2 & 2.2 & 2.2 & 2.2 & 2.2 & 2.2 & 2.2 & 2.2 & 2.2 & 2.2 & 2.2 & 2.2 & 2.2 & 2.2 & 87.0 & \\
\hline & B & 0.0 & 0.0 & 0.0 & 0.0 & 0.0 & 0.0 & 0.0 & 0.0 & 0.0 & 0.0 & 0.0 & 0.0 & 0.0 & 0.0 & 0.0 & 3.9 & \\
\hline & $\mathbf{F}$ & 0.0 & 0.0 & 0.0 & 0.0 & 0.0 & 0.0 & 0.0 & 0.0 & 0.0 & 0.0 & 0.0 & 0.0 & 0.0 & 0.0 & 0.0 & 2.9 & \\
\hline Tot:alexRLOSIVES & & 2.7 & 2.7 & 2.7 & 2.7 & 2.7 & 2.7 & 2.7 & 2.7 & 2.7 & 2.7 & 2.1 & 2.7 & 2.7 & 2.7 & 2.7 & 206.1 & \\
\hline \multirow[t]{10}{*}{ IN ORG LO/SLG } & $A$ & 0.5 & 0.5 & 0.5 & 0.5 & 0.5 & 0.5 & $n .5$ & 0.5 & 0.5 & 0.5 & 0.5 & 0.5 & 0.5 & 0.5 & 0.5 & 20.7 & \\
\hline & AEDF & 3.7 & 3.7 & 3.7 & 3.7 & 3.7 & 3.7 & 3.7 & 3.7 & 3.7 & 3.7 & 3.7 & 3.7 & 3.7 & 3.7 & 3.7 & 123.0 & \\
\hline & AEEG & 1.4 & 1.1 & 1.1 & 1.4 & 1.4 & 1.4 & 1.4 & 1.4 & 1.4 & 1.4 & 1.4 & 1.4 & 1.4 & 1.4 & 1.4 & $\begin{array}{l}\cdot 43.9 \\
\end{array}$ & \\
\hline & AEG & $19 R .2$ & 241.8 & 309.8 & 387.2 & 484.0 & 605.1 & 756.3 & 915.4 & $1101 . \theta$ & 1177.3 & 1846.6 & 2308.3 & 2805.4 & 3606.7 & 1508.4 & 22514.3 & \\
\hline & $B$ & 0.5 & 0.5 & 0.5 & 0.5 & 0.5 & 0.5 & 0.5 & 0.5 & 0.5 & 0.5 & 0.5 & 0.5 & 0.5 & 0.3 & 0.5 & 19.2 & \\
\hline & $B G$ & n.0 & 0.0 & 0.0 & 0.0 & 0.0 & 0.0 & 0.0 & 0.0 & 0.0 & 0.0 & 0.0 & 0.0 & 0.0 & 0.0 & 0.0 & 12.1 & \\
\hline & $c$ & 0.1 & 0.4 & 0.1 & 0.4 & 0.4 & 0.1 & 0.1 & 0.4 & 0.1 & 0.1 & 0.1 & 0.1 & 0.4 & 0.4 & 0.1 & 10.1 & \\
\hline & D & 0.7 & 0.7 & 0.7 & 0.7 & 0.7 & 0.7 & 0.7 & 0.7 & 0.7 & 0.7 & 0.7 & 0.7 & 0.7 & 0.7 & 0.7 & 27.9 & \\
\hline & F & 0.0 & 0.0 & 0.0 & 0.0 & 0.0 & 0.1 & 0.0 & 0.0 & 0.0 & 0.0 & 0.0 & 0.0 & 0.0 & 0.0 & 0.0 & 7.8 & \\
\hline & 0 & 1.4 & 1.4 & 1.4 & 1.1 & 1.4 & 1.4 & 1.4 & 1.1 & 1.4 & 1.4 & 1.4 & 1.1 & 1.4 & 1.4 & 1.1 & 47.8 & \\
\hline
\end{tabular}




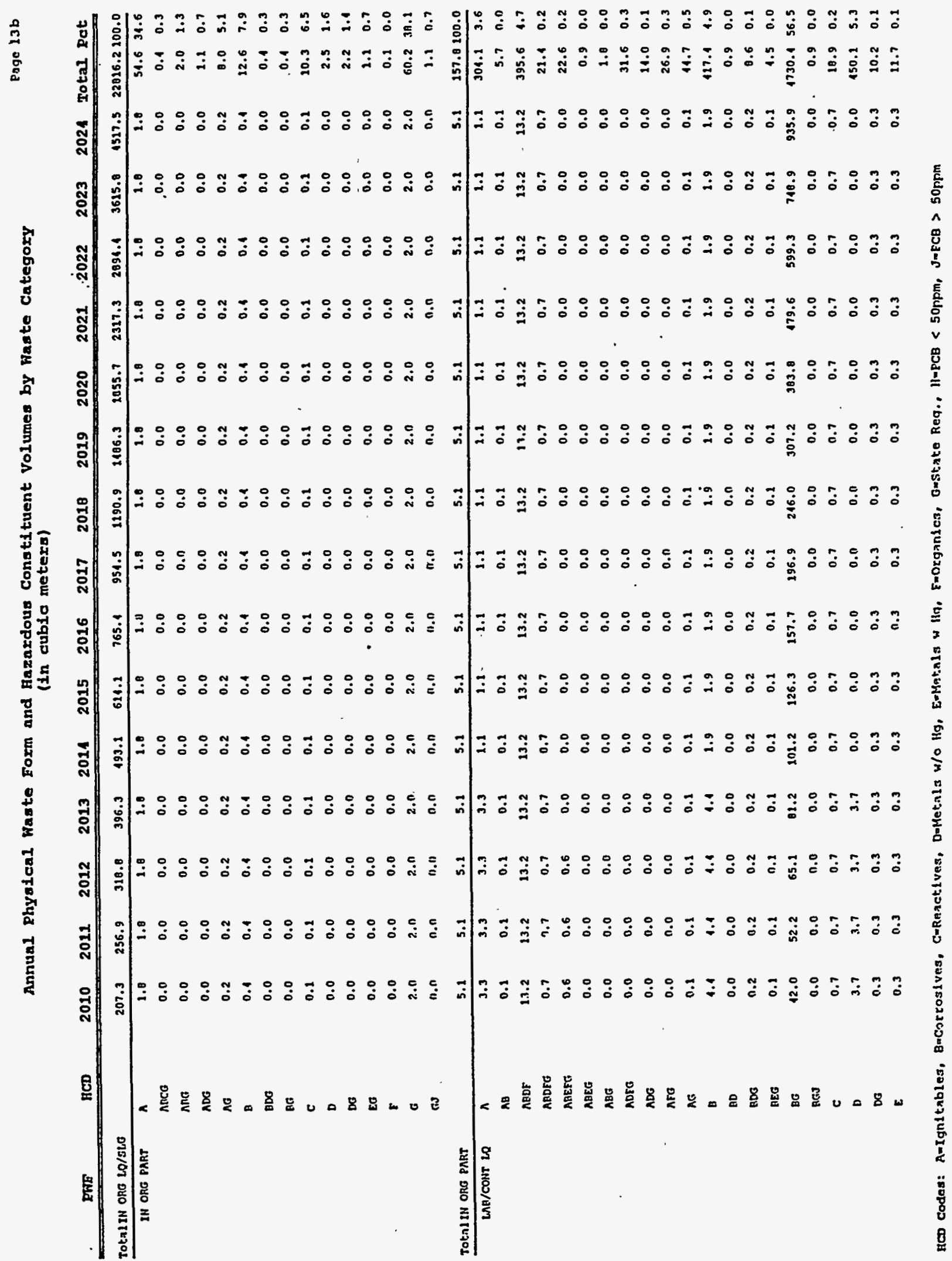


Annual Physical Faste Form and Bazardous Constituent Volumes by Haste Category

(in cubic meters)

\begin{tabular}{|c|c|c|c|c|c|c|c|c|c|c|c|c|c|c|c|c|c|}
\hline PWE & HCD & 2010 & 2011 & 2012 & 2013 & 2014 & 2015 & 2016 & 2017 & 2018 & 2019 & 2020 & 2021 & 2022 & 2023 & 2024 & Total Ret \\
\hline & EG & 0.1 & 0.1 & 0.1 & 0.1 & 0.1 & 0.1 & 0.1 & 0.1 & 0.1 & 0.1 & 0.1 & 0.1 & 0.1 & 0.1 & 0.1 & 3.1 \\
\hline & $\mathbf{F}$ & 3.4 & 3.1 & 3.1 & 3.4 & 2.4 & 2.1 & 2.1 & 2.1 & 2.1 & 2.1 & 2.4 & 2.1 & 2.4 & 2.1 & 2.1 & $172.3 \quad 2.0$ \\
\hline & EG & 10.0 & 12.5 & 15.6 & 19.1 & 24.3 & 30.3 & 37.9 & 47.3 & 59.2 & 73.9 & 92.4 & 115.5 & 211.3 & 180.1 & 225.5 & $1129.0 \quad 23.5$ \\
\hline & G & 12.0 & 12.0 & 12.0 & 12.8 & 12.0 & 12.0 & 12.8 & 12.8 & 12.0 & 12.0 & 12.8 & 22.8 & 12.8 & 12.0 & 12.0 & 161.8 \\
\hline & H & 0.8 & 0.6 & 0.0 & 0.8 & 0.3 & 0.3 & 0.3 & 0.3 & 0.3 & 0.3 & 0.3 & 0.3 & 0.3 & 0.3 & 0.3 & 65.9 \\
\hline & 3 & 0.3 & 0.3 & 0.3 & 0.3 & 0.3 & 0.3 & 0.3 & 0.3 & 0.3 & 0.3 & 0.3 & 0.3 & 0.3 & 0.3 & 0.3 & 6.1 \\
\hline TotalLAB/CONT LQ & & 99.3 & 111.0 & 127.0 & 146.3 & 161.3 & 192.1 & 231.1 & 280.1 & 310.9 & 117.0 & 512.0 & 630.9 & 779.1 & 965.1 & 1197.2 & $8362.4 \quad 99.9$ \\
\hline \multirow[t]{6}{*}{ LEAD } & $A D F$ & 0.3 & 0.3 & 0.3 & 0.3 & 0.3 & 0.3 & 0.3 & 0.3 & 0.3 & 0.3 & 0.3 & 0.3 & 0.3 & 0.3 & 0.3 & 11.30 .9 \\
\hline & BDG & 9.1 & 11.4 & 14.2 & 17.0 & 22.2 & 27.8 & 34.0 & 43.5 & 54.4 & 68.0 & 05.0 & 106.2 & 132.0 & 166.0 & 207.5 & $1036: 3 \quad 89.6$ \\
\hline & D & 0.7 & 0.7 & 0.7 & 0.7 & 0.0 & 0.0 & 0.0 & 0.0 & 0.0 & 0.0 & 0.0 & 0.0 & 0.0 & 0.0 & 0.0 & $80.4 \quad 7.6$ \\
\hline & $\approx$ & 0.5 & 0.5 & 0.5 & 0.5 & 0.5 & 0.5 & 0.5 & 0.5 & 0.5 & 0.5 & 0.5 & 0.5 & 0.5 & 0.5 & 0.5 & 15.6 \\
\hline & G & 0.1 & 0.1 & $\mathbf{n . 1}$ & 0.1 & 0.1 & 0.1 & 0.1 & 0.1 & 0.1 & 0.1 & 0.1 & 0.1 & 0.1 & 0.1 & 0.1 & 1.6 \\
\hline & UNKKOKN & 0.0 & 0.0 & 0.0 & 0.0 & 0.0 & 0.0 & 0.0 & 0.0 & 0.0 & 0.0 & 0.0 & 0.0 & 0.0 & 0.0 & 0.0 & 0.0 \\
\hline TOt:31LEAD & & 10.9 & 13.1 & 16.0 & 19.6 & 23.3 & 28.9 & 35.9 & $\$ 1.6$ & 55.4 & 69.0 & 06.0 & 107.3 & 133.9 & 167.2 & 200.6 & 1156.4100 .0 \\
\hline \multirow[t]{3}{*}{ MEREIJRY } & c & 0.0 & 0.0 & 0.0 & 0.0 & 0.0 & 0.0 & 0.0 & 0.0 & 0.0 & 0.0 & 0.0 & 0.0 & 0.0 & 0.0 & 0.0 & $2.9 \quad 0.7$ \\
\hline & D & 0.5 & 0.5 & 0.5 & 0.5 & 0.5 & 0.5 & 0.5 & 0.5 & 0.5 & 0.5 & 0.5 & 0.5 & 0.5 & 0.5 & 0.5 & $15.7 \quad 45.9$ \\
\hline & $\mathbf{z}$ & 0.5 & 0.5 & 0.5 & 0.5 & 0.5 & 0.5 & 0.5 & 0.5 & 0.5 & 0.5 & 0.5 & 0.5 & 0.3 & 0.5 & 0.3 & $\begin{array}{lll}15.5 & 15.3\end{array}$ \\
\hline Tot:alMERrouRY & & 1.1 & 1.1 & 1.1 & 1.1 & 1.1 & 1.1 & 1.1 & 1.1 & 1.1 & 1.1 & 1.1 & $1: 1$ & 1.1 & 1.1 & 1.1 & 31.2100 .0 \\
\hline \multirow[t]{14}{*}{ ORG ABS LQ/SLO } & $\lambda$ & 0.2 & 0.2 & 0.2 & 0.2 & 0.2 & 0.2 & 0.2 & 0.2 & 0.2 & 0.2 & 0.2 & 0.2 & 0.2 & 0.2 & 0.2 & 0.10 .0 \\
\hline & ABDE & 7.5 & 7.5 & 7.5 & 7.5 & 7.5 & 7.5 & 7.5 & 7.5 & 7.5 & 7.5 & 7.5 & 7.5 & 7.5 & 7.5 & 7.5 & $226.1 \quad 0.5$ \\
\hline & A:Fr & 356.9 & 116.1 & 557.6 & 697.1 & 871.3 & 1089.2 & 1361.5 & 1702.0 & 2127.3 & 2659.2 & 3323.9 & 1154.9 & 5193.7 & 6492.1 & 0113.1 & 40525.7 96.7 \\
\hline & $\boldsymbol{N F}$ & 0.2 & 0.2 & 0.2 & 0.2 & 0.2 & 0.2 & 0.2 & 0.2 & 0.2 & 0.2 & 0.2 & 0.2 & 0.2 & 0.2 & 0.2 & 6.9 \\
\hline & AFG & 0.3 & 0.3 & 0.3 & 0.3 & 0.3 & 0.3 & 0.3 & 0.3 & 0.3 & 0.3 & 0.3 & 0.3 & 0.3 & 0.3 & 0.3 & 9.0 \\
\hline & $\Lambda G$ & 0.0 & 0.0 & 0.0 & 0.0 & 0.0 & 0.0 & 0.0 & 0.0 & 0.0 & 0.0 & 0.0 & 0.0 & 0.0 & 0.0 & 0.0 & 1.4 \\
\hline & B & 0.1 & 0.1 & 0.1 & 0.1 & 0.1 & 0.1 & 0.1 & 0.1 & 0.1 & 0.1 & 0.1 & 0.1 & 0.1 & 0.1 & 0.1 & 3.2 \\
\hline & c & 0.0 & 0.0 & 0.0 & 0.0 & 0.0 & 0.0 & 0.0 & 0.0 & 0.0 & 0.0 & 0.0 & 0.0 & 0.0 & 0.0 & 0.0 & 0.7 \\
\hline & E. & 0.0 & 0.0 & 0.0 & 0.0 & 0.0 & 0.0 & .0 .0 & 0.0 & 0.0 & 0.0 & 0.0 & 0.0 & 0.0 & 0.0 & 0.0 & 1.8 \\
\hline & EG & 0.2 & 0.2 & 0.2 & 0.2 & 0.2 & 0.2 & 0.2 & 0.2 & 0.2 & 0.2 & 0.2 & 0.2 & 0.2 & 0.2 & 0.2 & 0.7 \\
\hline & $\mathbf{F}$ & 2.3 & 2.3 & 2.3 & 2.3 & 2.3 & 2.3 & 2.3 & 2.3 & 2.3 & 2.3 & 2.3 & 2.3 & 2.3 & 2.3 & 2.3 & 01.5 \\
\hline & 80 & 8.0 & 10.0 & 12.6 & 15.7 & 19.7 & 24.6 & 30.8 & 38.5 & 48.1 & 60.1 & 75.2 & 94.0 & 117.3 & 146.9 & 103.6 & $988.3 \quad 2.3$ \\
\hline & c & 0.2 & 0.2 & 0.2 & 0.2 & 0.2 & 0.2 & 0.2 & 0.2 & 0.2 & 0.2 & 0.2 & 0.2 & 0.2 & 0.2 & 0.2 & 30.8 \\
\hline & 3 & 0.0 & 0.0 & 0.0 & 0.0 & 0.0 & 0.0 & 0.0 & 0.0 & 0.0 & 0.0 & 0.0 & 0.0 & 0.0 & 0.0 & 0.0 & 1.10 .0 \\
\hline TOt:A1ORG ABS LQ/SLG & & 376.1 & 167.6 & 581.7 & 724.3 & 902.5 & 1125.3 & 1403.7 & 1751.0 & 2186.9 & 2730.8 & 3410.6 & 1260.1 & 5322.7 & 6650.5 & 0310.3 & $\$ 1900.1100 .0$ \\
\hline \multirow[t]{4}{*}{ ORG BART } & $\wedge$ & 0.8 & 0.0 & 0.8 & 0.8 & 0.0 & 0.0 & 0.0 & 0.0 & 0.0 & 0.0 & 0.0 & 0.0 & 0.0 & 0.0 & 0.0 & $102.6 \quad 13.5$ \\
\hline & $\triangle B D F$ & 1.0 & 1.0 & 1.0 & 1.0 & 1.0 & 1.0 & 1.8 & 1.8 & 1.8 & 1.8 & 1.0 & 1.0 & 1.0 & 1.0 & 1.0 & $36.5 \quad 7.1$ \\
\hline & B & 1.6 & 1.6 & 1.6 & 1.6 & 0.0 & 0.0 & 0.0 & 0.0 & 0.0 & 0.0 & 0.0 & 0.0 & 0.0 & 0.0 & 0.0 & 204.927 .0 \\
\hline & c & 0.0 & 0.0 & 0.0 & 0.0 & 0.0 & 0.0 & 0.0 & 0.0 & 0.0 & 0.0 & 0.0 & 0.0 & 0.0 & 0.0 & 0.0 & 3.9 \\
\hline
\end{tabular}

BCD Codes: AmIgnitables, BmCorroslves, Cmeactives, DwMetals w/O Hg, E-Hetals w Hg, F-Organlcs, G=State Reg., HaPCB < 50ppm, J=PCB > 50ppm 

(in cublo meters)

PWF \begin{tabular}{lllllllllllllllll}
2010 & 2011 & 2012 & 2013 & 2014 & 2015 & 2016 & 2017 & 2018 & 2019 & 2020 & 2021 & 2022 & 2023 & 2021 & Total Pat \\
\hline 2.2 & 2.2 & 2.2 & 2.2 & 0.0 & 0.0 & 0.0 & 0.0 & 0.0 & 0.0 & 0.0 & 0.0 & 0.0 & 0.0 & 0.0
\end{tabular}

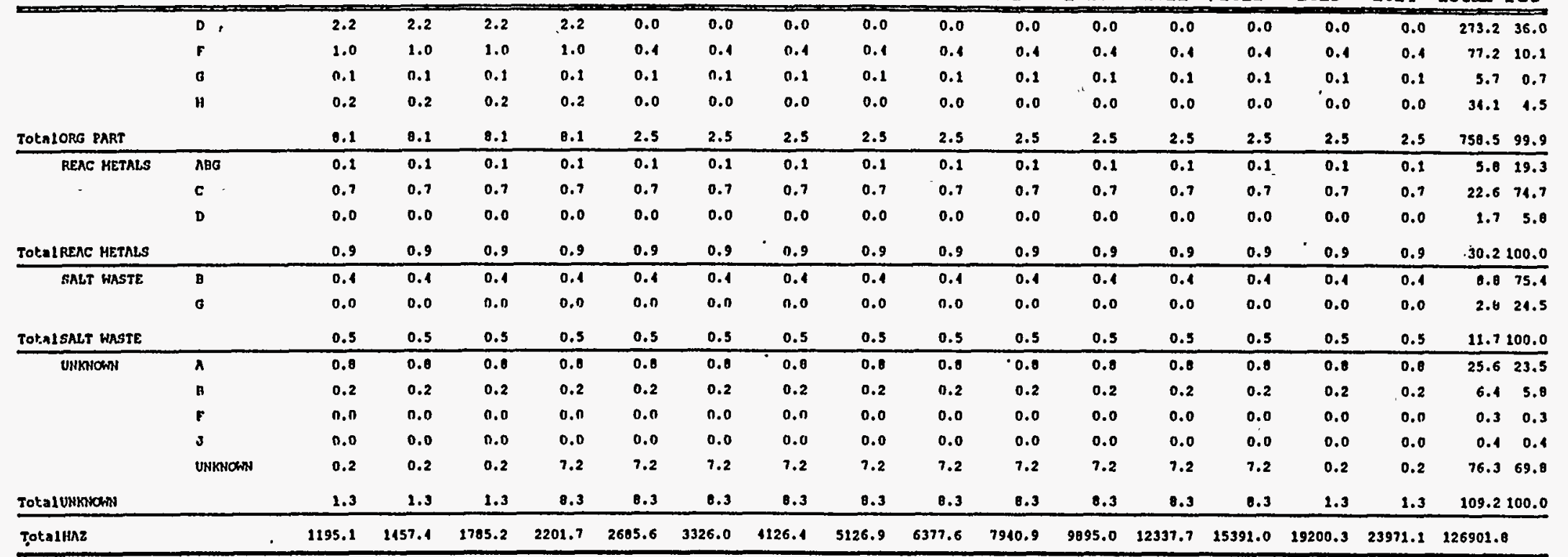

$\frac{\frac{\text { Totallinz }}{\text { Wasto Category: RH_LLW }}}{\text { p-Consust }}$

10.95 .1

\begin{tabular}{|c|c|c|c|c|c|c|c|c|c|c|c|c|c|c|c|}
\hline Ot:a1 1 D-COMBUST & 26.9 & 26.9 & 26.9 & 26.9 & 26.9 & 26.9 & 26.9 & 26.9 & 26.9 & 26.9 & 26.9 & 26.9 & 26.9 & 26.9 & 568.4100 .0 \\
\hline D-HET & 58.4 & 58.4 & 59.3 & 59.3 & 59.3 & 59.3 & 59.3 & 39.3 & 59.3 & 39.3 & 59.3 & 59.3 & 38.4 & 50.1 & 00.0 \\
\hline
\end{tabular}

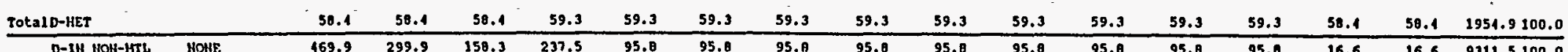

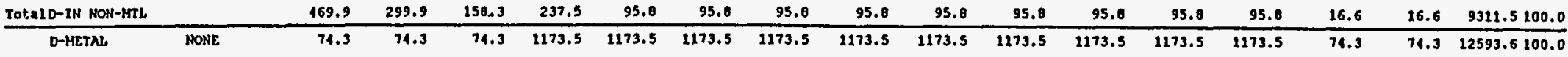

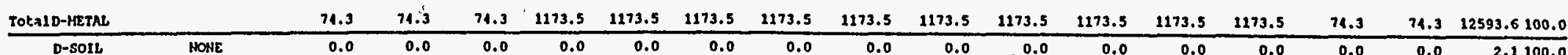

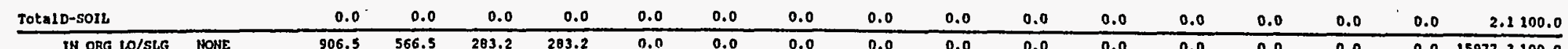

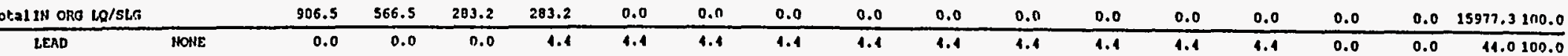

BCo Codes: A-Ignitables, B=Corrosives, CmRenctives, DeMetals w/O Hg, Emetnls w Hạ, Emorganlça, G=Sthte Reg., HmPCB < 50ppm, 1-PCA > 50ppm 

(in cubic meters)

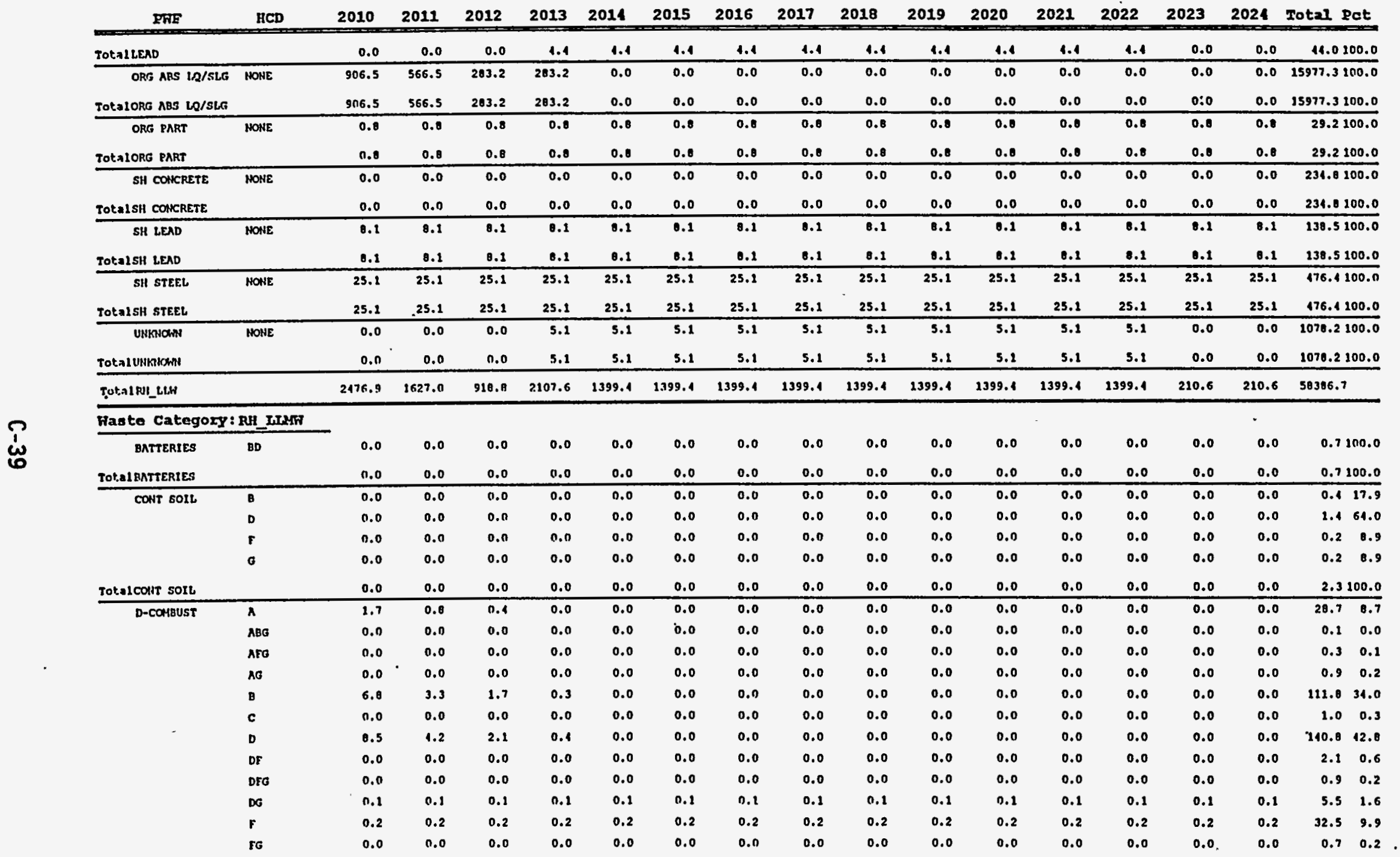

RCD Codas: A-Ignitables, B-Corrosives, C-Reactives, D-Metals w/o Hg, EoMetals w Hg, E-Organlcs, G-Statn Req., H=PCB < 50prm, J-FCB > 50ppm 


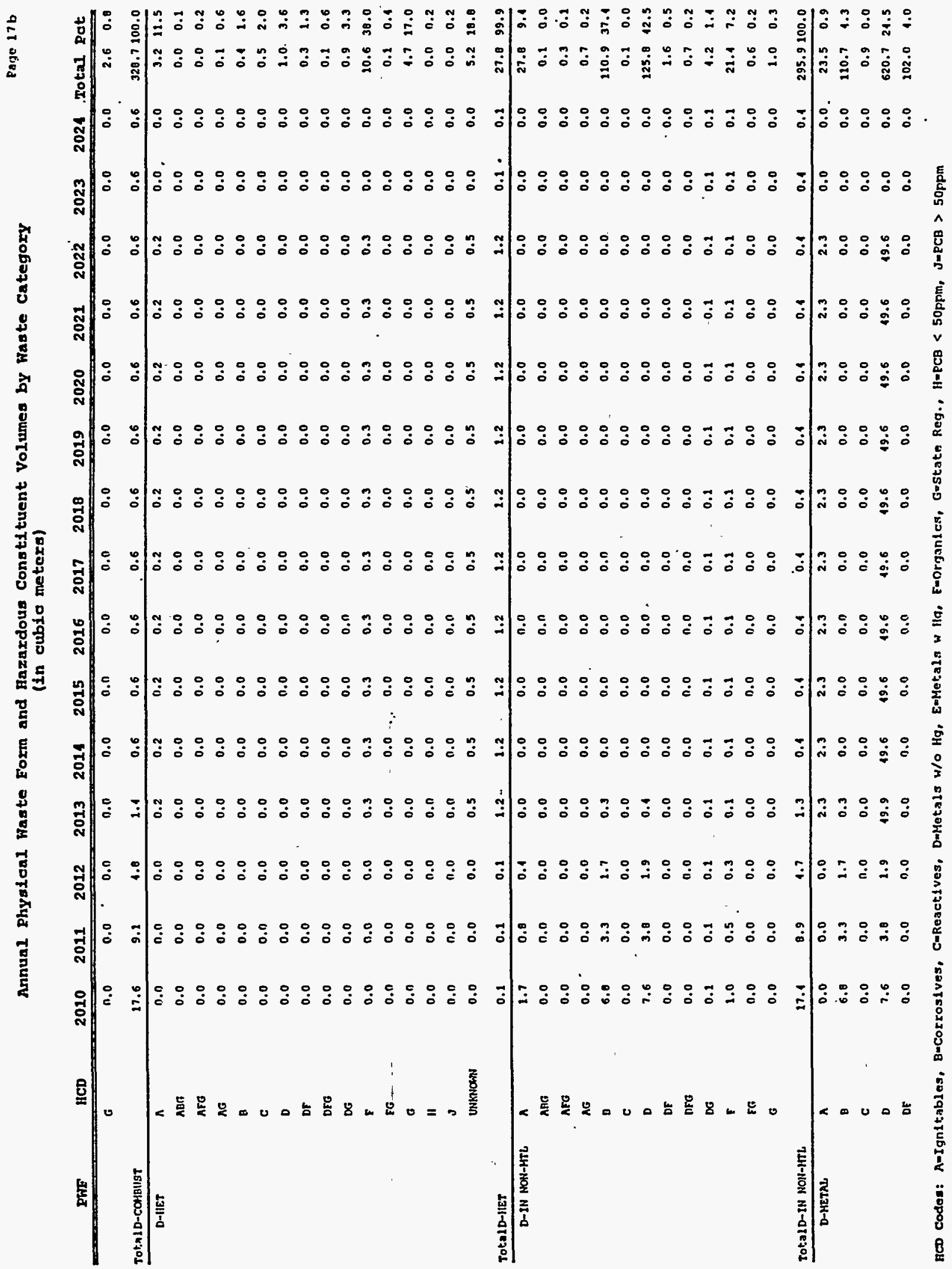




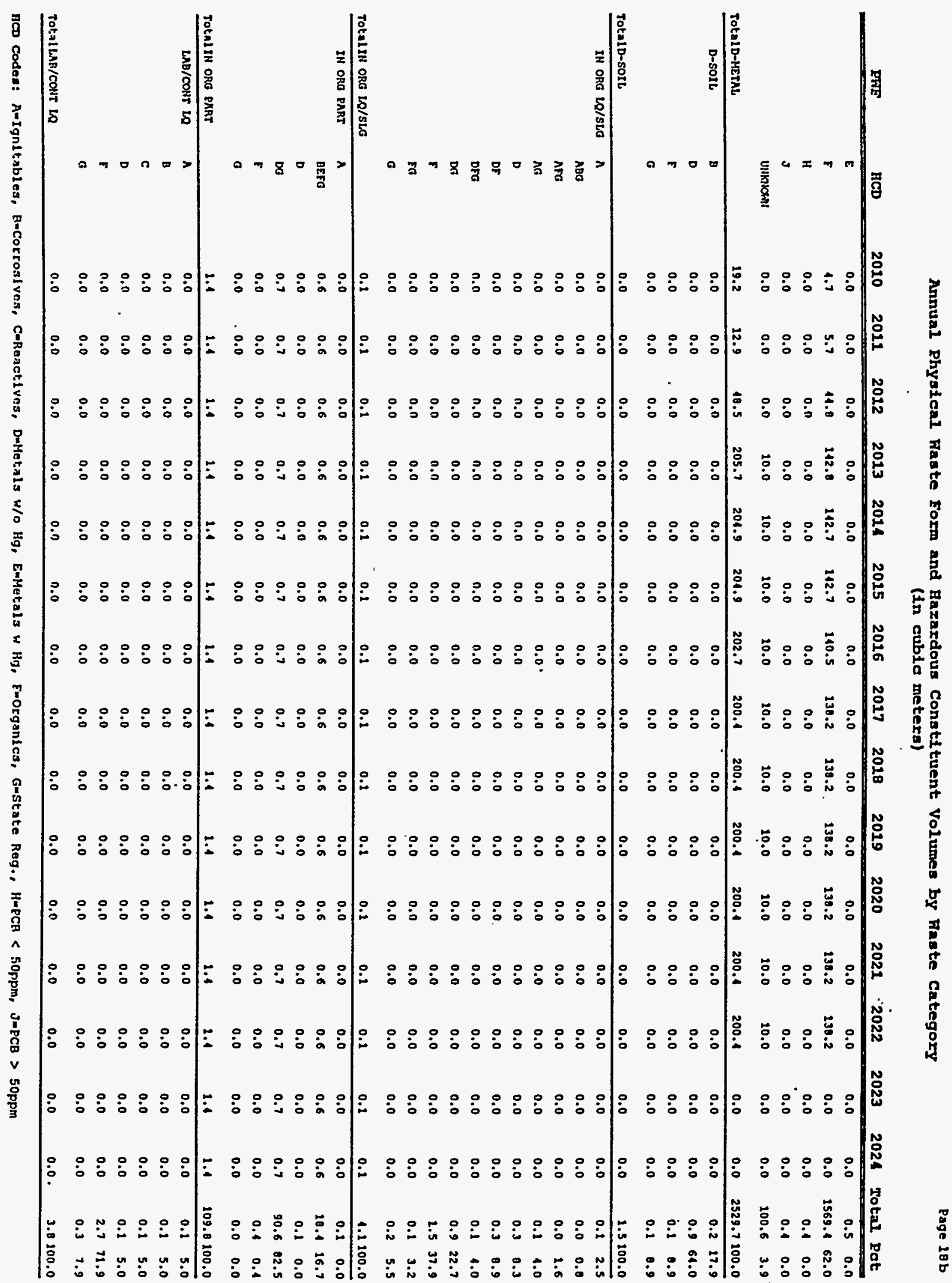


Annual Physical Waste Form and Hazardous Constituent Volumes by Faste Category

(in cubic meters)

\begin{tabular}{|c|c|c|c|c|c|c|c|c|c|c|c|c|c|c|c|c|c|c|}
\hline & PWF & $\mathrm{IICD}$ & 2010 & 2011 & 2012 & 2013 & 2014 & 2015 & 2016 & 2017 & 2018 & 2019 & 2020 & 2021 & 2022 & 2023 & 2024 & Total Pct \\
\hline & LEAD & D & 0.0 & 0.0 & 0.0 & 0.0 & 0.0 & 0.0 & 0.0 & 0.0 & 0.0 & 0.0 & 0.0 & 0.0 & 0.0 & 0.0 & 0.0 & 0.710 .2 \\
\hline & & INNHIOMIt & 0.0 & 0.0 & 0.0 & 0.6 & 0.6 & 0.6 & 0.6 & 0.6 & 0.6 & 0.6 & 0.6 & 0.6 & 0.6 & 0.0 & 0.0 & 6.789 .7 \\
\hline & TOt:aI LEAD & & 0.0 & 0.0 & 0.0 & 0.6 & 0.6 & 0.6 & 0.6 & 0.6 & 0.6 & 0.6 & 0.6 & 0.6 & 0.6 & .0 .0 & 0.0 & 1.4100 .0 \\
\hline & HERCURY & E & 0.0 & 0.0 & 0.0 & 0.0 & 0,0 & 0.0 & 0.0 & 0.0 & 0.0 & 0.0 & 0.0 & 0.0 & 0.0 & 0.0 & 0.0 & 0.7100 .0 \\
\hline & TOta IMERCURY & & 0.0 & 0.0 & 0.0 & 0.0 & 0.0 & 0.0 & 0.0 & 0.0 & 0.0 & 0.0 & 0.0 & 0.0 & 0.0 & 0.0 & 0.0 & 0.7100 .0 \\
\hline & ORG ABS LO/SLG & ABG & 0.0 & 0.0 & 0.0 & 0.0 & 0.0 & 0.0 & 0.0 & 0.0 & 0.0 & 0.0 & 0.0 & 0.0 & 0.0 & 0.0 & 0.0 & 0.0 \\
\hline & & $\mathrm{ArG}$ & 0.0 & 0.0 & 0.0 & 0.0 & 0.0 & 0.0 & 0.0 & 0.0 & 0.0 & 0.0 & 0.0 & 0.0 & 0.0 & 0.0 & 0.0 & 0.0 \\
\hline & & $\Lambda \mathbf{G}$ & 0.0 & 0.0 & 0.0 & 0.0 & 0.0 & 0.0 & 0.0 & 0.0 & 0.0 & 0.0 & 0.0 & 0.0 & 0.0 & 0.0 & 0.0 & 0.1 \\
\hline & & D & 0.0 & 0.0 & 0.0 & 0.0 & 0.0 & 0.0 & 0.0 & 0.0 & 0.0 & 0.0 & 0.0 & 0.0 & 0.0 & 0.0 & 0.0 & 0.2 \\
\hline & & DF & 0.0 & 0.0 & 0.0 & 0.0 & 0.0 & 0.0 & 0.0 & 0.0 & 0.0 & 0.0 & 0.0 & 0.0 & 0.0 & 0.0 & 0.0 & $13.1 \quad 17.0$ \\
\hline & & DFe & 0.0 & 0.0 & 0.0 & 0.0 & 0.0 & 0.0 & 0.0 & 0.0 & 0.0 & 0.0 & 0.0 & 0.0 & 0.0 & 0.0 & 0.0 & $0.1 \quad 0.2$ \\
\hline & & $D G$ & 0.0 & 0.0 & 0.0 & 0.0 & 0.0 & 0.0 & 0.0 & 0.0 & 0.0 & 0.0 & 0.0 & 0.0 & 0.0 & 0.0 & 0.0 & 0.9 \\
\hline & & r & 1.1 & 2.2 & 22.1 & 2.2 & 2.2 & 2.2 & 1.1 & 0.0 & 0.0 & 0.0 & 0.0 & 0.0 & 0.0 & 0.0 & 0.0 & $61.9 \quad 00.3$ \\
\hline & & FG & 0.0 & 0.0 & 0.0 & 0.0 & 0.0 & 0.0 & 0.0 & 0.0 & 0.0 & 0.0 & 0.0 & 0.0 & 0.0 & 0.0 & 0.0 & 0.10 .1 \\
\hline & & 6 & 0.0 & 0.0 & 0.0 & 0.0 & $0 . n$ & $n . n$ & 0,0 & 0.0 & 0.0 & 0.0 & 0.0 & 0.0 & 0.0 & 0.0 & 0.0 & 0.2 \\
\hline & TOt:n LORS AAS LQ/SLG & & 1.2 & 2.3 & 22,2 & 2.3 & 2.3 & 2.3 & 1.2 & 0.1 & 0.1 & 0.1 & 0.1 & 0.1 & 0.1 & 0.1 & 0.1 & 71.0100 .0 \\
\hline & ORG PART & 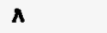 & 11.9 & 5.9 & 2.9 & 0.5 & 0.0 & 0.0 & n.o & 0.0 & 0.0 & 0.0 & 0.0 & 0.0 & 0.0 & 0.0 & 0.0 & $193.8 \quad 9.9$ \\
\hline & & B & 17.6 & 23.7 & 11.9 & 2.3 & 0.0 & 0.0 & 0.0 & 0.0 & 0.0 & 0.0 & 0.0 & 0.0 & 0.0 & 0.0 & 0.0 & $775.5 \quad 39.6$ \\
\hline & & BEFG & 0.6 & 0.6 & 0.6 & 0.6 & 0.6 & 0.6 & 0.6 & 0.6 & 0,6 & 0.6 & 0.6 & 0.6 & 0.6 & 0.6 & 0.6 & $\begin{array}{ll}18.4 & 0.9\end{array}$ \\
\hline & & D & 53.5 & 26.7 & 13.4 & 2.6 & 0.0 & 0.0 & 0.0 & 0.0 & 0.0 & 0.0 & 0.0 & 0.0 & 0.0 & 0.0 & 0.0 & $072.5 \quad 14.5$ \\
\hline & & $\mathbf{F}$ & 5.9 & 2.9 & 1.4 & 0.2 & 0.0 & 0.0 & 0.0 & 0.0 & 0.0 & 0.0 & 0.0 & 0.0 & 0.0 & 0.0 & 0.0 & $97.6 \quad 1.9$ \\
\hline & & G & 0.0 & 0.0 & 0.0 & 0.0 & 0.0 & 0.0 & 0.0 & 0.0 & 0.0 & 0.0 & 0.0 & 0.0 & 0.0 & 0.0 & 0.0 & $0.0 \quad 0.0$ \\
\hline & TOt:aloRg PART & & 119.7 & 50.0 & 30.4 & 6.5 & 0.6 & 0.6 & 0.6 & 0.6 & 0.6 & 0.6 & 0.6 & 0.6 & 0.6 & 0.6 & 0.6 & 1958.1100 .0 \\
\hline & SALT WASTE & 8 & 0.0 & 0.0 & 0.0 & 0.0 & 0.0 & 0.0 & 0.0 & 0.0 & 0.0 & 0.0 & 0.0 & 0.0 & 0.0 & 0.0 & 0.0 & 0.121 .9 \\
\hline & & $\mathbf{r}$ & 0.0 & 0.0 & 0.0 & 0.0 & 0.0 & 0.0 & 0.0 & 0.0 & 0.0 & 0.0 & 0.0 & 0.0 & 0.0 & 0.0 & 0.0 & $0.5 \quad 78.0$ \\
\hline & TOt:ALSALT WASTE & & 0.0 & 0.0 & 0.0 & 0.0 & 0.0 & 0.0 & 0.0 & 0.0 & 0.0 & 0.0 & 0.0 & 0.0 & 0.0 & 0.0 & 0.0 & 0.7100 .0 \\
\hline & SH LEND & ABa & 0.0 & 0.0 & 0.0 & 0.0 & 0.0 & 0.0 & 0.0 & 0.0 & 0.0 & 0.0 & 0.0 & 0.0 & 0.0 & 0.0 & 0.0 & $0.1 \quad 0.1$ \\
\hline & & AFG & 0.0 & 0.0 & 0.0 & 0.0 & 0.0 & 0.0 & 0.0 & 0.0 & 0.0 & 0.0 & 0.0 & 0.0 & 0.0 & 0.0 & 0.0 & $\begin{array}{ll}0.2 & 0.3\end{array}$ \\
\hline & & AG & 0.0 & 0.0 & 0.0 & 0.0 & 0.0 & 0.0 & 0.0 & 0.0 & 0.0 & 0.0 & 0.0 & 0.0 & 0.0 & 0.0 & 0.0 & 0.5 \\
\hline & & D & 0.8 & 0.0 & 0.8 & 0.0 & 0.8 & 0.0 & 0.8 & 0.8 & 0.8 & 0.0 & 0.8 & 0.8 & 0.8 & 0.0 & 0.0 & $59.2 \quad 05.0$ \\
\hline & & DE & 0.0 & 0.0 & 0.0 & 0.0 & 0.0 & 0.0 & 0.0 & 0.0 & 0.0 & 0.0 & 0.0 & 0.0 & 0.0 & 0.0 & 0.0 & 1.2 \\
\hline & & DFG & 0.0 & 0.0 & 0.0 & 0.0 & 0.0 & 0.0 & 0.0 & 0.0 . & 0.0 & 0.0 & 0.0 & 0.0 & 0.0 & 0.0 & 0.0 & 0.5 \\
\hline & & DG & 0.1 & 0.1 & 0.1 & 0.1 & 0.1 & 0.1 & 0.1 & 0.1 & 0.1 & $\cdot 0.1$ & 0.1 & 0.1 & 0.1 & 0.1 & 0.1 & 3.1 \\
\hline & & $\mathbf{F}$ & 0.1 & 0.1 & 0.1 & 0.1 & 0.1 & 0.1 & 0.1 & 0.1 & 0.1 & 0.1 & 0.1 & 0.1 & 0.1 & 0.1 & 0.1 & 3.5 \\
\hline & & FG & 0.0 & $n, n$ & 0.0 & 0.0 & 0.0 & $0 . n$ & 0.0 & 0.0 & 0.0 & 0.0 & 0.0 & 0.0 & 0.0 & 0.0 & 0.0 & 0.1 \\
\hline & & G & 0.0 & 0,0 & 0.0 & 0.0 & 0.0 & 0.0 & 0.0 & 0.0 & 0.0 & 0.0 & 0.0 & 0.0 & 0.0 & 0.0 & 0.0 & 0.3 \\
\hline
\end{tabular}

HCD Codes: A-Ignitables, B-Corrosives, CeReactives, DeMetals w/o Hg, E-Motals w Hg, Fuorganles, G-State Reg., HmPCB < 50prm, J-PCB > 50ppm 
Annual Physical Haste Form and gazaxdous Constituent Volumes by waste Category

(in cubio meters)

\begin{tabular}{|c|c|c|c|c|c|c|c|c|c|c|c|c|c|c|c|c|c|}
\hline PFE & RCD & 2010 & 2011 & 2012 & 2013 & 2014 & 2015 & 2016 & 2017 & 2018 & 2019 & 2020 & 2021 & 2022 & 2023 & 2024 & Total Pct \\
\hline Tota1SH LEAD & & 1.2 & 1.2 & 1.2 & 1.2 & 1.2 & 1.2 & 1.2 & 1.2 & 1.2 & 1.2 & 1.2 & 1.2 & 1.2 & 1.2 & 1.2 & 69.7100 .0 \\
\hline \multirow[t]{3}{*}{ SH STEEL } & D & 0.0 & 0.0 & 0.0 & 0.0 & 0.0 & 0.0 & 0.0 & 0.0 & 0.0 & 0.0 & 0.0 & 0.0 & 0.0 & 0.0 & 0.0 & $1.1 \quad 1.9$ \\
\hline & $D E$ & 0.0 & 0.0 & 0.0 & 0.0 & 0.0 & 0.0 & 0.0 & 0.0 & 0.0 & 0.0 & 0.0 & 0.0 & 0.0 & 0.0 & 0.0 & 12.722 .1 \\
\hline & UNKMOWN & 0.3 & 0.3 & 0.3 & 0.3 & 0.3 & 0.3 & 0.3 & 0.3 & 0.3 & 0.3 & 0.3 & 0.3 & 0.3 & 0.3 & 0.3 & 9.215 .9 \\
\hline TOtn1SH STEEL & & 0.3 & 0.3 & 0.3 & 0.3 & 0.3 & 0.3 & 0.3 & 0.3 & 0.3 & 0.3 & 0.3 & 0.3 & 0.3 & 0.3 & 0.3 & 57.6100 .0 \\
\hline \multirow[t]{2}{*}{ SII VOID } & DE & 0.0 & 0.0 & 0.0 & 0.0 & 0.0 & 0.0 & 0.0 & 0.0 & 0.0 & 0.0 & 0.0 & 0.0 & 0.0 & 0.0 & 0.0 & $127.5 \quad 14.5$ \\
\hline & $F$ & 0.0 & 0.0 & 0.0 & 0.0 & 0.0 & 0.0 & 0.0 & 0.0 & 0.0 & 0.0 & 0.0 & 0.0 & 0.0 & 0.0 & 0.0 & 105.112 .0 \\
\hline TotnisH Vold & & 19.0 & 37.9 & 376.0 & 37.9 & 37.9 & 37.9 & 19.0 & 0.0 & 0.0 & 0.0 & 0.0 & 0.0 & 0.0 & 0.0 & 0.0 & 875.1100 .0 \\
\hline UNKHOWNN & UNhNown & 0.0 & 0.0 & 0.0 & 5.9 & 5.9 & 5.9 & 5.9 & 3.9 & 5.9 & 5.9 & 5.9 & 3.9 & 5.9 & 0.0 & 0.0 & 314.7100 .0 \\
\hline TotalUNKNOWN & & 0.0 & 0.0 & 0.0 & 5.9 & 3.9 & 5.9 & 5.9 & 3.9 & 5.9 & 5.9 & 5.9 & 3.9 & 3.9 & 0.0 & 0.0 & 314.7100 .0 \\
\hline Ţota1PH_LLHW & & 197.5 & 134.6 & 490.0 & 266.2 & 257.8 & 257.8 & 235.5 & 213.1 & 213.1 & 213.1 & 213.1 & 213.1 & 213.1 & 5.0 & 3.0 & 6666.2 \\
\hline \multicolumn{18}{|c|}{ Waste Category: RH_TRU } \\
\hline D-COMBUSt & NONE & 10.3 & 10.3 & 10.3 & 10.3 & 10.3 & 10.3 & 19.3 & 10.3 & 10.3 & 10.3 & 20.3 & 10.3 & 10.3 & 10.3 & 10.3 & 176.4100 .0 \\
\hline Tot,n10-COHBUST & & 10.3 & 10.3 & 20.3 & 10.3 & 20.3 & 10.3 & 20.3 & 10.3 & 10.3 & 10.3 & 20.3 & 10.3 & 20.3 & 10.3 & 10.3 & $176.4 \cdot 100.0$ \\
\hline Tota1D-IIET & & 4.9 & 4.9 & 4.9 & 6.0 & 6.0 & 6.0 & 6.0 & 6.0 & 6.0 & 6.0 & 6.0 & 6.0 & 6.0 & 4.9 & 4.9 & 142.9100 .0 \\
\hline D-IN NOH-ATL & HONE & 5.3 & 5.3 & 5.3 & 14.7 & 14.7 & 24.7 & 14.7 & 24.7 & 14.7 & 14.7 & 14.7 & 11.7 & 24.7 & 5.3 & 3.3 & 233.4100 .0 \\
\hline TotalD-IN HON-HTL & & 5.3 & 5.3 & 5.3 & 14.7 & 14.7 & 14.7 & 14.7 & 14.7 & 14.7 & 11.7 & 24.7 & 21.7 & 14.7 & 5.3 & 3.3 & 233.4100 .0 \\
\hline D-HETAL & NOHE & 20.9 & 20.9 & 20.9 & 278.1 & 270.1 & 278.1 & 278.1 & 278.1 & 270.1 & 270.1 & 278.1 & 270.1 & 270.1 & 20.9 & 20.9 & 2939.4100 .0 \\
\hline Tot=10-METAL & & 20.9 & 20.9 & 20.9 & 270.1 & 278.1 & 278.1 & 270.1 & 270.1 & 270.1 & 278.1 & 278.1 & 278.1 & 270.1 & 20.9 & 20.9 & 2939.4100 .0 \\
\hline IH ORG PART & NONE & 0.0 & 0.0 & 0.0 & 0.0 & 0.0 & 0.0 & 0.0 & 0.0 & 0.0 & 0.0 & 0.0 & 0.0 & 0.0 & 0.0 & 0.0 & 24.9100 .0 \\
\hline Tota1IN ORG PART & & 0.0 & 0.0 & 0.0 & 0.0 & 0.0 & 0.0 & 0.0 & 0.0 & 0.0 & 0.0 & 0.0 & 0.0 & 0.0 & 0.0 & 0.0 & 24.9100 .0 \\
\hline LEAD & NONE & 0.0 & 0.0 & 0.0 & 13.8 & 13.0 & 13.0 & 13.0 & 13.0 & 13.8 & 13.0 & 13.0 & 13.0 & 13.8 & 0.0 & 0.0 & 238.2100 .0 \\
\hline TOtalLEAD & & 0.0 & 0.0 & 0.0 & 13.8 & 13.0 & 13.8 & 13.8 & 23.0 & 23.8 & 23.8 & 23.0 & 23.8 & 13.0 & 0.0 & 0.0 & 130.1100 .0 \\
\hline ORG PART & NOAE & 0.0 & 0.0 & 0.0 & 21.2 & 21.2 & 21.2 & 21.2 & 21.2 & 21.2 & 21.2 & 21.2 & 21.2 & 21.2 & 0.0 & 0.0 & 219.2100 .0 \\
\hline Tota1ORG PART & & 0.0 & 0.0 & 0.0 & 21.2 & 21.2 & 21.2 & 21.2 & 21.2 & 21.2 & 21.2 & 21.2 & 21.2 & 21.2 & 0.0 & 0.0 & 219.2100 .0 \\
\hline SH LEAD & NONE & 1.9 & 4.9 & 1.9 & 4.9 & 1.9 & 4.9 & 4.9 & 1.9 & 1.9 & 1.9 & 4.9 & 4.9 & 4.9 & 4.9 & 4.9 & 110.0100 .0 \\
\hline TotalsA LEAD & & 1.9 & 4.9 & 4.9 & 1.9 & 4.9 & 4.9 & 1.9 & 1.9 & 1.9 & 1.9 & 1.9 & 1.9 & 4.9 & 4.9 & 1.9 & 110.0100 .0 \\
\hline SH STEEL & HONE & 1.9 & 1.9 & 4.9 & 4.9 & 4.9 & 4.9 & 4.9 & 4.9 & 4.9 & 1.9 & 4.9 & 4.9 & 4.9 & 4.9 & 1.9 & 80.7100 .0 \\
\hline TOL:A1SI STEEL & & 1.9 & 1.9 & 1.9 & 1.9 & 1.9 & 4.9 & 1.9 & 1.9 & 1.9 & 1.9 & 4.9 & 4.9 & 4.9 & 4.9 & 1.9 & 00.7100 .0 \\
\hline
\end{tabular}

BCD Codes: A-Ignitables, B-Corrosives, C-Rexctives, D-Metals w/O Hg, Emetals w Hg, F-Organics, G-State Reg., H-PCB < 50ppm, J-PCB > 50ppm 


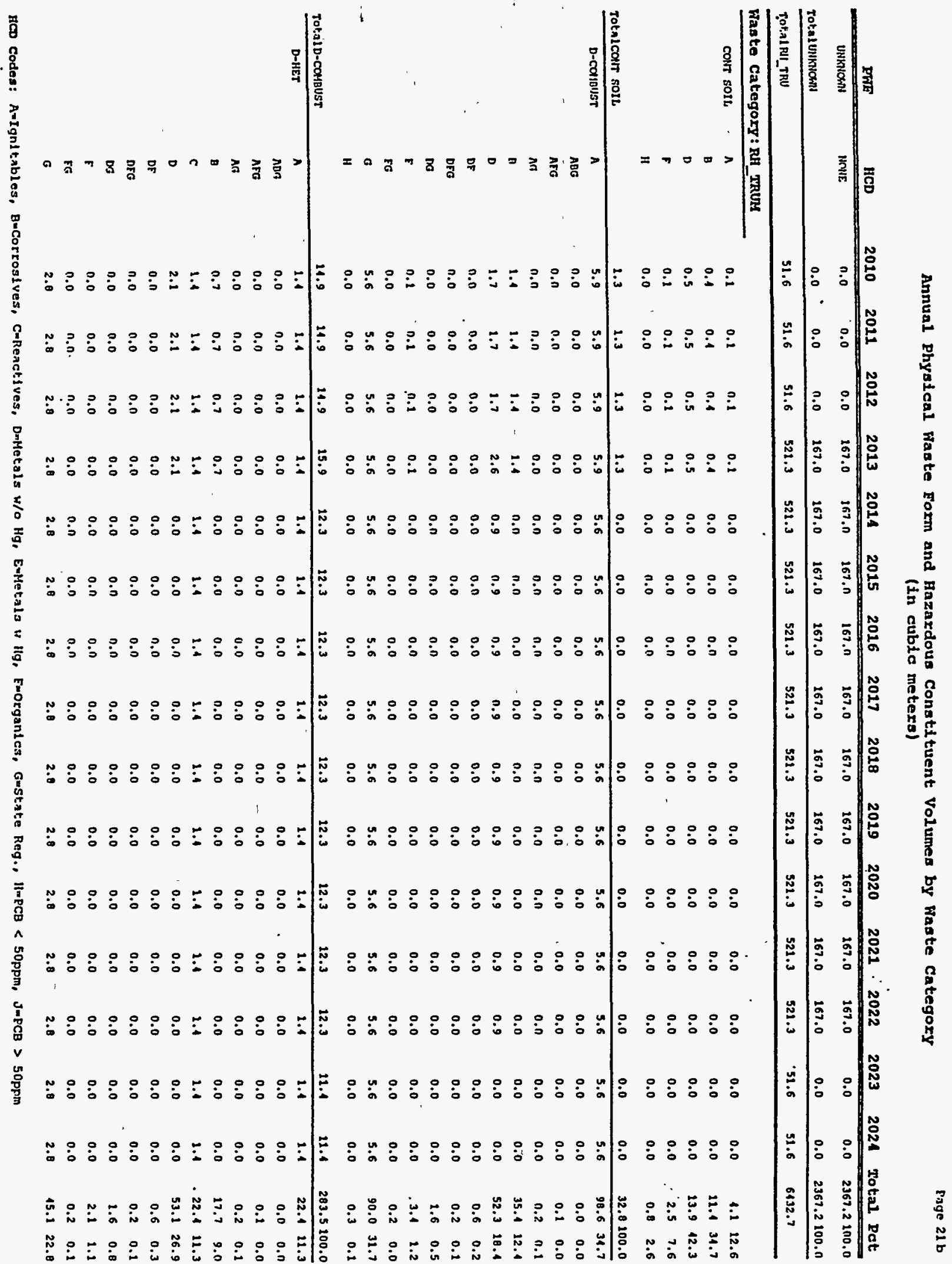




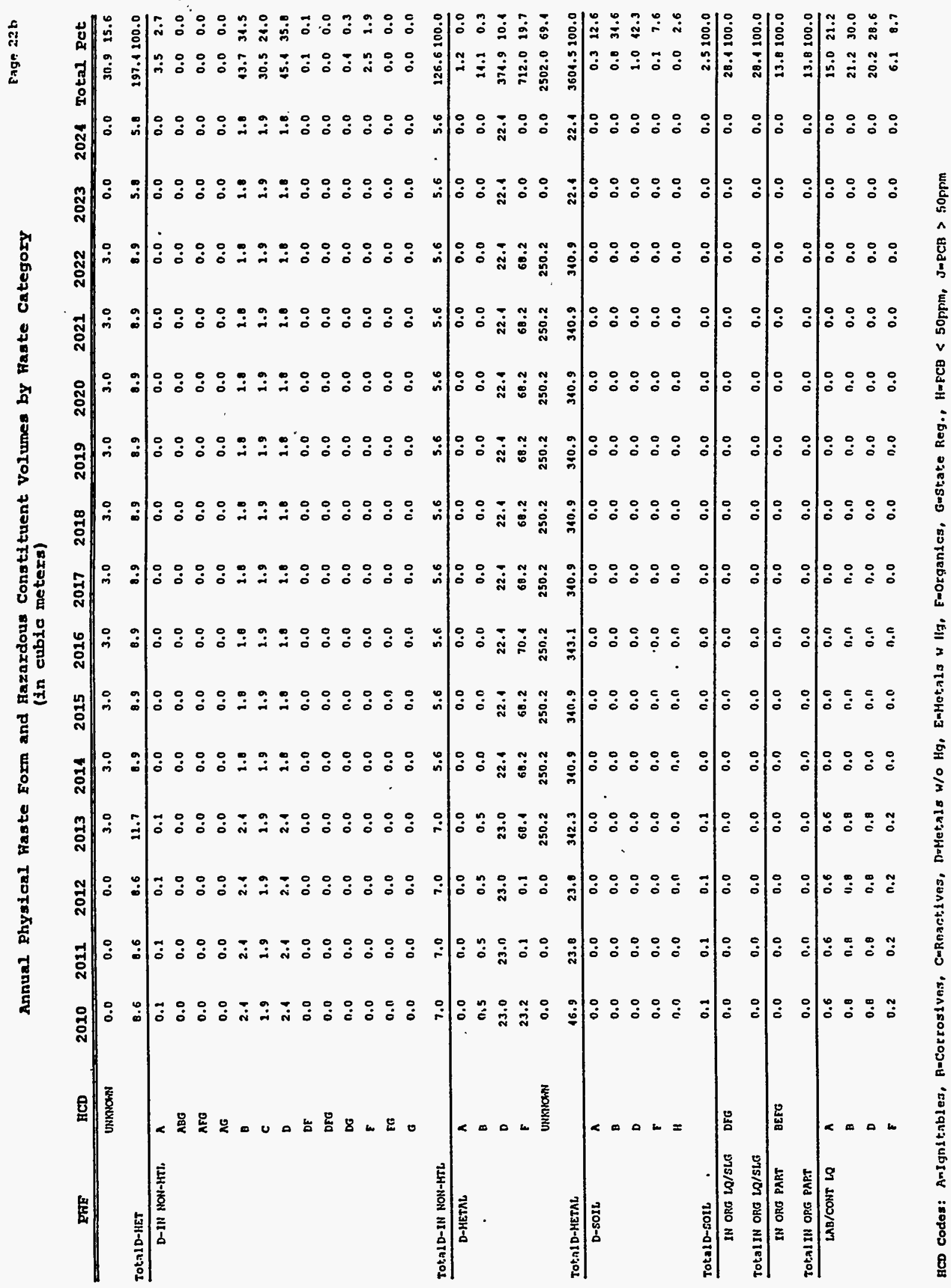



(in cuble meters)

\begin{tabular}{|c|c|c|c|c|c|c|c|c|c|c|c|c|c|c|c|c|c|c|}
\hline & PWE & IICD & 2010 & 2011 & 2012 & 2013 & 2014 & 2015 & 2016 & 2017 & 2018 & 2019 & 2020 & 2021 & 2022 & 2023 & 2021 & Total Pct \\
\hline & & $\mathrm{H}$ & 0.3 & 0.3 & 0.3 & 0.3 & 0.0 & 0.0 & 0.0 & 0.0 & 0.0 & 0.0 & 0.0 & 0.0 & 0.0 & 0.0 & 0.0 & 0.011 .3 \\
\hline & Tota1 LAB/CONT Lo & & 2.8 & 2.0 & 2.0 & 2.0 & 0.0 & 0.0 & 0.0 & 0.0 & 0.0 & 0.0 & 0.0 & 0.0 & 0.0 & 0.0 & 0.0 & 70.0100 .0 \\
\hline & LEAD & D & 0.0 & 0.1 & 0.0 & 2.7 & 2.7 & 2.7 & 2.7 & 2.7 & 2.7 & 2.7 & 2.7 & 2.7 & 2.7 & 0.0 & 0.0 & 27.6100 .0 \\
\hline & TOtaILEAD & & 0.0 & 0.0 & 0.0 & 2.7 & 2.7 & 2.7 & 2.7 & 2.7 & 2.7 & 2.7 & 2.7 & 2.7 & 2.7 & 0.0 & 0.0 & 21.6100 .0 \\
\hline \multirow[t]{12}{*}{ - } & \multirow{12}{*}{ ORG $A B S$ LQ/SLG } & $\lambda$ & 2.8 & 2.8 & 2.8 & 2.8 & 0.0 & 0.0 & 0.0 & 0.0 & 0.0 & 0.0 & 0.0 & 0.0 & 0.0 & 0.0 & 0.0 & $20.8 \quad 19.2$ \\
\hline & & $A B G$ & 0.0 & 0.0 & 0.0 & 0.0 & 0.0 & 0.0 & 0.0 & 0.0 & 0.0 & 0.0 & 0.0 & 0.0 & 0.0 & 0.0 & 0.0 & $0.0 \quad 0.0$ \\
\hline & & Arg & 0.0 & 0.0 & 0.0 & 0.0 & 0.0 & 0.0 & $0 . n$ & 0.0 & 0.0 & 0.0 & 0.0 & 0.0 & 0.0 & 0.0 & 0.0 & $0.0 \quad 0.0$ \\
\hline & & $\Lambda a$ & 0.0 & 0.0 & 0.0 & 0.0 & 0.0 & 0.0 & $0 . n$ & -0.0 & 0.0 & 0.0 & 0.0 & 0.0 & 0.0 & 0.0 & 0.0 & $0.0 \quad 0.0$ \\
\hline & & $B$ & 4.2 & 4.2 & 4.2 & 1.2 & 0.0 & 0.0 & 0.0 & 0.0 & 0.0 & 0.0 & 0.0 & 0.0 & 0.0 & 0.0 & 0.0 & 106.228 .8 \\
\hline & & $D$ & 5.6 & 5.6 & 3.6 & 5.6 & 0.0 & 0.0 & 0.0 & 0.0 & 0.0 & 0.0 & 0.0 & 0.0 & 0.0 & 0.0 & 0.0 & $141.7 \quad 36.4$ \\
\hline & & or & 0.0 & 0.0 & 0.0 & 0.0 & 0.0 & 0.0 & 0.0 & 0.0 & 0.0 & 0.0 & 0.0 & 0.0 & 0.0 & 0.0 & 0.0 & 0.10 .0 \\
\hline & & DrG & 0.0 & 0.0 & 0.0 & 0.0 & 0.0 & 0.0 & 0.0 & 0.0 & 0.0 & 0.0 & 0.0 & $\cdot 0.0$ & 0.0 & 0.0 & 0.0 & $0.0 \quad 0.0$ \\
\hline & & $D G$ & 0.0 & 0.0 & 0.0 & 0.0 & 0.0 & 0.0 & 0.0 & 0.0 & 0.0 & 0.0 & 0.0 & 0.0 & 0.0 & 0.0 & 0.0 & $\begin{array}{lll}0.4 & 0.1\end{array}$ \\
\hline & & $\mathbf{F}$ & 12.9 & 1.4 & 1.4 & 1.4 & 0.0 & 0.0 & 1.1 & 0.0 & 0.0 & 0.0 & 0.0 & 0.0 & 0.0 & 0.0 & 0.0 & $\begin{array}{lll}18.5 \quad 13.1\end{array}$ \\
\hline & & EG & 0.0 & 0.0 & 0.0 & 0.0 & 0.0 & 0.0 & 0.0 & 0.0 & 0.0 & 0.0 & 0.0 & 0.0 & 0.0 & 0.0 & 0.0 & $\begin{array}{lll}0.0 & 0.0\end{array}$ \\
\hline & & $\sigma$ & 0.0 & 0.0 & 0.0 & 0.0 & 0.0 & 0.0 & 0.0 & 0.0 & 0.0 & 0.0 & 0.0 & 0.0 & 0.0 & 0.0 & 0.0 & $\begin{array}{ll}0.0 & 0.0\end{array}$ \\
\hline & TotaloRG ABS LQ/SLG & & 25.7 & 14.2 & 11.2 & 11.2 & 0.0 & 0.0 & 1.1 & 0.0 & 0.0 & 0.0 & 0.0 & 0.0 & 0.0 & 0.0 & 0.0 & 368.1100 .0 \\
\hline & \multirow[t]{4}{*}{ ORG PART } & $A$ & 0.2 & 0.2 & 0.2 & 0.2 & 0.0 & 0.0 & 0.0 & 0.0 & 0.0 & 0.0 & .0 .0 & 0.0 & 0.0 & 0.0 & 0.0 & 6.119 .6 \\
\hline & & P & 0.4 & 0.4 & 0.4 & 0.4 & 0.0 & 0.0 & 0.0 & 0.0 & 0.0 & 0.0 & 0.0 & 0.0 & 0.0 & 0.0 & 0.0 & $10.6 \quad 33.6$ \\
\hline & & BEFG & 0.0 & 0.0 & 0.0 & 0.0 & 0.0 & 0.0 & 0.0 & 0.0 & 0.0 & 0.0 & 0.0 & 0.0 & 0.0 & 0.0 & 0.0 & $13.8 \quad 13.9$ \\
\hline & & $D$ & 0.0 & 0.0 & 0.0 & 0.0 & 0.0 & 0.0 & 0.0 & 0.0 & 0.0 & 0.0 & 0.0 & 0.0 & 0.0 & 0.0 & 0.0 & $0.8 \quad 2.8$ \\
\hline & TotaloRG PART & & 0.7 & 0.7 & 0.7 & 0.7 & 0.0 & 0.0 & 0.0 & 0.0 & 0.0 & 0.0 & 0.0 & 0.0 & 0.0 & 0.0 & 0.0 & 31.5100 .0 \\
\hline & SH LEAD & D & 5.6 & 5.6 & 5.6 & 5.6 & 5.6 & 3.6 & 5.6 & 5.6 & 5.6 & 5.6 & 5.6 & 3.6 & 5.6 & 5.6 & 5.6 & 101.3100 .0 \\
\hline & Totalsh LEAD & & 5.6 & 5.6 & 5.6 & 5.6 & 5.6 & 5.6 & 5.6 & 5.6 & 5.6 & 5.6 & 5.6 & 5.6 & 5.6 & 5.6 & 5.6 & 101.3100 .0 \\
\hline & \multirow[t]{3}{*}{ SH STEEL } & $B$ & 2.8 & 2.0 & 2.8 & 2.8 & 2.8 & 2.8 & 2.8 & 2.0 & 2.8 & 2.8 & 2.8 & 2.8 & 2.8 & 2.0 & 2.0 & $11.0 \quad 16.4$ \\
\hline & & G & 2.0 & 2.0 & 2.8 & 2.0 & 2.8 & 2.1 & 2.0 & 2.0 & 2.0 & 2.8 & 2.8 & 2.0 & 2.0 & 2.0 & 2.0 & $\begin{array}{lll}11.8 & 16.4\end{array}$ \\
\hline & & Unerocent & 0.0 & 0.0 & 0.0 & 0.0 & 0.0 & 0.0 & 0.0 & 0.0 & 0.0 & 0.0 & 0.0 & 0.0 & 0.0 & 0.0 & 0.0 & $6.9 \quad 7.1$ \\
\hline & TOtalSH STEEL & & 5.6 & 5.6 & 5.6 & 5.6 & 5.6 & 5.6 & 5.6 & 5.6 & 5.6 & 5.6 & 5.6 & 5.6 & 5.6 & 5.6 & 5.6 & 96.6100 .0 \\
\hline & SH VOID & UNENOWN & 196.2 & 0.0 & 0.0 & 0.0 & 0.0 & 0.0 & 19.0 & 0.0 & 0.0 & 0.0 & 0.0 & 0.0 & 0.0 & 0.0 & 0.0 & 215.3100 .0 \\
\hline & Total sti voro & & 196.2 & 0.0 & 0.0 & 0.0 & 0.0 & 0.0 & 19.0 & 0.0 & 0.0 & 0.0 & 0.0 & 0.0 & 0.0 & 0.0 & 0.0 & 215.3100 .0 \\
\hline & Total RH_TRUM & & 315.8 & 04.9 & 01.9 & 110.1 & 381.8 & $301 . \mathrm{A}$ & 404.2 & 381.8 & 301.8 & 301.8 & 301.8 & 301.8 & 381.0 & 56.6 & 56.6 & 5201.3 \\
\hline & orand Tot: & & 36777.6 & 26601.1 & 23107.9 & 33442.2 & 14662.0 & 16128.5 & 33055.4 & 34360.3 & 386.0 & 30910.9 & 33114.5 & 36138.7 & 9610.2 & 10464.6 & 202.8 & 2824043.1 \\
\hline
\end{tabular}




\section{APPENDIX D}

ANNUAL VOLUMES OF PHYSICAL WASTE FORMS AND HAZARDOUS

CONSTITUENTS BY WASTE GENERATOR 
WHC-EP-0888

Intentionally Left Blank 


\begin{tabular}{|c|c|c|c|c|c|c|c|c|c|c|c|c|c|c|c|c|c|}
\hline PWF & HCD & 2010 & 011 & 2012 & 2013 & 2014 & 2015 & 2016 & 2017 & 2018 & 2019 & 2020 & 2021 & 2022 & 2023 & 024 & Total Pct \\
\hline BCHA1 D-METAL & & 0.0 & 0.0 & 0.0 & 14.7 & 24.7 & 14.7 & 14.7 & 14.7 & 14.7 & 34.7 & 11.7 & 14.7 & 34.7 & 0.0 & 0.0 & 147.2100 .0 \\
\hline Total Thatc_340 & & 0.0 & 0.0 & 0.0 & 22.0 & 22.0 & 22.0 & 22.0 & 22.0 & 22.0 & 22.0 & 22.0 & 22.0 & 22.0 & 0.0 & 0.0 & 220.8 \\
\hline \multicolumn{18}{|c|}{ Weste Genoratox:IFIHC_B_PLANT } \\
\hline \multirow[t]{2}{*}{ D-KETAL. } & D & 0.0 & 0.0 & 0.0 & 34.6 & 34.6 & 34.6 & 34.6 & 34.6 & 34.6 & 34.6 & 34.6 & 34.6 & 34.6 & 0.0 & 0.0 & $\begin{array}{rr}346.0 & 0.8 \\
12834.0 & 99.1\end{array}$ \\
\hline & NONE & 0.0 & 0.0 & 0.0 & 82000 & 8000. & 16030. & 1283.4 & 9283.4 & 1283.6 & 2283.4 & 203.4 & 1283.4 & 4283.4 & 0.0 & 0.0 & 12634.099 .1 \\
\hline ROta1D-HETAL & & 0.0 & 0.0 & 0.0 & 4318.0 & 4318.0 & 4318.0 & 4318.0 & 1318.0 & 4318.0 & 1318.0 & 4318.0 & 1310.0 & 1318.0 & 0.0 & 0.0 & $\$ 3100.0100 .0$ \\
\hline TOtal TWAC_B_PLANT & & 0.0 & 0.0 & 0.0 & 4318.0 & 4318.0 & 1318.0 & 1310.0 & 4318.0 & 1318.0 & 4318.0 & 4319.0 & 1318.0 & 4318.0 & 0.0 & 0.0 & 13180.0 \\
\hline \multicolumn{18}{|c|}{ Waste Genoratox:IFItC_PEP } \\
\hline D-HET & UNKNOWN & 0.0 & 0.0 & 0.0 & 15.4 . & 25.4 & 25.4 & 15.4 & 15.1 & 15.4 & 25.4 & 15.1 & 25.4 & 15.4 & 0.0 & 0.0 & 154.9100 .0 \\
\hline Tota1D-HET & & 0.0 & 0.0 & 0.0 & 25.4 & 15.1 & 15.1 & 15.1 & 15.1 & 15.1 & 15.4 & 15.1 & 15.4 & 15.1 & 0.0 & 0.0 & 154.9100 .0 \\
\hline \multirow[t]{2}{*}{ D-METAL } & MONE & 0.0 & 0.0 & 0.0 & 910.2 & 910.2 & 910.2 & 910.2 & 910.2 & 920.2 & 910.2 & 910.2 & 910.2 & 910.2 & 0.0 & 0.0 & $9102.5 \quad 12.3$ \\
\hline & Unknown & 0.0 & 0.0 & 0.0 & 1239.6 & 1239.6 & 1239.6 & 1239.6 & 1239.6 & 1239.6 & 1239.6 & 1239.6 & 1239.6 & 1239.6 & 0.0 & 0.0 & 12396.857 .6 \\
\hline Total D-METAL & & 0.0 & 0.0 & 0.0 & 2149.9 & 2149.9 & 2149.9 & 2149.9 & 2149.9 & 2149.9 & 2149.9 & 2149.9 & 2149.9 & 2149.9 & 0.0 & 0.0 & 21199.3100 .0 \\
\hline LEAD & MONE & 0.0 & 0.0 & 0.0 & 25.6 & 15.6 & 15.6 & 13.6 & 15.6 & 15.6 & 15.6 & 15.6 & 15.6 & 15.6 & 0.0 & 0.0 & 156.9100 .0 \\
\hline Tota1LEAD & & 0.0 & 0.0 & 0.0 & 15.6 & 15.6 & 15.6 & 15.6 & 25.6 & 25.6 & 15.6 & 15.6 & 15.6 & 15.6 & 0.0 & 0.0 & 156.9100 .0 \\
\hline UNKMOWN & NONE & 0.0 & 0.0 & 0.0 & 256.9 & 156.9 & 156.9 & 156.9 & 156.9 & 156.9 & 156.9 & 156.9 & 156.9 & 156.9 & 0.0 & 0.0 & 1569.4100 .0 \\
\hline Totalunkanowis & & 0.0 & 0.0 & 0.0 & 256.9 & 256.9 & 156.9 & 156.9 & 156.9 & 156.9 & 156.9 & 156.9 & 156.9 & 156.9 & 0.0 & 0.0 & 1569.4100 .0 \\
\hline Total This__PEP & & 0.0 & 0.0 & 0.0 & 2330.0 & 2338.0 & 2338.0 & 2338.0 & 2330.0 & 2338.0 & 2338.0 & 2338.0 & 2338.0 & 2338.0 & 0.0 & 0.0 & 23380.6 \\
\hline \multicolumn{3}{|c|}{ Faste Generatox:IFHC_PUREX } & & & & & & & & & & & & & & & - \\
\hline D-combust & D & 0.0 & 0.0 & 0.0 & 0.9 & 0.9 & 0.9 & 0.9 & 0.9 & 0.9 & 0.9 & 0.9 & 0.9 & 0.9 & 0.0 & 0.0 & 9.5100 .0 \\
\hline rotalD-CoMBUST & & 0.0 & 0.0 & 0.0 & 0.9 & 0.9 & 0.9 & 0.9 & 0.9 & 0.9 & 0.9 & 0.9 & 0.9 & 0.9 & 0.0 & 0.0 & 9.5100 .0 \\
\hline \multirow[t]{2}{*}{ D-IN NON-MTL } & $D$ & 0.0 & 0.0 & 0.0 & 0.0 & 0.0 & 0.0 & 0.0 & 0.0 & 0.0 & 0.0 & 0.0 & 0.0 & 0.0 & 0.0 & 0.0 & $0.0 \quad 0.0$ \\
\hline & MONE & 0.0 & 0.0 & 0.0 & 241.1 & 141.1 & 111.1 & 141.1 & 111.1 & 241.1 & 141.1 & 141.1 & 141.1 & 141.1 & 0.0 & 0.0 . & $1411.5 \quad 99.9$ \\
\hline Totz1D-IN NON-MTL & & 0.0 & 0.0 & 0.0 & 111.1 & 241.1 & 111.1 & 141.1 & 111.1 & 141.1 & 141.1 & 141.1 & 111.1 & 111.1 & 0.0 & 0.0 & 1121.5100 .0 \\
\hline D-METAL & D & 0.0 & 0.0 & 0.0 & 0.6 & 0.6 & 0.6 & 0.6 & 0.6 & 0.6 & 0.6 & 0.6 & 0.6 & 0.6 & 0.0 & 0.0 & 6.30 .1 \\
\hline - & HONE & 0.0 & 0.0 & 0.0 & 526.2 & 526.2 & 526.2 & 526.2 & 526.2 & 526.2 & 326.2 & 526.2 & 526.2 & 526.2 & 0.0 & 0.0 & $5262.0 \quad 99.0$ \\
\hline Tota 1 D-METAL & & 0.0 & 0.0 & 0.0 & 526.9 & 526.9 & 526.9 & 526.9 & 526.9 & 526.9 & 526.9 & 526.9 & 526.9 & 526.9 & 0.0 & 0.0 & 5269.1200 .0 \\
\hline LEAD & D & 0.0 & 0.0 & 0.0 & 2.1 & 2.1 & 2.4 & 2.4 & 2.1 & 2.4 & 2.4 & 2.4 & 2.4 & 2.4 & 0.0 & 0.0 & 21.7100 .0 \\
\hline Tota1lead & & 0.0 & 0.0 & 0.0 & 2.4 & 2.4 & 2.4 & 2.4 & 2.4 & 2.4 & 2.4 & 2.1 & 2.1 & 2.1 & 0.0 & 0.0 & 24.7100 .0 \\
\hline UNknOWNA & UNMNOWN & 0.0 & 0.0 & 0.0 & 29.5 & 29.5 & 29.5 & 29.5 & 29.5 & 29.5 & 29.5 & 29.5 & 29.5 & 29.5 & 0.0 & 0.0 & 295.4100 .0 \\
\hline Totalunisnown & & 0.0 & 0.0 & 0.0 & 29.5 & 29.5 & 29.5 & 29.5 & 29.5 & 29.5 & 29.5 & 29.5 & 29.5 & 29.5 & 0.0 & 0.0 & 295.4100 .0 \\
\hline
\end{tabular}

HCD Codes: A-Ignitables, B-Corrosives, C-Reactives, D-Metals w/O Hg, E-Metals w Hg, E-Organlcs, G-State Reg., H-PCB < 50ppm, J-PCB > 50ppm 
Annual Phyalcal Waste Form and Bazardous Constituent Volumes by Waste Generatox

(in cubia meters)

\begin{tabular}{|c|c|c|c|c|c|c|c|c|c|c|c|c|c|c|c|c|c|}
\hline EWE & $\mathrm{HCD}$ & 2010 & 2011 & 2012 & 2013 & 2014 & 2015 & 2016 & 2017 & 2018 & 2019 & 2020 & 2021 & 2022 & 2023 & 2024 & Total Pot \\
\hline rot:al TWHE_PUREX & & 0.0 & 0.0 & 0.0 & 701.0 & 701.0 & 701.0 & 701.0 & 701.0 & 701.0 & 701.0 & 701.0 & 201.0 & 701.0 & 0.0 & 0.0 & 7010.1 \\
\hline Waste Generato & :IFTRC_P & $X_{X} T$ T & & & & & & & & & & & & & & & \\
\hline Unkiown & NONE & 0.0 & 0.0 & 0.0 & 0.0 & 0.0 & 0.0 & 0.0 & 0.0 & 0.0 & 0.0 & 0.0 & 0.0 & 0.0 & 0.0 & 0.0 & $1719.0 \quad 07.6$ \\
\hline & UNKNOWN & 0.0 & 0.0 & 0.0 & 0.0 & 0.0 & 0.0 & 0.0 & 0.0 & 0.0 & 0.0 & 0.0 & 0.0 & 0.0 & 0.0 & 0.0 & $242.2 \quad 12.3$ \\
\hline rotalunkNOWN & & 0.0 & 0.0 & .0 .0 & 0.0 & 0.0 & 0.0 & 0.0 & 0.0 & 0.0 & 0.0 & 0.0 & 0.0 & 0.0 & 0.0 & 0.0 & 1961.2100 .0 \\
\hline SOtal ThHC_RUREX_TH & & 0.0 & 0.0 & 0.0 & 0.0 & 0.0 & 0.0 & 0.0 & 0,0 & 0.0 & 0.0 & 0.0 & 0.0 & 0.0 & 0.0 & 0.0 & 1961.2 \\
\hline
\end{tabular}

Wasto Genarator:IFHC_PX_CO18B

\begin{tabular}{|c|c|c|c|c|c|c|c|c|c|c|c|c|c|c|c|c|c|}
\hline D-COABUST & UNhMOCWN & 0.0 & 0.0 & 0.0 & 1.5 & 1.5 & 1.5 & 1.5 & 1.5 & 1.5 & 1.5 & 1,5 & 1.5 & 1.5 & 0.0 & 0.0 & 15.0100 .0 \\
\hline ota1 D-combust & & 0.0 & 0.0 & 0.0 & 1.5 & 1.5 & 1.5 & 1.5 & 1.5 & 1.5 & 1.5 & 1.5 . & 1.5 & 1.5 & 0.0 & 0.0 & 15.0100 .0 \\
\hline D-HET & UNERTCWN & 0.0 & 0.0 & 0.0 & 1.5 & 1.5 & 1.5 & 1.5 & 1.5 & 1.5 & 1.5 & 1.5 & 1.5 & 1.5 & 0.0 & 0.0 & $15.0100,0$ \\
\hline CotalD-HET & & 0.0 & 0.0 & 0.0 & 1.5 & 1.5 & 1.5 & 1.5 & 1.5 & 1.5 & 2.5 & 1.5 & 1.5 & 1.5 & 0.0 & 0.0 & 15.0100 .0 \\
\hline D- KETAS & UNKAOWN & 0.0 & 0.0 & 0.0 & 156.8 & 156.8 & 156.0 & 156.8 & 156.8 & 156.0 & 156.8 & 256.0 & 156.8 & 156.0 & 0.0 & 0.0 & 1569.9100 .0 \\
\hline otalD-HETAL & & 0.0 & 0.0 & 0.0 & 156.8 & 136.8 & 256.8 & 156.8 & 156.8 & 156.8 & 156.8 & 156.0 & 156.8 & 256.0 & 0.0 & 0.0 & 1568.9100 .0 \\
\hline otal THAC_PX_C & & 0.0 & 0.0 & 0.0 & 139.9 & 159.9 & 159.9 & 159.9 & 159.9 & 159.9 & 159.9 & 159.9 & 159.9 & 159.9 & 0.0 & 0.0 & 1599.0 \\
\hline
\end{tabular}

\section{Faste Generator:Twhe TRUsar}

\begin{tabular}{|c|c|c|c|c|c|c|c|c|c|c|c|c|c|c|c|c|c|}
\hline D-HET & UNGNOWN & 0.0 & 0.0 & 0.0 & 0.7 & 0.7 & 0.7 & 0.7 & 0.7 & 0.7 & 0.7 & 0.7 & 0.7 & 0.7 & 0.0 & 0.0 & 7.1100 .0 \\
\hline rotalD-HET & & 0.0 & 0.0 & 0.0 & 0.7 & 0.7 & 0.7 & 0.7 & 0.7 & 0.7 & 0.7 & 0.7 & 0.7 & 0.7 & 0.0 & 0.0 & 7.1100 .0 \\
\hline D-METAL & UNAKNoCWN & 0.0 & 0.0 & 0.0 & 0.6 & 0.6 & 0.6 & 0.6 & 0.6 & 0.6 & 0.6 & 0.6 & 0.6 & 0.6 & 0.0 & 0.0 & 6.2100 .0 \\
\hline TotalD-METnL & & 0.0 & 0.0 & 0.0 & 0.6 & 0.6 & 0.6 & 0.6 & 0.6 & 0.6 & 0.6 & 0.6 & 0.6 & 0.6 & 0.0 & 0.0 & 6.2100 .0 \\
\hline Total TWAE_TRUSAF & & 0.0 & 0.0 & 0.0 & 1.3 & 1.3 & 1.3 & 1.3 & 1.3 & 1.3 & 1.3 & 1.3 & 1.3 & 1.3 & 0.0 & 0.0 & 13.4 \\
\hline
\end{tabular}

Faste Gonorator:Imuc_I_PLAN

\begin{tabular}{|c|c|c|c|c|c|c|c|c|c|c|c|c|c|c|c|c|c|}
\hline D-METAL & $\begin{array}{l}\text { F } \\
\text { NONE }\end{array}$ & $\begin{array}{l}0.0 \\
0.0\end{array}$ & $\begin{array}{l}0.0 \\
0.0\end{array}$ & $\begin{array}{l}0.0 \\
0.0\end{array}$ & $\begin{array}{r}1019.8 \\
893.0\end{array}$ & $\begin{array}{r}1019.8 \\
893.0\end{array}$ & $\begin{array}{r}1019.8 \\
893.0\end{array}$ & $\begin{array}{r}1019.8 \\
893.0\end{array}$ & $\begin{array}{r}1019.0 \\
893.0\end{array}$ & $\begin{array}{r}1019.8 \\
893.0\end{array}$ & $\begin{array}{r}1019.8 \\
893.0\end{array}$ & $\begin{array}{r}2019.8 \\
893.0\end{array}$ & $\begin{array}{r}1019.8 \\
893.0\end{array}$ & $\begin{array}{r}1019.8 \\
893.0\end{array}$ & $\begin{array}{l}0.0 \\
0.0\end{array}$ & $\begin{array}{l}0.0 \\
0.0\end{array}$ & $\begin{array}{rl}10198.0 & 53.3 \\
8930.1 & 46.6\end{array}$ \\
\hline TOtaLD-METAL & & 0.0 & 0.0 & 0.0 & 1912.8 & 1912.8 & 1912.8 & 1912.0 & 1912.8 & 1912.0 & 1912.8 & 1912.0 & 1912.8 & 1912.8 & 0.0 & 0.0 & 19128.1100 .0 \\
\hline ORG PART & NONE & 0.0 & 0.0 & 0.0 & 105.2 & 106.2 & 106.2 & 106.2 & 106.2 & 206.2 & 106.2 & 106.2 & 106.2 & 106.2 & 0.0 & 0.0 & 1062.0100 .0 \\
\hline TOta10RG PART & & 0.0 & 0.0 & .0 .0 & 106.2 & 106.2 & 106.2 & 106.2 & 106.2 & 106.2 & 106.2 & 106.2 & 106.2 & 106.2 & 0.0 & 0.0 & 1062.0100 .0 \\
\hline TOta1 TWAC_T_PLANT & & 0.0 & 0.0 & 0.0 & 2019.0 & 2019.0 & 2019.0 & 2019.0 & 2019.0 & 2019.0 & 2019.0 & 2019.0 & 2019.0 & 2019.0 & 0.0 & 0.0 & 20190.1 \\
\hline \multicolumn{3}{|c|}{ Faste Generator:U_U } & & & & & & & & & & & & & & & \\
\hline \multirow[t]{2}{*}{ D-HET } & $\mathbf{F}$ & 0.3 & 0.3 & 0.3 & 0.3 & 0.3 & 0.3 & 0.3 & 0.3 & 0.3 & 0.3 & 0.3 & 0.3 & 0.3 & 0.3 & 0.3 & 9.050 .0 \\
\hline & NONE & 0.3 & 0.3 & 0.3 & 0.3 & 0.3 & 0.3 & 0.3 & 0.3 & 0.3 & 0.3 & 0.3 & 0.3 & 0.3 & 0.3 & 0.3 & 9.050 .0 \\
\hline
\end{tabular}

BCD Codes: A-Ignltables, B-Corrosives, C-Reactives, D-Metals w/O Hg, E-Metals w Hg, F-Organlcs, G-State Reg., H-PCB < 50ppm, JmPCB > 50ppm 


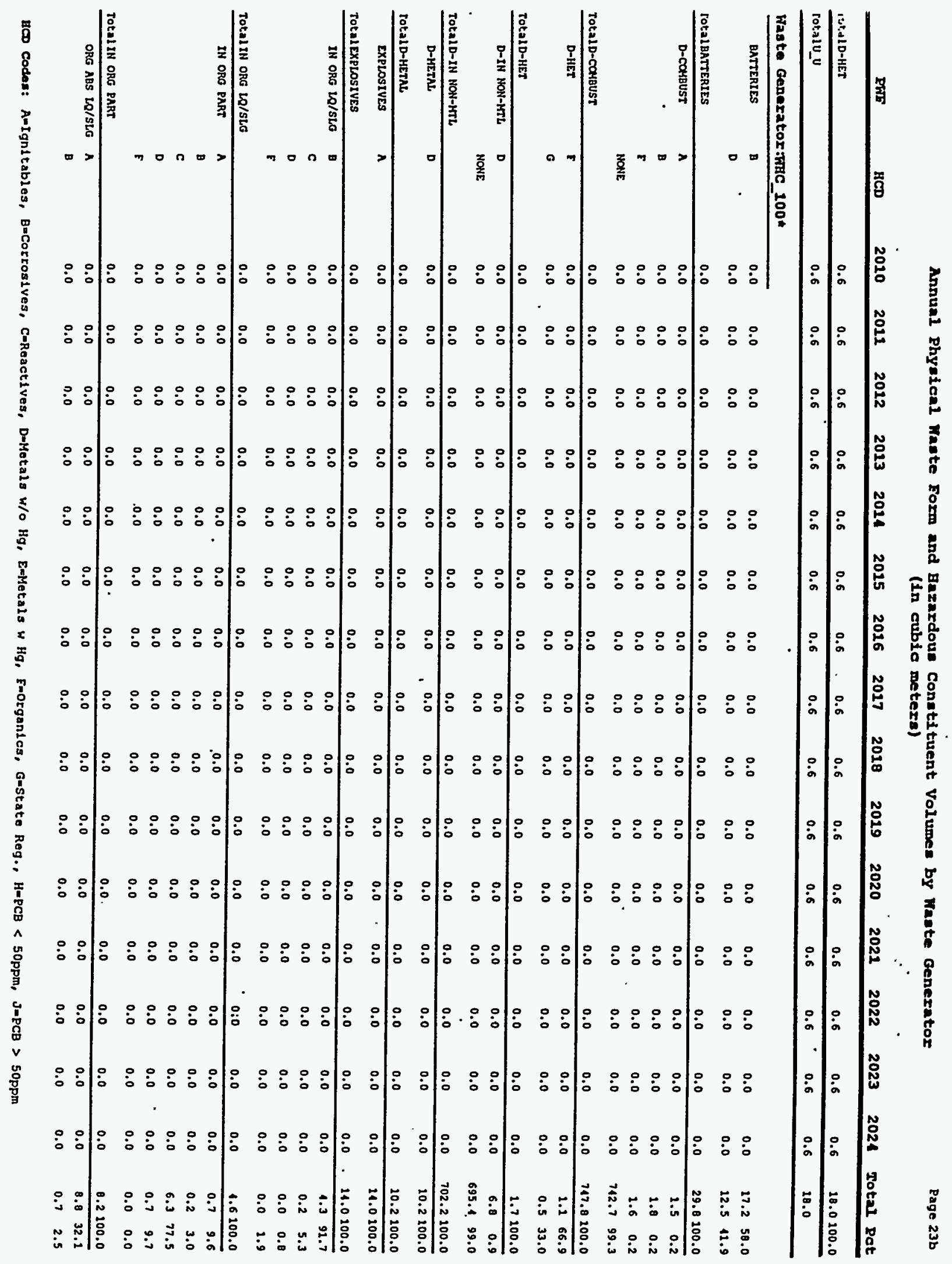




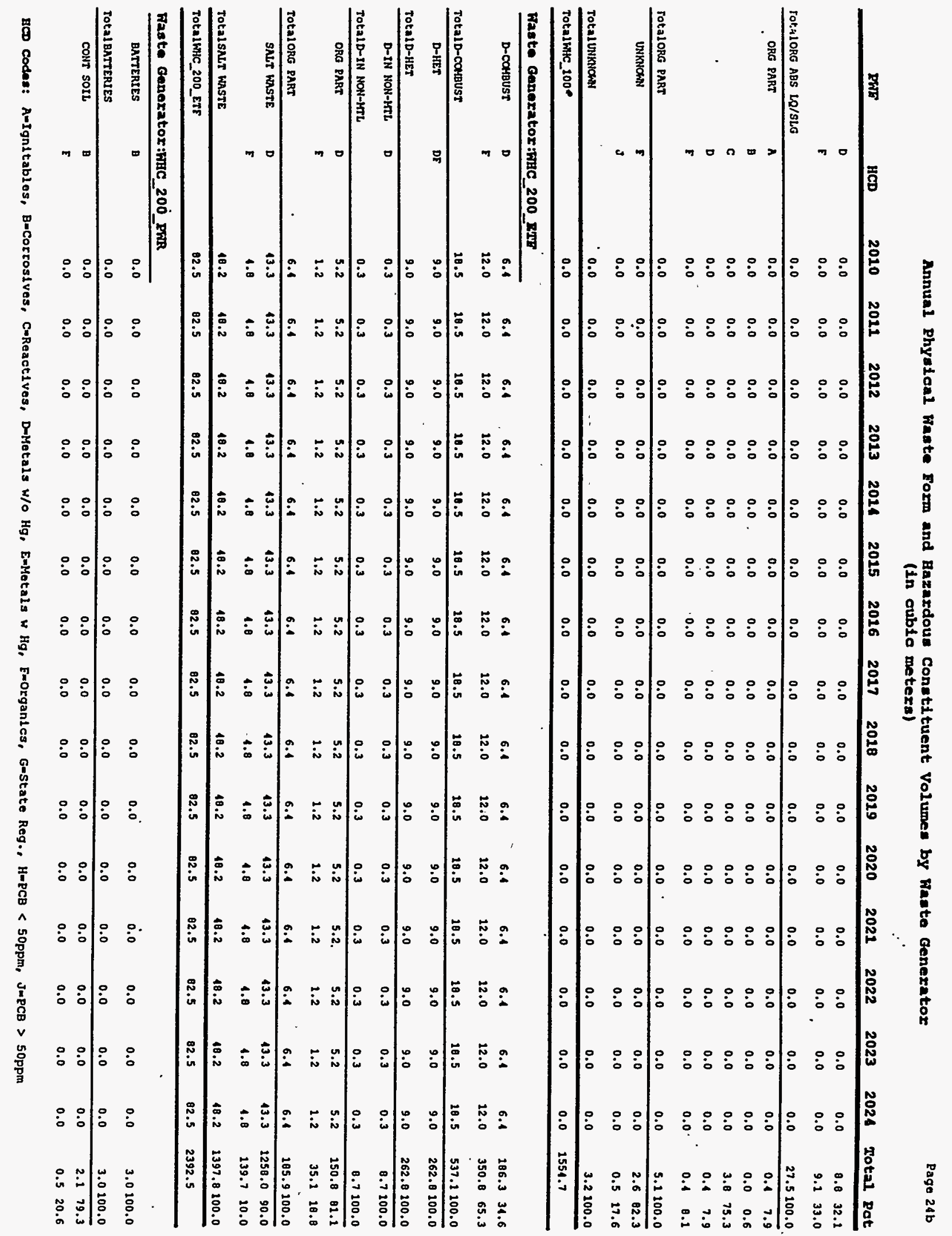




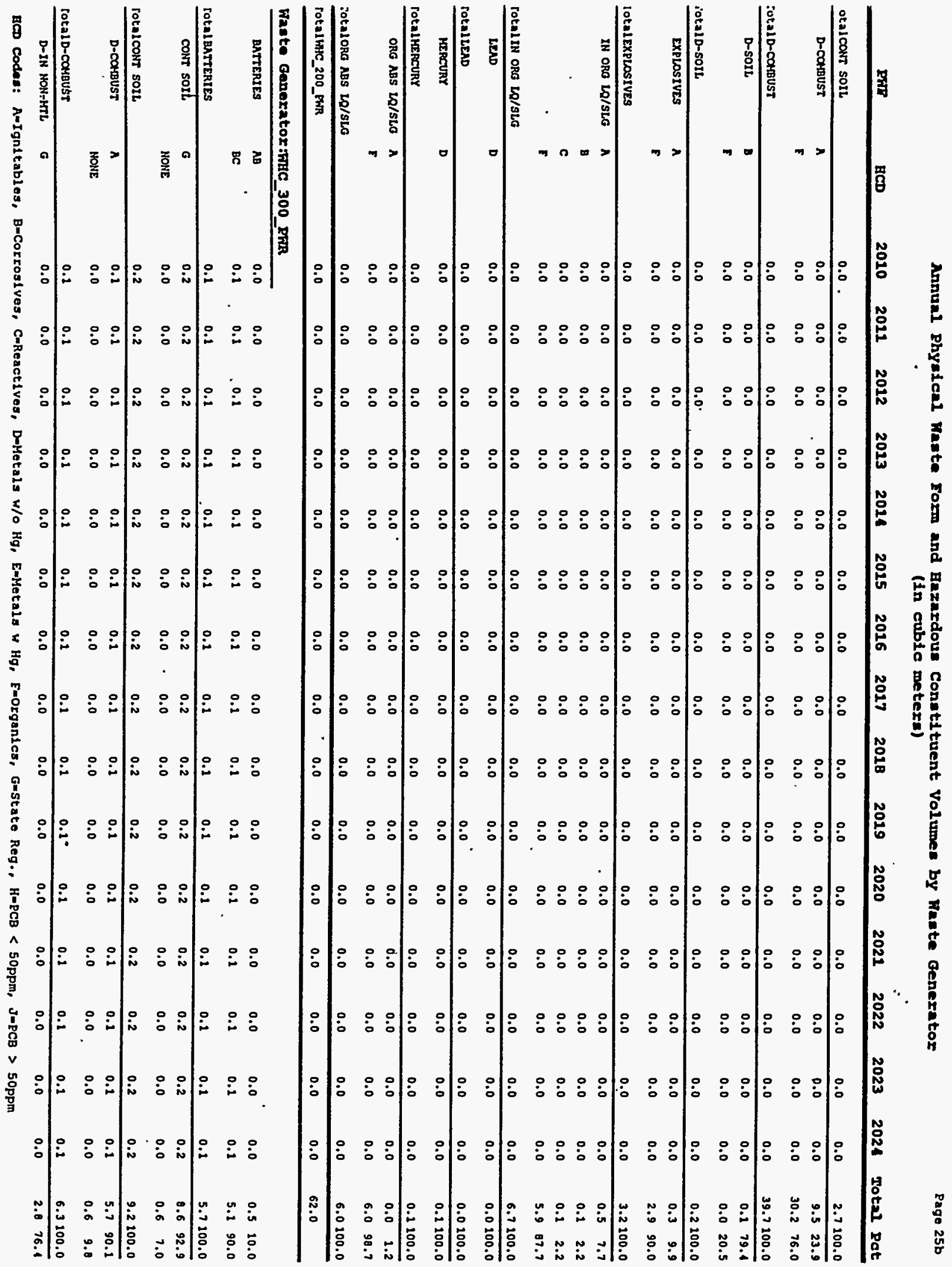




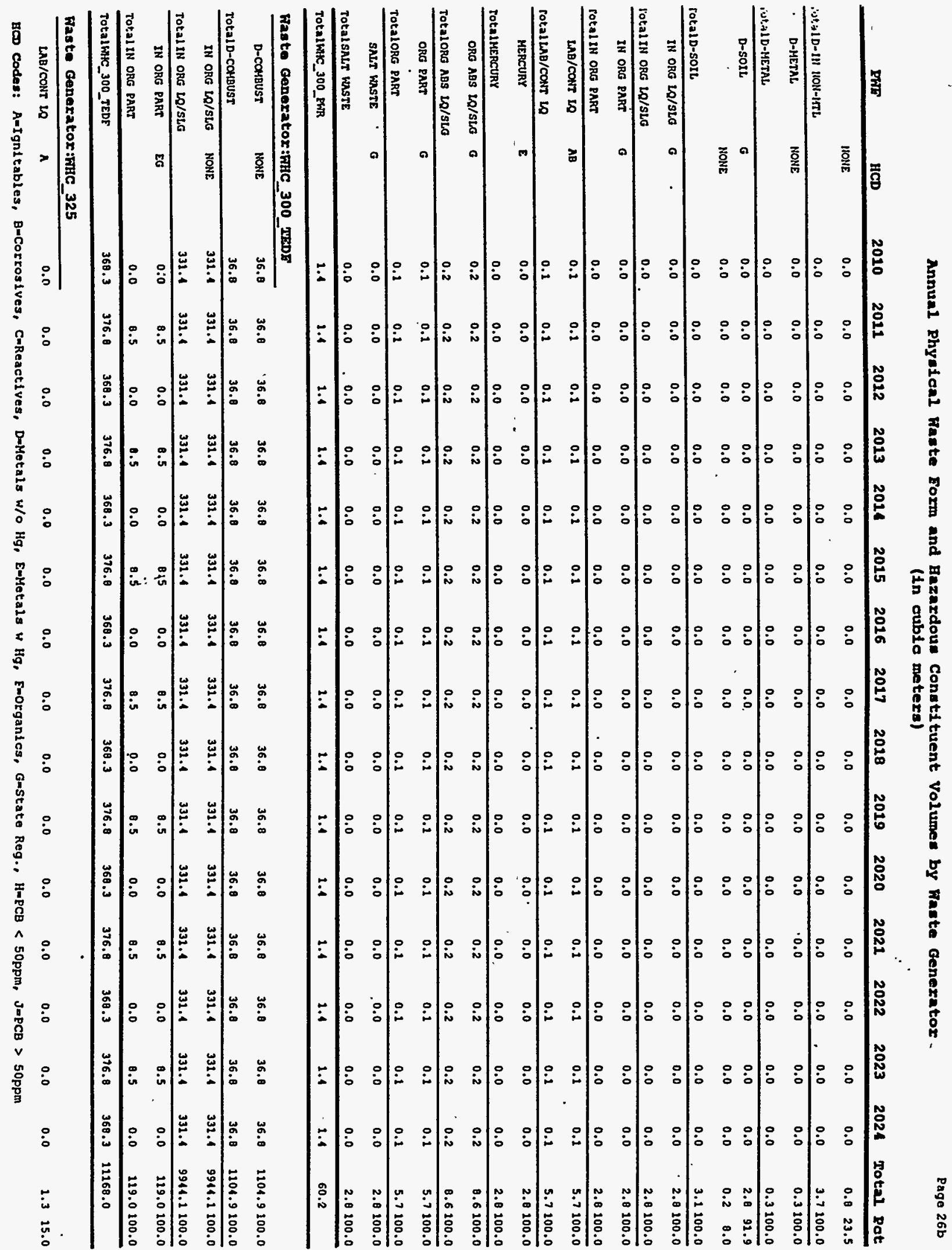




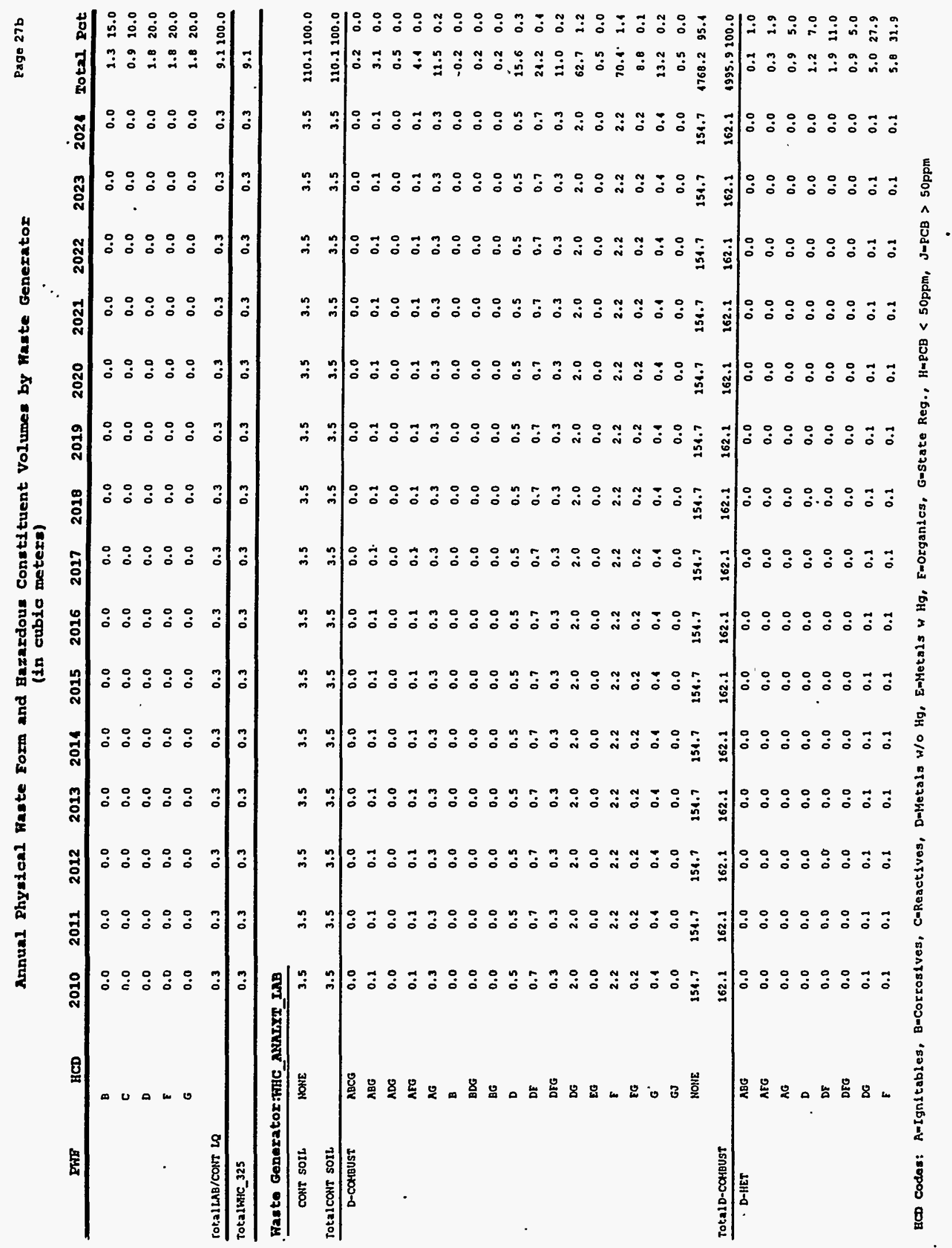



(In cublo meters)

\begin{tabular}{|c|c|c|c|c|c|c|c|c|c|c|c|c|c|c|c|c|c|}
\hline PWF & HCD & 2010 & 2011 & 2012 & 2013 & 2014 & 2015 & 2016 & 2017 & 2018 & 2019 & 2020 & 2021 & 2022 & 2023 & 2024 & Total Rot \\
\hline & FG & 0.0 & 0.0 & 0.0 & 0.0 & 0.0 & 0.0 & 0.0 & 0.0 & 0.0 & 0.0 & 0.0 & 0.0 & 0.0 & 0.0 & 0.0 & 0.7 \\
\hline - & G & 0.0 & 0.0 & 0.0 & 0.0 & 0.0 & 0.0 & 0.0 & 0.0 & 0.0 & 0.0 & 0.0 & 0.0 & 0.0 & 0.0 & 0.0 & 0.9 \\
\hline |'DT:A|D-HET & & 0.5 & 0.5 & 0.5 & 0.5 & 0.5 & 0.5 & 0.5 & 0.5 & 0.5 & 0.5 & 0.5 & 0.5 & 0.5 & 0.5 & 0.5 & 18.1100 .0 \\
\hline D-IN NON-MTLL & $A B G$ & 0.0 & 0.0 & 0.0 & 0.0 & 0.0 & 0.0 & 0.0 & 0.0 & 0.0 & 0.0 & 0.0 & 0.0 & 0.0 & 0.0 & 0.0 & 1.1 \\
\hline & AFG & 0.0 & 0.0 & 0.0 & 0.0 & 0.0 & 0.0 & 0.0 & 0.0 & 0.0 & 0.0 & 0.0 & 0.0 & 0.0 & 0.0 & 0.0 & $2.9 \quad 0.2$ \\
\hline & $A G$ & 0.2 & 0.2 & 0.2 & 0.2 & 0.2 & 0.2 & 0.2 & 0.2 & 0.2 & 0.2 & 0.2 & 0.2 & 0.2 & 0.2 & 0.2 & $\begin{array}{ll}7.1 & 0.7\end{array}$ \\
\hline & D & 0.3 & 0.3 & 0.3 & 0.3 & 0.3 & 0.3 & 0.3 & 0.3 & 0.3 & 0.3 & 0.3 & 0.3 & 0.3 & 0.3 & 0.3 & $10.1 \quad 1.0$ \\
\hline & Dr & 0.5 & 0.5 & 0.5 & 0.5 & 0.5 & 0.5 & 0.5 & 0.5 & 0.5 & 0.5 & 0.5 & 0.5 & 0.5 & 0.5 & 0.5 & $\begin{array}{lll}16.4 & 1.5\end{array}$ \\
\hline & orG & 0.2 & 0.2 & 0.2 & 0.2 & 0.2 & 0.2 & 0.2 & 0.2 & 0.2 & 0.2 & 0.2 & 0.2 & 0.2 & 0.2 & 0.2 & $.7 .4 \quad 0.7$ \\
\hline & DE & 1.3 & 1.3 & 1.3 & 1.3 & 1.3 & 1.3 & 1.3 & 1.3 & 1.3 & 1.3 & 1.3 & 1.3 & 1.3 & 1.3 & 1.3 & 31.0 \\
\hline & $\mathbf{F}$ & 1.5 & 1.5 & 1.5 & 1.5 & 1.5 & 1.5 & 1.5 & 1.5 & 1.5 & 1.5 & 1.5 & 1.5 & 1.5 & 1.5 & 1.5 & 17.7 \\
\hline & ro & 0.1 & 0.1 & 0.1 & 0.1 & 0.1 & 0.1 & 0.1 & 0.1 & 0.1 & 0.1 & 0.1 & 0.1 & 0.1 & 0.1 & 0.1 & 5.9 \\
\hline & G & 0.2 & 0.2 & 0.2 & 0.2 & 0.2 & 0.2 & 0.2 & 0.2 & 0.2 & 0.2 & 0.2 & 0.2 & 0.2 & 0.2 & 0.2 & 7.4 \\
\hline & NONE & 28.6 & 28.6 & 28.6 & 20.6 & 28.6 & 28.6 & 28.6 & 28.6 & 28.6 & 28.6 & 28.6 & 28.6 & 28.6 & 20.6 & 28.6 & 001.605 .5 \\
\hline Tota1D-IN NON-MTL & & 33.4 & 33.4 & 33.1 & 33.4 & 33.4 & 33.4 & 33.4 & 33.4 & 33.4 & 33.4 & 33.4 & 33.4 & 33.1 & 33.4 & 33.4 & 1030.9100 .0 \\
\hline D-HETAL & ABCG & 0.0 & 0.0 & 0.0 & 0.0 & 0.0 & 0.0 & 0.0 & 0.0 & 0.0 & 0.0 & 0.0 & 0.0 & 0.0 & 0.0 & 0.0 & $0.0 \quad 0.0$ \\
\hline & ABG & 0.0 & 0.0 & 0.0 & 0.0 & 0.0 & 0.0 & 0.0 & 0.0 & 0.0 & 0.0 & 0.0 & 0.0 & 0.0 & 0.0 & 0.0 & 0.10 .0 \\
\hline & ADG & 0.0 & 0.0 & 0.0 & 0.0 & 0.0 & 0.0 & 0.0 & 0.0 & 0.0 & 0.0 & 0.0 & 0.0 & 0.0 & 0.0 & 0.0 & $0.0 \quad 0.0$ \\
\hline & $M G^{\circ}$ & 0.0 & 0.0 & 0.0 & 0.0 & 0.0 & 0.0 & 0.0 & 0.0 & 0.0 & 0.0 & 0.0 & 0.0 & 0.0 & 0.0 & 0.0 & $0.0 \quad 0.0$ \\
\hline & $B$ & 0.0 & 0.0 & 0.0 & 0.0 & 0.0 & 0.0 & 0.0 & 0.0 & 0.0 & 0.0 & 0.0 & 0.0 & 0.0 & 0.0 & 0.0 & $0.0 \quad 0.0$ \\
\hline & $B D G$ & 0.0 & 0.0 & 0.0 & 0.0 & 0.0 & 0.0 & 0.0 & 0.0 & 0.0 & 0.0 & 0.0 & 0.0 & 0.0 & 0.0 & 0.0 & $0.0 \quad 0.0$ \\
\hline & BG & 0.0 & 0.0 & 0.0 & 0.0 & 0.0 & 0.0 & 0.0 & 0.0 & 0.0 & 0.0 & 0.0 & 0.0 & 0.0 & 0.0 & 0.0 & $0.0 \quad 0.0$ \\
\hline & 0 & 0.0 & 0.0 & 0.0 & 0.0 & 0.0 & 0.0 & 0.0 & 0.0 & 0.0 & 0.0 & 0.0 & 0.0 & 0.0 & 0.0 & 0.0 & 0.0 \\
\hline & $D G$ & 0.0 & 0.0 & 0.0 & 0.0 & 0.0 & 0.0 & 0.0 & 0.0 & 0.0 & 0.0 & 0.0 & 0.0 & 0.0 & 0.0 & 0.0 & 0.1 \\
\hline & EG & 0.0 & 0.0 & 0.0 & 0.0 & 0.0 & 0.0 & 0.0 & 0.0 & 0.0 & 0.0 & 0.0 & 0.0 & 0.0 & 0.0 & 0.0 & 0.0 \\
\hline & G & 0.0 & 0.0 & 0.0 & 0.0 & 0.0 & 0.0 & 0.0 & 0.0 & 0.0 & 0.0 & 0.0 & 0.0 & 0.0 & 0.0 & 0.0 & $0.1 \cdot 0.0$ \\
\hline - & GJ & 0.0 & 0.0 & 0.0 & 0.0 & 0.0 & 0.0 & 0.0 & .0 .0 & 0.0 & 0.0 & 0.0 & 0.0 & 0.0 & 0.0 & 0.0 & $0.0 \quad 0.0$ \\
\hline & MONE & 125.4 & 125.4 & 125.4 & 125.4 & 125.4 & 125.4 & 225.4 & 125.1 & 125.4 & 125.1 & 125.4 & 225.4 & 125.4 & 125.4 & 125.4 & $3865.9 \quad 99.9$ \\
\hline Tota1D-METAL & & 125.1 & 125.4 & 125.4 & 125.4 & 125.4 & 125.1 & 125.1 & 125.4 & 125.4 & 125.4 & 125.4 & 125.4 & 125.4 & 225.4 & 125.4 & 3867.2100 .0 \\
\hline IN ORG LR/SLG & $A B G$ & 0.0 & 0.0 & 0.0 & 0.0 & 0.0 & 0.0 & 0.0 & 0.0 & 0.0 & 0.0 & 0.0 & 0.0 & 0.0 & 0.0 & 0.0 & $0.0 \quad 1.0$ \\
\hline & A5G & 0.0 & 0.0 & 0.0 & 0.0 & 0.0 & 0.0 & 0.0 & 0.0 & 0.0 & 0.0 & 0.0 & 0.0 & 0.0 & 0.0 & 0.0 & 0.0 \\
\hline & $A G$ & 0.0 & 0.0 & 0.0 & 0.0 & 0.0 & 0.0 & 0.0 & 0.0 & 0.0 & 0.0 & 0.0 & 0.0 & 0.0 & 0.0 & 0.0 & 0.1 \\
\hline & $D$ & 0.0 & 0.0 & 0.0 & 0.0 & 0.0 & 0.0 & 0.0 & 0.0 & 0.0 & 0.0 & 0.0 & 0.0 & 0.0 & 0.0 & 0.0 & $0.2 \quad 7.0$ \\
\hline & $\mathrm{DE}$ & 0.0 & 0.0 & 0.0 & 0.0 & 0.0 & 0.0 & 0.0 & 0.0 & 0.0 & 0.0 & 0.0 & 0.0 & 0.0 & 0.0 & 0.0 & 0.311 .0 \\
\hline & DFG & 0.0 & 0.0 & 0.0 & 0.0 & 0.0 & 0.0 & 0.0 & 0.0 & $0: 0$ & 0.0 & 0.0 & 0.0 & 0.0 & 0.0 & 0.0 & $0.1 \quad 4.9$ \\
\hline & $D G$ & 0.0 & 0.0 & 0.0 & 0.0 & 0.0 & 0.0 & 0.0 & 0.0 & 0.0 & 0.0 & 0.0 & 0.0 & 0.0 & 0.0 & 0.0 & $0.9 \quad 27.9$ \\
\hline & $\varepsilon$ & 0.0 & 0.0 & 0.0 & 0.0 & 0.0 & 0.0 & 0.0 & 0.0 & 0.0 & 0.0 & 0.0 & 0.0 & 0.0 & 0.0 & 0.0 & $1.0 \quad 32.0$ \\
\hline & EG & 0.0 & 0.0 & 0.0 & 0.0 & 0.0 & 0.0 & 0.0 & 0.0 & 0.0 & 0.0 & 0.0 & 0.0 & 0.0 & 0.0 & $0.0^{\circ}$ & $0.1 \quad 3.9$ \\
\hline
\end{tabular}

HCD Coden: A-Ignitables, B-Corrosives, CaReactives, DaMetals w/O Hg, E-Metals w Hg, E-Organics, G-State Reg., H-PCB < 50ppm, J-PCB > 50ppm 


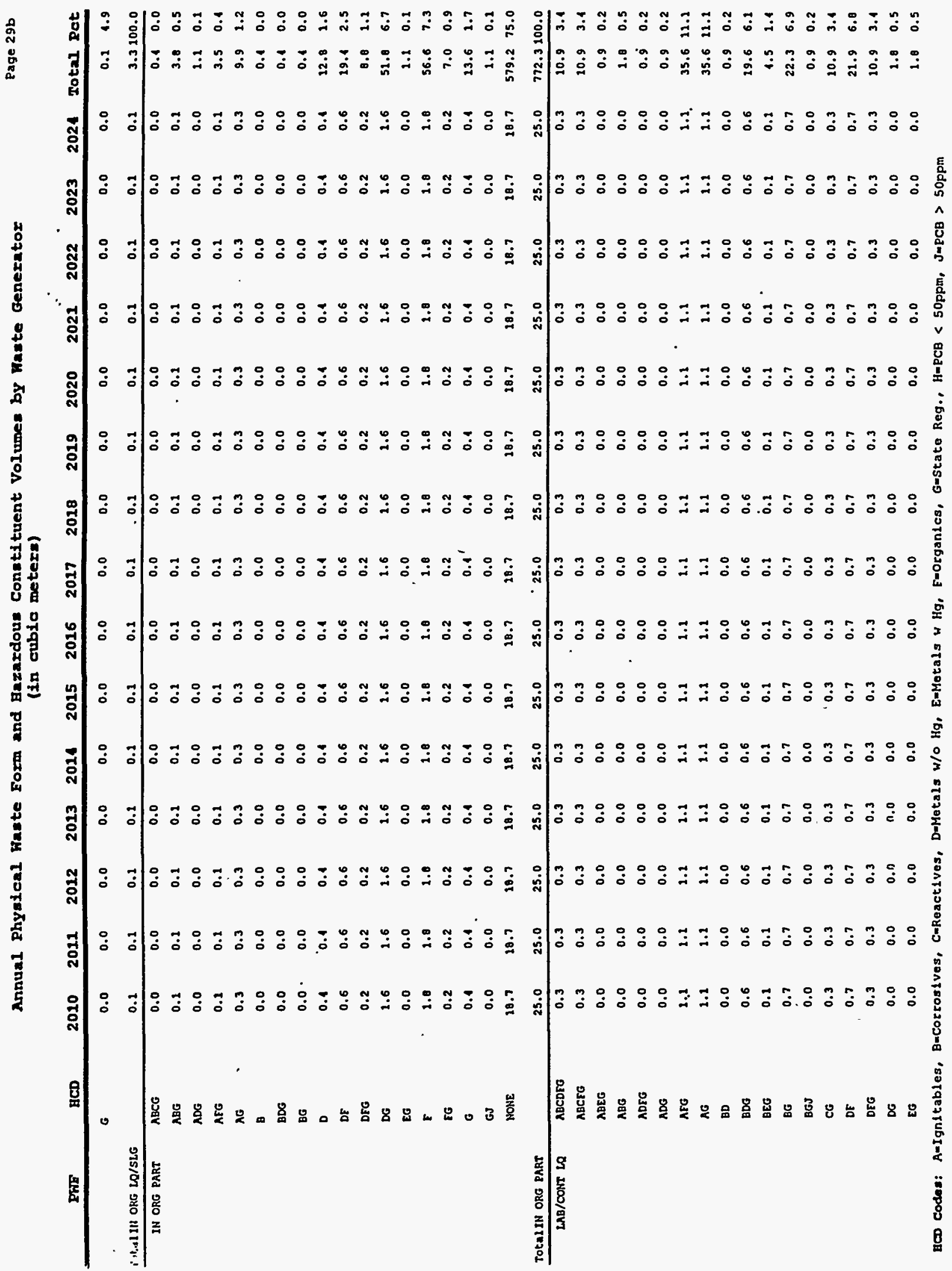



(In cubia meters)

\begin{tabular}{|c|c|c|c|c|c|c|c|c|c|c|c|c|c|c|c|c|c|}
\hline FWE & HCD & 2010 & 2011 & 2012 & 2013 & 2014 & 2015 & 2016 & 2017 & 2018 & 2019 & 2020 & 2021 & 2022 & 2023 & 2024 & Rotal Pot \\
\hline & $\mathbf{F}$ & 1.5 & 3.5 & 1.5 & 1.5 & 1.5 & 1.5 & 1.5 & 1.5 & 1.5 & 1.5 & 1.5 & 1.5 & 1.5 & 1.5 & .2 .5 & $16.6 \quad 14.5$ \\
\hline & EG & 0.7 & 0.7 & 0.7 & 0.7 & 0.7 & 0.7 & 0.7 & 0.7 & 0.7 & 0.7 & 0.7 & 0.7 & 0.1 & .0 .7 & 0.7 & $22.8 \quad 7.1$ \\
\hline & $\sigma$ & 1.8 & 1.8 & 1.8 & 1.8 & 1.8 & 1.8 & 1.8 & 1.8 & 1.8 & 1.8 & 1.0 & 1.8 & 1.8 & 1.8 & 1.0 & $56.6 \quad 17.7$ \\
\hline \multirow[t]{12}{*}{ TOLaILAB/CONT LQ } & & 10.3 & 20.3 & 10.3 & 10.3 & 20.3 & 20.3 & 10.3 & 10.3 & 10.3 & 10.3 & 10.3 & 10.3 & 10.3 & 10.3 & 10.3 & 319.8100 .0 \\
\hline & ABG & 0.0 & 0.0 & 0.0 & 0.0 & 0.0 & 0.0 & 0.0 & 0.0 & 0.0 & 0.0 & 0.0 & 0.0 & 0.0 & 0.0 & 0.0 & 0.1 \\
\hline & AFG & 0.0 & 0.0 & 0.0 & 0.0 & 0.0 & 0.0 & 0.0 & 0.0 & 0.0 & 0.0 & 0.0 & 0.0 & 0.0 & 0.0 & 0.0 & 0.2 \\
\hline & MG & 0.0 & 0.0 & 0.0 & 0.0 & 0.0 & 0.0 & 0.0 & 0.0 & 0.0 & 0.0 & 0.0 & 0.0 & 0.0 & 0.0 & 0.0 & 0.6 \\
\hline & D & 0.0 & 0.0 & 0.0 & 0.0 & 0.0 & 0.0 & 0.0 & 0.0 & 0.0 & 0.0 & 0.0 & 0.0 & 0.0 & 0.0 & 0.0 & 0.9 \\
\hline & DE & 0.0 & 0.0 & 0.0 & 0.0 & 0.0 & 0.0 & 0.0 & 0.0 & 0.0 & 0.0 & 0.0 & 0.0 & 0.0 & 0.0 & 0.0 & 1.5 \\
\hline & DEG & 0.0 & $0: 0$ & 0.0 & 0.0 & 0.0 & 0.0 & 0.0 & 0.0 & 0.0 & 0.0 & 0.0 & 0.0 & 0.0 & 0.0 & 0.0 & $0.6 \quad 2.2$ \\
\hline & DG & 0.1 & 0.1 & 0.1 & 0.1 & 0.1 & 0.1 & 0.1 & 0.1 & 0.1 & 0.1 & 0.1 & 0.1 & 0.1 & 0.1 & 0.1 & $3.8 \quad 12.1$ \\
\hline & $\mathbf{F}$ & 0.1 & 0.1 & 0.1 & 0.1 & 0.1 & 0.1 & 0.1 & 0.1 & 0.1 & 0.1 & 0.1 & 0.1 & 0.1 & 0.2 & 0.1 & $4.3 \quad 14.1$ \\
\hline & FG & 0.0 & 0.0 & 0.0 & 0.0 & 0.0 & 0.0 & .0 .0 & 0.0. & 0.0 & 0.0 & 0.0 & 0.0 & 0.0 & 0.0 & 0.0 & $\begin{array}{ll}0.5 & 1.7\end{array}$ \\
\hline & 0 & 0.0 & 0.0 & 0.0 & 0.0 & 0.0 & 0.0 & 0.0 & 0.0 & 0.0 & 0.0 & 0.0 & 0.0 & 0.0 & 0.0 & 0.0 & $0.6 \quad 2.2$ \\
\hline & HONE. & 0.5 & 0.5 & 0.5 & 0.5 & 0.5 & 0.5 & 0.5 & 0.5 & 0.5 & 0.5 & 0.5 & 0.5 & 0.5 & 0.5 & 0.5 & $17.1 \quad 55.6$ \\
\hline TOtalORG ABS LQ/SLG & & 1.0 & 1.0 & 1.0 & 1.0 & 1.0 & 1.0 & 1.0 & 1.0 & 1.0 & 1.0 & 1.0 & 1.0 & 1.0 & 1.0 & 1.0 & 30.0100 .0 \\
\hline \multirow[t]{10}{*}{ SH LEND } & $A B G$ & 0.0 & 0.0 & 0.0 & 0.0 & 0.0 & 0.0 & 0.0 & 0.0 & 0.0 & 0.0 & 0.0 & 0.0 & 0.0 & 0.0 & 0.0 & $0.9 \quad 2.0$ \\
\hline & AFG & 0.0 & 0.0 & 0.0 & 0.0 & 0.0 & 0.0 & 0.0 & 0.0 & 0.0 & 0.0 & 0.0 & 0.0 & 0.0 & 0.0 & 0.0 & 1.92 .0 \\
\hline & $\mathbf{A G}$ & 0.1 & 0.1 & 0.2 & 0.1 & 0.1 & 0.1 & 0.1 & 0.1 & 0.1 & 0.1 & 0.1 & 0.1 & 0.1 & 0.1 & 0.1 & 4.9 \\
\hline & D. & 0.2 & 0.2 & 0.2 & 0.2 & 0.2 & 0.2 & 0.2 & 0.2 & 0.2 & 0.2 & 0.2 & 0.2 & 0.2 & 0.2 & 0.2 & 6.97 .0 \\
\hline & DE & 0.3 & 0.3 & 0.3 & 0.3 & 0.3 & 0.3 & 0.3 & 0.3 & 0.3 & 0.3 & 0.3 & 0.3 & 0.3 & 0.3 & 0.3 & $10.9 \cdot 11.0$ \\
\hline & DEG & 0.1 & 0.1 & 0.2 & 0.1 & 0.1 & 0.1 & 0.1 & 0.1 & 0.1 & 0.1 & 0.1 & 0.1 & 0.2 & 0.1 & 0.1 & 4.95 .0 \\
\hline & $D G$ & 0.9 & 0.9 & $0.9^{\circ}$ & 0.9 & 0.9 & 0.9 & 0.9 & 0.9 & 0.9 & 0.9 & 0.9 & 0.9 & 0.9 & 0.9 & 0.9 & 27.928 .0 \\
\hline & $\mathbf{E}$ & 1.0 & 1.0 & 1.0 & 1.0 & 2.0 & 2.0 & 1.0 & 1.0 & 1.0 & 1.0 & 1.0 & 1.0 & 1.0 & 1.0 & 1.0 & 31.932 .0 \\
\hline & FG & 0.1 & 0.1 & 0.1 & 0.1 & 0.1 & 0.1 & 0.1 & 0.1 & 0.1 & 0.1 & 0.1 & 0.1 & 0.1 & 0.1 & 0.1 & $3.9 \quad 4.0$ \\
\hline & $\sigma$ & 0.1 & 0.1 & 0.1 & 0.1 & 0.1 & 0.1 & 0.1 & 0.1 & 0.1 & 0.1 & 0.1 & 0.1 & 0.1 & 0.1 & 0.1 & 4.9 .5 .0 \\
\hline TOta1SH LEAD & & 3.2 & 3.2 & 3.2 & 3.2 & 3.2 & 3.2 & 3.2 & 3.2 & 3.2 & 3.2 & 3.2 & 3.2 & 3.2 & 3.2 & 3.2 & 99.7100 .0 \\
\hline TOtaLLAC_NAALYT_LAB & & 365.0 & 365.0 & 365.0 & 365.0 & 365.0 & 365.0 & 365.0 & 365.0 & 365.0 & 365.0 & 365.0 & 365.0 & 365.0 & 365.0 & 365.0 & 11248.6 \\
\hline \multicolumn{18}{|c|}{ Wasto Gonorator:FHC_BQUIP } \\
\hline \multirow[t]{5}{*}{ D-METAL } & $\boldsymbol{A}$ & 0.0 & 0.0 & 0.0 & 0.0 & 0.0 & 0.0 & 0.1 & 0.0 & 0.0 & 0.0 & 0.0 & 0.1 & 0.0 & 0.0 & 0.0 & 2.20 .0 \\
\hline & B & 0.4 & 0.4 & 0.5 & 0.5 & 0.6 & 0.7 & 0.0 & 0.4 & 0.1 & 0.5 & 0.7 & 0.8 & 0.1 & 0.0 & 0.0 & 18.5 \\
\hline & D & 26.6 & 26.6 & 35.5 & 35.5 & 39.9 & 14.3 & 53.2 & 31.0 & 31.0 & 35.5 & 11.3 & 53.2 & 26.6 & 0.0 & 0.0 & $1160.0 \quad 15.6$ \\
\hline & $\mathrm{F}$ & 312.8 & 212.8 & 190.3 & 190.3 & 214.2 & 238.0 & 285.5 & 166.5 & 166.5 & 290.3 & 238.0 & 205.5 & $112 . \theta$ & 0.0 & 0.0 & 6219.084 .0 \\
\hline & G & 0.0 & 0.0 & 0.0 & 0.0 & 0.0 & 0.0 & 0.1 & 0.0 & 0.0 & 0.0 & 0.0 & 0.1 & 0.0 & 0.0 & 0.0 & $2.2 \quad 0.0$ \\
\hline Tota1D-METAL & & 169.9 & 169.9 & 226.5 & 226.5 & 254.9 & 283.2 & 339.8 & 198.2 & 198.2 & 226.5 & $2 \theta 3.2$ & 339.8 & 169.9 & 0.0 & 0.0 & 7401.9100 .0 \\
\hline Tota IWAC_BQUIP & & 169.9 & 169.9 & 226.5 & 226.5 & 254.9 & 283.2 & 339.8 & 198.2 & 198.2 & 226.5 & 283.2 & 339.8 & 169.9 & 0.0 & 0.0 & 7101.9 \\
\hline
\end{tabular}

BCo codes: A-Ignitables, B-Corroslves, C=Reactives, D=Metals w/O Hg, E-Metals w Hg, F-Organics, G=State Reg., H=PCB < 50ppm, J-PCB > 50ppm 

(In cublo meters)

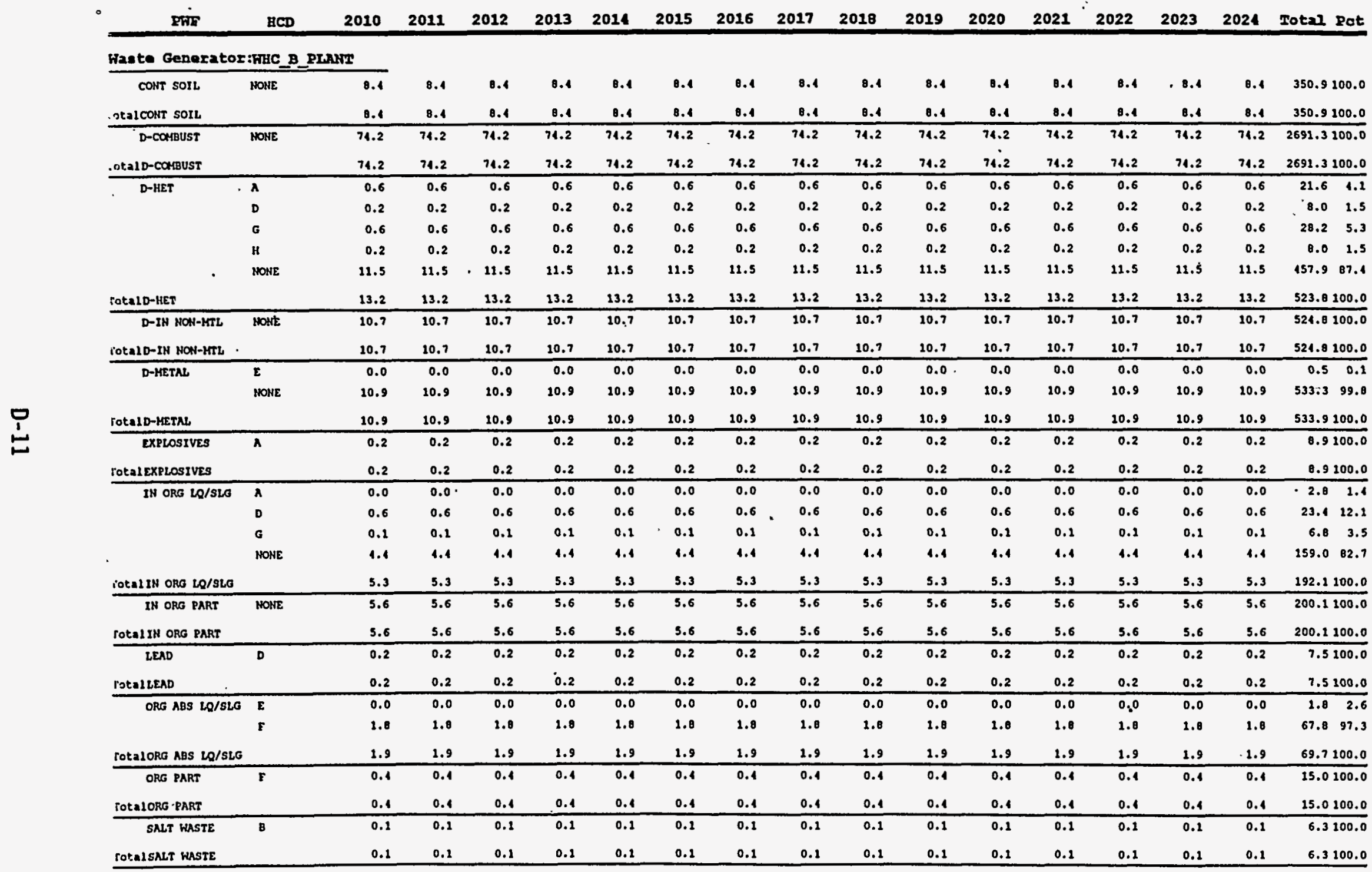

yCD Codes: A-Ignitables, B-Corrosives, C-Reactlves, D-Metals W/O Hg, E-Metals w Hg, F-Organles, G-State Reg., H-PCB < 50ppm, J-PCB > 50ppm 

(In cubic meters)

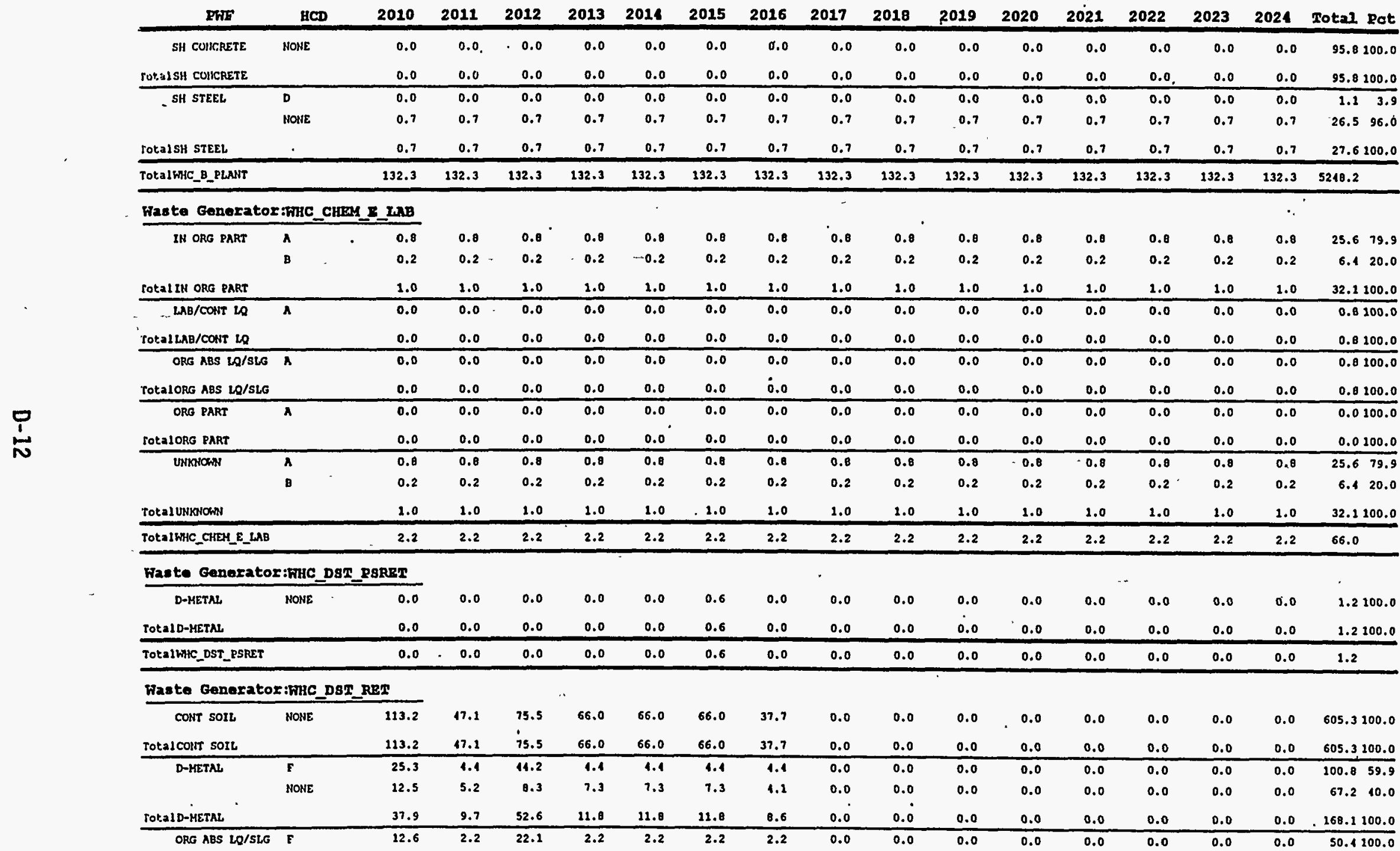



(in cubic meters)

\begin{tabular}{|c|c|c|c|c|c|c|c|c|c|c|c|c|c|c|c|c|c|}
\hline EFE & HCD & 2010 & 2011 & 2012 & 2013 & 2014 & 2015 & 2016 & 2017 & 2018 & - 2019 & 2020 & 2021 & 2022 & 2023 & 2021 & Total Pot \\
\hline IDt.alsh voID & & 12.6 & 2.2 & 22.1 & 2.2 & 2.2 & 2.2 & 2.2 & 0.0 & 0.0 & 0.0 & 0.0 & 0.0 & 0.0 & 0.0 & 0.0 & 50.4100 .0 \\
\hline SH VOID & UNKAOCWNA & 215.3 & 37.9 & 376.0 & 37.9 & 37.9 & 37.9 & 38.0 & 0.0 & 0.0 & 0.0 & 0.0 & 0.0 & 0.0 & 0.0 & 0.0 & 857.3100 .0 \\
\hline rot:a1SH voID & & 215.3 & 37.9 & 376.0 & 37.9 & 37.9 & 37.9 & 38.0 & 0.0 & 0.0 & 0.0 & 0.0 & 0.0 & 0.0 & 0.0 & 0.0 & 857.3100 .0 \\
\hline TOtalWHA_DST_RET & & 379.1 & 97.1 & 526.3 & 118.1 & 118.1 & 118.1 & 86.7 & 0.0 & 0.0 & 0.0 & 0.0 & 0.0 & 0.0 & 0.0 & 0.0 & 1681.3 \\
\hline \multicolumn{18}{|c|}{ Waste Generator:kHC_EN_F112 } \\
\hline \multirow[t]{2}{*}{ CONT SOIL } & $\bar{E}$ & 0.0 & 0.0 & 0.0 & 0.0 & 0.0 & 0.0 & 0.0 & 0.0 & 0.0 & 0.0 & 0.0 & 0.0 & 0.0 & 0.0 & 0.0 & $0.7 \quad 2.0$ \\
\hline & NONE & 0.0 & 0.0 & 0.0 & 0.0 & 0.0 & 0.0 & 0.0 & 0.0 & 0.0 & 0.0 & 0.0 & 0.0 & 0.0 & 0.0 & 0.0 & $35.5 \quad 97.9$ \\
\hline rotalcont sort & & 0.0 & 0.0 & 0.0 & 0.0 & 0.0 & 0.0 & 0.0 & 0.0 & 0.0 & 0.0 & 0.0 & 0.0 & 0.0 & 0.0 & 0.0 & 36.2100 .0 \\
\hline D-COABUST & 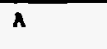 & 0.0 & 0.0 & 0.0 & 0.0 & 0.0 & 0.0 & 0.0 & 0.0 & 0.0 & 0.0 & 0.0 & 0.0 & 0.0 & 0.0 & 0.0 & 0.2100 .0 \\
\hline Tot21D-COMBUST & & 0.0 & 0.0 & 0.0 & 0.0 & 0.0 & 0.0 & 0.0 & 0.0 & 0.0 & 0.0 & 0.0 & 0.0 & 0.0 & 0.0 & 0.0 & 0.2100 .0 \\
\hline \multirow[t]{2}{*}{ D-sort } & 5 & 0.0 & 0.0 & 0.0 & 0.0 & 0.0 & 0.0 & 0.0 & 0.0 & 0.0 & 0.0 & 0.0 & 0.0 & 0.0 & 0.0 & 0.0 & $0.0 \quad 2.0$ \\
\hline & NONE & 0.0 & 0.0 & 0.0 & 0.0 & 0.0 & 0.0 & 0.0 & 0.0 & 0.0 & 0.0 & 0.0 & 0.0 & $0.0^{\circ}$ & 0.0 & 0.0 & 2.797 .9 \\
\hline TotalD-SOIL & & 0.0 & 0.0 & 0.0 & 0.0 & 0.0 & 0.0 & 0.0 & 0.0 & 0.0 & 0.0 & 0.0 & 0.0 & 0.0 & 0.0 & 0.0 & 2.8100 .0 \\
\hline UNKNOAN & $\mathbf{F}$ & 0.0 & 0.0 & 0.0 & 0.0 & 0.0 & 0.0 & 0.0 & 0.0 & 0.0 & 0.0 & 0.0 & 0.0 & 0.0 & 0.0 & 0.0 & 0.3100 .0 \\
\hline Total UNKn+ChN & & 0.0 & 0.0 & 0.0 & 0.0 & 0.0 & 0.0 & 0.0 & 0.0 & 0.0 & 0.0 & 0.0 & 0.0 & 0.0 & 0.0 & 0.0 & 0.3100 .0 \\
\hline Tota 1WHC_ER_W112 & & 0.0 & 0.0 & 0.0 & 0.0 & 0.0 & 0.0 & 0.0 & 0.0 & 0.0 & 0.0 & 0.0 & 0.0 & 0.0 & 0.0 & 0.0 & 39.7 \\
\hline \multicolumn{18}{|c|}{ Fanto Generator:FIRC_RirC_UIIL } \\
\hline \multirow[t]{3}{*}{ BATTERIES } & B & 0.5 & 0.5 & 0.5 & 0.5 & 0.5 & 0.5 & 0.5 & 0.5 & 0.5 & 0.5 & 0.5 & 0.5 & 0.3 & 0.5 & 0.5 & $17.5 \quad 37.5$ \\
\hline & $\mathrm{BE}$ & 0.1 & 0.1 & 0.1 & 0.1 & 0.1 & 0.1 & 0.1 & 0.1 & 0.1 & 0.1 & 0.1 & 0.1 & 0.1 & 0.1 & 0.1 & $\begin{array}{lll}5.6 & 12.5\end{array}$ \\
\hline & $\varepsilon$ & 0.7 & 0.7 & 0.7 & 0.7 & 0.7 & 0.7 & 0.7 & 0.7 & 0.7 & 0.7 & 0.7 & 0.7 & 0.7 & 0.7 & 0.7 & 23.450 .0 \\
\hline TOt=1BATTERIES & & 1.5 & 1.5 & 1.5 & 1.5 & 1.5 & 1.5 & 1.5 & 1.5 & 1.5 & 1.5 & 1.5 & 1.5 & 1.5 & 1.5 & 1.5 & 16.0100 .0 \\
\hline \multirow[t]{2}{*}{ D-METAL } & $\lambda$ & 0.3 & 0.3 & 0.3 & 0.3 & 0.3 & 0.3 & 0.3 & 0.3 & 0.3 & 0.3 & 0.3 & 0.3 & 0.3 & 0.3 & 0.3 & 11.775 .0 \\
\hline & D & 0.1 & 0.1 & 0.1 & 0.1 & 0.1 & 0.1 & 0.1 & 0.1 & 0.1 & 0.1 & 0.1 & 0.2 & 0.1 & 0.1 & 0.1 & 3.925 .0 \\
\hline Tota1D-METAL & & 0.5 & 0.5 & 0.5 & 0.5 & 0.5 & 0.5 & 0.5 & 0.5 & 0.5 & 0.5 & 0.3 & 0.5 & 0.5 & 0.5 & 0.5 & 15.6100 .0 \\
\hline \multirow[t]{4}{*}{ LAB/CONT LQ } & $\AA$ & 0.3 & 0.3 & 0.3 & 0.3 & 0.3 & 0.3 & 0.3 & 0.3 & 0.3 & 0.3 & 0.3 & 0.3 & 0.3 & 0.3 & 0.3 & 11.725 .0 \\
\hline & B & 0.3 & 0.3 & 0.3 & 0.3 & 0.3 & 0.3 & 0.3 & 0.3 & 0,3 & 0.3 & 0.3 & 0.3 & 0.3 & 0.3 & 0.3 & 11.725 .0 \\
\hline & c & 0.3 & 0.3 & 0.3 & 0.3 & 0.3 & 0.3 & 0.3 & 0.3 & 0.3 & 0.3 & 0.3 & 0.3 & 0.3 & 0.3 & 0.3 & $11.7 \quad 25.0$ \\
\hline & $\varepsilon$ & 0.3 & 0.3 & 0.3 & 0.3 & 0.3 & 0.3 & 0.3 & 0.3 & 0.3 & 0.3 & 0.3 & 0.3 & 0.3 & 0.3 & 0.3 & $11.7 \quad 25.0$ \\
\hline TOta1 LAB/CONT LQ & & 1.5 & 1.5 & 1.5 & 1.5 & 1.5 & 1.5 & 1.5 & 2.5 & 1.5 & 2.5 & 3.5 & 2.5 & 1.5 & 1.5 & 1.5 & 16.0100 .0 \\
\hline IEAD & $\bar{E}$ & 0.5 & 0.5 & 0.5 & 0.5 & 0.5 & 0.5 & 0.3 & 0.5 & 0.5 & 0.3 & 0.5 & 0.5 & 0.5 & 0.5 & 0.5 & 25.6100 .0 \\
\hline TOtaI LEAD & & 0.5 & 0.5 & 0.5 & 0.5 & 0.5 & 0.5 & 0.5 & 0.5 & 0.5 & 0.5 & 0.5 & 0.5 & 0.5 & 0.5 & 0.5 & 15.6100 .0 \\
\hline MERCURY & D & 0.5 & 0.5 & 0.5 & 0.5 & 0.5 & 0.5 & 0.5 & 0.5 & 0.5 & 0.5 & 0.5 & 0.5 & 0.5 & 0.5 & 0.5 & 15.6100 .0 \\
\hline
\end{tabular}

HOD Codes: A-Ignitables, BwCortosives, C-Reactives, D-Metals w/O Hg, E-Metals w Hg, E-Organics, GaState Reg. H-PCB < 50ppm, J=PCB > 50ppm 
(in cubia meters)

\begin{tabular}{|c|c|c|c|c|c|c|c|c|c|c|c|c|c|c|c|c|c|}
\hline PWF & HCD & 2010 & 2011 & 2012 & 2013 & 2014 & 2015 & 2016 & 2017 & 2018 & 2019 & 2020 & 2021 & 2022 & 2023 & 2024 & Total Pat \\
\hline rota I MEREURY & & 0.5 & 0.5 & 0.5 & 0.5 & 0.5 & 0.5 & 0.5 & 0.5 & 0.5 & 0.5 & 0.5 & 0.5 & 0.5 & 0.5 & 0.5 & 15.6100 .0 \\
\hline REAC METALS & c & 0.5 & 0.5 & 0.5 & 0.5 & 0.5 & 0.5 & 0.5 & 0.5 & 0.5 & 0.5 & 0.5 & 0.5 & 0.5 & 0.5 & 0.5 & 15.6100 .0 \\
\hline TOtalREAC METALS & & 0.5 & 0.5 & 0.5 & 0.5 & 0.5 & 0.5 & 0.5 & 0.5 & 0.5 & 0.5 & 0.5 & 0.5 & 0.5 & 0.5 & 0.5 & 15.6100 .0 \\
\hline Tota 1 WHE_ELEC_UTIL & & 5.2 & 5.2 & 5.2 & 3.2 & 5.2 & 5.2 & 5.2 & 5.2 & 5.2 & 5.2 & 5.2 & 3.2 & 5.2 & 5.2 & 5.2 & 156.0 \\
\hline \multicolumn{18}{|c|}{ Waste Generatox:FIRC_ENG_DRV } \\
\hline D-combust & AF & 1.5 & 1.5 & 1.5 & 1.3 & 1.5 & 1.5 & 1.5 & 1.5 & 1.5 & 1.5 & 1.5 & 1.5 & 1.5 & 1.5 & 1.5 . & 50.0100 .0 \\
\hline Total D-COHBusT & & 1.5 & 1.5 & 1.5 & 1.5 & 1.5 & 1.5 & 1.5 & 1.5 & 1.5 & 1.5 & 1.5 & 1.5 & 1.5 & 1.5 & 1.5 & 50.0100 .0 \\
\hline \multirow[t]{3}{*}{ LAB/CONT LQ } & 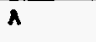 & 0.2 & 0.2 & 0.2 & 0.2 & 0.2 & 0.2 & 0.2 & 0.2 & 0.2 & 0.2 & $0: 2$ & 0.2 & 0.2 & 0.2 & 0.2 & $6.1 \quad 17.7$ \\
\hline & B & 0.7 & 0.7 & 0.7 & 0.7 & 0.7 & 0.7 & 0.7 & 0.7 & 0.7 & 0.7 & 0.7 & 0.7 & 0.7 & 0.7 & 0.7 & 21.763 .0 \\
\hline & $\mathbf{F}$ & 0.2 & 0.2 & 0.2 & 0.2 & 0.2 & 0.2 & 0.2 & 0.2 & 0.2 & 0.2 & 0.2 & 0.2 & 0.2 & 0.2 & 0.2 & 6.619 .2 \\
\hline TOta1 LAB/CONT LQ & & 1.1 & 1.1 & 1.1 & 1.1 & 1.1 & 1.1 & 1.1 & 1.1 & 1.1 & 1.1 & 1.1 & 1.1 & 1.1 & 1.1 & 1.1 & 31.5100 .0 \\
\hline Tota1WHC_ENG_DEV & & 2.7 & 2.7 & 2.7 & 2.7 & 2.7 & 2.7 & 2.7 & 2.7 & 2.7 & 2.7 & 2.7 & 2.7 & 2.7 & 2.7 & 2.7 & 04.6 \\
\hline \multicolumn{18}{|c|}{ Wasta Generator:FHC_mNG_IFBT } \\
\hline \multirow[t]{3}{*}{ IN ORG PART } & A & 0.0 & 0.0 & 0.0 & 0.0 & 0.0 & 0.0 & 0.0 & 0.0 & 0.0 & 0.0 & 0.0 & 0.0 & 0.0 & 0.0 & 0.0 & 2.234 .0 \\
\hline & B & 0.0 & 0.0 & 0.0 & 0.0 & 0.0 & 0.0 & 0.0 & 0.0 & 0.0 & 0.0 & 0.0 & 0.0 & 0.0 & 0.0 & 0.0 & 2.133 .0 \\
\hline & G & 0.0 & 0.0 & 0.0 & 0.0 & 0.0 & 0.0 & 0.0 & 0.0 & 0.0 & 0.0 & 0.0 & 0.0 & 0.0 & 0.0 & 0.0 & $2.1 \quad 33.0$ \\
\hline Tota1 IN ORG EART & & 0.2 & 0.2 & 0.2 & 0.2 & 0.2 & 0.2 & 0.2 & 0.2 & 0.2 & 0.2 & 0.2 & 0.2 & 0.2 & 0.2 & 0.2 & 6.5100 .0 \\
\hline TotalkHC_ENG_IRST & & 0.2 & 0.2 & 0.2 & 0.2 & 0.2 & 0.2 & 0.2 & 0.2 & 0.2 & 0.2 & 0.2 & 0.2 & 0.2 & 0.2 & 0.2 & 6.5 \\
\hline \multicolumn{18}{|c|}{ Wasto Generator:FHC_FRD } \\
\hline D-COMBUST & $\boldsymbol{\varepsilon}$ & 0.0 & 0.0 & 0.0 & 0.0 & 0.0 & 0.0 & 0.0 & 0.0 & 0.0 & 0.0 & 0.0 & 0.0 & 0.0 & 0.0 & 0.0 & 0.1200 .0 \\
\hline Tota1D-CONBust & & 0.0 & 0.0 & 0.0 & 0.0 & 0.0 & 0.0 & 0.0 & 0.0 & 0.0 & 0.0 & 0.0 & 0.0 & 0.0 & 0.0 & 0.0 & 0.4100 .0 \\
\hline \multirow[t]{2}{*}{ IN ORG LQ/SLG } & $\boldsymbol{A}$ & 0.0 & 0.0 & 0.0 & 0.0 & 0.0 & 0.0 & 0.0 & 0.0 & 0.0 & 0.0 & 0.0 & 0.0 & 0.0 & 0.0 & 0.0 & 3.475 .0 \\
\hline & D & 0.0 & 0.0 & 0.0 & 0.0 & 0.0 & 0.0 & 0.0 & 0.0 & 0.0 & 0.0 & 0.0 & 0.0 & 0.0 & 0.0 & 0.0 & 1.124 .9 \\
\hline TotaLIN ORG LQ/SLG & & 0.0 & 0.0 & 0.0 & 0.0 & 0.0 & 0.0 & 0.0 & 0.0 & 0.0 & 0.0 & 0.0 & 0.0 & 0.0 & 0.0 & 0.0 & 1.5100 .0 \\
\hline TotalWHAC_FED & & 0.0 & 0.0 & 0.0 & 0.0 & 0.0 & 0.0 & 0.0 & 0.0 & 0.0 & 0.0 & 0.0 & 0.0 & 0.0 & 0.0 & 0.0 & 5.0 \\
\hline \multicolumn{18}{|c|}{ Waste Generator:WHC_EETE_MRIN } \\
\hline \multirow[t]{4}{*}{ D-COMBUST } & $\mathbf{r}$ & 0.0 & 0.0 & 0.0 & 0.0 & 0.0 & 0.0 & 0.0 & 0.0 & 0.0 & 0.0 & 0.0 & 0.0 & 0.0 & 0.0 & 0.0 & $4.1 \quad 5.7$ \\
\hline & H & 0.0 & 0.0 & 0.0 & 0.0 & 0.0 & 0.0 & 0.0 & 0.0 & 0.0 & 0.0 & 0.0 & 0.0 & 0.0 & 0.0 & 0.0 & $2.0 \quad 2.0$ \\
\hline & J & 0.0 & 0.0 & 0.0 & 0.0 & 0.0 & 0.0 & 0.0 & 0.0 & 0.0 & 0.0 & 0.0 & $0.0^{\circ}$ & 0.0 & 0.0 & 0.0 & $4.1 \quad 5.7$ \\
\hline & NOHE & 0.0 & 0.0 & 0.0 & 0.0 & 0.0 & 0.0 & 0.0 & 0.0 & 0.0 & 0.0 & 0.0 & 0.0 & 0.0 & 0.0 & 0.0 & $61.2 \quad 85.5$ \\
\hline Total D-COHBusT & & 0.0 & 0.0 & 0.0 & 0.0 & 0.0 & 0.0 & 0.0 & 0.0 & 0.0 & 0.0 & 0.0 & 0.0 & 0.0 & 0.0 & 0.0 & 71.6100 .0 \\
\hline
\end{tabular}

HCD Codes: A-Ignitables, B-Corrosives, C=Reactives, D-Metals w/O Hg, E-Metals w Hg, F-Organlcs, G-State Reg., H-PCB < 50ppm, J-PCB > 50ppm 
Annual Phyeical Fante Form and Baiardous Conatituent Volumen by Haste Generator

(In cubio metera)

\begin{tabular}{|c|c|c|c|c|c|c|c|c|c|c|c|c|c|c|c|c|c|}
\hline ENE & HCD & 2010 & 2011 & 2012 & 2013 & 2014 & 2015 & 2016 & 2017 & 2018 & 2019 & 2020 & 2021 & 2022 & 2023 & 2024 & Total Pot \\
\hline D-HET & $\varepsilon$ & 0.0 & 0.0 & 0.0 & 0.0 & 0.0 & 0.0 & 0.0 & 0.0 & 0.0 & 0.0 & 0.0 & 0.0 & 0.0 & 0.0 & 0.0 & 2.0 is.0 \\
\hline & MONE & 0.0 & 0.0 & 0.0 & 0.0 & 0.0 & 0.0 & 0.0 & 0.0 & 0.0 & 0.0 & 0.0 & 0.0 & 0.0 & 0.0 & 0.0 & $11.2 \quad 84.9$ \\
\hline FUta1D-HET & & 0.0 & 0.0 & 0.0 & 0.0 & 0.0 & 0.0 & 0.0 & 0.0 & 0.0 & 0.0 & 0.0 & 0.0 & 0.0 & 0.0 & 0.0 & 13.2100 .0 \\
\hline \multirow[t]{2}{*}{ DAEETAS } & F & 0.0 & 0.0 & 0.0 & 0.0 & 0.0 & 0.0 & 0.0 & 0.0 & 0.0 & 0.0 & 0.0 & 0.0 & 0.0 & 0.0 & 0.0 & $0.0 \quad 2.0$ \\
\hline & MONE & 0.0 & 0.0 & 0.0 & 0.0 & 0.0 & 0.0 & 0.0 & 0.0 & 0.0 & 0.0 & 0.0 & 0.0 & 0.0 & 0.0 & 0.0 & $31.0 \quad 97.1$ \\
\hline COt210-METAL & & 0.0 & 0.0 & 0.0 & 0.0 & 0.0 & 0.0 & 0.0 & 0.0 & 0.0 & 0.0 & 0.0 & 0.0 & 0.0 & 0.0 & 0.0 & 31.9100 .0 \\
\hline LEAD & D & 0.0 & 0.0 & 0.0 & 0.0 & 0.0 & 0.0 & 0.0 & 0.0 & 0.0 & 0.0 & 0.0 & 0.0 & 0.0 & 0.0 & 0.0 & 2.3100 .0 \\
\hline lotalLEAD & & 0.0 & 0.0 & 0.0 & 0.0 & 0.0 & 0.0 & 0.0 & 0.0 & 0.0 & 0.0 & 0.0 & 0.0 & 0.0 & 0.0 & 0.0 & 2.3100 .0 \\
\hline ORG $A B S$ LQ/SLG & J & 0.0 & 0.0 & 0.0 & 0.0 & 0.0 & 0.0 & 0.0 & 0.0 & 0.0 & 0.0 & 0.0 & 0.0 & 0.0 & 0.0 & 0.0 & 1.4100 .0 \\
\hline rotalORG ABS LR/SLG & & 0.0 & 0.0 & 0.0 & 0.0 & 0.0 & 0.0 & 0.0 & 0.0 & 0.0 & 0.0 & 0.0 & 0.0 & 0.0 & 0.0 & 0.0 & 1.1100 .0 \\
\hline REAC METAIS. & c & 0.0 & 0.0 & 0.0 & 0.0 & 0.0 & 0.0 & 0.0 & 0.0 & 0.0 & 0.0 & 0.0 & 0.0 & 0.0 & 0.0 & 0.0 & 2.3100 .0 \\
\hline TOtalREAC METALS & & 0.0 & 0.0 & 0.0 & 0.0 & 0.0 & 0.0 & 0.0 & 0.0 & 0.0 & 0.0 & 0.0 & 0.0 & 0.0 & 0.0 & 0.0 & 2.3100 .0 \\
\hline SH CONCRETE & HONE & 0.0 & 0.0 & 0.0 & 0.0 & 0.0 & 0.0 & 0.0 & 0.0 & 0.0 & 0.0 & 0.0 & 0.0 & 0.0 & 0.0 & 0.0 & 13.4100 .0 \\
\hline PotalsH COHCRETE & & 0.0 & 0.0 & 0.0 & 0.0 & 0.0 & 0.0 & 0.0 & 0.0 & 0.0 & 0.0 & 0.0 & 0.0 & 0.0 & 0.0 & 0.0 & 43.4100 .0 \\
\hline SH STEEL & NONE & 0.0 & 0.0 & 0.0 & 0.0 & 0.0 & 0.0 & 0.0 & 0.0 & 0.0 & 0.0 & 0.0 & 0.0 & 0.0 & 0.0 & 0.0 & 1.4100 .0 \\
\hline rotalsH STEEL & & 0.0 & 0.0 & 0.0 & 0.0 & 0.0 & 0.0 & 0.0 & 0.0 & 0.0 & 0.0 & 0.0 & 0.0 & 0.0 & 0.0 & 0.0 & 1.4100 .0 \\
\hline TOLalWAC_ETTE_MAIN & & 0.0 & 0.0 & 0.0 & 0.0 & 0.0 & 0.0 & 0.0 & 0.0 & 0.0 & 0.0 & 0.0 & 0.0 & 0.0 & 0.0 & 0.0 & 270.0 \\
\hline
\end{tabular}

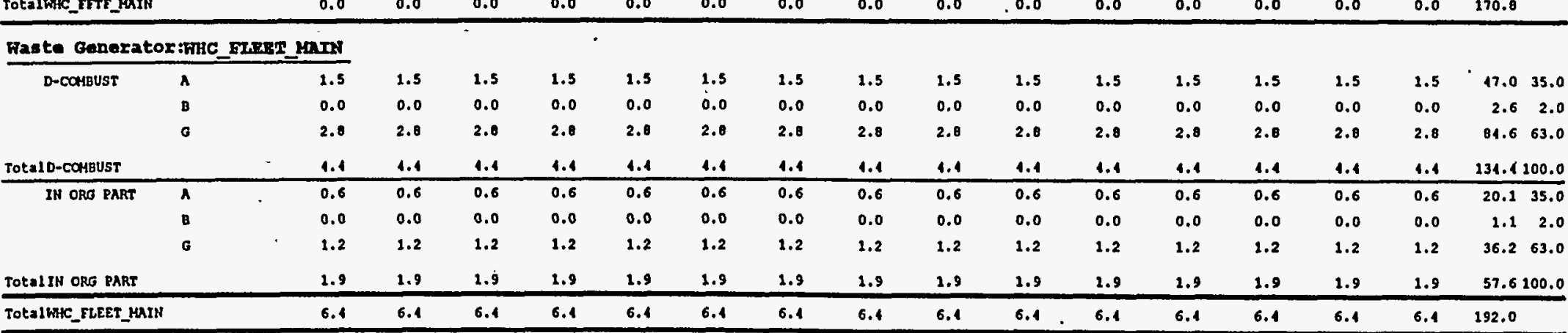

\section{Faste Generator:WHC_RMR}

\begin{tabular}{|c|c|c|c|c|c|c|c|c|c|c|c|c|c|c|c|c|c|}
\hline BATTERIES & BG & 0.1 & 0.1 & 0.1 & 0.1 & 0.1 & 0.1 & 0.1 & 0.2 & 0.2 & 0.1 & 0.1 & 0.1 & 0.1 & 0.1 & 0.1 & 3.9100 .0 \\
\hline otal gATTERIES & & 0.1 & 0.1 & 0.1 & 0.1 & 0.1 & 0.1 & 0.1 & 0.1 & 0.1 & 0.1 & 0.1 & 0.1 & 0.1 & 0.2 & 0.1 & 3.9100 .0 \\
\hline \multirow{3}{*}{ IN ORG LQ/SLG } & $A$ & 0.0 & 0.0 & 0.0 & 0.0 & 0.0 & 0.0 & 0.0 & 0.0 & 0.0 & 0.0 & 0.0 & 0.0 & 0.0 & 0.0 & 0.0 & 1.010 .6 \\
\hline & B & 0.0 & 0.0 & 0.0 & 0.0 & 0.0 & 0.0 & 0.0 & 0.0 & 0.0 & 0.0 & 0.0 & 0.0 & 0.0 & 0.0 & 0.0 & 2.313. \\
\hline & G & 0.1 & 0.1 & 0.1 & 0.4 & 0.1 & 0.4 & 0.4 & 0.1 & 0.1 & 0.4 & 0.4 & 0.4 & 0.4 & 0.4 & 0.4 & $13.3 \quad 76$ \\
\hline
\end{tabular}

gCD Codes: AmIgnitables, B-Corrosives, C-Reactives, D-Metals w/O Hg, Emotals w Hg, Emorganles, GmState Reg., H-PCB < 50ppm, J-PCB > 50ppm 


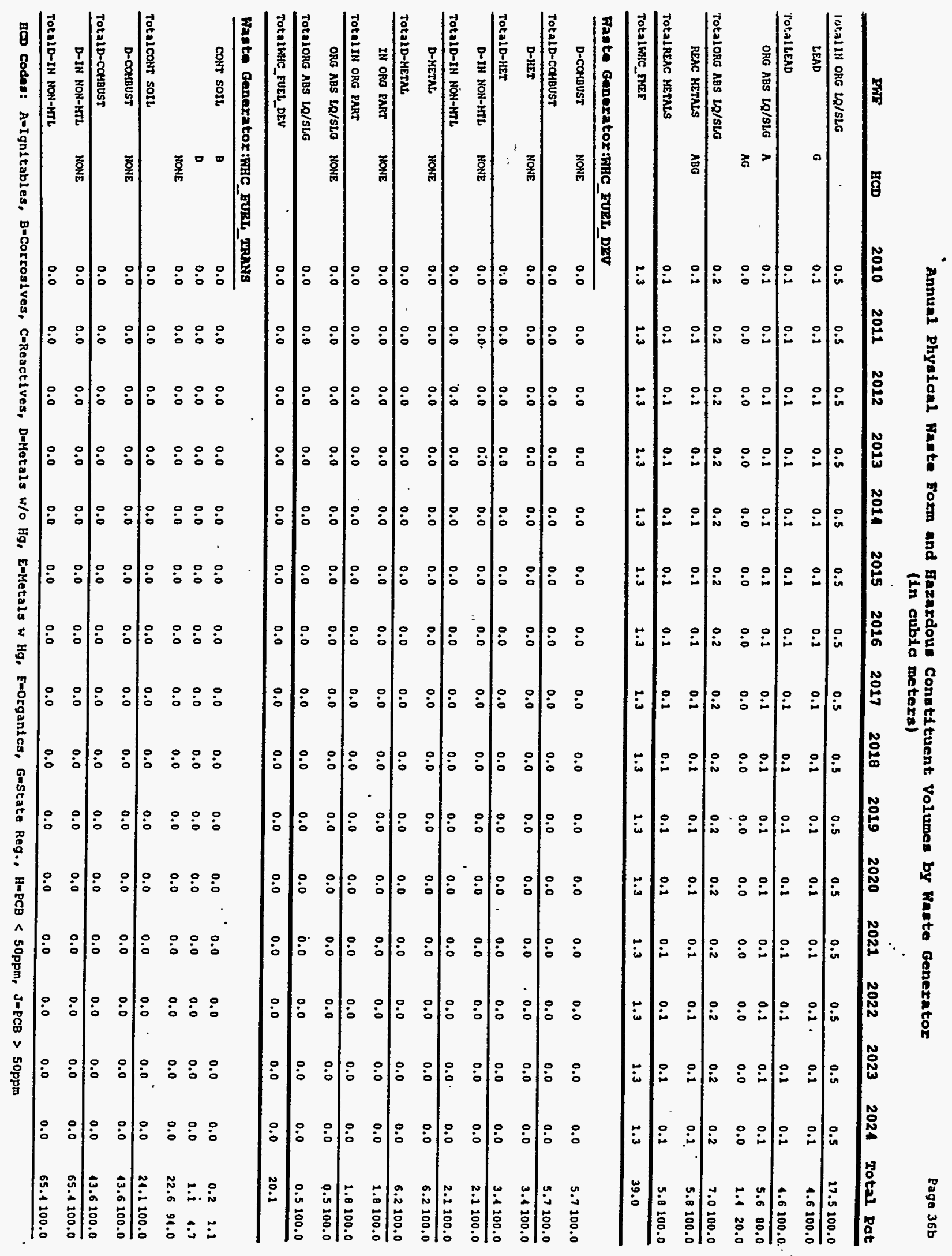


(in cublo meters)

$\begin{array}{lllllllllllllllllll}\text { PFF } & \text { BCD } & 2010 & 2011 & 2012 & 2013 & 2014 & 2015 & 2016 & 2017 & 2018 & 2019 & 2020 & 2021 & 2022 & 2023 & 2024 & \text { Total PCt }\end{array}$

\begin{tabular}{|c|c|c|c|c|c|c|c|c|c|c|c|c|c|c|c|c|c|}
\hline D-METAL & MONE & 0.0 & 0.0 & 0.0 & 0.0 & 0.0 & 0.0 & 0.0 & 0.0 & 0.0 & $0: 0$ & 0.0 & 0.0 & 0.0 & 0.0 & 0.0 & 68.1100 .0 \\
\hline OL:AI D-METAL & & 0.0 & 0.0 & 0.0 & 0.0 & 0.0 & 0.0 & 0.0 & 0.0 & 0.0 & 0.0 & 0.0 & 0.0 & 0.0 & 0.0 & 0.0 & 88.1100 .0 \\
\hline \multirow{3}{*}{ EXPLOSIVES } & $\boldsymbol{A}$ & 0.0 & 0.0 & 0.0 & 0.0 & 0.0 & 0.0 & 0.0 & 0.0 & 0.0 & 0.0 & 0.0 & 0.0 & 0.0 & 0.0 & 0.0 & $4.6 \quad 50.0$ \\
\hline & B & 0.0 & 0.0 & 0.0 & 0.0 & 0.0 & 0.0 & 0.0 & 0.0 & 0.0 & 0.0 & 0.0 & 0.0 & 0.0 & 0.0 & 0.0 & $1.5 \quad 18.4$ \\
\hline & D & 0.0 & 0.0 & 0.0 & 0.0 & 0.0 & 0.0 & 0.0 & 0.0 & 0.0 & 0.0 & 0.0 & 0.0 & 0.0 & 0.0 & 0.0 & 0.121 .5 \\
\hline TOtal EXPLOSIVES & & 0.0 & 0.0 & 0.0 & 0.0 & 0.0 & 0.0 & 0.0 & 0.0 & 0.0 & 0.0 & 0.0 & 0.0 & 0.0 & 0.0 & 0.0 & 9.3200 .0 \\
\hline \multirow[t]{4}{*}{ IN ORG LQ/SLG } & $\mathbf{A}$ & 0.0 & 0.0 & 0.0 & 0.0 & 0.0 & 0.0 & 0.0 & 0.0 & 0.0 & 0.0 & 0.0 & 0.0 & 0.0 & 0.0 & 0.0 & $\begin{array}{ll}1.1 & 9.2\end{array}$ \\
\hline & B & 0.0 & 0.0 & 0.0 & 0.0 & 0.0 & 0.0 & 0.0 & 0.0 & 0.0 & 0.0 & 0.0 & 0.0 & 0.0 & 0.0 & 0.0 & $2.7 \quad 22.1$ \\
\hline & D & 0.0 & 0.0 & 0.0 & 0.0 & 0.0 & 0.0 & 0.0 & 0.0 & 0.0 & 0.0 & 0.0 & 0.0 & 0.0 & 0.0 & 0.0 & $7.3 \quad 59.2$ \\
\hline & J & 0.0 & 0.0 & 0.0 & 0.0 & 0.0 & 0.0 & 0.0 & 0.0 & 0.0 & 0.0 & 0.0 & 0.0 & 0.0 & 0.0 & 0.0 & 1.19 .2 \\
\hline rotal IN ORG LQ/SLG & & 0.0 & 0.0 & 0.0 & 0.0 & 0.0 & 0.0 & 0.0 & 0.0 & 0.0 & 0.0 & 0.0 & 0.0 & 0.0 & 0.0 & 0.0 & 12.3100 .0 \\
\hline rota 1LAC_EUEL_TRANS & & 0.0 & 0.0 & 0.0 & 0.0 & 0.0 & 0.0 & 0.0 & 0.0 & 0.0 & 0.0 & 0.0 & 0.0 & 0.0 & 0.0 & 0.0 & 213.0 \\
\hline
\end{tabular}

Waste Generator:HHC_GNB_NORTB

\begin{tabular}{|c|c|c|c|c|c|c|c|c|c|c|c|c|c|c|c|c|c|}
\hline BATTERIES & B & 1.3 & 1.3 & 1.3 & 1.3 & 1.3 & 1.3 & 1.3 & 1.3 & 1.3 & $1.3^{\circ}$ & 1.3 & 1.3 & 1.3 & 1.3 & 1.3 & $\$ 1.5100 .0$ \\
\hline TOta1 BATTERIES & & 1.3 & 1.3 & 1.3 & 1.3 & 1.3 & 1.3 & 1.3 & 1.3 & 1.3 & 1.3 & 1.3 & 1.3 & 1.3 & 1.3 & 1.3 & 11.5100 .0 \\
\hline D-COABUST & $\lambda 0$ & 3.3 & 3.3 & 3.3 & $\mathbf{3 . 3}$ & 3.3 & 3.3 & 3.3 & 3.3 & 3.3 & 3.3 & 3.3 & 3.3 & 3.3 & 3.3 & 3.3 & 99.9100 .0 \\
\hline fota10-consust & & 3.3 & 3.3 & 3.3 & 3.3 & 3.3 & 3.3 & 3.3 & 3.3 & 3.3 & 3.3 & 3.3 & 3.3 & 3.3 & 3.3 & 3.3 & 99.9100 .0 \\
\hline $\mathrm{D}-\mathrm{HET}$ & H & 1.1 & 1.1 & 1.1 & 1.1 & 1.1 & 1.1 & 1.1 & 1.1 & 1.1 & 1.1 & 1.1 & 1.1 & 1.1 & 1.1 & 1.1 & 34.9100 .0 \\
\hline rotalD-HET & & 1.1 & 2.1 & 1.1 & 1.1 & 1.1 & 1.1 & 1.1 & 1.1 & 1.1 & 1.2 & 1.1 & 1.1 & 1.1 & 1.1 & 1.1 & 34.9100 .0 \\
\hline D-METAL & H & 1.0 & 1.0 & 1.0 & 1.0 & 1.0 & 1.0 & 1.0 & 1.0 & 1.0 & 1.0 & 1.0 & 1.0 & 1.0 & 1.0 & 2.0 & 31.7100 .0 \\
\hline rot=1D-METAL & & 1.0 & 1.0 & 1.0 & 2.0 & 1.0 & 1.0 & 1.0 & 1.0 & 1.0 & 1.0 & 3.0 & 1.0 & 1.0 & 1.0 & 1.0 & 31.7100 .0 \\
\hline LAB/CONT LQ & G & 12.3 & 12.3 & 12.3 & 12.3 & 12.3 & 12.3 & .12 .3 & 12.3 & 12.3 & 12.3 & 12.3 & 12.3 & 12.3 & 12.3 & 12.3 & 371.0200 .0 \\
\hline TOtaLLAB/CONT LR & & 12.3 & 12.3 & 12.3 & 12.3 & 12.3 & 12.3 & 12.3 & 12.3 & 12.3 & 12.3 & 12.3 & 12.3 & 12.3 & 12.3 & 12.3 & 371.0100 .0 \\
\hline ORG ABS LQ/SLG & EG & 0.2 & 0.2 & 0.2 & 0.2 & 0.2 & 0.2 & 0.2 & 0.2 & 0.2 & 0.2 & 0.2 & 0.2 & 0.2 & 0.2 & 0.2 & 0.7100 .0 \\
\hline rotalORG ABS LQ/SLG & & 0.2 & 0.2 & 0.2 & 0.2 & 0.2 & 0.2 & 0.2 & 0.2 & 0.2 & 0.2 & 0.2 & 0.2 & 0.2 & 0.2 & 0.2 & 8.7100 .0 \\
\hline TOtAlWHC_GAS_NORTH & & 19.6 & 19.6 & 19.6 & 19.6 & 19.6 & 19.6 & 19.6 & 19.6 & 19.6 & 19.6 & 19.6 & 19.6 & 19.6 & 19.6 & 19.6 & 500.0 \\
\hline
\end{tabular}

\section{Faste Generator:FHC_Qns_souTh}

\begin{tabular}{|c|c|c|c|c|c|c|c|c|c|c|c|c|c|c|c|c|c|}
\hline BATTERIES & $B D G$ & 0.1 & 0.1 & 0.1 & 0.2 & 0.1 & 0.1 & 0.1 & 0.1 & 0.1 & 0.1 & 0.1 & 0.1 & 0.1 & 0.1 & 0.1 & $3.5 \quad 16.3$ \\
\hline & BEG & 0.6 & 0.6 & 0.6 & 0.6 & 0.6 & 0.6 & 0.6 & 0.6 & 0.6 & 0.6 & 0.6 & 0.6 & 0.6 & $0.6^{\circ}$ & 0.6 & $18.3 \quad 03.6$ \\
\hline rotạl BATTERIES & & 0.7 & 0.7 & 0.7 & 0.7 & 0.7 & 0.7 & 0.7 & 0.7 & 0.7 & 0.7 & 0.7 & 0.7 & 0.7 & 0.7 & 0.7 & 21.0100 .0 \\
\hline D-IN NON-MTL & ABCEG & 1.4 & 1.4 & 1.4 & 1.4 & 1.4 & 1.1 & 1.4 & 1.4 & 1.1 & 1.4 & 1.4 & 1.4 & 1.4 & 2.1 & 1.4 & 13.9100 .0 \\
\hline rotalD-IN NON-HTL & & 1.4 & 3.4 & 1.4 & 1.4 & 1.4 & 2.1 & 3.1 & 2.4 & 2.1 & 3.4 & 1.1 & 1.4 & 1.4 & 1.4 & 1.4 & 13.9100 .0 \\
\hline
\end{tabular}


(In cubio metera)

\begin{tabular}{|c|c|c|c|c|c|c|c|c|c|c|c|c|c|c|c|c|c|}
\hline PWE & HCD & 2010 & 2011 & 2012 & 2013 & 2014 & 2015 & 2016 & 2017 & 2018 & 2019 & 2020 & 2021 & 2022 & 2023 & 2024 & Total Pot \\
\hline D-HETAL & GJ & 1.4 & $1: 4$ & 1.4 & 1.4 & 1.4 & 1.1 & 2.1 & 1.1 & 1.1 & 1.1 & 1.4 & 1.4 & 1.4 & 1.1 & 1.4 & 42.8100 .0 \\
\hline DOT:ALD-METAL & & 1.4 & 1.1 & 1.1 & 1.4 & 1.4 & 1.1 & 1.4 & 1.4 & 1.1 & 1.4 & 1.1 & 1.1 & 1.4 & 1.4 & 1.1 & 12.0100 .0 \\
\hline IN ORG LQ/SLG & AEFG & 1.4 & 1.4 & 1.4 & 1.4 & 1.4 & 1.4 & 1.1 & 1.1 & 1.1 & 1.4 & 1.1 & 1.4 & 2.1 & 1.1 & 1.1 & 13.9100 .0 \\
\hline IUt:Bl1N ORG LQ/SRG & & 1.4 & 1.4 & 1.4 & 1.4 & 1.1 & 2.1 & 1.1 & 1.1 & 1.1 & 1.1 & 1.4 & 1.1 & 1.4 & 1.1 & 1.1 & 43.9100 .0 \\
\hline LAB/CONT LQ & ABDFG & 0.7 & 0.7 & 0.7 & 0.7 & 0.7 & 0.7 & 0.7 & 0.7 & 0.7 & 0.7 & 0.7 & 0.7 & 0.7 & 0.7 & 0.7 & 21.1100 .0 \\
\hline TOr.aILAB/CONT LQ & & 0.7 & 0.7 & 0.7 & 0.7 & 0.7 & 0.7 & 0.7 & 0.7 & 0.7 & 0.7 & $0.7^{\circ}$ & 0.7 & 0.7 & 0.7 & 0.7 & $21 .+100.0$ \\
\hline ORG ABS LQ/SLG & AFG & 0.3 & 0.3 & 0.3 & 0.3 & 0.3 & 0.3 & 0.3 & 0.3 & 0.3 & 0.3 & 0.3 & 0.3 & 0.3 & 0.3 & 0.3 & 9.0100 .0 \\
\hline Pota1ORG ABS LR/SLG & & 0.3 & 0.3 & 0.3 & 0.3 & 0.3 & 0.3 & 0.3 & 0.3 & 0.3 & 0.3 & 0.3 & 0.3 & 0.3 & 0.3 & 0.3 & 9.0100 .0 \\
\hline rotalWhic_GRS_SOUTH & & 6.1 & 6.1 & 6.1 & 6.1 & 6.1 & 6.1 & 6.1 & 6.1 & 6.1 & 6.1 & 6.1 & 6.1 & 6.1 & 6.1 & 6.1 & 183.0 \\
\hline \multicolumn{18}{|c|}{ Waste Generator:FHC_GROTRC_IMB } \\
\hline D-COMBUST & HONE & 0.1 & 0.1 & 0.1 & 0.1 & 0.1 & 0.1 & 0.2 & 0.1 & 0.1 & 0.1 & 0.1 & 0.1 & 0.1 & 0.1 & 0.1 & 6.2100 .0 \\
\hline rotalD-cossaust & & 0.1 & 0.1 & 0.1 & 0.1 & 0.1 & 0.1 & 0.1 & 0.1 & 0.1 & 0.1 & 0.1 & 0.1 & 0.1 & 0.1 & 0.1 & 6.2100 .0 \\
\hline D-HET & NONE & 0.2 & 0.2 & 0.2 & 0.2 & 0.2 & 0.2 & 0.2 & 0.2 & 0.2 & 0.2 & 0.2 & 0.2 & 0.2 & 0.2 & 0.2 & 9.3100 .0 \\
\hline rota2D-HET & & 0.2 & 0.2 & 0.2 & 0.2 & 0.2 & 0.2 & 0.2 & 0.2 & 0.2 & 0.2 & 0.2 & 0.2 & 0.2 & 0.2 & 0.2 & 9.3100 .0 \\
\hline D-METAL & MONE & 0.3 & 0.3 & 0.3 & 0.3 & 0.3 & 0.3 & 0.3 & 0.3 & 0.3 & 0.3 & 0.3 & 0.3 & 0.3 & 0.3 & 0.3 & 15.6100 .0 \\
\hline Tota1D-METAL & & 0.3 & 0.3 & 0.3 & 0.3 & 0.3 & 0.3 & 0.3 & 0.3 & 0.3 & 0.3 & 0.3 & 0.3 & 0.3 & 0.3 & 0.3 & 15.6100 .0 \\
\hline IN ORG LQ/SLG & $B$ & 0.2 & 0.2 & 0.2 & 0.2 & 0.2 & 0.2 & 0.2 & 0.2 & 0.2 & 0.2 & 0.2 & 0.2 & 0.2 & 0.2 & 0.2 & 6.9100 .0 \\
\hline rota1IN ORG LQ/SLG & & 0.2 & 0.2 & 0.2 & 0.2 & 0.2 & 0.2 & 0.2 & 0.2 & 0.2 & 0.2 & 0.2 & 0.2 & 0.2 & 0.2 & 0.2 & 6.9100 .0 \\
\hline \multirow[t]{4}{*}{ LAB/CONT LQ } & $\wedge$ & 0.0 & 0.0 & 0.0 & 0.0 & 0.0 & 0.0 & 0.0 & 0.0 & 0.0 & 0.0 & 0.0 & 0.0 & 0.0 & 0.0 & 0.0 & 2.525 .0 \\
\hline & B & 0.0 & 0.0 & 0.0 & 0.0 & 0.0 & 0.0 & 0.0 & 0.0 & 0.0 & 0.0 & 0.0 & 0.0 & 0.0 & $0.0^{\circ}$ & 0.0 & 2.525 .0 \\
\hline & $\mathbf{F}$ & 0.0 & 0.0 & 0.0 & 0.0 & 0.0 & 0.0 & 0.0 & 0.0 & 0.0 & 0.0 & 0.0 & 0.0 & 0.0 & 0.0 & 0.0 & $2.0 \quad 19.9$ \\
\hline & G & 0.1 & 0.1 & 0.1 & 0.1 & 0.1 & 0.1 & 0.1 & 0.1 & 0.1 & 0.1 & 0.1 & 0.1 & 0.1 & 0.1 & 0.1 & $\begin{array}{lll}3.0 & 29.9\end{array}$ \\
\hline Tota LAB/CONT LQ & & 0.3 & 0.3 & 0.3 & 0.3 & 0.3 & 0.3 & 0.3 & 0.3 & 0.3 & 0.3 & 0.3 & 0.3 & 0.3 & 0.3 & 0.3 & 10.0100 .0 \\
\hline ORG ABS LQ/SLG & $B$ & 0.2 & 0.2 & 0.2 & 0.2 & 0.2 & 0.2 & 0.2 & 0.2 & 0.2 & 0.2 & 0.2 & 0.2 & 0.2 & 0.2 & 0.2 & 6.9100 .0 \\
\hline Tota1 ORG AES LQ/SLG & & 0.2 & 0.2 & 0.2 & 0.2 & 0.2 & 0.2 & 0.2 & 0.2 & 0.2 & 0.2 & 0.2 & 0.2 & 0.2 & 0.2 & 0.2 & 6.9100 .0 \\
\hline TotalkHC_GEOTEC_LAB & & 2.5 & 1.5 & 1.5 & 1.5 & 1.5 & 1.5 & 1.5 & 1.5 & 1.5 & 1.5 & 1.5 & 1.5 & 1.5 & 1.5 & 1.5 & 55.3 \\
\hline Waste Generator & ᄃ:FHC_B & . & & & & & & . & & & & & & & & & \\
\hline \multirow[t]{2}{*}{ BATTERIES } & B & 0.6 & 0.6 & 0.6 & 0.6 & 0.6 & 0.6 & 0.6 & 0.6 & 0.6 & 0.6 & 0.6 & 0.6 & 0.6 & 0.6 & 0.6 & $11.0 \quad 50.0$ \\
\hline & D & 0.6 & 0.6 & 0.6 & 0.6 . & 0.6 & 0.6 & 0.6 & 0.6 & 0.6 & 0.6 & 0.6 & 0.6 & 0.6 & 0.6 & 0.6 & $11.0 \quad 50.0$ \\
\hline TOt.alBATTERIES & & 1.3 & 1.3 & 1.3 & 1.3 & 1.3 & 1.3 & 1.3 & 1.3 & 1.3 & 1.3 & 1.3 & 1.3 & 1.3 & 1.3 & 1.3 & 22.0200 .0 \\
\hline \multirow[t]{2}{*}{ D-COABUST } & 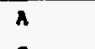 & 23.3 & 23.3 & 23.3 & 23.3 & 23.3 & 23.3 & 23.3 & 23.3 & 23.3 & 23.3 & 23.3 & 23.3 & 23.3 & 23.3 & 23.3 & $373.2 \quad 2.5$ \\
\hline & G & 19.0 & 19.0 & 19.0 & 19.0 & 29.0 & 19.0 & 19.0 & 19.0 & 19.0 & 19.0 & 29.0 & 19.0 & 19.0 & 19.0 & 19.0 & 304.1 \\
\hline
\end{tabular}

HCD Codes: A-Ignitables, BmCorrosives, C-Reactives, D-Metals w/O Hg, E-Matals w Hg, EmorganlCs, G-State Reg., H-PCB < 50ppm, JmPCB > 50ppm 
Annual Physical Faste Form and Hazardous Constituent Volumea by Faste Generator

Pagu 39b

(in cibic meters)

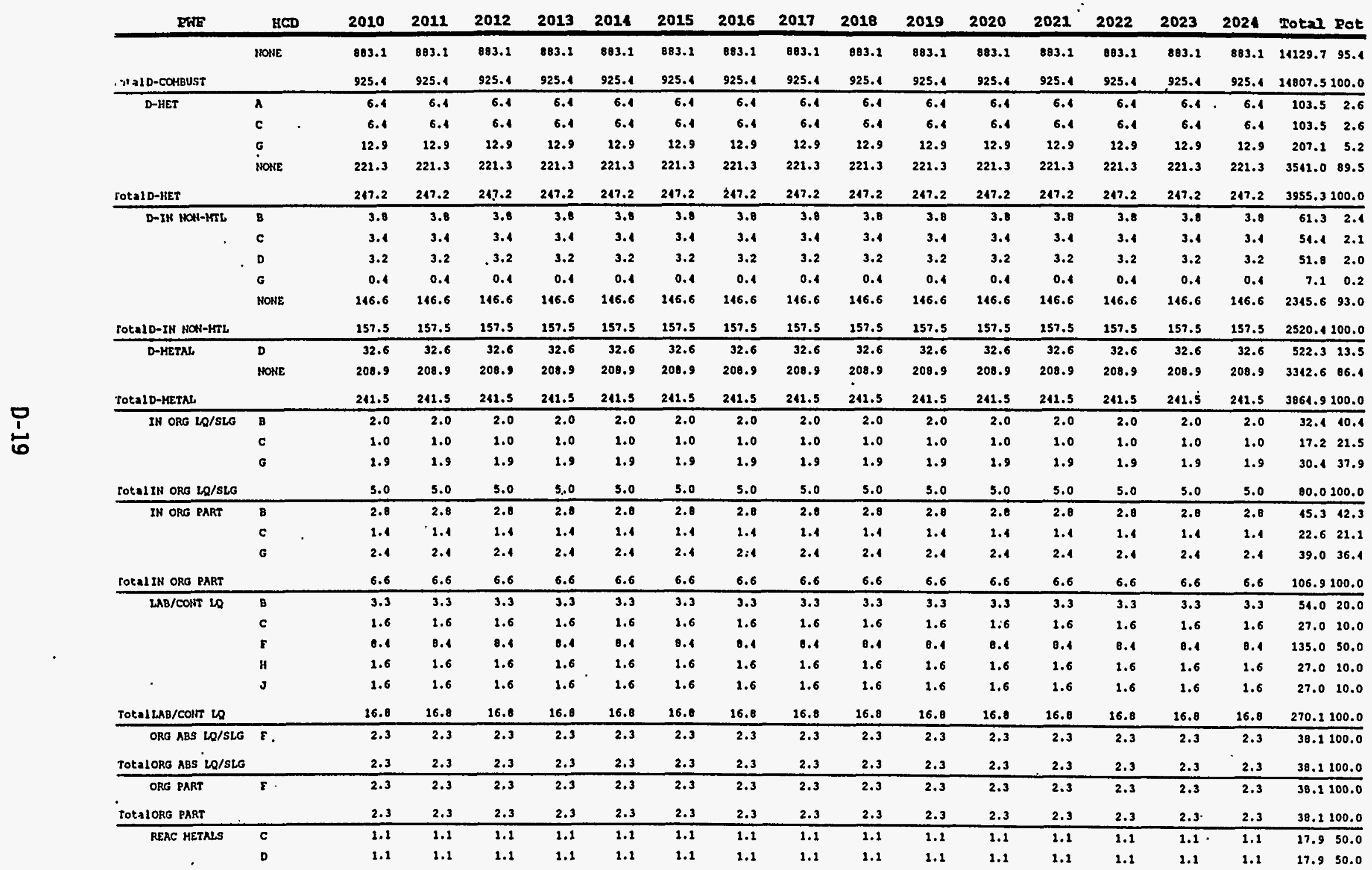

aco Codas: A-Ignitables, B-Corrosives, C-Reactives, D-Motals w/O Hg, Emetals w Hg, F-Organics, G-State Reg., H-PCB < 50ppm, J-PCB > 50ppm 


\begin{tabular}{|c|c|c|c|c|c|c|c|c|c|c|c|c|c|c|c|c|c|}
\hline EWE & HCD & 2010 & 2011 & 2012 & 2013 & 2014 & 2015 & 2016 & 2017 & 2018 & 2019 & 2020 & 2021 & 2022 & 2023 & 2024 & Total Pot \\
\hline It,atREAC METALS & & 2.2 & 2.2 & 2.2 & 2.2 & 2.2 & 2.2 & 2.2 & 2.2 & 2.2 & 2.2 & 2.2 & 2.2 & 2.2 & 2.2 & 2.2 & 35.9100 .0 \\
\hline SALT WASTE & B & 2.3 & 2.3 & 2.3 & 2.3 & 2.3 & 2.3 & 2.3 & .2 .3 & 2.3 & 2.3 & 2.3 & 2.3 & 2.3 & 2.3 & 2,3 & 38.1100 .0 \\
\hline IAISALT WASTE & & 2.3 & 2.3 & 2.3 & 2.3 & 2.3 & 2.3 & 2.3 & 2.3 & 2.3 & 2.3 & 2.3 & 2.3 & 2.3 & 2.3 & 2.3 & 38.1100 .0 \\
\hline \multirow[t]{2}{*}{ SH LEAD } & D & 5.6 & 5.6 & 5.6 & 5.6 & 5.6 & 5.6 & 5.6 & 5.6 & 5.6 & 5.6 & 3.6 & 5.6 & 5.6 & 5.6 & 5.6 & $89.7 \quad 33.1$ \\
\hline & MONE & 11.3 & 11.3 & 11.3 & 11.3 & 11.3 & 12.3 & 11.3 & 11.3 & 11.3 & 21.3 & 11.3 & 21.3 & 11.3 & 11.3 & 11.3 & $201.3 \quad 66.8$ \\
\hline I0t.a1SH LEAD & & 26.9 & 16.9 & 16.9 & 16.9 & 26.9 & 16.9 & 16.9 & 16.9 & 16.9 & 16.9 & 16.9 & 16.9 & 26.9 & 16.9 & 16.9 & 271.1100 .0 \\
\hline \multirow[t]{3}{*}{ SH STEEL } & $B$ & 2.8 & 2.8 & 2.8 & 2.0 & 2.8 & 2.8 & 2.8 & 2.8 & 2.8 & 2.8 & 2.8 & 2.8 & 2.0 & 2.8 & 2.8 & $\begin{array}{lll}46.8 & 9.1\end{array}$ \\
\hline & $G$ & 2.8 & 2.0 & 2.8 & 2.8 & 2.8 & 2.8 & 2.8 & 2.8 & 2.8 & 2.8 & 2.8 & 2.8 & 2.0 & 2.8 & 2,8 & 11.89 .1 \\
\hline & NONE & 24.0 & 24.0 & 28.0 & 24.0 & 24.0 & 24.0 & 24.0 & 24.0 & 24.0 & 24.0 & 24.0 & 21.0 & 24.0 & 21.0 & 24.0 & 384.481 .0 \\
\hline lotalSH STEEL & & 29.6 & 29.6 & 29.6 & 29.6 & 29.6 & 29.6 & 29.6 & 29.6 & 29.6 & 29.6 & 29.6 & 29.6 & 29.6 & 29.6 & 29.6 & 174.1100 .0 \\
\hline rotalWBA__HLVP & & 1657.7 & 1657.7 & 1657.1 & 1657.7 & 1657.7 & 1657.7 & 2657.7 & 1657.7 & 1657.7 & 1657.7 & 1657.7 & 657.7 & 657.7 & 1657.7 & 657.7 & 26523.2 \\
\hline
\end{tabular}

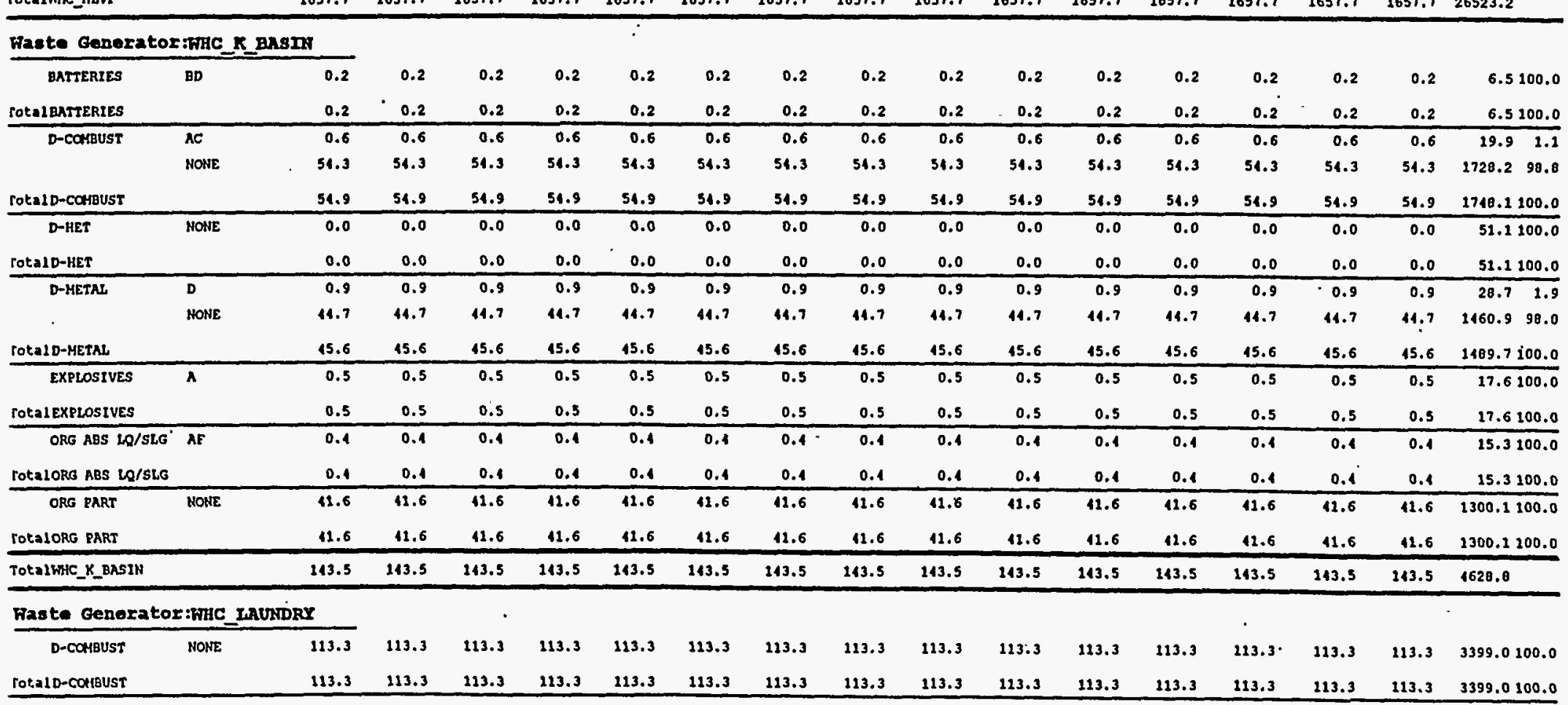

BCD Codes: A-Ignitables, B-Coriosives, C-Reactives, D-Metals w/O Hg, E-Metals w Hg, E-organics, G-State Reg., H-PCB < 50ppm, JuPCB > 50ppm 


\begin{tabular}{|c|c|c|c|c|c|c|c|c|c|c|c|c|c|c|c|c|c|}
\hline PWF & BCD & 2010 & 2011 & 2012 & 2013 & 2014 & 2015 & 2016 & 2017 & 2018 & 2019 & 2020 & 2021 & 2022 & 2023 & 2024 & Total Pet \\
\hline D-HET & B & 0.3 & 0.3 & 0.3 & 0.3 & 0.3 & 0.3 & 0.3 & 0.3 & 0.3 & 0.3 & 0.3 & 0.3 & 0.3 & 0.3 & 0.3 & 9.0100. \\
\hline Sot:a10-HET & & 0.3 & 0.3 & 0.3 & 0.3 & 0.3 & 0.3 & 0.3 & 0.3 & 0.3 & 0.3 & 0.3 & 0.3 & 0.3 & .0 .3 & 0.3 . & 9.0100. \\
\hline TOE:a IWHE_LAUUHDRY & & 113.6 & 113.6 & 113.6 & 113.6 & 113.6 & 113.6 & 113.6 & 113.6 & 123.6 & 113.6 & 213.6 & 113.6 & 113.6 & 113.6 & 113.6 & 3408.0 \\
\hline \multicolumn{18}{|c|}{ Waste Generator:KHC_ITBB } \\
\hline CONT SOIL & NONE & 0.4 & 0.4 & 0.1 & 0.4 & 0.1 & 0.1 & 0.4 & 0.1 & 0.4 & 0.1 & 0.1 & 0.1 & 0.1 & 0.1 & 0.1 & 12.9100. \\
\hline TotalCONT SOLL & & 0.4 & 0.4 & 0.1 & 0.1 & 0.1 & 0.1 & 0.1 & 0.4 & 0.4 & 0.4 & 0.1 & 0.1 & 0.1 & 0.1 & 0.1 & 12.9100 .0 \\
\hline D-coreust & MONE & 5.6 & 5.6 & 5.6 & 5.6 . & 5.6 & 5.6 & 5.6 & 5.6 & 5.6 & 5.6 & 5.6 & 5.6 & 5.6 & 5.6 & 5.6 & 170.3100 .0 \\
\hline rotalD-CorBust & & 5.6 & 5.6 & 5.6 & 5.6 & 5.6 & 5.6 & 5.6 & 5.6 & 5.6 & 5.6 & 5.6 & 5.6 & 5.6 & 5.6 & 5.6 & 170.3100 .0 \\
\hline D-HET & G & 0.0 & 0.0 & 3.1 & 0.0 & 0.0 & 3.4 & 0.0 & 0.0 & 3.4 & 0.0 & 0.0 & 3.4 & 0.0 & 0.0 & 0.0 & 30.6100 .0 \\
\hline Total D-KET & & 0.0 & 0.0 & 3.4 & 0.0 & 0.0 & 3.4 & 0.0 & 0.0 & 3.4 & 0.0 & 0.0 & 3.1 & 0.0 & 0.0 & 0.0 & 30.6100 .0 \\
\hline D-METAL & NONE & 0.1 & 0.1 & 0.1 & 0.1 & 0.1 & 0.1 & 0.1 & 0.1 & 0.1 & 0.1 & 0.1 & 0.1 & 0.1 & 0.1 & 0.1 & 1.6100 .0 \\
\hline Tota1 D-HETRL & & 0.1 & 0.1 & 0.1 & 0.1 & 0.1 & 0.2 & 0.1 & 0.1 & 0.1 & 0.1 & 0.1 & 0.1 & 0.1 & 0.1 & 0.1 & 4.6100 .0 \\
\hline D-SOIL & MONE & 0.0 & 0.0 & 0.0 & 0.0 & 0.0 & 0.0 & 0.0 & 0.0 & 0.0 & 0.0 & 0.0 & 0.0 & 0.0 & 0.0 & 0.0 & 2.0100 .0 \\
\hline Tota1D-sorL & & 0.0 & 0.0 & 0.0 & 0.0 & 0.0 & 0.0 & 0.0 & 0.0 & 0.0 & 0.0 & 0.0 & 0.0 & 0.0 & 0.0 & 0.0 & 1.0100. \\
\hline TotalWHC_LLBG & & 6.3 & 6.3 & 9.7 & 6.3 & 6.3 & 9.7 & 6.3 & 6.3 & 9.7 & 6.3 & 6.3 & 9.7 & 6.3 & 6.3 & 6.3 & 219.6 \\
\hline \multicolumn{18}{|c|}{ Faste Genexator:FHIC_IlWP } \\
\hline \multirow[t]{2}{*}{ BATTERIES } & B & 0.2 & 0.2 & 0.2 & 0.2 & 0.2 & 0.2 & 0.2 & 0.2 & 0.2 & 0.2 & 0.2 & 0.2 & 0.2 & 0.2 & 0.2 & 1.250 \\
\hline & D & 0.2 & 0.2 & 0.2 & 0.2 & 0.2 & 0.2 & 0.2 & 0.2 & 0.2 & 0.2 & 0.2 & 0.2 & 0.2 & 0.2 & 0.2 & 4.250 .0 \\
\hline Total BATTERIES & & 0.4 & 0.1 & 0.1 & 0.1 & 0.1 & 0.4 & 0.4 & 0.1 & 0.1 & 0.1 & 0.1 & 0.4 & 0.1 & 0.1 & 0.1 & 0.1100 .0 \\
\hline \multirow[t]{3}{*}{ D-COABUST } & $\AA$ & 0.3 & 0.3 & 0.3 & 0.3 & 0.3 & 0.3 & 0.3 & 0.3 & 0.3 & 0.3 & 0.3 & 0.3 & 0.3 & 0.3 & 0.3 & $7.6 \quad 0.1$ \\
\hline & G & 0.2 & 0.2 & 0.2 & 0.2 & $0.2^{\circ}$ & 0.2 & 0.2 & 0.2 & 0.2 & 0.2 & 0.2 & 0.2 & 0.2 & 0.2 & 0.2 & $4.1 \cdot 0.0$ \\
\hline & NONE & 371.9 & 371.9 & 371.9 & 371.9 & 371.9 & 371.9 & 371.9 & 371.9 & 371.9 & 371.9 & 371.9 & 371.9 & 371.9 & 371.9 & 371.9 & $7439.0 \quad 99.8$ \\
\hline Tota1 D-CORBUST & & 372.5 & 372.5 & 372.5 & 372.5 & 372.5 & 372.5 & 372.5 & 372.5 & 372.5 & 372.5 & 372.5 & 322.5 & 372.5 & 372.5 & 372.5 & 7451.0100 .0 \\
\hline \multirow[t]{4}{*}{ D-HET } & 8 & 0.0 & 0.0 & 0.0 & 0.0 & 0.0 & 0.0 & 0.0 & 0.0 & 0.0 & $0.0^{\circ}$ & 0.0 & 0.0 & 0.0 & 0.0 & 0.0 & $1.8 \quad 0.1$ \\
\hline & c & 0.0 & 0.0 & 0.0 & 0.0 & 0.0 & 0.0 & 0.0 & 0.0 & 0.0 & 0.0 & 0.0 & 0.0 & 0.0 & 0.0 & 0.0 & $1.8 \quad 0.2$ \\
\hline & G & 0.1 & 0.1 & 0.1 & 0.1 & 0.1 & 0.1 & 0.1 & 0.1 & 0.1 & 0.1 & 0.1 & 0.1 & 0.1 & 0.1 & 0.1 & $\begin{array}{ll}3.7 & 0.2\end{array}$ \\
\hline & HONE & 87.2 & 87.2 & 87.2 & 87.2 & 87,2 & 07.2 & 87.2 & 87.2 & 87.2 & 87.2 & 97.2 & 87.2 & 87.2 & 87.2 & 87.2 & $1745.2 \quad 99.5$ \\
\hline TotalD-HET & & 87.6 & 07.6 & 87.6 & 07.6 & 87.6 & 87.6 & 87.6 & 07.6 & 07.6 & 87.6 & 07.6 & 87.6 & 87.6 & 87.6 & 07.6 & 1752.7100 .0 \\
\hline \multirow[t]{4}{*}{ D-IN NON-HTL } & 8 & 0.1 & 0.1 & 0.1 & 0.1 & 0.1 & 0.1 & 0.1 & 0.2 & 0.1 & 0.1 & 0.1 & 0.1 & 0.1 & 0.1 & 0.1 & $2.4 \quad 0.2$ \\
\hline & c & 0.0 & 0.0 & 0,0 & 0.0 & 0.0 & 0.0 & 0.0 & 0.0 & 0.0 & 0.0 & 0.0 & 0.0 & 0.0 & 0.0 & 0.0 & 1.2 \\
\hline & G & 0.1 & 0.1 & 0.1 & 0.1 & 0.1 & 0.1 & 0.1 & 0.1 & 0.1 & 0.1 & 0.1 & 0.1 & 0.1 & 0.1 & 0.1 & 2.2 \\
\hline & NONE & 58.9 & 50.9 & 58.9 & 58.9 & 58.9 & 58.9 & 58.9 & 58.9 & 58.9 & 58.9 & 58.9 & 58.9 & 58.9 & 58.9 & 58.9 & $2178.0 \quad 99.4$ \\
\hline TOt:A1 D-1N NON-HTL & & 59.2 & 59.2 & 59.2 & 59.2 & 59.2 & 59.2 & 59.2 & 59.2 & 59.2 & 59.2 & 59.2 & 59.2 & 39.2 & 59.2 & 59.2 & 1184.0100 .0 \\
\hline
\end{tabular}

BCD Codes: A-Ignitables, B-Corrosives, C-Reactives, D-Metals w/O Hg, E-Motals w Hg, F-Organics, G-State Reg., H-PCB < 50ppm, J-PCB > 50ppm 


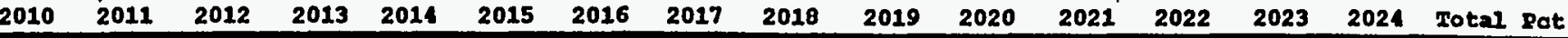

\begin{tabular}{|c|c|c|c|c|c|c|c|c|c|c|c|c|c|c|c|c|c|}
\hline D-METAL & $\begin{array}{l}\text { D } \\
\text { MONE }\end{array}$ & $\begin{array}{r}0.2 \\
66.5\end{array}$ & $\begin{array}{r}0.2 \\
66.5\end{array}$ & $\begin{array}{r}0.2 \\
66.5\end{array}$ & $\begin{array}{r}0.2 \\
66.5\end{array}$ & $\begin{array}{r}0.2 \\
66.5\end{array}$ & $\begin{array}{r}0.2 \\
66.5\end{array}$ & $\begin{array}{r}0.2 \\
66.5\end{array}$ & $\begin{array}{r}0.2 \\
66.5\end{array}$ & $\begin{array}{r}0.2 \\
66.5\end{array}$ & $\begin{array}{r}0.2 \\
66.5\end{array}$ & $\begin{array}{r}0.2 \\
66.5\end{array}$ & $\begin{array}{r}0.2 \\
66.5\end{array}$ & $\begin{array}{r}0.2 \\
66.5\end{array}$ & $\begin{array}{r}0.2 \\
66.5\end{array}$ & $\begin{array}{r}0.2 \\
66.5\end{array}$ & $\begin{array}{rr}5.8 & 0 \\
1330.6 & 99\end{array}$ \\
\hline OL.A1 L-METAL & & 66.8 & 66.8 & 66.8 & 66.0 & 66.0 & 66.8 & 66.8 & 66.0 & 66.0 & 66.8 & 66.0 & 66.8 & 66.8 & 66.8 & 66.8 & 1336.4100 \\
\hline IN ORG LQ/SLG & B & 0.2 & 0.2 & 0.2 & 0.2 & 0.2 & 0.2 & 0.2 & 0.2 & 0.2 & 0.2 & 0.2 & 0.2 & 0.2 & 0.2 & 0.2 & 4.731 \\
\hline & c & 0.1 & 0.1 & 0.1 & 0.1 & 0.1 & 0.1 & 0.1 & 0.1 & 0.1 & 0.1 & 0.1 & 0.1 & 0.1 & 0.1 & 0.1 & 3.6 \\
\hline & G & 0.3 & 0.3 & 0.3 & 0.3 & 0.3 & 0.3 & 0.3 & 0.3 & 0.3 & 0.3 & 0.3 & 0.3 & 0.3 & 0.3 & 0.3 & 6.9 \\
\hline
\end{tabular}

\begin{tabular}{|c|c|c|c|c|c|c|c|c|c|c|c|c|c|c|c|c|}
\hline rotal IN ORG LQ/SLG & 0.7 & 0.7 & 0.7 & 0.7 & 0.7 & 0.7 & 0.7 & 0.7 & 0.7 & 0.7 & 0.7 & 0.7 & 0.1 & 0.7 & 0.7 & 15.4200 .0 \\
\hline 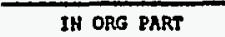 & 0.2 & 0.2 & 0.2 & 0.2 & 0.2 & 0.2 & 0.2 & 0.2 & 0.2 & 0.2 & 0.2 & 0.2 & 0.2 & 0.2 & 0.2 & 9.237 \\
\hline
\end{tabular}

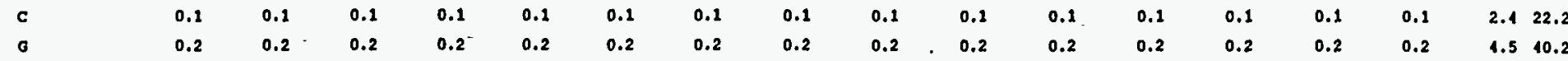

\begin{tabular}{|c|c|c|c|c|c|c|c|c|c|c|c|c|c|c|c|c|}
\hline TOtalIN ORG PART & 0.5 & 0.5 & 0.5 & 0.5 & 0.5 & 0.5 & 0.5 & 0.5 & 0.5 & 0.5 & 0.5 & 0.5 & 0.5 & 0.5 & 0.5 & 11.2100 .0 \\
\hline
\end{tabular}

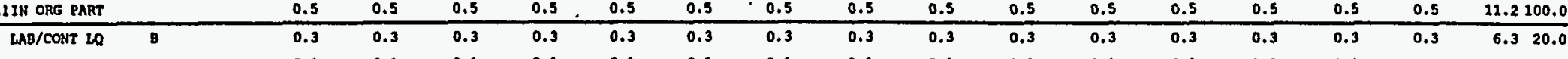

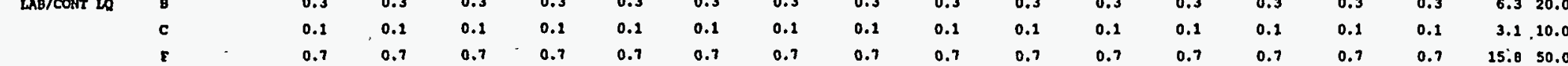

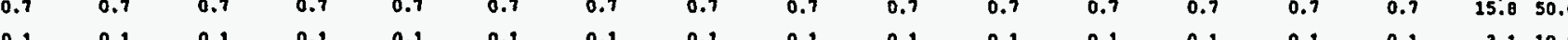

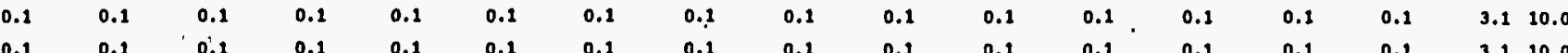

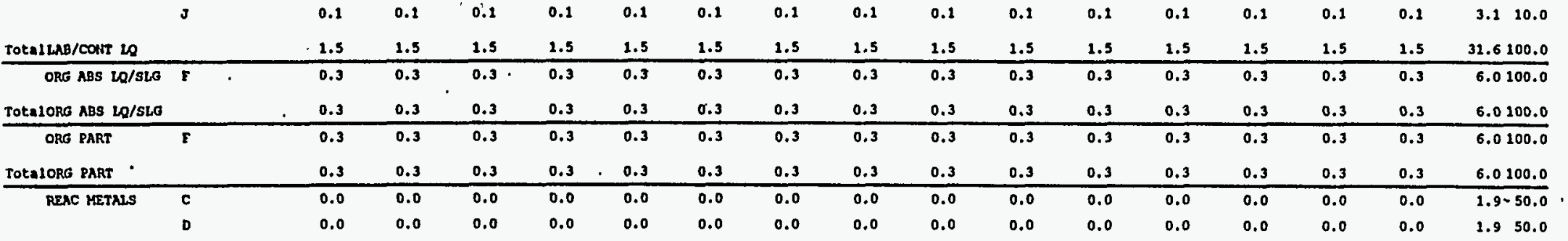

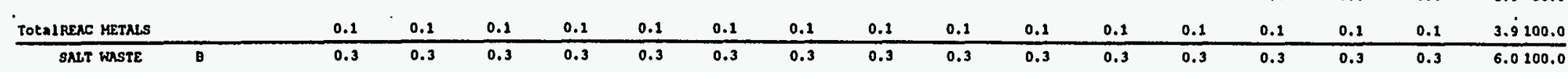

\begin{tabular}{cccccccccccccccccc} 
TOtaLSALT WASTE & & 0.3 & 0.3 & 0.3 & 0.3 & 0.3 & 0.3 & 0.3 & 0.3 & 0.3 & 0.3 & 0.3 & 0.3 & 0.3 & 0.3 & 0.3 & 6.0100 .0 \\
\hline SH LEAD & NONE & 1.0 & 1.0 & 1.0 & 1.0 & 1.0 & 1.0 & 1.0 & 1.0 & 1.0 & 1.0 & 1.0 & 1.0 & 1.0 & 1.0 & 1.0 & 21.0100 .0
\end{tabular}

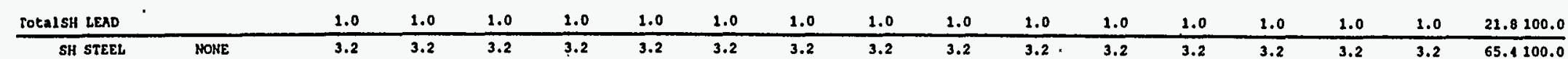

\begin{tabular}{|c|c|c|c|c|c|c|c|c|c|c|c|c|c|c|c|c|}
\hline TOtalSH STEEL & 3.2 & 3.2 & 3.2 & 3.2 & 3.2 & 3.2 & 3.2 & 3.2 & 3.2 & 3.2 & 3.2 & 3.2 & 3.2 & 3.2 & 3.2 & 65.4100 .0 \\
\hline Tota1WAE_LLVP & 595.0 & 595.0 & 595.0 & 595.0 & 595.0 & 595.0 & 595.0 & 595.0 & 595.0 & 595.0 & 595.0 & 595.0 & 595.0 & 595.0 & 595.0 & 11900.0 \\
\hline
\end{tabular}

Faste Generator:FHC_PEP

\begin{tabular}{|c|c|c|c|c|c|c|c|c|c|c|c|c|c|c|c|c|c|}
\hline & BDG & $\begin{array}{l}0.0 \\
0.0\end{array}$ & $\begin{array}{l}0.0 \\
0.0\end{array}$ & $\begin{array}{l}0.0 \\
0.0\end{array}$ & $\begin{array}{l}0.0 \\
0.0\end{array}$ & $\begin{array}{l}0.0 \\
0.0\end{array}$ & $\begin{array}{l}0.0 \\
0.0\end{array}$ & $\begin{array}{l}0.0 \\
0.0\end{array}$ & $\begin{array}{l}0.0 \\
0.0\end{array}$ & $\begin{array}{l}0.0 \\
0.0\end{array}$ & $\begin{array}{l}0.0 \\
0.0\end{array}$ & $\begin{array}{l}0.0 \\
0.0\end{array}$ & $\begin{array}{l}0.0 \\
0.0\end{array}$ & $\begin{array}{l}0.0 \\
0.0\end{array}$ & $\begin{array}{l}0.0 \\
0.0\end{array}$ & $\begin{array}{l}0.0 \\
0.0\end{array}$ & $\begin{array}{rr}11.1 & 38.6 \\
6.5 & 22.7\end{array}$ \\
\hline
\end{tabular}

gCD Codes: A-Ignitables, B-Corrosives, C=Reactives, DmMetals w/O Hg, E-Metals w Hg, E-Organics, G=State Reg., H-PCB < 50ppm, JaPCB > 50ppm 
Annuel Physical Haste Form and gazardous Constituent Volumes by Haste Generator

(in cubio metera)

PWT

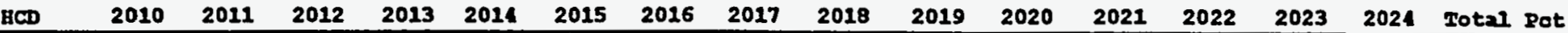

\begin{tabular}{|c|c|c|c|c|c|c|c|c|c|c|c|c|c|c|c|c|c|}
\hline & $\varepsilon$ & 0.0 & 0.0 & 0.0 & 0.0 & 0.0 & 0.0 & 0.0 & 0.0 & 0.0 & 0.0 & 0.0 & 0.0 & 0.0 & 0.0 & 0.0 & 11.138 .6 \\
\hline . Lal BATTERIES & & 0.0 & 0.0 & 0.0 & 0.0 & 0.0 & 0.0 & 0.0 & 0.0 & 0.0 & 0.0 & 0.0 & 0.0 & 0.0 . & 0.0 & 0.0 & 28.7100 .0 \\
\hline CONT SOIL & HONE & 1.0 & 1.0 & 1.0 & 1.0 & 1.0 & 0.6 & 0.6 & 0.6 & 0.6 & 0.6 & 0.6 & 0.6 & 0.6 & 0.6 & 0.6 & 139.3100 .0 \\
\hline
\end{tabular}

\begin{tabular}{|c|c|c|c|c|c|c|c|c|c|c|c|c|c|c|c|}
\hline J.alcont soll & 1.0 & 1.0 & 1.0 & 1.0 & 0.6 & 0.6 & 0.6 & 0.6 & 0.6 & 0.6 & 0.6 & 0.6 & 0.6 & 0.6 & 139.3100 .0 \\
\hline D-colitust & 0.0 & 0.0 & 0.0 & 0.0 & 0.0 & 0.0 & 0.0 & 0.0 & 0.0 & 0.0 & 0.0 & 0.0 & 0.0 & 0.0 & $\begin{array}{ll}11.1 & 0.2\end{array}$ \\
\hline
\end{tabular}

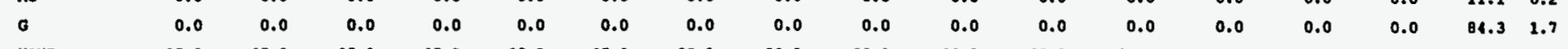

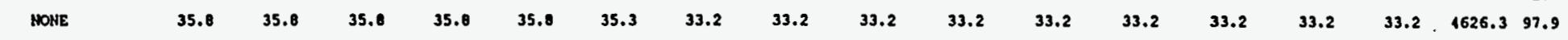

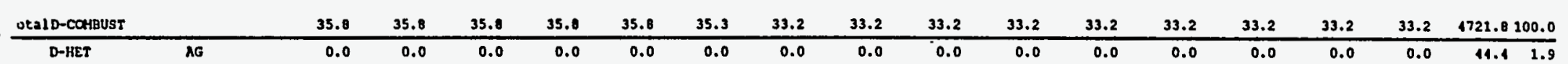

\begin{tabular}{|c|c|c|c|c|c|c|c|c|c|c|c|c|c|c|c|c|c|}
\hline \multirow[t]{2}{*}{ D-KET } & AG & 0.0 & 0.0 & 0.0 & 0.0 & 0.0 & 0.0 & 0.0 & 0.0 & 0.0 & 0.0 & 0.0 & 0.0 & 0.0 & 0.0 & 0.0 & 14.4 \\
\hline & D & 0.0 & 0.0 & 0.0 & 0.0 & 0.0 & 0.0 & 0.0 & 0.0 & 0.0 & 0.0 & 0.0 & 0.0 & 0.0 & 0.0 & 0.0 & $388.5 \quad 17.0$ \\
\hline
\end{tabular}

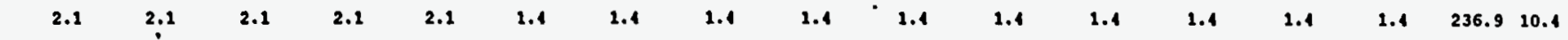

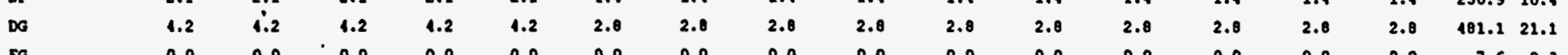

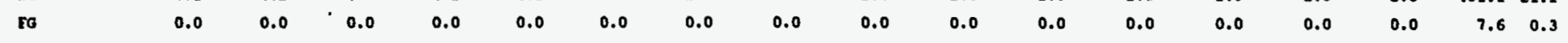

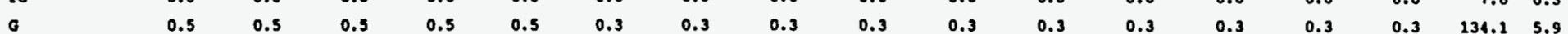

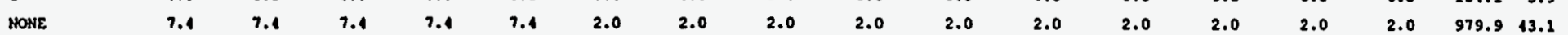

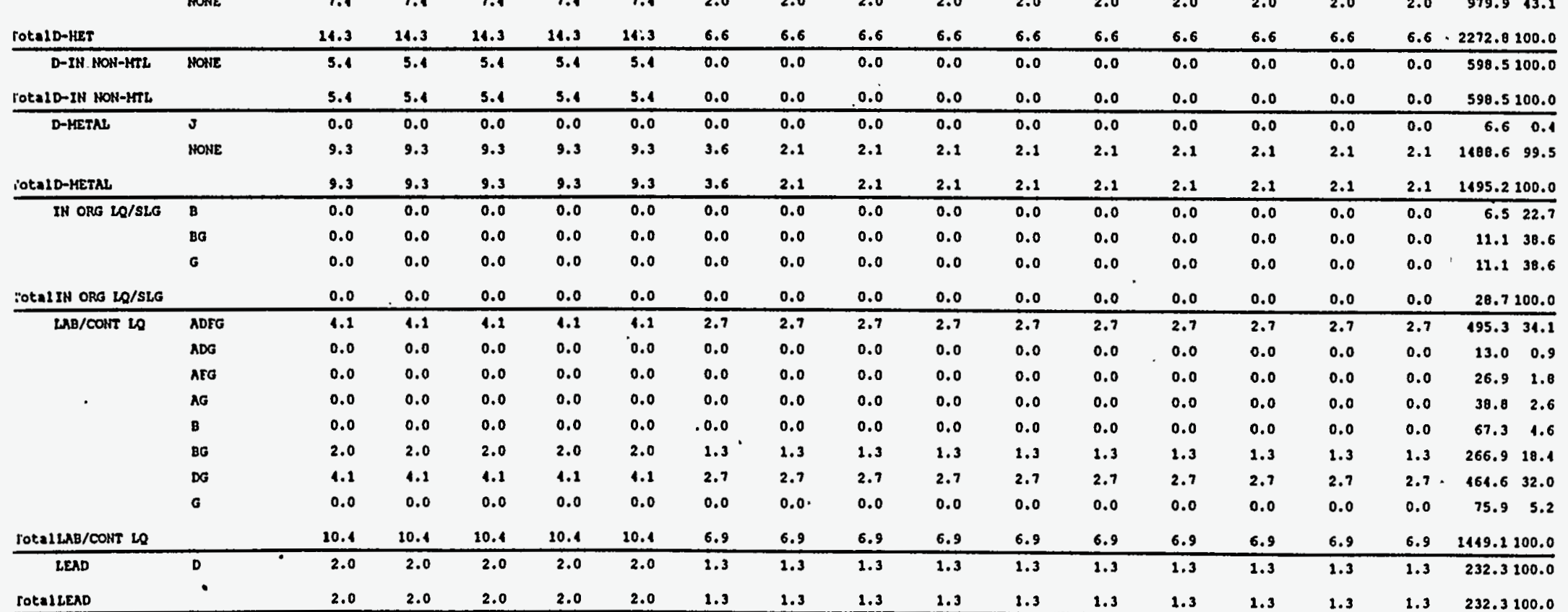

gCD Codes: A-Ignitables, B-Corrosives, C-Reactives, D-Metals w/O Hg, E-Metals w Hg, E-Organics, G-State Reg., HmPCB < 50ppm, J-PCB > 50ppm 


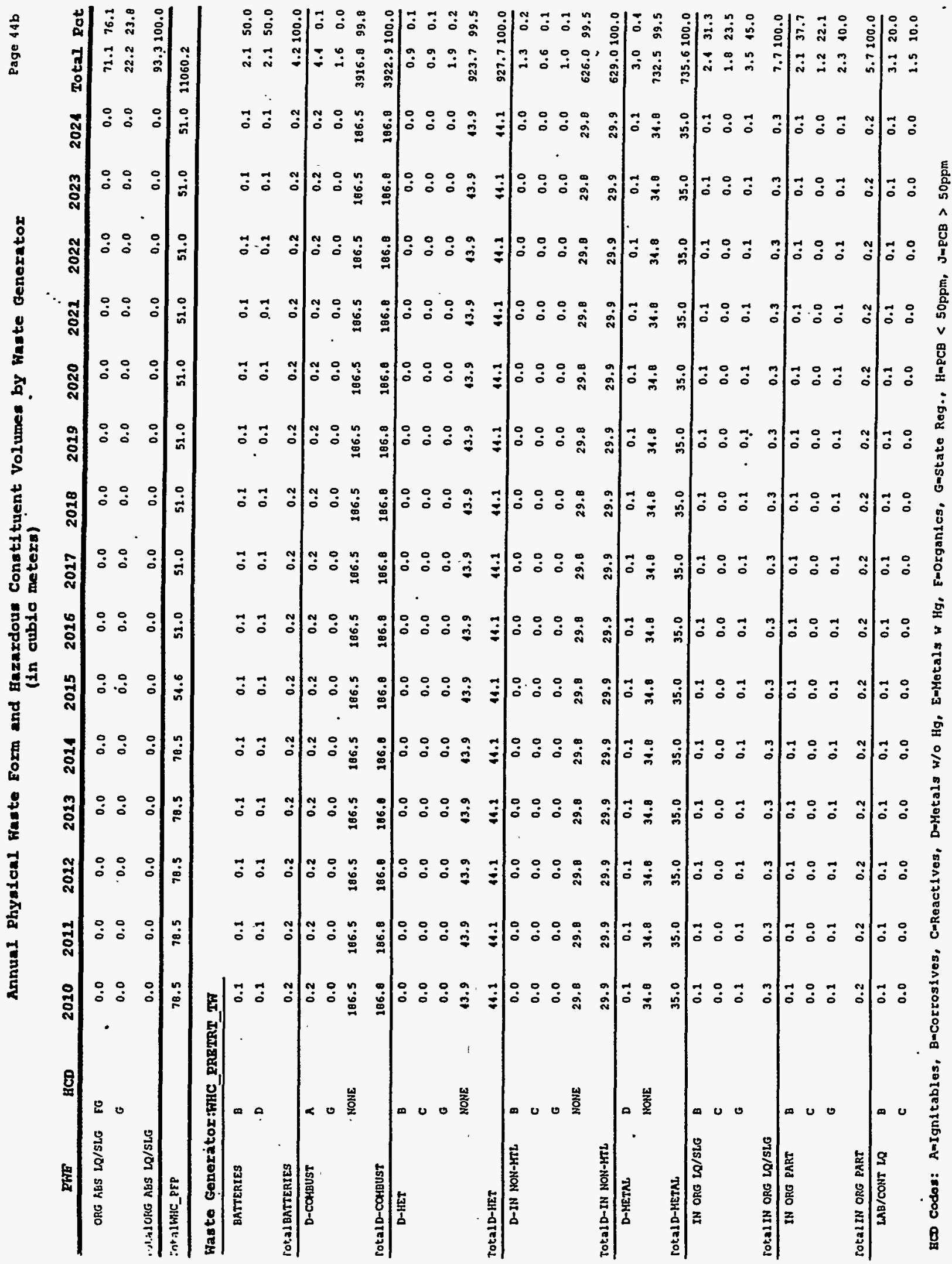




\begin{tabular}{|c|c|c|c|c|c|c|c|c|c|c|c|c|c|c|c|c|c|}
\hline & 8 & 0.3 & 0.3 & 0.3 & 0.3 & 0.3 & 0.3 & 0.3 & 0.3 & 0.3 & 0.3 & 0.3 & 0.3 & 0.3 & 0.3 & 0.3 & 7.950 .0 \\
\hline & н & 0.0 & 0.0 & 0.0 & 0.0 & 0.0 & 0.0 & 0.0 & 0.0 & 0.0 & 0.0 & 0.0 & 0.0 & 0.0 & 0.0 & 0.0 & $\begin{array}{lll}1.5 & 10.0\end{array}$ \\
\hline & $\mathbf{J}$ & 0.0 & 0.0 & 0.0 & 0.0 & 0.0 & 0.0 & 0.0 & 0.0 & 0.0 & 0.0 & 0.0 & 0.0 & 0.0 & 0.0 & 0.0 & 1.510 .0 \\
\hline . Otal LAB/COIT LQ & & 0.7 & 0.7 & 0.7 & 0.7 & 0.7 & 0.7 & 0.7 & 0.7 & 0.7 & 0.7 & 0.7 & 0.7 & 0.7 & 0.7 & 0.1 & 15.8100 .0 \\
\hline ORG ABS LQ/SLG & $\bar{F}$ & 0.1 & 0.1 & 0.1 & 0.1 & 0.1 & 0.1 & 0.1 & 0.1 & 0.1 & 0.1 & 0.1 & 0.1 & 0.1 & 0.1 & 0.1 & 3.0100 .0 \\
\hline rotalORG ABS LQ/SLG & & 0.1 & 0.1 & 0.1 & 0.1 & 0.1 & 0.1 & 0.1 & 0.1 & 0.1 & 0.1 & 0.1 & 0.1 & 0.1 & 0.1 & 0.1 & 3.0100 .0 \\
\hline ORG PART & $\bar{E}$ & 0.1 & 0.1 & 0.1 & 0.1 & 0.1 & 0.1 & 0.1 & 0.1 & 0.1 & 0.2 & 0.1 & 0.1 & 0.1 & 0.1 & 0.1 & 3.0100 .0 \\
\hline PotaloRg PART & & 0.1 & 0.1 & 0.1 & 0.1 & 0.1 & 0.1 & 0.1 & 0.1 & 0.1 & 0.1 & 0.1 & 0.1 & 0.1 & 0.1 & 0.1 & 3.0100 .0 \\
\hline REAC METALS & D & 0.0 & 0.0 & 0.0 & 0.0 & 0.0 & 0.0 & 0.0 & 0.0 & 0.0 & 0.0 & 0.0 & 0.0 . & 0.0 & 0.0 & 0.0 & 1.050 .0 \\
\hline rotal REAC METRLS & & 0.0 & 0.0 & 0.0 & 0.0 & 0.0 & 0.0 & 0.0 & 0.0 & 0.0 & 0.0 & 0.0 & 0.0 & 0.0 & 0.0 & 0.0 & 2.0100 .0 \\
\hline SALT WASTE & B & 0.1 & 0.1 & 0.1 & 0.1 & 0.1 & 0.1 & 0.1 & 0.1 & 0.1 & 0.1 & 0.1 & 0.1 & 0.1 & 0.1 & 0.1 & 3.0100 .0 \\
\hline Total SALT kaste & & 0.1 & 0.1 & 0.1 & 0.1 & 0.1 & 0.1 & 0.1 & 0.1 & 0.1 & 0.1 & 0.2 & 0.1 & 0.1 & 0.1 & 0.2 & 3.0200 .0 \\
\hline SH LEAD & NONE & 0.7 & 0.7 & 0.7 & 0.7 & 0.7 & 0.7 & 0.7 & 0.7 & 0.7 & 0.7 & 0.7 & 0.7 & 0.7 & 0.7 & 0.7 & 15.2100 .0 \\
\hline PotalsH LEAD & & 0.7 & 0.7 & 0.7 & 0.7 & 0.7 & 0.7 & 0.7 & 0.7 & 0.7 & 0.7 & 0.7 & 0.7 & 0.7 & 0.7 & 0.7 & 15.2100 .0 \\
\hline SH STEEL & NOWE & 2.1 & 2.1 & 2.1 & 2.1 & 2.1 & 2.1 & 2.1 & 2.1 & 2.1 & 2.1 & 2.1 & 2.1 & 2.1 & 2.1 & 2.1 & .45 .6100 .0 \\
\hline TotalSH STEEL & & 2.1 & 2.1 & 2.1 & 2.1 & 2.1 & 2.1 & 2.1 & 2.1 & 2.1 & 2.1 & 2.1 & 2.1 & 2.1 & 2.1 & 2.1 & 45.6100 .0 \\
\hline \multicolumn{18}{|c|}{ Haste Generator:WHC_PROCURMRMT } \\
\hline \multirow[t]{3}{*}{ CONT SOIL } & $\mathbf{A}$ & 0.0 & 0.0 & 0.0 & 0.0 & 0.0 & 0.0 & 0.0 & 0.0 & 0.0 & 0.0 & 0.0 & 0.0 & 0.0 & 0.0 & 0.0 & $0.0,50.2$ \\
\hline & 8 & 0.0 & 0.0 & 0.0 & 0.0 & 0.0 & 0.0 & 0.0 & 0.0 & 0.0 & 0.0 & 0.0 & 0.0 & 0.0 & 0.0 & 0.0 & 0.024 .0 \\
\hline & $c$ & 0.0 & 0.0 & 0.0 & 0.0 & 0.0 & 0.0 & 0.0 & 0.0 & 0.0 & 0.0 & 0.0 & 0.0 & 0.0 & 0.0 & 0.0 & 0.024 .8 \\
\hline Totalcont soIt & & 0.0 & 0.0 & 0.0 & 0.0 & 0.0 & 0.0 & 0.0 & 0.0 & 0.0 & 0.0 & 0.0 & 0.0 & 0.0 & 0.0 & 0.0 & 0.1100 .0 \\
\hline$i^{\text {Decangust }}$ & $A$ & 0.0 & 0.0 & 0.0 & 0.0 & 0.0 & 0.0 & 0.0 & 0.0 & 0.0 & 0.0 & 0.0 & 0.0 & 0.0 & 0.0 & 0.0 & 2.1100 .0 \\
\hline Total D-comaust & & 0.0 & 0.0 & 0.0 & 0.0 & 0.0 & 0.0 & 0.0 & 0.0 & 0.0 & 0.0 & 0.0 & 0.0 & 0.0 & 0.0 & 0.0 & 2.1200 .0 \\
\hline \multirow[t]{3}{*}{ D-HET } & $A$ & 0.2 & 0.2 & 0.2 & 0.2 & 0.2 & 0.2 & 0.2 & 0.2 & 0.2 & 0.2 & 0.2 & 0.2 & 0.2 & 0.2 & $\overline{0.2}$ & 7.154 .7 \\
\hline & 8 & 0.0 & 0.0 & 0.0 & 0.0 & 0.0 & 0.0 & 0.0 & 0.0 & 0.0 & 0.0 & 0.0 & 0.0 & 0.0 & 0.0 & 0.0 & $1.2 \quad 9.5$ \\
\hline & c & 0.1 & 0.1 & 0.1 & 0.1 & 0.1 & 0.1 & 0.1 & 0.1 & 0.1 & 0.1 & 0.1 & 0.1 & 0.1 & 0.1 & 0.1 & 4.635 .6 \\
\hline Total D-HET & & 0.1 & 0.4 & 0.1 & 0.1 & 0.1 & 0.1 & 0.4 & 0.4 & 0.4 & 0.4 & 0.4 & 0.1 & 0.1 & 0.4 & 0.1 & 12.9100 .0 \\
\hline \multirow[t]{3}{*}{ D-IN NOH-HTL } & $\lambda$ & 0.0 & 0.0 & 0.0 & 0.0 & 0.0 & 0.0 & 0.0 & 0.0 & 0.0 & 0.0 & 0.0 & 0.0 & 0.0 & 0.0 & 0.0 & 0.024 .8 \\
\hline & 8 & 0.0 & 0.0 & 0.0 & 0.0 & 0.0 & 0.0 & 0.0 & 0.0 & 0.0 & 0.0 & 0.0 & 0.0 & 0.0 & 0.0 & 0.0 & 0.150 .3 \\
\hline & c & 0.0 & 0.0 & 0.0 & 0.0 & 0.0 & 0.0 & 0.0 & 0.0 & 0.0 & 0.0 & 0.0 & 0.0 & 0.0 & 0.0 & 0.0 & 0.024 .8 \\
\hline TOt:aLD-IN NON-MTL & · & 0.0 & 0.0 & 0.0 & 0.0 & 0.0 & 0.0 & 0.0 & 0.0 & 0.0 & 0.0 & 0.0 & 0.0 & 0.0 & 0.0 & 0.0 & 0.2100 .0 \\
\hline D-METAL & 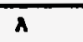 & 0.0 & 0.0 & 0.0 & 0.0 & 0.0 & 0.0 & 0.0 & 0.0 & 0.0 & 0.0 & 0.0 & 0.0 & 0.0 & 0.0 & 0.0 & 1.448 .8 \\
\hline
\end{tabular}

aCD Codes: A-Ignitables, B-Corrosives, C-Reactives, D-Metals w/O Hg, E-Metals w Hg, E-Organics, G-State Reg., H-PCB < 50ppm, JmPCB > 50ppm 


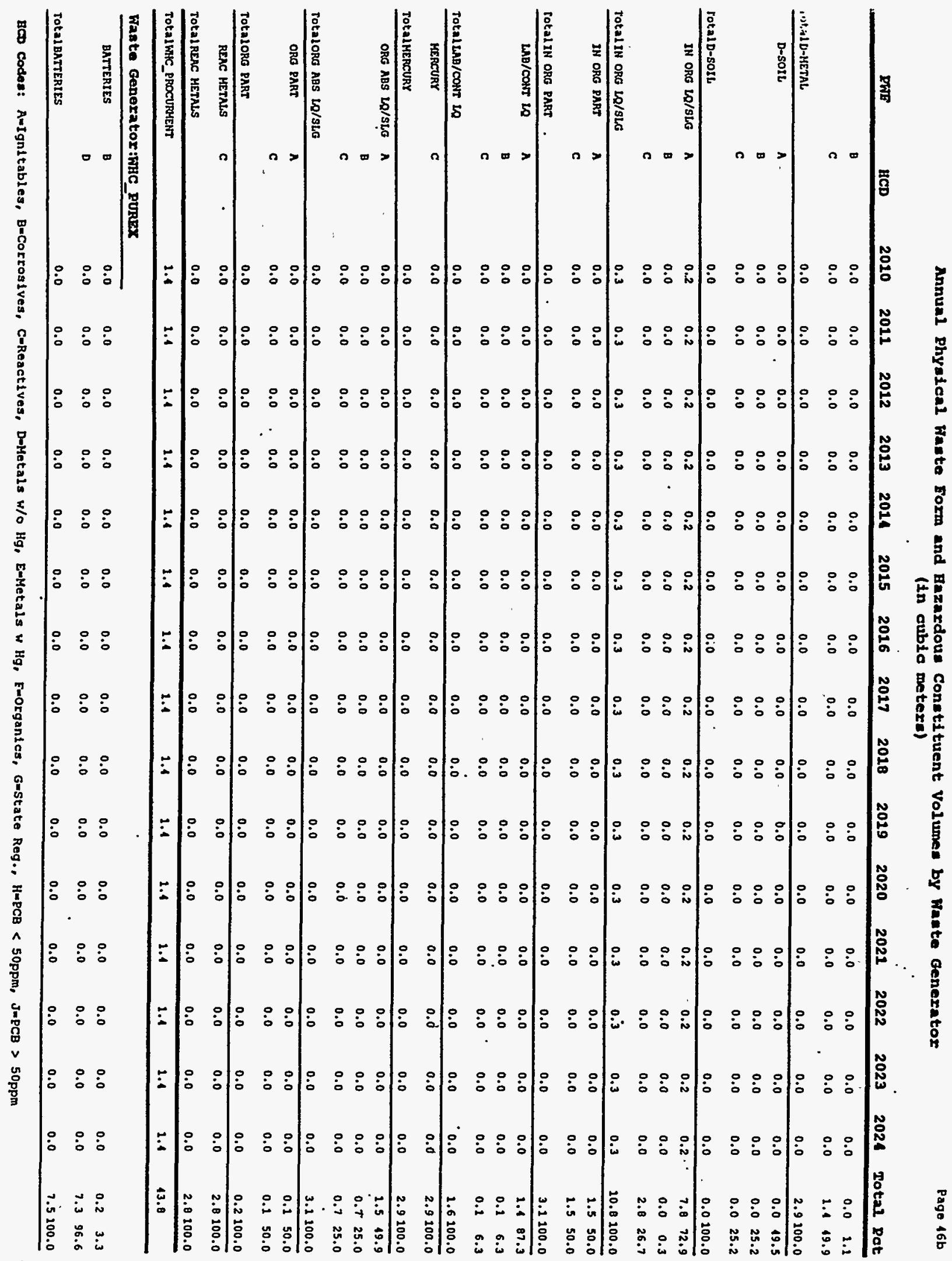




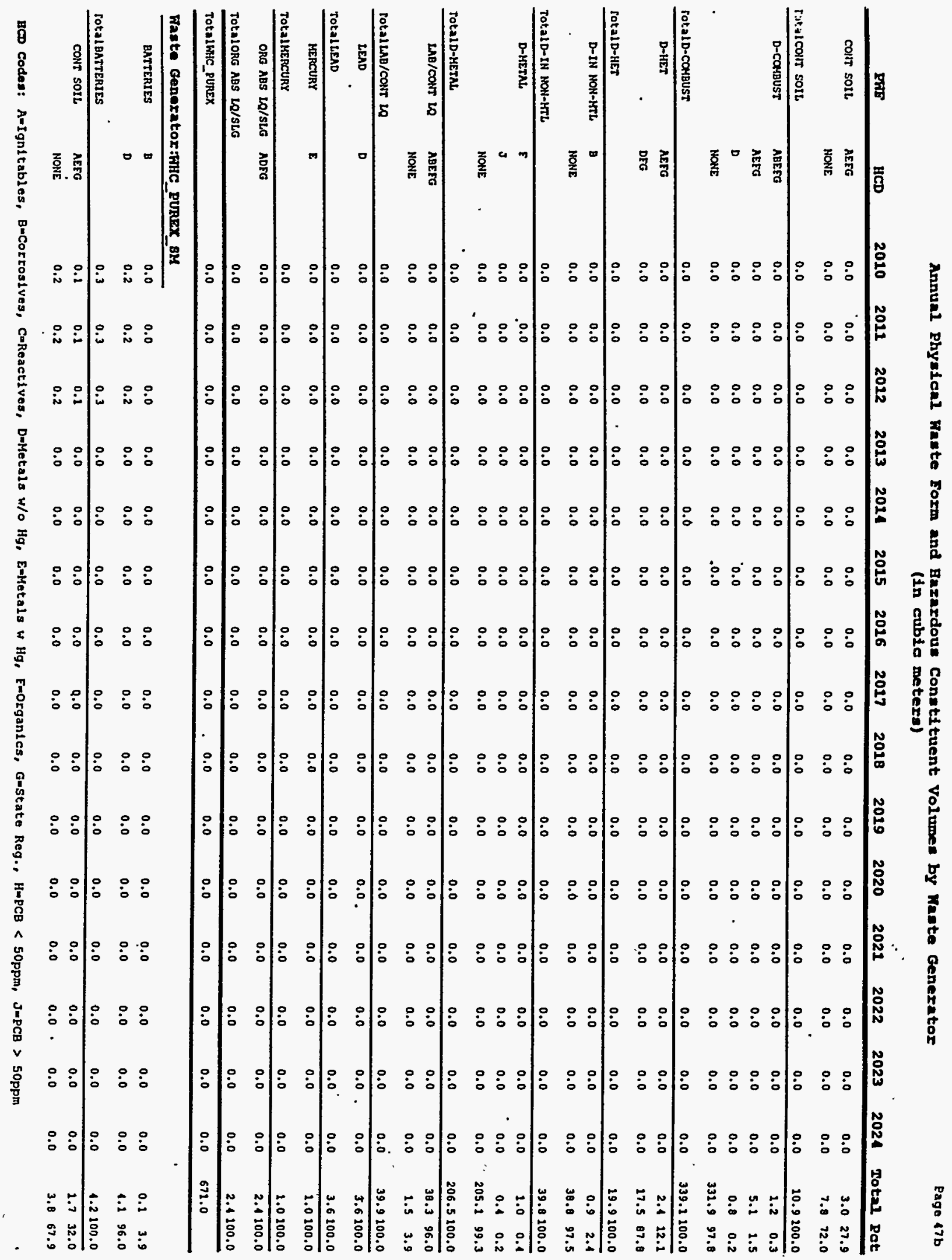




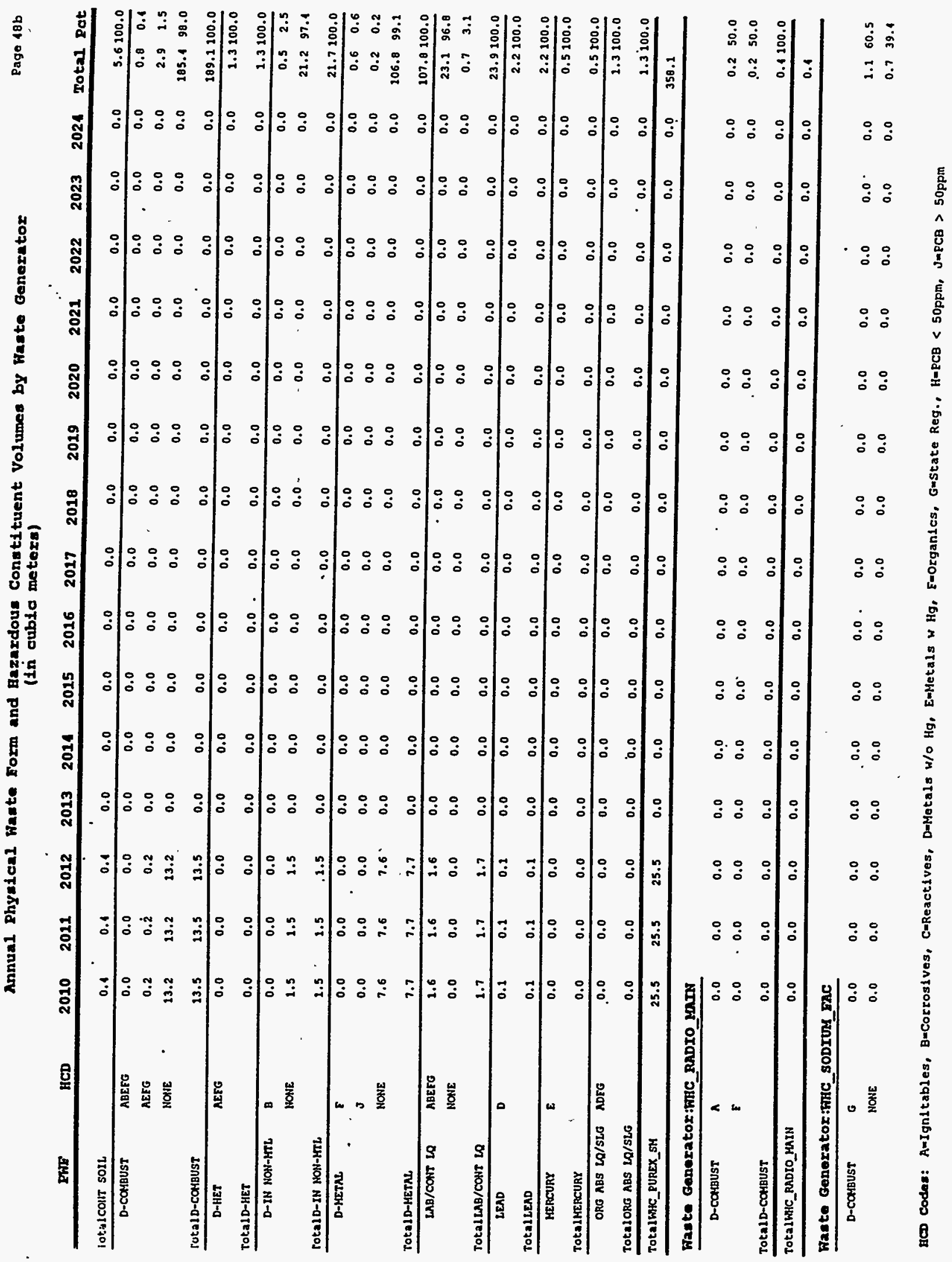




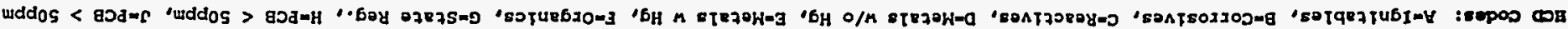

\begin{tabular}{|c|c|c|c|c|c|c|c|c|c|c|c|c|c|c|c|c|c|}
\hline $0.0018 .16 \mathrm{I}$ & 0.0 & 0.0 & 0.0 & 0.0 & 0.0 & 0.0 & 0.0 & $t \cdot 92$ & $7 \cdot 92$ & 5.92 & $2 \cdot 2 z$ & $2 \cdot \theta I$ & 9.51 & $0 . \varepsilon t$ & $1 \cdot 0 t$ & & 7LH-NON NI-OTEZOJ \\
\hline โ.ร9 & 0.0 & 0.0 & 0.0 & 0.0 & 0.0 & 0.0 & 0.0 & 6.51 & 6.55 & 6.51 & 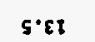 & $\tau \cdot \tau$ & 5.6 & $6 . \mathrm{L}$ & $\varepsilon \cdot 9$ & 3NON & - \\
\hline 8. BE $9.5 L$ & 0.0 & 0.0 & 0.0 & 0.0 & 0.0 & 0.0 & 0.0 & $\cdot \imath \cdot 0 \pi$ & r.or & t.0t & 9.8 & T.L & 0.9 & 0.5 & 0.1 & 30 & IIH-NON NI-Q \\
\hline $0.0018 \cdot z \varepsilon$ & 0.0 & 0.0 & 0.0 & 0.0 & 0.0 & 0.0 & 0.0 & $\varepsilon \cdot 1$ & $\varepsilon \cdot b$ & {$[\cdot 1$} & $9 \cdot \varepsilon$ & $0 \cdot \varepsilon$ & $9 \cdot 2$ & $1 \cdot z$ & $\overline{L I}$ & & $13 \mathrm{H}-91270 \mathrm{~J}$ \\
\hline $0.001 r \cdot z \varepsilon$ & 0.0 & 0.0 & 0.0 & 0.0 & 0.0 & 0.0 & 0.0 & $\varepsilon \cdot 1$ & $\varepsilon \cdot \vartheta$ & $\varepsilon \cdot 1$ & $9 \cdot \varepsilon$ & $0 . \varepsilon$ & $9 \cdot 2$ & $\because 2$ & 2.7 & 30 & $23 H-a$ \\
\hline $0.001+2 \angle L 6$ & 0.0 & 0.0 & 0.0 & 0.0 & 0.0 & 0.0 & 0.0 & S.OET & $5 \cdot 0$ t & S'0ET & 6.011 & c.16 & $E \cdot \theta L$ & $2 \cdot 59$ & $2 \cdot z s$ & & ISn8Ho0-atezoj \\
\hline H.1z $9 \cdot<\varepsilon z$ & 0.0 & 0.0 & 0.0 & 0.0 & 0.0 & 0.0 & 0.0 & $6 \cdot \mathrm{te}$ & $6 \cdot 1 \varepsilon$ & $6 \cdot \mathrm{te}$ & $\cdot x \cdot 2 z$ & $\varepsilon \cdot 22$ & $t \cdot 61$ & 6.51 & $2 \cdot z$ & 3NON & \\
\hline S.SL B.VEL & 0.0 & 0.0 & 0.0 & 0.0 & 0.0 & 0.0 & 0.0 & $9 \cdot 86$ & $9 \cdot 96$ & $9 \cdot 96$ & $\theta \cdot \varepsilon \theta$ & 0.69 & $r .65$ & c.6r & $1.6 \varepsilon$ & 30 & ISก9н100- $a$ \\
\hline $0.00 \tau 2 \cdot 16$ & 0.0 & 0.0 & 0.0 & 0.0 & 0.0 & 0.0 & 0.0 & $0 . \varepsilon 1$ & $0 \cdot \varepsilon \mathrm{II}$ & 0.81 & 0.41 & 7.6 & $8 \%$ & $5 \cdot 9$ & $2 \cdot 9$ & & TIOS IHOSTEzOJ \\
\hline $0.0022 . \angle 6$ & 0.0 & 0.0 & 0.0 & 0.0 & 0.0 & 0.0 & 0.0 & $0 \cdot \varepsilon \mathfrak{l}$ & $0 . \mathrm{EI}$ & $0.8 t$ & $0 . \pi$ & 1.6 & Q.2 & 5.9 & $2 \cdot 5$ & so & 710S 21100 \\
\hline
\end{tabular}

\begin{tabular}{|c|c|c|c|c|c|c|c|c|c|c|c|c|c|c|c|c|c|}
\hline $6 .\{080 \theta$ & $2 \cdot 16 L \varepsilon$ & $2 \cdot 16 L E$ & $2 \cdot 76 L \varepsilon$ & o.srze & $\varepsilon \cdot 6 L L Z$ & L'EErz & 6.0602 & O.590L & $\varepsilon \cdot 0989$ & $2.6 r 59$ & s.soos & $6 \cdot 8 \angle 01$ & 9.5otr & O.LQLE & $0.16 L 2$ & & 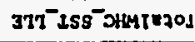 \\
\hline $0.00 R E .82869$ & $\varepsilon \cdot 6 L 2 \varepsilon$ & $8.6 L 2 E$ & $\varepsilon \cdot 6 \angle Z E$ & $9 \cdot 1002$ & $\tau \cdot z 012$ & resotz & $\tau \cdot \angle 0 \theta \tau$ & $6.90 t 9$ & 8.6265 & .0995 & $9 \cdot 2$ TOS & s.212t & g. LT9E & $T \cdot \varepsilon L Z \varepsilon$ & s.stbz & & T33LS HSTEวOJ \\
\hline $0.00 t \varepsilon .0 Z 869$ & $E \cdot 6 L 2 \varepsilon$ & $\varepsilon .6 \iota z \varepsilon$ & $\varepsilon \cdot \sigma L 2 \varepsilon$ & $9 \cdot 1082$ & $x \cdot 2012$ & $1 \cdot$ rotz & $t \cdot 200 t$ & 6.9019 & 8.6265 & 1.0995 & $9 \cdot 2105$ & s.2t2! & 9.2TgE & $t \cdot \varepsilon<2 \varepsilon$ & s.sirz & 30 & 73329 HS \\
\hline $0.00 t s . s L 60 t$ & $8 \cdot 115$ & $0 \cdot 615$ & o.hts & E'orl & $\tau^{\circ} L L E$ & $z \cdot 0 \varepsilon \varepsilon$ & $L^{\circ} \Sigma \theta Z$ & 0.856 & 6.086 & L.808 & $0 . \angle B L$ & $c \cdot 199$ & 6.295 & D.ETs & $8 \cdot 628$ & & TKLSBk-al=zol \\
\hline $0.00 t 5.5 L 60 t$ & o.ris & 8.415 & B.his & E.arn & T. LLE & $z \cdot 0 \varepsilon \varepsilon$ & L.E्ट & $8 \cdot 856$ & 6.086 & ८००08 & $\theta \cdot \angle \theta L$ & $\varepsilon \cdot 199$ & 6.295 & O.ETs & $2.62 E$ & sa & TVג3H- $\mathbf{a}$ \\
\hline
\end{tabular}

\begin{tabular}{|c|c|c|c|c|c|c|c|c|c|c|c|c|c|c|c|c|c|}
\hline $9 \cdot \varepsilon$ & 0.0 & 0.0 & 0.0 & 0.0 & 0.0 & 0.0 & 0.0 & 0.0 & 0.0 & 0.0 & 0.0 & 0.0 & 0.0 & 0.0 & 0.0 & & 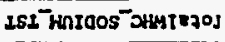 \\
\hline 0.0020 .1 & 0.0 & 0.0 & 0.0 & 0.0 & 0.0 & 0.0 & 0.0 & 0.0 & 0.0 & 0.0 & 0.0 & 0.0 & 0.0 & 0.0 & 0.0 & & $\left.T V 13 H-a[=\} O_{1}\right]$ \\
\hline 0.0010 .1 & 0.0 & 0.0 & 0.0 & 0.0 & 0.0 & 0.0 & 0.0 & 0.0 & 0.0 & 0.0 & 0.0 & 0.0 & 0.0 & 0.0 & 0.0 & $v$ & TVL3ßk-a \\
\hline $0.00 t 0.1$ & 0.0 & 0.0 & 0.0 & 0.0 & 0.0 & 0.0 & 0.0 & 0.0 & 0.0 & 0.0 & 0.0 & 0.0 & 0.0 & 0.0 & 0.0 & & TLH-NOH NI-QTEZOJ \\
\hline 0.0010 .1 & 0.0 & 0.0 & 0.0 & 0.0 & 0.0 & 0.0 & 0.0 & 0.0 & 0.0 & 0.0 & 0.0 & 0.0 & 0.0 & 0.0 & 0.0 & $v$ & TLH-HON NI-G \\
\hline $0.00 t+\cdot \tau$ & 0.0 & 0.0 & 0.0 & 0.0 & 0.0 & 0.0 & 0.0 & 0.0 & 0.0 & 0.0 & 0.0 & 0.0 & 0.0 & 0.0 & $0 \% 0$ & & $23 \mathrm{H}-\mathrm{Q}\{27 \mathrm{O}$ \\
\hline $0.05 \quad 2.0$ & 0.0 & 0.0 & 0.0 & 0.0 & 0.0 & 0.0 & 0.0 & 0.0 & 0.0 & 0.0 & 0.0 & 0.0 & 0.0 & 0.0 & 0.0 & a & \\
\hline $0.05 \quad 1.0$ & 0.0 & 0.0 & 0.0 & 0.0 & 0.0 & 0.0 & 0.0 & 0.0 & 0.0 & 0.0 & 0.0 & 0.0 & 0.0 & 0.0 & 0.0 & $\mathbf{v}$ & $\mathbf{L} 3 \mathrm{H}-\mathrm{G}$ \\
\hline
\end{tabular}

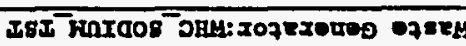

\begin{tabular}{|c|c|c|c|c|c|c|c|c|c|c|c|c|c|c|c|c|c|}
\hline $8 \cdot \varepsilon$ & $0 \% 0$ & 0.0 & 0.0 & 0.0 & 0.0 & 0.0 & 0.0 & 0.0 & 0.0 & 0.0 & 0.0 & 0.0 & 0.0 & 0.0 & 0.0 & & 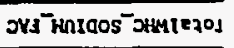 \\
\hline 0.0016 .8 & 0.0 & 0.0 & 0.0 & 0.0 & 0.0 & 0.0 & 0.0 & 0.0 & 0.0 & 0.0 & 0.0 & 0.0 & 0.0 & 0.0 & 0.0 & & I3H-QIEZC: \\
\hline$\because 6 \varepsilon \quad\llcorner .0$ & 0.0 & 0.0 & 0.0 & 0.0 & 0.0 & 0.0 & 0.0 & 0.0 & 0.0 & 0.0 & 0.0 & 0.0 & 0.0 & 0.0 & 0.0 & SMON & \\
\hline S.09 $\tau$ & 0.0 & 0.0 & 0.0 & 0.0 & 0.0 & 0.0 & 0.0 & 0.0 & 0.0 & 0.0 & 0.0 & 0.0 & 0.0 & 0.0 & 0.0 & 9 & $\stackrel{13 H-Q}{-Q}$ \\
\hline 0.0016 .1 & 0.0 & 0.0 & 0.0 & 0.0 & 0.0 & 0.0 & 0.0 & 0.0 & 0.0 & 0.0 & 0.0 & 0.0 & 0.0 & 0.0 & 0.0 & & I \\
\hline
\end{tabular}

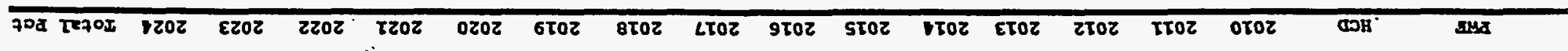

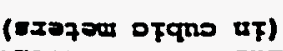

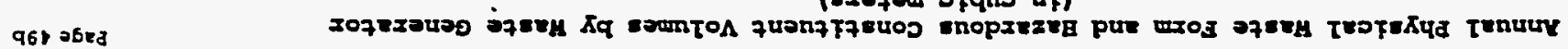




\begin{tabular}{|c|c|c|c|c|c|c|c|c|c|c|c|c|c|c|c|c|c|}
\hline PWF & HCD & 2010 & 2011 & 2012 & 2013 & 2014 & 2015 & 2016 & 2017 & 2018 & 2019 & 2020 & 2021 & 2022 & 2023 & 2024 & Total Pot \\
\hline D-METAL & DE & 6.3 & 7.9 & 9.5 & 11.1 & 13.5 & 15.9 & 25.9 & 15.9 & 0.0 & 0.0 & 0.0 & 0.0 & 0.0 & 0.0 & 0.0 & $118.8 \quad 10.7$ \\
\hline . & NONE & 9.2 & 11.6 & 13.9 & 16.2 & 19.7 & 23.2 & 23.2 & 23.2 & 0.0 & 0.0 & 0.0 & 0.0 & 0.0 & 0.0 & 0.0 & $172.8 \quad 59.2$ \\
\hline 'Bt,aLU-METAL & & 15.6. & 29.5 & 23.4 & 27.4 & $33 . \dot{2}$ & 39.1 & 39.1 & 39.1 & 0.0 & 0.0 & 0.0 & 0.0 & 0.0 & 0.0 & 0.0 & 291.7100 .0 \\
\hline \multirow[t]{2}{*}{ IN ORG LQ/SLG } & $D F$ & 0.5 & 0.7 & 0.8 & 1.0 & 1.2 & 1.4 & 1.1 & 1.4 & 0.0 & 0.0 & 0.0 & 0.0 & 0.0 & 0.0 & 0.0 & $10.8 \quad 66.6$ \\
\hline & MONE & 0.2 & 0.3 & 0.1 & 0.5 & 0.6 & 0.7 & 0.7 & 0.7 & 0.0 & $0: 0$ & 0.0 & 0.0 & 0.0 & 0.0 & 0.0 & 5.433 .3 \\
\hline IOt,al IN ORG LQ/SLG & & 0.8 & 1.0 & 1.3 & 2.5 & 1.8 & 2.1 & 2.1 & 2.1 & 0.0 & 0.0 & 0.0 & 0.0 & 0.0 & 0.0 & 0.0 & 16.2100 .0 \\
\hline \multirow[t]{2}{*}{ ORG PART } & DE & 0.5 & 0.7 & 0.0 & 1.0 & 1.2 & 1.4 & 1.4 & 1.4 & 0.0 & 0.0 & 0.0 & 0.0 & 0.0 & 0.0 & 0.0 & $10.8 \quad 66.6$ \\
\hline & NONE & 0.2 & 0.3 & 0.1 & 0.5 & 0.6 & 0.7 & 0.1 & 0.7 & 0.0 & 0.0 & 0.0 & 0.0 & 0.0 & 0.0 & 0.0 & 5.133 .3 \\
\hline rotaloRG PART & & 0.0 & 1.0 & 2.3 & 1.5 & 1.0 & 2.1 & 2.1 & 2.1 & 0.0 & 0.0 & 0.0 & 0.0 & 0.0 & 0.0 & 0.0 & 16.2100 .0 \\
\hline TOt,aLWHC_SST_PET & & 97.0 & 108.0 & 130.5 & 152.3 & 184.9 & 217.5 & 217.5 & 217.5 & 0.0 & 0.0 & 0.0 & 0.0 & 0.0 & 0.0 & 0.0 & 1620.8 \\
\hline \multicolumn{18}{|c|}{ Waste Generator:FHC_s8_mn } \\
\hline \multirow[t]{2}{*}{ BATTERIES } & B & 0.0 & 0.0 & 0.0 & 0.0 & 0.0 & 0.0 & 0.0 & 0.0 & 0.0 & 0.0 & 0.0 & 0.0 & 0.0 & 0.0 & 0.0 & $2.2 \quad 69.9$ \\
\hline & D & 0.0 & 0.0 & 0.0 & .0 .0 & 0.0 & 0.0 & 0.0 & 0.0 & 0.0 & 0.0 & 0.0 & 0.0 & 0.0 & 0.0 & 0.0 & 0.930 .0 \\
\hline rotal BATTERIES & & 0.1 & 0.1 & 0.1 & 0.1 & 0.1 & 0.1 & 0.1 & 0.1 & 0.1 & 0.1 & 0.1 & 0.1 & 0.2 & 0.2 & 0.1 & 3.2100 .0 \\
\hline \multirow[t]{3}{*}{ LAB/CONT LQ } & $A$ & 0.1 & 0.1 & 0.1 & 0.1 & 0.1 & 0.1 & 0.1 & 0.2 & 0.1 & 0.1 & 0.1 & 0.1 & 0.1 & 0.1 & 0.1 & $\begin{array}{ll}5.5 & 17.4\end{array}$ \\
\hline & B & 0.0 & 0.0 & 0.0 & 0.0 & 0.0 & 0.0 & 0.0 & 0.0 & 0.0 & 0.0 & 0.0 & 0.0 & 0.0 & 0.0 & 0.0 & $0.1 \quad 4.1$ \\
\hline & $\mathbf{r}$ & 0.2 & 0.1 & 0.1 & 0.2 & 0.1 & 0.1 & 0.1 & 0.1 & 0.1 & 0.1 & 0.1 & 0.1 & 0.1 & 0.1 & 0.1 & $5.6 \quad 48.4$ \\
\hline Tota1 LAB/CONT LQ & & 0.3 & 0.3 & 0.3 & 0.3 & 0.3 & 0.3 & 0.3 & 0.3 & 0.3 & 0.3 & 0.3 & 0.3 & 0.3 & 0.3 & 0.3 & 11.7100 .0 \\
\hline TotalWHIC_SS_MAIN & & 0.5 & 0.5 & 0.5 & 0.5 & 0.5 & 0.5 & 0.5 & 0.5 & 0.5 & 0.5 & 0.5 & 0.5 & 0.5 & 0.5 & 0.5 & 15.0 \\
\hline \multicolumn{18}{|c|}{ Faste Generatox:FHC_sTRND_LNB } \\
\hline \multirow[t]{2}{*}{ BATTERIES } & B & 0.0 & 0.0 & 0.0 & 0.0 & 0.0 & 0.0 & 0.0 & 0.0 & 0.0 & 0.0 & 0.0 & 0.0 & 0.0 & 0.0 & 0.0 & $2.8 \quad 10.0$ \\
\hline & D & 0.0 & 0.0 & 0.0 & 0.0 & 0.0 & 0.0 & 0.0 & 0.0 & 0.0 & 0.0 & 0.0 & 0.0 & 0.0 & 0.0 & 0.0 & 1.230 .0 \\
\hline TOtal BATTERIES & & 0.1 & 0.1 & 0.1 & 0.2 & 0.1 & 0.2 & 0.1 & -0.1 & 0.1 & 0.1 & 0.1 & 0.1 & 0.2 & 0.1 & 0.1 & 1.0100 .0 \\
\hline \multirow[t]{2}{*}{ D-COMBUST } & $B$ & 0.0 & 0.0 & 0.0 & 0.0 & 0.0 & 0.0 & 0.0 & 0.0 & 0.0 & 0.0 & 0.0 & 0.0 & 0.0 & 0.0 & 0.0 & 2.050 .0 \\
\hline & D & 0.0 & 0.0 & 0.0 & 0.0 & 0.0 & 0.0 & 0.0 & 0.0 & 0.0 & 0.0 & 0.0 & 0.0 & 0.0 & 0.0 & 0.0 & 1.025 .0 \\
\hline . & $\mathbf{F}$ & 0.0 & 0.0 & 0.0 & 0.0 & 0.0 & 0.0 & 0.0 & 0.0 & 0.0 & 0.0 & 0.0 & 0.0 & 0.0 & 0.0 & 0.0 & 1.025 .0 \\
\hline rota1D-COMBUST & & 0.1 & 0.1 & 0.1 & 0.1 & 0.1 & 0.1 & 0.1 & 0.1 & 0.1 & 0.1 & 0.1 & 0.1 & 0.1 & 0.1 & 0.1 & 4.0100 .0 \\
\hline \multirow[t]{2}{*}{ IN ORG LO/SLG } & D & 0.0 & 0.0 & 0.0 & 0.0 & 0.0 & 0.0 & 0.0 & 0.0 & 0.0 & 0.0 & 0.0 & 0.0 & 0.0 & 0.0 & 0.0 & 1.750 .0 \\
\hline & $\mathbf{E}$ & 0.0 & 0.0 & 0.0 & 0.0 & 0.0 & 0.0 & 0.0 & 0.0 & 0.0 & 0.0 & 0.0 & 0.0 & 0.0 & 0.0 & 0.0 & 1.750 .0 \\
\hline rotal IN ORG LQ/SLG & & 0.1 & 0.1 & 0.1 & 0.1 & 0.1 & 0.1 & 0.1 & 0.1 & 0.1 & 0.1 & 0.1 & 0.1 & 0.1 & 0.1 & 0.1 & 3.5100 .0 \\
\hline \multirow[t]{2}{*}{ IN ORG PART } & D & 0.0 & 0.0 & 0.0 & 0.0 & 0.0 & 0.0 & 0.0 & 0.0 & 0.0 & 0.0 & 0.0 & 0.0 & 0.0 & 0.0 & 0.0 & 0.150 .0 \\
\hline & $\mathbf{F}$ & 0.0 & 0.0 & 0.0 & 0.0 & 0.0 & 0.0 & 0.0 & 0.0 & 0.0 & 0.0 & 0.0 & 0.0 & 0.0 & 0.0 & 0.0 & 0.150 .0 \\
\hline Total IN ORG PART & & 0.0 & 0.0 & 0.0 & 0.0 & 0.0 & 0.0 & 0.0 & 0.0 & 0.0 & 0.0 & 0.0 & 0.0 & 0.0 & 0.0 & 0.0 & 0.2100 .0 \\
\hline
\end{tabular}

gCD Codas: A-Ignitables, B-Corrosives, C=Reactives, D-Metals w/O Hg, Em-Metals w Hg, F-Organics, G=State Reg.. H-PCB < 50ppm, J-PCB > 50ppm 
Annual Phyaical Fante Form and Bazardous Constituent Volumes by Waste Generator

(in cublo weters)

\begin{tabular}{|c|c|c|c|c|c|c|c|c|c|c|c|c|c|c|c|c|c|}
\hline FWE & HCD & 2010 & 2011 & 2012 & 2013 & 2014 & 2015 & 2016 & 2017 & 2018 & 2019 & 2020 & 2021 & 2022 & 2023 & 2024 & Total Pot \\
\hline \multirow[t]{3}{*}{ LAB/CONT LO } & $\boldsymbol{A}$ & 0.1 & 0.1 & 0.2 & 0.1 & 0.1 & 0.1 & 0.1 & 0.1 & 0.1 & 0.1 & 0.1 & 0.1 & 0.1 & 0.1 & 0.1 & $5.5 \quad 15.6$ \\
\hline & B & 0.0 & 0.0 & 0.0 & 0.0 & 0.0 & 0.0 & 0.0 & 0.0 & 0.0 & 0.0 & 0.0 & 0.0 & 0.0 & 0.0 & 0.0 & $0.8 \quad 7.2$ \\
\hline & $\mathbf{E}$ & 0.2 & 0.1 & 0.1 & 0.1 & 0.1 & 0.1 & 0.1 & 0.1 & 0.1 & 0.1 & 0.1 & 0.1 & 0.1 & 0.1 & 0.1 & $5.7 \quad 17.1$ \\
\hline \multicolumn{2}{|l|}{ SOt.alLAB/CONT LQ } & 0.4 & 0.4 & 0.4 & 0.4 & 0.1 & 0.4 & 0.4 & 0.1 & 0.1 & 0.4 & 0.4 & 0.1 & 0.1 & 0.1 & 0.4 & 12.1100 .0 \\
\hline \multicolumn{2}{|l|}{ TOt, IKHC_STAND_LAB } & 0.0 & 0.0 & 0.8 & 0.0 & 0.0 & 0.8 & 0.0 & 0.8 & 0.0 & 0.8 & 0.0 & 0.0 & 0.8 & 0.8 & 0.0 & 24.0 \\
\hline \multicolumn{18}{|c|}{ Waste Generator:FriC_sURPLs_mac } \\
\hline \multirow[t]{6}{*}{ CONT SOIL } & A & 3.6 & 2.4 & 1.9 & 1.1 & 0.0 & 0.0 & 0.0 & 0.0 & 0.0 & 0.0 & 0.0 & 0.0 & 0.0 & 0.0 & 0.0 & $i 66.6$ \\
\hline & 8 & 12.3 & 7.6 & 5.2 & 3.3 & 0.0 & 0.0 & 0.0 & 0.0 & 0.0 & 0.0 & 0.0 & 0.0 & 0.0 & 0.0 & 0.0 & 410.5 \\
\hline & D & 14.3 & 9.0 & 6.4 & 4.2 & 0.0 & 0.0 & 0.0 & 0.0 & 0.0 & 0.0 & 0.0 & 0.0 & 0.0 & 0.0 & 0.0 & 522.5 \\
\hline & $\mathbf{r}$ & 2.0 & 1.4 & 2.1 & 0.9 & 0.0 & 0.0 & 0.0 & 0.0 & 0.0 & 0.0 & 0.0 & 0.0 & 0.0 & 0.0 & 0.0 & 103.9 \\
\hline & H & 0.3 & 0.3 & 0.3 & 0.3 & 0.0 & 0.0 & 0.0 & 0.0 & 0.0 & 0.0 & 0.0 & 0.0 & 0.0 & 0.0 & 0.0 & $\begin{array}{ll}11.3 & 0.0\end{array}$ \\
\hline & MONE & 416.8 & 75.7 & 35.3 & 33.3 & 0.0 & 0.0 & 0.0 & 0.0 & 0.0 & 0.0 & 0.0 & 0.0 & 0.0 & 0.0 & 0.0 & $146910.6 \quad 99.1$ \\
\hline \multicolumn{2}{|l|}{ rotalcont soIt } & 179.6 & 96.7 & 30.1 & 45.9 & 0.0 & 0.0 & 0.0 & 0.0 & 0.0 & 0.0 & 0.0 & 0.0 & 0.0 & 0.0 & 0.0 & 148171.6100 .0 \\
\hline \multirow[t]{6}{*}{ D-carbust } & $\lambda$ & 4.0 & 2.0 & 1.8 & 1.0 & 0.0 & 0.0 & 0.0 & 0.0 & 0.0 & 0.0 & 0.0 & 0.0 & 0.0 & 0.0 & 0.0 & 104.12 .9 \\
\hline & B & 19.2 & 11.3 & 7.4. & 4.3 & 0.0 & 0.0 & 0.0 & 0.0 & 0.0 & 0.0 & 0.0 & 0.0 & 0.0 & 0.0 & 0.0 & 116.711 .6 \\
\hline & D & 23.6 & 23.0 & 9.0 & 3.0 & 0.0 & 0.0 & 0.0 & 0.0 & 0.0 & 0.0 & 0.0 & 0.0 & 0.0 & 0.0 & 0.0 & 194.913 .0 \\
\hline & $\mathbf{r}$ & 0.2 & 0.2 & 0.2 & 0.2 & 0.0 & 0.0 & 0.0 & 0.0 & 0.0 & 0.0 & 0.0 & 0.0 & 0.0 & 0.0 & 0.0 & 20.90 .5 \\
\hline & s & 0.0 & 0.0 & 0.0 & 0.0 & 0.0 & 0.0 & 0.0 & 0.0 & 0.0 & 0.0 & 0.0 & 0.0 & 0.0 & 0.0 & 0.0 & $\begin{array}{ll}4.9 & 0.1\end{array}$ \\
\hline & NONE & 7.7 & 1.3 & 0.6 & 0.6 & 0.0 & 0.0 & 0.0 & 0.0 & 0.0 & 0.0 & 0.0 & 0.0 & 0.0 & 0.0 & 0.0 & 2532.970 .9 \\
\hline \multicolumn{2}{|l|}{ rotalD-Carbust } & 55.7 & 29.7 & 19.3 & 21.4 & 0.0 & 0.0 & 0.0 & 0.0 & 0.0 & 0.0 & 0.0 & 0.0 & 0.0 & 0.0 & 0.0 & 3574.7100 .0 \\
\hline \multirow[t]{4}{*}{ D-HET } & B & 1.5 & 1.5 & 1.5 & 1.5 & 0.0 & 0.0 & 0.0 & 0.0 & 0.0 & 0.0 & 0.0 & 0.0 & 0.0 & 0.0 & 0.0 & $19.1,2.9$ \\
\hline & $D$ & 3.1 & 1.7 & 1.5 & 1.1 & 0.0 & 0.0 & 0.0 & 0.0 & 0.0 & 0.0 & 0.0 & 0.0 & 0.0 & 0.0 & 0.0 & $136.7^{\circ} \quad 0.1$ \\
\hline & $\mathbf{F}$ & 0.0 & 0.0 & 0.0 & 0.0 & 0.0 & 0.0 & 0.0 & 0.0 & 0.0 & 0.0 & 0.0 & 0.0 & 0.0 & 0.0 & 0.0 & $0.7 \quad 0.0$ \\
\hline & NONE & 9.5 & 6,3 & 5.9 & 5.9 & 0.0 & 0.0 & 0.0 & 0.0 & 0.0 & 0.0 & 0.0 & 0.0 & 0.0 & 0.0 & 0.0 & $1497.3 \quad 08.9$ \\
\hline \multicolumn{2}{|l|}{ Tota1 D-HET } & 16.3 & 12.6 & 12.1 & 11.9 & 0.0 & 0.0 & 0.0 & 0.0 & 0.0 & 0.0 & 0.0 & 0.0 & 0.0 & 0.0 & 0.0 & 1683.0100 .0 \\
\hline \multirow[t]{5}{*}{ D-IN NON-MII } & $A$ & 4.6 & 2.5 & 1.4 & 0.6 & 0.0 & 0.0 & 0.0 & 0.0 & 0.0 & 0.0 & 0.0 & 0.0 & 0.0 & 0.0 & 0.0 & 91.30 .0 \\
\hline & B & 18.7 & 20.2 & 5.9 & 2.5 & 0.0 & 0.0 & 0.0 & 0.0 & 0.0 & 0.0 & 0.0 & 0.0 & 0.0 & 0.0 & 0.0 & 377.5 \\
\hline & D & 20.9 & 11.3 & 6.6 & 2.7 & 0.0 & 0.0 & 0.0 & 0.0 & 0.0 & 0.0 & 0.0 & 0.0 & 0.0 & 0.0 & 0.0 & 415.6 \\
\hline & $\mathbf{E}$ & 2.4 & 1.3 & 0.8 & 0.4 & 0.0 & 0.0 & 0.0 & 0.0 & 0.0 & 0.0 & 0.0 & 0.0 & 0.0 & 0.0 & 0.0 & $\begin{array}{ll}56.2 & 0.0\end{array}$ \\
\hline & None & 13500.7 & 3242.1 & 1578.4 & 1646.0 & 0.0 & 0.0 & 0.0 & 0.0 & 0.0 & 0.0 & 0.0 & 0.0 & 0.0 & 0.0 & & 745211.299 .8 \\
\hline \multicolumn{2}{|l|}{ TOtalD-IN NON-HTL } & 13635.5 & 3267.8 & 1593.3 & 2652.3 & 0.0 & 0.0 & 0.0 & 0.0 & 0.0 & 0.0 & 0.0 & 0.0 & 0.0 & 0.0 & 0.0 & 746055.0100 .0 \\
\hline \multirow[t]{4}{*}{ D-METAL } & $A$ & 0.2 & 0.2 & 0.2 & 0.2 & 0.0 & 0.0 & 0.0 & 0.0 & 0.0 & 0.0 & 0.0 & 0.0 & 0.0 & 0.0 & 0.0 & $19.6 \quad 3.3$ \\
\hline & B & 10.2 & 5.9 & 3.0 & 2.2 & 0.0 & 0.0 & 0.0 & 0.0 & 0.0 & 0.0 & 0.0 & 0.0 & 0.0 & 0.0 & 0.0 & $235.2 \quad 40.0$ \\
\hline & $D$ & 11.5 & 6.6 & 4.2 & 2.2 & 0.0 & 0.0 & 0.0 & 0.0 & $0.0^{\circ}$ & 0.0 & 0.0 & 0.0 & 0.0 & 0.0 & 0.0 & $257.5 \quad 43.7$ \\
\hline & $\mathbf{r}$ & 3.5 & 2.0 & 1.2 & 0.6 & 0.0 & 0.0 & 0.0 & 0.0 & 0.0 & 0.0 & 0.0 & 0.0 & 0.0 & 0.0 & 0.0 & $75.7 \quad 12.8$ \\
\hline Tot:AI D-METAL & & 25.3 & 14.0 & 9.5 & 5.2 & 0.0 & 0.0 & 0.0 & 0.0 & 0.0 & 0.0 & 0.0 & 0.0 & 0.0 & 0.0 & 0.0 & 580.0100 .0 \\
\hline
\end{tabular}

BCO Codes: A-Ignitables, 8-Corţosives, C-Reactivos, D-Motals w/O Hg, E-Metals w Hg, E-Organics, G-Stato Reg.. H-PCB < 50ppm, J-PCB > 50ppm 


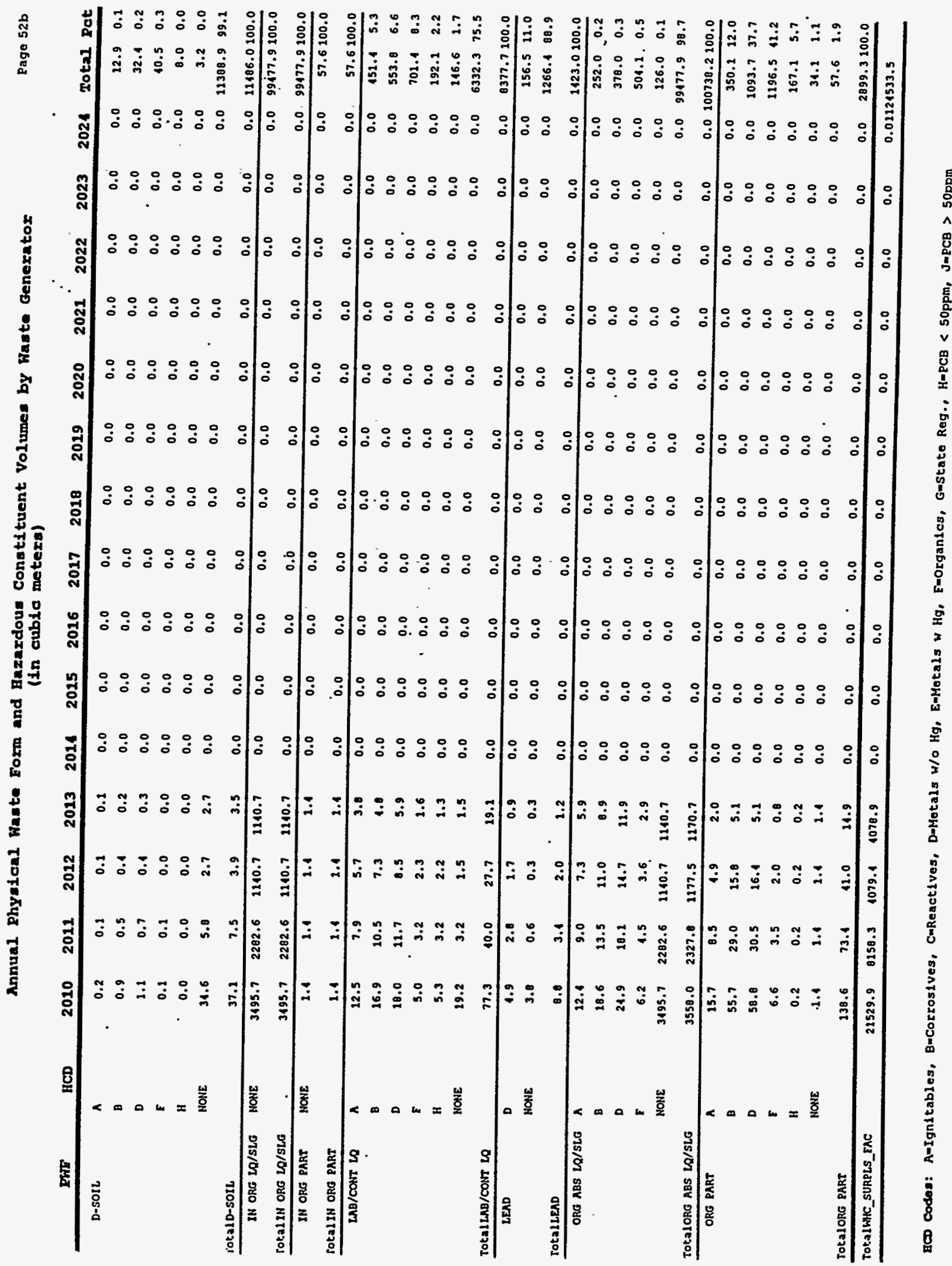




\begin{tabular}{|c|c|c|c|c|c|c|c|c|c|c|c|c|c|c|c|c|c|}
\hline BATTERIES & BDF & 0.7 & 0.7 & 0.7 & 0.7 & 0.7 & 0.7 & 0.7 & 0.7 & 0.7 & 0.7 & 0.7 & 0.7 & 0.7 & - 0.7 & 0.7 & 22.6200 .0 \\
\hline Total BATTERIES & & 0.7 & 0.7 & 0.7 & 0.7 & 0.7 & 0.7 & 0.7 & 0.7 & 0.7 & 0.7 & 0.7 & 0.7 & 0.7 & 0.7 & 0.7 & 22.6100 .0 \\
\hline \multirow[t]{2}{*}{ CONT SOIL } & Ae & 3.0 & 3.0 & 3.0 & $\cdot 3.0$ & 3.0 & 3.0 & 3.0 & 3.0 & 3.0 & 3.0 & 3.0 & 3.0 & 3.0 & 3.0 & 3.0 & $92.1 \quad 30.0$ \\
\hline & Dr & 7.2 & 7.2 & 7.2 & 7.2 & 7.2 & 7.2 & 7.2 & 7.2 & 7.2 & 7.2 & 7.2 & 7.2 & 7.2 & 7.2 & 7.2 & 215.770 .0 \\
\hline rotalCoNt soll & & 10.3 & 10.3 & 10.3 & 20.3 & 10.3 & 10.3 & 10.3 & 10.3 & 10.3 & 10.3 & 20.3 & 10.3 & 10.3 & 10.3 & 10.3 & 308.1100 .0 \\
\hline \multirow[t]{3}{*}{ D-carisust } & $\mathrm{ABDF}$ & 7.5 & 7.5 & 7.5 & 7.5 & 7.5 & 7.5 & 7.5 & 7.5 & 7.5 & 7.5 & 7.5 & 7.5 & 7.5 & 7.5 & 7.5 & 226.13 .0 \\
\hline & DE & 75.2 & 75.2 & 75.2 & 75.2 & 75.2 & 75.2 & 75.2 & 75.2 & 25.2 & 75.2 & 75.2 & 75.2 & 75.2 & 75.2 & 75.2 & $2250.6 \quad 30.2$ \\
\hline & MONE & 166.3 & 166.3 & 166.3 & 166.3 & 166.3 & 166.3 & 166.3 & 166.3 & 166.3 & 166.3 & 166.3 & 166.3 & 166.3 & 166.3 & 166.3 & $1974.8 \quad 66.7$ \\
\hline rotalD-COABUST & & 249.1 & 219.1 & 219.1 & 249.1 & 249.1 & 249.1 & 249.1 & 249.1 & 219.1 & 249.1 & 249.1 & 219.1 & 249.1 & 249.1 & 249.1 & 7451.5100 .0 \\
\hline D-HET & DE & 1.8 & 4.8 & .4 .0 & 1.0 & 4.8 & 1.0 & 1.0 & 1.8 & 4.8 & 1.8 & 4.8 & 4.8 & 1.8 & 4.0 & 4.8 & 114.0100 .0 \\
\hline TotalD-HET & & 1.8 & 4.8 & 1.8 & 1.8 & 4.0 & 4.8 & 4.0 & 1.8 & 1.8 & 1.8 & 1.8 & 4.8 & 1.8 & 4.8 & 4.8 & 114.8100 .0 \\
\hline \multirow[t]{3}{*}{ D-IN HON-HTL } & $\lambda c$ & 2.5 & 2.5 & 2.5 & 2.5 & 2.5 & 2.5 & 2.5 & 2.5 & 2.5 & 2.5 & 2.5 & 2.5 & 2.5 & 2.5 & 2.5 & $77.5 \quad 2.8$ \\
\hline & Dr & 35.0 & 35.0 & 35.0 & 35.0 & 35.0 & 35.0 & 35.0 & 35.0 & 35.0 & 35.0 & 35.0 & 35.0 & 35.0 & 35.0 & 35.0 & $1047.7 \quad 39.0$ \\
\hline & NONE & 52.0 & 52.0 & 52.0 & 52.0 & 52.0 & $52.0^{\circ}$ & 52.0 & 52.0 & 52.0 & 52.0 & 52.0 & 52.0 & 52.0 & 52.0 & 32.0 & $1557.6 \quad 38.0$ \\
\hline rotalD-IN NON-HTL & & 09.7 & 89.7 & 09.7 & 09.7 & 89.7 & 99.7 & 69.7 & 89.7 & 89.7 & 89.7 & 89.7 & 89.7 & 09.7 & 89.7 & 09.7 & 2682.9100 .0 \\
\hline \multirow[t]{2}{*}{ D-METAL } & DF & 55.2 & 55.2 & 55.2 & 55.2 & 55.2 & 55.2 & 55.2 & 55.2 & 55.2 & 55.2 & 35.2 & 55.2 & 55.2 & 35.2 & 35.2 & $2652.1 \quad 12.1$ \\
\hline & NONE & 75.7 & 75.7 & 25.7 & 75.7 & 75.7 & 75.7 & 75.7 & 75.7 & 75.7 & 75.7 & 75.7 & 75.7 & 75.7 & 75.7 & 75.7 & $2265.7 \quad 57.8$ \\
\hline Total D-METAL & & 230.9 & 230.9 & 130.9 & 230.9 & 130.9 & 230.9 & 130.9 & 130.9 & 230.9 & 130.9 & 130.9 & 130.9 & 130.9 & 130.9 & 130.9 & 3917.0100 .0 \\
\hline EXPLOSIVES & ABDF & 2.2 & 2.2 & 2.2 & 2.2 & 2.2 & 2.2 & 2.2 & 2.2 & 2.2 & 2.2 & 2.2 & 2.2 & 2.2 & 2.2 & 2.2 & 67.8100 .0 \\
\hline TotalexpLosives & & 2.2 & 2.2 & 2.2 & 2.2 & 2.2 & 2.2 & 2.2 & 2.2 & 2.2 & 2.2 & 2.2 & 2.2 & 2.2 & 2.2 & 2.2 & 67.0 i00.0 \\
\hline \multirow[t]{3}{*}{ IN ORG LQ/SLG } & AGDF & 3.7 & 3.7 & 3.1 & 3.7 & 3.7 & 3.7 & 3.7 & 3.7 & 3.7 & 3.7 & 3.7 & 3.7 & 3.7 & 3.7 & 3.7 & $113.0 \quad 17.1$ \\
\hline & $D E$ & 1.0 & 1.0 & 1.0 & 1.0 & 1.8 & 1.0 & 1.0 & 1.0 & 1.8 & 1.8 & 1.0 & 1.0 & 1.8 & 1.8 & 1.8 & $55.9^{\circ} 23.3$ \\
\hline & NONE & 2.3 & 2.3 & 2.3 & 2.3 & 2.3 & 2.3 & 2.3 & 2.3 & 2.3 & 2.3 & 2.3 & 2.3 & 2.3 & 2.3 & 2.3 & $70.8 \quad 29.5$ \\
\hline Total IN ORG LQ/SLG & & 0.0 & 8.0 & 8.0 & 8.0 & 0.0 & 0.0 & 0.0 & 0.0 & 0.0 & 0.0 & 8.0 & 0.0 & 0.0 & 0.0 & 0.0 & 239.7100 .0 \\
\hline LAB/CONT LO & ABOF & 13.2 & 13.2 & 13.2 & 13.2 & 13.2 & 13.2 & 23.2 & 23.2 & 13.2 & 23.2 & 13.2 & 13.2 & 13.2 & 13.2 & 13.2 & 395.6100 .0 \\
\hline TOtA1 LAB/CONT LQ & & 23.2 & 13.2 & 13.2 & 13.2 & 23.2 & 13.2 & 13.2 & 13.2 & 13.2 & 13.2 & 13.2 & 23.2 & 13.2 & 13.2 & 13.2 & 395.6100 .0 \\
\hline LEAD & ADF & 0.3 & 0.3 & 0.3 & 0.3 & 0.3 & 0.3 & 0.3 & 0.3 & 0.3 & 0.3 & 0.3 & 0.3 & 0.3 & 0.3 & 0.3 & 21.3100 .0 \\
\hline Total LEAD. & & 0.3 & 0.3 & 0.3 & 0.3 & 0.3 & 0.3 & 0.3 & 0.3 & 0.3 & 0.3 & 0.3 & 0.3 & 0.3 & 0.3 & 0,3 & 11.3100 .0 \\
\hline MERCURY & $\bar{E}$ & 0.3 & 0.3 & 0.3 & 0.3 & 0.3 & 0.3 & 0.3 & 0.3 & 0.3 & 0.3 & 0.3 & 0.3 & 0.3 & 0.3 & 0.3 & 21.3100 .0 \\
\hline TOtalMERCURY & . & 0.3 & 0.3 & 0.3 & 0.3 & 0.3 & 0.3 & 0.3 & 0.3 & 0.3 & 0.3 & 0.3 & 0.3 & 0.3 & 0.3 & 0.3 & 21.3100 .0 \\
\hline ORG ABS LQ/SLG & $A B D E$ & 7.3 & 7.5 & 7.5 & 7.5 & 7.5 & 7.5 & 7.5 & 7.5 & 7.5 & 7.5 & 7.5 & 7.5 & 7.5 & 7.5 & 7.5 & 226.1100 .0 \\
\hline TOtalORG ABS LQ/SLG & & 7.5 & 7.5 & 7.5 & 7.5 & 7.5 & 7.5 & 7.5 & 7.5 & 7.5 & 7.5 & 7.5 & 7.5 & 7.5 & 7.5 & 7.5 & 226.1100 .0 \\
\hline ORG PART & ABDF & 1.8 & 1.8 & 1.0 & 1.8 & 1.8 & 1.8 & 2.0 & 1.8 & 1.8 & 1.8 & 1.0 & 1.8 & 1.8 & 1.8 & 1.0 & $56.5 \quad 30.8$ \\
\hline
\end{tabular}

BCD Codes: A-Ignitables, B-Cortosives, C-Reactives, D-Metals w/O Hg, E-Metals w Hg, Em-Organles, G-State Reg., H-PCB < 50ppm, J-PCB > 50ppm 
(In cubic meters)

\begin{tabular}{|c|c|c|c|c|c|c|c|c|c|c|c|c|c|c|c|c|c|}
\hline PWE & HCD & 2010 & 2011 & 2012 & 2013 & 2014 & 2015 & 2016 & 2017 & 2018 & 2019 & 2020 & 2021 & 2022 & 2023 & 2024 & Total Pot \\
\hline & DE & 1.8 & 1.0 & 1.0 & 1.0 & $1 . \dot{8}$ & 1.0 & 1.8 & 1.0 & 1.8 & 1.0 & 1.0 & 1.0 & 1.0 & 1.8 & 1.8 & $55.9 \quad 30.5$ \\
\hline & MONE & 2.3 & 2.3 & 2.3 & 2.3 & 2.3 & 2.3 & 2.3 & 2.3 & 2.3 & 2.3 & 2.3 & 2.3 & 2.3 & 2.3 & 2.3 & $70.0 \quad 30.6$ \\
\hline IOL.al GP.G PART & & 6.1 & 6.1 & 6.1 & 6.1 & 6.1 & 6.1 & 6.1 & 6.1 & 6.1 & 6.1 & 6.1 & 6.1 & 6.1 & 6.1 & 6.1 & 103.2100 .0 \\
\hline rot:alWHC_TE_OPER & & 523.7 & 523.7 & 523.7 & 523.7 & 323.7 & 523.7 & 523.7 & 323.7 & 523.7 & 523.7 & 523.7 & 523.7 & 523.7 & 523.7 & 323.7 & 15663.3 \\
\hline
\end{tabular}

Waste Generator:FHC_IPANSPORT

\begin{tabular}{|c|c|c|c|c|c|c|c|c|c|c|c|c|c|c|c|c|c|}
\hline \multirow[t]{2}{*}{ D-cambust } & AG & 0.5 & 0.5 & 0.5 & 0.5 & 0.5 & 0.5 & 0.5 & 0.5 & 0.5 & 0.5 & 0.5 & 0.5 & 0.5 & 0.5 & 0.5 & $16.2 \quad 43.0$ \\
\hline & G & 0.7 & 0.7 & 0.7 & 0.7 & 0.7 & 0.7 & 0.7 & 0.7 & 0.7 & 0.7 & 0.7 & 0.7 & 0.7 & 0.7 & 0.7 & $21.5 \quad 57.0$ \\
\hline Tota1D-ConBust & & 1.2 & 1.2 & 1.2 & 1.2 & 1.2 & 1.2 & 1.2 & 1.2 & 1.2 & 1.2 & 1.2 & 1.2 & 1.2 & 1.2 & 1.2 & 37.8100 .0 \\
\hline IN ORG PART & AG & 0.2 & 0.2 & 0.2 & 0.2 & 0.2 & 0.2 & 0.2 & 0.2 & 0.2 & 0.2 & 0.2 & 0.2 & 0.2 & 0.2 & 0.2 & $6.9 \quad 13.0$ \\
\hline • & $6^{-}$ & 0.3 & 0.3 & 0.3 & 0.3 & 0.3 & 0.3 & 0.3 & 0.3 & 0.3 & 0.3 & 0.3 & 0.3 & 0.3 & 0.3 & 0.3 & 9.257 .0 \\
\hline rOtal IN ORE PART & & 0.5 & 0.5 & 0.5 & 0.5 & 0.5 & 0.5 & 0.5 & 0.5 & 0.5 & 0.5 & 0.5 & 0.5 & 0.5 & 0.5 & 0.5 & 16.2100 .0 \\
\hline TotalWHE_TRANSPORT & & 1.8 & 1.0 & 1.8 & 1.0 & 1.0 & 1.8 & 1.0 & 1.0 & 1.8 & 1.8 & 1.8 & 1.0 & 1.8 & 1.8 & 1.8 & 58.0 \\
\hline
\end{tabular}

\section{Waste Generator:FHC TRUSAT}

\begin{tabular}{|c|c|c|c|c|c|c|c|c|c|c|c|c|c|c|c|c|c|}
\hline D-CoHbust & NONE & 0.0 & 0.0 & 0.0 & 0.0 & 0.0 & 0.0 & 0.0 & 0.0 & 0.0 & 0.0 & 0.0 & 0.0 & 0.0 & 0.0 & 0.0 & 9.5100 .0 \\
\hline Tota1D-COMBUST & & 0.0 & 0.0 & 0.0 & 0.0 & 0.0 & 0.0 & 0.0 & 0.0 & 0.0 & 0.0 & 0.0 & 0.0 & 0.0 & 0.0 & 0.0 & 9.5100 .0 \\
\hline \multirow[t]{2}{*}{ D-HET } & c & 0.0 & 0.0 & 0.0 & 0.0 & 0.0 & 0.0 & 0.0 & 0.0 & 0.0 & 0.0 & 0.0 & 0.0 & 0.0 & 0.0 & 0.0 & $2.9 \quad 87.6$ \\
\hline & NONE & 0.0 & 0.0 & 0.0 & 0.0 & 0.0 & 0.0 & 0.0 & 0.0 & 0.0 & 0.0 & 0.0 & 0.0 & 0.0 & 0.0 & 0.0 & $0.4 \quad 12.3$ \\
\hline Tota1D-HET & & 0.0 & 0.0 & 0.0 & 0.0 & 0.0 & 0.0 & 0.0 & 0.0 & 0.0 & 0.0 & 0.0 & 0.0 & 0.0 & 0.0 & 0.0 & 3.3100 .0 \\
\hline D-METAL & MONE & 0.0 & 0.0 & 0.0 & 0.0 & 0.0 & 0.0 & 0.0 & 0.0 & 0.0 & 0.0 & 0.0 & 0.0 & 0.0 & 0.0 & 0.0 & 0.6100 .0 \\
\hline Tot=1D-METAL & & 0.0 & 0.0 & 0.0 & 0.0 & 0.0 & 0.0 & 0.0 & 0.0 & 0.0 & 0.0 & 0.0 & 0.0 & 0.0 & 0.0 & 0.0 & 0.6100 .0 \\
\hline TotalKAC_TRUSAF & & 0.0 & 0.0 & 0.0 . & 0.0 & 0.0 & 0.0 & 0.0 & 0.0 & 0.0 & 0.0 & 0.0 & 0.0 & 0.0 & 0.0 & 0.0 & .13 .5 . \\
\hline \multicolumn{18}{|c|}{ Waste Generator:FHC_IFP_H028 } \\
\hline CONT SOIL & NONE & 0.0 & 0.0 & 0.0 & 0.0 & 0.0 & 0.0 & 0.0 & 0.0 & 0.0 & 0.0 & 0.0 & 0.0 & 0.0 & 0.0 & 0.0 & 5.9100 .0 \\
\hline TotalcoNT soIr & & 0.0 & 0.0 & 0.0 & 0.0 & 0.0 & 0.0 & 0.0 & 0.0 & 0.0 & 0.0 & 0.0 & 0.0 & 0.0 & 0.0 & 0.0 & 5.9100 .0 \\
\hline D-METAR & NONE & 0.0 & 0.0 & 0.0 & 0.0 & 0.0 & 0.0 & 0.0 & 0.0 & 0.0 & 0.0 & 0.0 & 0.0 & 0.0 & 0.0 & 0.0 & 1.4100 .0 \\
\hline TOtal D-METAL & & 0.0 & 0.0 & 0.0 & 0.0 & 0.0 & 0.0 & 0.0 & 0.0 & 0.0 & 0.0 & 0.0 & 0.0 & 0.0 & 0.0 & 0.0 & 1.1100 .0 \\
\hline TOta1WAC_TWP_WO2O & & 0.0 & 0.0 & 0.0 & 0.0 & 0.0 & 0.0 & 0.0 & 0.0 & 0.0 & 0.0 & 0.0 & 0.0 & 0.0 & 0.0 & 0.0 & 7.4 \\
\hline \multicolumn{18}{|c|}{ Faste Generator:FHC_TWP_F030 } \\
\hline CONT SOIL & NONE & 0.0 & 0.0 & 0.0 & 0.0 & 0.0 & 0.0 & 0.0 & 0.0 & 0.0 & 0.0 & 0.0 & 0.0 & 0.0 & 0.0 & 0.0 & 9.9100 .0 \\
\hline Totalcont soll & & 0.0 & 0.0 & 0.0 & 0.0 & 0.0 & 0.0 & 0.0 & 0.0 & 0.0 & 0.0 & 0.0 & 0.0 & 0.0 & 0.0 & 0.0 & 9.9100 .0 \\
\hline D-COHBUST & NONE & 0.0 & 0.0 & 0.0 & 0.0 & 0.0 & 0.0 & 0.0 & 0.0 & 0.0 & 0.0 & 0.0 & 0.0 & 0.0 & 0.0 & 0.0 & 4.3100 .0 \\
\hline
\end{tabular}

HCD Coden: A-Ignitables, B=Corrosives, C-Reactives, DeMetals w/O Hg, E=Metals W Hg, F-Organles, G-State Reg., H=PCB < 50ppm, J-PCB > 50ppm 
(In cubia meter:)

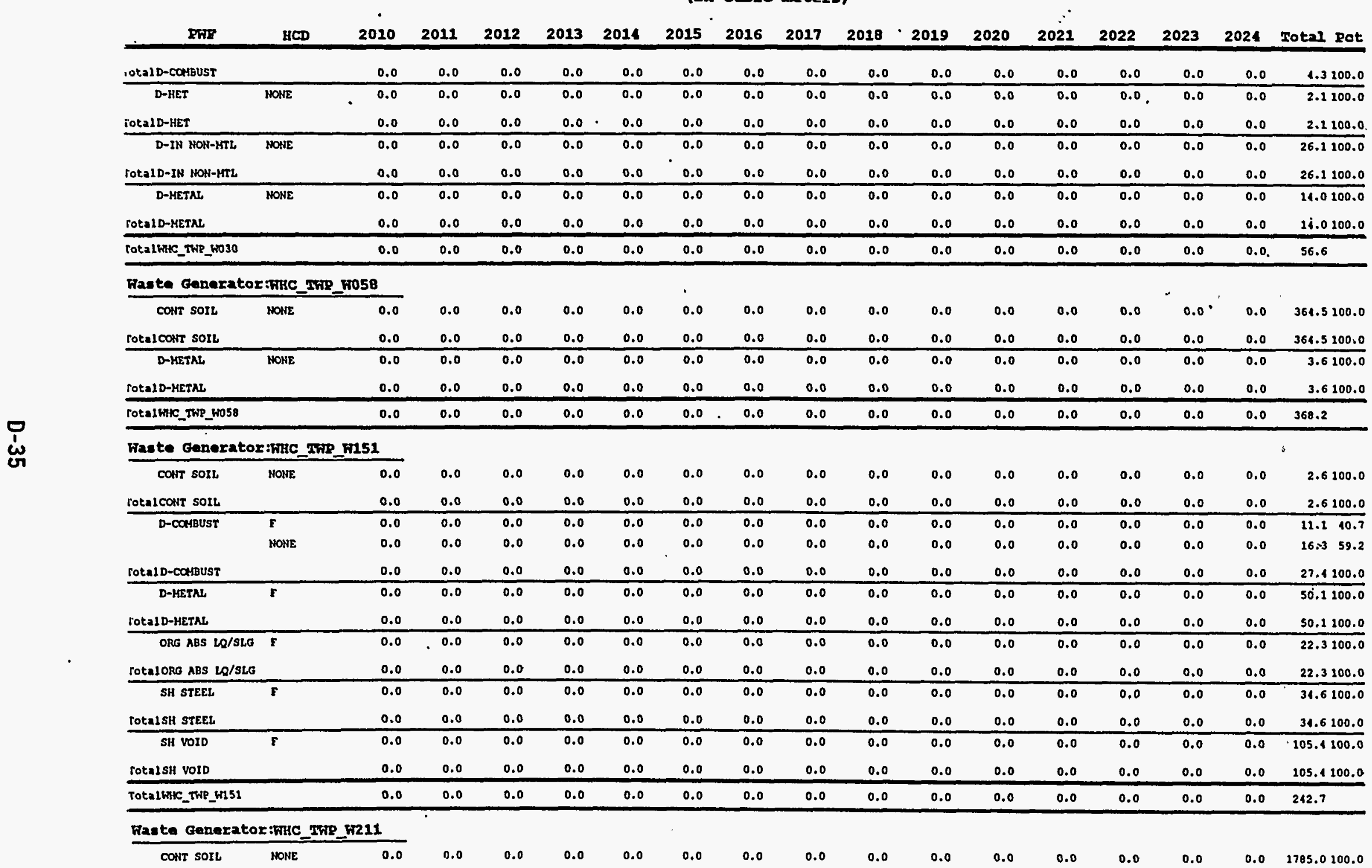

HCD Codas: A-Ignitables, B-Corrosives, C-Reactives, D-Metals w/O Hg, E-Metals w Hg, F-Organles, G-State Reg., H-PCB < 50ppm, J-PCB > 50ppm 


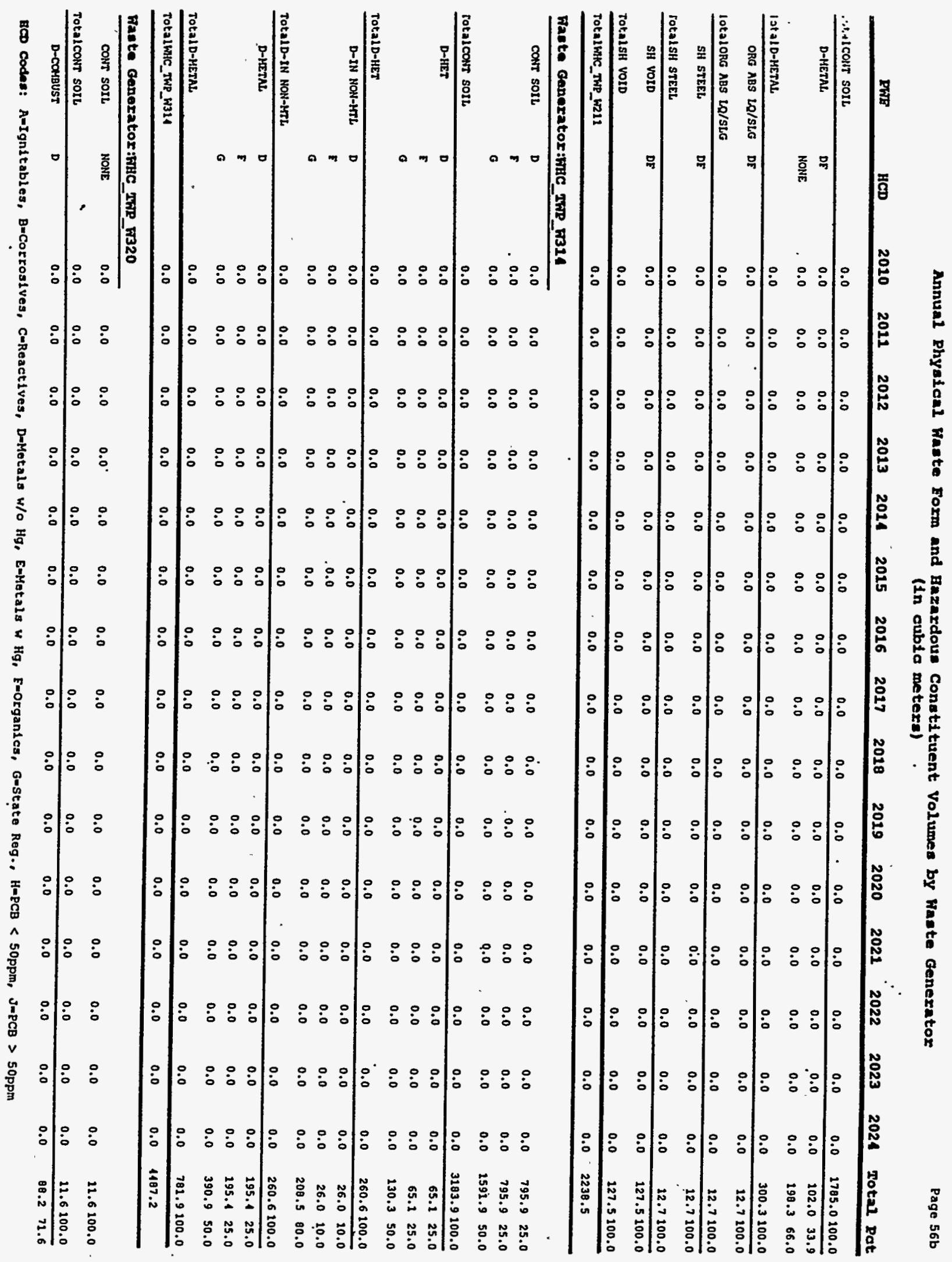




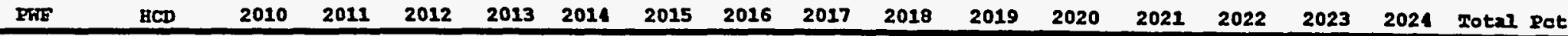

\begin{tabular}{|c|c|c|c|c|c|c|c|c|c|c|c|c|c|c|c|c|c|}
\hline & & & & & & & & & & & $\ldots$ & $20<0$ & 2062 & 2022 & 2023 & 2024 & Totn Pot \\
\hline & WONE & 0.0 & 0.0 & 0.0 & 0.0 & $0 ; 0$ & 0.0 & 0.0 & 0.0 & 0.0 & 0.0 & 0.0 & 0.0 & 0.0 & 0.0 & 0.0 & 31.928 .3 \\
\hline ot:.10-consust & & 0.0 & 0.0 & 0.0 & 0.0 & 0.0 & 0.0 & 0.0 & 0.0 & 0.0 & 0.0 & 0.0 & 0.0 & 0.0 & 0.0 & 0.0 & 123.2100 .0 \\
\hline \multirow[t]{2}{*}{ D-IM NON-MTL } & D & 0.0 & 0.0 & 0.0 & 0.0 & 0.0 & 0.0 & 0.0 & 0.0 & 0.0 & 0.0 & 0.0 & 0.0 & 0.0 & 0.0 & 0.0 & 29.429 .6 \\
\hline & NONE & 0.0 & 0.0 & 0.0 & 0.0 & 0.0 & 0.0 & 0.0 & 0.0 & 0.0 & 0.0 & 0.0 & 0.0 & 0.0 & 0.0 & 0.0 & 69.970 .3 \\
\hline Fut:a10-IN HON-MTL & & 0.0 & 0.0 & 0.0 & 0.0 & 0.0 & 0.0 & 0.0 & 0.0 & 0.0 & 0.0 & 0.0 & 0.0 & 0.0 & 0.0 & 0.0 & 99.3100 .0 \\
\hline \multirow[t]{2}{*}{ D-METAL } & D & 0.0 & 0.0 & 0.0 & 0.0 & 0.0 & 0.0 & 0.0 & 0.0 & 0.0 & 0.0 & 0.0 & 0.0 & 0.0 & 0.0 & 0.0 & 29.129 .6 \\
\hline & MONE & 0.0 & 0.0 & 0.0 & 0.0 & 0.0 & 0.0 & 0.0 & 0.0 & 0.0 & 0.0 & 0.0 & 0.0 & 0.0 & 0.0 & 0.0 & $69.9 \quad 70.3$ \\
\hline Tota1 D-METnS & & 0.0 & 0.0 & 0.0 & 0.0 & 0.0 & 0.0 & 0.0 & 0.0 & 0.0 & 0.0 & 0.0 & 0.0 & 0.0 & 0.0 & 0.0 & 99.3100 .0 \\
\hline D-soIh & NONE & 0.0 & 0.0 & 0.0 & 0.0 & 0.0 & 0.0 & 0.0 & 0.0 & 0.0 & 0.0 & 0.0 & 0.0 & 0.0 & 0.0 & 0.0 & 2.5100 .0 \\
\hline rota1D-solt & & 0.0 & 0.0 & 0.0 & 0.0 & 0.0 & 0.0 & 0.0 & 0.0 & 0.0 & 0.0 & 0.0 & 0.0 & 0.0 & 0.0 & 0.0 & 2.5100 .0 \\
\hline IN ORG PART & MONE & 0.0 & 0.0 & 0.0 & 0.0 & 0.0 & 0.0 & 0.0 & 0.0 & 0.0 & 0.0 & 0.0 & 0.0 & 0.0 & 0.0 & 0.0 & 2.5200 .0 \\
\hline TOta1 IN ORG PART & & 0.0 & 0.0 & 0.0 & 0.0 & 0.0 & 0.0 & 0.0 & 0.0 & 0.0 & 0.0 & 0.0 & 0.0 & 0.0 & 0.0 & 0.0 & 2.5100 .0 \\
\hline SH CONCRETE & D & 0.0 & 0.0 & 0.0 & 0.0 & 0.0 & 0.0 & 0.0 & 0.0 & 0.0 & 0.0 & 0.0 & 0.0 & 0.0 & 0.0 & 0.0 & 117.6200 .0 \\
\hline TOtalSH CONCRETE & & 0.0 & 0.0 & 0.0 & 0.0 & 0.0 & 0.0 & 0.0 & 0.0 & 0.0 & 0.0 & 0.0 & 0.0 & 0.0 & 0.0 & 0.0 & 117.6100 .0 \\
\hline SH LEAD & D & 0.0 & 0.0 & 0.0 & 0.0 & 0.0 & 0.0 & 0.0 & 0.0 & 0.0 & 0.0 & 0.0 & 0.0 & 0.0 & 0.0 & 0.0 & 29.4100 .0 \\
\hline TOtaISH LEAD & & 0.0 & 0.0 & 0.0 & 0.0 & 0.0 & 0.0 & 0.0 & 0.0 & 0.0 & 0.0 & 0.0 & 0.0 & 0.0 & 0.0 & 0.0 & 29.1200 .0 \\
\hline TotalWHAC_TWP_W320 & & 0.0 & 0.0 & 0.0 & 0.0 & 0.0 & 0.0 & 0.0 & 0.0 & 0.0 & 0.0 & 0.0 & 0.0 & 0.0 & 0.0 & 0.0 & 485.8 \\
\hline
\end{tabular}

\section{Wasto Generator:FHC_TWP_H310}

\begin{tabular}{|c|c|c|c|c|c|c|c|c|c|c|c|c|c|c|c|c|c|}
\hline conts sorl & NONE & 0.0 & 0.0 & 0.0 & 0.0 & 0.0 & 0.0 & 0.0 & 0.0 & 0.0 & 0.0 & 0.0 & 0.0 & 0.0 & 0.0 & 0.0 & 7.8100 .0 \\
\hline rotalcont sort & & 0.0 & 0.0 & 0.0 & 0.0 & 0.0 & 0.0 & 0.0 & 0.0 & 0.0 & 0.0 & 0.0 & 0.0 & 0.0 & 0.0 & 0.0 & 7.0100 .0 \\
\hline D-IN NON-MTLL & NONE & 0.0 & 0.0 & 0.0 & 0.0 & 0.0 & 0.0 & 0.0 & 0.0 & 0.0 & 0.0 & 0.0 & 0.0 & 0.0 & 0.0 & 0.0 & 6.1100 .0 \\
\hline rotal D-IN MON-MTL & & 0.0 & 0.0 & 0.0 & 0.0 & 0.0 & 0.0 & 0.0 & 0.0 & 0.0 & 0.0 & 0.0 & 0.0 & 0.0 & 0.0 & 0.0 & 6.1100 .0 \\
\hline TotelWHE_THP_W340 & & 0.0 & 0.0 & 0.0 & 0.0 & 0.0 & 0.0 & 0.0 & 0.0 & 0.0 & 0.0 & 0.0 & 0.0 & 0.0 & 0.0 & 0.0 & 33.9 \\
\hline
\end{tabular}

\section{TotalWiC_TWP_W340}

\begin{tabular}{|c|c|c|c|c|c|c|c|c|c|c|c|c|c|c|c|c|c|}
\hline BATTERIES & BD & 0.1 & 0.1 & 0.1 & 0.1 & 0.1 & 0.1 & 0.1 & 0.1 & 0.1 & 0.1 & 0.1 & 0.1 & 0.1 & 0.1 & 0.1 & 3.9200 .0 \\
\hline TOLaL BATTERIES & & 0.1 & 0.1 & 0.1 & 0.1 & 0.1 & 0.1 & 0.1 & 0.1 & 0.1 & 0.1 & 0.1 & 0.1 & 0.1 & 0.1 & 0.1 & 3.9100 .0 \\
\hline \multirow[t]{5}{*}{ CONT SOIL } & B & 0.0 & 0.0 & 0.0 & 0.0 & 0.0 & 0.0 & 0.0 & 0.0 & 0.0 & 0.0 & 0.0 & 0.0 & 0.0 & 0.0 & 0.0 & $\begin{array}{ll}1.7 \quad 5.7 \\
\end{array}$ \\
\hline & D & 0.1 & 0.1 & 0.1 & 0.2 & 0.1 & 0.1 & 0.1 & 0.1 & 0.1 & 0.1 & 0.1 & 0.1 & 0.1 & 0.1 & 0.1 & 6.320 .3 \\
\hline & $\boldsymbol{E}$ & 0.0 & 0.0 & 0.0 & 0.0 & 0.0 & 0.0 & 0.0 & 0.0 & 0.0 & 0.0 & 0.0 & 0.0 & 0.0 & 0.0 & 0.0 & $0.7 \quad 2.3$ \\
\hline & o & 0.0 & 0.0 & 0.0 & 0.0 & 0.0 & 0.0 & 0.0 & 0.0 & 0.0 & 0.0 & 0.0 & 0.0 & 0.0 & 0.0 & 0.0 & 1.0 \\
\hline & NOHE & 0.7 & 0.7 & 0.7 & 0.7 & 0.7 & 0.7 & 0.7 & 0.7 & 0.7 & 0.7 & 0.7 & 0.1 & 0.7 & 0.7 & 0.7 & $21.3 \quad 60.1$ \\
\hline
\end{tabular}

BCD Codes: A-Ignitables, B-Corrosives, C-Reactives, D-Metals w/O Hg, E-Metals w Hg, E-Organles, G-State Reg., H-PCB < 50ppm, J-PCB > 50ppm 


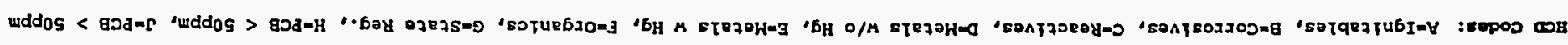

\begin{tabular}{|c|c|c|c|c|c|c|c|c|c|c|c|c|c|c|c|c|c|c|}
\hline $9 \cdot 0$ & $2 \cdot 0$ & 0.0 & 0.0 & 0.0 & 0.0 & 0.0 & 0.0 & 0.0 & 0.0 & 0.0 & 0.0 & 0.0 & 0.0 & 0.0 & 0.0 & 0.0 & 3 & \\
\hline $1 \cdot 0$ & $\tau \cdot 0$ & 0.0 & $0 \cdot 0$ & 0.0 & 0.0 & 0.0 & 0.0 & 0.0 & 0.0 & 0.0 & 0.0 & 0.0 & 0.0 & 0.0 & 0.0 & 0.0 & g & \\
\hline $0 \cdot \tau \pi$ & 5.1 & $\tau \cdot 0$ & $\tau \cdot 0$ & $t \cdot 0$ & $I \because 0$ & $7.0^{\circ}$ & $T \cdot 0$ & t.0 & $\tau \cdot 0$ & $x \cdot 0$ & $1 \cdot 0$ & $\tau \cdot 0$ & $I 00$ & $\because 00$ & $t \cdot 0$ & $r \cdot 0$ & $x$ & D7S/07 จ4о $\mathrm{HI}$ \\
\hline 0.001 & 16.62 & 8.0 & 0.0 & $8 \cdot 0$ & 8.0 & 0.0 & 8.0 & $8 \cdot 0$ & 8.0 & 8.0 & 0.0 & $8 \cdot 0$ & 8.0 & $0 \cdot 0$ & $8 \cdot 0$ & 0.0 & & 7IOS-a18701 \\
\hline $2 \cdot 9 L$ & $\varepsilon \cdot 1 z$ & $L \cdot 0$ & $2 \cdot 0$ & L.O & $\iota \cdot 0$ & L.O & $\iota \cdot 0$ & $\iota \cdot 0$ & $L \cdot O$ & L.O & $2 \cdot 0$ & $L \cdot 0$ & L.0 & $2 \cdot 0$ & $L \cdot 0$ & $L \cdot 0$ & 3NON & \\
\hline$s \cdot 2$ & $L \cdot 0$ & 0.0 & 0.0 & 0.0 & 0.0 & 0.0 & 0.0 & 0.0 & 0.0 & 0.0 & 0.0 & 0.0 & 0.0 & 0.0 & 0.0 & 0.0 & o & \\
\hline$\llcorner\cdot I$ & $1 \cdot 0$ & 0.0 & 0.0 & 0.0 & 0.0 & 0.0 & 0.0 & 0.0 & 0.0 & 0.0 & 0.0 & 0.0 & 0.0 & 0.0 & 0.0 & 0.0 & $\mathbf{3}$ & \\
\hline$\tau \cdot S \tau$ & $2 \cdot 1$ & $I \cdot 0$ & $\tau \cdot 0$ & $\boldsymbol{r} \cdot 0$ & $\tau \cdot 0$ & $1 \cdot 0$ & r.0 & $\tau \cdot 0$ & $r \cdot 0$ & $1 \cdot 0$ & $r \cdot 0$ & $\tau \cdot 0$ & $2 \cdot 0$ & $1 \cdot 0$ & t.o & 100 & a & \\
\hline $2 \cdot 1$ & $t \cdot \tau$ & 0.0 & 0.0 & 0.0 & 0.0 & 0.0 & 0.0 & 0.0 & 0.0 & 0.0 & 0.0 & 0.0 & 0.0 & 0.0 & 0.0 & 0.0 & a & 7IOS-ם \\
\hline 0.008 & $2 \cdot \theta<T$ & $6 . \varepsilon$ & 6.8 & $6 \cdot \varepsilon$ & 6.8 & $6 . \varepsilon$ & $6 . \varepsilon$ & $\sigma^{\prime} \cdot \varepsilon$ & $6 . \varepsilon$ & 6.8 & 6.8 & $6 . \varepsilon$ & $6 . \varepsilon$ & $6 . \varepsilon$ & $6 \cdot \varepsilon$ & $6 \cdot \varepsilon$ & & TUL3H-a1=20L \\
\hline$\llcorner\cdot 65$ & $s \cdot 90 t$ & $t \cdot 2$ & $t \cdot z$ & $\pi \cdot z$ & $\tau \cdot z$ & $t \cdot z$ & $I \cdot z$ & 1.2 & $\tau \cdot \tau$ & $t \cdot z$ & $\tau \cdot z$ & $r \cdot 2$ & $r \cdot z$ & $t \cdot z$ & $x \cdot 2$ & $t \cdot z$ & 3NON & \\
\hline$\vdash \cdot 8 \varepsilon$ & $S \cdot 89$ & $9 \cdot t$ & $9 \cdot 1$ & $9 \cdot \tau$ & $9 \cdot \tau$ & $9 \cdot \tau$ & $9 \cdot t$ & $9 \cdot \tau$ & $9 \cdot 1$ & $9 \cdot \tau$ & $\overline{9} \cdot \tau$ & $9 \cdot t$ & $9 \cdot 1$ & $9 \cdot 1$ & $9 \cdot 1$ & $9 \cdot 1$ & 3 & \\
\hline$L \cdot t$ & $i \cdot \varepsilon$ & $1 \cdot 0$ & $I \cdot 0$ & $t \cdot 0$ & $\tau \cdot 0$ & $I \cdot 0$ & $\tau \cdot 0$ & $1 \cdot 0$ & $\tau \cdot 0$ & $I \cdot 0$ & $1 \cdot 0$ & $\tau \cdot 0$ & I.0 & $t \cdot 0$ & $\tau \cdot 0$ & $t \cdot 0$ & $a$ & TVL3พW-A \\
\hline $0.00 \pi$ & I.2t & $\tau \cdot \tau$ & $T \cdot t$ & $I \cdot \tau$ & $I \cdot T$ & $\boldsymbol{\imath} \tau$ & โโ & $\tau \cdot \tau$ & $\tau \cdot t$ & $I \cdot T$ & $T \cdot T$ & $t \cdot t$ & $t \cdot t$ & $t \cdot t$ & $I \cdot T$ & $t \cdot t$ & & TIH-HON HI-QTEJOL \\
\hline 9.09 & $s \cdot s z$ & $L \cdot O$ & $\iota \cdot 0$ & $\iota \cdot 0$ & $\iota \cdot 0$ & $2 \cdot 0$ & $\angle \cdot 0$ & $\iota \cdot 0$ & $2 \cdot 0$ & $2 \cdot 0$ & $L \cdot O$ & $L \cdot 0$ & $2 \cdot 0$ & L.O & $L \cdot 0$ & $L \cdot 0$ & INOK & \\
\hline $2 \cdot 1$ & $\cdot \cdot r$ & 0.0 & 0.0 & 0.0 & 0.0 & 0.0 & 0.0 & 0.0 & 0.0 & 0.0 & 0.0 & 0.0 & 0.0 & 0.0. & 0.0 & 0.0 & 0 & \\
\hline $6 \cdot z 2$ & 9.6 & $2 \cdot 0$ & $2: 0$ & $2 \cdot 0$ & $z \cdot 0$ & $z \cdot 0$ & $2 \cdot 0$ & $2 \cdot 0$ & $z \cdot 0$ & $z \cdot 0$ & $2 \cdot 0$ & $2 \cdot 0$ & $2 \cdot 0$ & $2 \cdot 0^{\circ}$ & $2 \cdot 0$ & $2 \cdot 0$ & $\mathbf{3}$ & \\
\hline t.5 & $x \cdot z$ & 0.0 & 0.0 & 0.0 & 0.0 & 0.0 & 0.0 & 0.0 & 0.0 & 0.0 & 0.0 & 0.0 & 0.0 & 0.0 & 0.0 & 0.0 & a & \\
\hline$\varepsilon \cdot 2$ & $6 \cdot 0$ & 0.0 & 0.0 & 0.0 & 0.0 & 0.0 & 0.0 & 0.0 & 0.0 & 0.0 & 0.0 & 0.0 & 0.0 & 0.0 & 0.0 & 0.0 & o & \\
\hline$\varepsilon \cdot z$ & 6.0 & 0.0 & 0.0 & 0.0 & 0.0 & 0.0 & 0.0 & 0.0 & 0.0 & 0.0 & 0.0 & 0.0 & 0.0 & 0.0 & 0.0 & 0.0 & 8 & \\
\hline$\varepsilon \cdot z$ & 6.0 & $0: 0$ & 0.0 & 0.0 & 0.0 & 0.0 & 0.0 & 0.0 & 0.0 & 0.0 & 0.0 & 0.0 & 0.0 & 0.0 & 0.0 & 0.0 & $\mathbf{v}$ & THH-NON NI-ם \\
\hline $0.00 \tau$ & $2 \cdot \varepsilon 0 \varepsilon$ & 8.8 & 8.8 & 0.0 & $\theta \cdot \theta$ & $\theta \cdot 8$ & 8.8 & $\theta \cdot \theta$ & $\theta \cdot \theta$ & $\theta \cdot \theta$ & $0 \cdot 0$ & $8 \cdot \theta$ & 0.0 & 0.8 & $9 \cdot 8$ & $\theta \cdot \theta$ & & Lath-a $1=701$ \\
\hline$\tau \cdot \angle \theta$ & $r \cdot r 92$ & 8.2 & $\theta \cdot L$ & B.L & 8.2 & $\theta \cdot L$ & 8.2 & $\theta \cdot L$ & $\theta . L$ & $\theta \cdot 2$ & $\theta \cdot \iota$ & $\theta \cdot 2$ & 0.2 & 0.2 & $8 \cdot 2$ & $\theta \cdot L$ & 3NON & \\
\hline $\boldsymbol{I} \cdot I$ & $s \cdot \varepsilon$ & I.0 & $1 \cdot 0$ & $I .0$ & $\tau=0$ & $\pi 0$ & $\pi \cdot 0$ & $\pi 0$ & $t \cdot 0$ & $\tau \cdot 0$ & $I \cdot 0$ & $\tau \cdot 0$ & $t \cdot 0$ & I.0 & $t \cdot 0$ & $\tau \cdot 0$ & o & \\
\hline 0.8 & $z \cdot r z$ & s.o & $5 \cdot 0$ & s.o & $s \cdot 0$ & s.o & $5 \cdot 0$ & $s \cdot 0$ & s.0 & s.o & s.o & S.0 & s.o & $5 \cdot 0$ & $5 \cdot 0$ & s.o & 3 & \\
\hline 6.0 & $6 \cdot 2$ & 0.0 & 0.0 & 0.0 & 0.0 & 0.0 & 0.0 & 0.0 & 0.0 & 0.0 & 0.0 & 0.0 & 0.0 & 0.0 & 0.0 & 0.0 & $a$ & \\
\hline $9 \cdot 0$ & $8 \cdot \tau$ & 0.0 & 0.0 & 0.0 & 0.0 & 0.0 & 0.0 & 0.0 & 0.0 & 0.0 & 0.0 & 0.0 & 0.0 & 0.0 & 0.0 & 0.0 & D & \\
\hline 0.0 & $s \cdot z$ & 0.0 & 0.0 & 0.0 & 0.0 & 0.0 & 0.0 & 0.0 & 0.0 & 0.0 & 0.0 & 0.0 & 0.0 & 0.0 & 0.0 & 0.0 & g & \\
\hline$\tau \cdot \tau$ & $6 \cdot \varepsilon$ & 1.0 & $I \cdot 0$ & $t \cdot 0$ & $\tau \cdot 0$ & $\tau \because 0$ & $t \cdot 0$ & 1.0 & $1 \cdot 0$ & $\tau \cdot 0$ & I.0 & $I \cdot 0$ & $1 \cdot 0$ & $\tau \cdot 0$ & $\tau 0$ & $\therefore 0$ & $v$ & $\mathbf{2} 3 \mathrm{H}-\mathbf{a}$ \\
\hline$\overline{0.00 t}$ & $\theta \cdot c z t$ & $\overline{S \cdot E}$ & $s \cdot c$ & $S \cdot \varepsilon$ & $5 \cdot \varepsilon$ & $\overline{s \cdot \varepsilon}$ & $S^{\prime} \varepsilon$ & $S^{\prime} \varepsilon$ & $s \cdot c$ & $\overline{s \cdot \varepsilon}$ & $s \cdot \varepsilon$ & $s^{\circ} \varepsilon$ & $s \cdot \varepsilon$ & $S^{\circ} E$ & S.E & $s^{\prime} \cdot \boldsymbol{c}$ & & 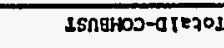 \\
\hline $9 \cdot 0 z$ & s.sz & $\iota \cdot 0$ & $\iota \cdot 0$ & L.O & $L \cdot 0$ & $L \cdot O$ & $L \cdot 0$ & $2 \cdot 0$ & $\iota \cdot 0$ & $\iota \cdot 0$ & $i \cdot 0$ & $८ 0$ & $L \cdot O$ & $\boldsymbol{L} \cdot 0$ & $\iota \cdot 0$ & $2 \cdot 0$ & 3NON & \\
\hline$\iota \cdot L$ & 9.6 & E.0 & $\varepsilon \cdot 0$ & $\varepsilon \cdot 0$ & $\varepsilon \cdot 0$ & $\varepsilon \cdot 0$ & $\varepsilon \cdot 0$ & $\varepsilon \cdot 0$ & $\varepsilon \cdot 0$ & $\varepsilon \cdot 0$ & $\varepsilon .0$ & $E \cdot 0$ & $\varepsilon \cdot 0$ & c.o & $\varepsilon \cdot 0$ & E.0 & D & \\
\hline $0 . n$. & $9 \cdot 45$ & $\varepsilon \cdot I$ & $\varepsilon \cdot \tau$ & $\varepsilon \cdot \tau$ & $E \cdot \tau$ & $E \cdot \tau$ & $\varepsilon \cdot \tau$ & $\varepsilon \cdot \tau$ & $\varepsilon \cdot \tau$ & $\varepsilon \cdot \tau$ & $\varepsilon \cdot \tau$ & $\varepsilon \cdot t$ & $E \cdot 1$ & E't & $t \cdot \tau$ & $\varepsilon \cdot \tau$ & $\mathbf{J}$ & \\
\hline$\varepsilon \cdot \iota$ & $1 \cdot 6$ & $\varepsilon \cdot 0$ & $\varepsilon \cdot 0$ & $\varepsilon \cdot 0$ & $\varepsilon \cdot 0$ & $\varepsilon \cdot 0$ & $\varepsilon \cdot 0$ & E.0 & $\varepsilon \cdot 0$ & E.0 & $\varepsilon \cdot 0$ & $\varepsilon \cdot 0$ & $\varepsilon \cdot 0$ & $\varepsilon \cdot 0$ & E.O & $\varepsilon \cdot 0$ & a & \\
\hline$L \cdot C$ & $9 \cdot r$ & $1 \% 0$ & $r \cdot 0$ & I.O & $t \cdot 0$ & $\tau \cdot 0$ & $\tau \cdot 0$ & 100 & $\tau \cdot 0$ & $\tau \circ 0$ & $I \cdot 0$ & $\tau \cdot 0$ & $1 \cdot 0$ & T.0 & $1 \cdot 0$ & I.0 & 0 & \\
\hline 6.5 & $\varepsilon \cdot\llcorner$ & $2 \cdot 0$ & $2 \cdot 0$ & $2 \cdot 0$ & $2 \cdot 0$ & $2 \cdot 0$ & $2 \cdot 0$ & $2 \cdot 0$ & $2 \cdot 0$ & $2 \cdot 0$ & $2 \cdot 0$ & $2 \cdot 0$ & $2 \cdot 0$ & $z \cdot 0$ & $2 \cdot 0$ & $z \cdot 0$ & a & \\
\hline ror & $6.2 T$ & s.0 & $s \cdot 0$ & $s \cdot 0$ & s.o & $s \cdot 0$ & s.0 & $s \cdot 0$ & s.o & s.o & s.o & $s \cdot 0$ & $s \cdot 0$ & s.0 & s.o & $5 \cdot 0$ & $v$ & LSTIEHOJ-a - \\
\hline $70 d$ & TE70I & 1202 & $E Z 0 Z$ & 2202 & $\tau 20 Z$ & 0202 & 6T0Z & $8 T 02$ & LTOZ & 9т0Z & STOZ & tToz & ETOZ & zTOz & TTOZ & oroz & COH & IMd \\
\hline
\end{tabular}




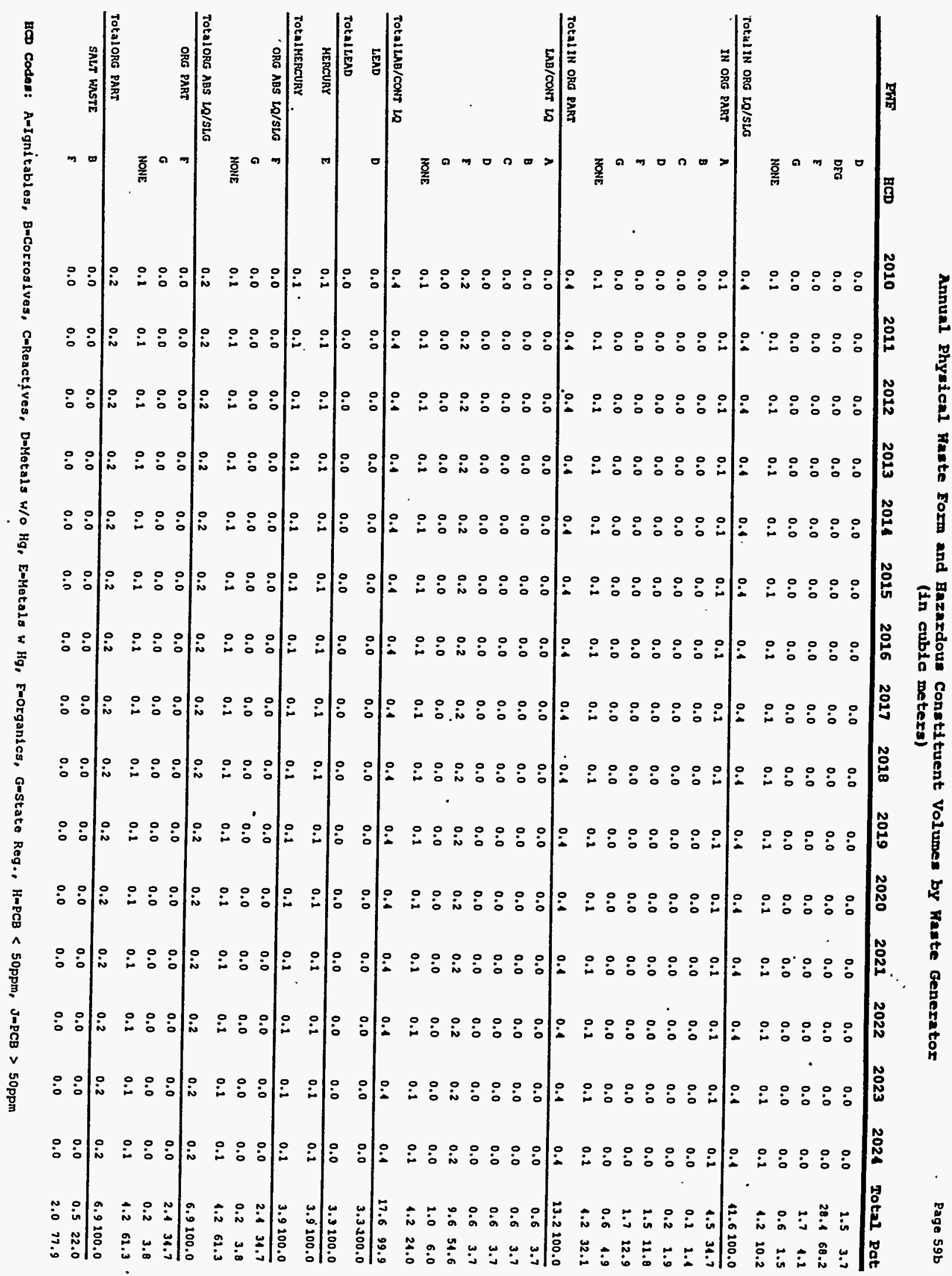


waddos < 80j-L 'mddos > 803-H

\begin{tabular}{|c|c|c|c|c|c|c|c|c|c|c|c|c|c|c|c|c|c|}
\hline$t \cdot \varepsilon \cos z B$ & $\theta \cdot z \theta z 5 \varepsilon$ & $9 \cdot 69108$ & $z \cdot 0 T 96 \varepsilon$ & $L \cdot B \varepsilon t 9 \varepsilon$ & S.PItEE & $6.0160 \varepsilon$ & $0.98 \varepsilon 0 \varepsilon$ & c.ogere & r.ssoes & S. & 0.29911 & z. zU1CE & 6. Lotez & I' โย992 & $9 \cdot \angle 0 L 98$ & & OI purzo \\
\hline 0.052 & $9 \cdot 0$ & $9 \cdot 8$ & $9 \cdot 9$ & $9 \cdot 8$ & $9 \cdot 8$ & $9 \cdot 8$ & $9 \cdot 8$ & $9 \cdot 8$ & $9 \cdot \theta$ & 9.8 & $9 \cdot 8$ & $9 \cdot 0$ & $9 \cdot 0$ & $9 \cdot 8$ & $9 \cdot 0$ & & วะם \\
\hline $0.0016 .2 \mathrm{t}$ & $\because \cdot 0$ & $1 \cdot 0$ & 1.0 & $\cdot \cdot 0$ & $\because 0$ & $\because \cdot 0$ & $\because \cdot 0$ & 1.0 & $r \cdot 0$. & $P \cdot 0$ & b.0 & $1 \cdot 0$ & $1 \%$ & $1 \cdot 0$ & $1 \cdot 0$ & & 2IOS-atezoL \\
\hline I.SI $6 . t$ & 0.0 & 0.0 & 0.0 & 0.0 & 0.0 & 0.0 & 0.0 & 0.0 & 0.0 & 0.0 & 0.0 & 0.0 & 0.0 & 0.0 & 0.0 & 3NON & \\
\hline $0.68 \quad 6.01$ & $\varepsilon \cdot 0$ & $\varepsilon \cdot 0$ & $\varepsilon \cdot 0$ & $\varepsilon \cdot 0$ & $\varepsilon \cdot 0$ & $\varepsilon \cdot 0$ & $E \cdot 0$ & $\varepsilon \cdot 0$ & $\varepsilon \cdot 0$ & $8 \cdot 0$ & $\varepsilon \cdot 0$ & $E \cdot 0$ & $\varepsilon \cdot 0$ & $\varepsilon \cdot 0$ & $E \cdot 0$ & 3 & $7105-9$ \\
\hline 0.0016 .25 & 1.0 & 1.0 & $\cdot 0$ & $r \cdot 0$ & 1.0 & 10. & $1 \cdot 0$ & 100 & 1.0 & $1 \cdot 0$ & 1.0 & $1 \cdot 0$ & $7 \cdot 0$ & $1 \cdot 0$ & 1.0 & & $T V 13 H-a 1=701$ \\
\hline $\mathfrak{T} \cdot S t \quad 6 \cdot \tau$ & 0.0 & 0.0 & 0.0 & 0.0 & 0.0 & 0.0 & 0.0 & 0.0 & 0.0 & 0.0 & 0.0 & 0.0 & 0.0 & 0.0 & 0.0 & 3NON & \\
\hline $0.68 \quad 6.01$ & $\varepsilon \cdot 0$ & $\varepsilon \cdot 0$ & $\varepsilon \cdot 0$ & $\varepsilon \cdot 0$ & $\varepsilon \cdot 0$ & $\varepsilon \cdot 0$ & $E \cdot 0$ & $\varepsilon \cdot 0$ & $E \cdot 0$ & $E \cdot 0$ & E.O & $\varepsilon \cdot 0$ & $\varepsilon \cdot 0$ & $\varepsilon \cdot 0$ & $E \cdot 0$ & 3 & $T N 23 H-a$ \\
\hline 0.0018 .52 & $8 \cdot 0$ & 0.0 & 8.0 & $\theta \cdot 0$ & $8 \cdot 0$ & 0.0 & $8 \cdot 0$ & 8.0 & 0.0 & $9 \cdot 0$ & 8.0 & $0 \cdot 0$ & $\theta \cdot 0$ & 0.0 & $9 \cdot 0$ & & 7IH-HON AI- $-41 E 701$ \\
\hline I.ร่ป $\quad 6 . \varepsilon$ & $\tau \cdot 0$ & $1 \% 0$ & $r \cdot 0$ & 1.0 & 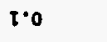 & $1 \cdot 0$ & $\tau \cdot 0$ & $1 \cdot 0$ & $I=0$ & $t \cdot 0$ & $r 00$ & $t \cdot 0$ & $t \cdot 0$ & I.0 & r.o & 3HON & \\
\hline $8.38 \quad 6.12$ & $\llcorner\cdot 0$ & $\llcorner\cdot 0$ & $\llcorner\cdot 0$ & $\llcorner\cdot 0$ & $\angle \cdot 0$ & $\llcorner\cdot 0$ & $\angle \cdot 0$ & $2 \cdot 0$ & L.0 & $6 \cdot 0$ & $2 \cdot 0$ & L.O & $6 \cdot 0$ & ¿०0 & $2 \cdot 0$ & 3 & TIH-NON NI-a \\
\hline 0.0011 .902 & $8 \cdot 9$ & 8.9 & 0.9 & 8.9 & 8.9 & $9 \cdot 9$ & $8 \cdot 9$ & $8 \cdot 9$ & 0.9 & 0.9 & 8.9 & 0.9 & 8.9 & 0.9 & 8.9 & & THOS LNOOTETOL \\
\hline I.ST $\tau \cdot t \varepsilon$ & $0 . t$ & $0 \cdot \tau$ & $0 \cdot I$ & $0 . \tau$ & $0 . \tau$ & $0 . \tau$ & $0 . \tau$ & $0 . \tau$ & $0 . \tau$ & $0 . \tau$ & $0 \cdot t$ & 0.1 & 0.5 & 0.1 & זיט & 3NON & \\
\hline
\end{tabular}

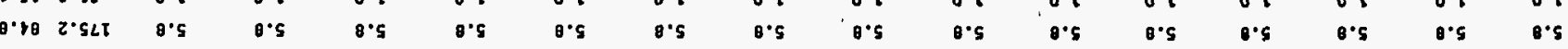

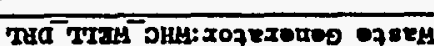

\begin{tabular}{|c|c|c|c|c|c|c|c|c|c|c|c|c|c|c|c|c|c|c|}
\hline & S.059 & $s \cdot \imath$ & $s \cdot \tau z$ & $5 \cdot t z$ & s.tz & s.te & $s \cdot t z$ & $s \cdot \tau z$ & $s \cdot \tau z$ & $s \cdot \tau z$ & 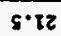 & $s \cdot \tau z$ & $s \cdot u z$ & s.tz & s.tz & $s \cdot \tau z$ & & 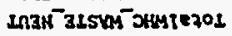 \\
\hline 0.00 & $\varepsilon \cdot \varepsilon s$ & $\llcorner\cdot \boldsymbol{I}$ & $\iota \cdot \tau$ & L'T & $\llcorner\cdot t$ & $L \cdot t$ & $\iota \cdot \tau$ & $\llcorner\cdot \tau$ & $\iota \cdot \tau$ & $L \cdot T$ & $\iota \cdot \tau$ & $\iota \cdot I$ & $L \cdot 1$ & $\omega \cdot \tau$ & $L \cdot 1$ & $L \cdot 8$ & & TVLIH-a \\
\hline $9 \cdot 26$ & r.6r & 9.1 & $9 \cdot \tau$ & 9.1 & $9 \cdot \tau$ & 9.1 & $9 \cdot \tau$ & $9 \cdot 1$ & $9 \cdot \tau$ & $9 \cdot \mathrm{I}$ & $9 \cdot t$ & $9 \cdot \tau$ & $9 \cdot 1$ & $9 \cdot 1$ & $9 \cdot \mathbf{I}$ & $9 \cdot 1$ & 3NON & \\
\hline$\varepsilon \cdot L$ & $6 \cdot \varepsilon$ & I.0 & $1 \cdot 0$ & $\mathfrak{r} \cdot 0$ & I.0 & $\tau \cdot 0$ & 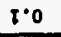 & $\tau \cdot 0$ & $\because \cdot 0$ & $I \cdot 0$ & I.0 & $\boldsymbol{r} \cdot 0$ & $\tau \cdot 0$ & r.0 & $1 \cdot 0$ & I.0 & 33 & TV23H-a \\
\hline 0.00 & T. 509 & L.6T & $\iota \cdot 6 \pi$ & 0.67 & . .61 & $L \cdot 6 x$ & $L \cdot 6 \mathrm{~T}$ & 2.62 & $2 \cdot 6 \pi$ & $2.6 t$ & 6.65 & t*6 $6 \mathrm{~T}$ & $\therefore \cdot 6 T^{\circ}$ & 2.65 & .6 .61 & $\angle \cdot 6 \tau$ & & 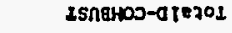 \\
\hline I. 16 & $0.0<5$ & S'0t & $s \cdot \theta t$ & $S^{\prime} \theta t$ & $s^{\prime} \theta$ r & $5 \cdot 0 \pi$ & $s^{\circ} \theta t$ & $5 \cdot 81$ & S'Bt & S'at & $s \cdot \theta t$ & S'Bt & 5.61 & 5. $8 \mathrm{t}$ & $5 \cdot 0 \mathfrak{r}$ & $s \cdot \theta t$ & 3HON & \\
\hline$\theta \cdot 5$ & T'SE & $\boldsymbol{I} \cdot \mathbf{I}$ & $\tau$ & $\tau \cdot \tau$ & $t \cdot \tau$ & $\tau \cdot \tau$ & $\tau$ & $\mathfrak{t} \cdot \mathfrak{t}$ & $\tau \cdot \tau$ & $I \cdot I$ & $\tau \cdot \tau$ & $\boldsymbol{t} \cdot \tau$ & $\tau \cdot \tau$ & $\tau \cdot \tau$ & $\boldsymbol{I} \cdot \boldsymbol{\tau}$ & $\mathfrak{I} \cdot \tau$ & 1 & 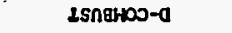 \\
\hline
\end{tabular}

\begin{tabular}{|c|c|c|c|c|c|c|c|c|c|c|c|c|c|c|c|c|c|}
\hline \multirow[b]{2}{*}{ B.006 } & \multirow[b]{2}{*}{$\tau \cdot \boldsymbol{L}$} & \multirow[b]{2}{*}{$\tau \cdot r z$} & \multirow[b]{2}{*}{ I.vz } & \multirow[b]{2}{*}{$r \cdot r 2$} & \multirow[b]{2}{*}{$I \cdot 12$} & \multirow[b]{2}{*}{$r \cdot v z$} & \multirow[b]{2}{*}{ t've } & \multirow[b]{2}{*}{$\tau \cdot \boldsymbol{r}$} & \multirow[b]{2}{*}{$\tau \cdot \boldsymbol{\eta} z$} & \multirow[b]{2}{*}{$\tau \cdot 12$} & \multirow[b]{2}{*}{$r \cdot h 2$} & \multirow[b]{2}{*}{$I \cdot \operatorname{rz}$} & \multirow[b]{2}{*}{$\tau \cdot r z$} & \multirow[b]{2}{*}{$\tau \cdot \boldsymbol{r}$} & \multicolumn{3}{|c|}{ 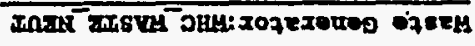 } \\
\hline & & & & & & & & & & & & & & & $\tau \cdot \boldsymbol{r}$ & & LNMA'I"FMTEZOL \\
\hline $0.0016 .8 L$ & $\tau \cdot z$ & $\tau \cdot \tau$ & $\tau \cdot z$ & $1 \cdot 2$ & $I \cdot z$ & $r \cdot z$ & $r \cdot 2$ & $\tau \cdot \tau$ & $I \cdot z$ & t'2 & $1 \cdot 2$ & $\tau \cdot z$ & $\tau \cdot z$ & $t \cdot 2$ & $r \cdot z$ & & NHORYNnTEzol \\
\hline $9 . \angle 9+E S$ & $\bullet \tau$ & $\bullet \tau$ & $r \cdot 1$ & $\because \tau$ & $r \cdot \mathrm{t}$ & $\cdot 1$ & $\bullet 1$ & $1 \cdot \tau$ & $\cdot r$ & $1 \cdot 1$ & $1 \cdot 8$ & .1 & $\bullet 1$ & $\bullet \cdot \tau$ & $r \tau$ & N20:4MNn & \\
\hline$\varepsilon \cdot 2 \varepsilon s \cdot s z$ & $\llcorner\cdot 0$ & $\llcorner\cdot 0$ & $L \cdot 0$ & ¿.0 & $L \cdot 0$ & $2 \cdot 0$ & $\llcorner\cdot 0$ & $\angle \cdot 0$ & $\llcorner\cdot 0$ & $\iota \cdot 0$ & $\iota \cdot 0$ & $\llcorner\cdot 0$ & $\llcorner\cdot 0$ & $\angle \cdot 0$ & $\llcorner\cdot 0$ & 3NON & КЕоКИЕ \\
\hline 0.0016 .41 & E.0 & $\varepsilon^{\circ} 0$ & $\varepsilon \cdot 0$ & $\varepsilon \cdot 0$ & $\varepsilon \cdot 0$ & $\varepsilon * 0$ & E.0 & E.0 & $\varepsilon \cdot 0$ & $\varepsilon \cdot 0$ & $\varepsilon \cdot 0$ & $\varepsilon \cdot 0$ & $\varepsilon^{\circ} 0$ & $\varepsilon \cdot 0$ & $8 \cdot 0$ & & av37 Hล1::0.1 \\
\hline 0.0006 .12 & $\varepsilon \cdot 0$ & $\varepsilon \cdot 0^{\circ}$ & $\varepsilon \cdot 0$ & E.0. & $\varepsilon \cdot 0$ & $\varepsilon \cdot 0$ & $\varepsilon \cdot 0$ & $\varepsilon \cdot 0$ & $\varepsilon^{\circ} 0$ & $\varepsilon \cdot 0$ & E.O & $\varepsilon \cdot 0$ & $E \cdot 0$ & $\varepsilon \cdot 0$ & $\varepsilon \cdot 0$ & a & av3? HS \\
\hline 0.0049 .2 & 0.0 & 0.0 & 0.0 & 0.0 & 0.0 & 0.0 & 0.0 & 0.0 & 0.0 & 0.0 & 0.0 & 0.0 & 0.0 & 0.0 & 0.0 & & 315VM LTVS /E'T\%! \\
\hline
\end{tabular}

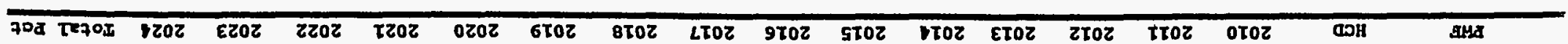




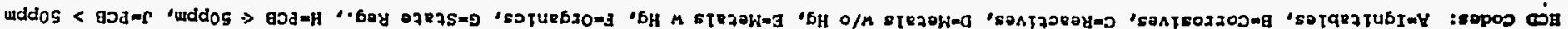

\begin{tabular}{|c|c|c|c|c|c|c|c|c|c|c|c|c|c|c|c|c|c|}
\hline s.s2 & s.sz & 5.52 & s.5z & 5.52 & 5.52 & s.5z & s.sz & s'br & $0^{\circ} 85 \pi$ & $0.10 t$ & $6 \cdot 19$ & 8.69 & 0.05 & $0 . \angle \varepsilon$ & 0.0 & & TVL3H-A TEJOL \\
\hline$r \cdot s z$ & 1.52 & 1.52 & r.sz & 1.52 & $\cdot \cdot s z$ & $1 \cdot s z$ & r.s 2 & E.H & O.LST & $6.50 \mathrm{t}$ & 9.ra & $5 \cdot 69$ & 9.05 & $\theta \cdot 9 \varepsilon$ & 0.0 & 3NON & \\
\hline r.0 & t.0 & t.0 & $\mathfrak{\tau} \cdot 0$ & I.0 & $8 \cdot 0$ & $r \cdot 0$ & $\tau \cdot 0$ & r.0 & $\tau=0$ & $1 \cdot 0$ & $\tau \cdot 0$ & $7 \cdot 0$ & I.0 & $\tau \cdot 0$ & 0.0 & $a$ & TVL3H-a \\
\hline .0 .52 & 0.52 & 8.52 & 8.5z & $\theta \cdot s z$ & 8.52 & 9.52 & $\theta^{\prime} \varsigma z$ & 9.91 & $\angle \cdot 0 L T$ & L'ttI & 8.05 & 8.89 & 8. Is & $2 \cdot 0 \varepsilon$ & 0.0 & & TL1-NON NI-O TEZOL \\
\hline O.sz & $\theta \cdot 52$ & $\theta \cdot 5 z$ & $8 \cdot 5 z$ & 0.52 & $\theta \cdot 52$ & 0.52 & $0 \cdot 5 z$ & 9.98 & L.0LI & L.tII & 8.05 & 0.69 & 8.15 & $2 \cdot \theta \varepsilon$ & 0.0 & 3NON & TLH-NON NI- \\
\hline $2 \cdot 16$ & ใ. โ6 & $\llcorner\cdot 16$ & C.16 & $L^{\circ}+6$ & c.t6 & $L^{\circ} 76$ & $L^{\circ} 16$ & I'bst & 0.065 & $r \cdot \angle 9 \varepsilon$ & $5 \cdot 06 t$ & $9 \cdot \varepsilon 1 z$ & $0.9 \varepsilon t$ & 6.901 & 0.0 & & D3H-A TEZOL \\
\hline$\varepsilon \cdot t \theta$ & $\varepsilon \cdot 1 \theta$ & $\varepsilon+1 \theta$ & E. 18 & E. 18 & E.18 & $8 \cdot 18$ & c.te & $6.05 t$ & o.rss & $6.09 \varepsilon$ & 0.501 & $\varepsilon \cdot 10 z$ & 0.221 & 8.ह6 & 0.0 & 3NON & \\
\hline ror & ror & $1 \cdot 0 \mathrm{r}$ & ror & 1.0 & 5.02 & r.or & $r \cdot 0 \tau$ & 2.5 & $0 \cdot \iota \varepsilon$ & 2.9 & $1 \cdot 5$ & $2 \cdot 21$ & Iอย & $\tau \cdot \varepsilon \tau$ & 0.0 & a & $133 H-a$ \\
\hline 0.5I & e.st & Q'st & B.ST & O.ST & 0.5ז & 8.5i & B.9 & $z \cdot \theta z$ & s.20t & $2 \cdot 49$ & $6 \cdot b \varepsilon$ & 6.28 & $2 \cdot+2$ & L.4t & 0.0 & & LSABHOJ-G YEJOL \\
\hline 0.51 & $8.5 t$ & O.SI & 8.5t & 8.5 & 8.5t & $8.5 \mathrm{t}$ & $\theta .5 \tau$ & $2 \cdot 82$ & s.20r & $2 \cdot 49$ & 6.18 & $6 \cdot L \varepsilon$ & $2 \cdot 12$ & $L \cdot L T$ & 0.0 & 3NON & ISก \\
\hline $6 \cdot 19$ & $6 \cdot 19$ & 6.19 & $6 \cdot 19$ & 6.19 & $6 \cdot 19$ & 6.79 & $6 \cdot 79$ & $\tau^{2} \mathrm{Ett}$ & $9 \cdot 2 t 8$ & S'rLz & 2.015 & E'EST & $Z \cdot T S E$ & L.LLZ & 0.0 & & 71OS 2 NOSTEZOL \\
\hline $9 \cdot 19$ & $9 \cdot 19$ & פז & $9 \cdot 29$ & $9 \cdot 19$ & $9 \cdot 19$ & פז 9 & $9 \cdot 19$ & O.ET & 9.021 & 1.122 & seort & 6.251 & $6.09 \varepsilon$ & $E \cdot L L Z$ & 0.0 & 3NON & \\
\hline 0.0 & 0.0 & 0.0 & 0.0 & 0.0 & 0.0 & 0.0 & 0.0 & 0.0 & $2 \cdot 0$ & 0.0 & 0.0 & 0.0 & 0.0 & 0.0 & 0.0 & f & \\
\hline 100 & $1 \cdot 0$ & 80 & I.0 & 1.0 & $\tau \cdot 0$ & $1 \cdot 0$ & I.0 & 0.0 & $9 \cdot 0$ & $1 \cdot 0$ & 0.0 & $\mathfrak{r} \cdot 0$ & $2 \cdot 0$ & $2 \cdot 0$ & 0.0 & 3 & \\
\hline 0.0 & 0.0 & 0.0 & 0.0 & 0.0 & 0.0 & 0.0 & $0 \cdot 0$ & 0.0 & $2 \cdot 0$ & 0.0 & 0.0 & 0.0 & 0.0 & 0.0 & 0.0 & a & TIOS LNOOO \\
\hline
\end{tabular}

\begin{tabular}{|c|c|c|c|c|c|c|c|c|c|c|c|c|c|c|c|c|c|}
\hline $2 \cdot \varepsilon$ & $2 \cdot \varepsilon$ & $2 \cdot E$ & $z \cdot \varepsilon$ & $2 \cdot \varepsilon$ & $\because \cdot \varepsilon$ & $z \cdot \varepsilon$ & $\boldsymbol{Z} \cdot \mathbf{\varepsilon}$ & $z \cdot \mathbf{E}$ & $\tau \cdot \varepsilon$ & $c \cdot 9$ & $\varepsilon \cdot 9$ & 1.9 & 1.9 & 1.9 & $t \cdot 9 t$ & & S3W TEZOL \\
\hline 0.0 & 0.0 & 0.0 & 0.0 & 0.0 & 0.0 & 0.0 & 0.0 & 0.0 & 0.0 & 0.0 & 0.0 & I.0 & $I \cdot 0$ & $t \cdot 0$ & $2 \cdot 0$ & & 67 LNOO/GWI TEJOL \\
\hline 0.0 & 0.0 & 0.0 & 0.0 & 0.0 & 0.0 & 0.0 & 0.0 & 0.0 & 0.0 & 0.0 & 0.0 & 0.0 & 0.0 & 0.0 & $2 \cdot 0$ & ag & \\
\hline 0.0 & 0.0 & 0.0 & 0.0 & 0.0 & 0.0 & 0.0 & 0.0 & 0.0 & 0.0 & 0.0 & 0.0 & I.0 & $\tau \cdot 0$ & $t \cdot 0$ & 0.0 & 8 & or LNOOS/AV7 \\
\hline 0.0 & 0.0 & 0.0 & 0.0 & 0.0 & 0.0 & 0.0 & 0.0 & 0.0 & 0.0 & 0.0 & 0.0 & $0 \cdot 0$ & 0.0 & 0.0 & $8 \cdot 1$ & & LUVd פYO NI TEZOL \\
\hline 0.0 & 0.0 & 0.0 & 0.0 & 0.0 & 0.0 & 0.0 & 0.0 & 0.0 & 0.0 & 0.0 & 0.0 & 0.0 & 0.0 & 0.0 & $0 . \tau$ & 3MON & IrYY DSO NI \\
\hline 0.0 & 0.0 & 0.0 & 0.0 & 0.0 & 0.0 & 0.0 & 0.0 & 0.0 & 0.0 & 0.0 & 0.0 & 0.0 & 0.0 & 0.0 & $2 \cdot 0$ & & TKL3H-O TOZOL \\
\hline 0.0 & 0.0 & 0.0 & 0.0 & 0.0 & 0.0 & 0.0 & 0.0 & 0.0 & 0.0 & 0.0 & $0.0^{\circ}$ & 0.0 & 0.0 & 0.0 & $2 \cdot 0$ & a & TN $13 H-0$ \\
\hline$z \cdot \varepsilon$ & $2 \cdot \varepsilon$ & $\tau \cdot \varepsilon$ & $2 \cdot \varepsilon$ & $\overline{2 \cdot \varepsilon}$ & $\overline{2 \cdot \varepsilon}$ & $2 \cdot \varepsilon$ & $\overline{\tau \cdot \varepsilon}$ & $\overline{z \cdot \varepsilon}$ & $\overline{2 \cdot \varepsilon}$ & $E^{\prime} \cdot 9$ & $\varepsilon \cdot 9$ & $5 \cdot 9$ & E.9 & $8 \cdot 9$ & '. $\varepsilon$ &. & TIH-NOH NI-Q IEJOL \\
\hline$z \cdot \varepsilon$ & $z \cdot \varepsilon$ & $2 \cdot \varepsilon$ & $2 \cdot \varepsilon$ & $2 \cdot 5$ & $\tau \cdot \varepsilon$ & $z \cdot \varepsilon$ & $2 \cdot \varepsilon$ & $2 \cdot \varepsilon$ & $z \cdot \varepsilon$ & $\varepsilon \cdot 9$ & $\varepsilon \cdot 9$ & $\varepsilon \cdot 9$ & $\varepsilon \cdot 9$ & $\varepsilon \cdot 9$ & L.e & 3MON & T2H-NON NI- \\
\hline$\overline{0.0}$ & 0.0 & 0.0 & 0.0 & 0.0 & $2 \cdot 0$ & 0.0 & 0.0 & 0.0 & 0.0 & 0.0 & 0.0 & 0.0 & 0.0 & 0.0 & $2 \cdot 8$ & & IJH-Q TEYOI \\
\hline 0.0 & 0.0 & 0.0 & 0.0 & 0.0 & $t \cdot 0$ & 0.0 & 0.0 & 0.0 & 0.0 & 0.0 & 0.0 & 0.0 & 0.0 & 0.0 & $5 \cdot 8$ & 3NON & \\
\hline 0.0 & $0 \div 0$ & 0.0 & 0.0 & 0.0 & 0.0 & 0.0 & 0.0 & 0.0 & 0.0 & 0.0 & 0.0 & 0.0 & 0.0 & 0.0 & 0.0 & $a$ & \\
\hline 0.0 & 0.0 & 0.0 & 0.0 & 0.0 & 0.0 & 0.0 & 0.0 & 0.0 & 0.0 & 0.0 & 0.0 & 0.0 & 0.0 & 0.0 & 0.0 & 8 & $13 \mathrm{H}-\mathrm{G}$ \\
\hline 0.0 & 0.0 & 0.0 & 0.0 & 0.0 & 0.0 & 0.0 & 0.0 & 0.0 & 0.0 & 0.0 & 0.0 & 0.0 & 0.0 & 0.0 & $5 \cdot \pi$ & & ISnahroo-d IejoI \\
\hline 0.0 & 0.0 & 0.0 & 0.0 & 0.0 & 0.0 & 0.0 & 0.0 & 0.0 & 0.0 & 0.0 & 0.0 & 0.0 & 0.0 & 0.0 & S.T & 3MON & $\sin a n+0 j-a$ \\
\hline
\end{tabular}

8ร1\%:

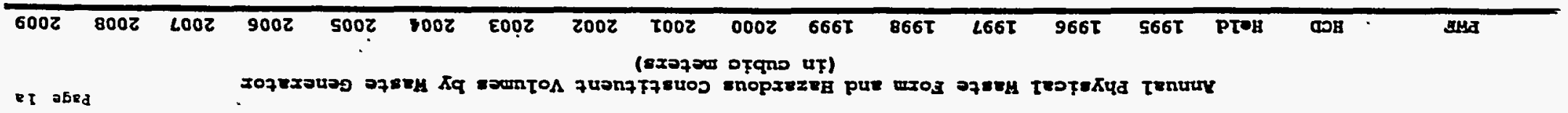


Annual Phyaical Faste Form and Hazardous Constituent Volumes by Haste Generator

(In oubio meters)

\begin{tabular}{|c|c|c|c|c|c|c|c|c|c|c|c|c|c|c|c|c|c|}
\hline EWE & HCD & Held & 1995 & 1996 & 1997 & 1998 & 1999 & 2000 & 2001 & 2002 . & 2003 & 2004 & 2005 & 2006 & 2007 & 2008 & 2009 \\
\hline \multirow[t]{4}{*}{ III ORG LQ/SLG } & A & 0.0 & 0.0 & 0.0 & 0.0 & 0.0 & 0.0 & 0.0 & 0.0 & 0.0 & 0.0 & 0.0 & 0.0 & 0.0 & 0.0 & 0.0 & 0.0 \\
\hline & B & 0.0 & 0.0 & 0.0 & 0.0 & 0.0 & 0.0 & 0.0 & 0.0 & 0.0 & 0.0 & 0.0 & 0.0 & 0.0 & 0.0 & 0.0 & 0.0 \\
\hline & $D$ & 0.0 & 0.7 & 0.7 & 0.7 & 0.3 & 0.1 & 2.0 & 0.3 & 0.6 & 0.6 & 0.6 & 0.6 & 0.6 & 0.6 & 0.6 & 0.6 \\
\hline & MOUE & 0.0 & 5.5 & 11.5 & 9.5 & 0.9 & 15.4 & 22.4 & 7.6 & 5.1 & 5.2 & 5.1 & 5.1 & 5.1 & 5.1 & 5.1 & 5.1. \\
\hline Total III ORG LQ/sLG & & 0.0 & 6.1 & 22.4 & 10.3 & 9.1 & 15.9 & 24.6 & 8.0 & 5.0 & 3.0 & 5.8 & 5.8 & 5.8 & 5.0 & 5.8 & 5.8 \\
\hline IN ORG PART & NONE & 0.0 & 1.0 & 2.7 & 3.8 & 3.5 & 6.7 & 10.3 & 2.8 & 1.6 & 1.6 & 1.6 & 1.6 & 1.6 & 1.6 & 1.6 & 1.6 \\
\hline Tot.al IN ORG PART & & 0.0 & 1.8 & 2.7 & 3.0 & 3.5 & 6.7 & 10.3 & 2.0 & 1.6 & 1.6 & 1.6 & 1.6 & 1.6 & 1.6 & 1.6 & 1.6 \\
\hline \multirow[t]{4}{*}{ LAB/CONT LQ } & a & 0.0 & 2.8 & 2.8 & 2.6 & 1.1 & 1.3 & 8.1 & 1.1 & 2.2 & 2.2 & 2.2 & 2.2 & 2.2 & 2.2 & 2,2 & 2.2 \\
\hline & B & 0.0 & 0.1 & 0.1 & 0.1 & 0.0 & 0.0 & 0.5 & 0.0 & 0.1 & 0.1 & 0.1 & 0.1 & 0.1 & 0.1 & 0.1 & 0.1 \\
\hline & D & 0.0 & 0.0 & 0.0 & 0.0 & 0.0 & 0.0 & 0.2 & 0.0 & 0.0 & 0.0 & 0.0 & 0.0 & 0.0 & 0.0 & 0.0 & 0.0 \\
\hline & $F$ & 0.0 & 0.0 & 0.0 & 0.0 & 0.0 & 0.0 & 0.0 & 0.0 & 0.0 & 0.0 & 0.0 & 0.0 & 0.0 & 0.0 & 0.0 & 0.0 \\
\hline \multicolumn{2}{|l|}{ Total LAB/CONT LQ } & 0.0 & 3.2 & 3.2 & 2.9 & 2.3 & 1.5 & 9.0 & 2.2 & 2.5 & 2.5 & 2.5 & 2.5 & 2.5 & 2.5 & 2.5 & 2.5 \\
\hline LEAD & D & 0.0 & 311.8 & 311.8 & 26.8 & 7.4 & 0.6 & 51.0 & 7.1 & 0.2 & 0.2 & 0.2 & 0.2 & 0.2 & 0.2 & 0.2 & 0.2 \\
\hline TOta1 LEAD & & 0.0 & 311.8 & 311.8 & 16.8 & 7.1 & 8.6 & 52.0 & 7.1 & 0.2 & 0.2 & 0.2 & 0.2 & 0.2 & 0.2 & 0.2 & 0.2 \\
\hline ORG ABS LO/SLG & NONE & 0.0 & 1.6 & 2.1 & 3.6 & 3.3 & 6.6 & 10.1 & 2.7 & 1.1 & 1.4 & 1.1 & 1.4 & 1.4 & 1.4 & 2.4 & 1.4 \\
\hline \multicolumn{2}{|l|}{ TOtal ORG ABS LQ/SLG } & 0.0 & 1.6 & 2.1 & 3.6 & 3.3 & 6.6 & 10.1 & 2.7 & 1.4 & 1.4 & 1.1 & 1.1 & 2.4 & 1.1 & 1.4 & 1.4 \\
\hline ORG PART & NONE & 0.0 & 1.6 & 2.1 & 3.6 & 3.3 & 6.6 & 10.1 & 2.7 & 2.4 & 1.4 & 1.4 & 1.4 & 1.4 & 1.4 & 1.4 & 1.4 \\
\hline \multicolumn{2}{|l|}{ Total ORG PART } & 0.0 & 1.6 & 2.1 & 3.6 & 3.3 & 6.6 & 10.1 & $2.7^{\circ}$ & 1.4 & 2.4 & 1.4 & 1.4 & 1.1 & 1.1 & 1.4 & 1.4 \\
\hline \multicolumn{2}{|l|}{ Tote1 ANL_E } & 0.0 & 804.3 & 966.7 & 579.5 & 518.4 & 970.9 & 1558.3 & 121.7 & 234.3 & 234.3 & 231.3 & 234.3 & 234.3 & 234.3 & 234.3 & 234.3 \\
\hline \multicolumn{18}{|c|}{ Was te Generator:BAPI } \\
\hline CONT SOIL & Fo & 0.0 & 0.2 & 0.9 & 0.1 & 0.5 & 0.5 & 0.5 & 0.0 & $0.0^{\circ}$ & 0.0 & 0.0 & 0.0 & 0.0 & 0.0 & 0.0 & 0.0 \\
\hline Total cont soll & & 0.0 & 0.2 & 0.9 & 0.7 & 0.5 & 0.5 & 0.5 & 0.0 & 0.0 & 0.0 & 0.0 & 0.0 & 0.0 & 0.0 & 0.0 & 0.0 \\
\hline \multirow[t]{2}{*}{ D-COMBUST } & G & 0.0 & 0.0 & 0.0 & 0.0 & 0.0 & 0.0 & 0.0 & 0.0 & 0.0 & 0.0 & 0.0 & 0.0 & 0.0 & 0.0 & 0.0 & 0.0 \\
\hline & NONE & 0.0 & $4.5^{\circ}$ & 2.0 & 2.0 & 2.0 & 1.1 & 1.1 & 0.0 & 0.0 & 0.0 & 0.0 & 0.0 & 0.0 & 0.0 & 0.0 & 0.0 \\
\hline Tota1 D-COMBUST & & 0.0 & 4.5 & 2.0 & 2.0 & 2.0 & 1.1 & 1.1 & 0.0 & 0.0 & 0.0 & 0.0 & 0.0 & 0.0 & 0.0 & 0.0 & 0.0 \\
\hline \multirow[t]{3}{*}{ D-HET } & EG & 0.0 & 0.0 & 0.0 & 0.0 & 0.0 & 0.0 & 0.0 & 0.0 & 0.0 & 0.0 & 0.0 & 0.0 & 0.0 & 0.0 & 0.0 & 0.0 \\
\hline & FG & 0.0 & 1.1 & 4.6 & 3.8 & 2.6 & 2.6 & 2.6 & 0.3 & 0.3 & 0.3 & 0.3 & 0.3 & 0.3 & 0.3 & 0.3 & 0.3 \\
\hline & MONE & 0.0 & 3.2 & 1.0 & 1.0 & 1.0 & 1.0 & 1.0 & 0.0 & 0.0 & 0.0 & 0.0 & 0.0 & 0.0 & 0.0 & 0.0 & 0.0 \\
\hline Total D-HET & & 0.0 & 1.7 & 5.7 & 4.8 & 3.6 & 3.6 & 3.6 & 0.3 & 0.3 & 0.3 & 0.3 & 0.3 & 0.3 & 0.3 & 0.3 & 0.3 \\
\hline D-1N NON-HTL & NONE & 0.0 & 1.7 & 1.1 & 1.1 & 1.1 & 0.2 & .0 .2 & 0.0 & 0.0 & 0.0 & 0.0 & 0.0 & 0.0 & 0.0 & 0.0 & 0.0 \\
\hline Tota1 D-IN MON-MTL & & 0.0 & 1.7 & 1.2 & 1.1 & 1.1 & 0.2 & 0.2 & 0.0 & 0.0 & 0.0 & 0.0 & 0.0 & 0.0 & 0.0 & 0.0 & 0.0 \\
\hline D-METAL & NOIE & 0.0 & 45.5 & 18.8 & 18.8 & 18.8 & 12.1 & 12.1 & 0.0 & 0.0 & 0.0 & 0.0 & 0.0 & 0.0 & 0.0 & 0.0 & 0.0 \\
\hline Total D-METAL & & 0.0 & 45.5 & 18.8 & 18.8 & 18.8 & 12.1 & 12.1 & 0.0 & 0.0 & 0.0 & 0.0 & 0.0 & 0.0 & 0.0 & 0.0 & 0.0 \\
\hline IN ORG LQ/SLG & D & 0.0 & 0.0 & 0.0 & 0.0 & 0.0 & 0.0 & 0.0 & 0.0 & 0.0 & 0.0 & 0.0 & 0.0 & 0.0 & 0.0 & 0.0 & 0.0 \\
\hline
\end{tabular}

gco Codes: A-Ignitables, B-Corrosives, C-Reactives, D-Metals w/O Hg, E-Metals w Hg, F-Organles, G-State Reg.. H-PCB < 50ppm, J-PCB > 50ppm 
Annual Physical Haste Form and gazardous Constituent volumes by Faste Generator

(In cublo metera)

PHE

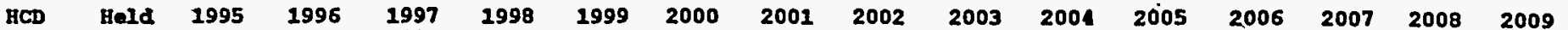

\begin{tabular}{|c|c|c|c|c|c|c|c|c|c|c|c|c|c|c|c|c|c|}
\hline Tot.21 aN ORG LQ/SLG & & 0.0 & 0.0 & 0.0 & 0.0 & 0.0 & 0.0 & 0.0 & 0.0 & 0.0 & 0.0 & 0.0 & 0.0 & 0.0 & 0.0 & 0.0 & 0.0 \\
\hline LEAD & D & 0.0 & 8.0 & 25.8 & 21.1 & 14.4. & 16.4 & 14.4 & 1.9 & 1.9 & 1.9 & $1: 9$ & 1.9 & 1.9 & 1.9 & 1.9 & 1.9 \\
\hline TDtaI LEAD & & 0.0 & 8.0 & 25.8 & 21.1 & 14.1 & 14.1 & 11.1 & 1.9 & 1.9 & 1.9 & 1.9 & 1.9 & 1.9 & 1.9 & 1.9 & 1.9 \\
\hline \multirow[t]{5}{*}{ ORG AES LO/SLG } & D & 0.0 & 0.3 & 3.0 & 0.0 & 0.5 & 0.5 & 0.5 & 0.0 & 0.0 & 0.0 & 0.0 & 0.0 & 0.0 & 0.0 & 0.0 & 0.0 \\
\hline & E & 0.0 & 0.2 & 0.6 & 0.5 & 0.3 & 0.3 & 0.3 & 0.0 & 0.0 & 0.0 & 0.0 & 0.0 & 0.0 & 0.0 & 0.0 & 0.0 \\
\hline & $\mathbf{r}$ & 0.0 & 0.0 & 0.0 & 0.0 & 0.0 & 0.0 & 0.0 & 0.0 & 0.0 & 0.0 & 0.0 & 0.0 & 0.0 & 0.0 & 0.0 & 0.0 \\
\hline & 6 & 0.0 & 0.2 & 0.9 & 0.7 & 0.5 & 0.5 & 0.5 & 0.0 & 0.0 & 0.0 & 0.0 & 0.0 & 0.0 & 0.0 & 0.0 & 0.0 \\
\hline & 3 & 0.0 & 0.2 & 0.5 & 0.1 & 0.3 & 0.3 & 0.3 & 0.0 & 0.0 & 0.0 & 0.0 & 0.0 & 0.0 & 0.0 & 0.0 & 0.0 \\
\hline Total ORG ABS LQ/SLG & & 0.0 & 1.0 & 3.2 & 2.6 & 1.0 & 1.8 & 1.8 & 0.2 & 0.2 & 0.2 & 0.2 & 0.2 & 0.2 & 0.2 & 0.2 & 0.2 \\
\hline ORG PART & $\mathbf{E}$ & 0.0 & 0.0 & 0.1 & 0.0 & 0.0 & 0.0 & 0.0 & 0.0 & 0.0 & 0.0 & 0.0 & 0.0 & 0.0 & 0.0 & 0.0 & 0.0 \\
\hline Total ORG PART & & 0.0 & 0.0 & 0.1 & 0.0 & 0.0 & 0.0 & 0.0 & 0.0 & 0.0 & 0.0 & 0.0 & 0.0 & 0.0 & 0.0 & 0.0 & 0.0 \\
\hline Total BAPL & & 0.0 & 66.1 & 57.9 & 51.6 & 42.5 & 34.1 & 34.1 & 2.5 & 2.5 & 2.5 & 2.5 & 2.5 & 2.5 & 2.5 & 2.5 & 2.5 \\
\hline
\end{tabular}

Waste Generatox:BuPL sHIPYDs

$\begin{array}{llllllllllllllllllll}\text { D-METAL } & \text { NONE } & 0.0 & 1.5 & 0.0 & 0.0 & 0.0 & 0.0 & 0.0 & 0.0 & 0.0 & 0.0 & 0.0 & 0.0 & 0.0 & 0.0 & 0.0 & 0.0\end{array}$

\begin{tabular}{lllllllllllllllll} 
TOLAL D-HETAL & 0.0 & 1.5 & 0.0 & 0.0 & 0.0 & 0.0 & 0.0 & 0.0 & 0.0 & 0.0 & 0.0 & 0.0 & 0.0 & 0.0 & 0.0 & 0.0 \\
\hline Total BAPL_SHIPYDS & 0.0 & 1.5 & 0.0 & 0.0 & 0.0 & 0.0 & 0.0 & 0.0 & 0.0 & 0.0 & 0.0 & 0.0 & 0.0 & 0.0 & 0.0 & 0.0 \\
\hline
\end{tabular}

\section{Faste Generator:Bar_criss_ma}

$\begin{array}{llllllllllllllllllllll}\text { CONT SOIL } & \text { NONE } & . & 0.0 & 29.4 & 29.4 & 29.4 & 36.8 & 36.0 & 33.1 & 0.0 & 0.0 & 0.0 & 0.0 & 0.0 & 0.0 & 0.0 & 0.0 & 0.0\end{array}$

\begin{tabular}{|c|c|c|c|c|c|c|c|c|c|c|c|c|c|c|c|}
\hline rotal ConT & 29.4 & 29.1 & 29.1 & 36.8 & 36.8 & 33.1 & 0.0 & 0.0 & 0.0 & 0.0 & 0.0 & 0.0 & 0.0 & 0.0 & 0.0 \\
\hline D-COHBUST & 22.6 & 22.6 & 22.6 & 28.3 & 28.3 & 25.5 & 0.0 & 0.0 & 0.0 & 0.0 & 0.0 & 0.0 & 0.0 & 0.0 & 0.0 \\
\hline
\end{tabular}

\begin{tabular}{|c|c|c|c|c|c|c|c|c|c|c|c|c|c|c|c|}
\hline Total D-COHBUST & 22.8 & 22.6 & 22.6 & 28.3 & 20.3 & 25.5 & 0.0 & 0.0 & 0.0 & 0.0 & 0.0 & 0.0 & 0.0 & 0.0 & 0.0 \\
\hline NON-MTL & 90.6 & 90.6 & 90.6 & 128.4 & 127.1 & 115.0 & 0.0 & 0.0 & 0.0 & 0.0 & 0.0 & 0.0 & 0.0 & 0.0 & 0.0 \\
\hline
\end{tabular}

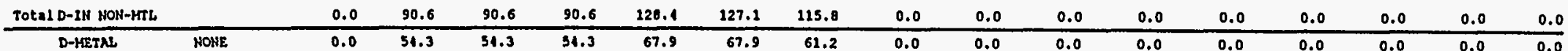

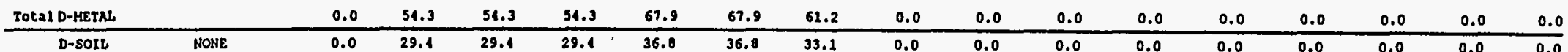

\begin{tabular}{|c|c|c|c|c|c|c|c|c|c|c|c|c|c|c|c|c|c|}
\hline Total D-SOIL & & 0.0 & 29.1 & 29.4 & 29.1 & 36.0 & 36.0 & 33.1 & 0.0 & 0.0 & 0.0 & 0.0 & 0.0 & 0.0 & 0.0 & 0.0 & 0.0 \\
\hline IN ORG PART & MONE & 0.0 & 0.0 & 0.0 & 0.0 & 8.8 & 6.0 & $\theta .0$ & 0.0 & 0.0 & 0.0 & 0.0 & 0.0 & 0.0 & 0.0 & 0.0 & 0,0 \\
\hline
\end{tabular}

\begin{tabular}{llllllllllllllllll} 
TOtaL IN ORG PART & 0.0 & 0.0 & 0.0 & 0.0 & 8.0 & 8.0 & 8.0 & 0.0 & 0.0 & 0.0 & 0.0 & 0.0 & 0.0 & 0.0 & 0.0 & 0.0 & \\
\hline & 0.0 & 0.3 & 0.3 & 0.3 & 0.3 & 0.0 & 0.0 & 0.0 & 0.0 & 0.0 & 0.0
\end{tabular}

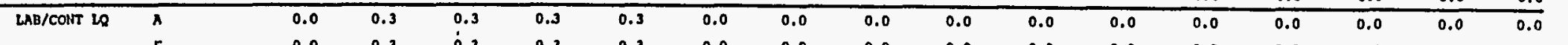

TOLAL LAB/CONT LO $\begin{array}{llllll}0.0 & 0.6 & 0.6 & 0.6 & 0.6 & 0.0\end{array}$ 0.0

$0.0 \quad 0.0$

HCD Codes: A=Ignitables, B-Corrosives, C-Reactives, De-Metals w/O Hg, E-Metals w Hg, F-Organics, G=State Reg., HuPCB < 50ppm, J-PCB > 50ppm 
Annual Phyaical Waste Form and Bazardous Constituent Volumes by Waste Generator

(In cubic meters)

\begin{tabular}{|c|c|c|c|c|c|c|c|c|c|c|c|c|c|c|c|c|c|}
\hline PFF & HCD & Held & 1995 & 1996 & 1997 & 1998 & 1999 & 2000 & 2001 & 2002 & 2003 & 2004. & $2 \dot{0005}$ & 2006 & 2007 & 2008 & 2009 \\
\hline \multirow[t]{3}{*}{ LEAD } & D & 0.0 & 1.2 & 4.2 & 4.2 & 4.2 & 0.0 & 0.0 & 0.0 & 0.0 & 0.0 & 0.0 & 0.0 & 0.0 & 0.0 & 0.0 & 0.0 \\
\hline & E & 0.0 & 0.0 & 0.0 & 0.0 & 0.8 & 0.0 & 0.0 & 0.0 & 0.0 & 0.0 & 0.0 & 0.0 & 0.0 & 0.0 & 0.0 & 0.0 \\
\hline & EH & 0.0 & 0.5 & 0.5 & 0.5 & 0.5 & 0.0 & 0.0 & 0.0 & 0.0 & 0.0 & 0.0 & 0.0 & 0.0 & 0.0 & 0.0 & 0.0 \\
\hline Total LEAD & & 0.0 & -5.6 & 3.6 & 5.6 & 5.6 & 0.0 & 0.0 & 0.0 & 0.0 & 0.0 & 0.0 & 0.0 & 0.0 & 0.0 & 0.0 & 0.0 \\
\hline \multirow[t]{2}{*}{ MERCURY } & $\bar{E}$ & 0.0 & 5.1 & 5.1 & 5.1 & 5.1 & 0.0 & 0.0 & 0.0 & 0.0 & 0.0 & 0.0 & 0.0 & 0.0 & 0.0 & 0.0 & $\overline{0.0}$ \\
\hline & EH & 0.0 & 0.5 & 0.5 & 0.5 & 0.5 & 0.0 & 0.0 & 0.0 & 0.0 & 0.0 & 0.0 & 0.0 & 0.0 & 0.0 & 0.0 & 0.0 \\
\hline TOt:al HERCURY & & 0.0 & 5.6 & 5.6 & 5.6 & 5.6 & 0.0 & 0.0 & 0.0 & 0.0 & 0.0 & 0.0 & 0.0 & 0.0 & 0.0 & 0.0 & 0.0 \\
\hline ORG ABS LQ/SLG & $r$ & 0.0 & 0.6 & 0.6 & 0.6 & 0.6 & 0.0 & 0.0 & $\cdot 0.0$ & 0.0 & 0.0 & 0.0 & 0.0 & 0.0 & 0.0 & 0.0 & $\overline{0.0}$ \\
\hline TOtal ORG ABS LR/SLG & & 0.0 & 0.6 & 0.6 & 0.6 & 0.6 & 0.0 & 0.0 & 0.0 & 0.0 & 0.0 & 0.0 & 0.0 & 0.0 & 0.0 & 0.0 & 0.0 \\
\hline SH LEAD & MONE & 0.0 & 0.0 & 0.0 & 0.0 & 1.2 & 1.1 & 1.1 & 0.0 & 0.0 & 0.0 & 0.0 & 0.0 & 0.0 & $\overline{0.0}$ & 0.0 & $\overline{0.0}$ \\
\hline Tota1 SH LEAD & & 0.0 & 0.0 & 0.0 & 0.0 & 1.2 & 1.1 & 1.1 & 0.0 & 0.0 & 0.0 & 0.0 & 0.0 & 0.0 & 0.0 & 0.0 & 0.0 \\
\hline TAta1 BAT_CLBS_LAB & & 0.0 & 239.2 & 239.2 & 239.2 & 321.1 & 305.4 & 278.1 & 0.0 & 0.0 & 0.0 & 0.0 & 0.0 & 0.0 & 0.0 & 0.0 & 0.0 \\
\hline \multicolumn{2}{|c|}{ Waste Generator:BRuruis } & & & & & & & & & & & & & & & & \\
\hline CONT SOIL & MONE & 0.0 & 24.1 & 26.3 & 17.1 & 10.0 & 10.9 & 19.0 & 20.8 & 21.9 & 23.0 & 24.1 & 25.3 & 26.6 & 27.9 & 29.3 & 30.8 \\
\hline Total cons sorh & & 0.0 & 24.4 & 16.3 & 17.1 & 18.0 & 10.9 & 19.8 & 20.0 & 21.9 & 23.0 & 24.1 & 25.3 & 26.6 & 27.9 & 29.3 & 30.8 \\
\hline D-HET & MONE & 0.0 & 123.5 & 61.0 & 85.1 & 99.3 & 93.8 & 98.5 & 303.4 & 108.6 & 114.0 & 119.7 & 125.7 & 132.0 & 138.6 & 145.5 & $\overline{152.0}$ \\
\hline Total D-HET & & 0.0 & 123.5 & 01.0 & 85.1 & 89.3 & 93.8 & 98.5 & 103.4 & 108.6 & 114.0 & 119.7 & 125.7 & $132.0^{\circ}$ & 138.6 & 115.5 & 152.8 \\
\hline D-IN NON-MTL & NONE & 0.0 & 36.9 & 24.5 & 23.7 & 27.0 & 28.4 & 29.8 & 31.3 & 32.8 & 34.5 & 36.2 & 38.0 & 39.9 & 11.9 & 11.0 & $\overrightarrow{16.2}$ \\
\hline Jotal D-IN NON-HTL & & 0.0 & 36.9 & 24.5 & 25.7 & 27.0 & 20.1 & 29.0 & 31.3 & 32.0 & 34.5 & 36.2 & 38.0 & 39.9 & 11.9 & 14.0 & $16.2^{\circ}$ \\
\hline D-METAL & $A$ & 0.0 & 0.1 & 0.2 & 0.1 & 0.1 & 0.1 & 0.1 & 0.1 & 0.1 & 0.1 & 0.1 & 0.1 & 0.1 & 0.1 & 0.1 & 0.1 \\
\hline$\cdot$ & NONE & 0.0 & 190.6 & 121.9 & 131.1 & 237.7 & 244.6 & 251.8 & 159.1 & 167.4 & 175.7 & 184.5 & 193.7 & 203.4 & 213.6 & 224.3 & 235.5 \\
\hline Total D-METAL & & 0.0 & 191.0 & 125.2 & 131.3 & 137.0 & 114.7 & 152.0 & 159.6 & 167.5 & 175.9 & 294.7 & 193.9 & 203.6 & 213.0 & 224.5 & 235.7 \\
\hline D-soll & MONE & 0.0 & 55.5 & 35.7 & 37.4 & 39.3 & 41.3 & 13.3 & 15.5 & 17.8 & 50.2 & 52.7 & 55.3 & 58.1 & 61.0 & 64.1 & 67.3 \\
\hline Tota1 D-sorL & & 0.0 & 35.5 & 35.7 & 37.4 & 39.3 & 41.3 & 13.3 & 15.5 & 47.8 & 50.2 & 32.7 & 55.3 & 58.1 & 61.0 & $\therefore \quad 64.1$ & 67.3 \\
\hline IN ORG LQ/SLG & MONE & 0.0 & 13.5 & 10.4 & 10.9 & 11.4 & 12.0 & 12.6 & 13.2 & 13.9 & 14.6 & $\overline{15.3}$ & $\overline{16.1}$ & 16.9 & 27.8 & 18.6 & 19.6 \\
\hline Total IN ORG LQ/SLG & & 0.0 & 15.5 & 10.1 & 10.9 & 11.4 & 12.0 & 12.6 & 13.2 & 23.9 & 24.6 & 15.3 & 16.1 & 16.9 & 17.0 & 18.6 & 19.6 \\
\hline LAB/CONT LQ & $\dot{\boldsymbol{\lambda}}$ & 0.0 & 1.9 & 1.1 & 0.6 & 0.6 & 0.6 & 0.6 & 0.6 & 0.6 & 0.6 & 0.6 & 0.6 & 0.6 & 0.6 & 0.6 & $\overline{0.6}$ \\
\hline & $A B$ & 0.0 & 0.9 & 0.5 . & 0.3 & 0.3 & 0.3 & 0.3 & 0.3 & 0.3 & 0.3 & 0.3 & 0.3 & 0.3 & 0.3 & 0.3 & 0.3 \\
\hline & $B$ & 0.0 & 0.9 & 0.5 & 0.3 & 0.3 & 0.3 & 0.3 & 0.3 & 0.3 & 0.3 & 0.3 & 0.3 & 0.3 & 0.3 & 0.3 & 0.3 \\
\hline & MOHE & 0.0 & 2.2 & 1.4 & 1.5 & 1.6 & 1.7 & 1.8 & 1.0 & 1.9 & 2.0 & 2.1 & 2.3 & 2.4 & 2.5 & 2.6 & 2.0 \\
\hline TOtal LAB/CONT LQ & & 0.0 & 6.0 & 3.7 & 2.9 & 2.9 & 3.0 & 3.1 & 3.2 & 3.3 & 3.1 & 3.5 & 3.6 & 3.7 & 3.9 & 4.0 & 1.1 \\
\hline LEAD & $D$ & 0.0 & 0.2 & 0.1 & 0.0 & 0.0 & 0.0 & 0.0 & 0.0 & 0.0 & 0.0 & 0.0 & 0.0 & 0.0 & 0.0 & 0.0 & .0 .0 \\
\hline TOtal LEAD & & 0.0 & 0.2 & 0.1 & 0.0 & 0.0 & 0.0 & 0.0 & 0.0 & 0.0 & 0.0 & 0.0 & 0.0 & 0.0 & 0.0 & 0.0 & 0.0 \\
\hline
\end{tabular}

BCo Codes: A-Ignitables, B-Corrosives, C-Reactives, D-Metals w/O Hg, E-Metals w Hg, F-Organics, G-State Reg., H-PCB < S0ppm, J-PCB > 50ppm 


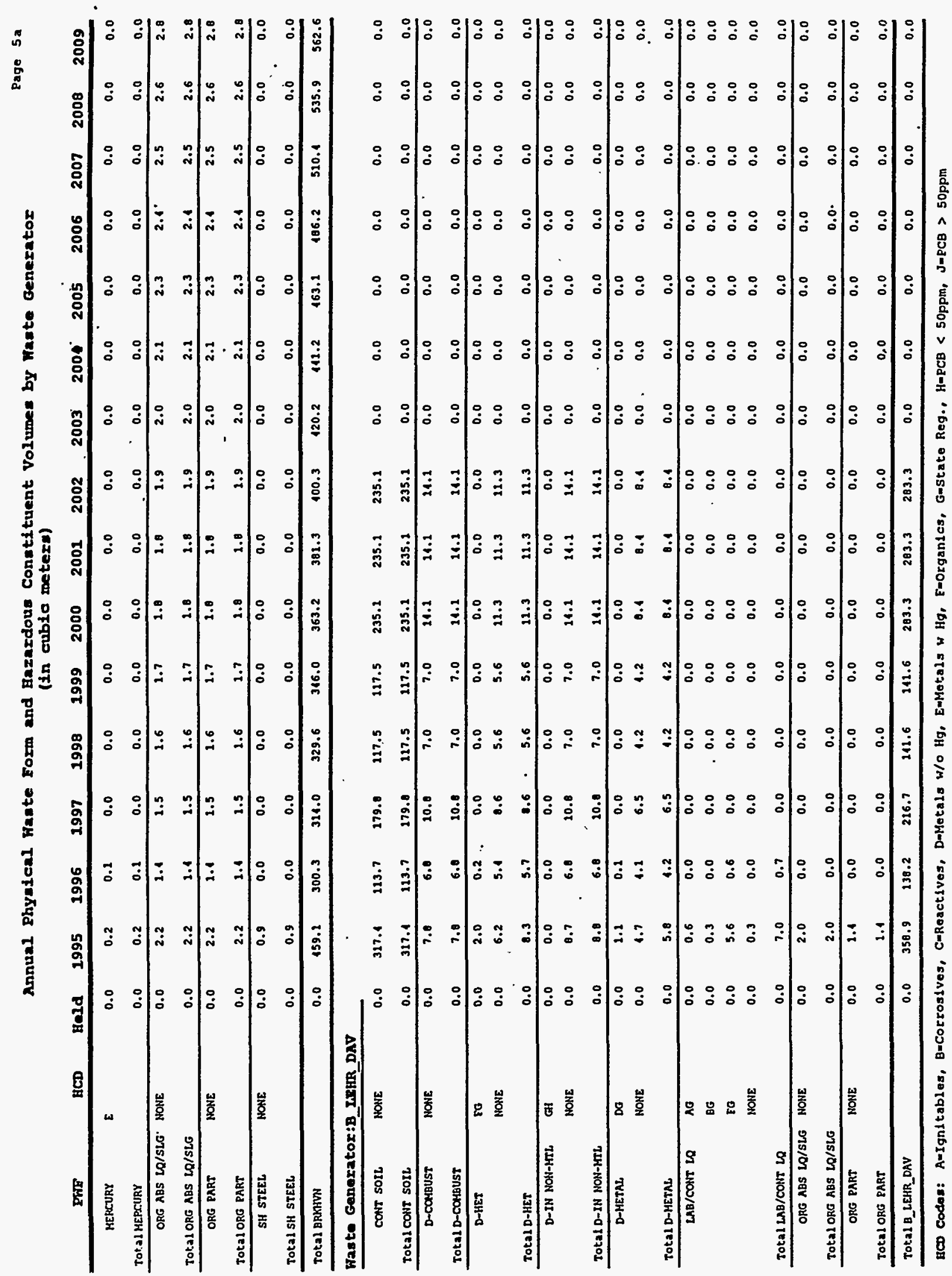


Annual Physical Haste Form and Bazardous Constituent Volumes by Faste Generator

(in cublo meters)

\begin{tabular}{|c|c|c|c|c|c|c|c|c|c|c|c|c|c|c|c|c|c|}
\hline PWF & HCD & Held & 1995 & 1996 & 1997 & 1998 & 1999 & 2000 & 2001 & 2002 & 2003 & 2001 & 2005 & 2006 & 2007 & 2008 & 2009 \\
\hline \multicolumn{18}{|c|}{ Waste Genorator:EGcG } \\
\hline D-CONBUST & NONE & 0.0 & 13.2 & 23.5 & 13.5 & 13.5 & 23.5 & 13.5 & 0.0 & 0.0 & 0.0 & 0.0 & 0.0 & 0.0 & 0.0 & 0.0 & 0.0 \\
\hline Tot:Al D-COMBUST & & 0.0 & 13.2 & 13.5 & 13.5 & 13.5 & 13.5 & 13.5 & 0.0 & 0.0 & 0.0 & 0.0 & 0.0 & 0.0 & 0.0 & 0.0 & 0.0 \\
\hline D-HET & HONE & 0.0 & 10.3 & 10.5 & 10.5 & 10.5 & 10.5 & 10.5 & 0.0 & 0.0 & 0.0 & 0.0 & 0.0 & 0.0 & 0.0 & 0.0 & 0.0 \\
\hline Tota I D-HET & & 0.0 & 10.3 & 20.5 & 10.5 & 10.5 & 20.5 & 20.5 & 0.0 & 0.0 & 0.0 & 0.0 & 0.0 & 0.0 & 0.0 & 0.0 & 0.0 \\
\hline D-IN NON-MTL & MOHE & 0.0 & .2 .6 & 2.7 & 2.7 & 2.7 & 2.7 & 2.7 & 0.0 & 0.0 & 0.0 & 0.0 & 0.0 & 0.0 & 0.0 & 0.0 & 0.0 \\
\hline Total D-IN HON-MTL & & 0.0 & 2.6 & 2.7 & 2.7 & 2.7 & 2.7 & 2.7 & 0.0 & 0.0 & 0.0 & 0.0 & 0.0 & 0.0 & 0.0 & 0.0. & 0.0 \\
\hline D-METAS & MONE & 0.0 & 0.2 & 0.2 & 0.2 & 0.2 & 0.2 & 0.2 & 0.0 & 0.0 & 0.0 & 0.0 & 0.0 & 0.0 & 0.0 & 0.0 & 0.0 \\
\hline TOtal D-METAL & & 0.0 . & 0.2 & 0.2 & 0.2 & 0.2 & 0.2 & 0.2 & 0.0 & 0.0 & 0.0 & 0.0 & 0.0 & 0.0 & 0.0 & 0.0 & 0.0 \\
\hline ORG $A B S$ LQ/SLG & MONE & 0.0 & 129.1 & 0.0 & 0.0 & 0.0 & 0.0 & 0.0 & 0.0 & 0.0 & 0.0 & 0.0 & 0.0 & 0.0 & 0.0 & 0.0 & 0.0 \\
\hline Total ORG ABS LQ/SLG & & 0.0 & 129.1 & 0.0 & 0.0 & 0.0 & 0.0 & 0.0 & 0.0 & 0.0 & 0.0 & 0.0 & 0.0 & 0.0 & 0.0 & 0.0 & 0.0 \\
\hline Tota1 EGGG & & 0.0 & 155.6 & 27.1 & 27.1 & 27.1 & 27.1 & 27.1 & 0.0 & 0.0 & 0.0 & 0.0 & 0.0 & 0.0 & 0.0 & .0 .0 & 0.0 \\
\hline \multicolumn{18}{|c|}{ Haste Generator: BRRMY } \\
\hline BATTERIES & D & 0.0 & 0.2 & 0.2 & 0.2 & 0.2 & 0.2 & 0.2 & 0.2 & 0.2 & 0.2 & 0.2 & 0.2 & 0.2 & 0.2 & 0.2 & 0.2 \\
\hline Total BATTERTES & & 0.0 & 0.2 & 0.2 & 0.2 & 0.2 & 0.2 & 0.2 & 0.2 & 0.2 & 0.2 & 0.2 & 0.2 & 0.2 & 0.2 & 0.2 & 0.2 \\
\hline Cont soth & NONE & 0.0 & .0 .5 & 0.5 & 0.5 & 0.5 & 0.5 & 0.5 & 0.3 & 0.5 & 0.5 & 0.5 & 0.5 & 0.5 & 0.5 & 0.5 & 0.5 \\
\hline Total conT SOIL & & 0.0 & 0.5 & 0.5 & 0.3 & 0.5 & 0.5 & 0.5 & 0.5 & 0.5 & 0.5 & 0.5 & 0.5 & 0.5 & 0.5 & 0.5 & 0.5 \\
\hline D-casBust & NONE & 0.1 & 3.3 & 3.3 & 3.3 & 3.3 & 3.3 & 3.3 & 3.3 & 3.3 & 3.3 & 3.3 & 3.3 & 3.3 & 3.3 & 3.3 & 3.3 \\
\hline Total D-conguss & & 0.1 & 3.3 & 3.3 & 3.3 & 3.3 & 3.3 & 3.3 & 3.3 & 3.3 & 3.3 & 3.3 & 3.3 & 3.3 & 3.3 & 3.3 & 3.3 \\
\hline D-HET & NONE & 0.1 & 1.4 & 1.4 & 1.4 & 1.4 & 1.4 & 1.1 & 1.1 & 1.4 & 1.4 & 1.4 & 1.1 & 1.1 & 1.4 & 1.4 & 1.4 \\
\hline Total D-HET & & 0.1 & 1.4 & 1.4 & 1.1 & 1.1 & 1.1 & 1.4 & 1.1 & 1.1 & 1.1 & 1.1 & 1.1 & 1.4 & 1.4 & 1.1 & 1.1 \\
\hline D-IN NON-HTL & NONE & 0.1 & 0.9 & 0.9 & 0.9 & 0.9 & 0.9 & 0.9 & 0.9 & 0.9 & 0.9 & 0.9 & 0.9 & 0.9 & 0.9 & 0.9 & 0.9 \\
\hline Total D-IN MON-MTL & & 0.1 & 0.9 & 0.9 & 0.9 & 0.9 & 0.9 & 0.9 & 0.9 & 0.9 & 0.9 & 0.9 & 0.9 & 0.9 & 0.9 & 0.9 & 0.9 \\
\hline D-METNL & NONE & 26.7 & 35.2 & 35.2 & 35.2 & 35.2 & 35.2 & 35.2 & 35.2 & 35.2 & 35.2 & 35.2 & 35.2 & 35.2 & 35.2 & 35.2 & 35.2 \\
\hline Total D-METAL & & 26.7 & 35.2 & 35.2 & 35.2 & 35.2 & 35.2 & 35.2 & 35.2 & 35.2 & 35.2 & 35.2 & 35.2 & 35.2 & 35.2 & 35.2 & 35.2 \\
\hline IN ORG LQ/SLG & NONE & 0.0 & 1.0 & 2.0 & 1.0 & 1.0 & 1.0 & 1.0 & 1.0 & 1.0 & 1.0 & 1.0 & 1.0 & 1.0 & 1.0 & 1.0 & 1.0 \\
\hline TOt=1 IN ORG LQ/SLG & & 0.0 & 1.0 & 1.0 & 1.0 & 1.0 & 3.0 & 1.0 & 1.0 & 1.0 & -1.0 & 1.0 & 1.0 & 1.0 & 1.0 & 1.0 & 1.0 \\
\hline IN ORG PART & NONE & 0.0 & .0 .6 & 0.6 & 0.6 & 0.6 & 0.6 & 0.6 & 0.6 & 0.6 & 0.6 & 0.6 & 0.6 & 0.6 & 0.6 & 0.6 & 0.6 \\
\hline TOtal IN ORG PART & & 0.0 & 0.6 & 0.6 & 0.6 & 0.6 & 0.6 & 0.6 & 0.6 & 0.6 & 0.6 & 0.6 & 0.6 & 0.6 & 0.6 & 0.6 & 0.6 \\
\hline LAB/CONT LQ & BD & 0.0 & 0.2 & 0.2 & 0.2 & 0.2 & 0.2 & 0.2 & 0.2 & 0.2 & 0.2 & 0.2 & 0.2 & 0.2 & 0.2 & 0.2 & 0.2 \\
\hline TOta1 LAB/CONT LQ & . & 0.0 & 0.2 & 0.2 & 0.2 & 0.2 & 0.2 & 0.2 & 0.2 & 0.2 & 0.2 & 0.2 & 0.2 & 0.2 & 0.2 & 0.2 & 0.2 \\
\hline LEND & D & 0.0 & 0.5 & 0.5 & 0.5 & 0.5 & 0.5 & 0.5 & 0.5 & 0.5 & 0.5 & 0.5 & 0.5 & 0.5 & 0.5 & 0.5 & 0.5 \\
\hline
\end{tabular}

BCo Codes: A-Ignitables, B-Corroslves, C-Reactives, D=Metals w/O Hg, E-Metils w Hg, E-Organlcs, G-Stato Reg., H-PCB < 50ppm, J-PCB > 50ppm 
(in cubic meters)

\begin{tabular}{|c|c|c|c|c|c|c|c|c|c|c|c|c|c|c|c|c|c|}
\hline PWE & HCD & Held & 1995 & 1996 & 1997 & 1998 & 1999 & 2000 & 2001 & 2002 & 2003 & 2004 & 2005 & 2006 & 2007 & 2008 & 2009 \\
\hline TOtal LEAD & & 0.0 & 0.5 & 0.5 & 0.5 & 0.5 & 0.5 & 0.5 & 0.5 & $\dot{0.5}$ & 0.5 & 0.5 & 0.5 & 0.5 & 0.5 & 0.5 & 0.5 \\
\hline ORG ABS LQ/SLG & MONE & 0.0 & 1.0 & 1.0 & 1.0 & 1.0 & 1.0 & 1.0 & 1.0 & 1.0 & 1.0 & 1.0 & 1.0 & 1.0 & 1.0 & 1.0 & 9.0 \\
\hline Total ORG ABS LQ/SLG & & 0.0 & 1.0 & 1.0 & 1.0 & 1.0 & 1.0 & 1.0 & 1.0 & 1.0 & 1.0 & 1.0 & 1.0 & 1.0 & 1.0 & 1.0 & 1.0 \\
\hline SALT WASTE & G & 0.0 & 1.5 & 1.5 & 1.5 & 1.5 & 1.5 & 1.5 & 1.5 & 1.5 & 1.5 & 1.5 & 1.5 & 1.5 & 1.5 & 1.5 & 1.5 \\
\hline TOtal SRLT WASTE & & 0.0 & 1.5 & 1.5 & 1.5 & 1.5 & 1.5 & 1.5 & 1.5 & 1.5 & 1.5 & 1.5 & 1.5 & 1.5 & 1.5 & 1.5 & 1.5 \\
\hline TOtal EERAI & & 27.2 & 46.9 & 16.9 & 16.9 & 46.9 & 16.9 & 16.9 & 16.9 & 16.9 & 46.9 & 46.9 & 16.9 & 46.9 & 16.9 & 16.9 & 16.9 \\
\hline \multicolumn{18}{|c|}{ Waste Genorator:IUSRIP } \\
\hline CONT SOIL & MONE & 0.0 & 2089.5 & 2809.5 & 2889.5 & 0.0 & 0.0 & 115.1 & 1007.0 & 1007.0 & 1007.0 & 2301.8 & 1294.7 & 0.0 & 0.0 & 0.0 & 0.0 \\
\hline Total cont soIt & & 0.0 & 2089.5 & 2889.5 & 2889.3 & 0.0 & 0.0 & 115.1 & 1007.0 & 1007.0 & 1007.0 & 2301.0 & 1294.7 & 0.0 & 0.0 & 0.0 & 0.0 \\
\hline D-IN NON-KTL & NONE & 0.0 & 506.8 & .505 .8 & 506.0 & 0.0 & 0.0 & 91.3 & 799.5 & 799.5 & 799.5 & 1827.5 & 2027.9 & 0.0 & 0.0 & 0.0 & 0.0 \\
\hline Total D-IN NON-HTL & & 0.0 & 506.0 & 506.0 & 506.0 & 0.0 & 0.0 & 91.3 & 799.5 & 799.5 & 799.5 & 1027.5 & 1027.9 & 0.0 & 0.0 & 0.0 & 0.0 \\
\hline D-SOIL & NONE & 0.0 & 223.9 & 223.9 & 223.9 & 0.0 & 0.0 & 8.9 & 78.0 & 78.0 & 78.0 & 178.1 & 100.3 & 0.0 & 0.0 & 0.0 & 0.0 \\
\hline Total D-soIh & & 0.0 & 223.9 & 223.9 & 223.9 & 0.0 & 0.0 & 0.9 & 70.0 & 78.0 & 78.0 & 178.1 & 100.3 & 0.0 & 0.0 & 0.0 & 0.0 \\
\hline IN ORG PART & MONE & 0.0 & 0.0 & 0.0 & 0.0 & 0.0 & 0.0 & 2.1 & 19.0 & 19.0 & 19.0 & 43.5 & 24.1 & 0.0 & 0.0 & 0.0 & 0.0 \\
\hline Total IN ORG PART & & 0.0 & 0.0 & 0.0 & 0.0 & 0.0 & 0.0 & 2.1 & 19.0 & 19.0 & 19.0 & 13.5 & 24.4 & 0.0 & 0.0 & 0.0 & 0.0 \\
\hline Total rusRAP & & 0.0 & 3620.1 & 3620.1 & 3620.4 & 0.0 & 0.0 & 217.6 & 1903.7 & 1903.7 & 1903.7 & 4351.3 & 2447.6 & 0.0 & 0.0 & 0.0 & 0.0 \\
\hline \multicolumn{18}{|c|}{ Waste Genorator:Gray_aros } \\
\hline CONT SOIL & D & 0.0 & 1.9 & 0.2 & 0.2 & 0.1 & 0.0 & 0.0 & 0.0 & 0.0 & 0.0 & 0.0 & 0.0 & 0.0 & 0.0 & 0.0 & 0.0 \\
\hline & NONE & 0.0 & 7.9 & 33.4 & 33.4 & 26.0 & 0.0 & 0.0 & 0.0 & 0.0 & 0.0 & 0.0 & 0.0 & 0.0 & 0.0 & 0.0 & 0.0 \\
\hline Total CONT SOIL & & 0.0 & 9.8 & 33.6 & 33.6 & 26.2 & 0.0 & 0.0 & 0.0 & 0.0 & 0.0 & 0.0 & 0.0 & 0.0 & 0.0 & 0.0 & 0.0 \\
\hline \multirow[t]{4}{*}{ D-HET } & D & 0.0 & 5.0 & 0.6 & 0.6 & 0.5 & 0.0 & 0.0 & 0.0 & 0.0 & 0.0 & 0.0 & 0.0 & 0.0 & 0.0 & 0.0 & 0.0 \\
\hline & 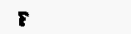 & 0.0 & 3.8 & 0.1 & 0.4 & 0.3 & 0.0 & 0.0 & 0.0 & 0.0 & 0.0 & 0.0 & 0.0 & 0.0 & 0.0 & 0.0 & 0.0 \\
\hline & G & 0.0 & 0.9 & 0.1 & 0.1 & 0.0 & 0.0 & 0.0 & 0.0 & 0.0 & 0.0 & 0.0 & 0.0 & 0.0 & 0.0 & 0,0 & 0.0 \\
\hline & NONE & 0.0 & 70.6 & 193.8 & 193.0 & 150.7 & 0.0 & 0.0 & 0.0 & 0.0 & 0.0 & 0.0 & 0.0 & 0.0 & 0.0 & 0.0 & 0.0 \\
\hline Total D-HET & & 0.0 & 81.2 & 295.0 & 195.0 & 151.7 & 0.0 & 0.0 & 0.0 & 0.0 & 0.0 & 0.0 & 0.0 & 0.0 & 0.0 & 0.0 & 0.0 \\
\hline D-IN MON-MTL & NONE & 0.0 & 0.9 & 11.5 & 11.3 & 8.9 & 0.0 & 0.0 & 0.0 & 0.0 & 0.0 & 0.0 & 0.0 & 0.0 & 0.0 & 0.0 & 0.0 \\
\hline Total D-2N NON-MTL & & 0.0 & 0.9 & 31.5 & 11.5 & 8.9 & 0.0 & 0.0 & 0.0 & 0.0 & 0.0 & 0.0 & 0.0 & 0.0 & 0.0 & 0.0 & 0.0 \\
\hline D-METAL & NOHE & 0.0 & 2.7 & 34.5 & 34.5 & 26.8 & 0.0 & 0.0 & 0.0 & 0.0 & 0.0 & 0.0 & 0.0 & 0.0 & 0.0 & 0.0 & 0.0 \\
\hline Total D-METAL & & 0.0 & 2.7 & 34.5 & 34.5 & 26.0 & 0.0 & 0.0 & 0.0 & 0.0 & 0.0 & 0.0 & 0.0 & 0.0 & 0.0 & 0.0 & 0.0 \\
\hline \multirow[t]{2}{*}{ IN ORG LR/SLG } & E & 0.0 & 0.2 & 0.0 & 0.0 & 0.0 & 0.0 & 0.0 & 0.0 & 0.0 & 0.0 & 0.0 & 0.0 & 0.0 & 0.0 & 0.0 & 0.0 \\
\hline & 3 & 0.0 & 0.2 & 0.0 & 0.0 & 0.0 & 0.0 & 0.0 & 0.0 & 0.0 & 0.0 & 0.0 & 0.0 & 0.0 & 0.0 & 0.0 & 0.0 \\
\hline Total IN ORG LQ/SLG & & 0.0 & 0.1 & 0.0 & 0.0 & 0.0 & 0.0 & 0.0 & 0.0 & 0.0 & 0.0 & 0.0 & 0.0 & 0.0 & 0.0 & 0.0 & 0.0 \\
\hline
\end{tabular}

HCD Codes: A-Ignitables, B-Corrosives, C-Reactives, D-Metals w/o Hg, E-Metsls w Hg, E-Organics, G-State Reg., HMPCB < 50ppm, J-PCB > 50ppm 
Annual Physical Waste Form and garardous Constituent Volumes by waste Generator (in cublo meters)

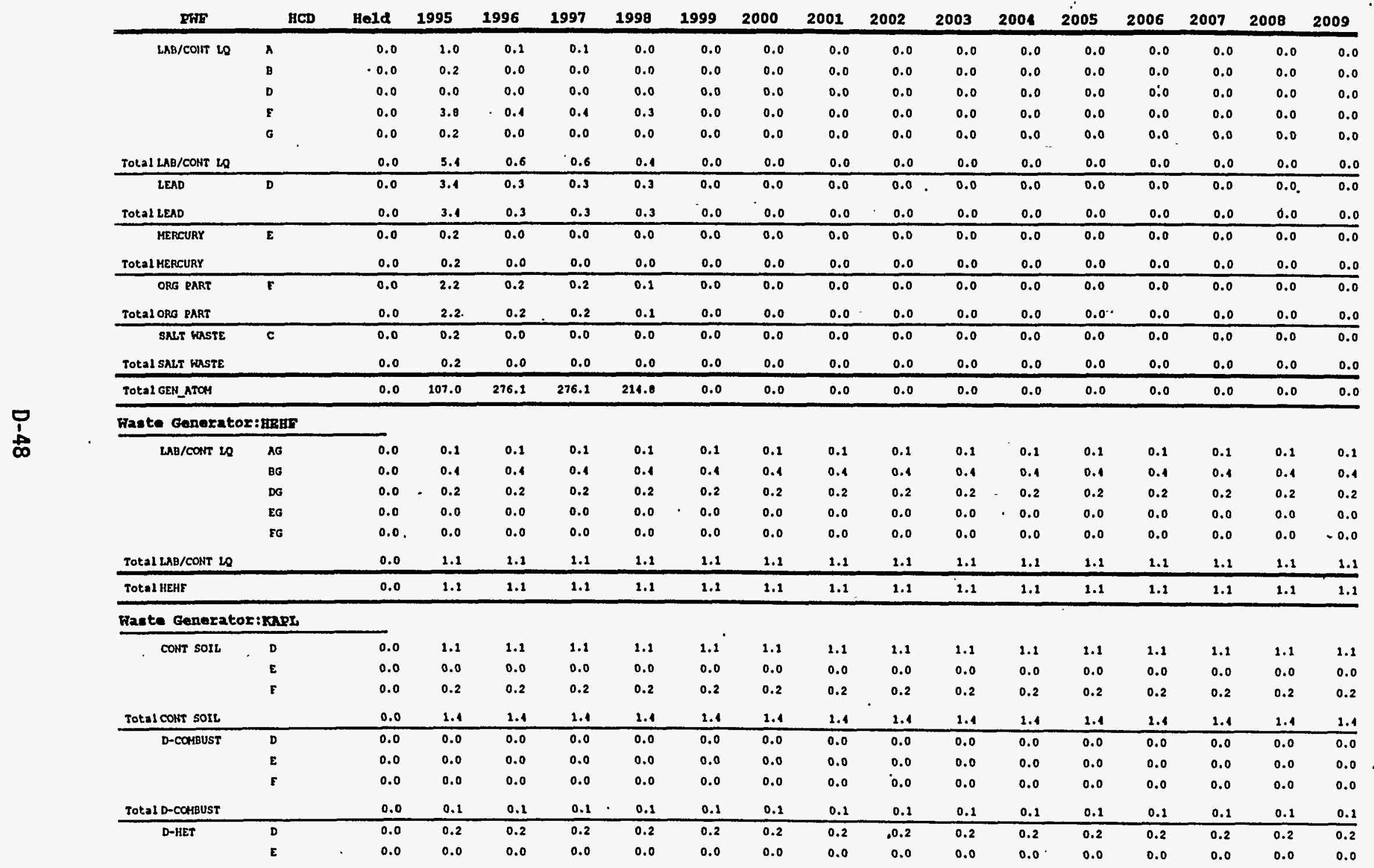

HCD Codes: AmIgnltables, B=Corrosives, C-Reactlves, D-Metals w/O Hg, E-Metals w Hg, F=Organles, G-State Reg., H-PCB < 50ppm, J-PCB > 50ppm 
Annual Physical Faste Form and Bazardous Constituent Volumes by waste Generator

(in cubic metera)

\begin{tabular}{|c|c|c|c|c|c|c|c|c|c|c|c|c|c|c|c|c|c|}
\hline PWE & HCD & Beld & 1995 & 1996 & 1997 & 1998 & 1999 & 2000 & 2001 & 2002 & 2003 & 2008 & 2005 & 2006 & 2007 & 2008 & 2009 \\
\hline & $\mathbf{r}$ & 0.0 & 0.0 & 0.0 & 0.0 & 0.0 & 0.0 & 0.0 & 0.0 & 0.0 & 0.0 & 0.0 & 0.0 & 0.0 & 0.0 & 0.0 & 0.0 \\
\hline Total D-HET & & 0.0 & 0.2 & 0.2 & 0.2 & 0.2 & 0.2 & 0.2 & 0.2 & 0.2 & 0.2 & 0.2 & 0.2 & 0.2 & 0.2 & 0.2 & 0.2 \\
\hline \multirow[t]{3}{*}{ D-IM NOH-STLL } & $\bar{D}$ & 0.0 & 0.3 & 0.3 & 0.3 & 0.3 & 0.3 & 0.3 & 0.3 & 0.3 & 0.3 & 0.3 & 0.3 & 0.3 & 0.3 & 0.3 & 0.3 \\
\hline & E & 0.0 & 0.0 & 0.0 & 0.0 & 0.0 & 0.0 & 0.0 & 0.0 & 0.0 & 0.0 & 0.0 & 0.0 & 0.0 & 0.0 & 0.0 & 0.0 \\
\hline & $\varepsilon$ & 0.0 & 0.0 & 0.0 & 0.0 & 0.0 & 0.0 & 0.0 & 0.0 & 0.0 & 0.0 & 0.0 & 0.0 & 0.0 & 0.0 & 0.0 & 0.0 \\
\hline Total D-IN NON-KTL' & & 0.0 & 0.1 & 0.1 & 0.1 & 0.4 & 0.4 & 0.1 & 0.4 & 0.4 & 0.1 & 0.1 & 0.4 & 0.4 & 0.4 & 0.1 & 0.1 \\
\hline \multirow[t]{2}{*}{ D-METAL } & D & 0.0 & 0.1 & 0.4 & 0.1 & 0.4 & 0.4 & 0.1 & 0.1 & 0.4 & 0.1 & 0.1 & 0.1 & 0.1 & 0.1 & 0.4 & 0.1 \\
\hline & $\mathbf{E}$ & 0.0 & 0.0 & 0.0 & 0.0 & 0.0 & 0.0 & 0.0 & 0.0 & 0.0 & 0.0 & 0.0 & 0.0 & 0.0 & 0.0 & 0.0 & 0.0 \\
\hline Total D-METAR & & 0.0 & 0.5 & 0.5 & 0.5 & 0.5 & 0.5 & 0.5 & 0.5 & 0.5 & 0.5 & 0.5 & 0.5 & 0.5 & 0.5 & 0.5 & 0.5 \\
\hline \multirow[t]{3}{*}{ IN ORG LQ/SLG } & D & 0.0 & 0.1 & 0.1 & 0.1 & 0.1 & 0.1 & 0.1 & 0.1 & 0.1 & 0.1 & 0.1 & 0.1 & 0.2 & 0.1 & 0.1 & 0.1 \\
\hline & $\varepsilon$ & 0.0 & 0.0 & 0.0 & 0.0 & 0.0 & 0.0 & 0.0 & 0.0 & 0.0 & 0.0 & 0.0 & 0.0 & 0.0 & 0.0 & 0.0 & 0.0 \\
\hline & 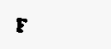 & 0.0 . & 0.0 & 0.0 & 0.0 & 0.0 & 0.0 & 0.0 & 0.0 & 0.0 & 0.0 & 0.0 & 0.0 & 0.0 & 0.0 & 0.0 & 0.0 \\
\hline TOtal IN ORG LQ/SLG & & 0.0 & 0.1 & 0.1 & 0.1 & 0.1 & 0.1 & 0.1 & 0.1 & 0.1 & 0.1 & 0.1 & 0.1 & 0.1 & 0.1 & 0.1 & 0.1 \\
\hline \multirow[t]{3}{*}{ IN ORG PART } & $\bar{D}$ & 0.0 & 0.0 & 0.0 & 0.0 & 0.0 & 0.0 & 0.0 & 0.0 & 0.0 & 0.0 & 0.0 & 0.0 & 0.0 & 0.0 & 0.0 & 0.0 \\
\hline & E & 0.0 & 0.0 & 0.0 & 0.0 & 0.0 & 0.0 & 0.0 & 0.0 & 0.0 & 0.0 & 0.0 & 0.0 & 0.0 & 0.0 & 0.0 & 0.0 \\
\hline & $\mathbf{E}$ & $0.0^{\circ}$ & 0.0 . & 0.0 & 0.0 & 0.0 & 0.0 & 0.0 & 0.0 & 0.0 & 0.0 & 0.0 & 0.0 & 0.0 & 0.0 & 0.0 & 0.0 \\
\hline TOtal IN ORO PART & & 0.0 & 0.0 & 0.0 & 0.0 & 0.0 & 0.0 & 0.0 & 0.0 & 0.0 & 0.0 & 0.0 & $0: 0$ & 0.0 & 0.0 & 0.0 & 0.0 \\
\hline \multirow[t]{6}{*}{ LAB/CONT 20} & $\pi$ & 0.0 & 0.0 & 0.0 & $\overline{0.0}$ & $\overline{0.0}$ & 0.0 & 0.0 & 0.0 & 0.0 & 0.0 & 0.0 & 0.0 & 0.0 & 0.0 & 0.0 & 0.0 \\
\hline & $\theta$ & 0.0 & 0.0 & 0.0 & 0.0 & 0.0 & 0.0 & 0.0 & 0.0 & 0.0 & 0.0 & 0.0 & 0.0 & 0.0 & 0.0 & 0.0 & 0.0 \\
\hline & c & 0.0 & 0.0 & 0.0 & 0.0 & 0.0 & 0.0 & 0.0 & 0.0 & 0.0 & 0.0 & 0.0 & 0.0 & 0.0 & 0.0 & 0.0 & 0.0 \\
\hline & D & 0.0 & 0.1 & 0.1 & 0.1 & 0.1 & 0.1 & 0.1 & 0.1 & 0.2 & 0.1 & 0.1 & 0.1 & 0.1 & 0.1 & 0.1 & 0.1 \\
\hline & $\varepsilon$ & 0.0 & 0.1 & 0.1 & 0.1 & 0.1 & 0.1 & 0.1 & 0.1 & 0.1 & 0.1 & 0.1 & 0.1 & 0.1 & 0.1 & 0.1 & 0.1 \\
\hline & $\mathbf{F}$ & 0.0 & 0.1 & 0.1 & 0.1 & 0.1 & 0.1 & 0.1 & 0.1 & 0.1 & 0.1 & 0.1 & 0.1 & 0.1 & 0.1 & 0.1 & 0.1 \\
\hline TOta1 LAB/CONT LO & & 0.0 & 0.6 & 0.6 & 0.6 & 0.6 & 0.6 & 0.6 & 0.6 & 0.6 & 0.6 & 0.6 & 0.6 & 0.6 & 0.6 & 0.6 & 0.6 \\
\hline MERCURY & $\varepsilon$ & 0.0 & 0.3 & 0.3 & 0.3 & 0.3 & 0.3 & 0.3 & 0.3 & 0.3 & 0.3 & 0.3 & 0.3 & 0.3 & 0.3 & 0.3 & $\overline{0.3}$ \\
\hline TOtal HERCURY & & 0.0 & 0.3 & 0.3 & 0.3 & 0.3 & 0.3 & 0.3 & 0.3 & 0.3 & .0 .3 & 0.3 & 0.3 & 0.3 & 0.3 & 0.3 & 0.3 \\
\hline ORG ABS LO/SLG & D & 0.0 & 0.1 & 0.1 & 0.1 & 0.1 & 0.1 & 0.1 & 0.1 & 0.1 & 0.1 & 0.1 & 0.1 & 0.1 & $\overline{0.1}$ & 0.1 & $\overline{0.1}$ \\
\hline TOtaL ORG ABS LQ/SLG & & 0.0 & 0.1 & 0.1 & 0.1 & 0.1 & 0.2 & 0.1 & 0.1 & 0.1 & 0.1 . & 0.1 & 0.1 & 0.1 & 0.1 & 0.1 & 0.1 \\
\hline \multirow[t]{3}{*}{ ORG PART } & $D$ & 0.0 & 0.0 & 0.0 & 0.0 & 0.0 & 0.0 & 0.0 & 0.0 & 0.0 & 0.0 & 0.0 & 0.0 & 0.0 & 0.0 & 0.0 & $\overline{0.0}$ \\
\hline & $\varepsilon$ & 0.0 & 0.0 & 0.0 & 0.0 & 0.0 & 0.0 & 0.0 & 0.0 & 0.0 & 0.0 & 0.0 & 0.0 & 0.0 & 0.0 & 0.0 & 0.0 \\
\hline & $\mathbf{r}$ & 0.0 & 0.0 & 0.0 & 0.0 & 0.0 & 0.0 & 0.0 & 0.0 & 0.0 & 0.0 & 0.0 & 0.0 & 0.0 & 0.0 & 0.0 & 0.0 \\
\hline Total ORG PART & & 0.0 & 0.0 & 0.0 & 0.0 & 0.0 & 0.0 & 0.0 & 0.0 & $0.0^{\circ}$ & 0.0 & 0.0 & 0.0 & 0.0 & 0.0 & 0.0 & 0.0 \\
\hline Tot.21 KAPL & & 0.0 & 4.2 & 1.2 & 1.2 & 4.2 & 1.2 & 4.2 & 1.2 & 4.2 & 1.2 & 1.2 & 4.2 & 1.2 & 1.2 & 4.2. & 4.2 \\
\hline
\end{tabular}

Faste Generator:KAPL_SHIPYDS

ECD Codos: A-Ignitables, B-Corrosives, C-React:Ives, D-Metels w/O Hg, E-Metals w Hg, Emorganics, G-State Reg., H-PCB < 50ppm, J-PCB > 50ppm 
(in cubic meters)

\begin{tabular}{|c|c|c|c|c|c|c|c|c|c|c|c|c|c|c|c|c|c|}
\hline PWE & HCD & Held & 1995 & 1996 & 1997 & 1998 & 1999 & 2000 & 2001 & 2002 & 2003 & 2004 & 2005 & 2006 & 2007 & 2008 & 2009 \\
\hline D-METAL & NOIE & 0.0 & 0.0 & 0.0 & 0.0 & 0.0 & 0.0 & 39.1 & 59.4 & 59.4 & 39.4 & 59.4 & 0.0 & 0.0 & 0.0 & 0.0 & 0.0 \\
\hline TOt:A1 U-HETAL & & 0.0 & 0.0 & 0.0 & 0.0 & 0.0 & 0.0 & 39.4 & 59.4 & 59.4 & 59.4 & 59.4 & 0.0 & 0.9 & 0.0 & 0.0 & 0.0 \\
\hline TOtal KAPL_SHIPYDS & & 0.0 & 0.0 & 0.0 & 0.0 & 0.0 & 0.0 & 59.4 & 59.1 & 59.4 & 59.1 & 59.4 & 0.0 & 0.0 & 0.0 & 0.0 & 0.0 \\
\hline Waste Generatox: & KEH_100 & & & & & & & & & & & & & & & & \\
\hline BATTERIES & BG & 0.0 & 0.0 & 0.0 & 0.0 & 0.0 & 0.1 & 0.1 & 0.1 & 0.2 & 0.2 & 0.3 & 0.4 & 0.5 & 0.6 & 0.8 & 1.0 \\
\hline TOtal BATTERIES & & 0.0 & 0.0 & 0.0 & 0.0 & 0.0 & 0.1 & 0.1 & 0.1 & 0.2 & 0.2 & 0.3 & 0.4 & 0.5 & 0.6 & 0.0 & 1.0 \\
\hline CONT SOll & D & 0.0 & 10.5 & 0.0 & 0.0 & 0.0 & 0.0 & 0.0 & 0.0 & 0.0 & 0.0 & 0.0 & 0.0 & 0.0 & 0.0 & 0.0 & 0.0 \\
\hline & MONE & 0.0 & 64.2 & 0.0 & 0.0 & 0.0 & 0.0 & 0.0 & 0.0 & 0.0 & 0.0 & 0.0 & 0.0 & 0.0 & 0.0 & 0.0 & 0.0 \\
\hline Total CONT SOIL & & 0.0 & 74.7 & 0.0 & 0.0 & 0.0 & 0.0 & 0.0 & 0.0 & 0.0 & 0.0 & 0.0 & 0.0 & 0.0 & 0.0 & 0.0 & 0.0 \\
\hline D-conbust & $A$ & 0.0 & 0.3 & 0.4 & 0.5 & 0.6 & 0.8 & 1.0 & 1.3 & 1.6 & 2.0 & 2.5 & 3.2 & 4.0 & 5.0 & 6.3 & 7.9 \\
\hline & AEO & 0.0 & 0.2 & 0.3 & 0.1 & 0.5 & 0.6 & 0.8 & 1.0 & 2.3 & 1.6 & 2.1 & 2.6 & 3.3 & 4.1 & 5.1 & 6.4 \\
\hline Tota1 D-COHBUST & & 0.0 & 0.6 & 0.7 & 0.9 & 1.2 & 1.5 & 2.9 & 2.4 & 3.0 & 3.7 & 4.7 & 5.8 & 7.3 & 9.2 & 11.5 & 14.3 \\
\hline D-IH NOH-HTL & NONE & 0.0 & 12.6 & 0.0 & 0.0 & 0.0 & 0.0 & 0.0 & 0.0 & 0.0 & 0.0 & 0.0 & 0.0 & 0.0 & 0.0 & 0.0 & 0.0 \\
\hline Total D-IN MON-MIL & & 0.0 & 12.6 & 0.0 & 0.0 & 0.0 & 0.0 & 0.0 & 0.0 & 0.0 & 0.0 & 0.0 & 0.0 & 0.0 & 0.0 & 0.0 & 0.0 \\
\hline D-METAL & DG & 0.0 & 13.7 & 17.1 & 21.4 & 26.8 & 33.5 & 11.8 & 32.3 & 65.4 & 01.0 & 102.2 & 127.8 & 159.8 & 199.7 & 219.6 & 312.1 \\
\hline & EG & 0.0 & 0.5 & 0.7 & 0.8 & 1.1 & 1.3 & 1.7 & 2.1 & 2.7 & 3.3 & 4.2 & 5.2 & 6.6 & 0.2 & 10.3 & 12.9 \\
\hline Tota1 D-METAL & & 0.0 & 14.2 & 17.8 & 22.3 & 27.9 & 34.8 & 13.6 & 51.5 & 68.1 & 85.2 & 106.5 & 133.1 & 266.1 & 208.0 & 260.0 & 325.0 \\
\hline IN ORG LQ/SLG & AEG & 0.0 & 6.9 & 8.7 & 10.9 & 13.6 & 27.0 & 21.2 & 26.6 & 33.2 & 41.5 & 51.9 & 64.9 & 01.2 & 101.5 & 126.8 & 158.6 \\
\hline Jotal IN ORG LR/SLG & & 0.0 & 6.9 & 8.7 & 20.9 & 23.6 & 17.0 & 21.2 & 26.6 & 33.2 & 41.5 & 51.9 & 64.9 & 01.2 & 101.5 & $126 . \theta$ & 158.6 \\
\hline LAB/CONT LQ & BG & 0.0 & 1.4 & 1.0 & 2.2 & 2.8 & 3.5 & 1.4 & 5.5 & 6.8 & 8.6 & 10.7 & 23.4 & 16.8 & 21.0 & 26.3 & 32.9 \\
\hline & Fo & 0.0 & 0.3 & 0.4 & 0.5 . & 0.6 & 0.0 & 1.0 & 1.3 & 1.6 & 2.0 & 2.5 & 3.2 & 4.0 & 5.0 & 6.3 & 7.9 \\
\hline Total LAB/CONT LQ & & 0.0 & 1.7 & 2.2 & 2.8 & 3.5 & 1.3 & 5.4 & 6.8 & 0.5 & 10.7 & 13.3 & 16.7 & 20.9 & 26.2 & 32.6 & $10.0^{\circ}$ \\
\hline LEAD & BDG & 0.0 & 0.3 & 0.4 & 0.5 & 0.6 & 0.7 & 0.9 & 1.2 & 1.5 & 1.9 & 2.3 & 2.9 & 3.7 & 4.6 & 5.8 & 7.3 \\
\hline & D & 0.0 & 0.7 & 0.0 & 0.0 & 0.0 & 0.0 & 0.0 & 0.0 & 0.0 & 0.0 & 0.0 & 0.0 & 0.0 & 0.0 & 0.0 & 0.0 \\
\hline Total LEAD & . & 0.0 & 1.1 & 0.1 & 0.5 & 0.6 & 0.7 & 0.9 & 1.2 & 1.5 & 1.9 & 2.3 & 2.9 & 3.7 & 1.6 & 5.8 & 7.3 \\
\hline ORG ABS LQR/SLG & AEFG & 0.0 & 12.5 & 15.6 & 29.6 & 24.5 & 30.6 & 38.3 & 17.9 & 59.8 & 74.8 & 93.5 & 116.9 & 146.1 & 182.7 & 228.4 & 285.5 \\
\hline & rG & 0.0 & 0.2 & 0.3 & 0.4 & 0.5 & 0.6 & 0.8 & 1.0 & 1.3 & 1.6 & 2.1 & 2.6 & 3.3 & 4.1 & 5.1 & 6.4 \\
\hline TOtaL ORG ABS LR/SLG & & 0.0 & 12.8 & 16.0 & 20.0 & 25.0 & 31.3 & 39.1 & 18.9 & 61.2 & 76.5 & 95.6 & 119.6 & 149.1 & 106.8 & 233.5 & 291.9 \\
\hline TOtal KEH_100 & & 0.0 & 125.1 & 16.1 & 57.7 & 72.1 & 90.1 & 112.6 & 140.8 & 176.0 & 220.0 & 275.0 & 363.8 & 429.7 & 537.1 & 671.4 & 839.3 \\
\hline Fiate Generator & :LBL & & & & & & & & & & & & & & & & \\
\hline D-1N NON-HTL & NONE & 0.0 & 80.3 & 23.3 & 6.7 & 6.8 & 6.9 & 2725.5 & 2725.5 & 2725.5 & 2725.5 & $2725.5^{\circ}$ & 7.0 & 7.0 & 7.0 & 7.0 & 1.0 \\
\hline Total D-IN NON-MTL & & 0.0 & 80.3 & 73.3 & 6.7 & 6.8 & 6.9 & 2725.5 & 2725.5 & 2725.5 & 2725.5 & 2725.5 & 7.0 & 7.0 & 7.0 & 7.0 & $\mathbf{7 . 0}$ \\
\hline
\end{tabular}

BCD Codes: A-Ignitables, B-Corrosives, C-Reactives, D-Metals w/O Hg, E-Metals w Hg, E-Organics, G-State Reg., H-PCB < 50ppm, J-PCB > 50ppm 
Annual Physical Haste Form and Barurdous Conatituent Volumes by Waste Generator (In cuble metera)

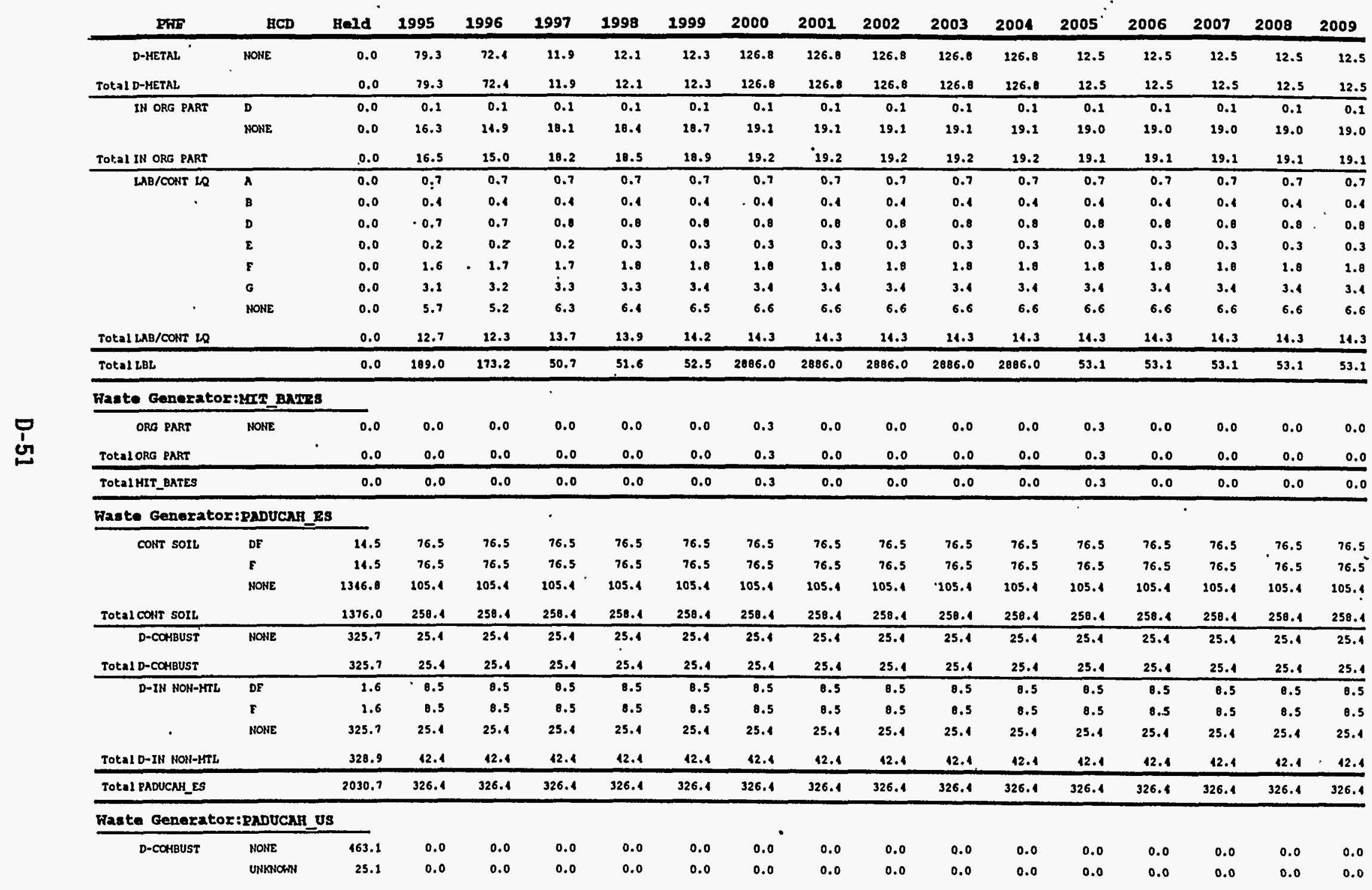

nCD Codes: A-Ignitables, BwCorrosives, C-Reactives, D-Metals w/O Hg, E-Metals w Hg, EmOrganics, GmState Reg., H-PCB < 50ppm, J-PCB > 50ppm 
Annual Physical Waste Form and Bazardous Constituent Volumea by Faste Generator

(in cubic metera)

\begin{tabular}{|c|c|c|c|c|c|c|c|c|c|c|c|c|c|c|c|c|c|}
\hline PWE & HCD & Hold & 1995 & 1996 & 1997 & 1998 & 1999 & 2000 & 2001 & 2002 & 2003 & 2004 & 2005 & 2006 & 2007 & 2008 & 2009 \\
\hline Tut:al D-COMBUST & . & 480.2 & 0.0 & 0.0 & 0.0 & 0.0 & 0.0 & 0.0 & 0.0 & 0.0 & 0.0 & 0.0 & 0.0 & 0.0 & 0.0 & 0.0 & 0.0 \\
\hline D-HETAL & NONE & 370.5 & 0.0 & 0.0 & 0.0 & 0.0 & 0.0 & 0.0 & 0.0 & 0.0 & 0.0 & 0.0 & 0.0 & 0.0 & 0.0 & 0.0 & $\overline{0.0}$ \\
\hline TOtal D-METAC & & 370.5 & 0.0 & 0.0 & 0.0 & 0.0 & 0.0 & 0.0 & 0.0 & 0.0 & 0.0 & 0.0 & 0.0 & 0.0 & 0.0 & 0.0 & 0.0 \\
\hline IN ORG PART & NONE & 92.6 & 0.0 & 0.0 & 0.0 & 0.0 & 0.0 & 0.0 & 0.0 & 0.0 & 0.0 & 0.0 & 0.0 & 0.0 & 0.0 & 0.0 & 0.0 \\
\hline Total IN ORG PART & & 92.6 & 0.0 & 0.0 & 0.0 & 0.0 & 0.0 & 0.0 & 0.0 & 0.0 & 0.0 & 0.0 & 0.0 & 0.0 & 0.0 & 0.0 & 0.0 \\
\hline Total PADUCAH_US & & 951.4 & 0.0 & 0.0 & 0.0 & 0.0 & 0.0 & 0.0 & 0.0 & 0.0 & 0.0 & 0.0 & 0.0 & 0.0 & 0.0 & 0.0 & 0.0 \\
\hline \multicolumn{18}{|c|}{ Waste Generator:PASI_PRAC_RRY } \\
\hline contr sort & B & 0.0 & 0.0 & 0.0 & 1061.1 & 843.2 & 1029.5 & 969.7 & 980.3 & 2054.1 & 2303.5 & 3423.0 & 2567.1 & 2644.4 & 1614.4 & 1651.4 & 1574.1 \\
\hline & D & 0.0 & 0.0 & 0.0 & 1061.1 & 843.2 & 1029.5 & 969.7 & 980.3 & 1054.1 & 1303.5 & 1423.0 & 1567.1 & 1611.4 & 1644.4 & 1651.4 & 1574.1 \\
\hline & MONE & 0.0 & 0.0 & 0.0 & 21.3 & 11.3 & 34.0 & 17.0 & 5.6 & 39.7 & 51.1 & 34.0 & 22.7 & 22.7 & 22.7 & 28.3 & 28.3 \\
\hline Total cont sols & & 0.0 & 0.0 & 0.0 & 2133.6 & 1697.9 & 2093.1 & 1956.6 & 1966.3 & 2147.9 & 2650.3 & $28 B 0.2$ & 3136.9 & 3311.5 & 3311.5 & 3331.3 & 3176.7 \\
\hline D-IN NOA-MTL & MONE, & 0.0 & 0.0 & 0.0 & 55.0 & 55.0 & 165.2 & 02.6 & 27.5 & 192.7 & 247.8 & 165.2 & 110.1 & 110.1 & 110.1 & 137.6 & 137.6 \\
\hline Total D-IN MON-MTC & & 0.0 & 0.0 & 0.0 & 55.0 & 55.0 & 165.2 & 82.6 & 27.5 & 192.7 & $217.8^{\circ}$ & 165.2 & 110.1 & 110.1 & 110.1 & 137.6 & 137.6 \\
\hline D-METAL & D & 0.0 & 0.0 & 0.0 & 23.0 & 18.3 & 22.4 & 21.1 & 21.3 & 22.9 & 28.3 & 30.9 & 34.1 & 35.7 & 35.7 & 35.9 & 34.2 \\
\hline & NONE & 0.0 & 0.0 & 0.0 & 55.0 & 55.0 & 165.2 & 82.6 & 27.5 & 192.7 & 217.8 & 165.2 & 130.1 & 110.1 & 110.2 & 237.6 & 137.6 \\
\hline Total D-METAL & & 0.0 & 0.0 & 0.0 & 78.1 & 73.1 & 187.6 & 103.1 & 48.8 & 215.6 & 276.1 & 196.1 & 144.2 & 145.9 & 115.9 & 173.6 & 371.9 \\
\hline D-soll & B & 0.0 & 0.0 & 0.0 & 82.2 & 63.3 & 79.8 & 75.1 & 75.9 & 81.7 & 101.0 & 110.3 & 221.4 & 127.4 & 127.1 & 128.0 & 122.0 \\
\hline & D & 0.0 & 0.0 & 0.0 & 02.2 & 65.3 & 79.8 & 75.1 & 75.9 & 81.7 & 101.0 & 210.3 & 221.4 & 127.4 & 127.4 & 128.0 & 122.0 \\
\hline . & NONE & 0.0 & 0.0 & 0.0 & 0.8 & 0.8 & 2.6 & 1.3 & 0.1 & 3.0 & 3.9 & 2.6 & 1.7 & 1.7 & 1.7 & 2.2 & 2.2 \\
\hline Total D-SOIL & & 0.0 & 0.0 & 0.0 & 163.3 & 131.6 & 162.2 & 151.6 & 152.4 & 166.5 & 206.0 & 223.2 & 244.1 & 256.7 & 256.7 & 258.2 & 216.2 \\
\hline Tota1 PAST_PRAC_REM & & 0.0 & 0.0 & 0.0 & 2432.2 & 2958.0 & 2600.2 & 2294.6 & 2195.1 & 2722.9 & 3388.3 & 3464.8 & 3656.0 & 3024.3 & 3824.3 & 3900.8 & 3732.5 \\
\hline Haste Gonerator & $x:$ p2Na & & & & & & & & & & & & & & & & \\
\hline Cont sort & NONE & 0.0 & 1.0 & 3.0 & 1.0 & 1.0 & 1.0 & 1.0 & 1.0 & 1.0 & 1.0 & 1.0 & 1.3 & 1.3 & 1.3 & 1.3 & 1.3 \\
\hline Total cont soll & & 0.0 & 1.0 & 1.0 & 1.0 & 1.0 & 1.0 & 1.0 & 1.0 & 1.0 & 1.0 & 1.0 & 1.3 & 1.3 & 1.3 & 1.3 & 1.3 \\
\hline D-CONBUST & MOHE & 13.3 & 28.3 & 20.0 & 28.0 & 28.0 & 28.0 & .28 .0 & 26.0 & 26.8 & 26.0 & 26.0 & 31.1 & 29.9 & 29.9 & 29.9 & 29.9 \\
\hline Total D-Compust & & 13.3 & 28.3 & 28.0 & 28.0 & 28.0 & 28.0 & 28.0 & $\therefore 26.8$ & 26.8 & 26.0 & 26.8 & 31.1 & 29.9 & 29.9 & 29.9 & 29.9 \\
\hline D-HET. & D & 0.0 & 0.6 & 0.6 & 0.6 & 0.6 & 0.6 & 0.7 & 0.7 & 0.7 & 0.7 & 0.7 & 0.7 & 0.2 & 0.7 & 0.7 & 0.7 \\
\hline & $\mathbf{F}$ & 0.0 & 1.2 & 1.2 & 1.2 & 1.2 & 1.2 & 1.5 & 1.5 & 1.5 & 1.5 & 1.5 & 1.5 & 1.5 & 1.5 & 2.5 & 1.5 \\
\hline & G & 0.0 & 1.8 & 1.8 & 1.8 & 1.8 & 2.8 & 2.2 & 2.2 & 2.2 & 2.2 & 2.2 & 2.2 & 2.2 & 2.2 & 2.2 & 2.2 \\
\hline & MONE & 56.8 & 688.2 & 687.0 & 607.0 & 687.0 & 607.0 & 647.3 & 617.3 & 647.3 & 647.3 & 617.3 & 623.0 & 623.0 & 623.0 & 623.0 & 623.0 \\
\hline Total D-HET & & 56.0 & 691.9 & 690.7 & 690.7 & 690.7 & 690.7 & 651.9 & 651.9 & 651.9 & 651.9 & 651.9 & 627.6 & 627.6 & 627.6 & 627.6 & 627.6 \\
\hline D-IN NON-MTL & B & 0.0 & 0.1 & 0.4 & 0.4 & 0.1 & 0.1 & 0.5 & 0.5 & 0.5 & 0.5 & 0.5 & 0.5 & 0.5 & 0.5 & 0.5 & 0.5 \\
\hline & $D$ & 0.0 & 1.7 & 1.7 & 1.7 & 1.7 & 1.7 & 2.6 & 2.6 & 2.6 & 2.6 & 2.6 & 2.6 & 2.6 & 2.6 & 2.6 & 2.6 \\
\hline
\end{tabular}

ECD Codes: A-Ignitables, B-Corrosives, C-Reactives, D-Metals w/O Hg, E-Metals w Hg, F=Organics, G-State Reg., H-PCB < 50ppm, J-ECB > 50ppm 
Annual Phyaical Waste Form and Hazardous Constituent Volumes by Waste Generator (in cubia meters)

\begin{tabular}{|c|c|c|c|c|c|c|c|c|c|c|c|c|c|c|c|c|c|}
\hline PWE & $\mathrm{BCD}$ & Held & 1995 & 1996 & 1997 & 1998 & 1999 & 2000 & 2001 & 2002 & 2003 & 2004 & 2005 & 2006 & 2007 & 2008 & 2009 \\
\hline & F & 0.0 & 0.0 & 0.0 & 0.0 & 0.0 & 0.0 & 0.1 & 0.1 & 0.1 & 0.1 & 0.1 & 0.1 & 0.1 & 0.1 & 0.1 & 0.1 \\
\hline & G & 0.0 & 1.7 & 1.7 & 1.2 & 1.7 & 1.7 & 2.6 & 2.6 & 2.6 & 2.6 & 2.6 & 2.6 & 2.6 & 2.6 & 2.6 & 2.6 \\
\hline & MONE & 4.8 & 0.0 & 0.0 & 0.0 & 0.0 & 0.0 & 0.0 & 0.0 & 0.0 & 0.0 & 0.0 & 0.0 & $0.0^{\circ}$ & 0.0 & 0.0 & 0.0 \\
\hline Total D-IN HON-MTL & & 1.8 & 4.0 & 4.0 & 4.0 & 1.0 & 1.0 & 5.9 & 5.9 & 5.9 & 5.9 & 5.9 & 5.9 & 5.9 & 5.9 . & 5.9 & 5.9 \\
\hline D-KETRE & HOWE & 0.0 & 1.0 & 1.0 & 1.0 & 1.0 & 1.0 & 1.0 & 0.2 & 0.2 & 0.2 & 0.2 & 0.2 & 0.2 & 0.2 & 0.2 & 0.2 \\
\hline TOLA1 D-METAL & & 0.0 & 1.0 & 1.0 & 1.0 & 1.0 & 1.0 & 1.0 & 0.2 & 0.2 & 0.2 & 0.2 & 0.2 & 0.2 & 0.2 & 0.2 & 0.2 \\
\hline \multirow[t]{3}{*}{ IN ORG PART } & BErG & 0.0 & 1.0 & 1.8 & 1.8 & 1.8 & 1.8 & 1.8 & 1.8 & 1.8 & 1.8 & 1.8 & 1.8 & 0.6 & 0.6 & 0.6 & $\overline{0.6}$ \\
\hline & DG & 0.0 & 0.0 & 0.0 & 0.0 & 0.0 & 0.0 & 7.9 & 7.9 & 7.9 & 7.9 & 7.9 & 1.9 & 7.9 & 7.9 & 7.9 & 7.9 \\
\hline & NONE & 1.1 & 0.6 & 0.5 & 0.5 & 0.5 & 0.5 & 0.5 & 0.3 & 0.3 & 0.3 & 0.3 & 0.3 & 0.2 & 0.2 & 0.2 & 0.2 \\
\hline Total IN ORG PART & & 1.4 & 2.1 & 2.3 & 2.3 & 2.3 & 2.3 & 10.3 & 10.1 & 10.1 & 10.1 & 10.1 & 10.1 & 0.8 & 8.8 & 0.0 & 0.8 \\
\hline \multirow[t]{6}{*}{ LAB/CONT LO } & $\AA$ & 0.0 & 1.0 & 1.8 & 1.0 & 1.8 & 1.8 & 2.2 & 2.2 & 2.2 & 2.2 & 2.2 & 2.2 & 2.2 & 2.2 & 2.2 & 2.2 \\
\hline & B & 0.0 & 1.0 & 1.0 & 1.0 & 1.0 & 1.0 & 2.2 & 2.2 & 2.2 & 2.2 & 2.2 & 2.2 & 2.2 & 2.2 & 2.2 & 2.2 \\
\hline & D & 0.0 & 2.5 & 2.5 & 2.5 & 2.5 & 2.5 & 3.2 & 3.2 & 3.2 & 3.2 & 3.2 & 3.2 & 3.2 & 3.2 & 3.2 & 3.2 \\
\hline & E & 0.0 & 0.1 & 0.1 & 0.1 & 0.1 & 0.1 & 0.2 & 0.2 & 0.2 & 0.2 & 0.2 & 0.2 & 0.2 & 0.2 & 0.2 & 0.2 \\
\hline & $\varepsilon$ & 0.0 & 0.9 & 0.9 & 0.9 & 0.9 & 0.9 & 1.1 & 1.1 & 1.2 & 1.1 & 1.1 & 1.1 & 1.1 & 1.1 & 1.1 & 1.1 \\
\hline & $G$ & 0.0 & 1.6 & 1.8 & 1.8 & 1.8 & 1.8 & 2.2 & 2.2 & 2.2 & 2.2 & 2.2 & 2.2 & 2.2 & 2.2 & 2.2 & 2.2 \\
\hline TOtal LAB/CONT LQ & & 0.0 & 9.2 & 9.2 & 9.2 & 9.2 & 9.2 & 11.4 & 11.4 & 11.1 & 11.4 & 11.4 & 11.4 & 11.4 & 21.4 & $11 . i$ & 11.1 \\
\hline LEAD & D & 0.0 & 2.3 & 2.3 & 2.3 & 2.3 & 2.3 & 2.2 & 2.2 & 2.2 & 2.2 & 2.2 & 2.2 & 2.2 & 2.2 & 2.2 & $\overline{2.2}$ \\
\hline TOta1 LEAD & & 0.0 & 2.3 & 2.3 & 2.3 & 2.3 & 2.3 & 2.2 & 2.2 & 2.2 & 2.2 & 2.2 & 2.2 & 2.2 & 2.2 & 2.2 & 2.2 \\
\hline \multirow[t]{3}{*}{ ORG ABS LO/SLG } & B & 0.0 & 0.1 & 0.1 & 0.1 & 0.1 & 0.1 & 0.2 & 0.2 & 0.2 & 0.2 & 0.2 & 0.2 & 0.2 & 0.2 & 0.2 & $\overline{0.2}$ \\
\hline & D & 0.0 & 0.2 & 0.2 & 0.2 & 0.2 & 0.2 & 0.3 & 0.3 & 0.3 & 0.3 & 0.3 & 0.3 & 0.3 & 0.3 & $0.3^{\circ}$ & 0.3 \\
\hline & G & 0.0 & 0.1 & 0.1 & 0.1 & 0.1 & 0.1 & 0.2 & 0.2 & 0.2 & 0.2 & 0.2 & 0.2 & .0 .2 & 0.2 & 0.2 & 0.2 \\
\hline TOtal ORO $A B S$ LQ/SLG & & 0.0 & 0.5 & 0.5 & 0.5 & 0.5 & 0.3 & 0.7 & 0.7 & 0.7 & 0.7 & 0.7 & 0.7 & 0.7 & 0.7 & 0.7 & 0.7 \\
\hline \multirow[t]{4}{*}{ ORG PART } & B & 0.0 & 0.1 & 0.1 & 0.1 & 0.1 & 0.1 & 0.1 & 0.1 & 0.1 & 0.1 & 0.1 & 0.1 & 0.1 & 0.1 & 0.2 & $\overrightarrow{0.1}$ \\
\hline & BEFo & 0.0 & 1.8 & 2.8 & 1.0 & 1.0 & 1.0 & 1.0 & 1.0 & 1.8 & 1.8 & 1.0 & 1.0 & 0.6 & 0.6 & 0.6 & 0.6 \\
\hline & $D$ & 0.0 & 0.1 & 0.1 & 0.1 . & 0.1 & .0 .1 & 0.1 & 0.1 & 0.1 & 0.1 & 0.1 & 0.1 & 0.1 & 0.1 & 0.1 & 0.1 \\
\hline & 6 & 0.0 & 0.2 & 0.1 & 0.1 & 0.1 & 0.1 & 0.1 & 0.1 & 0.1 & 0.1 & 0.2 & 0.1 & 0.1 & 0.1 & 0.1 & 0.1 \\
\hline Total ORG PART & & 0.0 & 2.1 & 2.1 & 2.1 & 2.1 & 2.1 & 2.2 & 2.2 & 2.2 & 2.2 & 2.2 & 2.2 & 1.0 & 1.0 & 1.0 & 1.0 \\
\hline SH CONCRETE & NONE & 0.0 & 19.1 & 19.1 & 19.1 & 19.1 & 19.1 & 0.0 & 0.0 & 0.0 & 0.0 & 0.0 & 0.0 & 0.0 & 0.0 & 0.0 & 0.0 \\
\hline Total SH CONCRETE & & 0.0 & 19.1 & 19.1 & 19.1 & 19.1 & 19.1 & 0.0 & 0.0 & 0.0 & 0.0 & 0.0 & 0.0 & 0.0 & 0.0 & 0.0 & 0.0 \\
\hline \multirow[t]{2}{*}{ SH LEAD } & $D$ & 0.0 & 1.5 & 1.5 & 1.5 & 3.5 & 1.5 & 4.9 & 1.9 & 1.9 & 1.9 & 4.9 & 4.9 & 3.9 & 3.9 & 3.9 & 3.9 \\
\hline & NONE & 0.0 & 2.9 & 2.1 & 2.1 & 2.1 & 2.1 & 2.1 & 1.4 & 1.4 & 1.1 & 1.4 & 1.4 & 1.4 & 1.4 & 1.4 & 1.4 \\
\hline Total SH LEAD & & 0.0 & 1.1 & 3.6 & 3.6 & 3.6 & 3.6 & 7.0 & 6.4 & 6.4 & 6.4 & 6.4 & 6.4 & 3.3 & 5.3 & 5.3 & 5.3 \\
\hline \multirow[t]{2}{*}{ SH STEEL } & NONE & 0.0 & 6.5 & 6.5 & 6.5 & 6.5 & 6.5 & 0.1 & 0.0 & 0.0 & 0.0 & 0.0 & 0.0 & 0.0 & 0.0 & 0.0 & 0.0 \\
\hline & UNKNOCNAN & 0.0 & 0.9 & 0.9 & 0.9 & 0.9 & 0.9 & 0.9 & 0.9 & 0.9 & 0.9 & 0.9 & 0.9 & 0.3 & 0.3 & 0.3 & 0.3 \\
\hline
\end{tabular}

BCo Codas: A-Ignitables, B-Corrosives, C-Reactives, D-Metals w/O Hg, E-Metals w Hg, F-Organics, G-State Reg., H-PCB < 50ppm, J-PCB > 50ppm 


\begin{tabular}{|c|c|c|c|c|c|c|c|c|c|c|c|c|c|c|c|c|c|}
\hline PWE & HCD & Held & 1995 & 1996 & 1997 & 1998 & 1999 & 2000 & 2001 & 2002 & 2003 & 2004 & 2005 & 2006 & 2007 & 2008 & 2009 \\
\hline TUt:Al SH STEEL & & 0.0 & 7.1 & 7.1 & 7.1 & 7.4 & 7.4 & 1.1 & 0.9 & 0.9 & 0.9 & 0.9 & 0.9 & 0.3 & 0.3 & 0.3 & 0.3 \\
\hline Total PNL & & 76.5 & 724.2 & 711.8 & 771.8 & 771.8 & 771.8 & 723.4 & 720.5 & 720.5 & 720.5 & 720.5 & 700.7 & 695.2 & 695.2 & 695.2 & 695.2 \\
\hline \multicolumn{18}{|c|}{ Waste Generatox:PORTSMOUTH_rs } \\
\hline CONT SOIL & HONE & 0.0 & 128.9 & 120.9 & 206.2 & 206.2 & 206.2 & 306.9 & 401.4 & 148.6 & 148.6 & 472.2 & 172.2 & 172.2 & 172.2 & 172.2 & 172.2 \\
\hline Total CONT SOIL & & 0.0 & 128.9 & 128.9 & 206.2 & 206.2 & 206.2 & 305.9 & 101.4 & 118.6 & 148.6 & 472.2 & 172.2 & 472.2 & 172.2 & 172.2 & 472.2 \\
\hline D-cohbust & NONE & 0.0 & 18.1 & 18.4 & 14.7 & 14.7 & 14.7 & 26.9 & 0.0 & 0.0 & 0.0 & 0.0 & 0.0 & 0.0 & 0.0 & 0.0 & 0.0 \\
\hline Total D-COMBUST & & 0.0 & 10.4 & 10.1 & 24.7 & 14.7 & 14.7 & 26.9 & 0.0 & 0.0 & 0.0 & 0.0 & 0.0 & 0.0 & 0.0 & $\ddot{0.0}$ & 0.0 \\
\hline D-IN NON-MTL & MONE & 0.0 & 18.1 & 10.4 & 14.7 & 14.7 & 14.7 & 80.9 & 53.9 & 0.0 & 0.0 & 0.0 & 0.0 & 0.0 & 0.0 & 0.0 & 0.0 \\
\hline Total D-IN NON-MTL & & 0.0 & 28.4 & 10.1 & 34.7 & 14.7 & .24 .7 & 80.9 & 53.9 & 0.0 & 0.0 & 0.0 & 0.0 & 0.0 & 0.0 & 0.0 & 0.0 \\
\hline D-METAL & MONE & 0.0 & 1.8 & 1.0 & 0.0 & 0.0 & 0.0 & 6.7 & 6.7 & 0.0 & 0.0 & 0.0 & 0.0 & 0.0 & 0.0 & 0.0 & 0.0 \\
\hline TOta1 D-METAL & & 0.0 & 1.8 & 1.8 & 0.0 & 0.0 & 0.0 & 6.7 & 6.7 & 0.0 & 0.0 & 0.0 & 0.0 & 0.0 & 0.0 & 0.0 & 0.0 \\
\hline IN ORG LO/SLG & NONE & 0.0 & 200.7 & 200.7 & 232.5 & 132.5 & 132.5 & 212.5 & 172.0 & 192.2 & 192.2 & 202.4 & 202.4 & 202.4 & 202.4 & 202.1 & 202.1 \\
\hline Total IN ORG LQ/SLG & & 0.0 & 200.7 & 200.7 & 132.5 & 132.5 & 132.5 & 212.5 & 172.0 & 192.2 & 192.2 & 202.4 & 202.4 & 202.4 & 202.4 & 202.1 & 202.4 \\
\hline IN ORG PART & NONE & 0.0 & 0.0 & 0.0 & 0.0 & 0.0 & 0.0 & 6.7 & 6.1 & 0.0 & 0.0 & 0.0 & 0.0 & 0.0 & 0.0 & 0.0 & 0.0 \\
\hline Total IN ORG PART & & 0.0 & 0.0 & 0.0 & 0.0 & 0.0 & 0.0 & 6.7 & 6.7 & 0.0 & 0.0 & 0.0 & 0.0 & 0.0 & 0.0 & 0.0 & 0.0 \\
\hline LAB/CONI LQ & NONE & 0.0 & 0.0 & 0.0 & 0.0 & 0.0 & 0.0 & 33.7 & 33.7 & 33.7 & 33.7 & 0.0 & 0.0 & 0.0 & 0.0 & 0.0 & 0.0 \\
\hline Total LAB/CONT LO & & 0.0 & 0.0 & 0.0 & 0.0 & 0.0 & 0.0 & 33.7 & 33.7 & 33.7 & 33.7 & 0.0 & 0.0 & 0.0 & 0.0 & 0.0 & 0.0 \\
\hline UNKNoWN & UNAHOWN & 0.0 & 0.0 & 0.0 & 0.0 & 0.0 & 0.0 & 309.2 & 309.2 & 309.2 & 309.2 & 309.2 & 309.2 & 309.2 & 309.2 & 309.2 & 309.2 \\
\hline Total UNKNOWN & & 0.0 & 0.0 & 0.0 & 0.0 & 0.0 & 0.0 & 309.2 . & 309.2 & 309.2 & 309.2 & 309.2 & 309.2 & 309.2 & 309.2 & 309.2 & 309.2 \\
\hline TOtal PORTSHOUTH_ES & 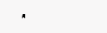 & 0.0 & 368.3 & 360.3 & 368.3 & 368.3 & 368.3 & 983.9 & 983.9 & 983.9 & 983.9 & 983.9 & 983.9 & 983.9 & 983.9 & 983.9 & 983.9 \\
\hline
\end{tabular}

\section{Waste Generator:PORTsMOUTH_us}

\begin{tabular}{|c|c|c|c|c|c|c|c|c|c|c|c|c|c|c|c|c|c|}
\hline D-carbust & NONE & 1420.5 & $0.0^{\circ}$ & 0.0 & 0.0 & 0.0 & 0.0 & 0.0 & 0.0 & 0.0 & 0.0 & 0.0 & 0.0 & 0.0 & 0.0 & 0.0 & 0.0 \\
\hline Total D-COMBUST & & 2420.5 & 0.0 & 0.0 & 0.0 & 0.0 & 0.0 & 0.0 & 0.0 & 0.0 & 0.0 & 0.0 & 0.0 & 0.0 & 0.0 & 0.0 & 0.0 \\
\hline D-METAL & NONE & 355.1 & 0.0 & 0.0 & 0.0 & 0.0 & 0.0 & 0.0 & 0.0 & 0.0 & 0.0 & 0.0 & 0.0 & .0 .0 & 0.0 & $\overline{0.0}$ & $\overline{0.0}$ \\
\hline TOtal D-HETAL & & 355.1 & 0.0 & 0.0 & 0.0 & 0.0 & 0.0 & 0.0 & 0.0 & 0.0 & 0.0 & 0.0 & 0.0 & 0.0 & 0.0 & 0.0 & 0.0 \\
\hline UNKNOWN & UNKNOWN & 213.5 & 0.0 & 0.0 & 0.0 & 0.0 & 0.0 & 0.0 & 0.0 & 0.0 & 0.0 & 0.0 & 0.0 & 0.0 & 0.0 & 0.0 & 0.0 \\
\hline Total UNKNOWN & & 213.5 & 0.0 & 0.0 & 0.0 & 0.0 & 0.0 & 0.0 & 0.0 & 0.0 & 0.0 & 0.0 & 0.0 & 0.0 & 0.0 & 0.0 & 0.0 \\
\hline TOtal PORTSHOUTH_US & & 1989.2 & 0.0 & 0.0 & 0.0 & 0.0 & 0.0 & 0.0 & 0.0 & 0.0 & 0.0 & 0.0 & 0.0 & 0.0 & 0.0 & 0.0 & 0.0 \\
\hline
\end{tabular}

$\begin{array}{lllllllllllllllllllllllll}\text { BERYLLIU } & D & 0.0 & 0.2 & 0.9 & 3.9 & 2.2 & 0.5 & 0.2 & 0.2 & 0.2 & 0.2 & 0.2 & 0.2 & 0.5 & 3.9 & 3.9 & 0.5\end{array}$

gco Codas: A-Ignitables, B-Corrosives, CmReactives, D-Metals w/O Hg, E-Motals w Hg, F-Organlcs, G-State Reg., H-PCB < 50ppm, J-PCB > 50ppm 
Annual Physical haste Form and Bazardoua Constituent Volumes by faste Generator

(in cubic meters)

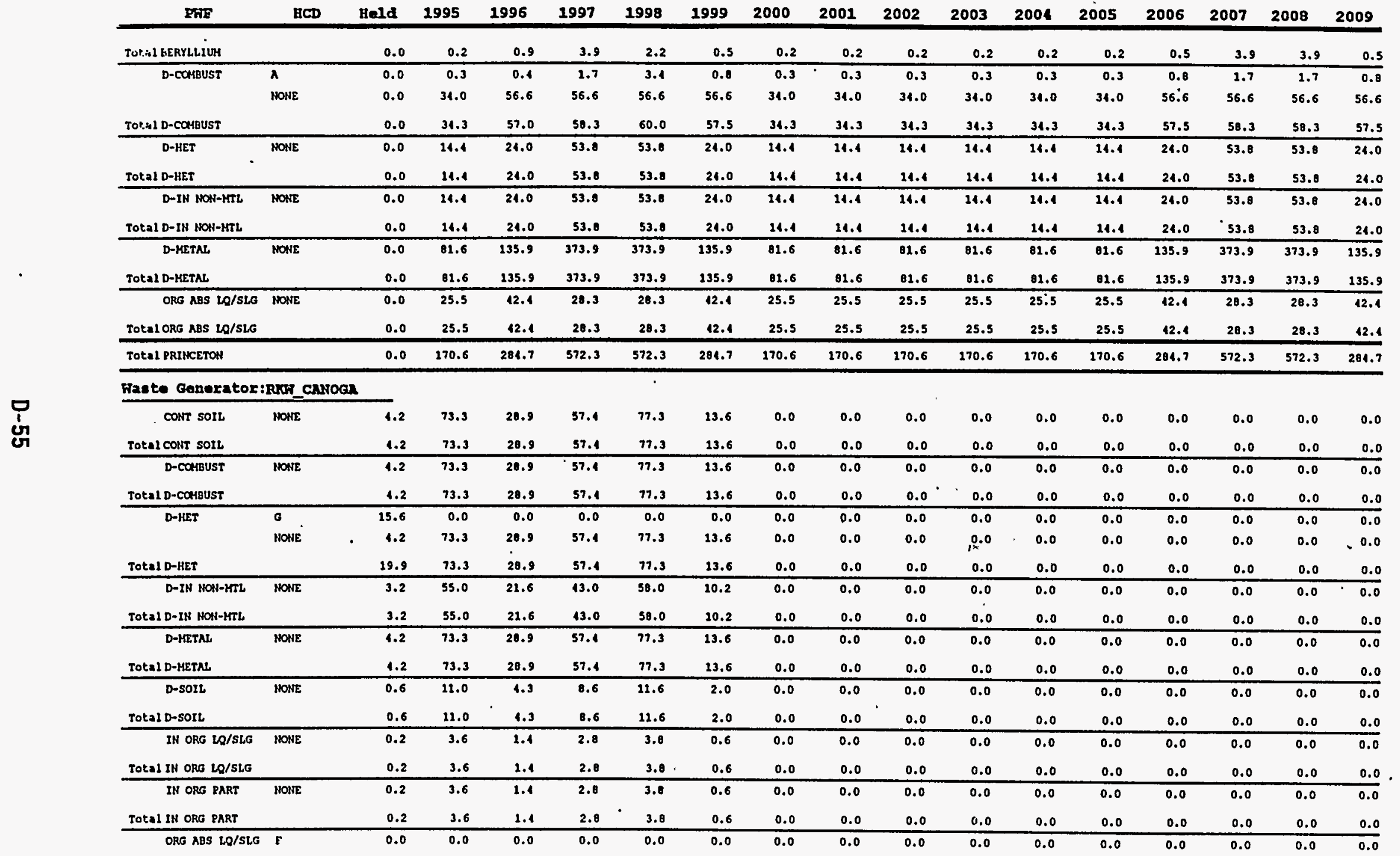

ECD Codes: A-Ignitables, B-Corrosives, C-Reactives, D-Metals H/O Hg, E-Metals w Hg, F-Organles, G=State Reg., H-PCB < 50ppm, J-PCB > 50ppm 


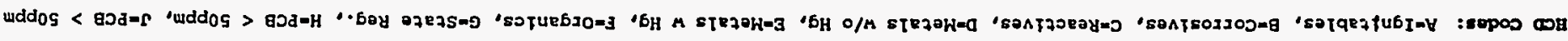

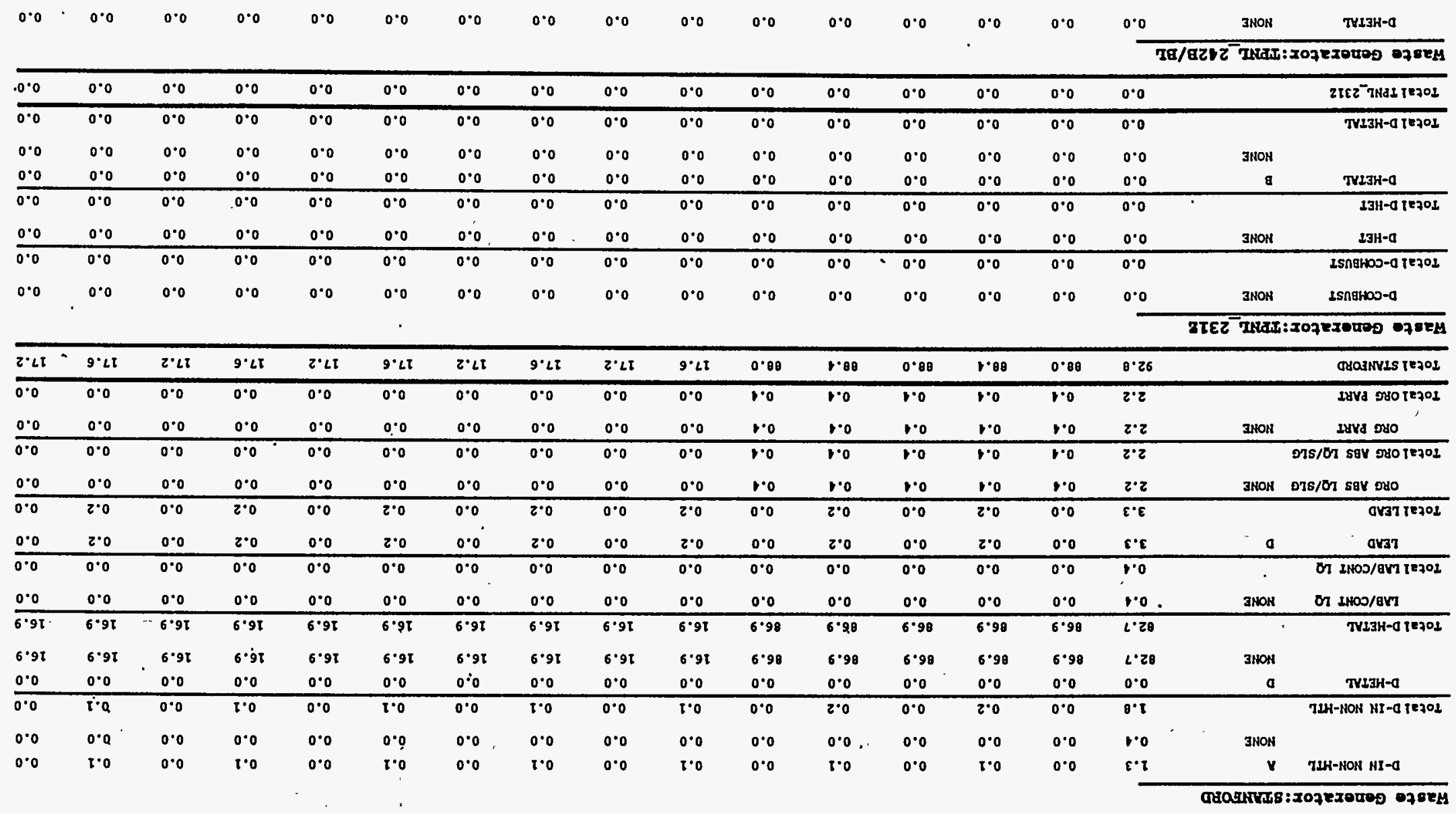

\begin{tabular}{|c|c|c|c|c|c|c|c|c|c|c|c|c|c|c|c|c|c|}
\hline 0.0 & 0.0 & 0.0 & 0.0 & 0.0 & 0.0 & 0.0 & 0.0 & 0.0 & 0.0 & i. & $L \cdot 9 \theta \varepsilon$ & $0 .\llcorner\theta 2$ & s.wt & $\iota \cdot 99 \varepsilon$ & $\tau \cdot \iota \varepsilon$ & & VoONYS \\
\hline 0.0 & 0.0 & 0.0 & 0.0 & 0.0 & 0.0 & 0.0 & 0.0 & 0.0 & 0.0 & 0.0 & 0.0 & 0.0 & 0.0 & 0.0 & 0.0 & & 3LSYM LTVS JEJOL \\
\hline 0.0 & 0.0 & 0.0 & 0.0 & 0.0 & 0.0 & 0.0 & 0.0 & 0.0 & 0.0 & 0.0 & 0.0 & 0.0 & 0.0 & 0.0 & 0.0 & $\infty$ & 315YM L7YS \\
\hline 0.0 & 0.0 & 0.0 & 0.0 & 0.0 & $0 \cdot 0$ & 0.0 & 0.0 & 0.0 & 0.0 & 0.0 & 0.0 & 0.0 & 0.0 & 0.0 & 0.0 & & 7 59у פو פ \\
\hline
\end{tabular}

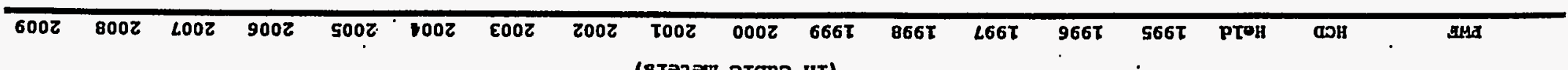

$\cot 06 \mathrm{~d}$

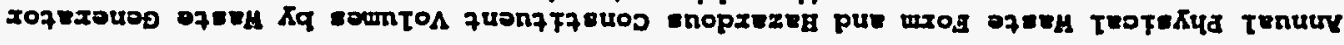


(In cubia meters)

\begin{tabular}{|c|c|c|c|c|c|c|c|c|c|c|c|c|c|c|c|c|c|}
\hline PFE & HCD & Hold & 1995 & 1996 & 1997 & 1998 & 1999 & 2000 & 2001 & 2002 & 2003 & 2004 & 2005 & 2006 & 2007 & 2008 & 2009 \\
\hline Tutal D-METAL & & 0.0 & 0.0 & 0.0 & 0.0 & 0.0 & 0.0 & 0.0 & 0.0 & 0.0 & 0.0 & 0.0 & 0.0 & 0.0 & 0.0 & 0.0 & 0.0 \\
\hline UNKNOWN & NONE & 0.0 & 0.0 & 0.0 & 0.0 & 0.0 & 0.0 & 0.0 & 0.0 & 0.0 & 0.0 & 0.0 & 0.0 & 0.0 & 0.0 & 0.0 & 0.0 \\
\hline Tot.al unknown & & 0.0 & 0.0 & 0.0 & 0.0 & 0.0 & 0.0 & 0.0 & 0.0 & 0.0 & 0.0 & 0.0 & 0.0 & 0.0 & 0.0 & 0.0 & 0.0 \\
\hline Total TPNL_212B/BL & & 0.0 & 0.0 & 0.0 & 0.0 & 0.0 & 0.0 & 0.0 & 0.0 & 0.0 & 0.0 & 0.0 & 0.0 & 0.0 & 0.0 & 0.0 & 0.0 \\
\hline \multicolumn{18}{|c|}{ Waste Generatox:MP2L_306 } \\
\hline D-HET & WONE & 0.0 & 0.0 & 0.0 & 0.0 & 0.0 & 0.0 & 0.0 & 0.0 & 0.0 & 0.0 & 0.0 & 0.0 & 0.0 & 0.0 & 0.0 & 0.0 \\
\hline D-METAL & NONE & 0.0 & 0.0 & 0.0 & 0.0 & 0.0 & 0.0 & 0.0 & 0.0 & 0.0 & 0.0 & 0.0 & 0.0 & 0.0 & 0.0 & 0.0 & 0.0 \\
\hline Total D-METAS & & 0.0 & 0.0 & 0.0 & 0.0 & 0.0 & 0.0 & 0.0 & 0.0 & 0.0 & 0.0 & 0.0 & 0.0 & 0.0 & 0.0 & 0.0 & 0.0 \\
\hline UWkHOWNS & NONE & 0.0 & 0.0 & 0.0 & 0.0 & 0.0 & 0.0 & 0.0 & 0.0 & 0.0 & 0.0 & 0.0 & 0.0 & 0.0 & 0.0 & 0.0 & 0.0 \\
\hline Total unarown & & 0.0 & 0.0 & 0.0 & 0.0 & 0.0 & 0.0 & 0.0 & 0.0 & 0.0 & 0.0 & 0.0 & 0.0 & 0.0 & 0.0 & 0.0 & 0.0 \\
\hline Total TPNZ__306W & & 0.0 & 0.0 & 0.0 & 0.0 & 0.0 & 0.0 & 0.0 & 0.0 & 0.0 & 0.0 & 0.0 & 0.0 & 0.0 & 0.0 & 0.0 & 0.0 \\
\hline \multicolumn{18}{|c|}{ Waste Generator:MRIL_314 } \\
\hline D-METAL & MONE & 0.0 & 0.0 & 0.0 & 0.0 & 0.0 & 0.0 & 0.0 & 0.0 & 0.0 & 0.0 & 0.0 & 0.0 & 0.0 & 0.0 & 0.0 & 0.0 \\
\hline Total TPNL_314 & & 0.0 & 0.0 & 0.0 & 0.0 & 0.0 & 0.0 & 0.0 & 0.0 & 0.0 & 0.0 & 0.0 & 0.0 & 0.0 & 0.0 & 0.0 & 0.0 \\
\hline
\end{tabular}

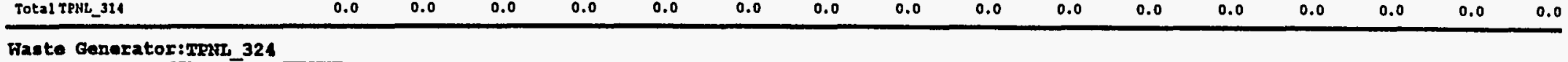

\begin{tabular}{|c|c|c|c|c|c|c|c|c|c|c|c|c|c|c|c|c|c|}
\hline D-HET & NONE & 0.0 & 0.0 & 0.0 & 0.0 & 0.0 & 0.0 & 0.0 & 0.0 & 0.0 & 0.0 & 0.0 & 0.0 & 0.0 & 0.0 & 0.0 & 0.0 \\
\hline Total D-HET & & 0.0 & 0.0 & 0.0 & 0.0 & 0.0 & 0.0 & 0.0 & 0.0 & 0.0 & 0.0 & 0.0 & 0.0 & 0.0 & 0.0 & 0.0 & 0.0 \\
\hline \multirow[t]{2}{*}{ D-METAL } & D & 0.0 & 0.0 & 0.0 & 0.0 & 0.0 & 0.0 & 0.0 & 0.0 & 0.0 & 0.0 & 0.0 & 0.0 & 0.0 & 0.0 & 0.0 & 0.0 \\
\hline & NONE & 0.0 & 0.0 & 0.0 & 0.0 & 0.0 & 0.0 & 0.0 & 0.0 & 0.0 & 0.0 & 0.0 & 0.0 & 0.0 & 0.0 & 0.0 & 0.0 \\
\hline TOtAL D-METAL & & 0.0 & 0.0 & 0.0 & 0.0 & 0.0 & 0.0 & 0.0 & 0.0 & 0.0 & 0.0 & 0.0 & 0.0 & 0.0 & 0.0 & 0.0 & 0.0 \\
\hline \multirow[t]{2}{*}{ LEAD } & D & 0.0 & 0.0 & 0.0 & 0.0 & 0.0 & 0.0 & 0.0 & 0.0 & 0.0 & 0.0 & 0.0 & 0.0 & 0.0 & 0.0 & 0.0 & 0.0 \\
\hline & HONE & 0.0 & 0.0 & 0.0 & 0.0 & 0.0 & 0.0 & 0.0 & 0.0 & 0.0 & 0.0 & 0.0 & 0.0 & 0.0 & 0.0 & 0.0 & 0.0 \\
\hline Total LEAD & & 0.0 & 0.0 & 0.0 & 0.0 & 0.0 & 0.0 & 0.0 & 0.0 & 0.0 & 0.0 & 0.0 & 0.0 & 0.0 & 0.0 & 0.0 & 0.0 \\
\hline Untentownt & NONE & 0.0 & 0.0 & 0.0 & 0.0 & 0.0 & 0.0 & 0.0 & 0.0 & 0.0 & 0.0 & 0.0 & 0.0 & 0.0 & 0.0 & 0.0 & 0.0 \\
\hline Total UNKNOWN & & 0.0 & 0.0 & 0.0 & 0.0 & 0.0 & 0.0 & 0.0 & 0.0 & 0.0 & 0.0 & 0.0 & 0.0 & 0.0 & 0.0 & 0.0 & 0.0 \\
\hline Total TPHL_324 & & 0.0 & 0.0 & 0.0 & 0.0 & 0.0 & 0.0 & 0.0 & 0.0 & 0.0 & 0.0 & 0.0 & 0.0 & 0.0 & 0.0 & 0.0 & $0.0^{\circ}$ \\
\hline
\end{tabular}

D-HET UNKNOWN

$0.0 \quad 0.0$

$\begin{array}{lll}0.0 & 0.0 & 0.0\end{array}$

0.0

$0.0 \quad 0.0$

$0.0 \quad 0.0$

HCD Codes: A-Ignltables, B-Corroslves, C=Reactlves, D-Metals w/O Hg, E-Metals w Hg, F-Organles, G=State Reg., H-PCB < 50ppm, JmPCB > 50ppm 


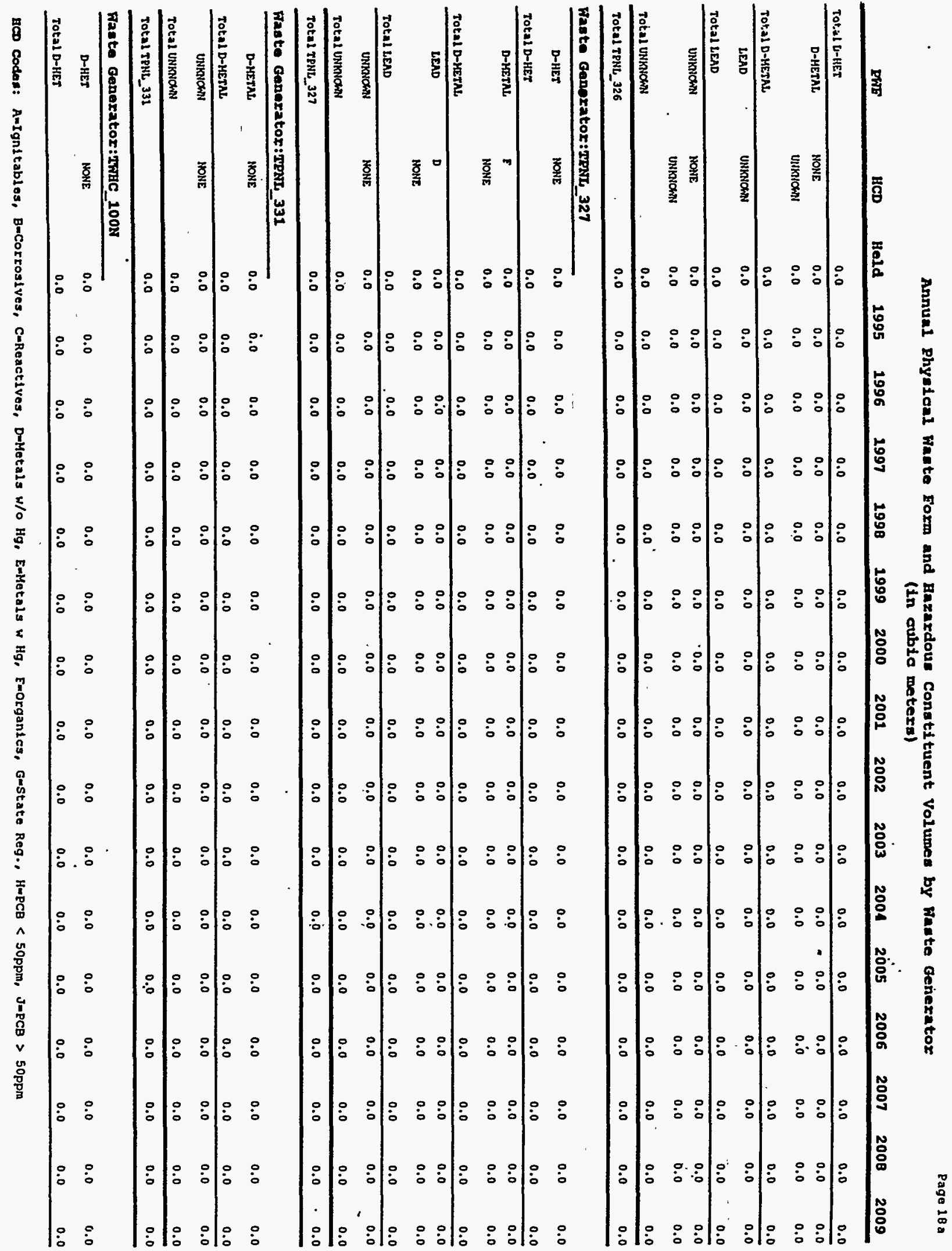




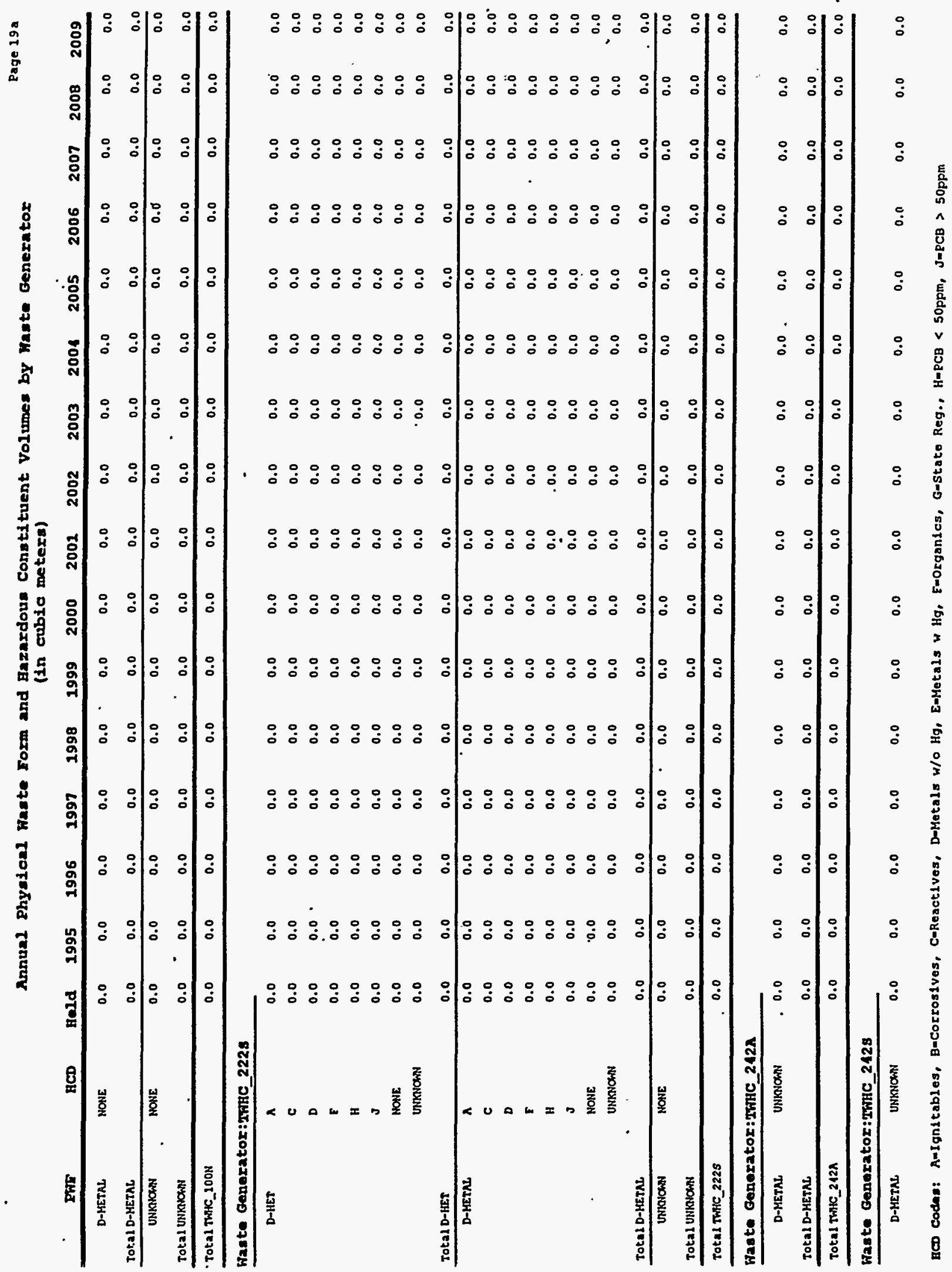


(in cubic metera)

\begin{tabular}{|c|c|c|c|c|c|c|c|c|c|c|c|c|c|c|c|c|c|}
\hline PFit & HCD & Held & 1995 & 1996 & 1997 & 1998 & 1999 & 2000 & 2001 & 2002 & 2003 & 2004 & .2005 & 2006 & 2007 & 2008 & 2009 \\
\hline TUL.ZI U-HETAL & & 0.0 & 0.0 & 0.0 & 0.0 & 0.0 & 0.0 & 0.0 & 0.0 & 0.0 & 0.0 & 0.0 & 0.0 & 0.0 & 0.0 & 0.0 & 0.0 \\
\hline Tot:A1 TWIC_242S & & -0.0 & 0.0 & 0.0 & 0.0 & 0.0 & 0.0 & 0.0 & 0.0 & 0.0 & 0.0 & 0.0 & .0 .0 & 0.0 & 0.0 & 0.0 & 0.0 \\
\hline \multicolumn{18}{|c|}{ Waste Gonerator: IFHC_212T } \\
\hline D-METAL & UNKNOWN & 0.0 & 0.0 & 0.0 & 0.0 & 0.0 & 0.0 & 0.0 & 0.0 & 0.0 & 0.0 & 0.0 & 0.0 & 0.0 & 0.0 & 0.0 & 0.0 \\
\hline TOt,a1 D-METAL & & 0.0 & 0.0 & 0.0 & 0.0 & 0.0 & 0.0 & 0.0 & 0.0 & 0.0 & 0.0 & 0.0 & 0.0 & 0.0 & 0.0 & 0.0 . & 0.0 \\
\hline Total TWHE_212T. & & 0.0 & 0.0 & 0.0 & 0.0 & 0.0 & 0.0 & 0.0 & 0.0 & 0.0 & 0.0 & 0.0 & 0.0 & 0.0 & 0.0 & 0.0 & 0.0 \\
\hline \multicolumn{18}{|c|}{ Waste Genorator:IFHC_306 } \\
\hline D-HET & MONE & 0.0 & 0.0 & 0.0 & 0.0 & 0.0 & 0.0 & 0.0 & 0.0 & 0.0 & 0.0 & 0.0 & 0.0 & 0.0 & 0.0 & 0.0 & 0.0 \\
\hline Total D-HET & & 0.0 & 0.0 & 0.0 & 0.0 & 0.0 & 0.0 & 0.0 & 0.0 & 0.0 & 0.0 & 0.0 & 0.0 & 0.0 & 0.0 & 0.0 & 0.0 \\
\hline \multirow[t]{2}{*}{ D-METAL } & MONE . & 0.0 & 0.0 & 0.0 & 0.0 & 0.0 & 0.0 & 0.0 & 0.0 & 0.0 & 0.0 & 0.0 & 0.0 & 0.0 & 0.0 & 0.0 & 0.0 \\
\hline & UHANOWN & 0.0 & 0.0 & 0.0 & 0.0 & 0.0 & 0.0 & 0.0 & 0.0 & 0.0 & 0.0 & 0.0 & 0.0 & 0.0 & 0.0 & 0.0 & 0.0 \\
\hline Total D-METAL & & 0.0 & 0.0 & 0.0 & 0.0 & 0.0 & 0.0 & 0.0 & 0.0 & 0.0 & 0.0 & 0.0 & 0.0 & 0.0 & 0.0 & 0.0 & 0.0 \\
\hline Total TWHC_306 & & 0.0 & 0.0 & 0.0 & 0.0 & 0.0 & 0.0 & 0.0 & 0.0 & 0.0 & 0.0 & 0.0 & 0.0 & 0.0 & 0.0 & 0.0 & 0.0 \\
\hline \multicolumn{18}{|c|}{ Fasto Genarator:4File_308 } \\
\hline D-COHBUST & HONE & 0.0 & 0.0 & 0.0 & 0.0 & 0.0 & 0.0 & 0.0 & 0.0 & 0.0 & 0.0 & 0.0 & 0.0 & 0.0 & 0.0 & 0.0 & 0.0 \\
\hline Tota1 D-COMBUST & & 0.0 & 0.0 & 0.0 & 0.0 & 0.0 & 0.0 & 0.0 & 0.0 & 0.0 & 0.0 & 0.0 & 0.0 & 0.0 & 0.0 & 0.0 & 0.0 \\
\hline D-HET & NONE & 0.0 & 0.0 & 0.0 & 0.0 & 0.0 & .0 .0 & $0 . \dot{0}$ & 0.0 & $0.0 \ldots$ & 0.0 & 0.0 & 0.0 & $0: 0$ & 0.0 & 0.0 & 0.0 \\
\hline Total D-HET & & 0.0 & 0.0 & 0.0 & 0.0 & 0.0 & 0.0 & 0.0 & 0.0 & 0.0 & 0.0 & 0.0 & 0.0 & 0.0 & 0.0 & 0.0 & 0.0 \\
\hline D-IN NON-HTL & NONE & 0.0 & 0.0 & 0.0 & 0.0 & 0.0 & 0.0 & 0.0 & 0.0 & 0.0 & 0.0 & 0.0 & 0.0 & 0.0 & 0.0 & 0.0 & 0.0 \\
\hline TOtal D-IN NON-HTL & & 0.0 & 0.0 & 0.0 & 0.0 & 0.0 & 0.0 & 0.0 & 0.0 & 0.0 & 0.0 & 0.0 & 0.0 & 0.0 & 0.0 & 0.0 & 0.0 \\
\hline D- METAL & NONE & 0.0 & 0.0 & 0.0 & 0.0 & 0.0 & 0.0 & 0.0 & 0.0 & 0.0 & 0.0 & 0.0 & 0.0 & 0.0 & 0.0 & 0.0 & 0.0 \\
\hline TOta1 D-METAL & & 0.0 & 0.0 & 0.0 & 0.0 & 0.0 & 0.0 & 0.0 & 0.0 & 0.0 & 0.0 & 0.0 & 0.0 & 0.0 & 0.0 & 0.0 & 0.0 \\
\hline \multirow[t]{2}{*}{ UNWNOWN } & NONE & 0.0 & 0.0 & 0.0 & 0.0 & 0.0 & 0.0 & 0.0 & 0.0 & 0.0 & 0.0 & 0.0 & 0.0 & 0.0 & 0.0 & 0.0 & 0.0 \\
\hline & UNKNOWN & 0.0 & 0.0 & 0.0 & 0.0 & 0.0 & 0.0 & 0.0 & 0.0 & 0.0 & 0.0 & 0.0 & 0.0 & 0.0 & 0.0 & 0.0 & 0.0 \\
\hline Total UNKNOWN & & 0.0 & 0.0 & 0.0 & 0.0 & 0.0 & 0.0 & 0.0 & 0.0 & 0.0 & 0.0 & 0.0 & 0.0 & 0.0 & 0.0 & 0.0 & 0.0 \\
\hline Totel TWHC_308 & & 0.0 & 0.0 & 0.0 & 0.0 & 0.0 & 0.0 & 0.0 & 0.0 & 0.0 & 0.0 & 0.0 & 0.0 & 0.0 & 0.0 & 0.0 & 0.0 \\
\hline \multicolumn{18}{|c|}{ Faste Generator:1FHC_340 } \\
\hline D-combust & HONE & 0.0 & 0.0 & 0.0 & 0.0 & 0.0 & 0.0 & 0.0 & 0.0 & 0.0 & 0.0 & 0.0 & 0.0 & 0.0 & 0.0 & 0.0 & 0.0 \\
\hline Total D-COMBUST & & 0.0 & 0.0 & 0.0 & 0.0 & 0.0 & 0.0 & 0.0 & 0.0 & 0.0 & 0.0 & 0.0 & 0.0 & 0.0 & 0.0 & 0.0 & 0.0 \\
\hline \multirow[t]{2}{*}{ D-KETAL } & MONE & 0.0 & 0.0 & 0.0 & 0.0 & 0.0 & 0.0 & 0.0 & 0.0 & 0.0 & 0.0 & 0.0 & 0.0 & 0.0 & 0.0 & 0.0 & 0.0 \\
\hline & unsoons: & 0.0 & 0.0 & 0.0 & 0.0 & 0.0 & 0.0 & 0.0 & 0.0 & 0.0 & 0.0 & 0.0 & 0.0 & 0.0 & 0.0 & 0.0 & 0.0 \\
\hline
\end{tabular}

BCD Codes: A=Ignitables, B-Corrosives, C-Reactives, D-Metals w/O Hg, E-Metals w Hg, E-Organles, G-State Reg., H-PCB < 50ppm, JuPCB > 50ppm 


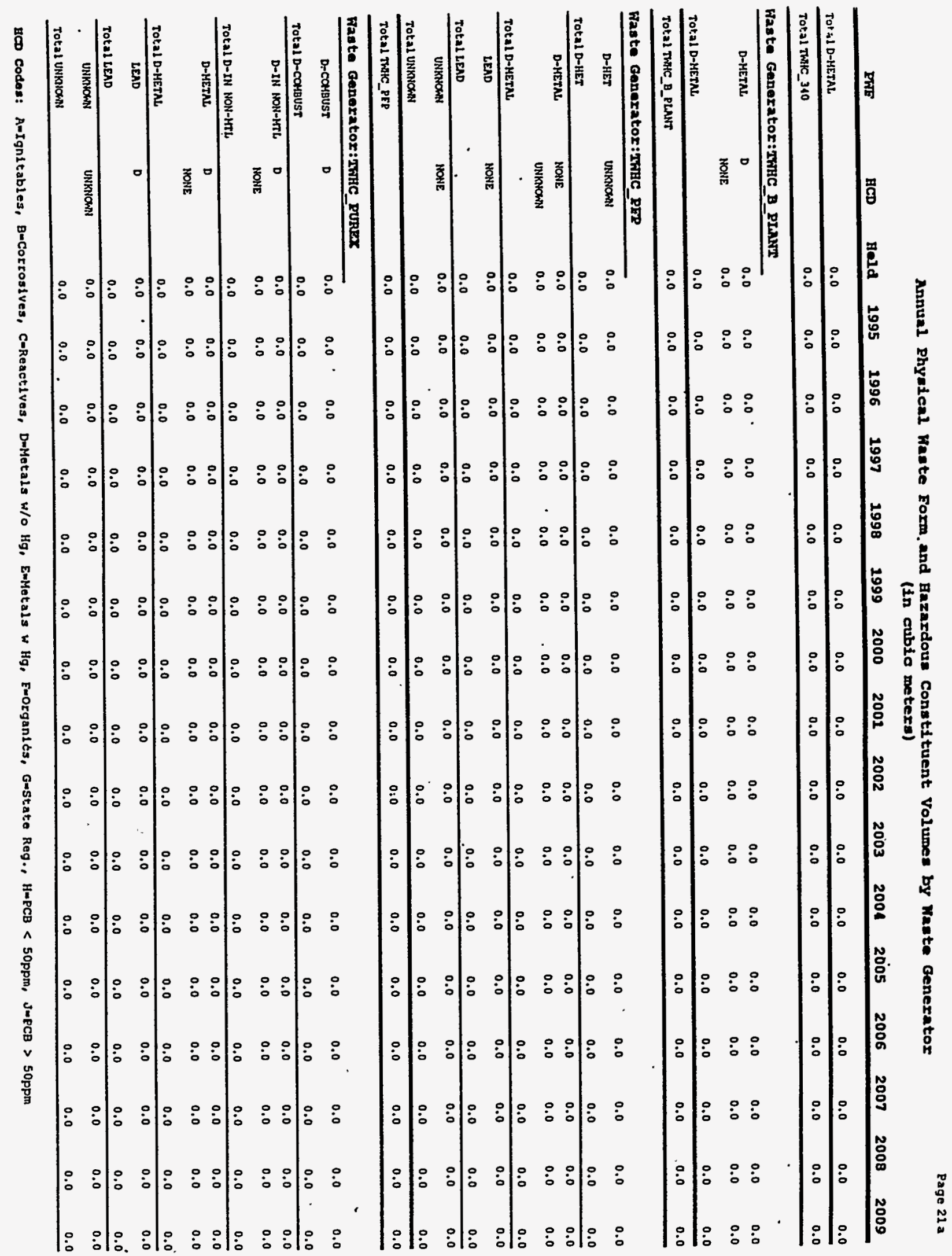


(in cubia meters)

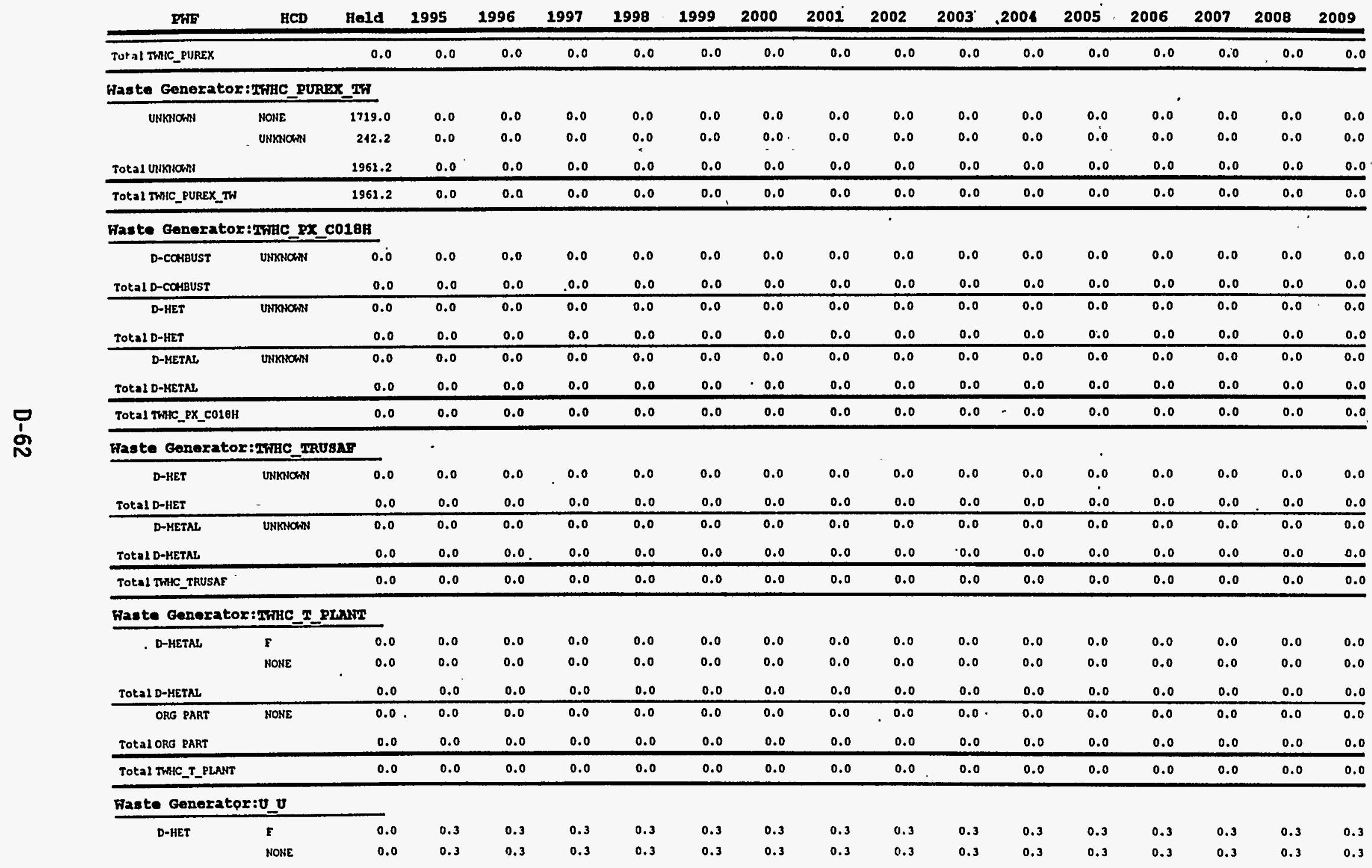

uCo Codes: A=Ignitables, B-Corrosives, C-Reactives, D-Metals w/O Hg, Emetals w Hg, Emorganics, G=State Reg., H-PCB < 50ppm, J-PCB > 50ppm 

Annual Physical Haste Forts and Bazardous Constituent Volumes by Waste Generator

Page $23 \mathrm{a}$

\begin{tabular}{|c|c|c|c|c|c|c|c|c|c|c|c|c|c|c|c|c|c|}
\hline PWE & HCD & Held & 1995 & 1996 & 1997 & 1998 & 1999 & 2000 & 2001 & 2002 & 2003 & 2004 & 2005 & 2006 & 2007 & 2008 & 2009 \\
\hline TOR 21 E-HET & & 0.0 & 0.6 & 0.6 & 0.6 & 0.6 & 0.6 & 0.6 & 0.6 & 0.6 & 0.6 & 0.6 & 0.6 & 0.6 & 0.6 & 0.6 & 0.6 \\
\hline Total U U & & 0.0 & 0.6 & 0.6 & 0.6 & 0.6 & 0.6 & 0.6 & 0.6 & 0.6 & 0.6 & 0.6 & 0.6 & 0.6 & 0.6 & 0.6 & 0.6 \\
\hline \multicolumn{18}{|c|}{ Waste Generator:FHC_100 } \\
\hline \multirow[t]{2}{*}{ BATTERIES } & B & 0.0 & 3.1 & 3.4 & 3.1 & 3.4 & 3.1 & 0.0 & 0.0 & 0.0 & 0.0 & 0.0 & 0.0 & 0.0 & 0.0 & 0.0 & 0.0 \\
\hline & D & 0.0 & 2.5 & 2.5 & 2.5 & 2.5 & 2.5 & 0.0 & 0.0 & 0.0 & 0.0 & 0.0 & 0.0 & 0.0 & 0.0 & 0.0 & 0.0 \\
\hline Tota1 BATTERIES & & 0.0 & 5.9 & 3.9 & 5.9 & 5.9 & 5.9 & 0.0 & 0.0 & 0.0 & 0.0 & 0.0 & 0.0 & 0.0 & 0.0 & 0.0 & 0.0 \\
\hline \multirow[t]{4}{*}{ D-COMBUST } & $\AA$ & 0.0 & 0.3 & 0.3 & 0.3 & 0.3 & 0.3 & 0.0 & 0.0 & 0.0 & 0.0 & 0.0 & 0.0 & 0.0 & 0.0 & 0.0 & 0.0 \\
\hline & - & 0.0 & 0.3 & 0.3 & 0.3 & 0.3 & 0.3 & 0.0 & 0.0 & 0.0 & 0.0 & 0.0 & 0.0 & 0.0 & 0.0 & 0.0 & 0.0 \\
\hline & E & 0.0 & 0.3 & 0.3 & 0.3 & 0.3 & 0.3 & 0.0 & 0.0 & 0.0 & 0.0 & 0.0 & 0.0 & 0.0 & 0.0 & 0.0 & 0.0 \\
\hline & NONE & 0.0 & 200.3 & 190.5 & 190.5 & 102.9 . & 58.2 & 0.0 & 0.0 & 0.0 & 0.0 & 0.0 & 0.0 & 0.0 & 0.0 & 0.0 & 0.0 \\
\hline Total D-COMBUST & & 0.0 & 201.3 & 191.5 & 191.5 & 104.0 & 59.2 & 0.0 & 0.0 & 0.0 & 0.0 & 0.0 & 0.0 & 0.0 & 0.0 & 0.0 & 0.0 \\
\hline \multirow[t]{2}{*}{ D-HET } & F & 0.0 & 0.2 & 0.2 & 0.2 & 0.2 & 0.2 & 0.0 & 0.0 & 0.0 & 0.0 & 0.0 & 0.0 & 0.0 & 0.0 & 0.0 & 0.0 \\
\hline & 0 & 0.0 & 0.1 & 0.1 & 0.1 & 0.1 & 0.1 & 0.0 & 0.0 & 0.0 & 0.0 & 0.0 & 0.0 & 0.0 & 0.0 & 0.0 & 0.0 \\
\hline Total D-HET & & 0.0 & 0.3 & 0.3 & 0.3 & 0.3 & 0.3 & 0.0 & 0.0 & 0.0 & 0.0 & 0.0 & 0.0 & 0.0 & 0.0 & 0.0 & 0.0 \\
\hline \multirow[t]{2}{*}{ D-IN HON-MTL } & D & 0.0 & 1.3 & 1.3 & 1.3 & 1.3 & 1.3 & 0.0 & 0.0 & 0.0 & 0.0 & 0.0 & 0.0 & 0.0 & 0.0 & 0.0 & 0.0 \\
\hline & HONE & 0.0 & 176.7 & 127.0 & 127.8 & 130.4 & 132.6 & 0.0 & 0.0 & 0.0 & 0.0 & 0.0 & 0.0 & 0.0 & 0.0 & 0.0 & 0.0 \\
\hline Total D-IN NON-HTL & & 0.0 & 278.1 & 129.2 & 129.2 & 131.7 & 133.9 & 0.0 & 0.0 & 0.0 & 0.0 & 0.0 & 0.0 & 0.0 & 0.0 & 0.0 & 0.0 \\
\hline D-METAL & D & 0.0 & 2.0 & 2.0 & 2.0 & 2.0 & 2.0 & 0.0 & 0.0 & 0.0 & 0.0 & 0.0 & 0.0 & 0.0 & 0.0 & 0.0 & 0.0 \\
\hline Total D-METAL & & 0.0 & 2.0 & 2.0 & 2.0 & 2.0 & 2.0 & 0.0 & 0.0 & 0.0 & 0.0 & 0.0 & 0.0 & 0.0 & 0.0 & 0.0 & 0.0 \\
\hline EXPLOSIVES & $\boldsymbol{A}$ & 0.0 & 2.8 & 2.8 & 2.8 & 2.8 & 2.8 & 0.0 & 0.0 & 0.0 & 0.0 & 0.0 & 0.0 & 0.0 & 0.0 & 0.0 & 0.0 \\
\hline Total ExpLosives & & 0.0 & 2.0 & 2.8 & 2.0 & 2.8 & 2.8 & 0.0 & 0.0 & 0.0 & 0.0 & 0.0 & 0.0 & 0.0 & 0.0 & 0.0 & 0.0 \\
\hline \multirow[t]{4}{*}{ IN ORG LQ/SLG } & B & 0.0 & 0.8 & 0.8 & 0.8 & 0.8 & 0.8 & 0.0 & 0.0 & 0.0 & 0.0 & 0.0 & 0.0 & 0.0 & 0.0 & 0.0 & 0.0 \\
\hline & c & 0.0 & 0.0 & 0.0 & 0.0 & 0.0 & 0.0 & 0.0 & 0.0 & 0.0 & 0.0 & 0.0 & 0.0 & 0.0 & 0.0 & 0.0 & 0.0 \\
\hline & D & 0.0 & 0.0 & 0.0 & 0.0 & 0.0 & 0.0 & 0.0 & 0.0 & 0.0 & 0.0 & 0.0 & 0.0 & 0.0 & 0.0 & 0.0 & 0.0 \\
\hline & $\mathbf{F}$ & 0.0 & 0.0 & 0.0 & 0.0 & 0.0 & 0.0 & 0.0 & 0.0 & 0.0 & 0.0 & 0.0 & 0.0 & 0.0 & 0.0 & 0.0 & 0.0 \\
\hline TOt=1 IN ORG LQ/SLG & & 0.0 & 0.9 & 0.9 & 0.9 & 0.9 & 0.9 & 0.0 & 0.0 & 0.0 & 0.0 & 0.0 & 0.0 & 0.0 & 0.0 & 0.0 & 0.0 \\
\hline \multirow[t]{5}{*}{ IN ORG PART } & $\Lambda$ & 0.0 & 0.1 & 0.1 & 0.1 & 0.1 & 0.1 & 0.0 & 0.0 & 0.0 & 0.0 & 0.0 & 0.0 & 0.0 & 0.0 & 0.0 & 0.0 \\
\hline & B & 0.0 & 0.0 & 0.0 & 0.0 & 0.0 & 0.0 & 0.0 & 0.0 & 0.0 & 0.0 & 0.0 & 0.0 & 0.0 & 0.0 & 0.0 & 0.0 \\
\hline & c & 0.0 & 1.2 & 1.2 & 1.2 & 1.2 & 1.2 & 0.0 & 0.0 & 0.0 & 0.0 & 0.0 & 0.0 & 0.0 & 0.0 & 0.0 & 0.0 \\
\hline & D & 0.0 & 0.1 & 0.1 & 0.1 & 0.1 & 0.1 & 0.0 & 0.0 & 0.0 & 0.0 & 0.0 & 0.0 & 0.0 & 0.0 & 0.0 & 0.0 \\
\hline & 8 & 0.0 & 0.0 & 0.0 & 0.0 & 0.0 & 0.0 & 0.0 & 0.0 & 0.0 & 0.0 & 0.0 & 0.0 & 0.0 & 0.0 & 0.0 & 0.0 \\
\hline Total IN ORG PART & & 0.0 & 1.6 & 1.6 & 1.6 & 1.6 & 1.6 & .0 .0 & 0.0 & 0.0 & 0.0 & 0.0 & 0.0 & 0.0 & 0.0 & 0.0 & 0.0 \\
\hline \multirow[t]{2}{*}{ ORG ABS LR/SLG } & $\boldsymbol{A}$ & 0.0 & 1.7 & 1.7 & $\cdot 1.7$ & 1.7 & 1.7 & 0.0 & 0.0 & 0.0 & 0.0 & 0.0 & 0.0 & 0.0 & 0.0 & 0.0 & $\overline{0.0}$ \\
\hline & $\mathbf{B}$ & 0.0 & 0.1 & 0.1 & 0.1 & 0.1 & 0.1 & 0.0 & 0.0 & 0.0 & 0.0 & 0.0 & 0.0 & 0.0 & 0.0 & 0.0 & 0.0 \\
\hline
\end{tabular}

HCo Codes: A-Ignitables, B-Corrosives, C-Reactives, D-Metals w/O Hg, E-Metals w Hg, Emorganles, G-State Reg., H-PCB < 50ppm, J-PCB > 50ppm 
(in cubia meters)

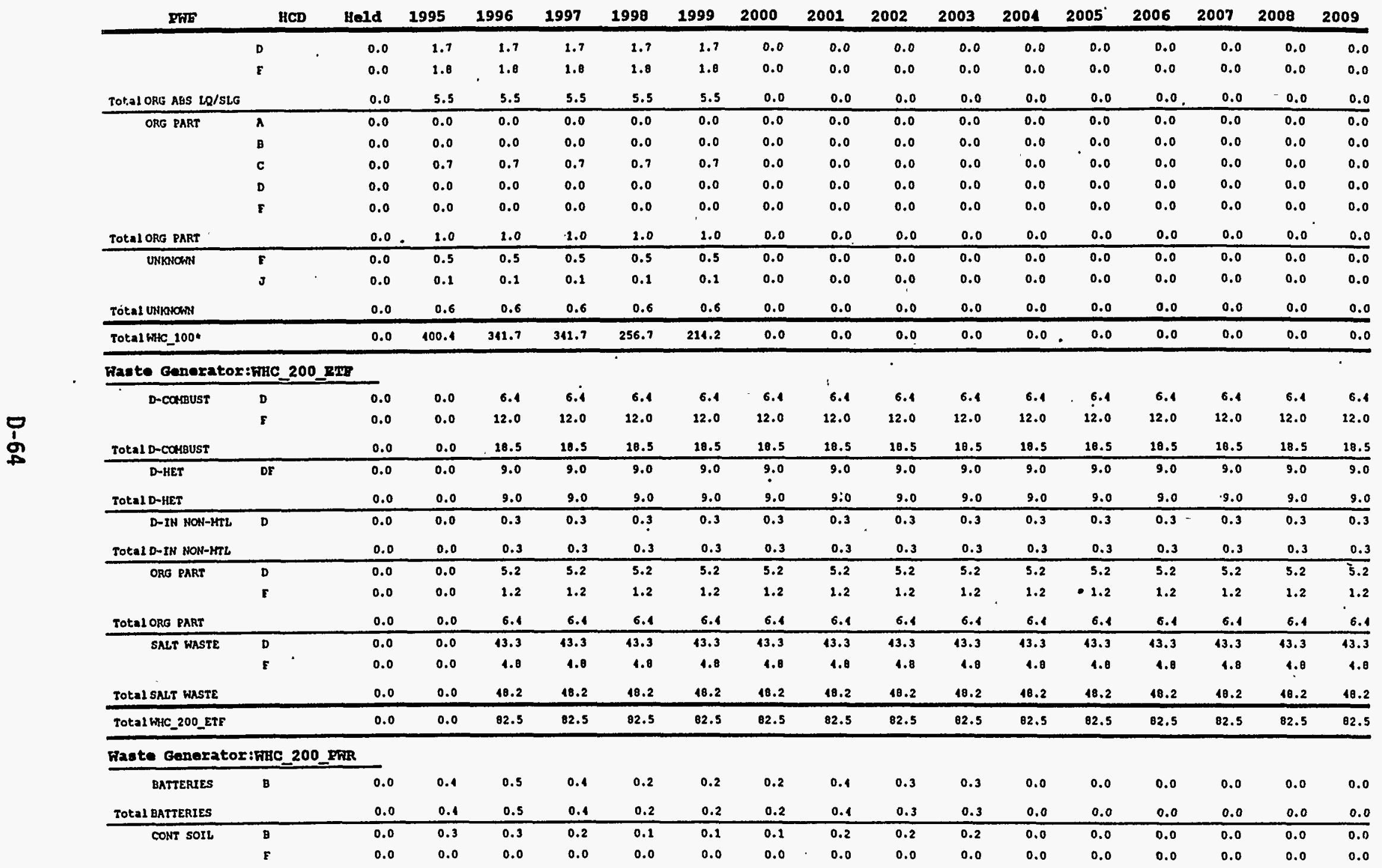

gCD codes: A-Ignitables, B-Corrosives, C=Reactives, D-Metals w/O Hg, E-Metals w Hg, F-Organlcs, G=State Reg., H=PCB < 50ppm, JaPCB > 50ppm 
Annual Phyaical Waste Form and Bazardous Constituent volumes by Haste Generator

(in cubic metera)

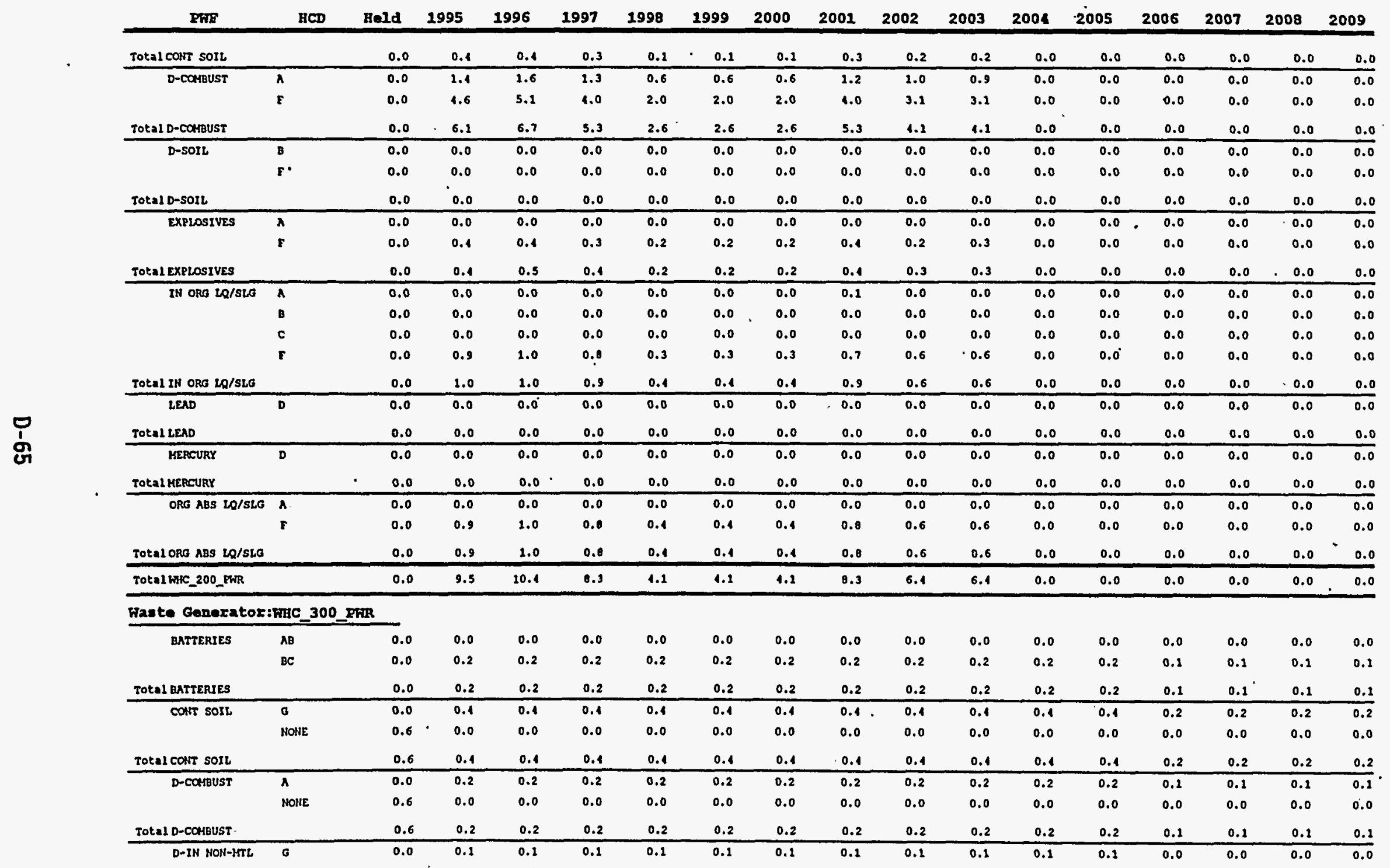

BCo Codes: A-Ignitables, B-Corrosives, C-Reactives; DeMetals w/O Hg, E-Metals w Hg, F-Organles, G-State Reg., H-PCB < 50ppm, J-PCB > 50ppm 
Annual Physical Faste Form and Hazardous Constituent Volumes by waste Generator

(In cubio metera)

\begin{tabular}{|c|c|c|c|c|c|c|c|c|c|c|c|c|c|c|c|c|c|}
\hline PHE & HCD & Held & 1995 & 1996 & 1997 & 1998 & 1999 & 2000 & 2001 & 2002 & 2003. & 2004 & $2005^{\circ}$ & 2006 & 2007 & 2008 & 2009 \\
\hline & HONE & 0.8 & 0.0 & 0.0 & 0.0 & 0.0 & 0.0 & 0.0 & 0.0 & 0.0 & 0.0 & 0.0 & 0.0 & 0.0 & 0.0 & 0.0 & 0.0 \\
\hline robint L-211 BOH-ATL & & 0.0 & 0.1 & 0.1 & 0.1 & 0.1 & 0.1 & 0.1 & 0.1 & 0.1 & 0.1 & 0.1 & 0.1 & 0.0 & 0.0 & 0.0 & 0.0 \\
\hline D-METAL & MOHE & 0.3 & 0.0 & 0.0 & 0.0 & 0.0 & 0.0 & 0.0 & 0.0 & 0.0 & 0.0 & 0.0 & 0.0 & 0.0 & 0.0 & 0.0 & 0.0 \\
\hline Tot:Al D-METAL & & 0.3 & 0.0 & 0.0 & 0.0 & 0.0 & 0.0 & 0.0 & 0.0 & 0.0 & 0.0 & 0.0 & 0.0 & 0.0 & 0.0 & 0.0 & 0.0 \\
\hline \multirow[t]{2}{*}{ D-soll } & G & 0.0 & 0.1 & 0.1 & 0.1 & 0.1 & 0.1 & 0.1 & 0.1 & 0.1 & 0.1 & 0.1 & 0.1 & 0.0 & 0.0 & 0.0 & 0.0 \\
\hline & NONE & 0.2 & 0.0 & 0.0 & 0.0 & 0.0 & 0.0 & 0.0 & 0.0 & 0.0 & 0.0 & 0.0 & 0.0 & 0.0 & 0.0 & 0.0 & 0.0 \\
\hline Total D-solh & & 0.2 & 0.1 & 0.1 & 0.1 & 0.1 & 0.1 & 0.1 & 0.1 & 0.1 & 0.1 & 0.1 & 0.2 & 0.0 & 0.0 & 0.0 & 0.0 \\
\hline IN ORG LQ/SLG & $G$ & 0.0 & 0.1 & 0.1 & 0.1 & 0.1 & 0.1 & 0.1 & 0.1 & 0.1 & 0.1 & 0.1 & 0.1 & 0.0 & 0.0 & 0.0 & 0.0 \\
\hline Total IN ORG LQ/SLG & & 0.0 & 0.1 & 0.1 & 0.1 & 0.1 & 0.1 & 0.1 & 0.1 & 0.1 & 0.1 & 0.1 & 0.1 & 0.0 & 0.0 & 0.0 & 0.0 \\
\hline IN ORG PART & $\sigma$ & 0.0 & 0.1 & 0.1 & 0.1 & 0.1 & 0.1 & 0.1 & 0.1 & 0.1 & 0.1 & 0.1 & 0.1 & 0.0 & 0.0 & 0.0 & 0.0 \\
\hline Total IM ORG PART & & 0.0 & 0.1 & 0.1 & 0.1 & 0.1 & 0.1 & 0.1 & 0.1 & 0.1 & 0.1 & 0.1 & 0.1 & 0.0 & 0.0 & 0.0 & 0.0 \\
\hline LAB/CONT $2 Q$ & $A B$ & 0.0 & 0.2 & 0.2 & 0.2 & 0.2 & 0.2 & 0.2 & 0.2 & 0.2 & 0.2 & 0.2 & 0.2 & 0.1 & 0.1 & 0.1 & 0.1 \\
\hline Totel LAB/CONT LQ & & 0.0 & 0.2 & 0.2 & 0.2 & 0.2 & 0.2 & 0.2 & 0.2 & 0.2 & 0.2 & 0.2 & 0.2 & 0.1 & 0.1 & 0.1 & 0.1 \\
\hline MEREURY & E & 0.0 & 0.1 & 0.1 & 0.1 & 0.1 & 0.1 & 0.1 & 0.1 & 0.1 & 0.1 & 0.1 & 0.1 & 0.0 & 0.0 & 0.0 & $\overline{0.0}$ \\
\hline TOta1 HERCURY & - & 0.0 & 0.1 & 0.1 & 0.1 & 0.1 & 0.1 & 0.1 & 0.1 & 0.1 & 0.1 & 0.1 & 0.1 & 0.0 & 0.0 & 0.0 & 0.0 \\
\hline ORO ABS LQ/SLG & G & 0.0 & 0.4 & 0.1 & 0.4 & 0.1 & 0.1 & 0.1 & 0.4 & 0.1 & 0.1 & 0.4 & 0.1 & 0.2 & 0.2 & 0.2 & 0.2 \\
\hline Total ORG ABS LQ/SLG & & 0.0 & 0.1 & 0.4 & 0.1 & 0.4 & 0.4 & 0.4 & 0.4 & 0.1 & 0.4 & 0.1 & 0.1 & 0.2 & 0.2 & 0.2 & 0.2 \\
\hline ORG PART & o & 0.0 & 0.2 & 0.2 & 0.2 & 0.2 & 0.2 & 0.2 & 0.2 & 0.2 & 0.2 & 0.2 & 0.2 & 0.1 & 0.1 & 0.1 & 0.1 \\
\hline Total ORS PART & & 0.0 & 0.2 & 0.2 & 0.2 & 0.2 & 0.2 & 0.2 & 0.2 & 0.2 & 0.2 & 0.2 & 0.2 & 0.1 & 0.1 & 0.1 & 0.1 \\
\hline SALT WASTE & G & 0.0 & .0 .1 & 0.1 & 0.1 & 0.1 & 0.1 & 0.1 & 0.1 & 0.1 & 0.1 & 0.1 & 0.1 & 0.0 & 0.0 & 0.0 & 0.0 \\
\hline TOta1 SALT WASTE & & 0.0 & 0.1 & 0.1 & 0.1 & 0.1 & 0.1 & 0.2 & 0.1 & 0.1 & 0.1 & 0.1 & 0.1 & 0.0 & 0.0 & 0.0 & $a, 0$ \\
\hline TOtal WHE $300_{-}$PWR & & 2.8 & 2.0 & 2.8 & 2.0 & 2.0 & 2.0 & 2.0 & 2.0 & 2.0 & 2.8 & 2.0 & 2.8 & 1.4 & 1.4 & 1.4 & 1.1 \\
\hline Faste Generator & :FHC_300 & YRDE & & & & . & & & & & & & & & & & \\
\hline D-conaust & NONE & 0.0 & 36.8 & 36.8 & 36.8 & 36.8 & 36.8 & 36.8 & 36.8 & $36 . \theta$ & 36.8 & 36.8 & 36.8 & 36.0 & 36.8 & 36.8 & 36.8 \\
\hline Total D-COMBUST & & 0.0 & 36.8 & 36.8 & 36.8 & 36.8 & 36.8 & 36.0 & 36.8 & 36.8 & 36.8 & 36.0 & 36.8 & 36.8 & 36.0 & 36.8 & 36.8 \\
\hline IN ORG LQ/SLG & NONE & 0.0 & 331.1 & 331.4 & 331.4 & .331 .4 & 331.4 & 331.4 & 331.4 & 331.4 & 331.4 & 331.4 & .331 .4 & 331.4 & 331.4 & 331.4 & 331.4 \\
\hline Total IN ORG LO/SLG & & 0.0 & 331.4 & 331.4 & 331.4 & 331.4 & 331.4 & 331.4 & 331.4 & 331.1 & 331.4 & 331.4 & 331.4 & 331.4 & 331.4 & 331.4 & 331.4 \\
\hline IN ORG PART & EG & 0.0 & 0.0 & 0.0 & 8.5 & 0.0 & 8.5 & 0.0 & 0.5 & 0.0 & 8.5 & 0.0 & 0.5 & 0.0 & 0.5 & 0.0 & 8.5 \\
\hline Total IN ORG PART & & 0.0 & 0.0 & 0.0 & 0.5 & 0.0 & 0.5 & 0.0 & 8.5 & 0.0 & 0.5 & 0.0 & 8.5 & 0.0 & 0.5 & 0.0 & 0.5 \\
\hline Total WHA_300_TEDE & & 0.0 & 366.3 & 368.3 & 376.8 & 368.3 & 376.8 & 368.3 & 376.8 & 368.3 & 376.8 & 368.3 & 376.8 & 368.3 & 376.0 & 360.3 & 376.8 \\
\hline Waste Generator & :FHC_325 & & & & & & & & & & & & & & & & \\
\hline LAB/CONT LQ & A & 0.0 & 0.0 & 0.0 & 0.0 & 0.0 & 0.0 & 0.0 & 0.0 & 0.0 & 0.0 & 0.0 & 0.0 & 0.0 & 0.0 & 0.0 & 0.0 \\
\hline
\end{tabular}

Cod Codes: A-Ignitables, B-Cörosives, C-Reactives, D-Motals w/O Hg, E-Metals w Hg, E-Organles, G-State Reg., H-PCB < 50ppm, J-PCB > 50ppm 


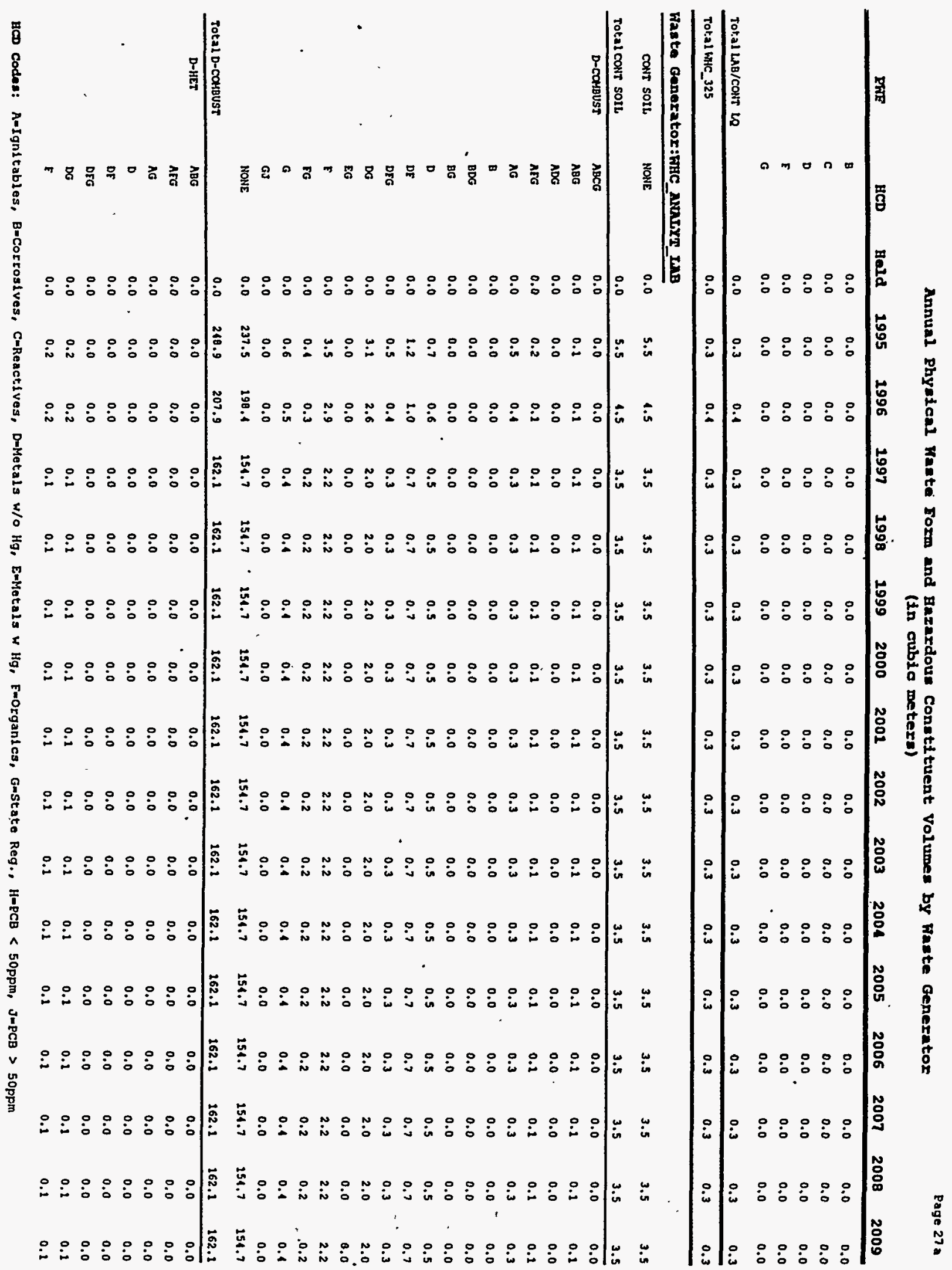


Annual Physical Faste Form and Gazardous Conatituent Volumes by Faste Generator

(in cubic metero)

\begin{tabular}{|c|c|c|c|c|c|c|c|c|c|c|c|c|c|c|c|c|c|}
\hline EWE & HCD & Hold & 1995 & 1996 & 1997 & 1998 & 1999 & 2000 & 2001 & 2002 & 2003 & 2004 & 2005 & 2006 & 2007 & 2008 & 2009 \\
\hline & EG & 0.0 & 0.0 & 0.0 & 0.0 & 0.0 & 0.0 & 0.0 & 0.0 & 0.0 & 0.0 & 0.0 & 0.0 & 0.0 & 0.0 & 0.0 & 0.0 \\
\hline & G & 0.0 & 0.0 & 0.0 & 0.0 & 0.0 & 0.0 & 0.0 & 0.0 & 0.0 & 0.0 & 0.0 & 0.0 & 0.0 & 0.0 & 0.0 & 0.0 \\
\hline Tot,al D-HET & & 0.0 & 0.1 & 0.7 & 0.5 & 0.5 & 0.5 & 0.5 & 0.5 & 0.5 & 0.5 & 0.5 & 0.5 & .0 .5 & 0.5 & 0.5 & 0. \\
\hline \multirow{11}{*}{ D-IN MON-MTL } & ABG & 0.0 & 0.0 & 0.0 & 0.0 & 0.0 & 0.0 & 0.0 & 0.0 & 0.0 & 0.0 & 0.0 & 0.0 & 0.0 & 0.0 & 0.0 & 0.0 \\
\hline & AEG & 0.0 & 0.1 & 0.1 & 0.0 & 0.0 & 0.0 & 0.0 & 0.0 & 0.0 & 0.0 & 0.0 & 0.0 & 0.0 & 0.0 & 0.0 & 0.0 \\
\hline & $A G$ & 0.0 & 0.3 & 0.3 & 0.2 & 0.2 & 0.2 & 0.2 & 0.2 & 0.2 & 0.2 & 0.2 & 0.2 & 0.2 & 0.2 & 0.2 & 0.2 \\
\hline & D & 0.0 & 0.5 & 0.4 & 0.3 & 0.3 & 0.3 & 0.3 & 0.3 & 0.3 & 0.3 & 0.3 & 0.3 & 0.3 & 0.3 & 0.3 & 0.3 \\
\hline & DE & 0.0 & 0.8 & 0.6 & 0.5 & 0.5 & 0.5 & 0.5 & 0.5 & 0.5 & 0.5 & 0.5 & 0.5 & 0.5 & 0.5 & 0.5 & 0.5 \\
\hline & DEG & 0.0 & 0.3 & 0.3 & 0.2 & 0.2 & 0.2 & 0.2 & 0.2 & 0.2 & 0.2 & 0.2 & 0.2 & 0.2 & 0.2 & 0.2 & 0.2 \\
\hline & DG & 0.0 & 2.1 & 1.7 & 1.3 & 1.3 & 1.3 & 1.3 & 1.3 & 1.3 & 1.3 & 1.3 & 1.3 & 1.3 & 1.3 & 1.3 & 1.3 \\
\hline & $F$ & 0.0 & 2.4 & 1.9 & 1.5 & 1.5 & 1.5 & 1.5 & 1.5 & 1.5 & 1.5 & 1.5 & 1.5 & 1.5 & 1.5 & 1.5 & 1.5 \\
\hline & EG & 0.0 & 0.3 & 0.2 & 0.1 & 0.1 & 0.1 & 0.1 & 0.1 & 0.1 & 0.1 & 0.1 & 0.1 & 0.1 & 0.2 & 0.1 & 0.1 \\
\hline & G & 0.0 & 0.3 & 0.3 & 0.2 & 0.2 & 0.2 & 0.2 & 0.2 & 0.2 & 0.2 & 0.2 & 0.2 & 0.2 & 0.2 & 0.2 & 0.2 \\
\hline & NONE & 0.0 & 43.9 & 36.6 & 28.6. & 28.6 & 28.6 & 28.6 & 28.6 & 28.6 & 28.6 & 28.6 & 28.6 & 28.6 & 28.6 & 28.6 & 28.6 \\
\hline Total D-IN NOH-MTL & & 0.0 & 51.1 & 42.9 & 33.4 & 33.4 & 33.1 & 33.4 & 33.4 & 33.4 & 33.4 & 33.4 & 33.4 & 33.4 & 33.4 & 33.4 & 33. \\
\hline \multirow[t]{11}{*}{ D-METAL } & ABCG & 0.0 & 0.0 & 0.0 & 0.0 & 0.0 & 0.0 & 0.0 & 0.0 & 0.0 & 0.0 & 0.0 & 0.0 & 0.0 & 0.0 & 0.0 & 0.0 \\
\hline & $A B G$ & 0.0 & 0.0 & 0.0 & 0.0 & 0.0 & 0.0 & 0.0 & 0.0 & 0.0 & 0.0 & 0.0 & 0.0 & 0.0 & 0.0 & 0.0 & 0.0 \\
\hline & ADG & 0.0 & 0.0 & 0.0 & 0.0 & 0.0 & 0.0 & 0.0 & 0.0 & 0.0 & 0.0 & 0.0 & 0.0 & 0.0 & 0.0 & 0.0 & 0.0 \\
\hline & $A G$ & 0.0 & 0.0 & 0.0 & 0.0 & 0.0 & 0.0 & 0.0 & 0.0 & 0.0 & 0.0 & 0.0 & 0.0 & 0.0 & 0.0 & 0.0 & 0.0 \\
\hline & B & 0.0 & 0.0 & 0.0 & 0.0 & 0.0 & 0.0 & 0.0 & 0.0 & 0.0 & 0.0 & 0.0 & 0.0 & 0.0 & 0.0 & 0.0 & 0.0 \\
\hline & BDG & 0.0 & 0.0 & 0.0 & 0.0 & 0.0 & 0.0 & 0.0 & 0.0 & 0.0 & 0.0 & 0.0 & 0.0 & 0.0 & 0.0 & 0.0 & 0.0 \\
\hline & BG & 0.0 & 0.0 & 0.0 & 0.0 & 0.0 & 0.0 & 0.0 & 0.0 & 0.0 & 0.0 & 0.0 & 0.0 & 0.0 & 0.0 & 0.0 & 0.0 \\
\hline & D & 0.0 & 0.0 & 0.0 & 0.0 & 0.0 & 0.0 & 0.0 & 0.0 & 0.0 & 0.0 & 0.0 & 0.0 & 0.0 & 0.0 & 0.0 & 0.0 \\
\hline & DG & 0.0 & 0.0 & 0.0 & 0.0 & 0.0 & 0.0 & 0.0 & 0.0 & 0.0 & 0.0 & 0.0 & 0.0 & 0.0 & 0.0 & 0.0 & 0.0 \\
\hline & EG & 0.0 & 0.0 & 0.0 & 0.0 & 0.0 & 0.0 & 0.0 & 0.0 & 0.0 & 0.0 & 0.0 & 0.0 & 0.0 & 0.0 & $0.0^{\circ}$ & 0.0 \\
\hline & $G$ & 0.0 & 0.0 & 0.0 & 0.0 & 0.0 & 0.0 & 0.0 & 0.0 & 0.0 & 0.0 & 0.0 & 0.0 & 0.0 & 0.0 & 0.0 & 0.0 \\
\hline \multirow[t]{2}{*}{$\ldots$} & GJ & 0.0 & 0.0 & 0.0 & 0.0 & 0.0 & 0.0 & 0.0 & 0.0 & 0.0 & 0.0 & 0.0 & 0.0 & 0.0 & 0.0 & 0.0 & 0.0 \\
\hline & NONE & 0.0 & 192.5 & 160.9 & 125.4 & 125.1 & 125.4 & 125.4 & 125.4 & 225.4 & 125.4 & 225.4 & 225.4 & 125.4 & 125.4 & 125.4 & 125.4 \\
\hline TOta1 D-METAL & & 0.0 & 192.6 & 260.9 & 125.1 & 125.4 & 125.4 & 125.4 & 125.4 & 125.4 & 125.4 & 125.1 & 225.1 & 125.4 & 125.4 & 125.4 & 125.4 \\
\hline \multirow[t]{9}{*}{ IN ORG LR/SLG } & ABG & 0.0 & 0.0 & 0.0 & 0.0 & 0.0 & 0.0 & 0.0 & 0.0 & 0.0 & 0.0 & 0.0 & 0.0 & 0.0 & 0.0 & 0.0 & 0.0 \\
\hline & AFO & 0.0 & 0.0 & 0.0 & 0.0 & 0.0 & 0.0 & 0.0 & 0.0 & 0.0 & 0.0 & 0.0 & 0.0 & 0.0 & 0.0 & 0.0 & 0.0 \\
\hline & AG & 0.0 & 0.0 & 0.0 & 0.0 & 0.0 & 0.0 & 0.0 & 0.0 & 0.0 & 0.0 & 0.0 & 0.0 & 0.0 & 0.0 & 0.0 & 0.0 \\
\hline & $D$ & 0.0 & 0.0 & 0.0 & 0.0 & 0.0 & 0.0 & 0.0 & 0.0 & 0.0 & 0.0 & 0.0 & 0.0 & 0.0 & 0.0 & 0.0 & 0.0 \\
\hline & DE & 0.0 & 0.0 & 0.0 & 0.0 & 0.0 & 0.0 & 0.0 & 0.0 & 0.0 & 0.0 & 0.0 & 0.0 & 0.0 & 0.0 & 0.0 & 0.0 \\
\hline & DFG & 0.0 & 0.0 & 0.0 & 0.0 & 0.0 & 0.0 & 0.0 & 0.0 & 0.0 & 0.0 & 0.0 & 0.0 & 0.0 & 0.0 & 0.0 & 0.0 \\
\hline & DG & 0.0 & 0.0 & 0.0 & 0.0 & 0.0 & 0.0 & 0.0 & 0.0 & 0.0 & 0.0 & 0.0 & 0.0 & 0.0 & 0.0 & 0.0 & 0.0 \\
\hline & $\varepsilon$ & 0.0 & 0.0 & 0.0 & 0.0 & 0.0 & 0.0 & 0.0 & 0.0 & 0.0 & 0.0 & 0.0 & 0.0 & 0.0 & 0.0 & 0.0 & 0.0 \\
\hline & FG & 0.0 & 0.0 & 0.0 & 0.0 & 0.0 & 0.0 & 0.0 & 0.0 & 0.0 & 0.0 & 0.0 & $0.0^{\circ}$ & 0.0 & 0.0 & 0.0 & 0.0 \\
\hline
\end{tabular}

BCD Codos: A=Ignitables, B-Corrosives, C-Reactives, D-Metals w/O Hg, E-Metals W Hg, F=Organics, G=State Reg., H-PCB < 50ppm, J-PCB > 50ppm 
Annual Physical waste rorm and Bazardous Constituent Volumea by Waste Generator

Page 29a (in cubic metere)

\begin{tabular}{|c|c|c|c|c|c|c|c|c|c|c|c|c|c|c|c|c|c|}
\hline PWE & HCD & Held & 1995 & 1996 & 1997 & 1998 & 1999 & 2000 & 2001 & 2002 & 2003 & 2004 & 2005 & 200.6 & 2007 & 2008 & 2009 \\
\hline & G & 0.0 & 0.0 & 0.0 & 0.0 & 0.0 & 0.0 & 0.0 & 0.0 & 0.0 & 0.0 & 0.0 & 0.0 & 0.0 & 0.0 & 0.0 & 0.0 \\
\hline Total III ORG LQ/SLG & 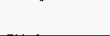 & 0.0 & 0.2 & 0.1 & 0.1 & 0.2 & 0.1 & 0.1 & 0.1 & 0.1 & 0.1 & 0.2 & 0.1 & 0.1 & 0.1 & 0.1 & 0.1 \\
\hline IN ORG PART & ABCG & 0.0 & 0.0 & 0.0 & 0.0 & 0.0 & 0.0 & 0.0 & 0.0 & 0.0 & 0.0 & 0.0 & 0.0 & 0.0 & 0.0 & 0.0 & 0.0 \\
\hline & ABG & 0.0 & 0.1 & 0.1 & 0.1 & 0.1 & 0.1 & 0.1 & 0.1 & 0.1 & 0.1 & $0.1^{\circ}$ & 0.1 & 0.1 & 0.1 & 0.1 & 0.1 \\
\hline & ADE & 0.0 & 0.0 & 0.0 & 0.0 & 0.0 & 0.0 & 0.0 & 0.0 & 0.0 & 0.0 & 0.0 & 0.0 & 0.0 & 0.0 & 0.0 & 0.0 \\
\hline & AFG & 0.0 & 0.1 & 0.1 & 0.1 & 0.1 & 0.1 & .0 .1 & 0.1 & 0.2 & 0.1 & 0.1 & 0.1 & 0.1 & 0.1 & 0.1 & 0.1 \\
\hline & AG & 0.0 & 0.1 & 0.1 & 0.3 & 0.3 & 0.3 & 0.3 & 0.3 & 0.3 & 0.3 & 0.3 & 0.3 & 0.3 & 0.3 & 0.3 & 0.3 \\
\hline & a & 0.0 & 0.0 & 0.0 & 0.0 & 0.0 & 0.0 & 0.0 & 0.0 & 0.0 & 0.0 & 0.0 & 0.0 & 0.0 & 0.0 & 0.0 & 0.0 \\
\hline & $B D G$ & 0.0 & 0.0 & 0.0 & 0.0 & 0.0 & 0.0 & 0.0 & 0.0 & 0.0 & 0.0 & 0.0 & 0.0 & 0.0 & 0.0 & 0.0 & 0.0 \\
\hline & BG & 0.0 & 0.0 & 0.0 & 0.0 & 0.0 & 0.0 & 0.0 & 0.0 & 0.0 & 0.0 & 0.0 & 0.0 & 0.0 & 0.0 & 0.0 & 0.0 \\
\hline & D & 0.0 & 0.6 & 0.5 & 0.1 & 0.1 & 0.1 & 0.4 & 0.4 & 0.4 & 0.4 & 0.4 & 0.4 & 0.1 & 0.1 & 0.1 & 0.1 \\
\hline & DE & 0.0 & 0.9 & 0.8 & 0.6 & 0.6 & 0.6 & 0.6 & 0.6 & 0.6 & 0.6 & 0.6 & 0.6 & 0.6 & 0.6 & 0.6 & 0.6 \\
\hline & DEG & 0.0 & 0.1 & 0.3 & 0.2 & 0.2 & 0.2 & 0.2 & 0.2 & 0.2 & 0.2 & 0.2 & 0.2 & 0.2 & 0.2 & 0.2 & 0.2 \\
\hline & $D G$ & 0.0 & 2.5 & 2.1 & 3.6 & 2.6 & 1.6 & 1.6 & 1.6 & 1.6 & 1.6 & 1.6 & 2.6 & 1.6 & 1.6 & 1.6 & 1.6 \\
\hline & EG & 0.0 & 0.0 & 0.0 & 0.0 & 0.0 & 0.0 & 0.0 & 0.0 & 0.0 & 0.0 & 0.0 & 0.0 & 0.0 & 0.0 & 0.0 & 0.0 \\
\hline & $F$ & 0.0 & 2.8 & 2.3 & 1.9 & 1.0 & 1.8 & 1.0 & 1.8 & 1.8 & 1.8 & 1.8 & 1.8 & 1.8 & 1.8 & 1.8 & 1.8 \\
\hline & EG & 0.0 & 0.3 & 0.2 & 0.2 & 0.2 & 0.2 & 0.2 & 0.2 & 0.2 & 0.2 & 0.2 & 0.2 & 0.2 & 0.2 & 0.2 & 0.2 \\
\hline & G & 0.0 & 0.6 & 0.5 & 0.1 & 0.1 & 0.1 & 0.1 & 0.4 & 0.4 & 0.1 & 0.1 & 0.1 & 0.1 & 0.4 & 0.4 & 0.1 \\
\hline & GJ & 0.0 & 0.0 & 0.0 & 0.0 & 0.0 & 0.0 & 0.0 & 0.0 & 0.0 & 0.0 & 0,0 & 0.0 & 0.0 & 0.0 & 0.0 & 0.0 \\
\hline & NONE & 0.0 & 28.8 & 24.1 & 18.7 & 18.7 & 18.7 & 18.7 & 18.7 & 28.7 & 28.7 & 18.7 & 18.7 & 28.7 & 18.7 & 18.7 & 18.7 \\
\hline Totai IN ORG PART & & 0.0 & 38.4 & 32.1 & 25.0 & 25.0 & 25.0 & 25.0 & 25.0 & 25.0 & 25.0 & 25.0 & 25.0 & 25.0 & 25.0 & 25.0 & 25.0 \\
\hline LAB/CONT LQ & ABCDFG & 0.0 & 0.5 & 0.1 & 0.3 & 0.3 & 0.3 & 0.3 & 0.3 & 0.3 & 0.3 & 0.3 & 0.3 & 0.3 & 0.3 & 0.3 & 0.3 \\
\hline & ABCEG & 0.0 & 0.5 & 0.1 & 0.3 & 0.3 & 0.3 & 0.3 & 0.3 & 0.3 & 0.3 & 0.3 & 0.3 & 0.3 & 0.3 & 0.3 & 0.3 \\
\hline & ABEG & 0.0 & 0.0 & 0.0 & 0.0 & 0.0 & 0.0 & 0.0 & 0.0 & 0.0 & 0.0 & 0.0 & 0.0 & 0.0 & 0.0 & 0.0 & $\cdot 0.0$ \\
\hline & ABG & 0.0 & 0.0 & 0.0 & 0.0 & 0.0 & 0.0 & 0.0 & 0.0 & 0.0 & 0.0 & 0.0 & 0.0 & 0.0 & 0.0 & 0.0 & 0.0 \\
\hline & ADEG & 0.0 & 0.0 & 0.0 & 0.0 & 0.0 & 0.0 & 0.0 & 0.0 & 0.0 & 0.0 & 0.0 & 0.0 & 0.0 & 0.0 & 0.0 & 0.0 \\
\hline & $A D G$ & 0.0 & 0.0 & 0.0 & 0.0 & 0.0 & 0.0 & 0.0 & 0.0 & 0.0 & 0.0 & 0.0 & 0.0 & 0.0 & 0.0 & 0.0 & 0.0 \\
\hline & AEG & 0.0 & 1.7 & 1.1 & 1.1 & 1.1 & 1.1 & 1.1 & 1.1 & 1.1 & 1.1 & 1.1 & 1.1 & 1.1 & 1.1 & 1.1 & 1.1 \\
\hline & AG & 0.0 & 1.7 & .1 .1 & 1.1 & 1.1 & 1.1 & 1.1 & 1.1 & 1.1 & 1.1 & 1.1 & 1.1 & 1.1 & 1.1 & 1.1 & 1.1 \\
\hline & BD & 0.0 & 0.0 & 0.0 & 0.0 & 0.0 & 0.0 & 0.0 & 0.0 & 0.0 & 0.0 & 0.0 & 0.0 & 0.0 & 0.0 & 0.0 & 0.0 \\
\hline & BDG & 0.0 & 0.9 & $0 . \theta$ & 0.6 & 0.6 & 0.6 & 0.6 & 0.6 & 0.6 & 0.6 & 0.6 & 0.6 & 0.6 & 0.6 & 0.6 & 0.6 \\
\hline & BEG & 0.0 & 0.2 & 0.1 & 0.1 & 0.1 & 0.1 & 0.1 & 0.1 & 0.1 & 0.1 & 0.1 & 0.1 & 0.1 & 0.1 & 0.2 & 0.1 \\
\hline & 80 & 0.0 & 1.0 & 0.9 & 0.7 & 0.7 & 0.7 & 0.7 & 0.7 & 0.7 & 0.7 & 0.7 & 0.7 & 0.7 & 0.7 & 0.7 & 0.7 \\
\hline & BGJ & 0.0 & 0.0 & 0.0 & 0.0 & 0.0 & 0.0 & 0.0 & 0.0 & 0.0 & 0.0 & 0.0 & 0.0 & 0.0 & 0.0 & 0.0 & 0.0 \\
\hline & CG & 0.0 & 0.5 & 0.4 & 0.3 & 0.3 & 0.3 & 0.3 & 0.3 & 0.3 & 0.3 & 0.3 & 0.3 & 0.3 & 0.3 & 0.3 & 0.3 \\
\hline & DF & 0.0 & 1.0 & 0.9 & 0.7 & 0.7 & 0.7 & 0.7 & 0.7 & 0.7 & 0.7 & 0.7 & 0.7 & 0.7 & 0.7 & 0.7 & 0.7 \\
\hline & DFG & 0.0 & 0.5 & 0.1 & 0.3 & 0.3 & 0.3 & 0.3 & 0.3 & 0.3 & 0.3 & 0.3 & 0.3 & 0.3 & 0.3 & 0.3 & 0.3 \\
\hline & $D G$ & 0.0 & 0.0 & 0.0 & 0.0 & 0.0 & 0.0 & 0.0 & 0.0 & 0.0 & 0.0 & 0.0 & 0.0 & 0.0 & 0.0 & 0.0 & 0.0 \\
\hline & EG & 0.0 & 0.0 & 0.0 & 0.0 & 0.0 & 0.0 & 0.0 & 0.0 & 0.0 & 0.0 & 0.0 & 0.0 & $0: 0$ & 0.0 & 0.0 & 0.0 \\
\hline
\end{tabular}

HCD Codes: A-Ignitables, B-Corrosives, C-Reactives, D-Metals w/O Hg, E-Metals w Hg, F-Organlcs, G-State Reg., H-PCB < 50ppm, J-PCB > 50ppm 


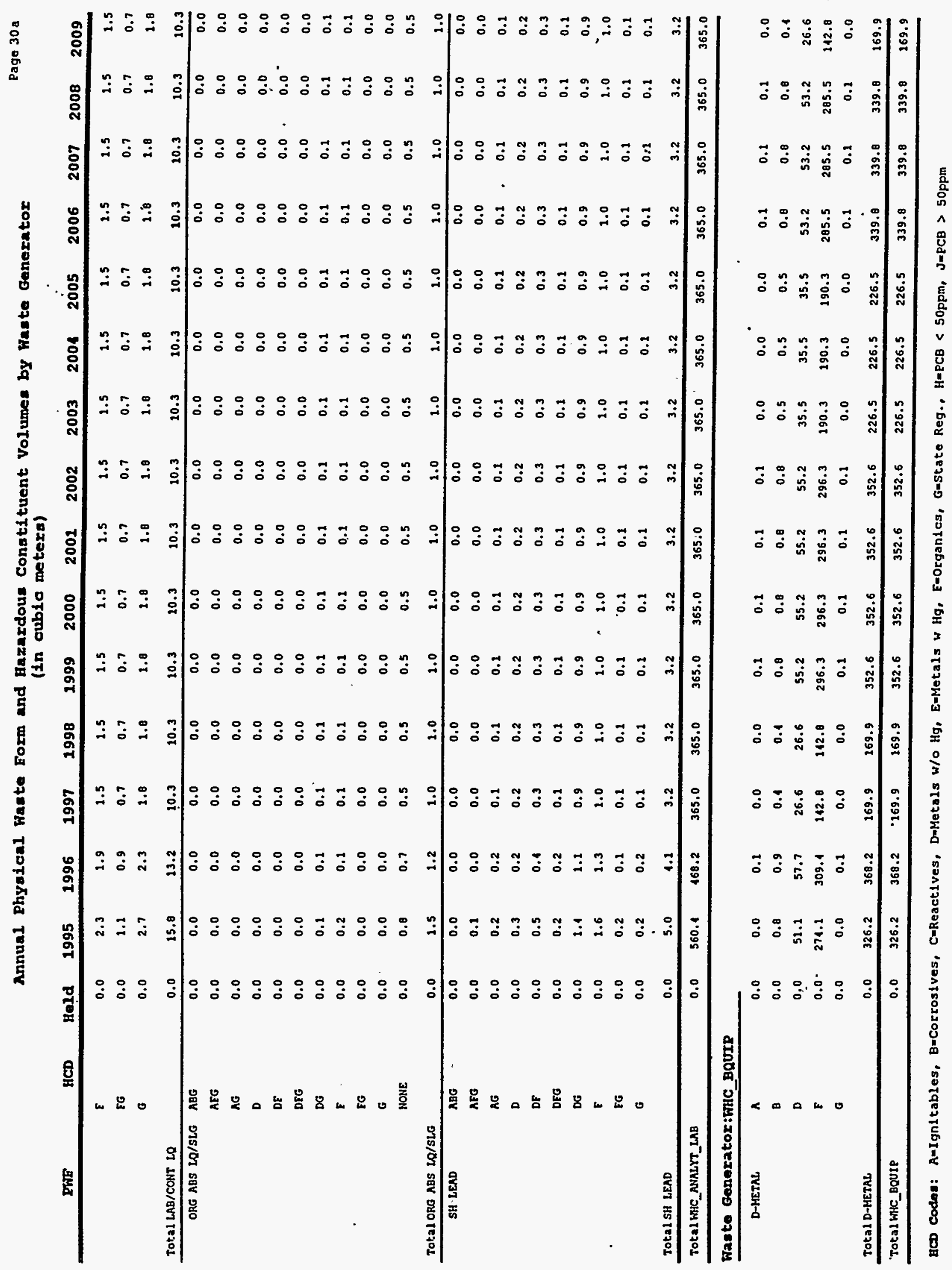


PW (in cubio meters)

\begin{tabular}{|c|c|c|c|c|c|c|c|c|c|c|c|c|c|c|c|c|c|}
\hline PWF & HCD & Held & 1995 & 1996 & 1997 & 1998 & 1999 & 2000 & 2001 & 2002 & 2003 & 2004 & $200 \dot{5}$ & 2006 & 2007 & 2008 & 2009 \\
\hline \multicolumn{18}{|c|}{ Waste Generator:FHC_B_PLANT } \\
\hline CONT SOIL & MONE & 0.1 & 12.5 & 10.6 & 25.2 & 14.3 & 23.5 & 12.6 & 11.8 & 10.9 & 20.1 & 9.3 & 8.4 & 0.4 & 8.4 & 8.4 & 8.1 \\
\hline Total COIT SOIL & & 0.1 & 12.5 & 10.6 & 15.2 & 14.3 & 13.5 & 12.6 & 11.8 & 10.9 & 10.1 & 9.3 & 8.1 & 0.4 & 8.4 & 0.1 & 0.4 \\
\hline D-COHBUST & MONE & 3.1 & 156.0 & 254.4 & 114.6 & 137.5 & 130.3 & 111.6 & 102.9 & 95.7 & 88.6 & 91.4 & 74.2 & 71.2 & 74.2 & 71.2 & 74.2 \\
\hline Total D-Corisust & & 3.1 & 156.0 & 154.4 & 144.6 & 137.5 & 130.3 & 211.6 & 102.9 & 95.7 & 88.6 & 81.1 & 74.2 & 74.2 & 74.2 & 74.2 & 74.2 \\
\hline \multirow[t]{5}{*}{ D-HET } & A & 0.1 & 1.2 & 1.1 & 1.0 & 1.0 & 0.9 & 0.9 & 0.8 & 0.7 & 0.7 & 0.6 & 0.6 & 0.6 & 0.6 & 0.6 & 0.6 \\
\hline & $D$ & 0.0 & 0.1 & 0.1 & 0.1 & 0.3 & 0.3 & 0.3 & 0.3 & 0.2 & 0.2 & 0.2 & 0.2 & 0.2 & 0.2 & 0.2 & 0.2 \\
\hline & G & 0.0 & 1.3 & 2.0 & 1.9 & 1.9 & 1.8 & 1.7 & 0.9 & 0.8 & 0.8 & 0.7 & 0.6 & 0.6 & 0.6 & 0.6 & 0.6 \\
\hline & H & 0.0 & 0.1 & 0.1 & 0.1 & 0.3 & 0.3 & 0.3 & 0.3 & 0.2 & 0.2 & 0.2 & 0.2 & 0.2 & 0.2 & .0 .2 & 0.2 \\
\hline & MONE & 0.1 & 32.3 & 31.9 & 30.5 & 29.1 & 28.3 & 17.3 & 15.9 & 14.8 & 13.7 & 12.6 & 11.5 & 11.5 & 11.5 & 11.5 & 11.5 \\
\hline Total D-HET & & 0.7 & 35.8 & 36.0 & 34.1 & 33.1 & 31.9 & 20.7 & 18.3 & 27.0 & 15.8 & 14.5 & 13.2 & 13.2 & 13.2 & 23.2 & 13.2 \\
\hline D-IN NON-MTL & NONE & 0.1 & 50.1 & 19.3 & 48.0 & 17.0 & 16.0 & 16.1 & 14.7 & 13.7 & 12.7 & 13.7 & $20: 7$ & 10.7 & 10.7 & 10.7 & $\overline{10.7}$ \\
\hline Total D-IN MON-HTL & & 0.1 & 50.1 & 19.3 & 10.0 & $17.0^{\circ}$ & 16.0 & 26.1 & 14.7 & 13.7 & 12.7 & 11.7 & 10.7 & 10.7 & 10.7 & 10.7 & 10.7 \\
\hline \multirow[t]{2}{*}{ D-HETAL } & E & 0.0 & 0.0 & 0.1 & 0.1 & 0.1 & 0.1 & 0.2 & 0.0 & 0.0 & .0 .0 & 0.0 & 0.0 & 0.0 & 0.0 & 0.0 & 0.0 \\
\hline & NONE & 0.4 & 31.0 & 49.8 & 48.1 & 97.4 & 16.4 & 16.4 & 15.1 & 14.0 & 13.0 & 11.9 & 10.9 & 10.9 & 10.9 & 10.9 & 10.9 \\
\hline Total D-KETAL & & 0.1 & 51.0 & 49.9 & 10.6 & 47.5 & 46.5 & 16.5 & 15.1 & 14.0 & 13.0 & 11.9 & 10.9 & 10.9 & 10.9 & 10.9 & 10.9 \\
\hline EXPLOSIVES & $\lambda$ & 0.0 & 0.5 & 0.1 & 0.4 & 0.1 & 0.4 & 0.3 & 0.3 & 0.3 & 0.3 & 0.2 & 0.2 & 0.2 & 0.2 & 0.2 & 0.2 \\
\hline Total EXPLOSIVES & . & 0.0 & 0.5 & 0.4 & 0.4 & 0.1 & 0.1 & 0.3 & 0.3 & 0.3 & 0.3 & 0.2 & 0.2 & 0.2 & 0.2 & 0.2 & 0.2 \\
\hline \multirow[t]{4}{*}{ IN ORG LQ/SLG } & 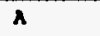 & 0.0 & 0.1 & 0.1 & 0.1 & 0.1 & 0.1 & 0.1 & 0.1 & 0.1 & 0.0 & 0.0 & 0.0 & 0.0 & 0.0 & 0.0 & 0.0 \\
\hline & D & 1.4 & 1.2 & 1.1 & 1.1 & 1.0 & 0.9 & 0.9 & 0.0 & 0.8 & 0.7 & 0.6 & 0.6 & 0.6 & 0.6 & .0 .6 & 0.6 \\
\hline & G & 0.4 & 0.3 & 0.3 & 0.3 & 0.3 & 0.2 & 0.2 & 0.2 & 0.2 & 0.2 & 0.1 & 0.2 & 0.1 & 0.1 & 0.1 & 0.1 \\
\hline & NONE & 0.2 & 9.0 & 8.6 & B.0 & 7.5 & 7.1 & 6.7 & 6.2 & 5.8 & 5.3 & 4.9 & 4.4 & 4.4 & 4.4 & 1.4 & $i .4$ \\
\hline Total IN ORG LO/SLG & & 2.2 & 10.7 & 10.2 & 9.6 & 9.0 & 0.5 & 8.0 & 7.4 & 6.9 & 6.1 & 5.8 & 5.3 & 5.3 & 5.3 & 5.3 & 5.3 \\
\hline IN ORO PART & MONE & 0.2 & 11.3 & 10.6 & 10.0 & 9.5 & 8.9 & 0.1 & $7.0^{\circ}$ & 7.3 & 6.7 & 6.1 & 5.6 & 5.6 & 5.6 & 5.6 & 5.6 \\
\hline Total IN ORG PART & & 0.2 & 11.3 & 20.8 & 20.0 & 9.5 & 8.9 & 8.4 & 7.8 & 7.3 & 6.7 & 6.1 & 5.6 & 5.6 & 5.6 & 5.6 & 5.6 \\
\hline LEAD & D & 0.0 & 0.1 & 0.1 & 0.3 & 0.3 & 0.3 & 0.3 & 0.3 & 0.2 & 0.2 & 0.2 & 0.2 & 0.2 & 0.2 & 0.2 & 0.2 \\
\hline Total LEAD & & 0.0 & 0.1 & 0.1 & 0.3 & 0.3 & 0.3 & 0.3 & 0.3 & 0.2 & 0.2 & 0.2 & 0.2 & 0.2 & 0.2 & 0.2 & 0.2 \\
\hline \multirow[t]{2}{*}{ ORG ABS LQ/SLG } & E & 0.0 & 0.1 & 0.1 & 0.0 & 0.0 & 0.0 & 0.0 & 0.0 & 0.0 & 0.0 & 0.0 & 0.0 & 0.0 & 0.0 & 0.0 & 0.0 \\
\hline & $\varepsilon$ & 0.1 & 3.8 & 3.6 & 3.4 & 3.2 & 3.0 & 2.8 & 2.6 & 2.1 & 2.2 & 2.1 & 1.0 & 1.8 & 1.8 & 1.8 & 1.0 \\
\hline Total ORG ABS LO/SLG & & 0.1 & 3.9 & 3.7 & 3.5 & 3.3 & 3.1 & 2.9 & 2.7 & 2.5 & 2.3 & 2.1 & 1.9 & 1.9 & 1.9 & 1.9 & 1.9 \\
\hline ORG PART & $\varepsilon$ & 0.0 & 0.8 & 0.8 & 0.7 & 0.7 & 0.6 & 0.6 & 0.6 & 0.5 & 0.5 & 0.1 & 0.1 & 0.4 & 0.1 & 0.1 & 0.1 \\
\hline Total ORG PART & & 0.0 & 0.8 & 0.8 & 0.7 & 0.7 & 0.6 & 0.6 & 0.6 & 0.5 & 0.5 & 0.4 & 0.1 & 0.4 & 0.4 & 0.1 & 0.4 \\
\hline SALT WASTE & 8 & 0.0 & 0.3 & 0.3 & 0.3 & 0.3 & 0.2 & 0.2 & 0.2 & 0.2 & 0.2 & 0.2 & 0.1 & 0.1 & 0.1 & 0.1 & 0.1 \\
\hline TOtal SALT WASTE & & 0.0 & 0.3 & 0.3 & 0.3 & 0.3 & 0.2 & 0.2 & 0.2 & 0.2 & 0.2 & 0.2 & 0.1 & 0.1 & 0.1 & 0.1 & 0.1 \\
\hline
\end{tabular}

GCD Codes: A-Ignitables, B-Corrosives, C-Reactives, D-Metals w/O Hg, E-Metals w Hg, Fmorganles, G=Stato Reg., HmPCB < 5Oppm, J-PCB > 50ppm 
(In cubio meters)

\begin{tabular}{|c|c|c|c|c|c|c|c|c|c|c|c|c|c|c|c|c|c|}
\hline PWE. & HCD & Hold & 1995 & 1996 & 1997 & 1998 & 1999 & 2000 & 2001 & 2002 & 2003 & 2004 & $2005^{\circ}$ & 2006 & 2007 & 2008 & 2009 \\
\hline SH COHCPETE & HONE & 0.0 & 19.8 & 28.9 & 18.9 & 20.9 & 10.9 & 0.0 & 0.0 & 0.0 & 0.0 & 0.0 & 0.0 & 0.0 & 0.0 & 0.0 & 0.0 \\
\hline IOL.A1 SH CONCRETE & & 0.0 & 19.8 & 18.9 & 10.9 & 10.9 & 10.9 & 0.0 & 0.0 & 0.0 & 0.0 & 0.0 & 0.0 & 0.0 & 0.0 & .0 .0 & 0.0 \\
\hline \multirow[t]{2}{*}{ SH STEEL } & D & 0.0 & 0.0 & 0.2 & 0.2 & 0.2 & 0.2 & 0.2 & 0.0 & 0.0 & 0.0 & 0.0 & 0.0 & 0.0 & 0.0 & 0.0 & $\overline{0.0}$ \\
\hline & HONE & 0.0 & 0.0 & 2.1 & 2.1 & 2.1 & 2.1 & 1.2 & 0.7 & 0.7 & 0.7 & 0.7 & 0.7 & 0.7 & 0.7 & 0.7 & 0.7 \\
\hline TOtal SH STEEL & & 0.0 & 0.0 & 2.3 & 2.3 & 2.3 & 2.3 & 1.5 & 0.7 & 0.7 & 0.7 & 0.7 & 0.7 & 0.7 & 0.7 & 0.7 & 0.7 \\
\hline TOt:a 1 WHC_B_PLANT & & 0.1 & 383.9 & 370.6 & 337.1 & 324.7 & 312.0 & 200.2 & 183.3 & 170.6 & 157.9 & 145.2 & 132.3 & 232.3 & 132.3 & 132.3 & 132.3 \\
\hline \multicolumn{18}{|c|}{ Was to Ganerator:FHC_CHEM_I_IAB } \\
\hline \multirow[t]{2}{*}{ IN ORG PART } & A & 0.0 & 0.0 & 0.8 & 0.8 & 0.8 & 0.8 & 0.8 & 0.8 & 0.0 & 0.8 & 0.8 & 0.8 & 0.8 & 0.0 & 0.8 & 0.0 \\
\hline & B & 0.0 & 0.2 & 0.2 & 0.2 & 0.2 & 0.2 & 0.2 & 0.2 & 0.2 & 0.2 & 0.2 & 0.2 & 0.2 & 0.2 & 0.2 & 0.2 \\
\hline Total IN ORG PART & & 0.0 & 1.0 & 1.0 & 1.0 & 1.0 & 1.0 & 1.0 & 1.0 & 1.0 & 1.0 & 1.0 & 1.0 & 1.0 & 1.0 & 1.0 & 1.0 \\
\hline LAB/CONT LQ & $\mathbf{A}$ & 0.0 & 0.0 & 0.0 & 0.0 & 0.0 & 0.0 & 0.0 & 0.0 & 0.0 & 0.0 & 0.0 & 0.0 & 0.0 & 0.0 & 0.0 & 0.0 \\
\hline TOta1 LAB/CONT LQ & & 0.0 & 0.0 & 0.0 & 0.0 & 0.0 & 0.0 & 0.0 & 0.0 & 0.0 & 0.0 & 0.0 & 0.0 & 0.0 & 0.0 & 0.0 & 0.0 \\
\hline ORG $\lambda B S$ LR/SLG & $\lambda$ & 0.0 & 0.0 & 0.0 & 0.0 & 0.0 & 0.0 & 0.0 & 0.0 & 0.0 & 0.0 & 0.0 & 0.0 & 0.0 & 0.0 & 0.0 & $\overline{0.0}$ \\
\hline Total ORG ABS LQ/SLG & & 0.0 & 0.0 & 0.0 & 0.0 & 0.0 & 0.0 & 0.0 & 0.0 & 0.0 & 0.0 & 0.0 & 0.0 & 0.0 & 0.0 & 0.0 & 0.0 \\
\hline ORG PART & 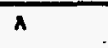 & 0.0 & 0.0 & 0.0 & 0.0 & 0.0 & 0.0 & 0.0 & 0.0 & 0.0 & 0.0 & 0.0 & 0.0 & 0.0 & 0.0 & 0.0 & $\overrightarrow{0.0}$ \\
\hline Total ORG PART & & 0.0 & 0.0 & 0.0 & 0.0 & 0.0 & 0.0 & 0.0 & 0.0 & 0.0 & 0.0 & 0.0 & 0.0 & 0.0 & 0.0 & 0.0 & 0.0 \\
\hline \multirow[t]{2}{*}{ UNRasown } & $\mathbf{A}$ & 0.0 & 0.8 & 0.8 & 0.8 & 0.8 & 0.8 & 0.8 & 0.8 & 0.8 & 0.8 & 0.8 & 0.8 & 0.8 & 0.0 & 0.8 & 0.8 \\
\hline & $\mathbf{8}$ & 0.0 & 0.2 & 0.2 & 0.2 & 0.2 & 0.2 & 0.2 & 0.2 & 0.2 & 0.2 & 0.2 & 0.2 & 0.2 & 0.2 & 0.2 & 0.2 \\
\hline Total UNANOWN & & 0.0 & 1.0 & 1.0 & 1.0 & 1.0 & 1.0 & 1.0 & 1.0 & 1.0 & 1.0 & 1.0 & 1.0 & 1.0 & $1.0^{\circ}$ & 1.0 & 1.0 \\
\hline TOta 1 WHC_CHEM_E_LAB & & 0.0 & 2.2 & 2.2 & 2.2 & 2.2 & 2.2 & 2.2 & 2.2 & 2.2 & 2.2 & 2.2 & 2.2 & 2.2 & 2.2 & 2.2 & 2.2 \\
\hline
\end{tabular}

Waste Generator:WHC_DST_PSRET

\begin{tabular}{|c|c|c|c|c|c|c|c|c|c|c|c|c|c|c|c|c|c|}
\hline D-METAL & NONE & 0.0 & 0.0 & 0.0 & 0.0 & 0.0 & 0.0 & 0.0 & 0.0 & 0.0 & 0.0 & 0.0 & 0.6 & 0.0 & 0.0 & 0.0 & 0.0 \\
\hline Total D-METAL & & 0.0 & 0.0 & 0.0 & 0.0 & 0.0 & 0.0 & 0.0 & 0.0 & 0.0 & 0.0 & 0.0 & 0.6 & 0.0 & 0.0 & 0.0 & 0.0 \\
\hline Total WHC_DST_PSRET & & 0.0 & 0.0 & 0.0 & 0.0 & 0.0 & 0.0 & 0.0 & 0.0 & 0.0 & 0.0 & 0.0 & 0.6 & 0.0 & 0.0 & 0.0 & 0.0 \\
\hline
\end{tabular}

Faste Generator:FHC_DBT_RET

\begin{tabular}{|c|c|c|c|c|c|c|c|c|c|c|c|c|c|c|c|c|c|}
\hline CONT SOIL & NONE & 0.0 & 0.0 & 0.0 & 0.0 & 0.0 & 0.0 & 0.0 & 0.0 & 0.0 & $\cdot 0.0$ & 0.0 & 0.0 & 0.0 & 0.0 & 66.0 & 67.5 \\
\hline TOtal CONT SOIL & & 0.0 & 0.0 & 0.0 & 0.0 & 0.0 & 0.0 & 0.0 & 0.0 & 0.0 & 0.0 & 0.0 & 0.0 & 0.0 & 0.0 & 66.0 & 67.5 \\
\hline \multirow[t]{2}{*}{ D-METAL } & $\Sigma$ & 0.0 & 0.0 & 0.0 & 0.0 & 0.0 & 0.0 & 0.0 & 0.0 & 0.0 & 0.0 & 0.0 & 0.0 & 0.0 & 0.0 & 4.1 & 9.1 \\
\hline & NONE & 0.0 & 0.0 & 0.0 & 0.0 & 0.0 & 0.0 & 0.0 & 0.0 & 0.0 & 0.0 & 0.0 & 0.0 & 0.0 & 0.0 & 7.3 & 7.5 \\
\hline Total D-METAL & & 0.0 & 0.0 & 0.0 & 0.0 & 0.0 & 0.0 & 0.0 & 0.0 & 0.0 & 0.0 & 0.0 & 0.0 & 0.0 & 0.0 & 11.0 & 11.9 \\
\hline
\end{tabular}

ECD Codes: A-Ignitables, BuCorrosives, C=Reactives, D-Metals w/O Hg, E-Metals w Hg, F-Organics, G=State Reg., H-PCB < 50ppm, J-PCB > 50ppm 


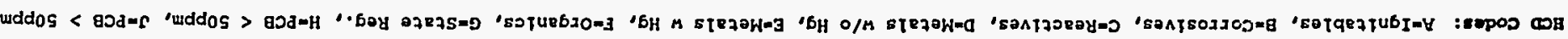

\begin{tabular}{|c|c|c|c|c|c|c|c|c|c|c|c|c|c|c|c|c|c|}
\hline 50 & s.0 & s.0 & $5 \%$ & s.0 & s.0 & s.0 & s.o & 5.0 & 5.0 & 5.0 & s.o & s.o & s.0 & s.o & 0.0 & a & 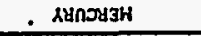 \\
\hline s.o & $5 \cdot 0$ & s'o & S.0 & $s \cdot 0$ & $5 \cdot 0$ & $s \cdot 0$ & 5.0 & $5 \cdot 0$ & 5.0 & 5.0 & s.0 & s:0 & 5.0 & 5.0. & 0.0 & & av37 It?01 \\
\hline $5 \cdot 0$ & 500 & s.0 & s.o & s.0 & s.o & s.o & s.o & s.0 & s.0 & s.0 & s.0 & 5.0 & s.o & $5 \cdot 0$ & 0.0 & 3 & av37 \\
\hline $\boldsymbol{S} \cdot \mathbf{t}$ & 5.8 & S'I & $s \cdot \tau$ & s.t & $s \cdot \tau$ & 5.1 & 5.1 & 5.1 & s.t & S'I & $2+1$ & $5 \cdot 1$ & S.t & $s \cdot 1$ & 0.0 & & 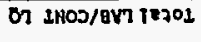 \\
\hline$\varepsilon \cdot 0$ & E.0 & E.0 & E.0 & E.0 & $\mathfrak{\varepsilon} \cdot 0$ & $\varepsilon \cdot 0$ & $\varepsilon \cdot 0$ & $\varepsilon \cdot 0$ & $8 \cdot 0$ & $\varepsilon \cdot 0$ & E.0 & $\varepsilon \cdot 0$ & $\varepsilon \cdot 0$ & $\varepsilon \cdot 0$ & 0.0 & $\mathbf{3}$ & \\
\hline$\varepsilon \cdot 0$ & E.0 & $\varepsilon \cdot 0$ & E.0 & c.0 & $c \cdot 0$ & $c \cdot 0$ & $\varepsilon \cdot 0$ & E.0 & E:0 & E.O & $\varepsilon \cdot 0$ & $\varepsilon \cdot 0$ & E.0 & $\varepsilon \cdot 0$ & 0.0 & 0 & \\
\hline$\varepsilon \cdot 0$ & c.0 0 & $\varepsilon 00$ & E.0 & $\varepsilon \cdot 0$ & $E \cdot 0$ & E.0 & $\varepsilon \cdot 0$ & $\varepsilon \cdot 0$ & $\varepsilon \cdot 0$ & $\varepsilon \cdot 0$ & $\varepsilon \cdot 0$ & $\varepsilon \cdot 0$ & $\varepsilon \cdot 0$ & $\varepsilon \cdot 0$ & 0.0 & g & \\
\hline$\varepsilon \cdot 0$ & E.0 & $\varepsilon^{\circ} 0$ & 5.0 & E.0 & c.0 & E.0 & $\varepsilon \cdot 0$ & $\varepsilon 0$ & E.0 & $\varepsilon \cdot 0$ & $\varepsilon \cdot 0$ & $\varepsilon \cdot 0$ & E.0 & E.0 & 0.0 & $x$ & or 11 100/gn \\
\hline s:o & s.0 & s:0 & s.0 & $s \cdot 0$ & 5.0 & s.o & $s \cdot 0$ & 5.0 & 5.0 & s.0 & s.0 & s:0 & s:0 & $5 \cdot 0$ & 0.0 & & THLISH-Q IEZOL \\
\hline r.o & I.0 & $r \cdot 0$ & I.0 & r.0 & I.0 & r.o & $1 \cdot 0$ & $2 \because 0$ & $r \cdot 0$ & $1 \% 0$ & $t \cdot 0$ & $1 \cdot 0$ & I.O & I.0 & 0.0 & $a$ & \\
\hline$\varepsilon \cdot 0$ & $\varepsilon \% 0$ & $\varepsilon \cdot 0$ & $\varepsilon \cdot 0$ & $\varepsilon \cdot 0$ & 8.0 & $\varepsilon \cdot 0$ & $\varepsilon \cdot 0$ & $E .0$ & $E \cdot 0$ & E.0 & E.O & $\varepsilon \cdot 0$ & $\varepsilon \cdot 0$ & $\varepsilon \cdot 0$ & 0.0 & $x$ & TV13H-a \\
\hline$s=\tau$ & $5 \cdot t$ & 5.1 & $5 \cdot x$ & s.t & $s \cdot \tau$ & S.t & S.t & S.t & s.t & S.I & S. & $5 \cdot 1$ & S.t & S't & 0.0 & & S3I83LIVY IRJOL \\
\hline $2^{\circ} 0^{\circ}$ & L.O & 4.0 & $\llcorner\cdot 0$ & 1.0 & $\llcorner\cdot 0$ & $2 \cdot 0$ & $2 \cdot 0$ & $L \cdot 0$ & $1 \cdot 0$ & $\iota \cdot 0$ & $\llcorner\cdot 0$ & $2 \cdot 0$ & L.O & ¿.O & 0.0 & $\mathbf{3}$ & \\
\hline I.0 & $1 \cdot 0$ & 100 & 1.0 & 100 & $1 \cdot 0$ & $1 \cdot 0$ & t०0 & $\dddot{0}$ & t०0 & IיO & $\tau \cdot 0$ & $1 \cdot 0$ & $1 \cdot 0$ & 100 & 0.0 & 3a & \\
\hline s.0 & s.o & $5 \cdot 0$ & $5 \cdot 0$ & s.o & s:o & s.0 & s.0 & 5.0 & s.0 & $5 \% 0$ & 500 & $5 \%$ & $s^{\circ} 0$ & 5.0 & 0.0 & g & 53183414 \\
\hline
\end{tabular}

\begin{tabular}{|c|c|c|c|c|c|c|c|c|c|c|c|c|c|c|c|c|c|}
\hline 0.0 & 0.0 & 0.0 & 0.0 & 0.0 & 0.0 & 0.0 & 0.0 & 0.0 & 0.0 & 0.0 & 0.0 & $\because$ & 0.0 & $\varepsilon \cdot \theta \varepsilon$ & 0.0 & & 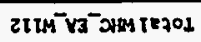 \\
\hline 0.0 & 0.0 & 0.0 & 0.0 & 0.0 & 0.0 & 0.0 & 0.0 & 0.0 & 0.0 & 0.0 & 0.0 & $\varepsilon \cdot 0$ & 0.0 & 0.0 & 0.0 & & MHOKYN TE7O2 \\
\hline 0.0 & 0.0 & 0.0 & 0.0 & 0.0 & 0.0 & 0.0 & 0.0 & 0.0 & 0.0 & 0.0 & 0.0 & $\varepsilon \cdot 0$ & 0.0 & 0.0 & 0.0 & 1 & Mondins \\
\hline 0.0 & 0.0 & 0.0 & 0.0 & 0.0 & 0.0 & 0.0 & 0.0 & 0.0 & 0.0 & 0.0 & 0.0 & 0.0 & 0.0 & $6 \cdot z$ & 0.0 & & $710 s-0$ tejod \\
\hline 0.0 & 0.0 & 0.0 & 0.0 & 0.0 & 0.0 & 0.0 & 0.0 & 0.0 & 0.0 & 0.0 & 0.0 & 0.0 & 0.0 & $\angle \cdot 2$ & 0.0 & INON & 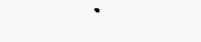 \\
\hline 0.0 & 0.0 & 0.0 & 0.0 & 0.0 & 0.0 & 0.0 & 0.0 & 0.0 & 0.0 & 0.0 & 0.0 & 0.0 & 0.0 & 0.0 & 0.0 & 3 & Tros-a \\
\hline 0.0 & 0.0 & 0.0 & 0.0 & 0.0 & 0.0 & 0.0 & 0.0 & 0.0 & 0.0 & 0.0 & 0.0 & $2 \cdot 0$ & 0.0 & 0.0 & 0.0 & & ISnEHOO-O TEjOL \\
\hline 0.0 & 0.0 & 0.0 & 0.0 & 0.0 & 0.0 & 0.0 & 0.0 & 0.0 & 0.0 & 0.0 & 0.0 & $2 \cdot 0$ & 0.0 & 0.0 & 0.0 & $x$ & ISnQh⿰丿丨- \\
\hline$\overline{0.0}$ & 0.0 & 0.0 & 0.0 & 0.0 & 0.0 & 0.0 & 0.0 & 0.0 & 0.0 & 0.0 & 0.0 & $\angle \cdot 0$ & 0.0 & $5 \cdot 58$ & 0.0 & & TIOS $1 \mathrm{~N} \times 00$ /E701 \\
\hline 0.0 & 0.0 & 0.0 & 0.0 & 0.0 & 0.0 & 0.0 & 0.0 & 0.0 & 0.0 & 0.0 & 0.0 & $0 \cdot 0$ & 0.0 & S.se & 0.0 & 3NON & \\
\hline 0.0 & 0.0 & 0.0 & 0.0 & 0.0 & 0.0 & 0.0 & 0.0 & 0.0 & 0.0 & 0.0 & 0.0 & $2 \cdot 0$ & 0.0 & 0.0 & 0.0 & $\mathbf{3}$ & TIOS L1100 \\
\hline
\end{tabular}

\begin{tabular}{|c|c|c|c|c|c|c|c|c|c|c|c|c|c|c|c|c|c|}
\hline \multirow[b]{2}{*}{$4.6 \pi \tau$} & \multirow[b]{2}{*}{$\tau \cdot 8 I t$} & \multirow[b]{2}{*}{0.0} & \multirow[b]{2}{*}{0.0} & \multirow[b]{2}{*}{0.0} & \multirow[b]{2}{*}{0.0} & \multirow[b]{2}{*}{0.0} & \multirow[b]{2}{*}{0.0} & \multirow[b]{2}{*}{0.0} & \multirow[b]{2}{*}{0.0} & \multirow[b]{2}{*}{0.0} & \multirow[b]{2}{*}{0.0} & \multirow[b]{2}{*}{0.0} & \multirow[b]{2}{*}{0.0} & \multirow[b]{2}{*}{0.0} & \multicolumn{3}{|c|}{ 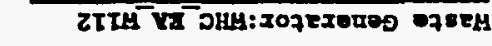 } \\
\hline & & & & & & & & & & & & & & & 0.0 & & $138^{-} I S A^{-}$OKM TEJOL \\
\hline 6.25 & 6.25 & 0.0 & 0.0 & 0.0 & 0.0 & 0.0 & 0.0 & 0.0 & 0.0 & 0.0 & 0.0 & 0.0 & 0.0 & 0.0 & 0.0 & & OLON HSTEIOL \\
\hline $6 \cdot \angle \varepsilon$ & $6^{\circ} \angle \varepsilon$ & 0.0 & 0.0 & 0.0 & 0.0 & 0.0 & 0.0 & 0.0 & 0.0 & 0.0 & 0.0 & 0.0 & 0.0 & 0.0 & 0.0 & MHONUAN & aros HS \\
\hline $2 \cdot 2$ & $2 \cdot z$ & 0.0 & 0.0 & 0.0 & 0.0 & 0.0 & 0.0 & 0.0 & 0.0 & 0.0 & 0.0 & 0.0 & 0.0 & 0.0 & 0.0 & & 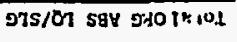 \\
\hline
\end{tabular}

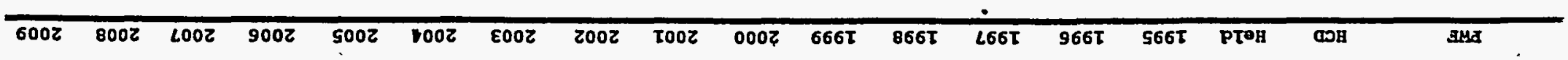
(

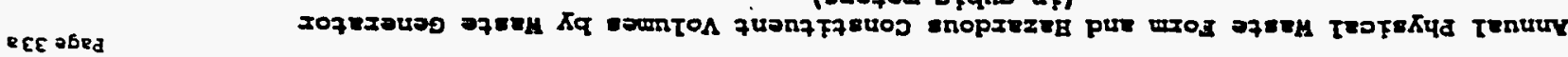




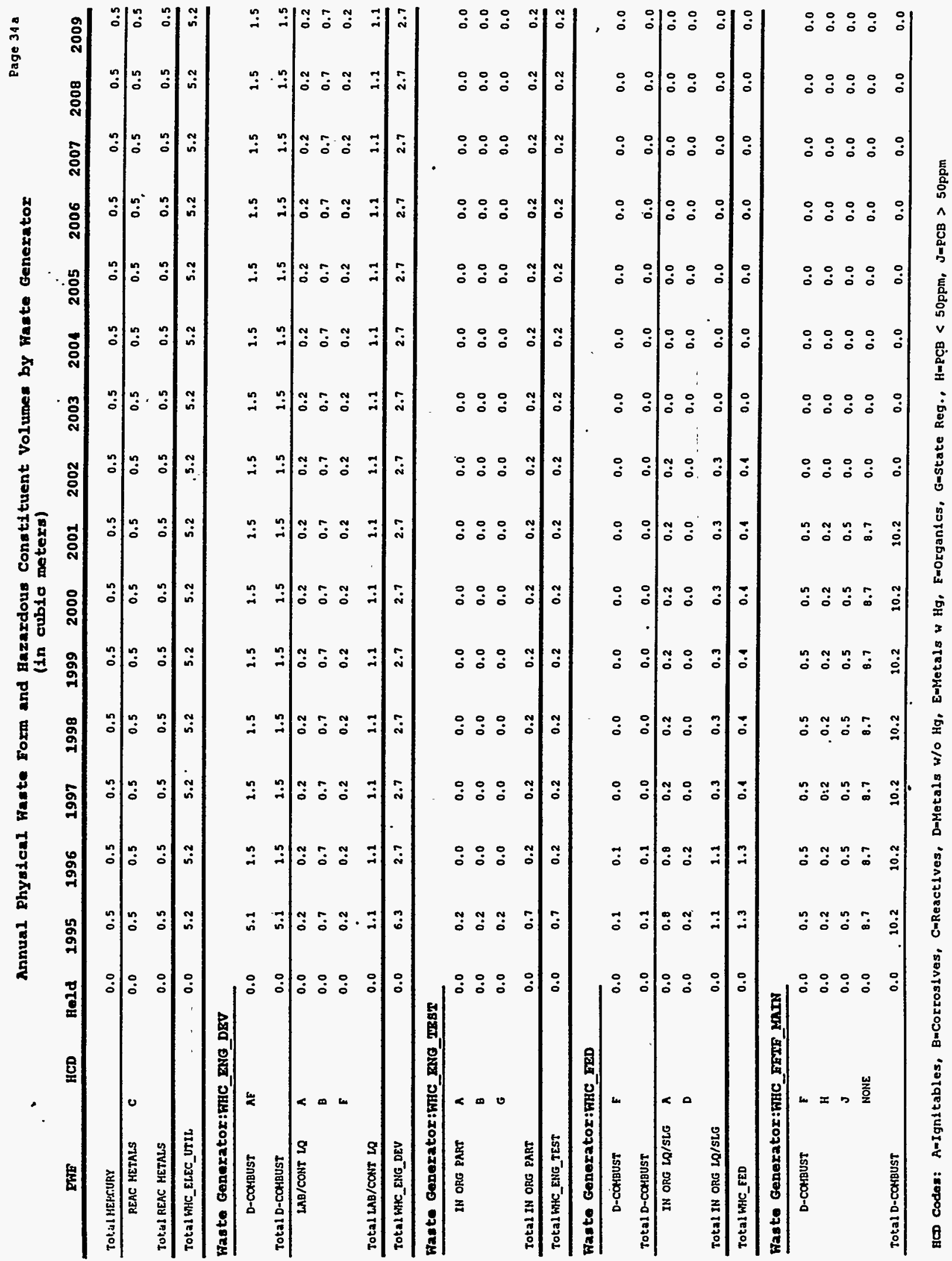


(in cubic meters)

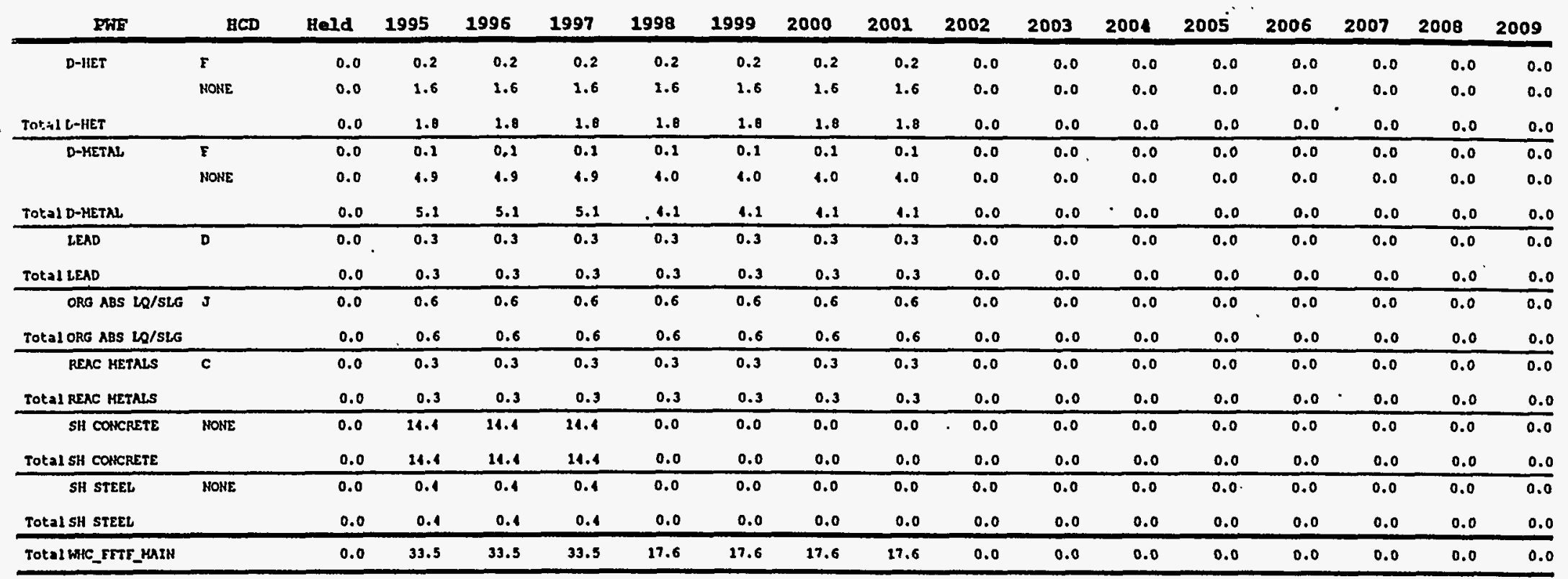

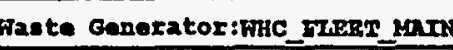

\begin{tabular}{|c|c|c|c|c|c|c|c|c|c|c|c|c|c|c|c|c|c|}
\hline \multirow[t]{3}{*}{ D-COMBUST } & a & 0.0 & 1.5 & 1.5 & 1.5 & 1.5 & 1.5 & 1.5 & 1.5 & 1.5 & 1.5 & 1.5 & $1.5^{\circ}$ & 1.5 & 2.5 & 1.5 & 1.5 \\
\hline & B & 0.0 & 0.0 & 0.0 & 0.0 & 0.0 & 0.0 & 0.0 & 0.0 & 0.0 & 0.0 & 0.0 & 0.0 & 0.0 & 0.0 & 0.0 & 0.0 \\
\hline & G & 0.0 & 2.0 & 2.8 & 2.8 & 2.0 & 2.8 & 2.8 & 2.8 & 2.0 & 2.0 & 2.8 & 2.8 & 2.8 & 2.8 & 2.8 & 2.8 \\
\hline Total D-COMBUST & & 0.0 & 1.1 & 1.1 & 1.4 & 4.4 & 1.1 & 4.4 & 4.4 & 1.4 & 1.1 & 1.1 & 1.4 & 4.4 & 1.4 & 4.4 & 1.4 \\
\hline \multirow[t]{3}{*}{ IN ORG PART } & $\wedge$ & 0.0 & 0.6 & 0.6 & 0.6 & 0.6 & 0.6 & 0.6 & 0.6 & 0.6 & 0.6 & 0.6 & 0.6 & 0.6 & 0.6 & 0.6 . & $\overrightarrow{0.6}$ \\
\hline & B & 0.0 & 0.0 & 0.0 & 0.0 & 0.0 & 0.0 & 0.0 & 0.0 & 0.0 & 0.0 & 0.0 & 0.0 & 0.0 & 0.0 & 0.0 & 0.0 \\
\hline & G & 0.0 & 1.2 & 1.2 & 1.2 & 1.2 & 1.2 & 1.2 & 1.2 & 1.2 & 1.2 & 1.2 & 1.2 & 1.2 & 1.2 & 1.2 & 1.2 \\
\hline \multicolumn{2}{|l|}{ Total IN ORG PART } & 0.0 & 2.9 & 1.9 & 1.9 & 1.9 & 1.9 & 2.9 & 1.9 & 1.9 & 1.9 & 1.9 & 1.9 & 1.9 & 1.9 & 1.9 & 1.9 \\
\hline \multicolumn{2}{|l|}{ TOtal WHE_ELEET_HAIN } & 0.0 & 6.4 & 6.4 & 6.1 & 6.4 & 6.1 & 6.4 & 6.4 & 6.4 & 6.4 & 6.4 & 6.1 & 6.1 & 6.4 & 6.1 & 6.4 \\
\hline \multicolumn{18}{|c|}{ Wasto Generatox:WHC_EMBE } \\
\hline BATTERIES & 86 & 0.0 & 0.1 & 0.1 & 0.1 & 0.1 & 0.1 & 0.1 & 0.1 & 0.1 & 0.1 & 0.1 & 0.1 & 0.1 & 0.1 & 0.1 & 0.1 \\
\hline Total BATTERIES & & 0.0 & 0.1 & 0.1 & 0.1 & 0.1 & 0.1 & 0.1 & 0.1 & 0.1 & 0.1 & 0.1 & 0.1 & 0.1 & 0.1 & 0.1 & 0.2 \\
\hline \multirow[t]{3}{*}{ IN ORE LQ/SLG } & $A$ & 0.0 & 0.0 & 0.0 & 0.0 & 0.0 & 0.0 & 0.0 & 0.0 & 0.0 & 0.0 & 0.0 & 0.0 & 0.0 & 0.0 & 0.0 & 0.0 \\
\hline & $B$ & 0.0 & 0.0 & 0.0 & 0.0 & 0.0 & 0.0 & 0.0 & 0.0 & 0.0 & 0.0 & 0.0 & 0.0 & 0.0 & 0.0 & 0.0 & 0.0 \\
\hline & G & 0.0 & 0.4 & 0.1 & 0.4 & 0.1 & 0.1 & 0.1 & 0.4 & 0.4 & 0.4 & 0.1 & 0.4 & 0.1 & 0.4 & 0.1 & 0.4 \\
\hline
\end{tabular}

HCD Codis: A-Ignitables, B-Corrosives, C-Reactives, DeMetals w/O Hg, E-Metals w Hq, F-Organlcs, G-State Reg., H-PCB < 50ppm, J-PCB > 50ppm 


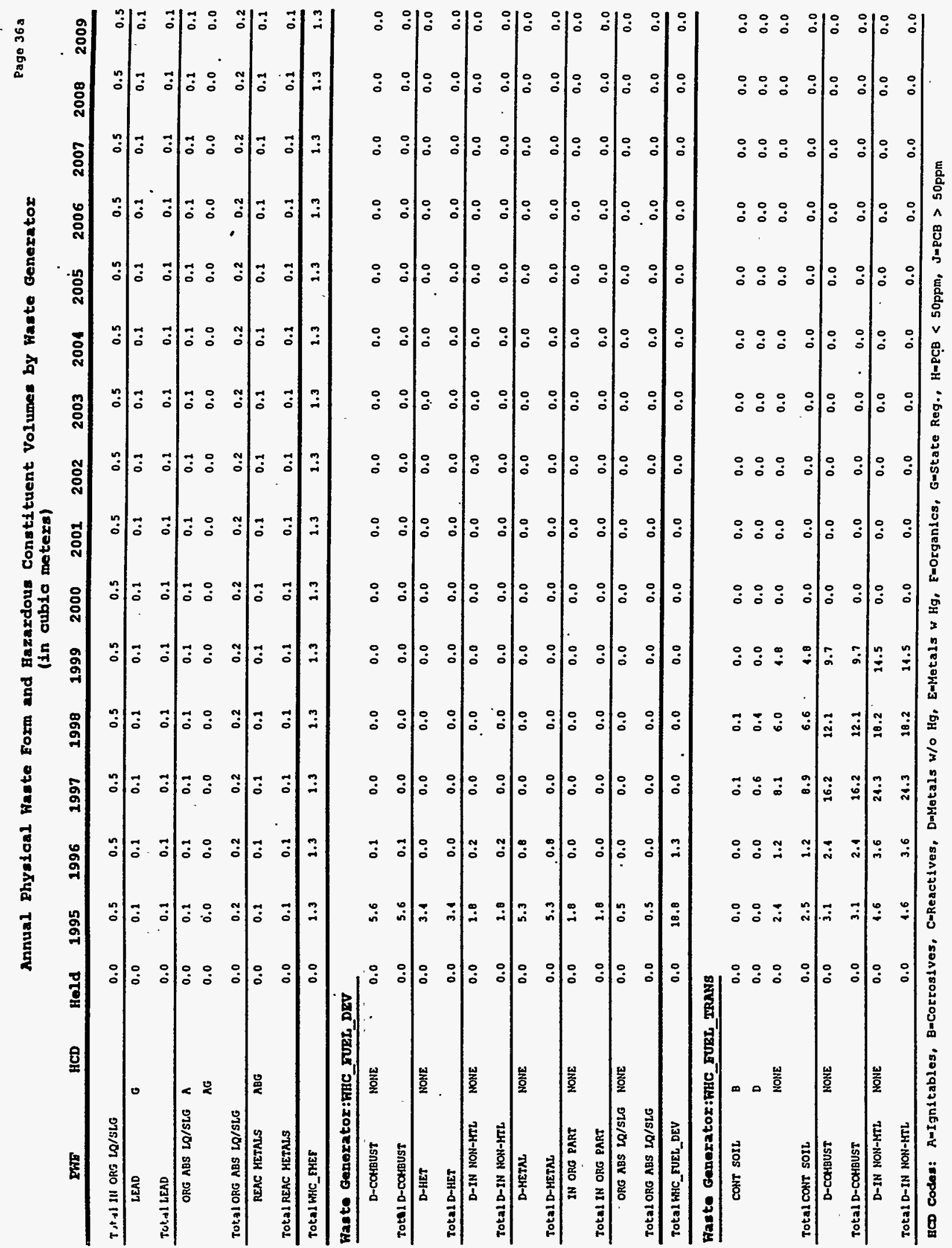


Annual Physical Waste Form and Bazardous Constituent Volumes by Haste Generator (in cubic meters)

\begin{tabular}{|c|c|c|c|c|c|c|c|c|c|c|c|c|c|c|c|c|c|}
\hline PWE & HCD & Beld & 1995 & 1996 & 1997 & 1998 & 2999 & 2000 & 2001 & 2002 & 2003 & 2004 & 2005 & 2006 & 2007 & 2008 & 2009 \\
\hline D-METAL & NONE & 0.0 & 7.0 & 4.8 & 32.4 & 24.3 & 19.4 & 0.0 & 0.0 & 0.0 & 0.0 & 0.0 & 0.0 & 0.0 & 0.0 & 0.0 & 0.0 \\
\hline TOt.a 1 D-METAL, & & 0.0 & 7.0 & 4.8 & 32.1 & 24.3 & 29.4 & 0.0 & 0.0 & 0.0 & 0.0 & 0.0 & 0.0 & 0.0 & 0.0 & 0.0 & 0.0 \\
\hline \multirow[t]{3}{*}{ EXPLOSIVES } & $\lambda$ & 0.0 & 0.1 & 0.0 & 2.7 & 1.1 & 0.0 & 0.0 & 0.0 & 0.0 & 0.0 & 0.0 & 0.0 & 0.0 & 0.0 & 0.0 & 0.0 \\
\hline & g & 0.0 & 0.1 & 0.0 & 2.6 & 1.3 & 0.0 & 0.0 & 0.0 & 0.0 & 0.0 & 0.0 & 0.0 & 0.0 & 0.0 & 0.0 & 0.0 \\
\hline & D & 0.0 & 0.0 & 0.0 & 0.0 & 0.0 & 0.0 & 0.0 & 0.0 & 0.0 & 0.0 & 0.0 & 0.0 & 0.0 & 0.0 & 0.0 & 0.0 \\
\hline Total EXPLOSIVES & & 0.0 & 0.9 & 0.0 & 5.5 & 2.8 & 0.0 & 0.0 & 0.0 & 0.0 & 0.0 & 0.0 & 0.0 & 0.0 & 0.0 & 0.0 & 0.0 \\
\hline \multirow[t]{4}{*}{ IN ORG LO/SLG } & $A$ & 0.0 & 0.0 & 0.0 & 0.6 & 0.1 & 0.0 & 0.0 & 0.0 & 0.0 & 0.0 & 0.0 & 0.0 & 0.0 & 0.0 & 0.0 & 0.0 \\
\hline & B & 0.0 & 0.1 & 0.0 & 1.5 & 0.9 & 0.0 & 0.0 & 0.0 & 0.0 & 0.0 & 0.0 & 0.0 & 0.0 & 0.0 & 0.0 & 0.0 \\
\hline & D & 0.0 & 0.4 & 0.0 & 4.2 & 2.6 & 0.0 & 0.0 & 0.0 & 0.0 & 0.0 & 0.0 & 0.0 & 0.0 & 0.0 & 0.0 & 0.0 \\
\hline & $\mathbf{J}$ & 0.0 & 0.0 & 0.0 & 0.6 & 0.1 & 0.0 & 0.0 & 0.0 & 0.0 & 0.0 & 0.0 & 0.0 & 0.0 & 0.0 & 0.0 & 0.0 \\
\hline Total IN ORG LQ/SLG & & 0.0 & 0.7 & 0.0 & 7.1 & 1.4 & 0.0 & 0.0 & 0.0 & 0.0 & 0.0 & 0.0 & 0.0 & 0.0 & 0.0 & 0.0 & 0.0 \\
\hline TOta1 WHA__EUEL_TRANS & & 0.0 & 19.1 & 12.2 & 94.5 & 60.6 & 10.6 & 0.0 & 0.0 & 0.0 & 0.0 & 0.0 & 0.0 & 0.0 & 0.0 & 0.0 & 0.0 \\
\hline
\end{tabular}

Waste Genorator:HHC Gas LrORTH

$\begin{array}{lllllllllllllllllll}\text { BATRERIES } & 8 & 0.0 & 1.3 & 1.3 & 1.3 & 1.3 & 1.3 & 1.3 & 1.3 & 1.3 & 1.3 & 1.3 & 1.3 & 1.3 & 1.3 & 1.3 & 1.3\end{array}$

\begin{tabular}{|c|c|c|c|c|c|c|c|c|c|c|c|c|c|c|c|c|c|}
\hline TOtal BATTERIES & & 0.0 & 1.3 & 2.3 & 1.3 & 1.3 & 1.3 & 2.3 & 1.3 & 1.3 & 1.3 & 1.3 & 1.3 & 1.3 & 1.3 & 1.3 & 1.3 \\
\hline D-Coribust & $A G$ & 0.0 & 3.3 & 3.3 & 3.3 & 3.3 & 3.3 & 3.3 & 3.3 & 3.3 & 3.3 & 3.3 & 3.3 & 3.3 & 3.3 & 3.3 & $\overline{3.3}$ \\
\hline Total D-Cangust & & 0.0 & 3.3 & 3.3 & 3.3 & 3.3 & 3.3 & 3.3 & 3.3 & 3.3 & 3.3 & 3.3 & 3.3 & 3.3 & 3.3 & 3.3 & 3.3 \\
\hline D-HET & $\mathbf{H}$ & 0.0 & 1.1 & 1.1 & 1.1 & 1.1 & 1.1 & 1.1 & 1.1 & 1.1 & 1.1 & 1.2 & 1.1 & 1.1 & 2.1 & 1.1 & 1.1 \\
\hline Total D-HET & & 0.0 & 1.1 & 1.1 & 1.1 & 1.1 & 1.1 & 1.1 & 1.1 & 1.1 & 1.1 & 1.1 & 1.1 & 1.1 & 1.1 & 1.1 & 1.1 \\
\hline D-METAL & H & 0.0 & 1.0 & 1.0 & 1.0 & 1.0 & 1.0 & 1.0 & 2.0 & 1.0 & 1.0 & 1.0 & 1.0 & 1.0 & 1.0 & 1.0 & 1.0 \\
\hline Total D-METAL & & 0.0 & 1.0 & 1.0 & 1.0 & 1.0 & 1.0 & 1.0 & 1.0 & 1.0 & 1.0 & 1.0 & 1.0 & 1.0 & 1.0 & 2.0 & 1.0 \\
\hline LAB/CONT LQ & G & 0.0 & 12.3 & 12.3 & 12.3 & 12.3 & 12.3 & 12.3 & 12.3 & 12.3 & 12.3 & 12.3 & 12.3 & 12.3 & 12.3 & 12.3 & 12.3 \\
\hline TOtaL LAB/CONT LQ & & 0.0 & 12.3 & 12.3 & 12.3 & 22.3 & 12.3 & 12.3 . & 12.3 & 12.3 & 12.3 & 12.3 & 12.3 & 12.3 & 12.3 & 12.3 & 12.3 \\
\hline ORG ABS LO/SLG & EG & 0.0 & 0.2 & 0.2 & 0.2 & 0.2 & 0.2 & 0.2 & 0.2 & 0.2 & 0.2 & 0.2 . & 0.2 & 0.2 & 0.2 & 0.2 & 0.2 \\
\hline Total ORG ABS LQ/SLG & & 0.0 & 0.2 & 0.2 & 0.2 & 0.2 & 0.2 & 0.2 & 0.2 & 0.2 & 0.2 & 0.2 & 0.2 & 0.2 & 0.2 & 0.2 & 0.2 \\
\hline TOtal WHC_GAS_NORTH & & 0.0 & 19.6 & 19.6 & 19.6 & 19.6 & 29.6 & 19.6 & 19.6 & 19.6 & 19.6 & 19.6 & 19.6 & 19.6 & 19.6 & 19.6 & 19.6 \\
\hline \multicolumn{18}{|c|}{ Waste Generator:FHC_GA8_sOUTH } \\
\hline BATTERIES & $B D G$ & 0.0 & 0.2 & 0.1 & 0.1 & 0.1 & 0.1 & 0.1 & 0.1 & 0.1 & 0.1 & 0.1 & 0.1 & 0.1 & 0.1 & 0.1 & 0.1 \\
\hline & BEG & 0.0 & 0.6 & 0.6 & 0.6 & 0.6 & 0.6 & 0.6 & 0.6 & 0.6 & 0.6 & 0.6 & 0.6 & 0.6 & 0.6 & 0.6 & 0.6 \\
\hline TOta1 BATIERIES & & 0.0 & 0.7 & 0.7 & 0.7 & 0.7 & 0.7 & 0.7 & 0.7 & 0.7 & 0.7 & 0.7 & 0.7 & 0.7 & 0.1 & 0.7 & 0.7 \\
\hline D-IN NON-HTL & ABCEG & 0.0 & 1.4 & 1.4 & 1.1 & 3.4 & 1.4 & 1.1 & 1.4 & 1.4 & 1.4 & 1.4 & 1.4 & 1.4 & 1.4 & 1.4 & 1.4 \\
\hline Total D-IN NON-MTL & & 0.0 & 2.1 & 1.4 & 1.1 & 1.4 & 1.4 & 2.1 & 1.1 & 1.4 & 1.1 & 1.4 & 1.4 & 1.1 & 1.1 & 1.4 & 1.4 \\
\hline
\end{tabular}

HCD Codes: A-Ignitables, B-Corroslves, C-Reactives, D-Metals w/O Hg, E-Metals w Hg, F-Organics, G-State Reg., H-FCB < 50ppm, J-PCB > 50ppm 


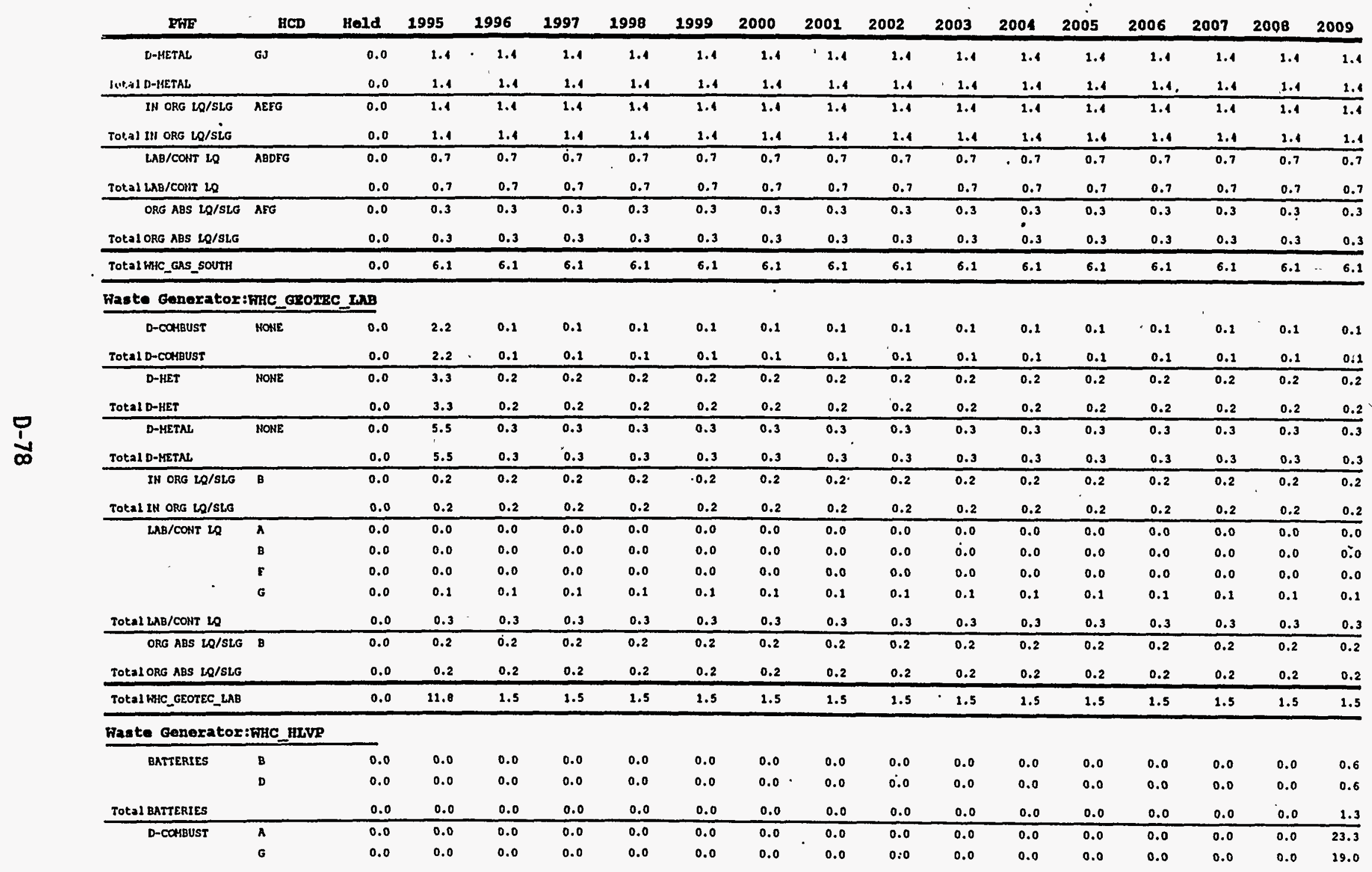

BCD Codes: A-Ignitables, B-Corrosives, C-Reactives, D-Metals w/O Hg, E-Metals w Hg, F-Organles, G-State Reg.. H-PCB < 50ppm, J-PCB > 50ppm 


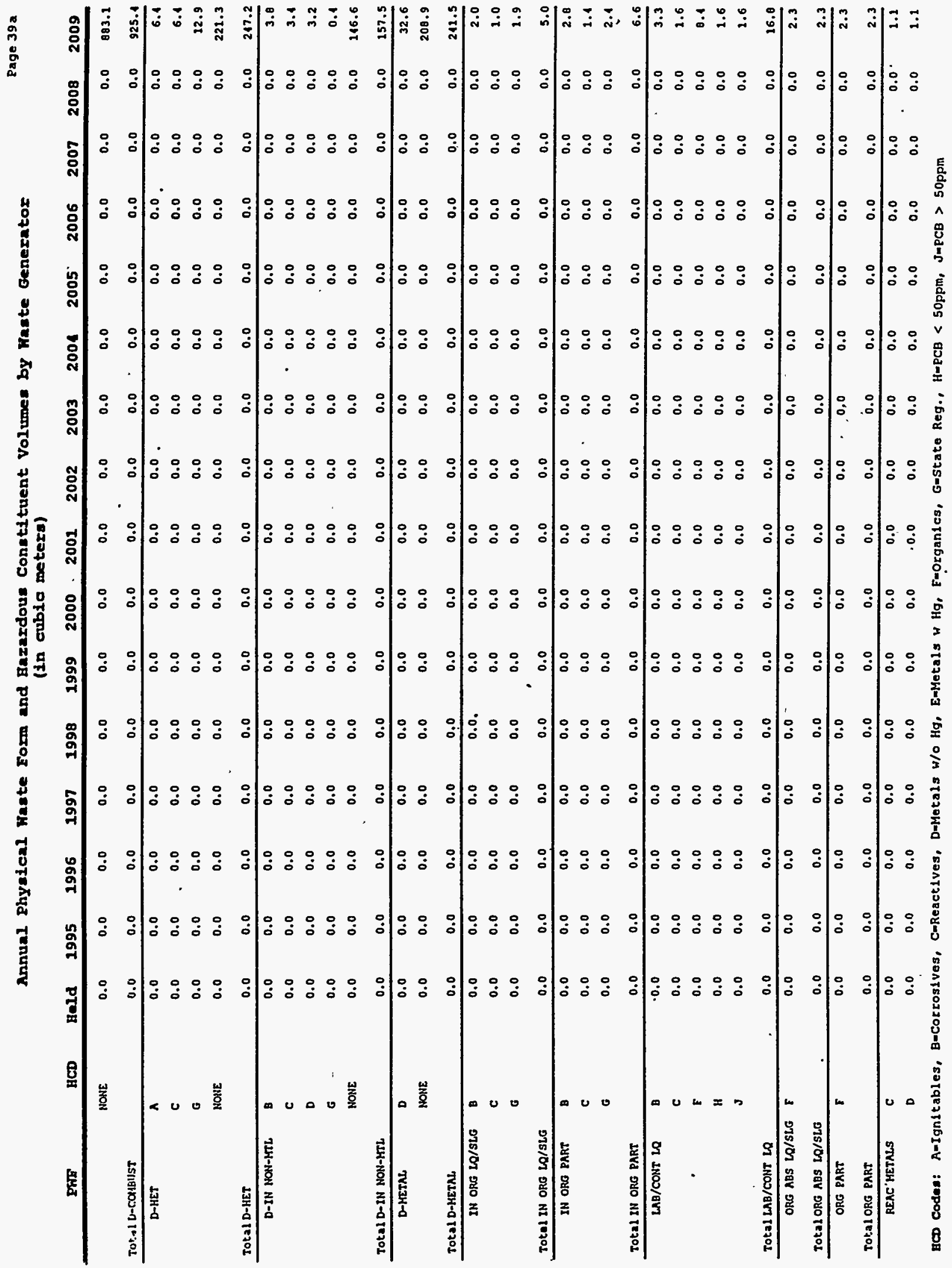


(In cubia meters)

\begin{tabular}{|c|c|c|c|c|c|c|c|c|c|c|c|c|c|c|c|c|c|}
\hline PWE & HCD & Held & 1995 & 1996 & 1997 & 1998 & 1999 & 2000 & 2001 & 2002 & 2003 & 2004 & 2005 & 2006 & 2007 & 2008 & 2009 \\
\hline TOI AI KEAC METALS & & 0.0 & 0.0 & 0.0 & 0.0 & 0.0 & 0.0 & 0.0 & 0.0 & 0.0 & 0.0 & 0.0 & 0.0 & 0.0 & 0.0 & 0.0 & 2.2 \\
\hline SALT WASTE & 8 & 0.0 & 0.0 & 0.0 & 0.0 & 0.0 & 0.0 & 0.0 & 0.0 & 0.0 & 0.0 & 0.0 & 0.0 & 0.0 & 0.0 & 0.0 & $\overrightarrow{2.3}$ \\
\hline TOL: 1 SALT WASTE & & 0.0 & 0.0 & 0.0 & 0.0 & 0.0 & 0.0 & 0.0 & 0.0 & 0.0 & 0.0 & 0.0 & 0.0 & 0.0 & 0.0 & 0.0 & 2.3 \\
\hline SH LEAD & D & 0.0 & 0.0 & 0.0 & 0.0 & 0.0 & 0.0 & 0.0 & 0.0 & 0.0 & 0.0 & 0.0 & 0.0 & 0.0 & 0.0 & 0.0 & 5.6 \\
\hline . & NONE : & 0.0 & 0.0 & 0.0 & 0.0 & 0.0 & 0.0 & 0.0 & 0.0 & 0.0 & 0.0 & 0.0 & 0.0 & 0.0 & 0.0 & 0.0 & 11.3 \\
\hline Tot21 SH LEAD & & 0.0 & 0.0 & 0.0 & 0.0 & 0.0 & 0.0 & 0.0 & 0.0 & 0.0 & 0.0 & 0.0 & 0.0 & 0.0 & 0.0 & 0.0 & 16.9 \\
\hline \multirow[t]{3}{*}{ SH STEEL } & B & 0.0 & 0.0 & 0.0 & 0.0 & 0.0 & 0.0 & 0.0 & 0.0 & 0.0 & 0.0 & 0.0 & 0.0 & 0.0 & 0.0 & 0.0 & 2.8 \\
\hline & G & 0.0 & 0.0 & 0.0 & 0.0 & 0.0 & 0.0 & 0.0 & 0.0 & 0.0 & 0.0 & 0.0 & 0.0 & 0.0 & 0.0 & 0.0 & 2.8 \\
\hline & MONE & 0.0 & 0.0 & 0.0 & 0.0 & 0.0 & 0.0 & 0.0 & 0.0 & 0.0 & 0.0 & 0.0 & 0.0 & 0.0 & 0.0 & 0.0 & 24.0 \\
\hline Tot:1 SH STEEL & & 0.0 & 0.0 & 0.0 & 0.0 & 0.0 & 0.0 & 0.0 & 0.0 & 0.0 & 0.0 & 0.0 & 0.0 & 0.0 & 0.0 & 0.0 & 29.6 \\
\hline Total WHC_HLVP & & 0.0 & 0.0 & 0.0 & 0.0 & 0.0 & 0.0 & 0.0 & 0.0 & 0.0 & 0.0 & 0.0 & 0.0 & 0.0 & 0.0 & 0.0 & 1657.7 \\
\hline \multicolumn{3}{|c|}{ Waste Gonerator:WHC_K_BMsI } & & . & $\cdots$ & & & & & - & & & - & & & & -- \\
\hline BATTERIES & BD & 0.0 & 0.2 & 0.2 & 0.2 & 0.2 & 0.2 & 0.2 & 0.2 & 0.2 & 0.2 & 0.2 & 0.2 & 0.2 & 0.2 & 0.2 & 0.2 \\
\hline Total BATTERIES & & 0.0 & 0.2 & 0.2 & 0.2 & 0.2 & 0.2 & 0.2 & 0.2 & 0.2 & 0.2 & 0.2 & 0.2 & 0.2 & 0.2 & 0.2 & 0.2 \\
\hline D-COABUST & & 0.0 & 0.9 & 0.9 & 0.0 & 0.8 & 0.6 & 0.6 & 0.6 & 0.6 & 0.6 & 0.6 & 0.6 & 0.6 & 0.6 & 0.6 & 0.6 \\
\hline & MONE & 0.0 & 99.9 & 117.1 & 54.3 & 54.3 & .54 .3 & 54.3 & 54.3 & 54.3 & 54.3 & 54.3 & 54.3 & 54.3 & 54.3 & 54.3 & 54.3 \\
\hline Total D-COMguST & & 0.0 & 90.9 & 118.1 & 55.1 & 55.1 & 54.9 & 54.9 & 54.9 & 54.9 & 54.9 & 54.9 & 54.9 & 54.9 & 54.9 & 54.9 & 54.9 \\
\hline D-HET & HONE & 0.0 & 51.1 & 0.0 & 0.0 & 0.0 & 0.0 & 0.0 & 0.0 & 0.0 & 0.0 & 0.0 & 0.0 & 0.0 & 0.0 & 0.0 & 0.0 \\
\hline Total D-HET & & 0.0 & 51.1 & $0: 0$ & 0.0 & 0.0 & 0.0 & 0.0 & 0.0 & 0.0 & 0.0 & 0.0 & 0.0 & 0.0 & 0.0 & 0.0 & 0.0 \\
\hline \multirow[t]{2}{*}{ D-METNL } & D & 0.0 & 1.4 & 1.1 & 1.1 & 1.1 & 0.9 & 0.9 & 0.9 & 0.9 & 0.9 & 0.9 & 0.9 & 0.9 & 0.9 & 0.9 & 0.9 \\
\hline & MONE & 0.0 & 114.2 & 93.8 & 41.7 & 41.7 & 14.7 & 14.7 & 14.7 & 14.7 & 14.7 & 14.7 & 14.7 & 14.7 & 14.7 & 14.7 & $M .7$ \\
\hline Total D-METAL & & 0.0 & 115.6 & 95.2 & 15.9 & 15.9 & 45.6 & 45.6 & 45.6 & 45.6 & 15.6 & 15.6 & 45.6 & 45.6 & 15.6 & 15.6 & 15.6 \\
\hline EXPLOSIVES & A & 0.0 & 0.8 & 0.8 & 0.7 & 0.7 & 0.5 & 0.5 & 0.5 & 0.5 & $0.5^{\prime}$ & 0.5 & 0.5 & 0.5 & 0.5 & 0.5 & 0.5 \\
\hline Total EXPLOSIVES & & 0.0 & 0.8 & 0.8 & 0.7 & 0.7 & 0.5 & 0.5 & 0.5 & 0.5 & 0.5 & 0.5 & 0.5 & 0.5 & 0.5 & 0.5 & 0.5 \\
\hline ORG ABS LQ/SLG & $A F$ & 0.0 & 0.7 & 0.7 & 0.5 & 0.5 & 0.4 & 0.4 & 0.4 & 0.4 & 0.4 & 0.1 & 0.1 & 0.4 & 0.4 & 0.4 & $\overline{0.4}$ \\
\hline TOt.2L ORG ABS LQ/SLG & & 0.0 & 0.7 & 0.7 & 0.5 & 0.5 & 0.1 & 0.4 & 0.1 & 0.4 & 0.1 & 0.1 & 0.1 & 0.1 & 0.1 & 0.1 & 0.4 \\
\hline ORG PART & NONE & 0.0 & 82.7 & 51.8 & $\$ 1.6$ & 42.6 & $\$ 1.6$ & 11.6 & 41.6 & 41.6 & 11.6 & 41.6 & 11.6 & $\$ 1.6$ & 41.6 & 11.6 & 11.6 \\
\hline TOt.21 ORG PART & & 0.0 & 82.7 & 51.8 & 11.6 & 11.6 & 11.6 & 11.6 & 11.6 & 41.6 & 41.6 & 41.6 & 41.6 & 11.6 & $\$ 1.6$ & 11.6 & $\$ 1.6$ \\
\hline Total WHE_K_BASIN & & 0.0 & 342.3 & 267.1 & 214.2 & 141.2 & 143.5 & 143.5 & 243.5 & 143.5 & 143.5 & 243.5 & 143.5 & 143.5 & 143.5 & 143.5 & 143.5 \\
\hline \multicolumn{18}{|c|}{ Faste Genorator:FHC_IAUNDRY } \\
\hline D-COHBUST & MONE & 0.0 & 113.3 & 113.3 & 113.3 & 113.3 & 113.3 & 213.3 & 113.3 & 113.3 & 113.3 & 113.3 & 113.3 & 113.3 & 113.3 & 113.3 & 113.3 \\
\hline TOta 1 D-COMBUST & & 0.0 & 113.3 & 113.3 & 113.3 & 213.3 & 113.3 & 113.3 & 113.3 & 113.3 & 213.3 & 113.3 & 113.3 & 113.3 & 113.3 & 113.3 & 113.3 \\
\hline
\end{tabular}

gCo Codos: A-Ignitables, B-Corrosives, C-Reactives, D=Metals w/o Hg, E-Metals w Hg, Fmorganlcs, G-State Reg.. H HCB < 50ppm, J-PCB > 50ppm 

(in cubio metera)

\begin{tabular}{|c|c|c|c|c|c|c|c|c|c|c|c|c|c|c|c|c|c|}
\hline PHE & HCD & Held & 1995 & 1996 & 1997 & 1998 & 1999 & 2000 & 2001 & 2002 & 2003 & 2004 & 2005 & 2006 & 2007 & 2008 & 2009 \\
\hline Q-HET. & B & 0.0 & 0.3 & 0.3 & 0.3 & 0.3 & 0.3 & 0.3 & 0.3 & 0.3 & 0.3 & 0.3 & 0.3 & 0.3 & 0.3 & 0.3 & 0.3 \\
\hline Total D-HET & & 0.0 & 0.3 & 0.3 & 0.3 & 0.3 & 0.3 & 0.3 & 0.3 & 0.3 & 0.3 & 0.3 & 0.3 & 0.3 & 0.3 & 0.3 & 0.3 \\
\hline TOta1 WHE_LAUMD & & 0.0 & 123.6 & 113.6 & 113.6 & 113.6 & 113.6 & 113.6 & 113.6 & 113.6 & 113.6 & 113.6 & 113.6 & 123.6. & 113.6 & 113.6 & 113.6 \\
\hline
\end{tabular}

Haste Genexator:HAC_LrBg

\begin{tabular}{|c|c|c|c|c|c|c|c|c|c|c|c|c|c|c|c|c|c|}
\hline Total CONT SOIL & & 0.0 & 0.4 & 0.1 & 0.1 & 0.4 & 0.1 & 0.1 & 0.1 & 0.1 & 0.1 & 0.1 & 0.1 & 0.4 & 0.4 & 0.1 & 0.1 \\
\hline D-COMBUST & MONE & 0.0 & 5.6 & 5.6 & 3.6 & 5.6 & 5.6 & 5.6 & 5.6 & 5.6 & 5.6 & 5.6 & 5.6 & 5.6 & 5.6 & 5.6 & 5.6 \\
\hline & & 0.0 & 5.6 & 5.6 & 5.6 & 5.6 & 5.6 & 5.6 & 5.6 & 5.6 & 5.6 & 5.6 & 5.6 & 5.6 & 5.6 & 5.6 & 5.6 \\
\hline
\end{tabular}

\begin{tabular}{|c|c|c|c|c|c|c|c|c|c|c|c|c|c|c|c|c|c|}
\hline Total D-CONBUST & & 0.0 & 5.6 & 5.6 & 5.6 & 5.6 & 5.6 & 5.6 & 5.6 & 5.6 & 5.6 & 5.6 & 5.6 & 5.6 & 5.6 & 5.6 & 5.6 \\
\hline D-HE & 6 & 0.0 & 0.0 & 0.0 & 3.4 & 0.0 & 0.0 & 3.4 & 0.0 & 0.0 & 3.4 & 0.0 & 0.0 & 3.4 & 0.0 & 0.0 & 3.4 \\
\hline
\end{tabular}

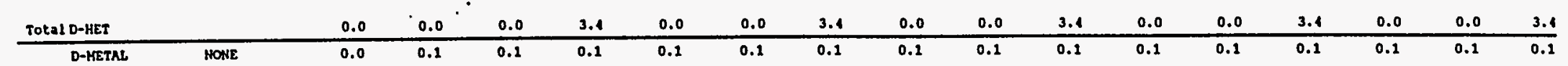

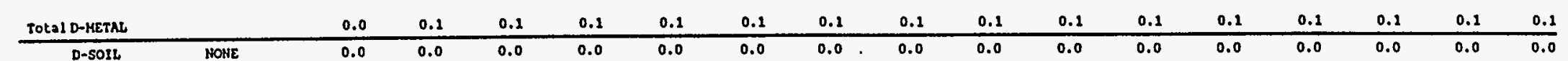

\begin{tabular}{|c|c|c|c|c|c|c|c|c|c|c|c|c|c|c|c|c|}
\hline Total D-sort & 0.0 & 0.0 & 0.0 & 0.0 & 0.0 & 0.0 & 0.0 & 0.0 & 0.0 & 0.0 & 0.0 & 0.0 & 0.0 & 0.0 & 0.0 & 0.0 \\
\hline Total WATC LLBG & 0.0 & 6.3 & 6.3 & 9.7 & 6.3 & 6.3 & 9.7 & 6.3 & 6.3 & 9.7 & 6.3 & 6.3 & 9.7 & 6.3 & 6.3 & 9.7 \\
\hline
\end{tabular}

Faste Ganorator:HIIC_LIVP

\begin{tabular}{|c|c|c|c|c|c|c|c|c|c|c|c|c|c|c|c|c|c|}
\hline BATTERIES & B & 0.0 & 0.0 & 0.0 & 0.0 & 0.0 & 0.0 & 0.0 & 0.0 & 0.0 & 0.0 & 0.0 & 0.2 & $\begin{array}{l}0.2 \\
0.2\end{array}$ & $\begin{array}{l}0.2 \\
0.2\end{array}$ & $\begin{array}{l}0.2 \\
0.2\end{array}$ & 0.2 \\
\hline & D & 0.0 & 0.0 & 0.0 & 0.0 & 0.0 & .0 .0 & 0.0 & 0.0 & 0.0 & 0.0 & 0.0 & 0.2 & 0.2 & & & \\
\hline Total aATterIES & & 0.0 & 0.0 & 0.0 & 0.0 & 0.0 & 0.0 & 0.0 & 0.0 & 0.0 & 0.0 & 0.0 & 0.1 & 0.1 & 0.1 & 0.1 & 0.4 \\
\hline \multirow[t]{3}{*}{ D-COMBUST } & $n$ & 0.0 & 0.0 & 0.0 & 0.0 & 0.0 & 0.0 & 0.0 & 0.0 & 0.0 & 0.0 & 0.0 & 0.3 & 0.3 & 0.3 & 0.3 & .0 .3 \\
\hline & G & 0.0 & 0.0 & 0.0 & 0.0 & 0.0 & 0.0 & 0.0 & 0.0 & 0.0 & 0.0 & 0.0 & 0.2 & 0.2 & 0.2 & 0.2 & 0.2 \\
\hline & NONE & 0.0 & 0.0 & 0.0 & 0.0 & 0.0 & 0.0 & 0.0 & 0.0 & 0.0 & 0.0 & 0.0 & 371.9 & 371.9 & 371.9 & 371.9 & 371.9 \\
\hline Total D-COHBUST & & 0.0 & 0.0 & 0.0 & 0.0 & 0.0 & 0.0 & 0.0 & 0.0 & 0.0 & 0.0 & 0.0 & 372.5 & 372.5 & 372.5 & 372.5 & 372.5 \\
\hline \multirow[t]{3}{*}{ D-HET } & B & 0.0 & 0.0 & 0.0 & 0.0 & 0.0 & 0.0 & 0.0 & 0.0 & 0.0 & 0.0 & 0.0 & 0.0 & 0.0 & 0.0 & 0.0 & 0.0 \\
\hline & c & 0.0 & 0.0 & 0.0 & 0.0 & 0.0 & 0.0 & 0.0 & 0.0 & 0.0 & 0.0 & 0.0 & 0.0 & 0.0 & 0.0 & 0.0 & 0.0 \\
\hline & HONE & 0.0 & 0.0 & 0.0 & 0.0 & 0.0 & 0.0 & 0.0 & 0.0 & 0.0 & 0.0 & 0.0 & 87.2 & 07.2 & 87.2 & 87.2 & 07.2 \\
\hline Total D-HET & & 0.0 & 0.0 & 0.0 & 0.0 & 0.0 & 0.0 & 0.0 & 0.0 & 0.0 & 0.0 & 0.0 & 07.6 & 87.6 & 07.6 & 07.6 & 87.6 \\
\hline \multirow[t]{4}{*}{ D-IN MON-MTL } & $B$ & 0.0 & 0.0 & 0.0 & 0.0 & 0.0 & 0.0 & 0.0 & 0.0 & 0.0 & 0.0 & 0.0 & 0.1 & 0.1 & 0.1 & 0.1 & $\overline{0.1}$ \\
\hline & c & 0.0 & 0.0 & 0.0 & 0.0 & 0.0 & 0.0 & 0.0 & 0.0 & 0.0 & 0.0 & 0.0 & 0.0 & 0.0 & 0.0 & 0.0 & 0.0 \\
\hline & 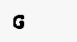 & 0.0 & 0.0 & 0.0 & 0.0 & 0.0 & 0.0 & 0.0 & 0.0 & 0.0 & 0.0 & 0.0 & 0.1 & 0.1 & 0.1 & 0.1 & 0.2 \\
\hline & HONE & 0.0 & 0.0 & 0.0 & 0.0 & 0.0 & 0.0 & 0.0 & 0.0 & 0.0 & 0.0 & 0.0 & 50.9 & 58.9 & 58.9 & 58.9 & 58.9 \\
\hline Total D-IN NON-MTL & & 0.0 & 0.0 & 0.0 & 0.0 & 0.0 & 0.0 & 0.0 & 0.0 & 0.0 & 0.0 & 0.0 & 59.2 & 59.2 & 59.2 & 59.2 & 59.2 \\
\hline
\end{tabular}

BCD Codes: A-Ignitables, BmCorrosives, C-Reactives, D-Metals w/O Hg, E-Metals w Hg, Fmorganles, GmState Reg., HmPCB < 50ppm, J-PCB > 50ppm 


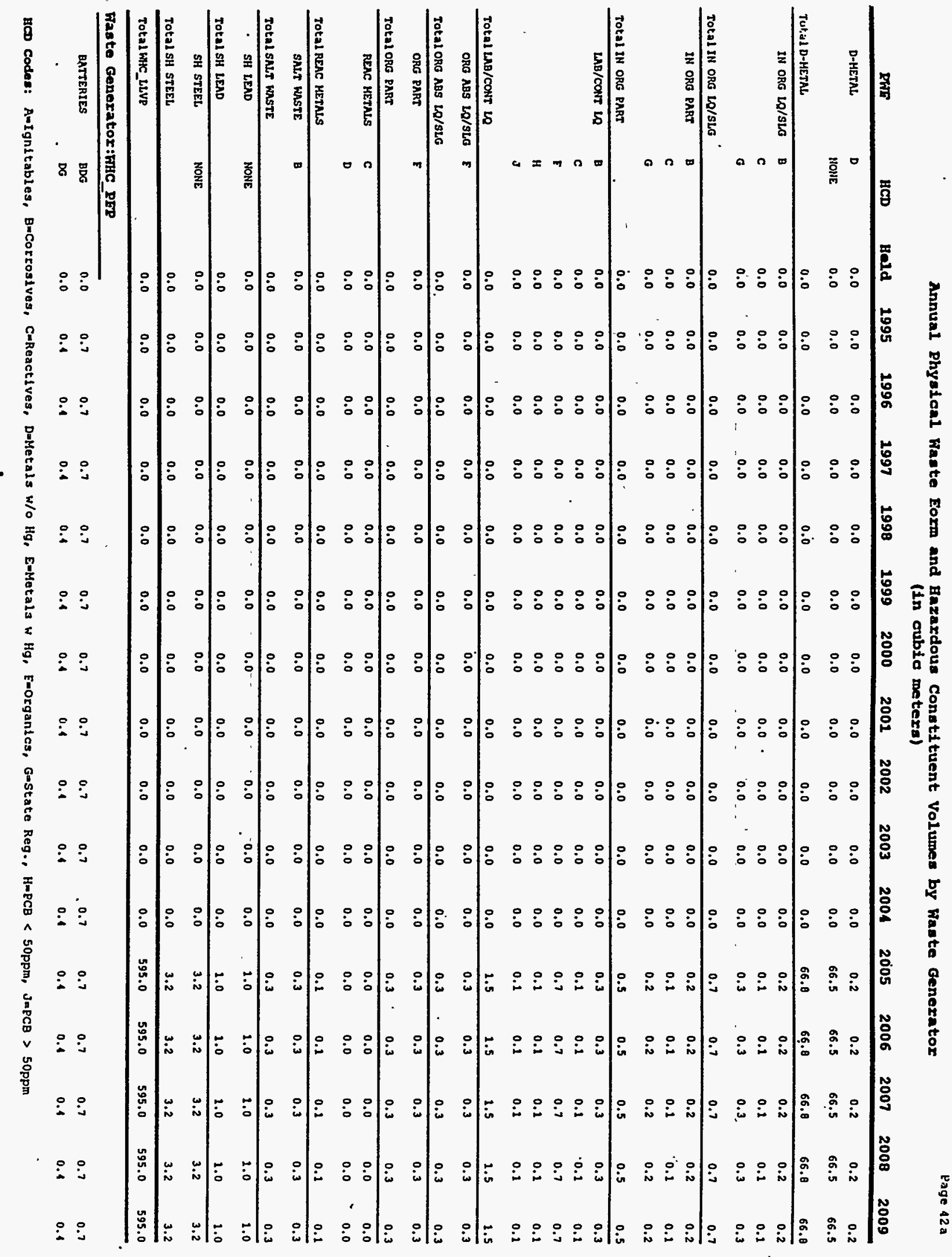


Annual Physical faste Form and Bazardous Constituent Volumes by Fante Generator

(in cubic meters)

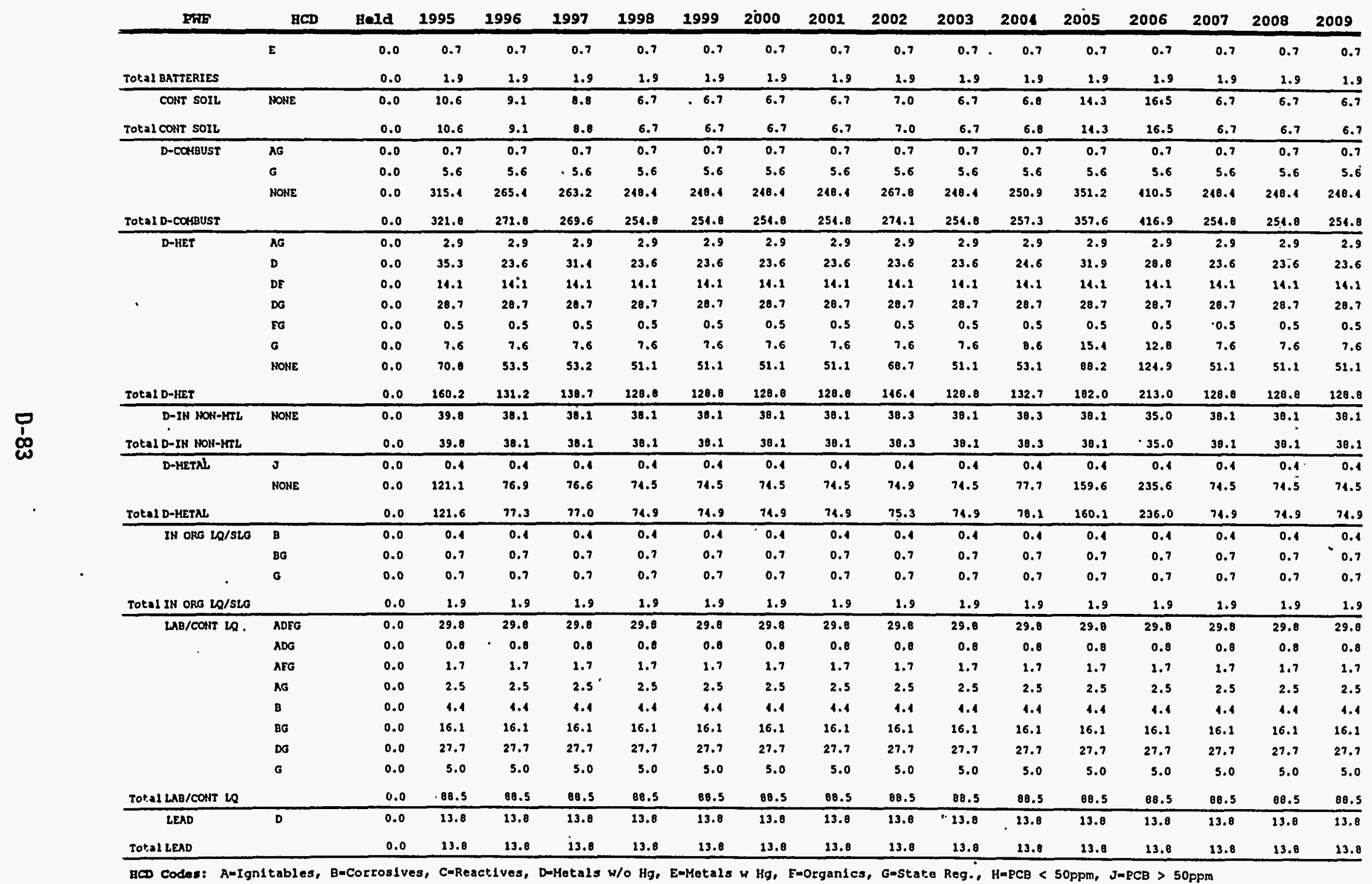


WHC-EP-0888

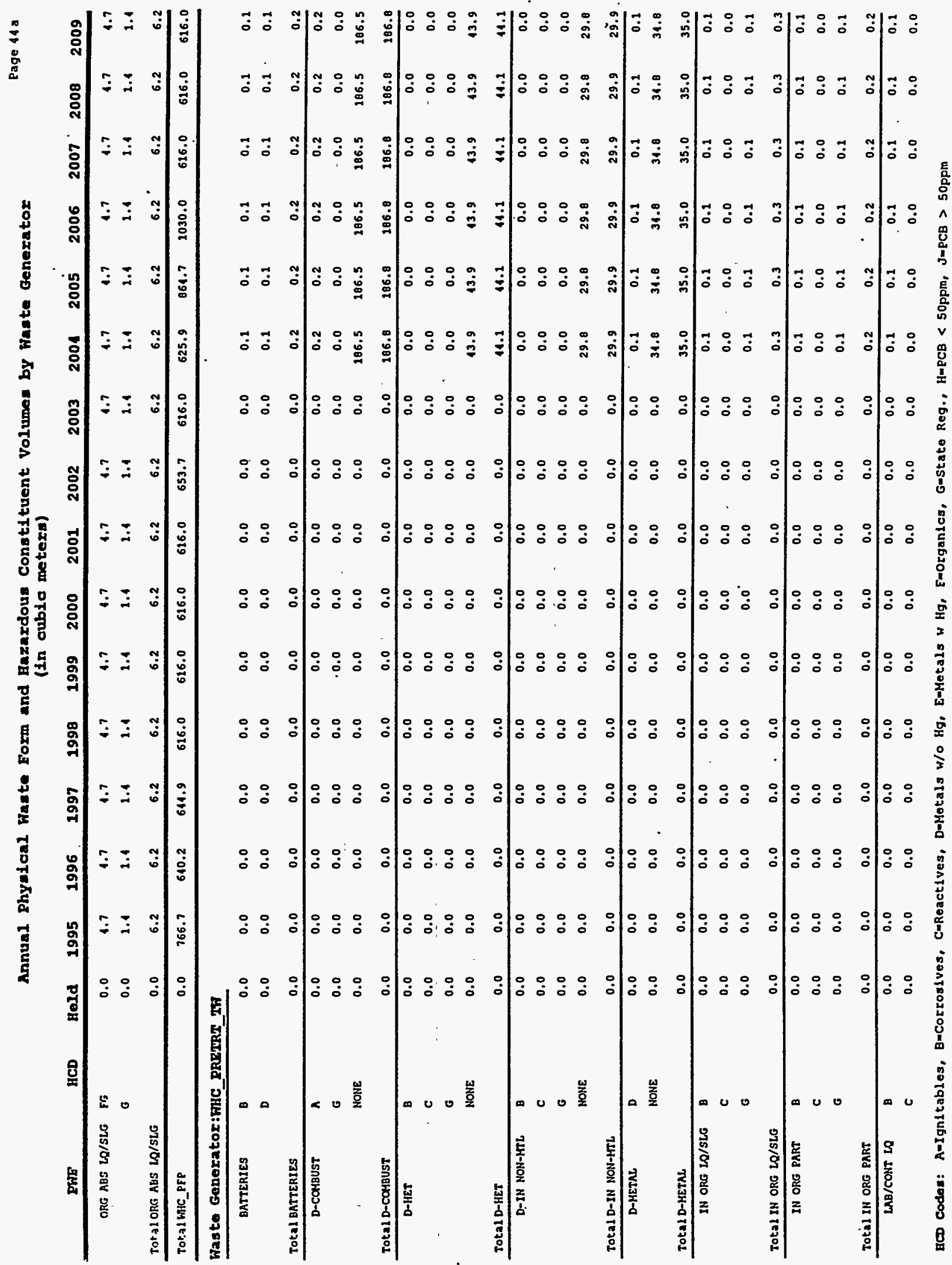




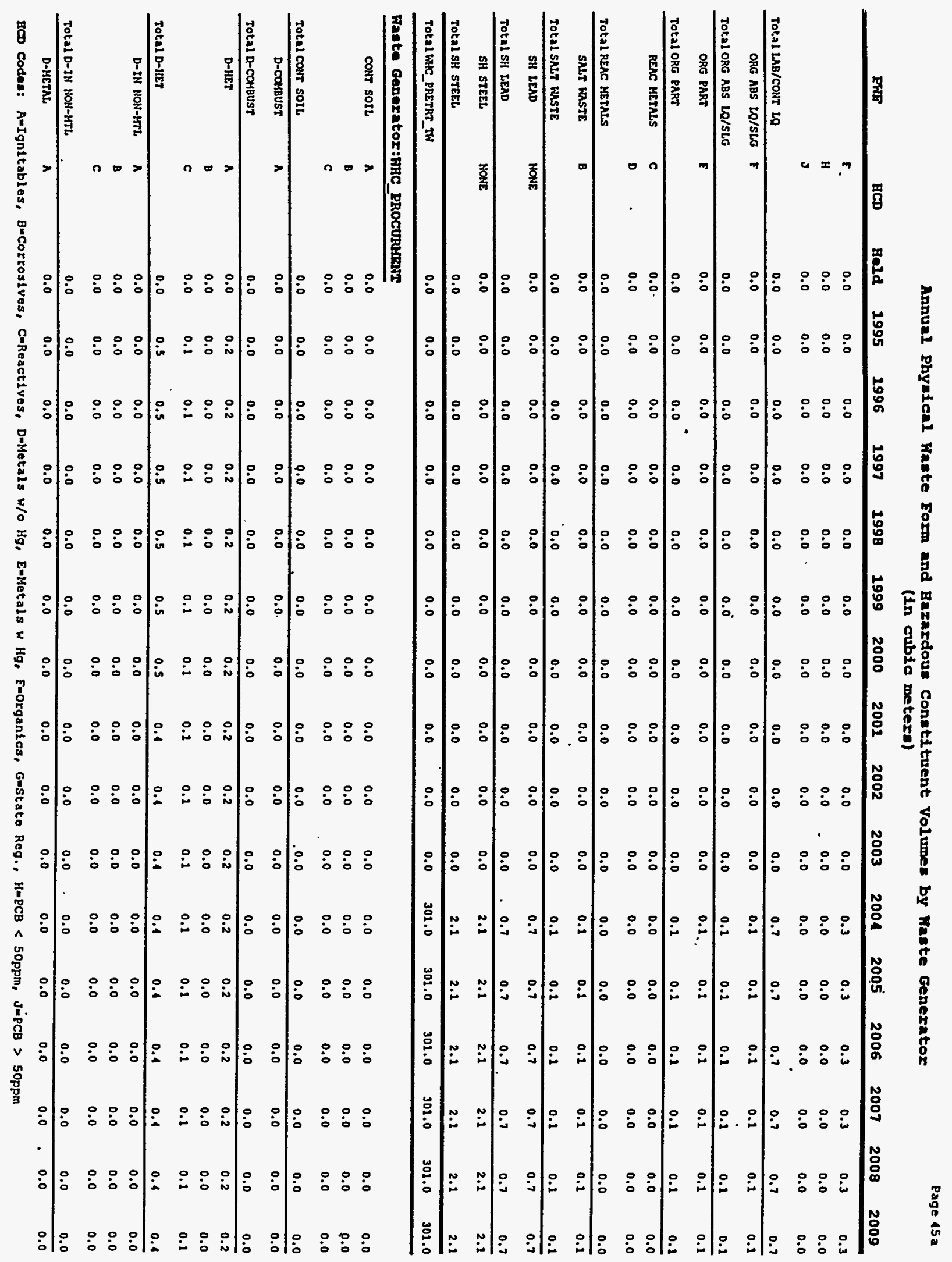



(in cubio meters)

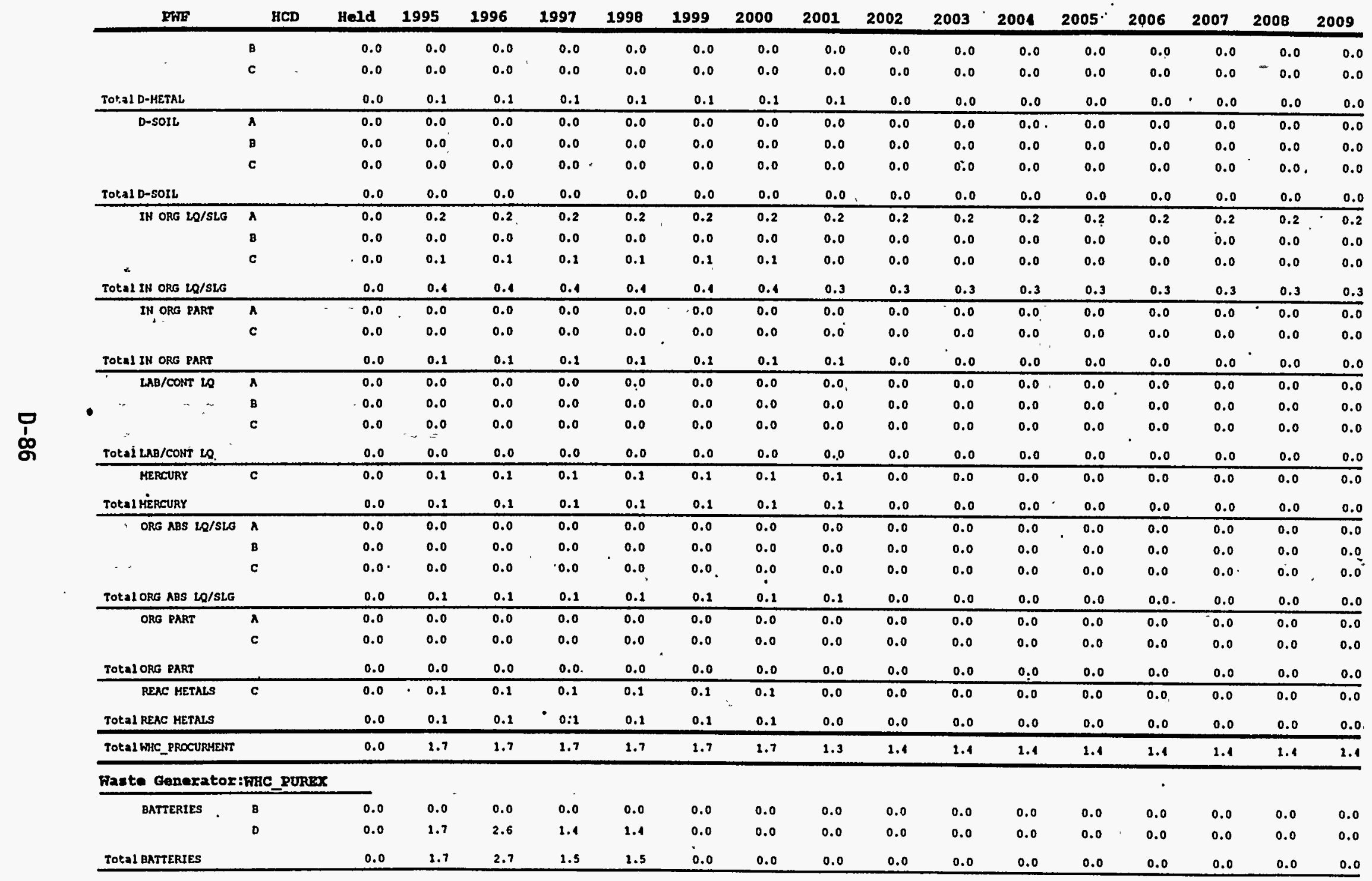

ECD Codas: A-Ignitables, B-Corrosives, C=Reactives, D-Metals w/O Hg, E-Matals w Hg, F-Organics, G-State Reg., H-PCB < 50ppm, J-PCB > 50ppm 


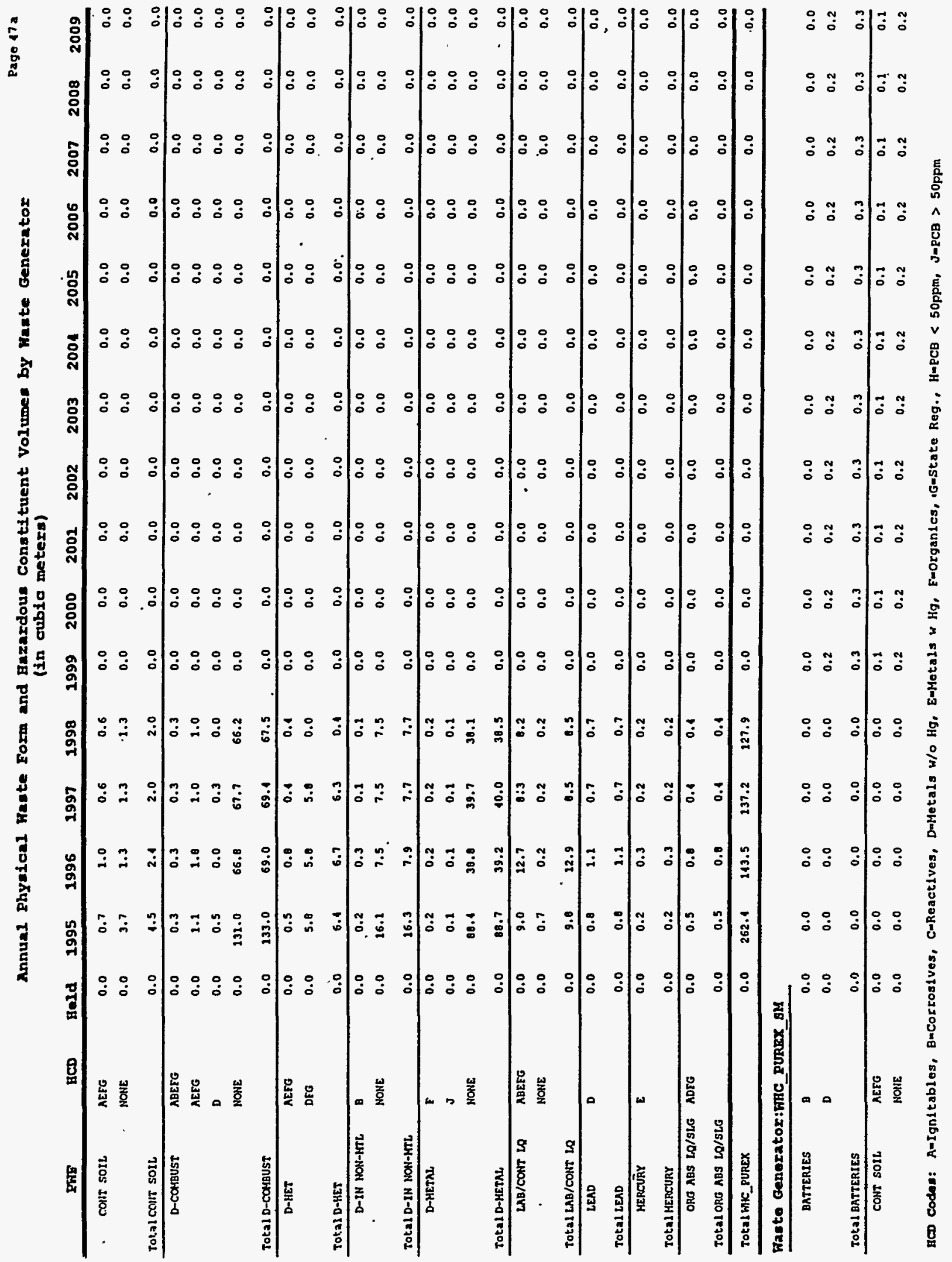


(in oublo metera)

\begin{tabular}{|c|c|c|c|c|c|c|c|c|c|c|c|c|c|c|c|c|c|}
\hline PHE & HCD & Held & 1995 & 1996 & 2997 & 1998 & 1999 & 2000 & 2001 & 2002 & 2003 & 2004 & $2005^{\circ}$ & 2006 & 2007 & 2008 & 2009 \\
\hline Tot.al COHT SOLL & & 0.0 & 0.0 & 0.0 & 0.0 & 0.0 & 0.1 & 0.4 & 0.4 & 0.4 & 0.1 & 0.1 & 0.1 & 0.1 & 0.1 & 0.4 & 0.1 \\
\hline \multirow[t]{3}{*}{ D-COHBUST } & ABEFG & 0.0 & 0.0 & 0.0 & 0.0 & 0.0 & 0.0 & 0.0 & 0.0 & 0.0 & 0.0 & 0.0 & 0.0 & 0.0 & 0.0 & 0.0 & 0.0 \\
\hline & AEEG & 0.0 & 0.0 & 0.0 & 0.0 & 0.0 & 0.2 & 0.2 & 0.2 & 0.2 & 0.2 & 0.2 & 0.2 & 0.2 & 0.2 & 0.2 & 0.2 \\
\hline & NONE & 0.0 & 0.0 & 0.0 & 0.0 & 0.0 & 13.2 & 13.2 & 13.2 & 13.2 & 13.2 & 13.2 & 13.2 & 23.2 & 13.2 & 13.2 & 13.2 \\
\hline Total D-COHBUST & & 0.0 & 0.0 & 0.0 & 0.0 & 0.0 & 13.5 & 13.5 & 13.5 & 13.5 & 13.5 & 13.5 & 13.5 & 13.5 & 13.5 & 13.5 & 23.5 \\
\hline D-HET & AEEG & 0.0 & 0.0 & 0.0 & 0.0 & 0.0 & 0.0 & 0.0 & 0.0 & 0.0 & 0.0 & 0.0 & 0.0 & 0.0 & 0.0 & 0.0 & 0.0 \\
\hline Total D-HET & & 0.0 & 0.0 & 0.0 & 0.0 & 0.0 & 0.0 & 0.0 & 0.0 & 0.0 & 0.0 & 0.0 & 0.0 & 0.0 & 0.0 & 0.0 & 0.0 \\
\hline \multirow[t]{2}{*}{ D-IN NON-HTL } & 8 & 0.0 & 0.0 & 0.0 & 0.0 & 0.0 & 0.0 & 0.0 & 0.0 & 0.0 & 0.0 & 0.0 & 0.0 & 0.0 & 0.0 & 0.0 & 0.0 \\
\hline & NONE ${ }^{\circ}$ & 0.0 & 0.0 & 0.0 & 0.0 & 0.0 & 1.5 & 1.5 & 1.5 & 1.5 & 1.5 & 1.5. & 1.5 & 1.5 & 1.5 & 1.5 & 1.5 \\
\hline Tota1 D-IH HON-HTL & & 0.0 & 0.0 & 0.0 & 0.0 & 0.0 & 1.5 & 1.5 & 1.5 & 1.5 & 1.5 & 1.5 & 1.5 & 1.5 & 1.5 & 1.5 & 1.5 \\
\hline \multirow[t]{3}{*}{ D-METAL } & F & 0.0 & 0.0 & 0.0 & 0.0 & 0.0 & 0.0 & 0.0 & 0.0 & 0.0 & 0.0 & 0.0 & 0.0 & 0.0 & 0.0 & 0.0 & 0.0 \\
\hline & J & 0.0 & 0.0 & 0.0 & 0.0 & 0.0 & 0.0 & 0.0 & 0.0 & 0.0 & 0.0 & 0.0 & 0.0 & 0.0 & 0.0 & 0.0 & 0.0 \\
\hline & NONE & 0.0 & 0.0 & 0.0 & 0.0 & 0.0 & 7.6 & 7.6 & 7.6 & 7.6 & 7.6 & 7.6 & 7.6 & 7.6 & 7.6 & 7.6 & 7.6 \\
\hline Total D-METAL & & 0.0 & 0.0 & 0.0 & 0.0 & 0.0 & 7.7 & 7.7 & 7.7 & 9.7 & 7.7 . & 7.7 & 7.7 & 7.7 & 7.7 & 7.7 & 7.7 \\
\hline \multirow[t]{2}{*}{ LAB/COHT LQ } & ABEFG & 0.0 & 0.0 & 0.0 & 0.0 & 0.0 & 1.6 & 1.6 & 1.6 & 1.6 & 1.6 & 1.6 & 1.6 & 1.6 & 1.6 & 2.6 & 1.6 \\
\hline & NONE & 0.0 & 0.0 & 0.0 & 0.0 & 0.0 & 0.0 & 0.0 & 0.0 & 0.0 & 0.0 & 0.0 & 0.0 & 0.0 & 0.0 & 0.0 & 0.0 \\
\hline Total LAD/CONT LQ & & 0.0 & 0.0 & 0.0 & 0.0 & 0.0 & 2.7 & 1.7 & 1.7 & 1.7 & 1.7 & 1.7 & 1.7 & 1.7 & 1.7 & 1.7 & 1.7 \\
\hline LEAD & $D$ & 0.0 & 0.0 & 0.0 & 0.0 & 0.0 & 0.1 & 0.1 & 0.1 & 0.1 & 0.1 & 0.1 & 0.1 & 0.1 & 0.1 & 0.1 & $\overline{0.1}$ \\
\hline TOtal LEAD & & 0.0 & 0.0 & 0.0 & 0.0 & 0.0 & 0.1 & 0.1 & 0.1 & 0.1 & 0.1 & 0.1 & 0.1 & 0.1 & 0.1 & 0.1 & 0.1 \\
\hline MEREURY & E & 0.0 & 0.0 & 0.0 & 0.0 & 0.0 & 0.0 & 0.0 & 0.0 & 0.0 & 0.0 & 0.0 & 0.0 & 0.0 & 0.0 & 0.0 & 0.0 \\
\hline TOta I HERCURY & & 0.0 & 0.0 & 0.0 & 0.0 & 0.0 & 0.0 & 0.0 & 0.0 & 0.0 & 0.0 & 0.0 & 0.0 & 0.0 & 0.0 & 0.0 & 0.0 \\
\hline ORG ABS LQ/SLG & ADEG & 0.0 & 0.0 & 0.0 & 0.0 & 0.0 & 0.0 & 0.0 & 0.0 & 0.0 & 0.0 & 0.0 & 0.0 & 0.0 & 0.0 & 0.0 & 0.0 \\
\hline TOtal ORG ABS LQ/SLG & & 0.0 & 0.0 & 0.0 & 0.0 & 0.0 & 0.0 & 0.0 & 0.0 & 0.0 & 0.0 & 0.0 & 0.0 & 0.0 & 0.0 & 0.0 & 0.0 \\
\hline TOtal WATC_PUREX_SH & & 0.0 & 0.0 & 0.0 & 0.0 & 0.0 & 25.5 & 25.5 & .25 .5 & 25.5 & 25.5 & 25.5 & 25.5 & 25.5 & 25.5 & 25.5 & 25.5 \\
\hline \multicolumn{18}{|c|}{ Faste Genorator:FHC_RADIO_kany } \\
\hline \multirow[t]{2}{*}{ D-conbust } & $\AA$ & 0.0 & 0.0 & 0.1 & 0.0 & 0.1 & 0.0 & 0.0 & 0.0 & 0.0 & 0.0 & 0.0 & 0.0 & 0.0 & 0.0 & 0.0 & 0.0 \\
\hline & $\mathbf{F}$ & 0.0 & 0.0 & 0.1 & 0.0 & 0.1 & 0.0 & 0.0 . & 0.0 & 0.0 & 0.0 & 0.0 & 0.0 & 0.0 & 0.0 & 0.0 & 0.0 \\
\hline Total D-carieust & & 0.0 & 0.0 & 0.2 & 0.0 & 0.2 & $0.0^{\circ}$ & 0.0 & 0.0 & 0.0 & 0.0 & 0.0 & 0.0 & 0.0 & 0.0 & 0.0 & 0.0 \\
\hline TOEal WHC_RADIO_MAIN & & 0.0 & 0.0 & 0.2 & 0.0 & 0.2 & 0.0 & 0.0 & 0.0 & 0.0 & 0.0 & 0.0 & 0.0 & 0.0 & 0.0 & 0.0 & 0.0 \\
\hline \multicolumn{18}{|c|}{ Waste Generator:FHC_soDIUM_EAC } \\
\hline D-combust & G & 0.0 & 0.0 & 0.7 & 0.4 & 0.0 & 0.0 & 0.0 & 0.0 & 0.0 & 0.0 & 0.0 & 0.0 & 0.0 & 0.0 & 0.0 & 0.0 \\
\hline & NONE & 0.0 & 0.0 & 0.5 & 0.2 & 0.0 & 0.0 & 0.0 & 0.0 & 0.0 & 0.0 & 0.0 & 0.0 & 0.0 & 0.0 & 0.0 & 0.0 \\
\hline
\end{tabular}

gCO Codes: A-Ignitables, B-Corrosives, C-Reactives, D-Metals w/O Hg, E-Metals $w$ Hg, E-Organics, G=State Reg., H=PCB < 50ppm, J-PCB > 50ppm 
(in cubic meters)

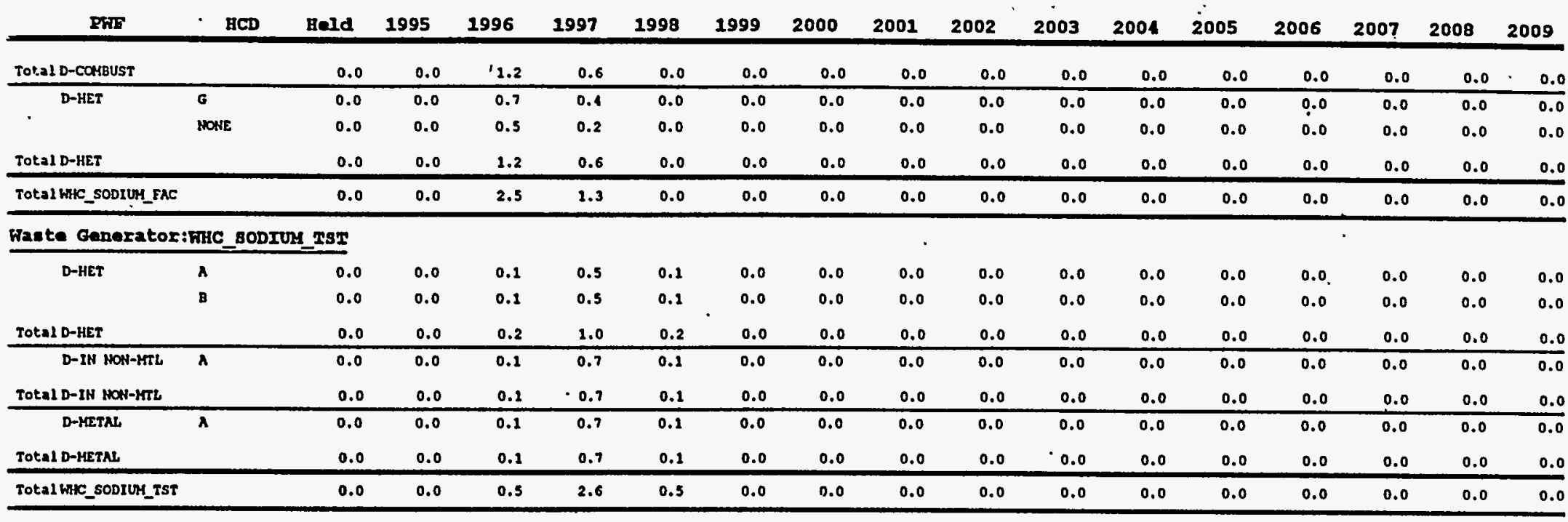

Fasto Generator:WHC_ssr_wre

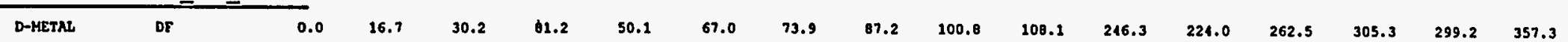

\begin{tabular}{|c|c|c|c|c|c|c|c|c|c|c|c|c|c|c|c|c|c|}
\hline Total D-METAL & & 0.0 & 16.7 & 30.2 & 01.2 & 50.1 & 67.0 & 73.9 & 87.2 & 100.8 & 108.1 & 246.3 & 224.0 & 262.5 & 305.3 & 299.2 & 357.3 \\
\hline SH STEEL & DF & 0.0 & 29.6 & 192.7 & 317.4 & 319.2 & 127.1 & 170.0 & 555.7 & 642.5 & 689.1 & 1568.8 & 1127.2 & 1671.9 & 1944.6 & 1906.3 & 2276.1 \\
\hline Total SH STEEL & & 0.0 & 29.6 & 192.7 & 517.1 & 319.2 & $\$ 27.1$ & 170.0 & 555.7 & 612.5 & 689.1 & 1560.0 & 1427.2 & 1671.9 & 1944.6 & 1906.3 & 2276.1 \\
\hline Total WHC_SST_LLE & & 0.0 & 46.4 & 223.0 & 598.7 & 369.4 & 194.2 & 514.8 & 643.0 & 713.1 & 797.3 & 1015.2 & 1651.3 & 1934.5 & 2250.0 & 2205.6 & 2633.5 \\
\hline
\end{tabular}

Waste Generator:HHC_ $88 \mathrm{R}$ RET

\begin{tabular}{|c|c|c|c|c|c|c|c|c|c|c|c|c|c|c|c|c|c|}
\hline CONT SOIL & DE & 0.0 & 0.0 & 0.0 & 0.6 & 0.0 & 0.0 & 0.0 & 0.0 & 0.0 & 0.0 & 1.9 & 1.9 & 2.6 & 3.2 & 3.2 & 4.5 \\
\hline otal CONT SOIL & & 0.0 & 0.0 & 0.0 & 0.6 & 0.0 & 0.0 & 0.0 & 0.0 & 0.0 & 0.0 & 1.9 & 1.9 & 2.6 & 3.2 & 3.2 & 1.5 \\
\hline \multirow[t]{2}{*}{ D-COMBUST } & DE & 0.0 & 0.0 & 0.0 & 4.9 & 0.0 & 0.0 & 0.0 & 0.0 & 0.0 & 0.0 & 14.8 & 14.0 & 19.7 & 24.6 & 24.6 & 34.5 \\
\hline & MONE & 0.0 & 0.0 & 0.0 & 1.5 & 0.0 & 0.0 & 0.0 & 0.0 & 0.0 & 0.0 & 4.7 & 4.7 & 6.3 & 7.9 & 7.9 & 11.1 \\
\hline otal D-COMBUST & & 0.0 & 0.0 & 0.0 & 6.5 & 0.0 & 0.0 & 0.0 & 0.0 & 0.0 & 0.0 & 19.6 & 19.6 & 26.1 & 32.6 & 32.6 & 15.7 \\
\hline D-HET & DE & 0.0 & 0.0 & 0.0 & 0.2 & 0.0 & 0.0 & 0.0 & 0.0 & 0.0 & 0.0 & 0.6 & 0.6 & 0.8 & 1.0 & 1.0 & $\overline{1.5}$ \\
\hline otal D HET & & 0.0 & 0.0 & 0.0 & 0.2 & 0.0 & 0.0 & 0.0 & 0.0 & 0.0 & 0.0 & 0.6 & 0.6 & 0.8 & 2.0 & 1.0 & 1.5. \\
\hline \multirow[t]{2}{*}{ D-IN NON-MTL } & DE & 0.0 & 0.0 & 0.0 & 0.5 & 0.0 & 0.0 & 0.0 & 0.0 & 0.0 & 0.0 & 1.5 & 1.5 & 2.0 & 2.5 & 2.5 & 3.5 \\
\hline & NONE & 0.0 & 0.0 & 0.0 & 0.7 & 0.0 & 0.0 & 0.0 & 0.0 & 0.0 & 0.0 & 2.3 & 2.3 & 3.1 & 3.9 & 3.9 & 5.5 \\
\hline Otal D-IN NON-MTL & & 0.0 & 0.0 & 0.0 & 1.3 & 0.0 & 0.0 & 0.0 & 0.0 & 0.0 & 0.0 & 3.9 & 3.9 & 5.2 & 6.5 & 6.5 & 9.1 \\
\hline
\end{tabular}

BCD Codes: A-Ignitables, B=Corrosives, C-Reactives, D-Metals w/O Hg, E-Metals w Hg, E-Organlcs, G=State Reg., H-PCB < 50ppm, JmPCB > 50ppm 


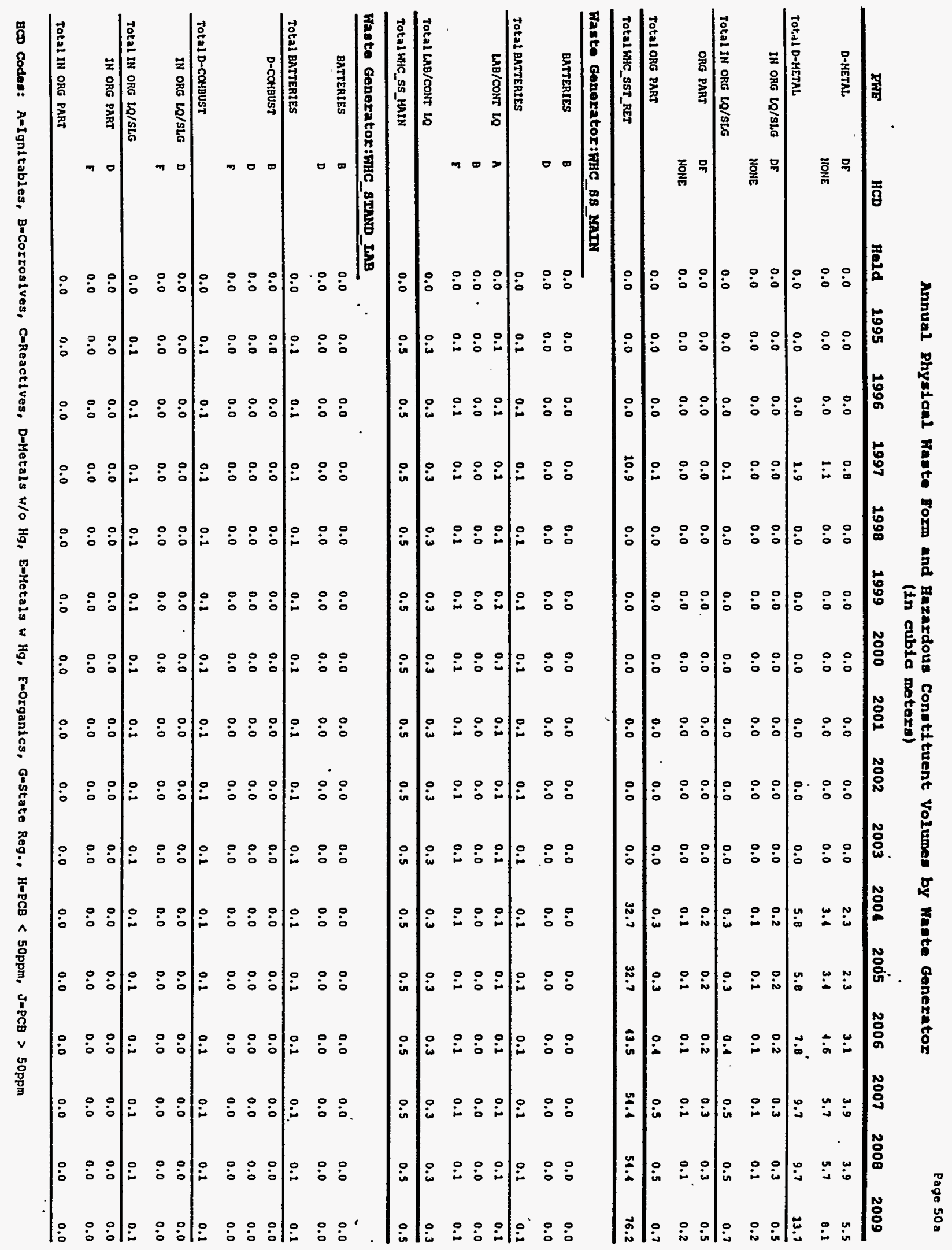




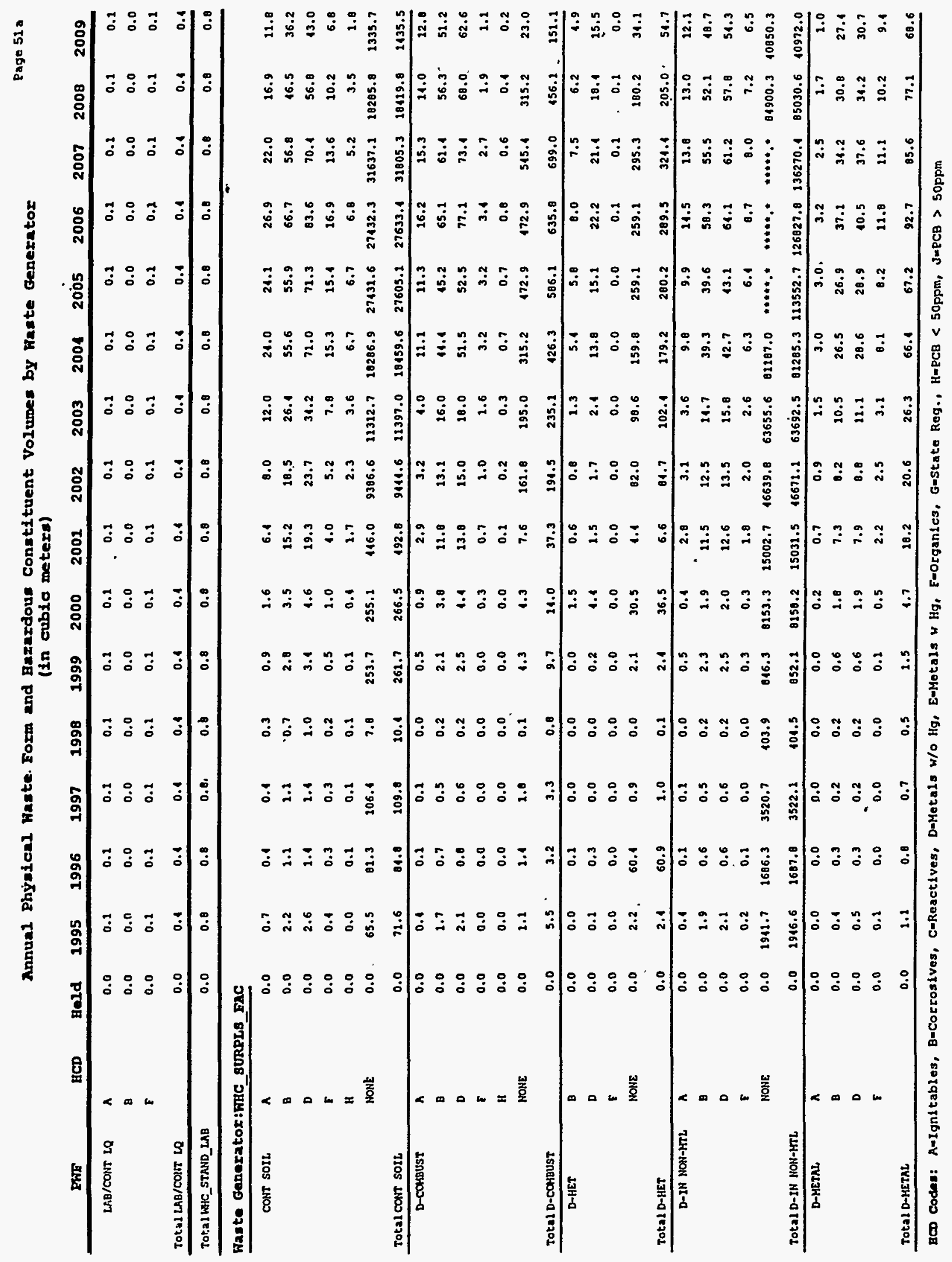



(in cubic metera)

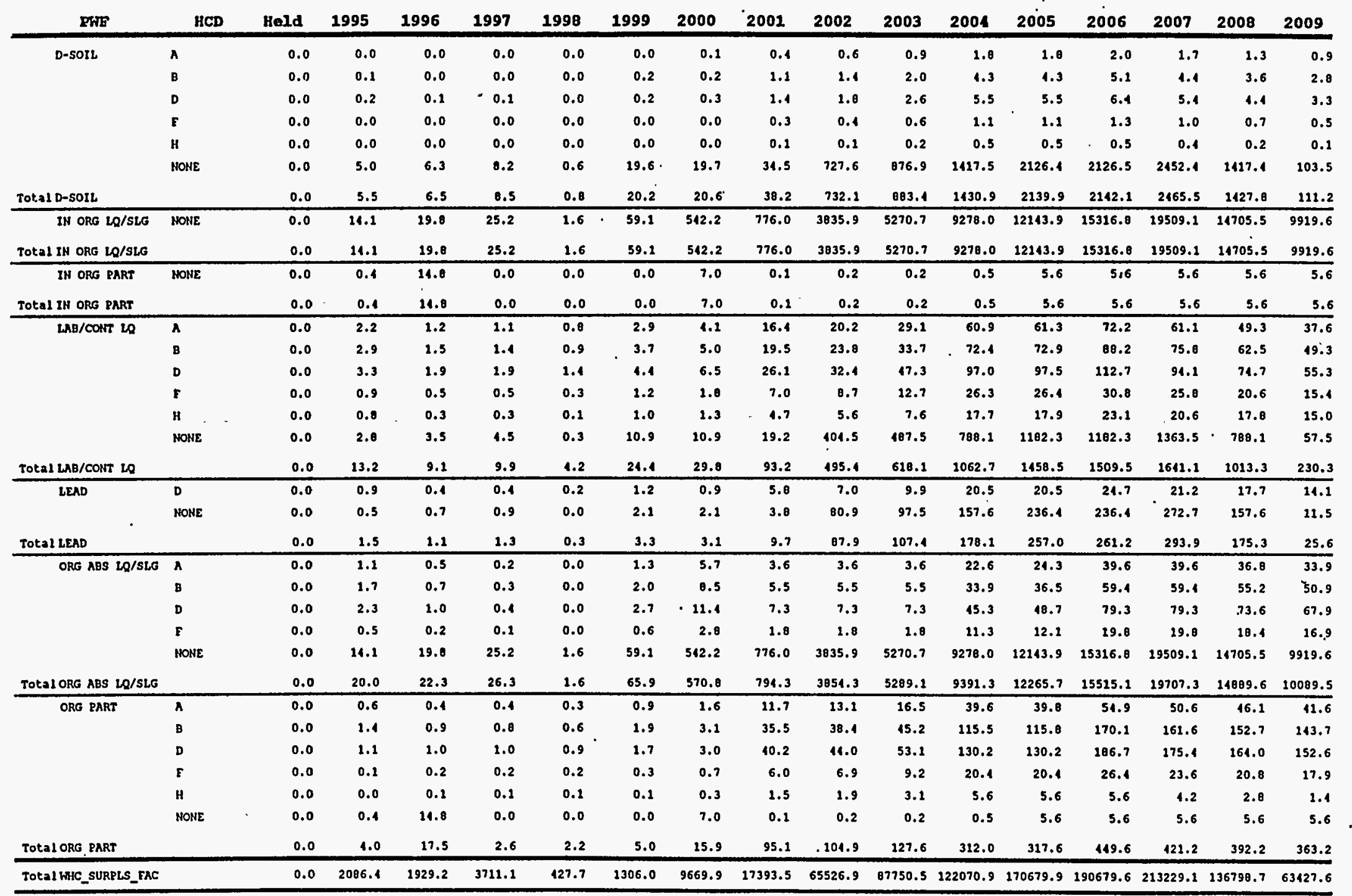


(in cubic meters)

\begin{tabular}{|c|c|c|c|c|c|c|c|c|c|c|c|c|c|c|c|c|c|}
\hline EWT & HCD & Held & 1995 & 1996 & 1997 & 1998 & 1999 & 2000 & 2001 & 2002 & 2003 & 2004 & 2005 & 2006 & 2007 & 2008 & 2009 \\
\hline \multicolumn{18}{|c|}{ Waste Generator:FHC_TS_OPBR } \\
\hline BATTERIES & BDE & 0.0 & 0.6 & 0.7 & 0.7 & 0.7 & 0.7 & 0.7 & 0.7 & 0.7 & 0.7 & 0.7 & 0.7 & 0.7 & 0.7 & 0.7 & 0.7 \\
\hline TUT:1 BATTERIES & & 0.0 & 0.6 & 0.1 & 0.7 & 0.7 & 0.7 & 0.7 & 0.7 & 0.7 & 0.7 & 0.7 & 0.7 & 0.7 & 0.7 & 0.7 & 0.7 \\
\hline \multirow[t]{2}{*}{ CONT SOIL } & AC & 0.0 & 2.8 & 3.0 & 3.0 & 3.0 & 3.0 & 3.0 & 3.0 & 3.0 & 3.0 & 3.0 & 3.0 & 3.0 & 3.0 & 3.0 & 3.0 \\
\hline & DF & 0.0 & 6.5 & 7.2 & 7.2 & 7.2 & 7.2 & 7.2 & 7.2 & 7.2 & 7.2 & 7.2 & 3.2 & 7.2 & 7.2 & 7.2 & 7.2 \\
\hline Total cont soll & & 0.0 & 9.3 & 10.3 & 10.3 & 10.3 & 10.3 & 10.3 & 10.3 & 10.3 & 10.3 & 10.3 & 10.3 & 10.3 & 10.3 & 10.3 & 20.3 \\
\hline \multirow[t]{3}{*}{ D-COASBUST } & ABDE & 0.0 & 6.8 & 7.5 & 7.5 & 7.5 & 7.5 & 7.5 & 7.5 & 7.5 & 7.5 & 7.5 & 7.5 & 7.5 & 7.5 & 7.5 & 7.5 \\
\hline & Dr & 0.0 & 68.4 & 75.2 & 75.2 & 75.2 & 75.2 & 75.2 & 75.2 & 75.2 & 75.2 & 75.2 & 75.2 & 75.2 & 75.2 & 75.2 & 75.2 \\
\hline & MONE & 0.0 & 251.3 & 166.3 & 365.3 & 166.3 & 166.3 & 166.3 & 266.3 & 368.3 & 166.3 & 166.3 & 166.3 & 166.3 & 366.3 & 166.3 & 166.3 \\
\hline Total D-Carbust & & 0.0 & 226.4 & 249.1 & 249.1 & 249.1 & 219.1 & 249.1 & 269.1 & 249.1 & 219.1 & 249.1 & 249.1 & 249.1 & 249.1 & 219.1 & 249.1 \\
\hline D-HET & DF & 0.0 & 4.1 & 1.8 & 4.0 & 1.8 & 4.8 & 1.8 & 4.8 & 4.0 & 1.8 & 1.8 & 4.8 & 1.8 & 1.0 & 1.8 & 1.8 \\
\hline Total D-HET & & 0.0 & 1.4 & 4.0 & 1.8 & 1.0 & 1.8 & 1.8 & 1.8 & 4.8 & 4.8 & 4.8 & 1.0 & 1.0 & 4.8 & 4.6 & 1.0 \\
\hline \multirow[t]{3}{*}{ D-IN MOH-MTL } & AC & 0.0 & 2.3 & 2.5 & 2.5 & 2.5 & 2.5 & 2.5 & 2.5 & 2.5 & 2.5 & 2.5 & 2.5 & 2.5 & 2.5 & 2.5 & 2.5 \\
\hline & DF & 0.0 & 31.0 & 35.0 & 35.0 & 35.0 & 35.0 & 35.0 & 35.0 & 35.0 & 35.0 & 33.0 & 35.0 & 35.0 & 35.0 & 35.0 & 35.0 \\
\hline & HONE & 0.0 & 47.3 & 52.0 & 32.0 & 52.0 & 52.0 & 52.0 & 32.0 & 52.0 & 52.0 & 52.0 & 52.0 & 52.0 & 52.0 & 52.0 & 32.0 \\
\hline Total D-IN MON-MTL & & 0.0 & 01.5 & 89.7 & 09.7 & 89.7 & 09.7 & 09.7 & 09.7 & 89.7 & 89.7 & 89.7 & 09.7 & 89.7 & 89.7 & 89.7 & 89.7 \\
\hline \multirow[t]{2}{*}{ DEMETAL } & DE & 0.0 & 50.2 & 55.2 & 35.2 & 35.2 & 35.2 & 55.2 & 55.2 & 55.2 & 55.2 & 55.2 & 55.2 & 55.2 & 55.2 & 55.2 & .55 .2 \\
\hline & MONE & 0.0 & 68.8 & 75.7 & 75.7 & 75.7 & 75.7 & 75.7 & 75.7 & 75.7 & 75.7 & 75.7 & 75.7 & 75.7 & 75.7 & 75.7 & 75.7 \\
\hline Total D-METAL & & 0.0 & 119.0 & 130.9 & 130.9 & 230.9 & 130.9 & 130.9 & 130.9 & 130.9 & 230.9 & 130.9 & 130.9 & 130.9 & 130.9 & 130.9 & $130: 9$ \\
\hline EXPLOSIVES & ABDF & 0.0 & 2.0 & 2.2 & 2.2 & 2.2 & 2.2 & 2.2 & 2.2 & 2.2 & 2.2 & 2.2 & 2.2 & 2.2 & 2.2 & 2.2 & 2.2 \\
\hline Totel EXPLOSIVES & & 0.0 & 2.0 & 2.2 & 2.2 & 2.2 & 2.2 & 2.2 & 2.2 & 2.2 & $2: 2$ & 2.2 & 2.2 & 2.2 & 2.2 & 2.2 & 2.2 \\
\hline \multirow[t]{3}{*}{ IN ORG LQ/SLG } & ABDF & 0.0 & 3.4 & 3.7 & 3.7 & 3.7 & 3.7 & 3.7 & 3.7 & 3.7 & 3.7 & 3.7 & 3.7 & 3.7 & 3.7 & 3.7 & 3.7 \\
\hline & DF & 0.0 & 1.7 & 1.8 & 1.8 & 1.0 & 1.0 & 1.0 & 1.8 & 1.8 & 1.0 & 1.8 & 1.8 & 1.8 & 2.6 & 1.0 & 1.8 \\
\hline & NONE & 0.0 & 2.1 & 2.3 & 2.3 & 2.3 & 2.3 & 2.3 & 2.3 & 2.3 & 2.3 & 2.3 & 2.3 & 2.3 & 2.3 & 2.3 & 2.3 \\
\hline TOtAL IN ORG LQ/SLG & & 0.0 & 7.2 & 0.0 & 0.0 & 8.0 & 8.0 & 0.0 & 8.0 & 8.0 & 8.0 & 8.0 & 0.0 & 0.0 & 0.0 & 0.0 & 8.0 \\
\hline LAB/CONT LQ & $A B D F$ & 0.0 & 12.0 & 13.2 & 23.2 & 13.2 & 13.2. & 13.2 & 23.2 & 13.2 & 13.2 & 13.2 & 13.2 & 13.2 & 13.2 & 13.2 & 13.2 \\
\hline TOtal LAB/CONT LQ & & 0.0 & 12.0 & 13.2 & 13.2 & 13.2 & 13.2 & 13.2 & 13.2 & 13.2 & 13.2 & 13.2 & 13.2 & 13.2 & 13.2 & 13.2 & 13.2 \\
\hline LEAD & ADF & 0.0 & 0.3 & 0.3 & 0.3 & 0.3 & 0.3 & 0.3 & 0.3 & 0.3 & 0.3 & 0.3 & 0.3 & 0.3 & 0.3 & 0.3 & 0.3 \\
\hline TOtal LEAD & & 0.0 & 0.3 & 0.3 & 0.3 & 0.3 & 0.3 & 0.3 & 0.3 & 0.3 & 0.3 & 0.3 & 0.3 & 0.3 & 0.3 & 0.3 & 0.3 \\
\hline MERCURY & $\varepsilon$ & 0.0 & 0.3 & 0.3 & 0.3 & 0.3 & 0.3 & 0.3 & 0.3 & 0.3 & 0.3 & 0.3 & 0.3 & 0.3 & 0.3 & 0.3 & 0.3 \\
\hline Total MERCURY & & 0.0 & 0.3 & 0.3 & 0.3 & 0.3 & 0.3 & 0.3 & 0.3 & 0.3 & 0.3 & 0.3 & 0.3 & 0.3 & 0.3 & 0.3 & 0.3 \\
\hline ORG ABS LQ/SLG & ABDF & 0.0 & 6.8 & 7.5 & 7.5 & 7.5 & 7.5 & 7.5 & 7.5 & 7.5 & 7.5 & 7.5 & .7 .5 & 7.5 & 7.5 & 7.5 & 7.5 \\
\hline TOtal ORG ABS LQ/SLG & & 0.0 & 6.8 & 7.5 & 7.5 & 7.5 & 7.5 & 7.5 & 7.5 & 7.5 & 7.5 & 7.5 & 7.5 & 7.5 & 7.5 & 7.5 & 1.5 \\
\hline ORG PART & $A B D F$ & 0.0 & 1.7 & 1.8 & 1.0 & 1.8 & 1.8 & 1.8 & 1.0 & 1.8 & 1.8 & 1.8 & 1.8 & 1.8 & 1.8 & 1.8 & 1.8 \\
\hline
\end{tabular}

zoo Codes: A-Ignitables, B-Corrosives, C=Reactlves, DeMetals w/O Hg, E-Metals w Hg, F-Organles, G=State Reg., H-PCB < 50ppm, J-PCB > 50ppm 
(In cubia metera)

\begin{tabular}{|c|c|c|c|c|c|c|c|c|c|c|c|c|c|c|c|c|c|}
\hline PFE & HCD & Hold & 1995 & 2996 & 1997 & 1998 & 1999 & 2000 & 2001 & 2002 & 2003 & 2004 & $2005^{\circ}$ & 2006 & 2007 & 2008 & 2009 \\
\hline & DF & 0.0 & 1.7 & 1.0 & 1.0 & 2.8 & 1.8 & 1.8 & 1.8 & 1.8 & 1.8 & 1.8 & 1.0 & 1.8 & 1.8 & 1.0 & 1.8 \\
\hline & NONE & 0.0 & 2.1 & 2.3 & 2.3 & 2.3 & 2.3 & 2.3 & 2.3 & 2.3 & 2.3 & 2.3 & 2.3 & 2.3 & 2.3 & 2.3 & 2.3 \\
\hline TEt:Al ORG PART & & 0.0 & 5.5 & 6.1 & 6.1 & 6.1 & 6.1 & 6.1 & 6.1 & 6.1 & 6.1 & 6.1 & 6.1 & 6.1 & 6.1 & 6.1 & 6.1 \\
\hline TOt. I WAC_TE_OPER & & 0.0 & $\$ 76.0$ & 523.7 & 523.7 & 523.7 & 523.7 & 523.7 & 523.7 & 523.7 & 523.7 & 523.7 & 523.7 & 523.7 & 523.7 & 523.7 & 523.7 \\
\hline \multicolumn{18}{|c|}{ Waste Generator:WHC_IRANSPORI } \\
\hline \multirow[t]{2}{*}{ D-Condust } & $\Lambda G$ & 0.0 & 0.5 & 0.5 & 0.5 & 0.5 & 0.5 & 0.5 & 0.5 & 0.5 & 0.5 & 0.5 & 0.5 & 0.3 & 0.5 & 0.5 & 0.5 \\
\hline & G & 0.0 & 0.7 & 0.7 & 0.7 & .0 .7 & 0.7 & 0.7 & 0.7 & 0.7 & 0.7 & 0.7 & 0.7 & 0.7 & 0.7 & 0.7 & 0.7 \\
\hline Total D-CoMBust & & 0.0 & 1.2 & 1.2 & 1.2 & 1.2 & 1.2 & 1.2 & 1.2 & 1.2 & 1.2 & 1.2 & 1.2 & 1.2 & 1.2 & 1.2 & 1.2 \\
\hline \multirow[t]{2}{*}{ IN ORG PART } & AG & 0.0 & 0.2 & 0.2 & 0.2 & 0.2 & 0.2 & 0.2 & 0.2 & 0.2 & 0.2 & 0.2 & 0.2 & 0.2 & 0.2 & 0.2 & $\overline{0.2}$ \\
\hline & G & 0.0 & 0.3 & 0.3 & 0.3 & 0.3 & 0.3 & 0.3 & 0.3 & 0.3 & 0.3 & 0.3 & 0.3 & 0.3 & 0.3 & 0.3 & 0.3 \\
\hline Total IN ORG PART & & 0.0 & 0.5 & 0.5 & 0.5 & 0.5 & 0.5 & 0.5 & 0.5 & 0.5 & 0.5 & 0.5 & 0.5 & 0.5 & 0.5 & 0.5 & 0.5 \\
\hline Total WHC_IRANSPORT & & 0.0 & 1.0 & 1.6 & 1.8 & 1.0 & 1.8 & 1.8 & 1.0 & 1.8 & 1.8 & 2.8 & 1.0 & 1.0 & 1.0 & 1.0 & 1.8 \\
\hline \multicolumn{18}{|c|}{ Waste Generator:WHC_TRUSAr } \\
\hline D-carbust & NONE, & 0.0 & 0.5 & 2.0 & 6.2 & 0.0 & 0.0 & 0.0 & 0.0 & 0.0 & 0.0 & 0.0 & $0: 0$ & 0.0 & 0.0 & 0.0 & 0.0 \\
\hline Tota1 D-COABUST & & 0.0 & 0.5 & 2.0 & 6.2 & 0.0 & 0.0 & 0.0 & 0.0 & 0.0 & 0.0 & 0.0 & 0.0 & 0.0 & 0.0 & 0.0 & 0.0 \\
\hline \multirow[t]{2}{*}{ D-HET } & G & 0.0 & 0.0 & 0.0 & 2.9 & 0.0 & 0.0 & 0.0 & 0.0 & 0.0 & 0.0 & 0.0 & 0.0 & 0.0 & 0.0 & 0.0 & 0.0 \\
\hline & NONE & 0.0 & 0.2 & 0.0 & 0.2 & 0.0 & 0.0 & 0.0 & 0.0 & 0.0 & $\cdot 0.0$ & 0.0 & 0.0 & 0.0 & 0.0 & 0.0 & 0.0 \\
\hline Total D-HET & & 0.0 & 0.2 & 0.0 & 3.1 & 0.0 & 0.0 & 0.0 & 0.0 & 0.0 & 0.0 & 0.0 & 0.0 & 0.0 & 0.0 & 0.0 & 0.0 \\
\hline D-HETAL & NONE & 0.0 & 0.3 & 0.0 & 0.3 & 0.0 & 0.0 & 0.0 & 0.0 & 0.0 & 0.0 & 0.0 & 0.0 & 0.0 & 0.0 & 0.0 & 0.0 \\
\hline Total D-METAL & & 0.0 & 0.3 & 0.0 & 0.3 & 0.0 & 0.0 & 0.0 & 0.0 & 0.0 & 0.0 & 0.0 & 0.0 & 0.0 & 0.0 & 0.0 & 0.0 \\
\hline TOtal WHAC_TRUSAF & & 0.0 & 1.0 & 2.0 & 9.7 & 0.0 & 0.0 & 0.0 & 0.0 & 0.0 & 0.0 & 0.0 & 0.0 & 0.0 . & 0.0 & 0.0 & 0.0 \\
\hline \multicolumn{3}{|c|}{ Waste Generator:FHC_THR_F028 } & & & & & & & & & & & & & - & & \\
\hline cont sorl & MONE & 0.0 & 0.0 & 2.9 & 2.9 & 0.0 & 0.0 & 0.0 & 0.0 & 0.0 & 0.0 & 0.0 & 0.0 & 0.0 & 0.0 & 0.0 & 0.0 \\
\hline Total cont solt & & 0.0 & 0.0 & 2.9 & 2.9 & 0.0 & 0.0 & 0.0 & 0.0 & 0.0 & 0.0 & 0.0 & 0.0 & 0.0 & 0.0 & 0.0 & 0.0 \\
\hline D-METAL & HONE & 0.0 & 0.0 & 0.7 & 0.7 & $0.0^{\circ}$ & 0.0 & 0.0 & 0.0 & 0.0 & 0.0 & 0.0 & 0.0 & 0.0 & 0.0 & 0.0 & 0.0 \\
\hline TOtal D-METAL & & 0.0 & 0.0 & 0.7 & 0.7 & 0.0 & 0.0 & 0.0 & 0.0 & 0.0 & 0.0 & 0.0 & 0.0 & 0.0 & 0.0 & 0.0 & 0.0 \\
\hline TOta1 KHE_TWP_WO2B & & 0.0 & 0.0 & 3.7 & 3.7 & 0.0 & 0.0 & 0.0 & 0.0 & 0.0 & 0.0 & 0.0 & 0.0 & 0.0 & 0.0 & 0.0 & 0.0 \\
\hline \multicolumn{18}{|c|}{ Waste Generator:WHC_IFP_W030 } \\
\hline cont soIL & NONE & 0.0 & 4.5 & 3.5 & 1.8 & 0.0 & 0.0 & 0.0 & 0.0 & 0.0 & 0.0 & 0.0 & 0.0 & 0.0 & 0.0 & 0.0 & 0.11 \\
\hline Total CONT SOIL & & 0.0 & 4.5 & 3.5 & 1.8 & 0.0 & 0.0 & 0.0 & 0.0 & 0.0 & 0.0 & 0.0 & 0.0 & 0.0 & 0.0 & 0.0 & 0.0 \\
\hline D-CONBUST & NONE & 0.0 & 1.6 & 1.5 & 1.0 & 0.0 & 0.0 & 0.0 & 0.0 & 0.0 & 0.0 & 0.0 & 0.0 & 0.0 & 0.0 & 0.0 & $\overline{0.0}$ \\
\hline
\end{tabular}

BCD Coden: A-Ignitables, BuCorrosives, CmReactives, Dmetals w/O Hg, Emetals w Hg, Fuorganlcs, GmState Reg.. H-PCB < 50ppm, J-PCB > 50ppm 
(in cubic meters)

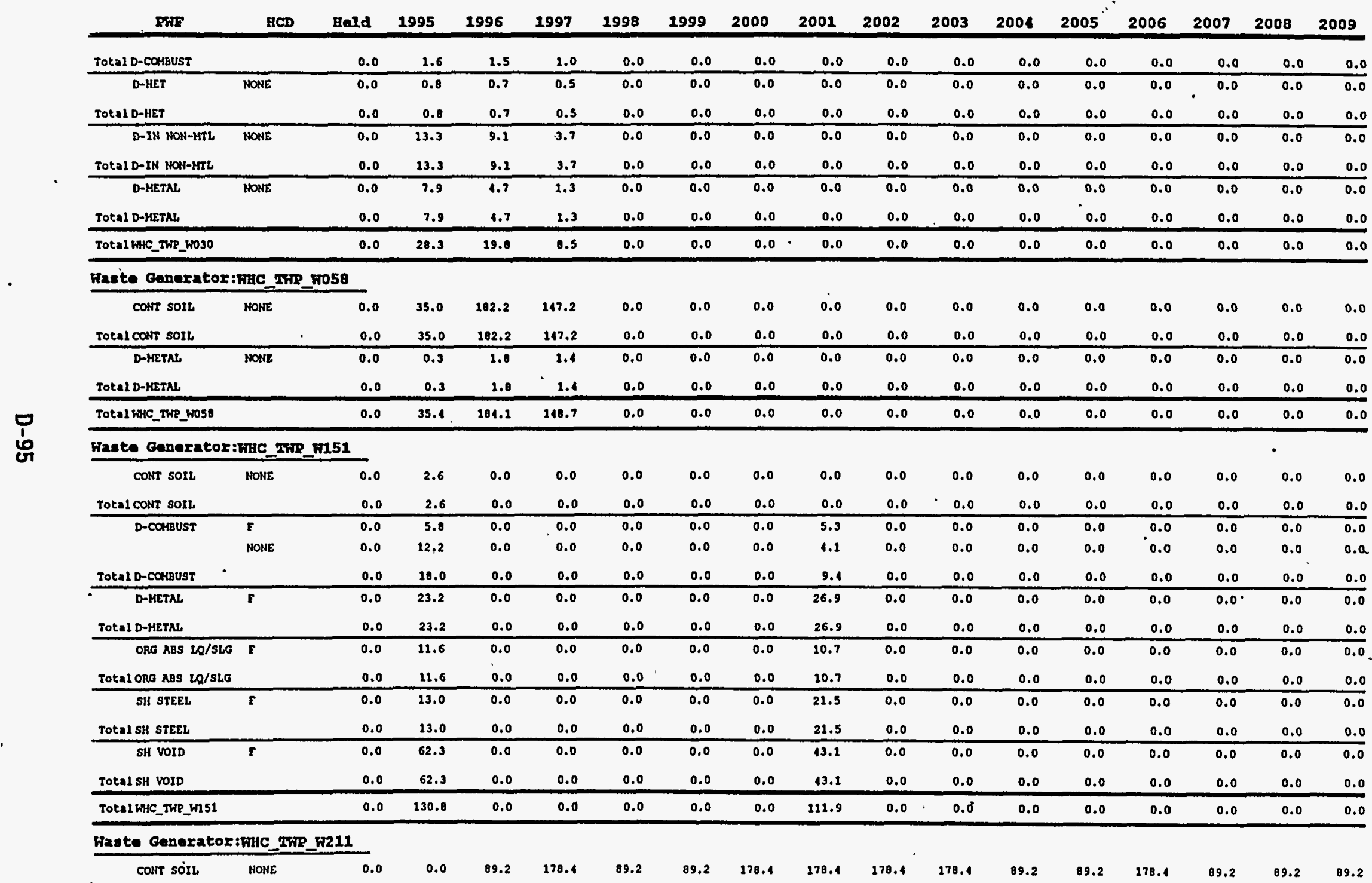

Had Codes: A-Ignitables, B-Corrosives, C=Reactives, D-Metals w/O Hg, E-Motals w Hg, F-Organles, G-State Reg., H-PCB < 50ppm, J-PCB > 50ppm 
(in cublo meters)

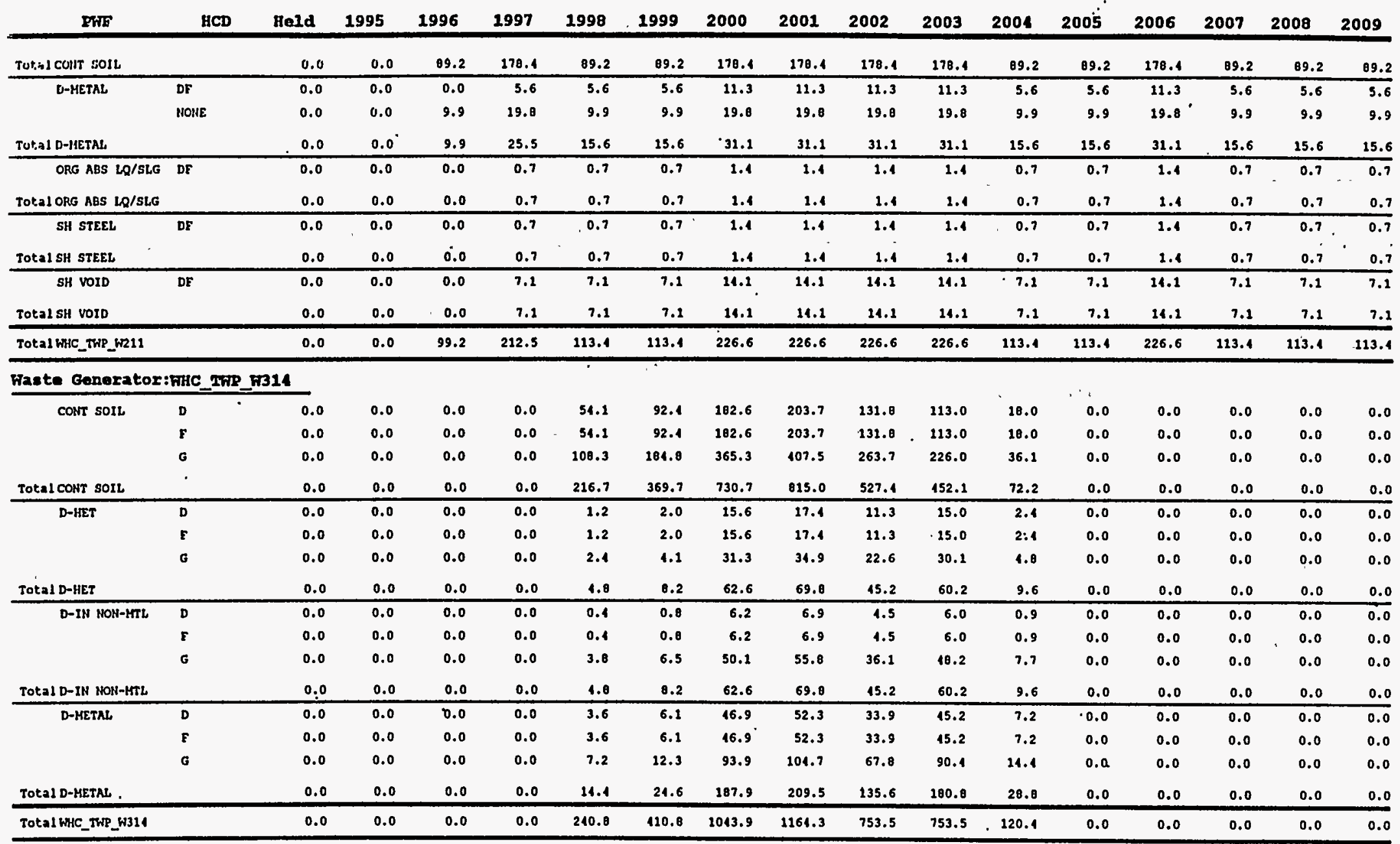

\section{Haste Generatox:WHC_THP_F320}

\begin{tabular}{|c|c|c|c|c|c|c|c|c|c|c|c|c|c|c|c|c|c|}
\hline Cont soll & HONE & 0.0 & 3.2 & 2.2 & 3.2 & 3.0 & 0.0 & 0.0 & 0.0 & 0.0 & 0.0 & 0.0 & 0.0 & 0.0 & 0.0 & 0.0 & 0.0 \\
\hline tal CONT SOIL & & 8.0 & 3.2 & 2.2 & 3.2 & 3.0 & 0.0 & 0.0 & 0.0 & 0.0 & 0.0 & 0.0 & 0.0 & 0.0 & 0.0 & 0.0 & 0.0 \\
\hline D-cambust & D & 0.0 & 12.0 & 0.7 & 60.1 & 7.3 & 0.0 & $0: 0$ & 0.0 & 0.0 & 0.0 & 0.0 & 0.0 & 0.0 & 0.0 & 0.0 & 0.0 \\
\hline
\end{tabular}

BCD Codos: A-Ignitables, B-Corrosives, C=Reactives, D-Metals w/O Hg, E-Metals w Hg, F-Organics, G=State Reg., H=PCB < 50ppm, J-PCB > 50ppm 


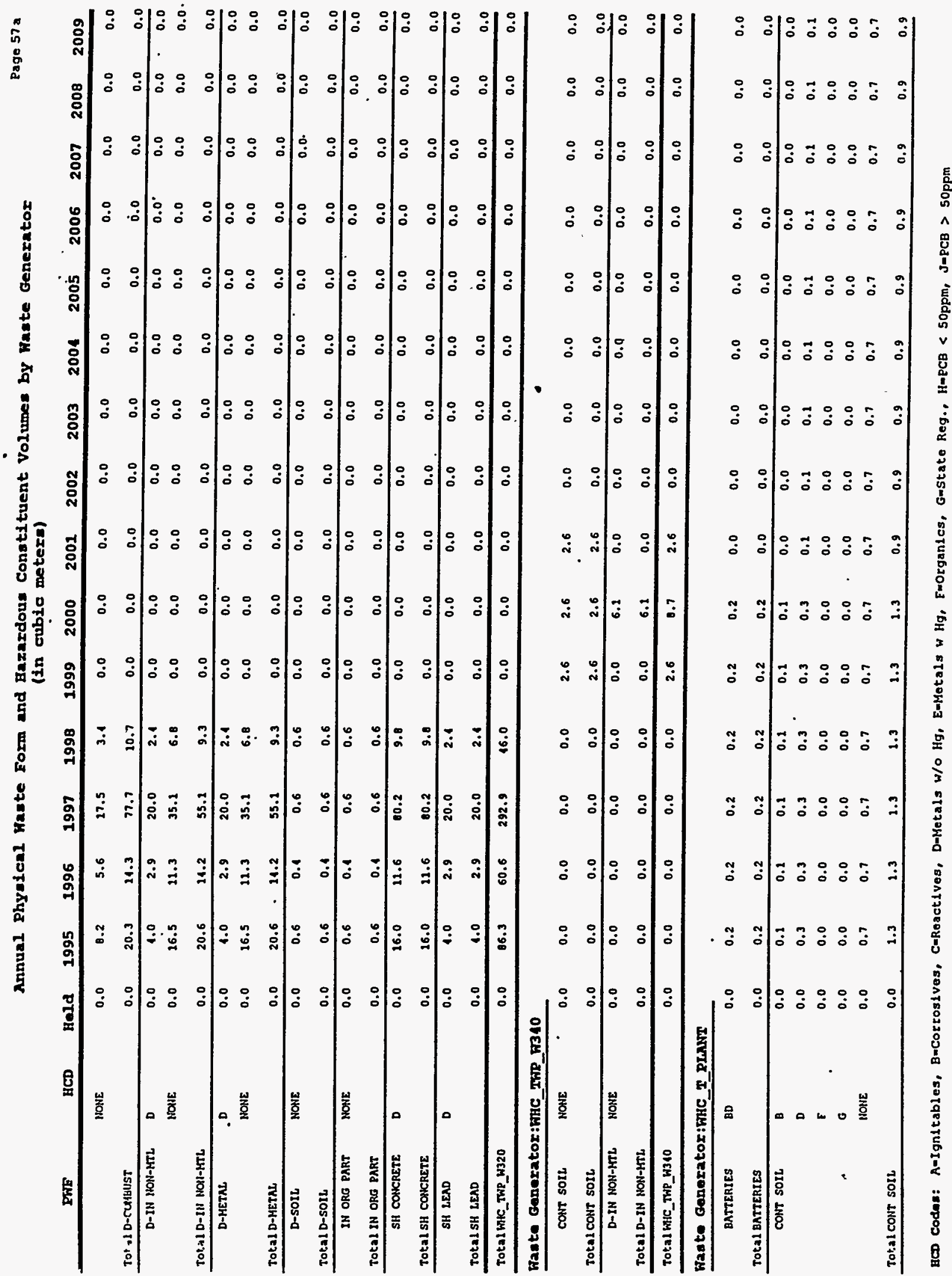




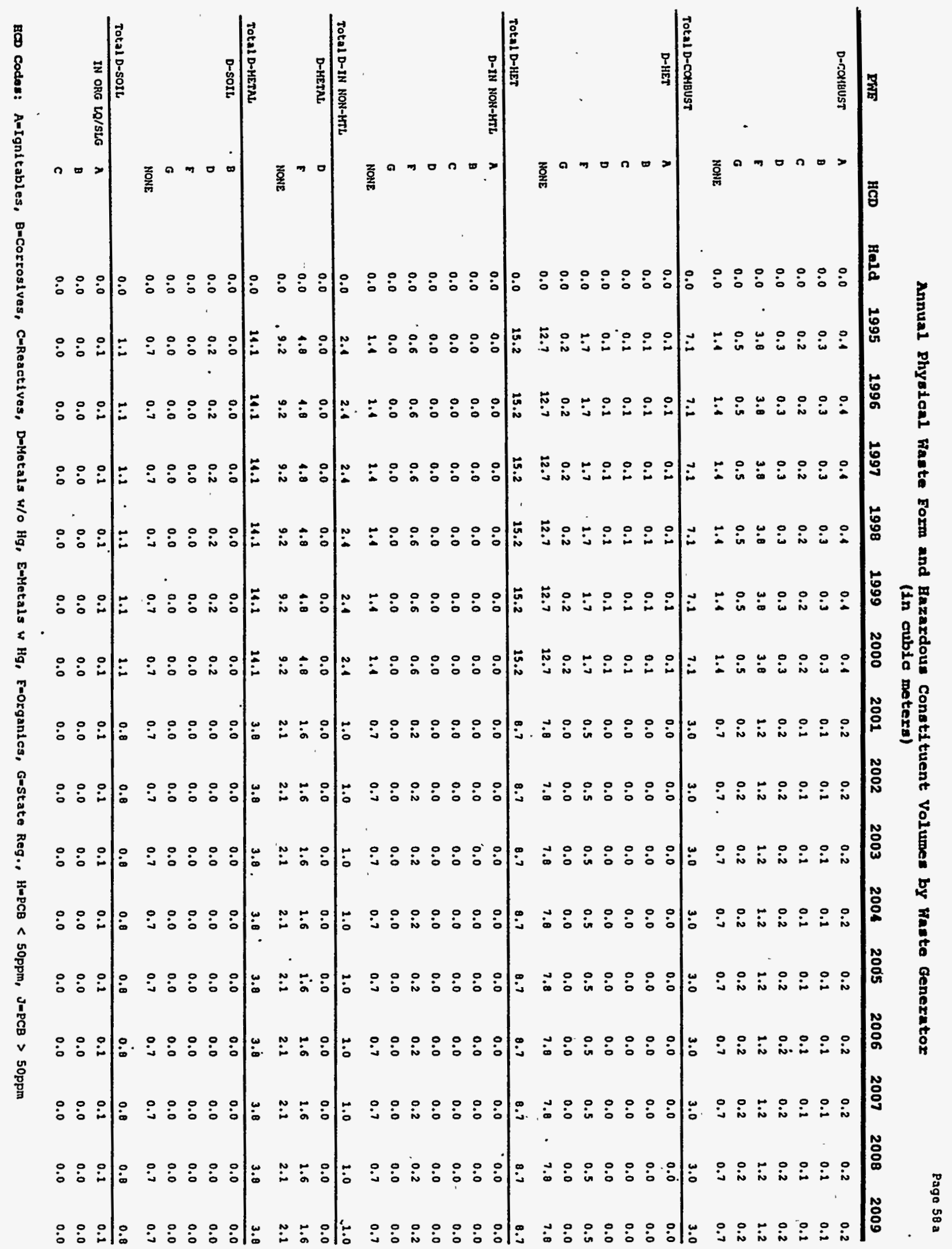




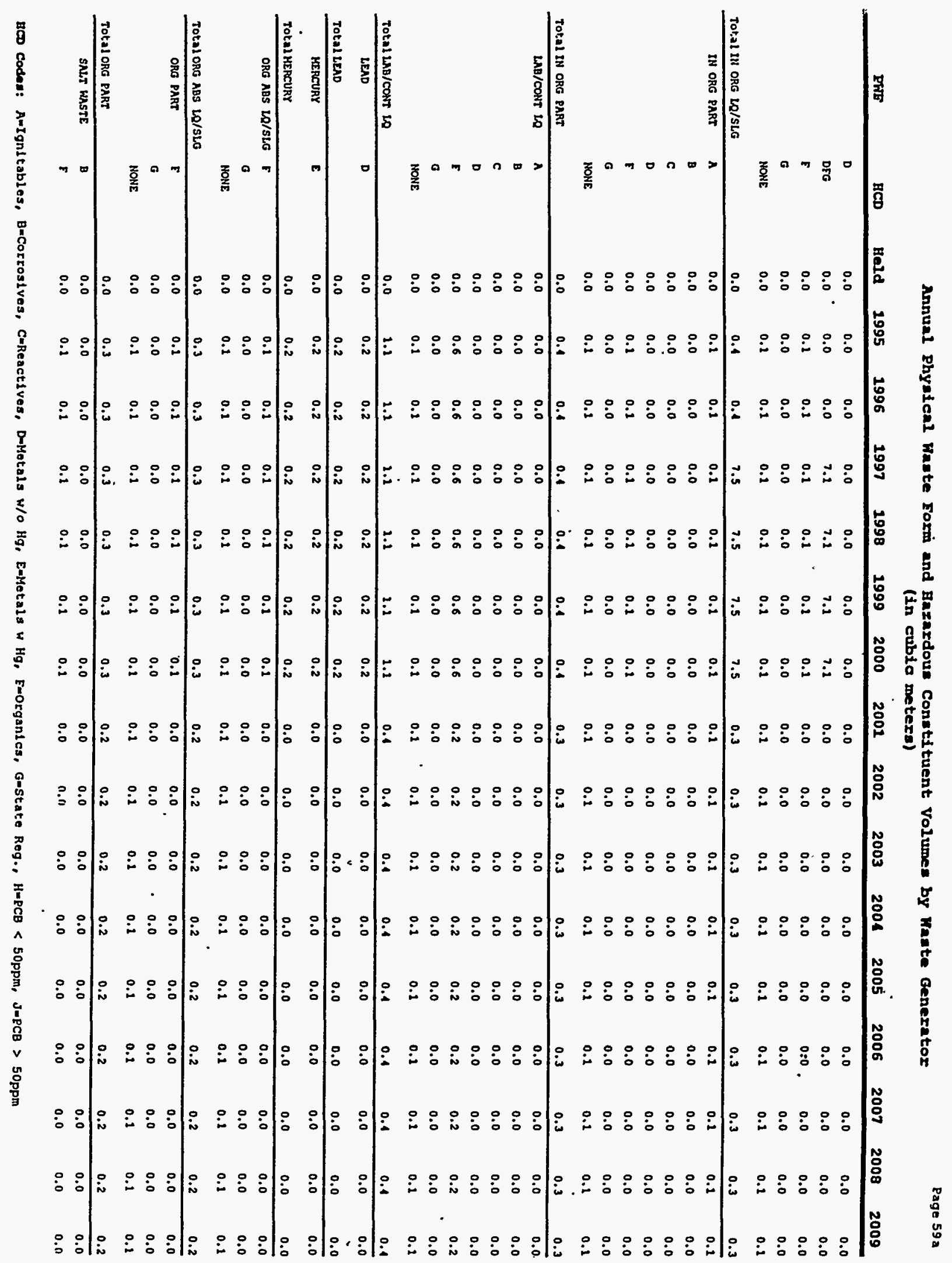


(In cubia metera)

\begin{tabular}{|c|c|c|c|c|c|c|c|c|c|c|c|c|c|c|c|c|c|}
\hline PWE & HCD & Held & 1995 & 1996 & 1997 & 1998 & 1999 & 2000 & 2001 & 2002 & 2003 & 2004 & 2005 & 2006 & 2007 & 2008 & 2009 \\
\hline TOtal SALT WASTE & & 0.0 & 0.1 & 0.1 & 0.1 & 0.1 & 0.1 & 0.1 & 0.0 & 0.0 & 0.0 & 0.0 & 0.0 & 0.0 & 0.0 & 0.0 & 0.0 \\
\hline SH LEAD & D & 0.0 & 1.0 & 1.0 & 1.0 & 1.0 & 1.0 & 1.0 & 0.3 & 0.3 & 0.3 & 0.3 & 0.3 & 0.3 & 0.3 & 0.3 & 0.3 \\
\hline Tut:al SH LEAD & & 0.0 & 1.0 & 1.0 & 1.0 & 1.0 & 1.0 & 1.0 & 0.3 & 0.3 & 0.3 & 0.3 & 0.3 & 0.3 & 0.3 & 0.3 & 0.3 \\
\hline UIHKIOWN & MONE & 0.0 & 2.1 & 1.1 & 1.4 & 1.4 & 1.4 & 1.4 & 0.7 & 0.7 & 0.7 & 0.7 & 0.7 & 0.7 & 0.7 & 0.7 & 0.7 \\
\hline & Unmsomst & 0.0 & 3.5 & 3.5 & 3.5 & 3.5 & 3.5 & 3.5 & 1.2 & 1.2 & 1.2. & 1.2 & 1.2 & 1.2 & 1.2 & 1.2 & 1.2 \\
\hline Total UNKNOWN & & 0.0 & 1.9 & 1.9 & 4.9 & 4.9 & 1.9 & 1.9 & 1.9 & 1.9 & 1.9 & 1.9 & 1.9 & 1.9 & 1.9 & 1.9 & 1.9 \\
\hline Tota1 WHC_T_PLANT & & 0.0 & 51.0 & 51.0 & 58.1 & 58.1 & 58.1 & 50.1 & 22.7 & 22.7 & 22.7 & 22.7 & 22.7 & 22.7 & 22.7 & 22.7 & 22.7 \\
\hline
\end{tabular}

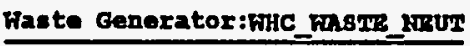

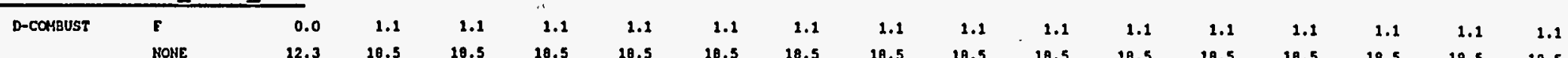

\begin{tabular}{|c|c|c|c|c|c|c|c|c|c|c|c|c|c|c|c|c|c|}
\hline & & 12.3 & 19.7 & 19.7 & 19.7 & 19.7 & 19.7 & 19.7 & 19.7 & 19.7 & 19.7 & 19.7 & 19.7 & 19.7 & 19.7 & 19.7 & 19.7 \\
\hline \multirow[t]{2}{*}{ D-HETAL } & EF & 0.0 & 0.1 & 0.1 & 0.1 & 0.1 & 0.1 & 0.1 & 0.1 & 0.1 & 0.1 & 0.1 & 0.1 & 0.1 & 0.1 & 0.1 & 0.1 \\
\hline & NOHE & 1.1 & 1.6 & 1.6 & 1.6 & 1.6 & 1.6 & 1.6 & 1.6 & - 1.6 & 1.6 & 1.6 & 1.6 & 1.6 & 1.6 & 1.6 & 1.6 \\
\hline Total D-KETAL & - & 1.1 & 1.7 & 1.7 & 1.7 & 1.7 & 1.7 & 1.7 & 1.7 & 1.7 & 1.7 & 1.7 & 1.7 & 1.7 & 1.7 & 1.7 & 1.7 \\
\hline Total WHC_ WAST & & 13.5 & 21.5 & 21.5 & 21.5 & 21.5 & 21.5 & 21.5 & 21.5 & 21.5 & 21.5 & 21.5 & 21.5 & 21.5 & 21.5 & 21.5 & 21.5 \\
\hline
\end{tabular}

Faste Generator:FRC_FRRI_ DRL

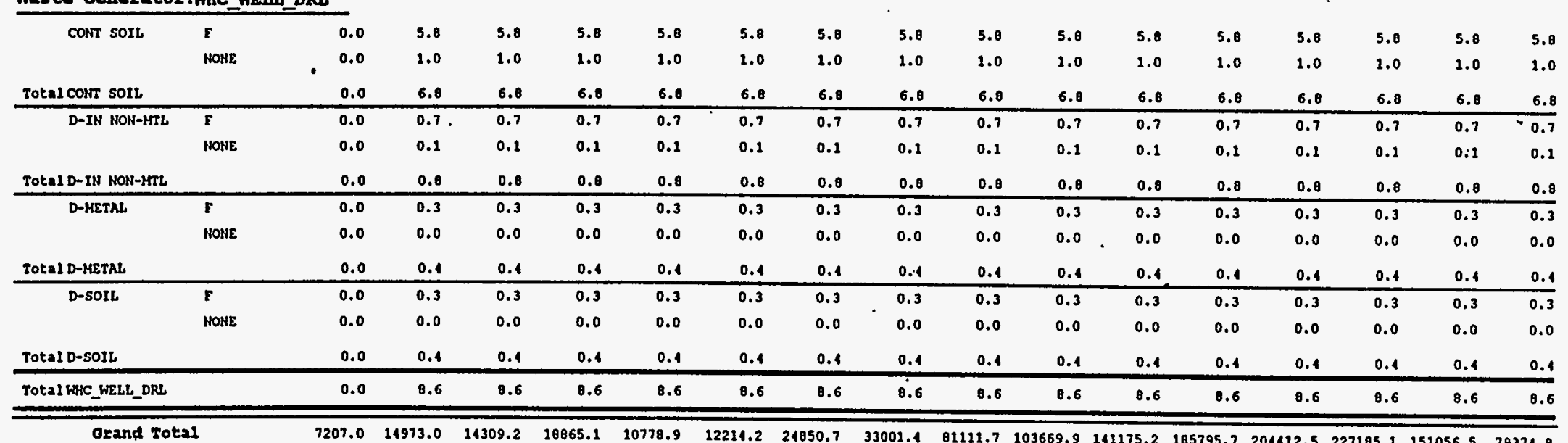

Orand Total

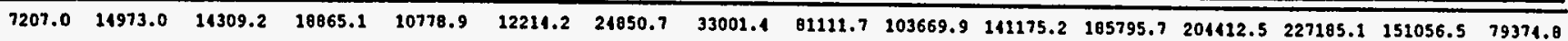


Waste Generator:Mugs

\begin{tabular}{|c|c|c|c|c|c|c|c|c|c|c|c|c|c|c|c|c|c|}
\hline D-CONBUST & NONE & 0.0 & 0.0 & 0.0 & 0.0 & 0.0 & 0.0 & 0.0 & 0.0 & 0.0 & 0.0 & 0.0 & 0.0 & 0.0 & 0.0 & 0.0 & 2.3100 .0 \\
\hline D...1D-COABUST & & 0.0 & 0.0 & 0.0 & 0.0 & 0.0 & 0.0 & 0.0 & 0.0 & 0.0 & 0.0 & 0.0 & 0.0 & 0.0 & 0.0 & 0.0 & 2.3100 .0 \\
\hline \multirow[t]{3}{*}{ D-HET } & B & 0.0 & 0.0 & 0.0 & 0.0 & 0.0 & 0.0 & 0.0 & 0.0 & 0.0 & 0.0 & 0.0 & 0.0 & 0.0 & 0.0 & 0.0 & 0.10 .1 \\
\hline & D & 0.0 & 0.0 & 0.0 & 0.0 & 0.0 & 0.0 & 0.0 & 0.0 & 0.0 & 0.0 & 0.0 & 0.0 & 0.0 & 0.0 & 0.0 & 0.1 \\
\hline & MONE & 1.1 & 1.1 & 1.1 & 1.1 & 1.2 & 1.1 & 1.1 & 1.1 & 0.5 & 0.5 & 0.5 & 0.5 & 0.5 & 0.5 & 0.5 & $22.5 \quad 99.1$ \\
\hline ota1D-HET & & 1.1 & 1.1 & 1.1 & 1.1 & 1.3 & 1.1 & 1.1 & 1.1 & 0.5 & 0.5 & 0.5 & 0.5 & 0.5 & 0.5 & 0.5 & 22.7100 .0 \\
\hline D-IN NON-RTL & NONE & 0.0 & 0.0 & 0.0 & 0.0 & 0.0 & 0.0 & 0.0 & 0.0 & 0.0 & 0.0 & 0.0 & 0.0 & 0.0 & 0.0 & 0.0 & 68.0100 .0 \\
\hline otalD-IN NON-MTL & & 0.0 & 0.0 & 0.0 & 0.0 & 0.0 & 0.0 & 0.0 & 0.0 & 0.0 & 0.0 & 0.0 & 0.0 & 0.0 & 0.0 & 0.0 & 60.0100 .0 \\
\hline D-METAL & D & 0.0 & 0.0 & 0.0 & 0.0 & 0.0 & 0.0 & 0.0 & 0.0 & 0.0 & 0.0 & 0.0 & 0.0 & 0.0 & 0.0 & 0.0 & 0.2100 .0 \\
\hline OtaLD-METAL & & 0.0 & 0.0 & 0.0 & 0.0 & 0.0 & 0.0 & 0.0 & 0.0 & 0.0 & 0.0 & 0.0 & 0.0 & 0.0 & 0.0 & 0.0 & 0.2100 .0 \\
\hline IN ORG PART & MONE & 0.2 & 0.2 & 0.2 & 0.2 & 0.2 & 0.2 & 0.2 & 0.2 & 0.1 & 0.1 & 0.1 & 0.1 & 0.1 & 0.1 & 0.1 & 1.7100 .0 \\
\hline OotalIN ORG PART & & 0.2 & 0.2 & 0.2 & 0.2 & 0.2 & 0.2 & 0.2 & 0.2 & 0.1 & 0.1 & 0.1 & 0.1 & 0.1 & 0.1 & 0.1 & 1.7100 .0 \\
\hline \multirow[t]{2}{*}{ LAB/CONT LQ } & B & 0.0 & 0.0 & 0.0 & 0.0 & 0.0 & 0.0 & 0.0 & 0.0 & 0.0 & 0.0 & 0.0 & 0.0 & 0.0 & 0.0 & 0.0 & 0.354 .5 \\
\hline & $B D$ & 0.0 & 0.0 & 0.0 & 0.0 & 0.0 & 0.0 & 0.0 & 0.0 & 0.0 & 0.0 & 0.0 & 0.0 & 0.0 & 0.0 & 0.0 & $0.2 \quad 45.4$ \\
\hline otal LAB/CONT LQ & & 0.0 & 0.0 & 0.0 & 0.0 & 0.0 & 0.0 & 0.0 & 0.0 & 0.0 & 0.0 & 0.0 & 0.0 & 0.0 & 0.0 & 0.0 & 0.5100 .0 \\
\hline OtaLAMES & & 1.6 & 1.6 & 1.6 & 1.6 & 1.8 & 1.6 & 1.6 & 1.6 & 0.0 & 0.8 & 0.8 & 0.0 & 0.8 & 0.8 & 0.8 & 98.7 \\
\hline
\end{tabular}

\section{Waste Generator:ANr_e}

\begin{tabular}{|c|c|c|c|c|c|c|c|c|c|c|c|c|c|c|c|c|c|}
\hline \multirow[t]{4}{*}{ CONT SOIL } & D & 0.0 & 0.0 & 0.0 & 0.0 & 0.0 & 0.0 & 0.0 & 0.0 & 0.0 & 0.0 & 0.0 & 0.0 & 0.0 & 0.0 & 0.0 & 1.7 \\
\hline & $\Sigma$ & 0.1 & 0.1 & 0.1 & 0.1 & 0.1 & 0.1 & 0.1 & 0.1 & 0.1 & 0.1 & 0.8 & 0.1 & 0.1 & 0.1 & 0.1 & 5.3 \\
\hline & J & 0.0 & 0.0 & 0.0 & 0.0 & 0.0 & 0.0 & 0.0 & 0.0 & 0.0 & 0.0 & 0.0 & 0.0 & 0.0 & 0.0 & 0.0 & 1.7 \\
\hline & NONE & 61.6 & 61.6 & 61.6 & 61.6 & 61.6 & 61.6 & 61.6 & 61.6 & 62.6 & 61.6 & 61.6 & 61.6 & 61.6 & 61.6 & 61.6 & 3157.499 .7 \\
\hline OtalCONT SOIL & & 61.9 & 61.9 & 61.9 & 61.9 & 61.9 & 61.9 & 61.9 & 61.9 & 61.9 & 61.9 & 61.9 & 61.9 & 61.9 & 61.9 & 61.9 & 3166.1100 .0 \\
\hline D-COHBUST & NONE & 13.0 & 15.8 & 15.8 & 15.0 & 15.8 & 15.0 & 15.0 & 15.8 & 15.8 & 15.8 & 25.0 & 15.8 & 15.0 & 15.8 & 25.8 & 677.9100 .0 \\
\hline otal D-COMBUST & & 15.0 & 15.0 & 15.8 & 15.8 & 15.0 & 25.8 & 15.8 & 15.8 & 15.8 & 15.0 & 15.0 & 15.0 & 15.0 & 15.8 & 25.8 & 677.9100 .0 \\
\hline \multirow[t]{2}{*}{ D-HET } & D & 10.4 & 10.4 & 10.1 & 10.4 & 10.1 & 10.1 & 10.1 & 20.4 & 10.4 & 20.4 & 10.1 & 10.4 & 10.1 & 10.4 & 10.4 & $332.1 \quad 8.5$ \\
\hline & NONE & 81.3 & 01.3 & 81.3 & 01.3 & 81.3 & 01.3 & 01.3 & 01.3 & 81.3 & 01.3 & 01.3 & 81.3 & 01.3 & 03.3 & 01.3 & 3537.291 .1 \\
\hline rotal D-HET & & 91.7 & 91.7 & 91.7 & 91.7 & 91.7 & 91.7 & 91.7 & 91.7 & 91.7 & 91.7 & 91.7 & 91.7 & 91.7 & 91.7 & 92.7 & 3869.6100 .0 \\
\hline D-IN NON-HTL & NONE & 23.8 & 25.8 & 23.8 & 25.8 & 25.0 & 25.8 & 25.8 & 25.0 & 25.8 & 25.8 & 25.8 & 25.8 & 25.8 & 25.0 & 25.8 & 2136.5100 .0 \\
\hline rota1D-IN NON-MTL & & 25.8 & 25.0 & 25.0 & 25.0 & 25.8 & 25.0 & 25.8 & 25.8 & 25.0 & 25.8 & 25.8 & 25.8 & 25.8 & 25.8 & 25.8 & 1136.5100 .0 \\
\hline \multirow[t]{2}{*}{ D-METAL } & D & 0.1 & 0.1 & 0.1 & 0.1 & 0.1 & 0.1 & 0.1 & 0.1 & 0.1 & 0.1 & 0.1 & 0.1 & 0.1 & 0.1 & 0.1 & 3.70 .3 \\
\hline & NONE & 25.4 & 25.1 & 25.4 & 25.4 & 25.4 & 25.4 & 25.4 & 25.1 & 25.4 & 25.4 & 25.4 & 25.4 & 25.1 & 25.4 & 23.1 & $1119.0 \quad 99.6$ \\
\hline Oota1D-METAL & & 25.5 & 25.5 & 25.5 & 25.5 & 25.5 & 25.5 & 25.5 & 25.5 & 25.5 & 25.5 & 25.5 & 25.5 & 25.5 & 25.5 & 25.5 & 1123.5100 .0 \\
\hline
\end{tabular}

HCD Codes: AmIgnitables, B-Corrosives, C-Reactlves, D-Metals w/O Hg, E-Metals w Hg, F-Organlcs, G-State Reg., H-PCB < 50ppm, J-PCB > 50ppm 
(in cubic meters)

\begin{tabular}{|c|c|c|c|c|c|c|c|c|c|c|c|c|c|c|c|c|c|}
\hline PWF & HCD & 2010 & 2011 & 2012 & 2013 & 2014 & 2015 & 2016 & 2017 & 2018 & $2019^{\circ}$ & 2020 & $2021^{\circ}$ & 2022 & 2023 & 2024 & Total pot \\
\hline \multirow[t]{4}{*}{ III ORG LQ/SLG } & A & 0.0 & 0.0 & 0.0 & 0.0 & 0.0 & 0.0 & 0.0 & 0.0 & 0.0 & 0.0 & 0.0 & 0.0 & 0.0 & 0.0 & 0.0 & 1.10 .5 \\
\hline & 8 & 0.0 & 0.0 & 0.0 & 0.0 & 0.0 & 0.0 & 0.0 & $0: 0$ & 0.0 & 0.0 & 0.0 & 0.0 & 0.0 & 0.0 & 0.0 & $0.7 \quad 0.3$ \\
\hline & D & 0.6 & 0.6 & 0.6 & 0.6 & 0.6 & 0.6 & 0.6 & 0.6 & 0.6 & 0.6 & 0.6 & 0.6 & 0.6 & 0.6 & 0.6 & $129.8 \quad 0.9$ \\
\hline & MORE & 5.1 & 5.1 & 5.1 & 5.1 & 5.1 & 5.1 & 5.1 & 5.1 & 5.1 & 5.1 & 5.1 & 5.1 & 5.1 & 5.1 & 5.1 & 199.990 .2 \\
\hline :OH il III ORG LQ/SLG & & 5.8 & 5.8 & 5.8 & 5.8 & 5.0 & 5.0 & 5.8 & 5.8 & 5.0 & 5.8 & 5.8 & 5.8 & 5.8 & 5.8 & 5.8 & 221.7100 .0 \\
\hline IN ORG PART & MONE & 1.6 & 1.6 & 1.6 & 1.6 & 1.6 & 1.6 & 1.6 & 1.6 & 1.6 & 1.6 & 1.6 & 1.6 & 1.6 & 1.6 & 1.6 & 69.9100 .0 \\
\hline IGtal IN ORG PART & & 1.6 & 1.6 & 1.6 & 1.6 & 1.6 & 1.6 & 1.6 & 1.6 & 1.6 & 1.6 & 1.6 & 1.6 & 1.6 & 1.6 & 1.6 & 69.9100 .0 \\
\hline \multirow[t]{4}{*}{ LAB/CONT LQ } & A & 2.2 & 2.2 & 2.2 & 2.2 & 2.2 & 2.2 & 2.2 & 2.2 & 2.2 & 2.2 & 2.2 & 2.2 & 2.2 & 2.2 & 2.2 & 72.790 .0 \\
\hline & B & 0.1 & 0.1 & 0.1 & 0.1 & 0.1 & 0.1 & 0.1 & 0.1 & 0.1 & 0.1 & 0.1 & 0.2 & 0.1 & 0.1 & 0.1 & $4.8 \quad 6.0$ \\
\hline & D & 0.0 & 0.0 & 0.0 & 0.0 & 0.0 & 0.0 & 0.0 & 0.0 & 0.0 & 0.0 & 0.0 & 0.0 & 0.0 & 0.0 & 0.0 & $2.4 \quad 2.9$ \\
\hline & $\mathbf{F}$ & 0.0 & 0.0 & 0.0 & 0.0 & 0.0 & 0.0 & 0.0 & 0.0 & 0.0 & 0.0 & 0.0 & 0.0 & 0.0 & 0.0 & 0.0 & 1.0 \\
\hline rot:a1 LAB/CONT LQ & & 2.5 & 2.5 & 2.5 & 2.5 & 2.5 & 2.5 & 2.5 & 2.5 & 2.5 & 2.5 & 2.5 & 2.5 & 2.5 & 2.5 & 2.5 & 00.0100 .0 \\
\hline LEAD & D & 0.2 & 0.2 & 0.2 & 0.2 & 0.2 & 0.2 & 0.2 & 0.2 & 0.2 & 0.2 & 0.2 & 0.2 & 0.2 & 0.2 & 0.2 & 721.4100 .0 \\
\hline BOtalLEAD & & 0.2 & 0.2 & 0.2 & 0.2 & 0.2 & 0.2 & 0.2 & 0.2 & 0.2 & 0.2 & 0.2 & 0.2 & 0.2 & 0.2 & 0.2 & 721.4100 .0 \\
\hline ORG ABS LO/SLG & NONE & 1.4 & 1.1 & 1.9 & 1.4 & 1.1 & 1.4 & 1.4 & 1.4 & 1.1 & 1.4 & 1.1 & 1.4 & 1.1 & 1.4 & 1.4 & 64.6100 .0 \\
\hline rotaloRo ABS LQ/SLG & & 1.1 & 1.1 & 1.4 & 1.4 & 1.1 & 1.4 & 1.4 & 1.4 & 1.1 & 1.1 & 1.1 & 1.4 & 1.1 & 1.1 & 1.4 & 64.6100 .0 \\
\hline ORG PART & NONE & 1.1 & 1.1 & 1.4 & 1.4 & 1.1 & 1.4 & 1.4 & 1.1 & 1.4 & 1.4 & 1.1 & 1.1 & 1.4 & 1.4 & $1.4^{\circ}$ & 64.6100 .0 \\
\hline rotaloRG PART & & 1.4 & 1.1 & 1.4 & 1.4 & 1.1 & 1.4 & 1.4 & 1.1 & 1.4 & 1.4 & 1.1 & 1.4 & 1.1 & 1.4 & 1.1 & 61.6100 .0 \\
\hline TotalANL_E & & 234.2 & 234.2 & 234.2 & 234.2 & 234.2 & 234.2 & 234.2 & 234.2 & 234.2 & 234.2 & 234.2 & 234.2 & 234.2 & 234.2 & 231.2 & 11197.3 \\
\hline \multicolumn{18}{|c|}{ Waste Generator:BnpL } \\
\hline CONT SOIL & FG & 0.0 & 0.0 & 0.0 & 0.0 & 0.0 & 0.0 & 0.0 & 0.0 & 0.0 & 0.0 & 0.0 & 0.0 & 0.0 & 0.0 & 0.0 & 5.0 .100 .0 \\
\hline TotalCONT SOIL & & 0.0 & 0.0 & 0.0 . & 0.0 & 0.0 & 0.0 & 0.0 & 0.0 & 0.0 & 0.0 & 0.0 & 0.0 & 0.0 & 0.0 & 0.0 & 5.0100 .0 \\
\hline \multirow[t]{2}{*}{ D-CaMBust } & G & 0.0 & 0.0 & 0.0 & 0.0 & 0.0 & 0.0 & 0.0 & 0.0 & 0.0 & 0.0 & 0.0 & 0.0 & 0.0 & 0.0 & 0.0 & $0.1 \quad 1.4$ \\
\hline & NONE & 0.0 & 0.0 & 0.0 & 0.0 & 0.0 & 0.0 & 0.0 & 0.0 & 0.0 & 0.0 & 0.0 & 0.0 & 0.0 & 0.0 & 0.0 & $12.9 \quad 98.5$ \\
\hline rotal D-COHBUST & & 0.0 & 0.0 & 0.0 & 0.0 & 0.0 & 0.0 & 0.0 & 0.0 & 0.0 & 0.0 & 0.0 & 0.0 & 0.0 & 0.0 & 0.0 & 13.1100 .0 \\
\hline \multirow[t]{3}{*}{ D-HET } & EG & 0.0 & 0.0 & 0.0 & 0.0 & 0.0 & 0.0 & 0.0 & 0.0 & 0.0 & 0.0 & 0.0 & 0.0 & 0.0 & 0.0 & 0.0 & $0.2 \quad 0.7$ \\
\hline & EG & 0.3 & 0.3 & 0.3 & 0.3 & 0.3 & 0.3 & 0.3 & 0.3 & 0.3 & 0.3 & 0.3 & 0.3 & 0.3 & 0.3 & 0.3 & 26.175 .0 \\
\hline & MONE & 0.0 & 0.0 & 0.0 & 0.0 & 0.0 & 0.0 & 0.0 & 0.0 & 0.0 & 0.0 & 0.0 & 0.0 & 0.0 & 0.0 & 0.0 & 8.124 .2 \\
\hline Tot.21D-HET & & 0.3 & 0.3 & 0.3 & 0.3 & 0.3 & 0.3 & 0.3 & 0.3 & 0.3 & 0.3 & 0.3 & 0.3 & 0.3 & 0.3 & 0.3 & 34.7100 .0 \\
\hline D-IN NON-MTL & NONE & 0.0 & 0.0 & 0.0 & 0.0 & 0.0 & 0.0 & 0.0 & 0.0 & 0.0 & 0.0 & 0.0 & 0.0 & 0.0 & 0.0 & $0 . \dot{0}$ & 5.7100 .0 \\
\hline Total D-1H NON-MTL & & 0.0 & 0.0 & 0.0 & 0.0 & 0.0 & 0.0 & 0.0 & 0.0 & 0.0 & 0.0 & 0.0 & 0.0 & 0.0 & 0.0 & 0.0 & 5.7100 .0 \\
\hline D-METAL & NONE & 0.0 & 0.0 & 0.0 & 0.0 & 0.0 & 0.0 & 0.0 & 0.0 & 0.0 & 0.0 & 0.0 & 0.0 & .0 .0 & 0.0 & 0.0 & 126.3100 .0 \\
\hline rotalD-METAL & & 0.0 & 0.0 & 0.0 & 0.0 & 0.0 & 0.0 & 0.0 & 0.0 & 0.0 & 0.0 & 0.0 & 0.0 & 0.0 & 0.0 & 0.0 & 126.3100 .0 \\
\hline IN ORG LQ/SLG & D & 0.0 & 0.0 & 0.0 & 0.0 & 0.0 & 0.0 & 0.0 & 0.0 & 0.0 & 0.0 & 0.0 & 0.0 & 0.0 & 0.0 & 0.0 & 0.1100 .0 \\
\hline
\end{tabular}

gCo Codes: A-Ignitables, B-Corrosives, C-Reactives, D-Metals w/O Hg, E-Metals w Hg, F-Organies, G-State Reg., H-PCB < 50ppm, J-PCB > 50ppm 


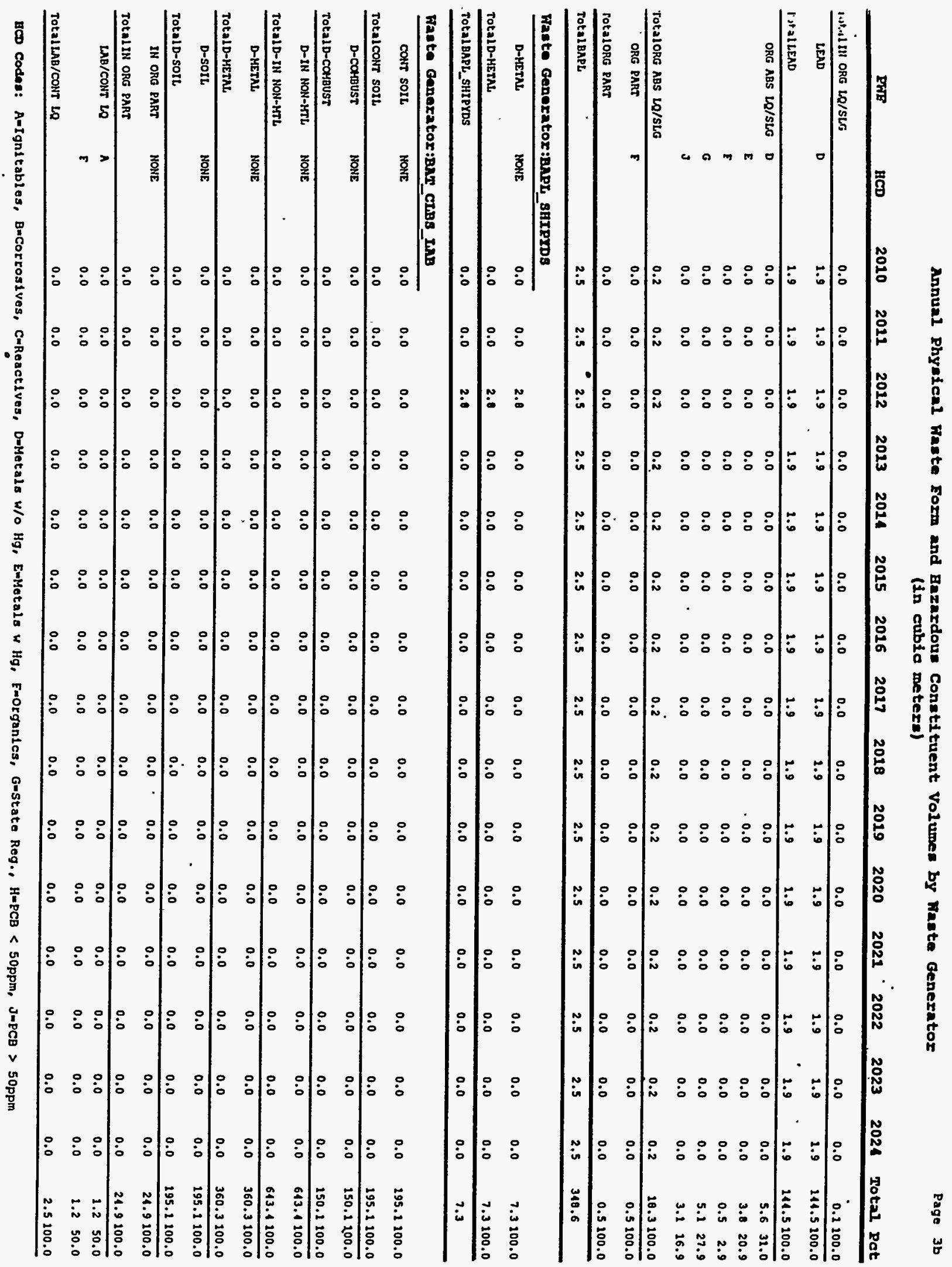


$\begin{array}{llllllllllllllllll}\text { FWE } & \text { HCD } & 2010 & 2011 & 2012 & 2013 & 2014 & 2015 & 2016 & 2017 & 2018 & 2019 & 2020 & 2021 & 2022 & 2023 & 2024 & \text { Total }\end{array}$

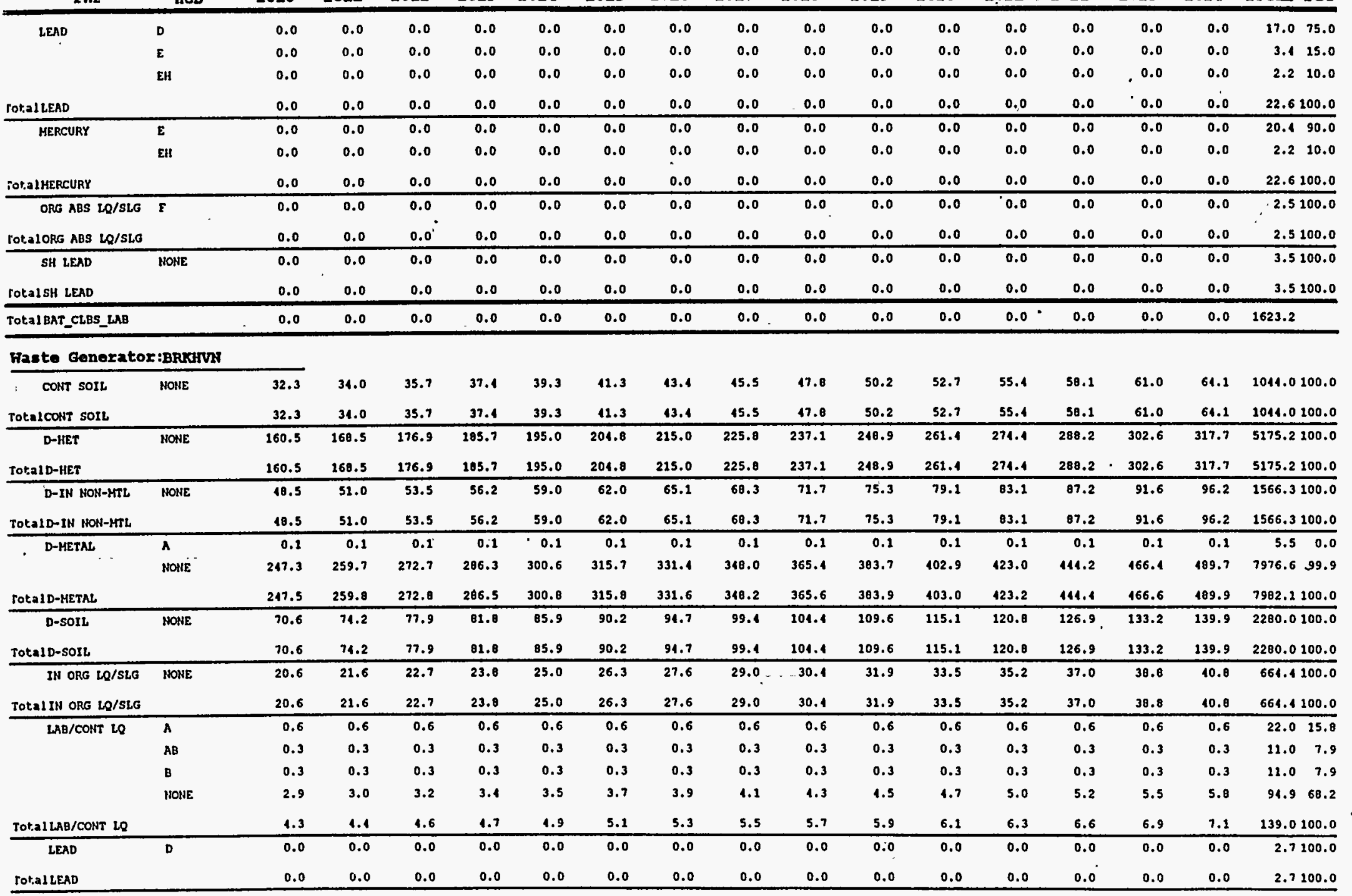

BCD Codes: A-Ignitables, B=Corrosives, CuReactives, D-Metals w/O Hg, E-Metals w Hg, FuOrganics, G=State Reg., H-PCB < 50ppm, J-PCB > 50ppm 


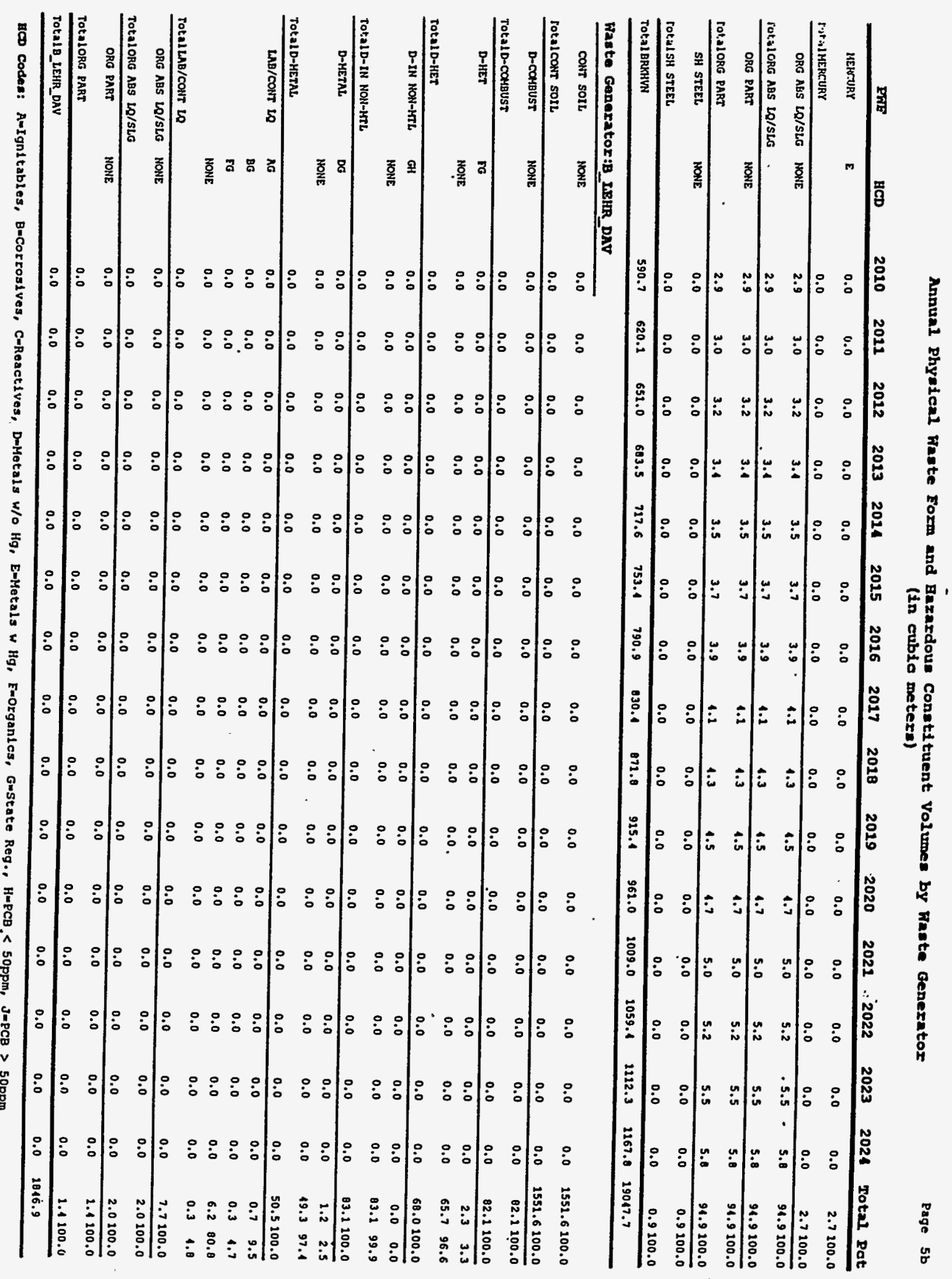




\section{Waste Generator:EGco}

\begin{tabular}{|c|c|c|c|c|c|c|c|c|c|c|c|c|c|c|c|c|c|}
\hline D-COMBUST & MONE & 0.0 & 0.0 & 0.0 & 0.0 & 0.0 & 0.0 & $0.0^{\circ}$ & 0.0 & 0.0 & 0.0 & 0.0 & 0.0 & 0.0 & 0.0 & 0.0 & 80.9100 .0 \\
\hline I.A1D-COXIGUST & & 0.0 & 0.11 & 0.0 & 0.0 & 0.0 & 0.0 & 0.0 & 0.0 & 0.0 & 0.0 & 0.0 & 0.0 & 0.0 & 0.0 & $0.0 \ldots$ & 80.9100 .0 \\
\hline D-HET & MONE & 0.0 & 0.0 & 0.0 & 0.0 & 0.0 & 0.0 & 0.0 & 0.0 & 0.0 & 0.0 & 0.0 & 0.0 & 0.0 & 0.0 & 0.0 & 63.1100 .0 \\
\hline t.al D-HET & & 0.0 & 0.0 & 0.0 & 0.0 & 0.0 & 0.0 & 0.0 & 0.0 & 0.0 & 0.0 & 0.0 & 0.0 & 0.0. & 0.0 & 0.0 & 63.1100 .0 \\
\hline D-IN NON-HTL & NOHE & 0.0 & 0.0 & 0.0 & 0.0 & 0.0 & 0.0 & 0.0 & 0.0 & 0.0 & 0.0 & 0.0 & 0.0 & 0.0 & 0.0 & 0.0 & 16.1100 .0 \\
\hline taID-IN NON-MTL & & 0.0 & 0.0 & 0.0 & 0.0 & 0.0 & 0.0 & 0.0 & 0.0 & 0.0 & 0.0 & 0.0 & 0.0 & 0.0 & 0.0 & 0.0 & 26.1100 .0 \\
\hline D-METAS & NONE & 0.0 & 0.0 & 0.0 & 0.0 & 0.0 & 0.0 & 0.0 & 0.0 & 0.0 & 0.0 & 0.0 & 0.0 & 0.0 & 0.0 & 0.0 & 1.6100 .0 \\
\hline tal D-HETAL & & 0.0 & 0.0 & 0.0 & 0.0 & 0.0 & 0.0 & 0.0 & 0.0 & 0.0 & 0.0 & 0.0 & 0.0 & 0.0 & 0.0 & 0.0 & 1.6100 .0 \\
\hline ORG ABS LQ/SLG & NONE & 0.0 & 0.0 & 0.0 & 0.0 & 0.0 & 0.0 & 0.0 & 0.0 & 0.0 & 0.0 & 0.0 & 0.0 & 0.0 & 0.0 & 0.0 & 129.1100 .0 \\
\hline ta1ORG ABS LQ/SLG & & 0.0 & 0.0 & 0.0 & 0.0 & 0.0 & 0.0 & 0.0 & 0.0 & 0.0 & 0.0 & 0.0 & 0.0 & 0.0 & 0.0 & 0.0 & 129.1100 .0 \\
\hline tal EG GG & & 0.0 & 0.0 & 0.0 & 0.0 & 0.0 & 0.0 & 0.0 & 0.0 & 0.0 & 0.0 & 0.0 & 0.0 & 0.0 & 0.0 & 0.0 & 291.1 \\
\hline
\end{tabular}

\section{Faste Genoratox:FrRMr}

\begin{tabular}{|c|c|c|c|c|c|c|c|c|c|c|c|c|c|c|c|c|c|}
\hline BATTERIES & D & 0.2 & 0.2 & 0.2 & 0.2 & 0.2 & 0.2 & 0.2 & 0.2 & 0.2 & 0.2 & 0.2 & 0.2 & 0.2 & 0.2 & 0.2 & 7.8100 .0 \\
\hline rotal BATTERIES & & 0.2 & 0.2 & 0.2 & 0.2 & 0.2 & 0.2 & 0.2 & 0.2 & 0.2 & 0.2 & 0.2 & 0.2 & 0.2 & 0.2 & 0.2 & 7.0100 .0 \\
\hline CONT SOIL & NONE & 0.5 & 0.5 & 0.5 & 0.5 & 0.5 & 0.5 & 0.5 & 0.5 & 0.5 & 0.5 & 0.5 & 0.5 & 0.5 & 0.5 & 0.5 & 16.4100 .0 \\
\hline rotalcont SOIL & & 0.5 & 0.5 & 0.5 & 0.5 & 0.5 & 0.5 & 0.5 & 0.5 & 0.5 & 0.5 . & 0.5 & 0.5 & 0.5 & 0.5 & 0.5 & 16.4100 .0 \\
\hline D-COMBUST & NONE & 3.3 & 3.3 & 3.3 & 3.3 & 3.3 & 3.3 & 3.3 & 3.3 & 3.3 & 3.3 & 3.3 & 3.3 & 3.3 & 3.3 & 3.3 & 101.5100 .0 \\
\hline TotalD-cOMBUST & & 3.3 & 3.3 & 3.3 & 3.3 & 3.3 & 3.3 & 3.3 & 3.3 & 3.3 & 3.3 & 3.3 & 3.3 & 3.3 & 3.3 & 3.3 & 101.5100 .0 \\
\hline D-HET & HONE & 1.4 & 1.4 & 1.4 & 1.4 & 1.4 & 1.4 & 1.4 & 1.4 & 1.4 & 1.4 & 1.4 & 1.4 & 1.4 & 1.4 & 1.4 & $\$ 3.1100 .0$ \\
\hline Total D-HET & & 1.1 & 1.4 & 1.4 & 1.4 & 1.4 & 1.4 & 1.1 & 1.4 & 1.4 & 1.4 & 1.1 & 1.1 & 1.4 & 1.4 & 1.1 & $\$ 3.1100 .0$ \\
\hline D-IN NON-HTL & MONE & 0.9 & 0.9 & 0.9 & 0.9 & 0.9 & 0.9 & 0.9 & 0.9 & 0.9 & 0.9 & 0.9 & 0.9 & 0.9 & 0.9 & 0.9 & 27.3100 .0 \\
\hline Tota1 D-1N NON-HTL & & 0.9 & 0.9 & 0.9 & 0.9 & 0.9 & 0.9 & 0.9 & 0.9 & 0.9 & 0.9 & 0.9 & 0.9 & 0.9 & 0.9 & 0.9 & 27.3100 .0 \\
\hline D-METAL & NONE & 35.2 & 35.2 & 35.2 & 35.2 & 35.2 & 35.2 & 35.2 & 35.2 & 35.2 & 35.2 & 35.2 & 35.2 & 35.2 & 35.2 & .35 .2 & 1083.2100 .0 \\
\hline rotaID-HETAL & & 35.2 & 35.2 & 35.2 & 35.2 & 35.2 & 35.2 & 35.2 & 35.2 & 35.2 & 35.2 & 35.2 & 35.2 & 35.2 & 35.2 & 35.2 & 1083.2100 .0 \\
\hline IN ORG LQ/SLG & MONE & 1.0 & 1.0 & 1.0 & 1.0 & 1.0 & 1.0 & 1.0 & 1.0 & 1.0 & 1.0 & 1.0 & 1.0 & 1.0 & 1.0 & 1.0 & 31.8100 .0 \\
\hline Total IN ORG LQ/SLG & & 1.0 & 2.0 & 1.0 & 1.0 & 1.0 & 1.0 & 1.0 & 1.0 & 1.0 & 1.0 & 1.0 & 1.0 & 1.0 & 1.0 & 1.0 & 31.0100 .0 \\
\hline IN ORG PART & NOHE & 0.6 & 0.6 & 0.6 & 0.6 & 0.6 & 0.6 & 0.6 & 0.6 & 0.6 & 0.6 & 0.6 & 0.6 & 0.6 & 0.6 & 0.6 & 20.6100 .0 \\
\hline TOtal IN ORG PART & & 0.6 & 0.6 & 0.6 & 0.6 & 0.6 & 0.6 & 0.6 & 0.6 & 0.6 & 0.6 & 0.6 & 0.6 & 0.6 & 0.6 & 0.6 & 20.6200 .0 \\
\hline LAB/CONT LQ & BD & 0.2 & 0.2 & 0.2 & 0.2 & 0.2 & 0.2 & 0.2 & 0.2 & 0.2 & 0.2 & 0.2 & 0.2 & 0.2 & 0.2 & 0.2 & 7.8100 .0 \\
\hline TOtal LAB/CONT LQ & & 0.2 & 0.2 & 0.2 & 0.2 & 0.2 & 0.2 & 0.2 & 0.2 & 0.2 & 0.2 & 0.2 & 0.2 & 0.2 & 0.2 & 0.2 & 7.8100 .0 \\
\hline LEAD & D & 0.5 & 0.5 & 0.5 & 0.5 & 0.5 & 0.5 & 0.5 & 0.5 & 0.5 & 0.5 & 0.5 & 0.5 & 0.5 & 0.5 & 0.5 & 15.6100 .0 \\
\hline
\end{tabular}

BCD Codes: A-Ignitables, BuCorrosives, C=Reactives, D-Metals w/O Hg, E-Metals w Hg, F-Organics, G-State Reg., H-PCB < 50ppm, J=PCB > 50ppm 


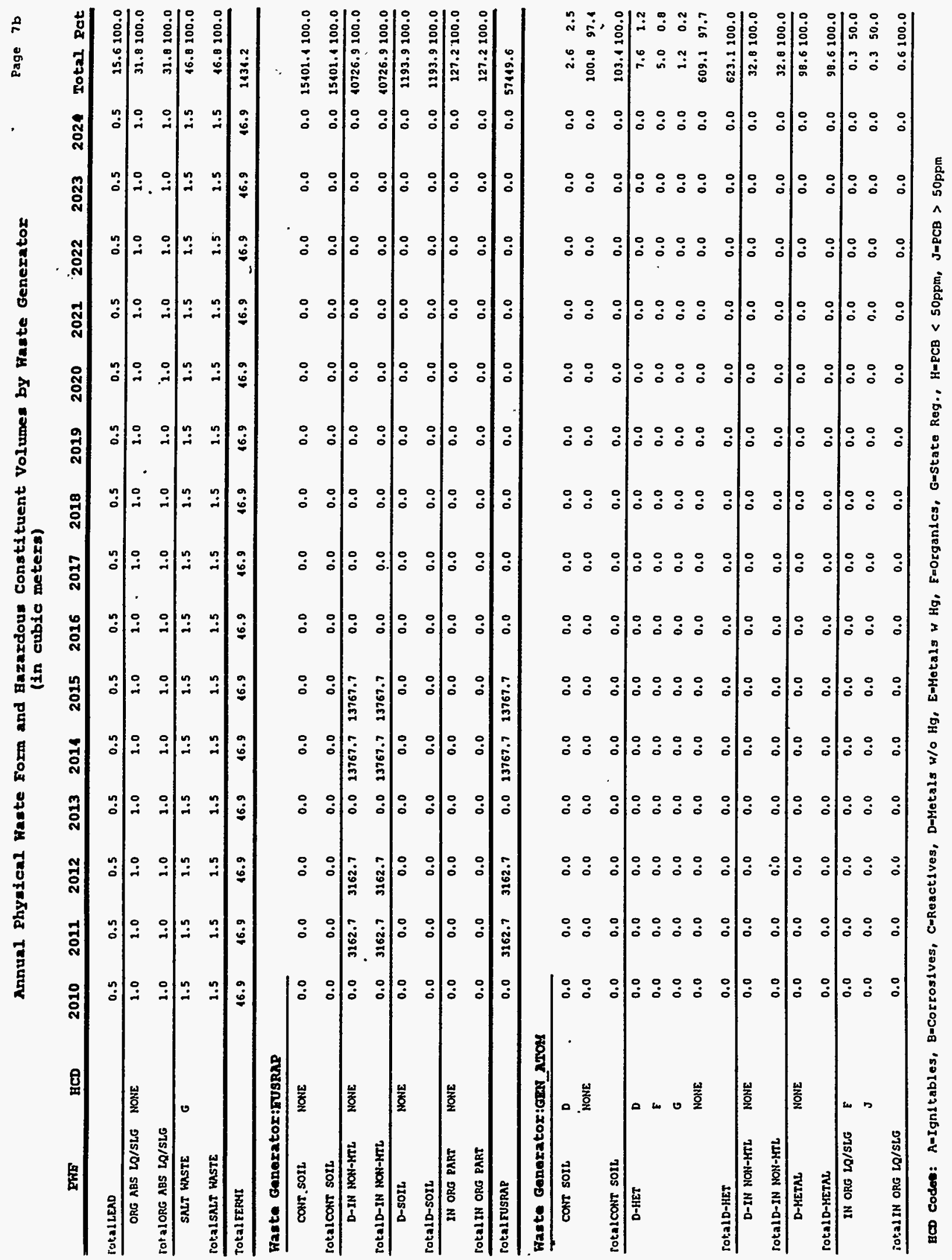




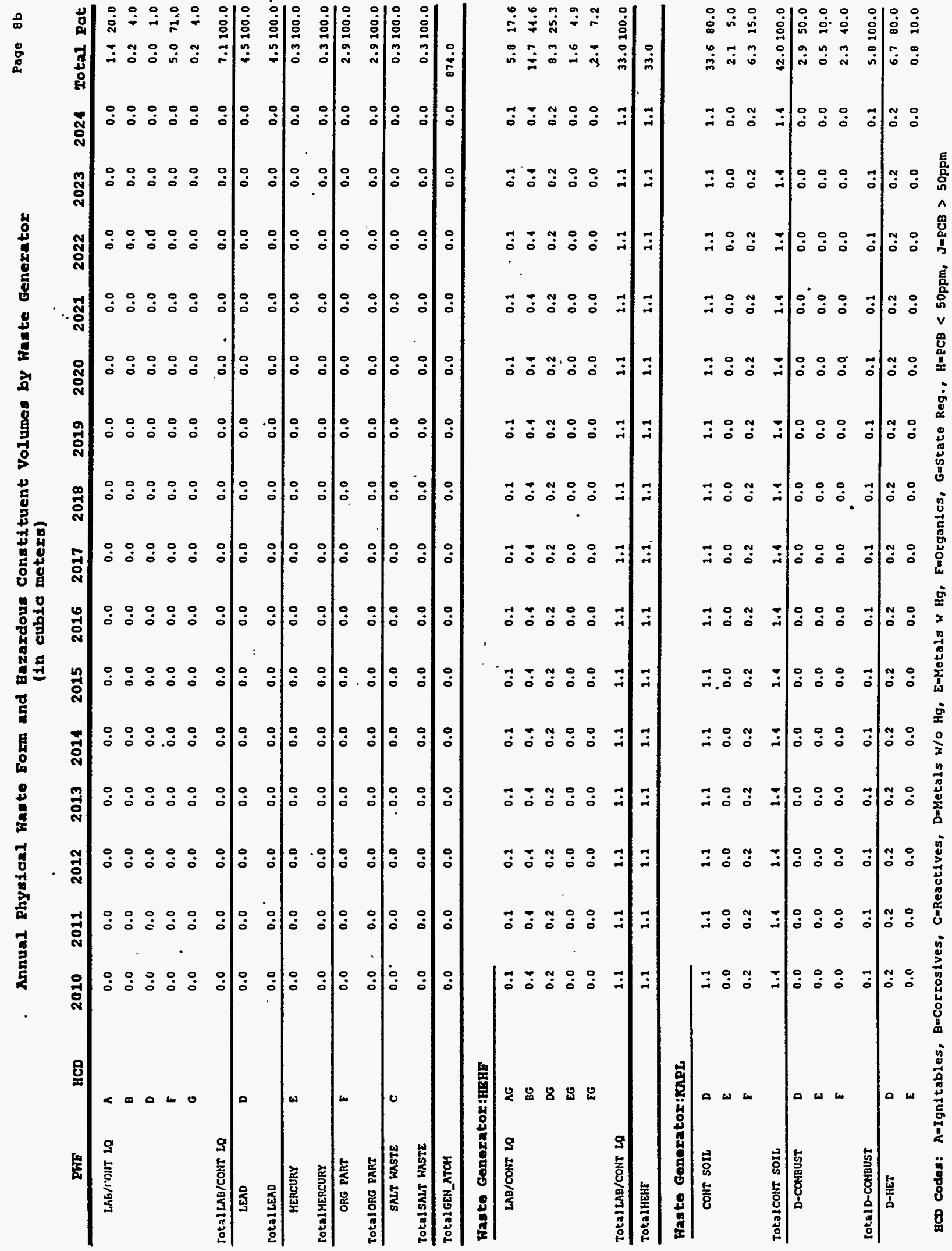




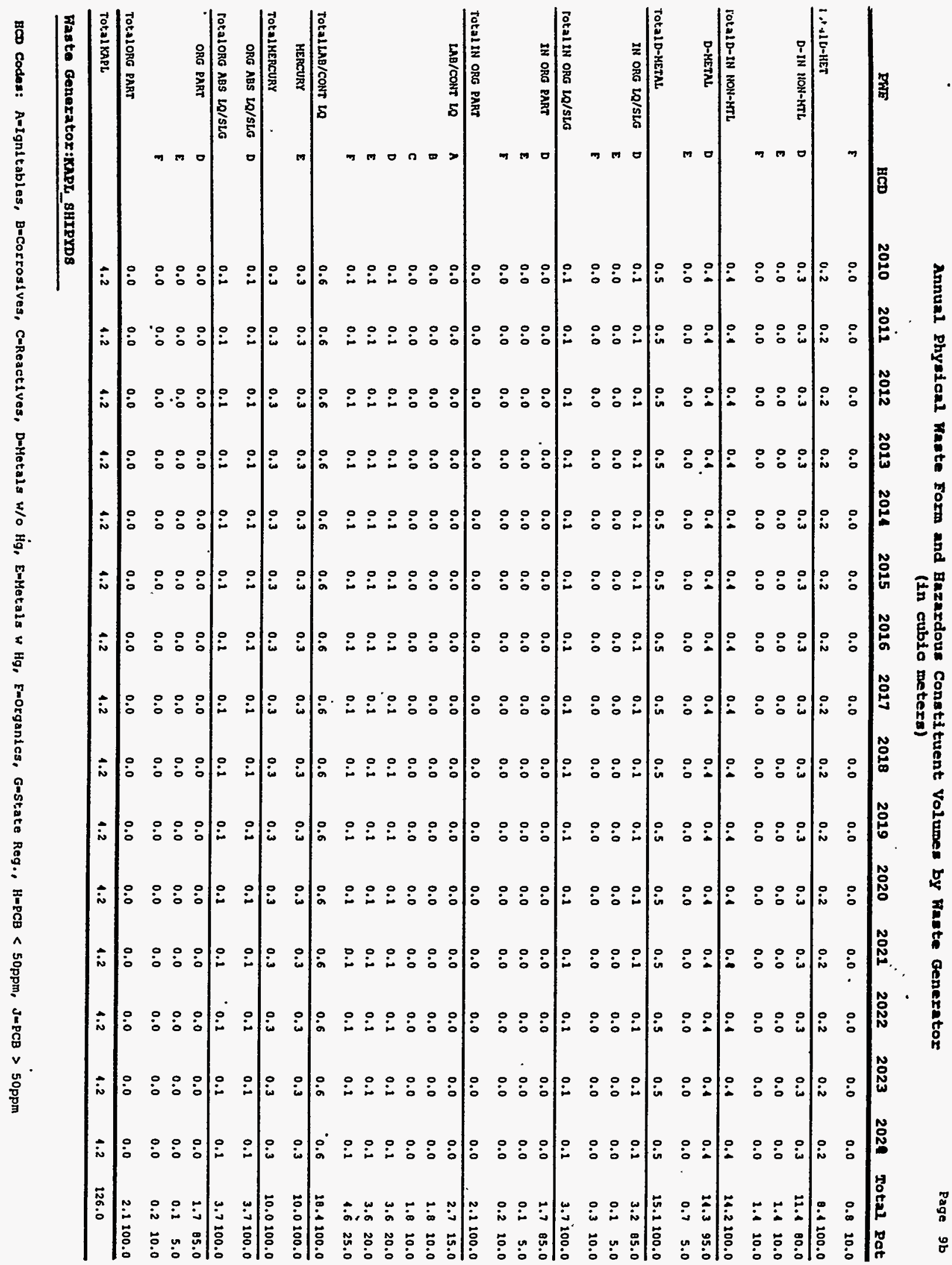




\begin{tabular}{|c|c|c|c|c|c|c|c|c|c|c|c|c|c|c|c|c|c|}
\hline PWE & HCD & 2010 & 2011 & 2012 & 2013 & 2014 & 2015 & 2016 & 2017 & 2018 & 2019 & 2020 & 2021 & 2022 & 2023 & 2024 & Total Pot \\
\hline D-METAL & nONE & 0.0 & 0.0 & 0.0 & 0.0 & 0.0 & 0.0 & 0.0 & 0.0 & 0.0 & 0.0 & 0.0 & 0.0 & 0.0 & 0.0 & 0.0 . & 297.0100 .0 \\
\hline INA,AID-BETAL & & 0.0 & 0.0 & 0.0 & 0.0 & 0.0 & 0.0 & 0.0 & 0.0 & 0.0 & 0.0 & 0.0 & 0.0 & 0.0 & 0.0 & 0.0 & 297.0100 .0 \\
\hline Fot.:A 1 KAPL_SHIPYDS & & 0.0 & 0.0 & 0.0 & 0.0 & 0.0 & 0.0 & 0.0 & 0.0 & 0.0 & 0.0 & 0.0 & 0.0 & 0.0 & 0.0 & 0.0 & 297.0 \\
\hline \multicolumn{18}{|c|}{ Waste Gonerator:KEH_100 } \\
\hline BATTERIES & BG & 1.3 & 1.7 & 2.1 & 2.6 & 3.3 & 1.1 & 5.2 & 6.5 & 8.1 & 10.1 & 12.7 & 15.0 & 19.8 & 24.8 & 31.0 & 251.0100 .0 \\
\hline Potal BATTERIES & & 1.3 & 1.7 & 2.1 & 2.6 & 3.3 & 1.1 & 5.2 & 6.5 & 8.2 & 10.1 & 12.7 & 15.8 & 19.8 & 24.8 & 31.0 & 154.8100 .0 \\
\hline \multirow[t]{2}{*}{ CONT SOIL } & D & 0.0 & 0.0 & 0.0 & 0.0 & 0.0 & 0.0 & 0.0 & 0.0 & .0 .0 & 0.0 & 0.0 & 0.0 & 0.0 & 0.0 & 0.0 & $10.5 \quad 14.0$ \\
\hline & NOHE & 0.0 & 0.0 & 0.0 & 0.0 . & 0.0 & 0.0 & 0.0 & 0.0 & 0.0 & 0.0 & 0.0 & 0.0 & 0.0 & 0.0 & 0.0 & $64.2 \quad 05.9$ \\
\hline Potalcont SOIL & & 0.0 & 0.0 & 0.0 & 0.0 & 0.0 & 0.0 & 0.0 & 0.0 & 0.0 & 0.0 & 0.0 & 0.0 & 0.0 & 0.0 & 0.0 & 74.7100 .0 \\
\hline \multirow[t]{2}{*}{ D-congust } & $\boldsymbol{A}$ & 9.9 & 12.3 & 15.4 & 29.3 & 24.2 & 30.2 & 37.8 & 47.2 & 59.0 & 73.8 & 92.3 & 115.4 & 111.2 & 180.3 & 225.4 & $0125.7 \quad 55.1$ \\
\hline & AFG & 8.0 & $10.0^{\circ}$ & 12.6 & 15.7 & 19.7 & 24.6 & 30.8 & 30.5 & 19.1 & 60.1 & 75.2 & 94.0 & 117.5 & 146.9 & 203.6 & 917.244 .8 \\
\hline [Ot:alD-COUBUST & & 17.9 & 22.4 & 28.1 & 35.1 & 43.9 & 54.9 & 68.6 & 05.7 & 107.2 & 134.0 & 167.5 & 209.4 & 261.8 & 327.2 & 109.0 & 2012.9100 .0 \\
\hline D-IN HON-HTL & NONE & 0.0 & 0.0 & 0.0 & 0.0 & 0.0 & 0.0 & 0.0 & 0.0 & 0.0 & 0.0 & 0.0 & 0.0 & 0.0 & 0.0 & 0.0 & 12.6100 .0 \\
\hline rota1D-IN NON-MTL & & 0.0 & 0.0 & 0.0 & 0.0 & 0.0 & 0.0 & 0.0 & 0.0 & 0.0 & 0.0 & 0.0 & 0.0 & 0.0 & 0.0 & 0.0 & 12.6100 .0 \\
\hline \multirow[t]{2}{*}{ D-METNL } & DG & 390.1 & 487.7 & 609.6 & 762.0 & 952.5 & 1190.7 & 1488.3 & 1860.4 & 2325.6 & 2906.9 & 3633.7 & 4542.1 & 5677.6 & 7097.1 & 8871.3 & 14301.996 .0 \\
\hline & EG & 16.1 & 20.1 & 25.2 & 31.5 & 39.4 & 19.3 & 61.6 & 77.0 & 96.3 & 120.3 & 150.4 & 188.0 & 235.1 & 293.8 & 367.3 & $1834.5 \quad 3.9$ \\
\hline rotaL D-METAL & & 106.3 & 507.9 & 634.0 & 793.6 & 991.9 & 1240.0 & 1550.0 & 1937.5 & 2421.9 & 3027.3 & 3784.1 & 1730.2 & 5912.7 & 7391.0 & $923 \theta .7$ & $1613 \dot{6} .4100 .0$ \\
\hline IN ORG LO/SLO & AEG & 190.2 & 247.0 & 309.8 & 307.2 & 184.0 & 605.1 & 756.3 & 945.4 & 1181.0 & 1477.3 & 1846.6 & 2300.3 & 2885.1 & 3606.7 & 4508.4 & 22514.3100 .0 \\
\hline rotal IN ORG LQ/SLG & & 198.2 & 247.8 & 309.8 & 387.2 & 184.0 & 605.1 & 756.3 & 945.1 & 1181.8 & 1477.3 & 1846.6 & 2308.3 & 2805.4 & 3606.7 & 4508.4 & 22514.3100 .0 \\
\hline \multirow[t]{2}{*}{ LAB/CONT LR } & 86 & 11.1 & 51.4 & 61.2 & 00.3 & 100.4 & 125.5 & 156.8 & 196.1 & 245.1 & 306.4 & 383.0 & 178.7 & 598.1 & 748.0 & 935.0 & $1669.6 \quad 80.5$ \\
\hline & EG & 9.9 & 12.3 & 15.4 & 19.3 & 24.2 & 30.2 & 37.8 & 17.2 & 59.0 & 73.8 & 92.3 & 215.1 & 141.2 & 180.3 & 225.4 & $1125.7 \quad 19.4$ \\
\hline rota1 LAB/CONT LO & & 51.0 & 63.7 & 79.7 & 99.6 & 124.6 & 155.7 & 194.7 & 243.3 & 301.2 & 380.2 & 175.3 & 594.1 & 742.7 & 928.4 & 1160.5 & 5795.3100 .0 \\
\hline \multirow[t]{2}{*}{ LEAD } & BDG & 9.1 & 11.4 & 14.2 & 17.8 & 22.2 & 27.8 & 34.8 & 13.5 & 54.4 & 68.0 & 85.0 & 106.2 & 132.8 & 166.0 & 207.5 & $1036.3 \quad 99.9$ \\
\hline & $D$ & 0.0 & 0.0 & 0.0 & 0.0 & 0.0 & 0.0 & 0.0 & 0.0 & 0.0 & 0.0 & 0.0 & 0.0 & 0.0 & 0.0 & 0.0 & $0.7 \quad 0.0$ \\
\hline Total LEAD & $\cdot$ & 9.1 & 11.4 & 11.2 & 17.0 & 22.2 & 27.8 & 34.8 & 13.5 & 54.4 & 68.0 & 85.0 & 206.2 & 132.8 & 166.0 & 207.5 & 1037.1100 .0 \\
\hline \multirow[t]{2}{*}{ ORG ABS LQ/SLG } & AEFG & 356.9 & 146.1 & 557.6 & 697.1 & 071.3 & 1089.2 & 1361.5 & 1701.8 & 2127.3 & 2659.2 & 3323.9 & 4154.9 & 5193.7 & 6492.1 & 0115.1 & $\begin{array}{lll}10525.7 & 97.7\end{array}$ \\
\hline & EG & 0.0 & 10.0 & 12.6 & 15.7 & 19.7 & 24.6 & 30.8 & 38.5 & 48.1 & 60.1 & 75.2 & 94.0 & 117.5 & 146.9 & 183.6 & $917.2 \quad 2.2$ \\
\hline rotal ORG ABS LQ/SLG & & 361.9 & 456.2 & 570.2 & 712.8 & 891.0 & 1113.8 & 1392.3 & 1740.1 & 2175.5 & 2719.3 & 3399.2 & 4219.0 & 5311.2 & 6639.1 & 0298.8 & 41643.0100 .0 \\
\hline Total KEH_100 & & 1049.1 & 1311.4 & 1639.1 & 2049.1 & 2561.3 & 3201.7 & 1002.1 & 5002.6 & 6253.3 & 7816.6 & 9770.7 & 12213.4 & 15266.7 & 19083.4 & 23854.2 & 119211.6 \\
\hline \multicolumn{18}{|c|}{ Faste Generator:IBL } \\
\hline D-IN NON-MTL & NONE & 7.0 & 7.0 & 7.0 & 7.0 & 7.0 & 7.0 & 7.0 & 7.0 & 1.0 & 7.0 & 7.0 & 7.0 & 7.0 & 7.0 & 7.0 & 13943.2100 .0 \\
\hline PO:.11D-IK NON-MTL & & 7.0 & 7.0 & 7.0 & 7.0 & 7.0 & 7.0 & 7.0 & 7.0 & 7.0 & 7.0 & 7.0 & 7.0 & 7.0 & 7.0 & 7.0 & 13943.2100 .0 \\
\hline
\end{tabular}

BCD Codes: A-Ignitables, B-Corrosives, C-Reactives, D-Metals w/O Hg, E-Metals w Hg, Em-Organics, G-State Reg., H-PCB < 50ppm, J-PCB > 50ppm 

(in cubio meters)

\begin{tabular}{|c|c|c|c|c|c|c|c|c|c|c|c|c|c|c|c|c|c|}
\hline PWE & HCD & 2010 & 2011 & 2012 & 2013 & 2014 & 2015 & 2016 & 2017 & 2018 & 2019 & 2020 & 2021 & 2022 & 2023 & 2024 & Total Pot \\
\hline D-METAJ. & NOIE & 12.5 & 12.5 & 12.5 & 12.5 & 12.5 & 12.5 & 12.5 & 12.5 & 12.5 & 12.5 & 12.5 & 12.5 & 12.5 & 12.5 & 12.5 & 1073.3100 .0 \\
\hline SUFA1D-METAL & & 12.5 & 12.5 & 12.5 & 12.5 & 12.5 & 12.5 & 12.5 & 12.5 & 12.5 & 12.5 & 12.5 & 12.5 & 12.5 & 12.5 & 12.5 & 1073.3100 .0 \\
\hline \multirow[t]{2}{*}{ III ORG PART } & D & 0.1 & 0.1 & 0.1 & 0.1 & 0.1 & 0.1 & 0.1 & 0.1 & 0.1 & 0.1 & 0.1 & 0.1 & 0.1 & 0.1 & 0.1 & $4.6 \quad 0.8$ \\
\hline & MOHE & 19.0 & 19.0 & 19.0 & 19.0 & 19.0 & 19.0 & 19.0 & 19.0 & 19.0 & 19.0 & 19.0 & 19.0 & 19.0 & 19.0 & .19 .0 & 562.799 .1 \\
\hline COt.aLIN ORG PART & & 19.1 & 19.1 & 19.1 & 29.1 & .19 .1 & 29.1 & 29.1 & 19.1 & 19.1 & 19.1 & 19.1 & 19.1 & 19.1. & 19.1 & 19.1 & 567.4100 .0 \\
\hline \multirow[t]{7}{*}{ LAB/CONT LQ } & $\pi$ & 0.7 & 0.7 & 0.7 & 0.7 & 0.7 & 0.7 & 0.7 & 0.7 & 0.7 & 0.7 & 0.7 & 0.7 & 0.7 & 0.7 & 0.7 & $22.7 \quad 5.3$ \\
\hline & B & 0.1 & 0.1 & 0.4 & 0.1 & 0.4 & 0.4 & 0.4 & 0.1 & 0.1 & 0.1 & 0.1 & 0.1 & 0.1 & 0.1 & 0.4 & 13.6 \\
\hline & D & 0.8 & 0.0 & 0.8 & 0.8 & 0.8 & 0.8 & 0.0 & 0.8 & 0.0 & 0.8 & 0.8 & 0.8 & 0.0 & 0.8 & 0.8 & 25.0 \\
\hline & $\varepsilon$ & 0.3 & 0.3 & 0.3 & 0.3 & 0.3 & 0.3 & 0.3 & 0.3 & 0.3 & 0.3 & 0.3 & 0.3 & 0.3 & 0.3 & 0.3 & 9.1 \\
\hline & $E$ & 1.0 & 1.8 & 1.8 & 1.8 & 1.8 & 1.0 & 1.8 & 1.0 & 1.8 & 1.8 & 1.8 & 1.8 & 1.8 & 1.8 & 1.0 & $54.7 \quad 12.8$ \\
\hline & G & 3.4 & 3.4 & 3.4 & 3.4 & 3.1 & 3.4 & 3.4 & 3.4 & 3.4 & 3.1 & 3.4 & 3.4 & 3.4 & 3.4 & 3.4 & 102.524 .1 \\
\hline & NOIE & 6.6 & 6.6 & 6.6 & 6.6 & 6.6 & 6.6 & 6.6 & 6.6 & 6.6 & 6.6 & 6.6 & 6.6 & 6.6 & 6.6 & 6.6 & $196.9 \quad 46.3$ \\
\hline TOta1LAB/CONT LQ & & 14.3 & 14.3 & 31.3 & 31.3 & 14.3 & 14.3 & 14.3 & 14.3 & 14.3 & 14.3 & 14.3 & 14.3 & 24.3 & 14.3 & 14.3 & 124.9100 .0 \\
\hline Total LBL & & 53.1 & 53.1 & 53.1 & 53.1 & .53 .1 & 53.1 & 53.1 & 53.1 & 53.1 & 53.1 & 53.1 & 53.1 & 53.1 & 53.1 & 53.1 & 16009.0 \\
\hline
\end{tabular}

\section{Haste Generator:MIT BATRs \\ Haste Generator:MII__BATRES
ORG PART NONE}

\begin{tabular}{llllllllllllllllll} 
TOtaloRg PART & 0.3 & 0.0 & 0.0 & 0.0 & 0.0 & 0.3 & 0.0 & 0.0 & 0.0 & 0.0 & 0.3 & 0.0 & 0.0 & 0.0 & 0.0 & 1.5100 .0 \\
\hline Tota1HIt_BATES & 0.3 & 0.0 & 0.0 & 0.0 & 0.0 & 0.3 & 0.0 & 0.0 & 0.0 & 0.0 & 0.3 & 0.0 & 0.0 & 0.0 & 0.0 & 1.5 &
\end{tabular}

\section{Faste Generator:PADUCAH_ng}

\begin{tabular}{|c|c|c|c|c|c|c|c|c|c|c|c|c|c|c|c|c|c|}
\hline \multirow[t]{3}{*}{ CONT SOIL } & DE & 76.5 & 16.5 & 76.5 & 76.5 & 76.5 & 76.5 & 76.5 & 76.5 & 76.5 & 76.5 & 76.5 & 76.5 & 76.5 & 76.5 & 76.5 & 2309.525 .3 \\
\hline & $\mathbf{F}$ & 76.5 & 76.5 & 76.5 & 76.5 & 76.5 & 76.5 & 76.5 & 76.5 & 76.5 & 76.5 & 76.5 & 76.5 & 76.3 & 76.5 & 76.5 & $2309.5 \quad 25.3$ \\
\hline & HONE & 105.4 & 105.4 & 105.4 & 203.4 & 105.1 & 205.4 & 105.1 & 105.1 & 105.4 & 105.4 & 105.1 & 105.4 & 105.4 & 105.4 & 105.4 & $4509.2 \quad 49.3$ \\
\hline Totalcont soIh & & 250.4 & 258.4 & 258.4 & 250.1 & 258.1 & 258.4 & 258.4 & 258.1 & 258.4 & 258.4 & 250.1 & 250.4 & 250.1 & 258.4 & 258.4 & 9128.4100 .0 \\
\hline D-COMBUST & NOHE & 25.4 & 25.4 & 25.4 & 25.4 & 25.4 & 25.4 & 25.4 & 25.1 & 25.4 & 25.4 & 25.4 & 25.4 & 25.1 & 25.4 & 25.4 & $1090.5 ! 100.0$ \\
\hline Total D-coHBUST & & 25.1 & 25.1 & 25.4 & 25.4 & 25.4 & 23.4 & 25.4 & 25.4 & 25.4 & 25.4 & 25.1 & 25.1 & 25.1 & 25.4 & 25.1 & 1090.5100 .0 \\
\hline D-IN NON-MTL & DE & 8.5 & 8.5 & 0.5 & 6.5 & 0.5 & 0.5 & 8.5 & B.5 & 0.5 & 8.5 & 8.5 & 0.5 & 0.5 & 8.5 & 8.5 & $256.6 \quad 16.0$ \\
\hline & $\mathbf{F}$ & 0.5 & 8.5 & 0.5 & 8.5 & 0.5 & 0.5 & .8 .5 & 6.5 & 8.5 & 8.5 & 0.5 & 8.5 & 8.5 & 8.5 & 0.5 & $256.6 \quad 16.0$ \\
\hline$\cdot$ & NONE & 25.4 & 25.1 & 25.1 & 25.4 & 25.1 & 23.4 & 23.1 & 25.4 & 25.4 & 25.4 & 25.1 & 25.4 & 25.4 & 25.4 & 25.4 & $1090.5 \quad 67.9$ \\
\hline TotaID-IN. NOH-MTL & & 12.4 & 12.1 & 12.1 & 12.1 & 42.4 & 12.4 & 12.4 & 42.4 & 12.4 & 12.4 & 12.4 & 42.4 & 12.4 & 12.4 & 42.4 & 1603.7100 .0 \\
\hline Fotal PADUCAH_ES & & 326.4 & 326.1 & 326.1 & 326.4 & 326.4 & 326.1 & 326.4 & 326.4 & 326.4 & 326.1 & 326.4 & 326.4 & 326.4 & 26.4 & 26.4 & 1822.7 \\
\hline
\end{tabular}

\section{Haste Generator:PADUCAH US}

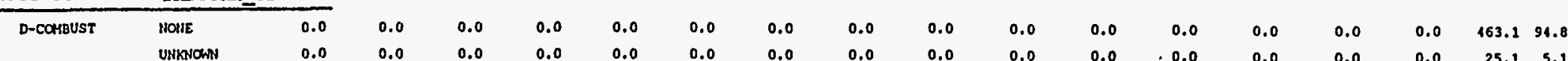

ged Codes: A-Ignitables, B-Cortosives, CwReactives, DeMetals w/O Hg, E-Metals w Hg, E-Organles, G-State Reg., H-PCB < 50ppm, J-PCB > 50ppm 


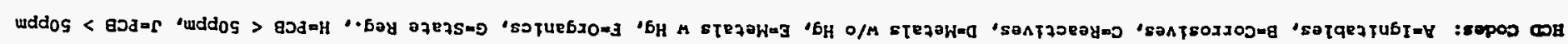

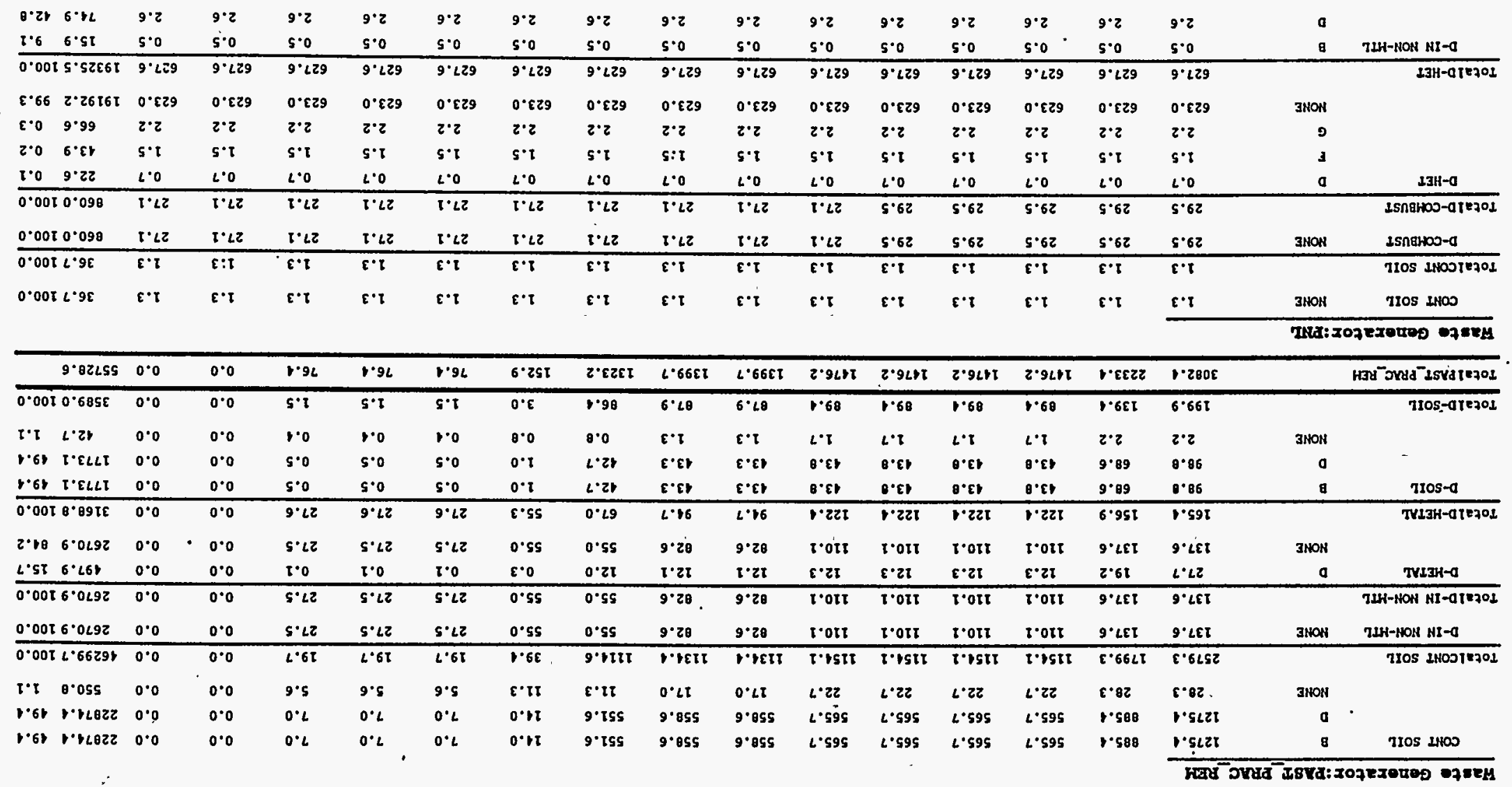

\begin{tabular}{|c|c|c|c|c|c|c|c|c|c|c|c|c|c|c|c|c|}
\hline . 256 & 0.0 & 0.0 & 0.0 & 0.0 & 0.0 & 0.0 & 0.0 & 0.0 & 0.0 & 0.0 & 0.0 & 0.0 & 0.0 & 0.0 & 0.0 & $\sin ^{-}$HYonavd IEzOL \\
\hline 0.0019 .26 & 0.0 & 0.0 & 0.0 & 0.0 & 0.0 & 0.0 & 0.0 & 0.0 & 0.0 & 0.0 & 0.0 & 0.0 & 0.0 & 0.0 & 0.0 & 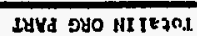 \\
\hline
\end{tabular}

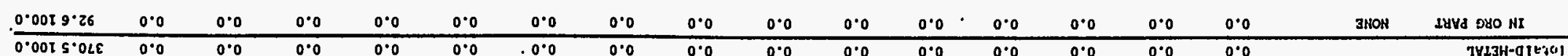

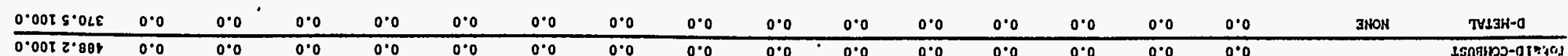

70d TеZ0I

qzT $250 \mathrm{~d}$

(8x070ux ofqno uT)

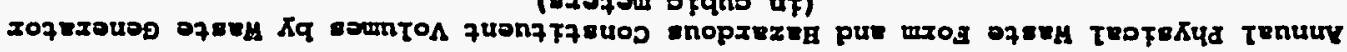




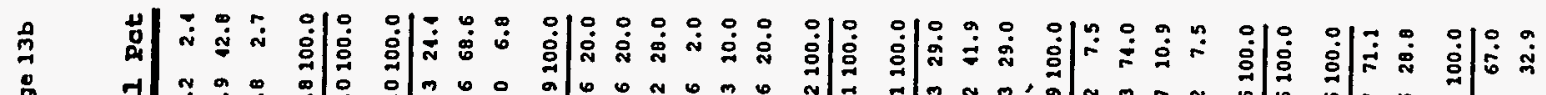

:

ลั่

可

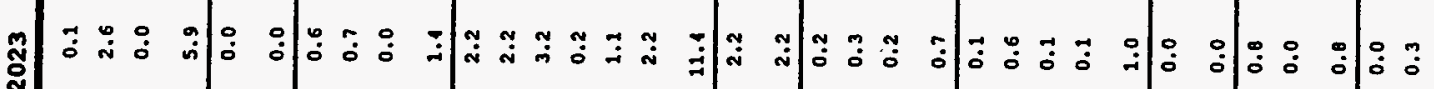

สู้

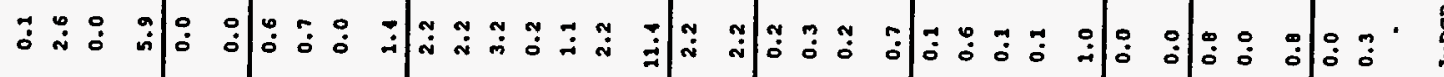

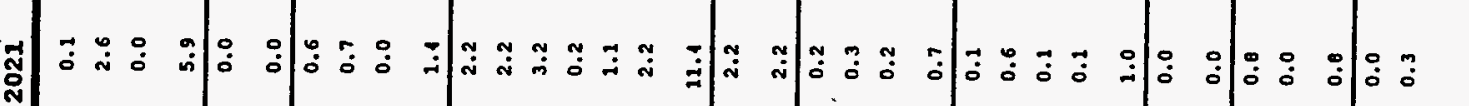

芯

究

象

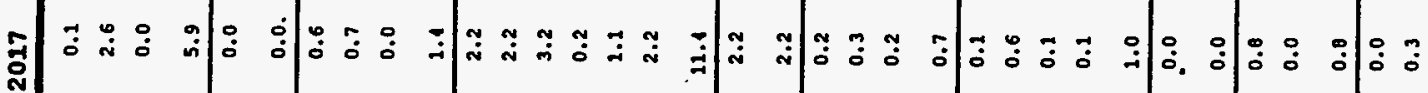

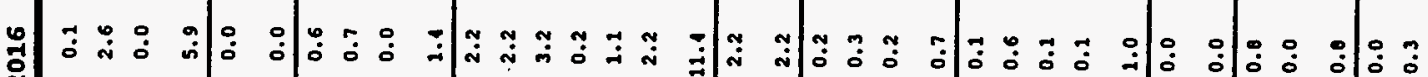

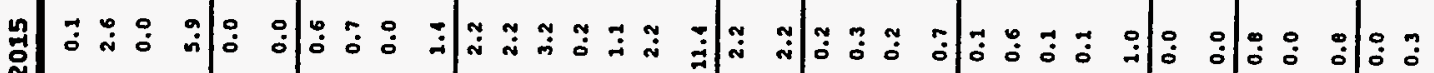

ב.

:

萑

究

羿

声

苟

오ำ

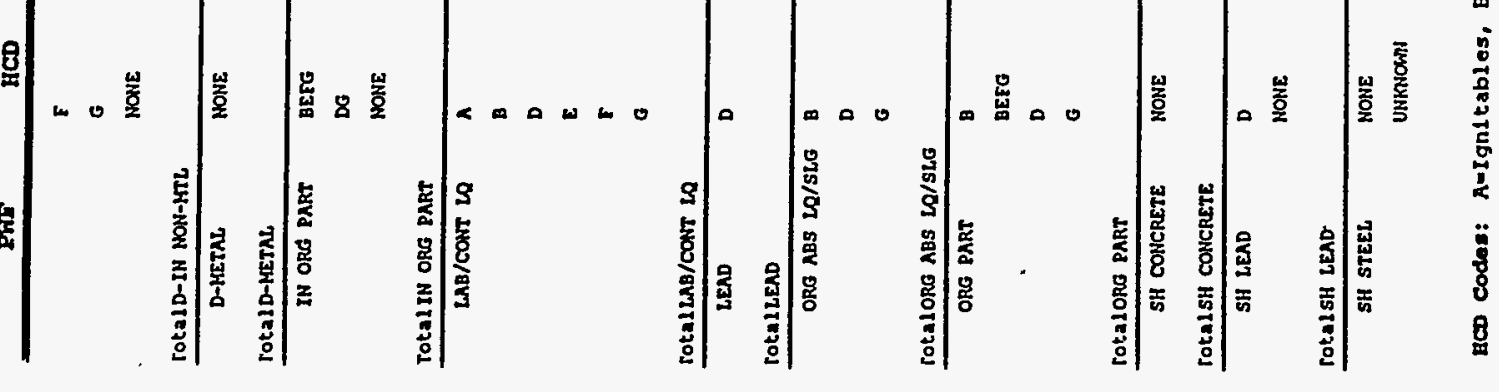




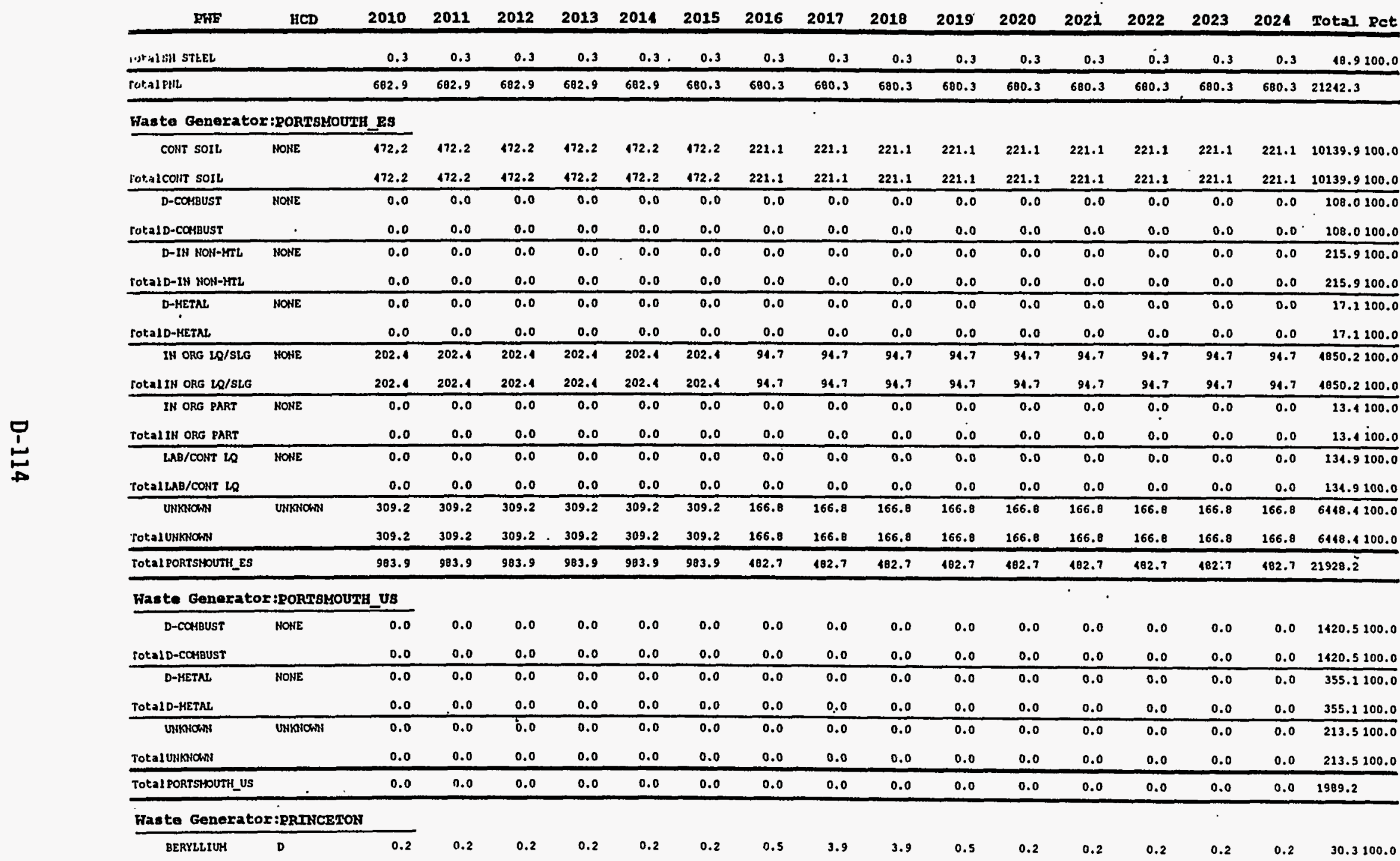

HOD Codes: A-Ignitables, B-Corrosives, C-Reactives, DmMetals w/O Hg, E-Metals w Hg, F-Organlcs, G-State Reg., H-PCB < 50ppm, J-PCB > 50ppm 
Arnual Physical Faste Form and gazaxdous Constituent Volumes by Maste Generator

Page 15b (in cuble metera)

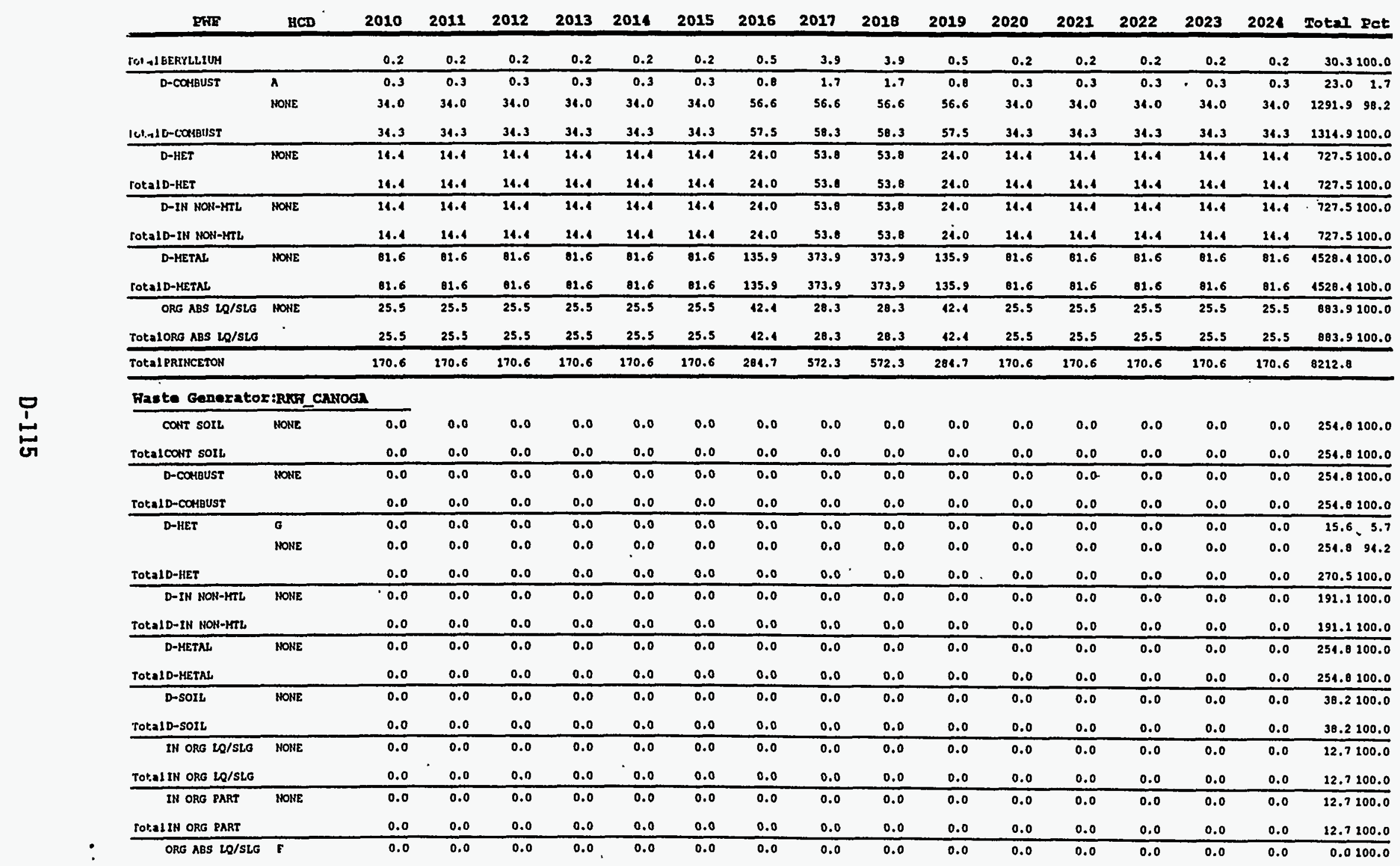

BCD Coden: A-Ignitables, B-Corrosives, C-Reactives, D-Metals w/O Hg, E-Metals w Hg, Em-Organles, G-State Reg., H-PCB $\leq$ 50ppm, JuPCB > 50ppm 
(in cubic meters)

\begin{tabular}{|c|c|c|c|c|c|c|c|c|c|c|c|c|c|c|c|c|c|}
\hline FWE & HCD & 2010 & 2011 & 2012 & 2013 & 2014 & 2015 & 2016 & 2017 & 2018 & 2019 & 2020 & 2021 & 2022 & 2023 & 2024 & Total Pot \\
\hline i,staloprs ABS LO/SLG & & 0.0 & 0.0 & 0.0 & 0.0 & 0.0 & 0.0 & 0.0 & 0.0 & 0.0 & 0.0 & 0.0 & 0.0 & 0.0 & 0.0 & 0.0 & 0.0100 .0 \\
\hline SALT WASTE & $D G$ & 0.0 & 0.0 & 0.0 & 0.0 & 0.0 & 0.0 & 0.0 & 0.0 & 0.0 & 0.0 & 0.0 & 0.0 & 0.0 & 0.0 & 0.0 & 0.0100 .0 \\
\hline DOt.hl SALT WASTE & & 0.0 & 0.0 & 0.0 & 0.0 & 0.0 & 0.0 & 0.0 & 0.0 & 0.0 & 0.0 & 0.0 & 0.0 & 0.0 & 0.0 & 0.0 & 0.0100 .0 \\
\hline rOt:al RKW_CANOGA & & 0.0 & 0.0 & 0.0 & 0.0 & 0.0 & 0.0 & 0.0 & 0.0 & 0.0 & 0.0 & 0.0 & 0.0 & 0.0 & 0.0 & 0.0 & 1290.1 \\
\hline \multicolumn{18}{|c|}{ Waste Generator:8MnNFORD } \\
\hline \multirow[t]{2}{*}{ D-IN MON-MTL } & $n$ & .0 .1 & 0.0 & 0.1 & 0.0 & 0.1 & 0.0 & 0.1 & 0.0 & 0.1 & 0.0 & 0.1 & 0.0 & 0.1 & 0.0 & 0.1 & 3.170 .5 \\
\hline & NOHE & 0.0 & 0.0 & 0.0 & 0.0 & 0.0 & 0.0 & 0.0 & 0.0 & 0.0 & 0.0 & 0.0 & 0.0 & 0.0 & 0.0 & 0.0 & 1.329 .1 \\
\hline TOta1D-IN NON-MTL & & 0.1 & 0.0 & 0.1 & 0.0 & 0.1 & 0.0 & 0.1 & 0.0 & 0.1 & 0.0 & 0.1 & 0.0 & 0.1 & 0.0 & 0.1 & 1.1100 .0 \\
\hline \multirow[t]{2}{*}{ D-METAL } & D & 0.0 & 0.0 & 0.0 & 0.0 & 0.0 & 0.0 & 0.0 & 0.0 & 0.0 & 0.0 & 0.0 & 0.0 & 0.0 & 0.0 & 0.0 & 0.10 .0 \\
\hline & NONE & 16.9 & 16.9 & 16.9 & 16.9 & 16.9 & 16.9 & 16.9 & 16.9 & 26.9 & 16.9 & 16.9 & 26.9 & 16.9 & 26.9 & 16.9 & $942.2 \quad 99.9$ \\
\hline Tota1D-METAL & & 16.9 & 16.9 & 16.9 & 16.9 & 16.9 & 16.9 & 16.9 & 16.9 & 16.9 & 16.9 & 16.9 & 16.9 & 26.9 & 16.9 & 16.9 & 942.3100 .0 \\
\hline LAB/CONT $L Q$ & NONE & .0 .0 & 0.0 & 0.0 & 0.0 & 0.0 & 0.0 & 0.0 & 0.0 & 0.0 & 0.0 & 0.0 & 0.0 & 0.0 & 0.0 & 0.0 & 1.3100 .0 \\
\hline Total LAB/CONT LQ & & 0.0 & 0.0 & 0.0 & 0.0 & 0.0 & 0.0 & 0.0 & 0.0 & 0.0 & 0.0 & 0.0 & 0.0 & 0.0 & 0.0 & 0.0 & 1.3100 .0 \\
\hline LEND & D & 0.2 & 0.0 & 0.2 & 0.0 & 0.2 & 0.0 & 0.2 & 0.0 & 0.2 & 0.0 & 0.2 & 0.0 & 0.2 & 0.0 & 0.2 & 7.5100 .0 \\
\hline TOtalLEAD & & 0.2 & 0.0 & 0.2 & 0.0 & 0.2 & 0.0 & 0.2 & 0.0 & 0.2 & 0.0 & 0.2 & 0.0 & 0.2 & 0.0 & 0.2 & 7.5100 .0 \\
\hline ORG ABS LO/SLG & MONE & 0.0 & 0.0 & 0.0 & 0.0 & 0.0 & 0.0 & 0.0 & 0.0 & 0.0 & 0.0 & 0.0 & 0.0 & 0.0 & 0.0 & 0.0 & 6.5100 .0 \\
\hline TOtalORG ABS LQ/SLG & & 0.0 & 0.0 & 0.0 & 0.0 & 0.0 & 0.0 & 0.0 & 0.0 & 0.0 & 0.0 & 0.0 & 0.0 & 0.0 & 0.0 & 0.0 & 6.5100 .0 \\
\hline ORG PART & MONE & 0.0 & 0.0 & 0.0 & 0.0 & 0.0 & 0.0 & 0.0 & 0.0 & 0.0 & 0.0 & 0.0 & 0.0 & 0.0 & 0.0 & 0.0 & 6.5100 .0 \\
\hline Totalorg PART & & 0.0 & 0.0 & 0.0 & 0.0 & 0.0 & 0.0 & 0.0 & 0.0 & 0.0 & 0.0 & 0.0 & 0.0 & 0.0 & 0.0 & 0.0 & 6.5100 .0 \\
\hline Total STANBORD & & 17.6 & 17.2 & 17.6 & 17.2 & 17.6 & 17.2 & 17.6 & 17.2 & 17.6 & 17.2 & 27.6 & 27.2 & 17.6 & 17.2 & 17.6 & 968.8 . \\
\hline Faste Generato & E:THRL & & & & & & & & & & & & & & & & \\
\hline D-COHBUST & NONE & 0.0 & 0.0 & 0.0 & 0.3 & 0.3 & 0.3 & 0.3 & 0.3 & 0.3 & 0.3 & 0.3 & 0.3 & 0.3 & 0.0 & 0.0 & 3.7100 .0 \\
\hline Tot.al D-consust & & 0.0 & 0.0 & 0.0 & 0.3 & 0.3 & 0.3 & 0.3 & 0.3 & 0.3 & 0.3 & 0.3 & 0.3 & 0.3 & 0.0 & 0.0 & 3.7100 .0 \\
\hline D-HET & NOHE & 0.0 & 0.0 & 0.0 & 0.5 & 0.5 & 0.5 & 0.5 & 0.5 & 0.5 & 0.5 & 0.5 & 0.5 & 0.5 & 0.0 & 0.0 & 5.4100 .0 \\
\hline TotalD-HET & & 0.0 & 0.0 & 0.0 & 0.5 & 0.5 & 0.5 & 0.5 & 0.5 & 0.5 & 0.5 & 0.5 & 0.5 & 0.5 & 0.0 & 0.0 & 5.1100 .0 \\
\hline D-HETAL & B & 0.0 & 0.0 & 0.0 & 0.2 & 0.2 & 0.2 & 0.2 & 0.2 & 0.2 & 0.2 & 0.2 & 0.2 & $\overline{0.2}$ & 0.0 & 0.0 & $2.0 \quad 7.0$ \\
\hline & NONE & 0.0 & 0.0 & 0.0 & 2.6 & 2.6 & 2.6 & 2.6 & 2.6 & 2.6 & 2.6 & 2.6 & 2.6 & 2.6 & 0.0 & 0.0 & $26.5 \quad 92.9$ \\
\hline TOL21D-HETAL & & 0.0 & .0 .0 & 0.0 & 2.0 & 2.8 & 2.8 & 2.0 & 2.8 & 2.8 & 2.8 & 2.0 & 2.0 & 2.8 & 0.0 & 0.0 & 28.5100 .0 \\
\hline Tota1TPNL_2312 & & 0.0 & 0.0 & 0.0 & 3.7 & 3.7 & 3.7 & 3.7 & 3.7 & 3.7 & 3.7 & 3.7 & 3.7 & 3.7 & 0.0 & 0.0 & 37.7 \\
\hline Faste Generato & $r: T P 2 \pi$ & 84 & & & & & & & & & & & & & & & \\
\hline D-METAL & NONE & 0.0 & 0.0 & 0.0 & 6.2 & 6.2 & 6.2 & 6.2 & 6.2 & 6.2 & 6.2 & 6.2 & 6.2 & 6.2 & 0.0 & 0.0 & 62.0100 .0 \\
\hline
\end{tabular}

nCD Codes: A-Ignitables, B-Corrosives, C-Reactives, DmMetals w/O Hg, EmMetals w Hg, F-Organics, G-State Reg., HuPCB < 50ppm, J-PCB > 50ppm 


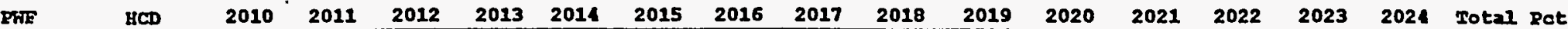

\begin{tabular}{|c|c|c|c|c|c|c|c|c|c|c|c|c|c|c|c|c|c|}
\hline otill U-METAL & & 0.0 & 0.0 & 0.0 & 6.2 & 6.2 & 6.2 & 6.2 & 6.2 & 6.2 & 6.2 & 6.2 & 6.2 & 6.2 & 0.0 & 0.0 & 62.0100 .0 \\
\hline UHKNOXWN & NONE & 0.0 & 0.0 & 0.0 & 3.3 & 3.3 & 3.3 & 3.3 & 3.3 & 3.3 & 3.3 & 3.3 & 3.3 & 3.3 & 0.0 & 0.0 & 33.9100 .0 \\
\hline 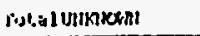 & & 0.0 & 0.0 & 0.0 & 3.3 & 3.3 & 3.3 & 3.3 & 3.3 & 3.3 & 3.3 & 3.3 & 3.3 & 3.3 & 0.0 & 0.0 & 33.9100 .0 \\
\hline Tot.alTPHL_262B/BL & & 0.0 & 0.0 & $0: 0$ & 9.6 & 9.6 & 9.6 & 9.6 & 9.6 & 9.6 & 9.6 & 9.6 & 9.6 & 9.6 & 0.0 & 0.0 & 96.0 \\
\hline \multicolumn{18}{|c|}{ Waste Generator:TH2⿰氵 $306 \mathrm{H}$} \\
\hline D-HET & MONE & 0.0 & 0.0 & 0.0 & 0.3 & 0.3 & 0.3 & 0.3 & 0.3 & 0.3 & 0.3 & 0.3 & 0.3 & 0.3 & 0.0 & 0.0 & 3.4100 .0 \\
\hline TotalD-HET & & 0.0 & 0.0 & 0.0 & 0.3 & 0.3 & 0.3 & 0.3 & 0.3 & 0.3 & 0.3 & 0.3 & 0.3 & 0.3 & 0.0 & 0.0 & 3.1100 .0 \\
\hline D-METAL & MONE & 0.0 & 0.0 & 0.0 & 4.4 & 4.4 & 4.4 & 4.4 & 4.4 & 4.4 & 4.4 & 4.4 & 6.4 & 4.4 & 0.0 & 0.0 & 14.2100 .0 \\
\hline TOta1D-KETAL & & 0.0 & $\cdot 0.0$ & 0.0 & 4.1 & 1.4 & 1.4 & 1.4 & 4.1 & 4.4 & 4.4 & 4.4 & 4.4 & 1.4 & 0.0 & 0.0 & 11.2100 .0 \\
\hline UMAarown & MONE & 0.0 & 0.0 & 0.0 & 0.3 & 0.3 & 0.3 & 0.3 & 0.3 & 0.3 & 0.3 & 0.3 & 0.3 & 0.3 & 0.0 & 0.0 & 3.1100 .0 \\
\hline Potal UNinkown & & 0.0 & 0.0 & 0.0 & 0.3 & 0.3 & 0.3 & 0.3 & 0.3 & 0.3 & 0.3 & 0.3 & 0.3 & 0.3 & 0.0 & 0.0 & 3.1100 .0 \\
\hline Total TPNL_306 & & 0.0 & 0.0 & 0.0 & 5.1 & 5.1 & 5.1 & 3.1 & 5.1 & 5.1 & 5.2 & 5.1 & 5.1 & 5.2 & 0.0 & 0.0 & 51.0 \\
\hline
\end{tabular}

\section{Faste Generator:TP2R_ 314}

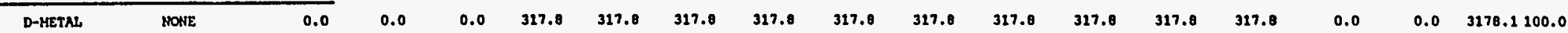

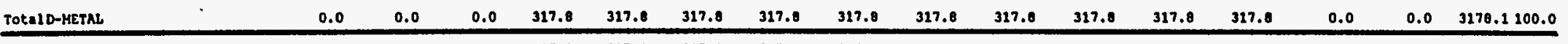

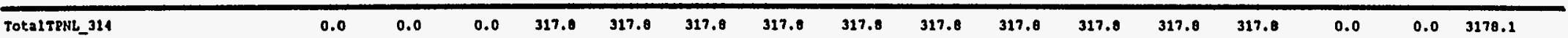

\section{- Faste Generator:TP2⿰_ 324}

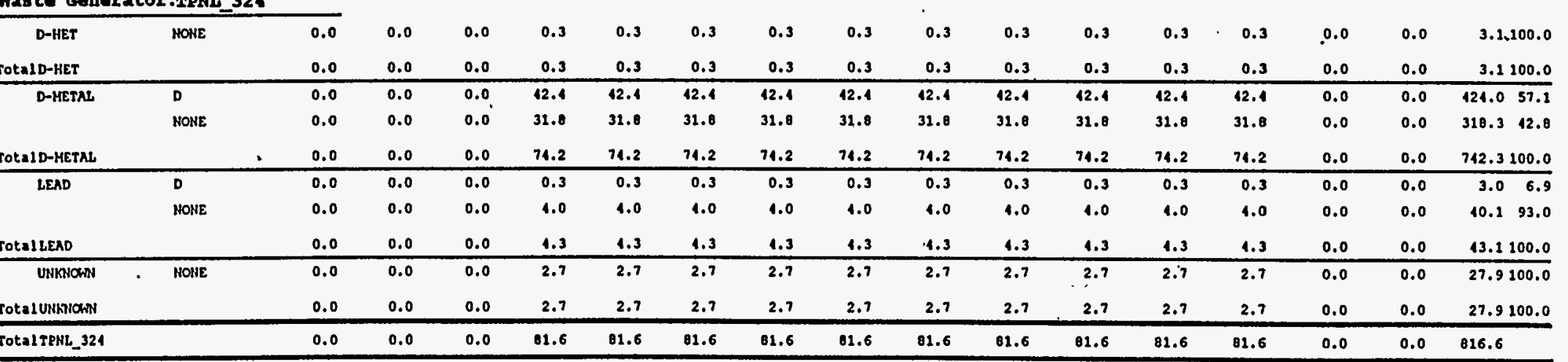

Haste Generator:TPRL 326

$\begin{array}{lllllllllllllllllll}\text { D-HET } & \text { UNKNOWN } & 0.0 & 0.0 & 0.0 & 0.3 & 0.3 & 0.3 & 0.3 & 0.3 & 0.3 & 0.3 & 0.3 & 0.3 & 0.3 & 0.0 & 0.0 & 3.8 & 100.0\end{array}$

gCo Codes: A-Ignitables, B=Corrosives, C-Reactives, D-Metals w/O Hg, E=-Metals w Hg, Emorganles, G=State Reg., H-PCB < 50ppm, J-PCB > 50ppm 


\begin{tabular}{|c|c|c|c|c|c|c|c|c|c|c|c|c|c|c|c|c|c|}
\hline PHF & HCD & 2010 & 2011 & 2012 & 2013 & 2014 & 2015 & 2016 & 2017 & 2018 & 2019 & 2020 & 2021 & 2022 & 2023 & 2024 & Total Rot \\
\hline . DH+1U-HET & & 0.0 & 0.0 & 0.0 & 0.3 & 0.3 & 0.3 & 0.3 & 0.3 & 0.3 & 0.3 & 0.3 & 0.3 & 0.3 & 0.0 & 0.0 & 3.8100 .0 \\
\hline \multirow[t]{2}{*}{ D-METAL } & HONE & 0.0 & 0.0 & 0.0 & 1.1 & 1.1 & 1.1 & 1.1 & 1.1 & 1.1 & 1.1 & 1.1 & 1.1 & 1.1 & 0.0 & 0.0 & $21.5 \quad 55.8$ \\
\hline & UNKNIOWI & 0.0 & 0.0 & 0.0 & 0.9 & 0.9 & 0.9 & 0.9 & 0.9 & 0.9 & 0.9 & 0.9 & 0.9 & 0.9 & 0.0 & 0.0 & $9.1 \quad 14.1$ \\
\hline HOt:al D-METAL & & 0.0 & 0.0 & 0.0 & 2.0 & 2.0 & 2.0 & 2.0 & 2.0 & 2.0 & 2.0 & 2.0 & 2.0 & 2.0 & 0.0 & 0.0 & 20.6100 .0 \\
\hline LEAD & UNKNOWN & 0.0 & 0.0 & 0.0 & 0.8 & 0.8 & 0.8 & 0.8 & 0.8 & 0.8 & 0.8 & 0.8 & 0.8 & 0.8 & 0.0 & 0.0 & 8.9100 .0 \\
\hline IOR:ALLEAD & & 0.0 & 0.0 & 0.0 & 0.0 & 0.8 & 0.8 & 0.8 & 0.8 & 0.8 & 0.8 & 0.0 & 0.8 & 0.8 & 0.0 & 0.0 & 8.9100 .0 \\
\hline \multirow[t]{2}{*}{ UNANOCA } & NONE & 0.0 & 0.0 & 0.0 & 0.1 & 0.4 & 0.4 & 0.1 & 0.4 & 0.4 & 0.1 & 0.4 & 0.1 & 0.1 & 0.0 & 0.0 & $4.5 \quad 59.5$ \\
\hline & UNGNOWN & 0.0 & 0.0 & 0.0 & 0.3 & 0.3 & 0.3 & 0.3 & 0.3 & 0.3 & 0.3 & 0.3 & 0.3 & 0.3 & 0.0 & 0.0 & $3.0 \quad 10.4$ \\
\hline Potal UNKNOWN & & 0.0 & 0.0 & 0.0 & 0.7 & 0.7 & 0.7 & 0.7 & 0.7 & 0.7 & 0.7 & 0.7 & 0.7 & 0.7 & 0.0 & 0.0 & 7.5100 .0 \\
\hline Total TPNL 326 & & 0.0 & 0.0 & 0.0 & 4.0 & 4.0 & 1.0 & 4.0 & 4.0 & 1.0 & 4.0 & 4.0 & 4.0 & 4.0 & 0.0 & 0.0 & 10.9 \\
\hline
\end{tabular}

\section{Waste Generator:Trisz 327}

\begin{tabular}{llllllllllllllllllllll}
\hline D-HET & HONE & & 0.0 & 0.0 & 0.0 & 1.6 & 1.6 & 1.6 & 1.6 & 1.6 & 1.6 & 1.6 & 1.6 & 1.6 & 1.6 & 0.0 & 0.0 & 16.9 & 100.0
\end{tabular}

\begin{tabular}{|c|c|c|c|c|c|c|c|c|c|c|c|c|c|c|c|c|c|}
\hline TotalD-HET & & 0.0 & 0.0 & 0.0 & 1.6 & 1.6 & 1.6 & 1.6 & 1.6 & 1.6 & 1.6 & 1.6 & 1.6 & 1.6 & 0.0 & 0.0 & 16.9100 .0 \\
\hline \multirow[t]{2}{*}{ D-METAL. } & $\mathbf{r}$ & 0.0 & 0.0 & 0.0 & 0.2 & 0.2 & 0.2 & 0.2 & 0.2 & 0.2 & 0.2 & 0.2 & 0.2 & 0.2 & 0.0 & 0.0 & $2.3 \quad 0.1$ \\
\hline & NONE & 0.0 & 0.0 & 0.0 & 197.3 & 187.3 & 187.3 & 187.3 & 187.3 & 207.3 & 287.3 & 187.3 & 187.3 & 187.3 & 0.0 & 0.0 & $1073.3 \quad 99.8$ \\
\hline Total D-METAL & & 0.0 & 0.0 & 0.0 & 107.5 & 197.5 & 107.5 & 187.5 & 107.5 & 187.5 & 187.5 & 187.5 & 107.5 & 187.5 & 0.0 & 0.0 & 1875.6100 .0 \\
\hline \multirow[t]{2}{*}{ LEAD } & D & 0.0 & 0.0 & 0.0 & 0.5 & 0.5 & 0.5 & 0.5 & 0.5 & 0.5 & 0.5 & 0.5 & 0.5 & 0.5 & 0.0 & 0.0 & 5.6 \\
\hline & HONE & 0.0 & 0.0 & 0.0 & 15.0 & 15.0 & 15.0 & 25.0 & 15.0 & 15.0 & 15.0 & 15.0 & 15.0 & 25.0 & 0.0 & 0.0 & 150.896 .3 \\
\hline TOta1LEAD & & 0.0 & 0.0 & 0.0 & 15.6 & 15.6 & 15.6 & 15.6 & 15.6 & 15.6 & 25.6 & 15.6 & 15.6 & 15.6 & 0.0 & 0.0 & 156.5100 .0 \\
\hline UNKAOCWN & MONE & 0.0 & 0.0 & 0.0 & 16.3 & 16.3 & 16.3 & 16.3 & 16.3 & 16.3 & 16.3 & 46.3 & 46.3 & 46.3 & 0.0 & 0.0 & 163.0100 .0 \\
\hline Total UNKNOWN & & 0.0 & 0.0 & 0.0 & 16.3 & 16.3 & 16.3 & 16.3 & 46.3 & 16.3 & 46.3 & 16.3 & 46.3 & 16.3 & 0.0 & 0.0 & 163.0100 .0 \\
\hline rotal TPNL_327 & & 0.0 & 0.0 & 0.0 & 251.2 & 251.2 & 251.2 & 251.2 & 251.2 & 251.2 & 251.2 & 251.2 & 251.2 & 251.2 & 0.0 & 0.0 & 2512.1 \\
\hline
\end{tabular}

Taste Generatorimang 331

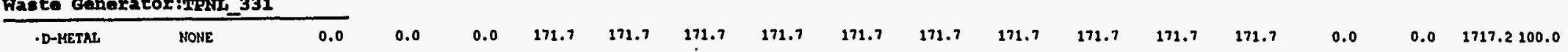

\begin{tabular}{|c|c|c|c|c|c|c|c|c|c|c|c|c|c|c|c|}
\hline ETAL & 0.0 & 0.0 & 171.7 & 171.7 & 171.7 & 171.7 & 171.7 & 171.7 & 171.7 & 171.7 & 171.7 & 171.7 & 0.0 & 0.0 & 2717.2100 .0 \\
\hline UNKNOWN & 0.0 & 0.0 & 19.0 & 19.0 & 29.0 & 19.0 & 19.0 & 19.0 & 19.0 & 19.0 & 19.0 & 19.0 & 0.0 & 0.0 & 100,0 \\
\hline
\end{tabular}

\begin{tabular}{|c|c|c|c|c|c|c|c|c|c|c|c|c|c|c|c|c|}
\hline rot & 0.0 & 0.0 & 0.0 & 19.0 & 29.0 & 19.0 & 19.0 & 19.0 & 19.0 & 19.0 & 19.0 & 19.0 & 19.0 & 0.0 & 0.0 & 190.8100 .0 \\
\hline & 0.0 & 0.0 & 0.0 & 190.0 & 190.8 & 190.8 & 190.8 & 190.8 & 190.8 & 190.8 & 190.8 & 190.8 & 190.8 & 0.0 & 0.0 & \\
\hline
\end{tabular}

Faste Generator:1WHC_100N

\begin{tabular}{|c|c|c|c|c|c|c|c|c|c|c|c|c|c|c|c|c|c|}
\hline D-HET & NONE & 0.0 & 0.0 & 0.0 & 314.3 & 314.3 & 314,3 & 314.3 & 314.3 & 314.3 & 314.3 & 314.3 & 314.3 & 311.3 & 0.0 & 0.0 & 3143.5100 .0 \\
\hline EAL D-HET & & 0.0 & 0.0 & 0.0 & 314.3 & 314.3 & $314.3^{n}$ & 314.3 & 314.3 & 314.3 & 314.3 & 314.3 & 314.3 & 314.3 & 0.0 & 0.0 & 3143.5200 .0 \\
\hline
\end{tabular}

ECO Codes: A-Ignitables, B-Corrosives, C-Reactives, D-Metals w/O Hg, E-Metals w Hg, F-Organlcs, G-State Reg., H-PCB < 50ppm, J-PCB > 50ppm 
Annual Phyaical waste Form and Bainardous Constituent Volumes by Waste Generator (in cubic meters)

\begin{tabular}{|c|c|c|c|c|c|c|c|c|c|c|c|c|c|c|c|c|c|}
\hline PWE & HCD & 2010 & 2011 & 2012 & 2013 & 2014 & 2015 & 2016 & 2017 & 2018 & 2019 & 2020 & 2021 & 2022 & 2023 & 2024 & Total Pot \\
\hline D-METAL & MOHE & 0.0 & 0.0 & 0.0 & 518.2 & 518.2 & 518.2 & 518.2 & 318.2 & 518.2 & 518.2 & 516.2 & 518.2 & 518.2 & 0.0 & 0.0 & 5182.5200 .0 \\
\hline Dt:alD-METAL & & 0.0 & 0.0 & 0.0 & 518.2 & 518.2 & 518.2 & 518.2 & 518.2 & 518.2 & 510.2 & 518.2 & 518.2 & 518.2 & $0: 0$ & 0.0 & 5182.5100 .0 \\
\hline UNKHOWN & MONE & 0.0 & 0.0 & 0.0 & 118.5 & 118.5 & 118.5 & 118.5 & 118.5 & 118.5 & 118.5 & 218.5 & 110.5 & 118.5 & 0.0 & 0.0 & 1185.8100 .0 \\
\hline iotalunknowias & & 0.0 & 0.0 & 0.0 & 110.5 & 118.5 & 218.5 & 118.5 & 118.5 & 318.5 & 118.5 & 118.5 & 118.5 & 210.5 & 0.0 & 0.0 & 1185.0100 .0 \\
\hline Tot:al TWHA__100N & & 0.0 & 0.0 & 0.0 & 951.1 & 951.1 & 951.1 & 951.1 & 951.1 & 951.1 & 951.1 & 951.1 & 951.1 & 951.1 & 0.0 & 0.0 & 9511.8 \\
\hline \multicolumn{3}{|c|}{ Haste Ganerator:1HHC_222s } & & & & & & & & & . & & & & & & \\
\hline \multirow[t]{8}{*}{ D-HET } & $A$ & 0.0 & 0.0 & 0.0 & 0.2 & 0.2 & 0.2 & 0.2 & 0.2 & 0.2 & 0.2 & 0.2 & 0.2 & 0.2 & 0.0 & 0.0 & 2.7 \\
\hline & c & 0.0 & 0.0 & 0.0 & 0.0 & 0.0 & 0.0 & 0.0 & 0.0 & 0.0 & 0.0 & 0.0 & 0.0 & 0.0 & 0.0 & 0.0 & 0.1 \\
\hline & D & 0.0 & 0.0 & 0.0 & 0.0 & 0.0 & 0.0 & 0.0 & 0.0 & 0.0 & 0.0 & 0.0 & 0.0 & 0.0 & 0.0 & 0.0 & 0.3 \\
\hline & $\mathbf{F}$ & 0.0 & 0.0 & 0.0 & 0.2 & 0.2 & 0.2 & 0.2 & 0.2 & 0.2 & 0.2 & 0.2 & 0.2 & 0.2 & 0.0 & 0.0 & 2.7 \\
\hline & H & 0.0 & 0.0 & 0.0 & 0.0 & 0.0 & 0.0 & 0.0 & 0.0 & 0.0 & 0.0 & 0.0 & 0.0 & 0.0 & 0.0 & 0.0 & 0.0 \\
\hline & J & 0.0 & 0.0 & 0.0 & 0.0 & 0.0 & 0.0 & 0.0 & 0.0 & 0.0 & 0.0 & 0.0 & 0.0 & 0.0 & 0.0 & 0.0 & 0.0 \\
\hline & NONE & 0.0 & 0.0 & 0.0 & 6.1 & 6.1 & 6.4 & 6.4 & 6.4 & 6.1 & 6.1 & 6.4 & 6.1 & 6.4 & 0.0 & 0.0 & $64.3 \quad 85.0$ \\
\hline & unknown: & 0.0 & 0.0 & 0.0 & 0.5 & 0.5 & 0.5 & 0.5 & 0.5 & 0.5 & 0.5 & 0.5 & 0.5 & 0.5 & 0.0 & 0.0 & $5.2 \quad 6.9$ \\
\hline rotalD-KET & & 0.0 & 0.0 & 0.0 & 1.5 & 1.5 & 7.5 & 7.5 & 7.5 & 7.5 & 7.5 & 7.5 & 7.5 & 7.5 & 0.0 & 0.0 & 75.6100 .0 \\
\hline \multirow[t]{8}{*}{ D-METAR } & $A$ & 0.0 & 0.0 & 0.0 & 3.3 & 3.3 & 3.3 & 3.3 & 3.3 & 3.3 & 3.3 & 3.3 & 3.3 & 3.3 & 0.0 & 0.0 & $33.6 \quad 0.2$ \\
\hline & c & 0.0 & 0.0 & 0.0 & 0.1 & 0.1 & 0.1 & 0.1 & 0.1 & 0.1 & 0.1 & 0.1 & 0.1 & 0.1 & 0.0 & 0.0 & 1.3 \\
\hline & D & 0.0 & 0.0 & 0.0 & 0.1 & 0.1 & 0.1 & 0.1 & 0.1 & 0.1 & 0.1 & 0.4 & 0.4 & 0.4 & 0.0 & 0.0 & 4.1 \\
\hline & $\mathbf{r}^{\prime}$ & 0.0 & 0.0 & 0.0 & 3.2 & 3.2 & 3.2 & 3.2 & 3.2 & 3.2 & 3.2 & 3.2 & 3.2 & 3.2 & 0.0 & 0.0 & 32.9 \\
\hline & H & 0.0 & 0.0 & 0.0 & 0.0 & 0.0 & 0.0 & 0.0 & 0.0 & 0.0 & 0.0 & 0.0 & 0.0 & 0.0 & 0.0 & 0.0 & 0.6 \\
\hline & ง & 0.0 & 0.0 & 0.0 & 0.0 & 0.0 & 0.0 & 0.0 & 0.0 & 0.0 & 0.0 & 0.0 & 0.0 & 0.0 & 0.0 & 0.0 & $0.6 \quad 0.0$ \\
\hline & NONE & 0.0 & 0.0 & 0.0 & 2103.7 & 1183.7 & 1183.7 & 1103.7 & 1183.7 & 1103.7 & 1183.7 & 1183.7 & 2183.7 & 2183.7 & $\cdot 0.0$ & 0.0 & 11837.698 .6 \\
\hline & UNKNOWN & 0,0 & 0.0 & 0.0 & 6.3 & 6.3 & 6.3 & 6.3 & 6.3 & 6.3 & 6.3 & 6.3 & 6.3 & 6.3 & 0.0 & 0.0 & $63.8 \quad 0.5$ \\
\hline lotal D-METAL & & 0.0 & 0.0 & 0.0 & 1197.5 & 1197.5 & 1197.5 & 1197.5 & 1197.5 & 1197.5 & 1197.5 & 1197.5 & 1197.5 & 1197.5 & 0.0 & 0.0 & 11975.0100 .0 \\
\hline Unkatown & HONE & 0.0 & 0.0 & 0.0 & 90.0 & 90.0 & 90.0 & 90.0 & 90.0 & 90.0 & 90.0 & 90.0 & 90.0 & 90.0 & 0.0 & 0.0 & 900.6100 .0 \\
\hline cotalunkasowas & & 0.0 & 0.0 & 0.0 & 90.0 & 90.0 & 90.0 & 90.0 & 90.0 & 20.0 & 90.0 & 90.0 & 90.0 & 90.0 & 0.0 & 0.0 & 900.6100 .0 \\
\hline rotal TwHE_ 2223 & & 0.0 & 0.0 & 0.0 & 1295.1 & 1295.1 & 1295.1 & 1295.1 & 1295.1 & 1295.1 & 1295.1 & 1295.1 & 1295.1 & 1295.1 & 0.0 & 0.0 & 12951.3 \\
\hline \multicolumn{3}{|c|}{ Waste Gonoratox:1FHC_242A } & & & & & & & & & & & & & & & \\
\hline DARTAL & Unknowns & 0.0 & 0.0 & 0.0 & 34.8 & 34.0 & 34.8 & 34.0 & 34.0 & 34.0 & 34.0 & 34.0 & 34.8 & 31.8 & 0.0 & 0.0 & 348.2100 .0 \\
\hline rotalD-HETAL & & 0.0 & 0.0 & 0.0 & 34.8 & 34.8 & 34.8 & 34.8 & 31.8 & 34.8 & 34.8 & 34.8 & 34.0 & 34.0 & 0.0 & 0.0 & $34 \theta .1100 .0$ \\
\hline rotal TWHAC_242A & & 0.0 & 0.0 & 0.0 & 34.0 & 34.8 & 34.9 & 34.0 & 34.8 & 34.8 & 34.8 & 34.0 & 34.0 & 34.8 & 0.0 & 0.0 & 340.1 \\
\hline \multicolumn{3}{|c|}{ Waste Generator:THHC_2128 } & & & & & & & & & & & & & & & \\
\hline D-METAL & UNMNOWN & 0.0 & 0.0 & 0.0 & 34.8 & 34.8 & 34.8 & 34.8 & 34.8 & 34.8 & 34.8 & 34.8 & 34.8 & 34.8 & 0.0 & 0.0 & 348.1100 .0 \\
\hline
\end{tabular}

BCD Codns: A=Ignitables, B-Corrosives, C-Reactives, D-Metals w/O Hg, E-Metals w Hg, Fmorganlcs, G-State Reg., H-PCB < 50ppm, J-PCB > 50ppm 


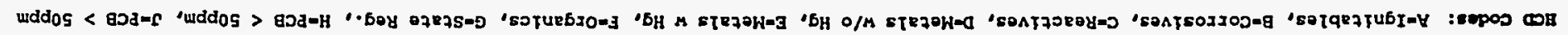

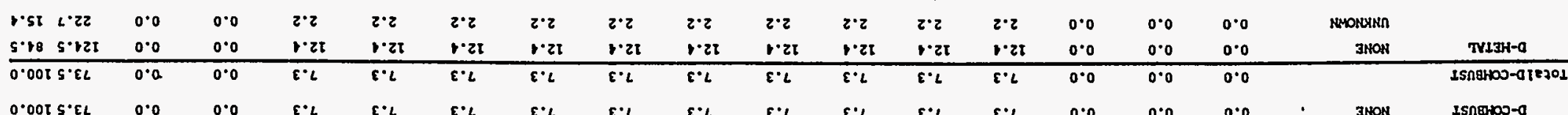

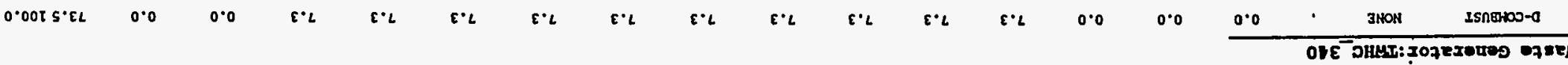

\begin{tabular}{|c|c|c|c|c|c|c|c|c|c|c|c|c|c|c|c|c|}
\hline$L^{\circ} \theta \theta 8 z$ & 0.0 & 0.0 & $\theta \cdot 8 z$ & $8 \cdot 82$ & 0.82 & $\theta \cdot \theta z$ & 0.82 & $\theta \cdot \theta z$ & $8 \cdot \theta 2$ & $\theta^{\circ} \cdot 0 z$ & $8 \cdot 82$ & 8.82 & 0.0 & 0.0 & 0.0 & OOE' OHMLTEJOL \\
\hline 0018.68 & 0.0 & 0.0 & $6 . \theta$ & 6.8 & 6.8 & 6.8 & 6.6 & 6.8 & 6.8 & 6.0 & 6.8 & 6.0 & 0.0 & 0.0 & 0.0 & MEYNYKR TE 701 \\
\hline
\end{tabular}

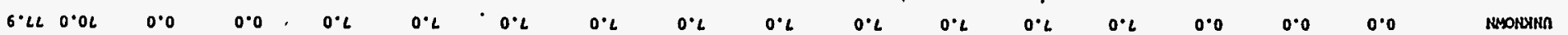

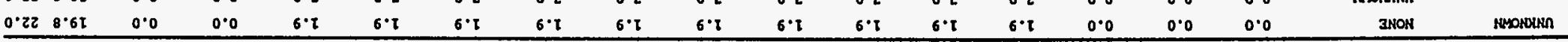

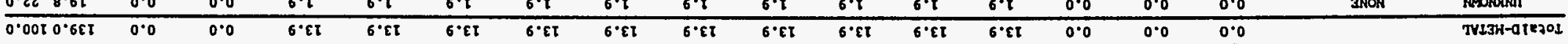

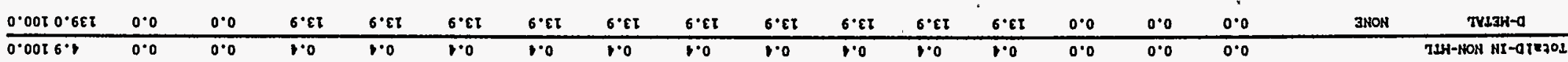

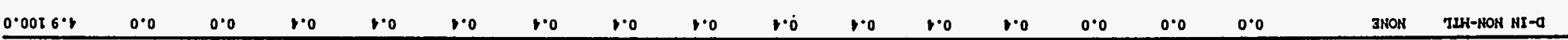

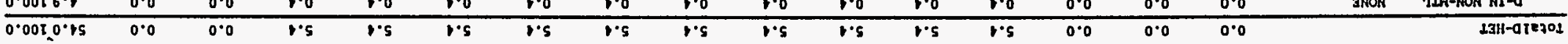

\begin{tabular}{|c|c|c|c|c|c|c|c|c|c|c|c|c|c|c|c|c|c|}
\hline & 0.0 & 0.0 & $\because \cdot s$ & 1.5 & $r \cdot s$ & 1.5 & $1 \cdot 5$ & 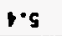 & $\because \mathbf{s}$ & 1.5 & $\because s$ & $\because \cdot s$ & 0.0 & 0.0 & 0.0 & 3NON & IJH-Q \\
\hline
\end{tabular}

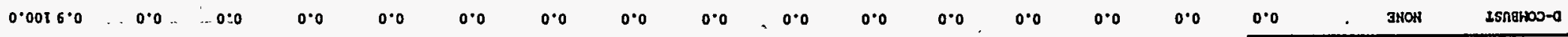

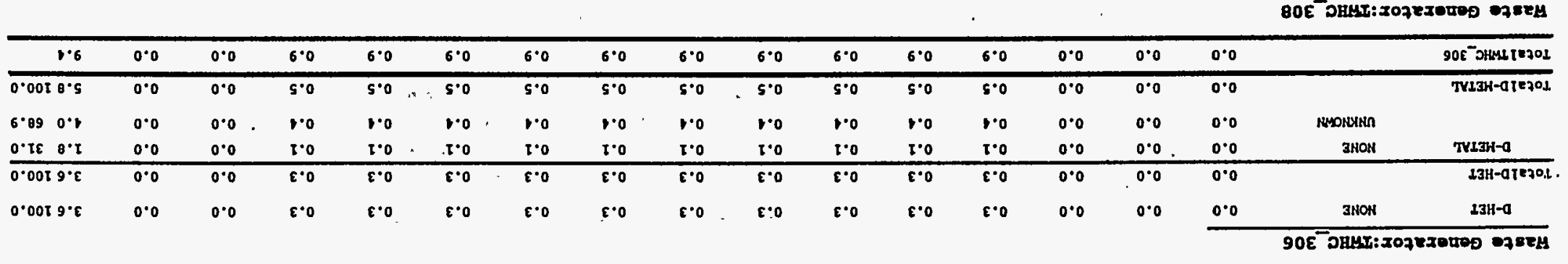

\begin{tabular}{|c|c|c|c|c|c|c|c|c|c|c|c|c|c|c|c|c|c|}
\hline $6.9 L$ & 0.0 & 0.0 & $5 \cdot 2$ & 5.2 & 5.2 & $S \cdot \mathrm{L}$ & $5 \cdot 2$ & $5 \cdot \mathrm{L}$ & $5 \cdot 2$ & $5 \cdot 2$ & S.L & $s \%$ & 0.0 & 0.0 & 0.0 & & 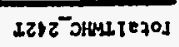 \\
\hline $0.0016 .5 L$ & 0.0 & 0.0 & S.L & $5 \cdot L$ & $5 \cdot 2$ & $S \cdot L$ & $5 \cdot h$ & $S \cdot 2$ & 5.2 & $5 \cdot 6$ & S.L & $s \cdot 2$ & 0.0 & 0.0 & 0.0 & & TVLJ3H-afE;OS \\
\hline $0.0016 . \mathrm{SL}$ & 0.0 & 0.0 & s.l & $5 \cdot 2$ & 5.2 & S.L & $s \cdot \mathrm{L}$ & S.L & $5 \cdot \iota$ & $5 \cdot 2$ & $S \cdot L$ & $5 \cdot 6$ & 0.0 & 0.0 & 0.0 & NYOHARI & TVג3H-O \\
\hline
\end{tabular}

\begin{tabular}{|c|c|c|c|c|c|c|c|c|c|c|c|c|c|c|c|c|}
\hline T.81E & 0.0 & 0.0 & o.1E & $\theta \cdot 8$ & O.1E & $\theta \cdot r \varepsilon$ & $\theta \cdot r \varepsilon$ & B.re & $\theta \cdot 0 \varepsilon$ & $\theta \cdot 8 \varepsilon$ & $8 \cdot r \varepsilon$ & $\theta \cdot v$ & 0.0 & 0.0 & 0.0 & 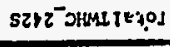 \\
\hline 0.001 I.8VE & 0.0 & 0.0 & Q.VE & $8 \cdot 1 \varepsilon$ & $\theta \cdot b \varepsilon$ & $\theta \cdot r \varepsilon$ & $0 \cdot r \varepsilon$ & $\theta \cdot b$ & $\theta \cdot 0 E$ & $0 \cdot 1 \varepsilon$ & $\theta \cdot r \varepsilon$ & $\theta \cdot 1 \varepsilon$ & 0.0 & 0.0 & 0.0 & 'TVL3H-Q Q \\
\hline
\end{tabular}

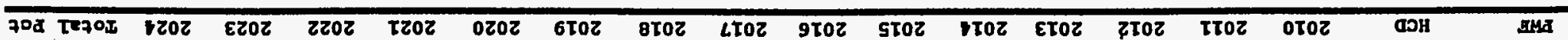

(8xo70u ofqno uT)

902 obed

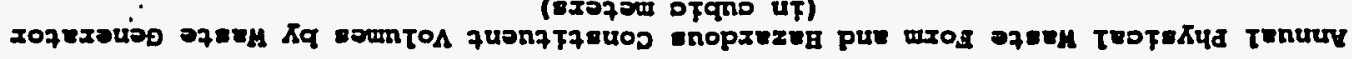


WHC-EP-0888

APPENDIX E

\section{WASTE GENERATOR ABBREVIATIONS}

E- $\mathbf{i}$ 
WHC-EP-0888

Intentionally Left Blank

$E-i i$ 
APPENDIX E

WASTE GENERATOR ABBREVIATIONS

Table E-1. Haste Generator List by Hanford Program Area

\begin{tabular}{|c|c|c|c|}
\hline Program Area & Waste Generator Name & 1994 Abbreviation & 1993 Abbreviation \\
\hline \multirow{2}{*}{$\begin{array}{l}\text { Analytical } \\
\text { Services }\end{array}$} & 222-S Analytical Laboratory & WHC ANALYT LAB & HHC 222S \\
\hline & 325 Radiochemistry Laboratory & HHC 325 & NHC 325 \\
\hline \multirow{27}{*}{$\begin{array}{l}\text { Enviromental } \\
\text { Restoration }\end{array}$} & 100 Area Transition (BHI) & UHC_100* & HHC_100 \\
\hline & $\begin{array}{l}\text { D\&D of } 200 \text { Area Effluent Treatment (Project } \\
\text { C018H) }\end{array}$ & THHC_PX_CO18H & THHC_PX_CO18H \\
\hline & D\&D of 222-S Analytical Laboratory & THHC_2225 & TWHC_222S \\
\hline & $\begin{array}{l}\text { D\&D of } 224 T \text { TRU Storage \& Assay Facility } \\
\text { (TRUSAF) }\end{array}$ & THHC_TRUSAF & TUHC_TRUSAF \\
\hline & D\&D of 242-A Evaporator & THHC 242A & THHC 242A \\
\hline & D\&D of 242-S Evaporator & THHC_242S & TWHC 2425 \\
\hline & D\&D of 242-T Evaporator & THHC_242T & THHC 242T \\
\hline & $\begin{array}{l}\text { D\&D of } 306 \text { Development/ } \\
\text { Fabrication/Test Lab. }\end{array}$ & THHC_306 & TWHC_306 \\
\hline & D\&D of 308 Fuels Development Laboratory & TWHC 308 & TWHC 308 \\
\hline & D\&D of 340 Waste Neutralization Facility & THHC 340 & TWHC 340 \\
\hline & DED of B Plant & THHC B PLANT & TWHC 225B \\
\hline & D\&D of $M$ Reactor & TWHC $100 \mathrm{~N}$ & TUHC $100 \mathrm{~N}$ \\
\hline & D\&D of PFP & TUHC PFP & TWHC 23452 \\
\hline & D\&D of PHL 231-Z Building & TPHL_2312 & TPNL_ 2312 \\
\hline & D\&D of PHL 242 Building & TPNL_242B/BL & TPHL 242B/BL \\
\hline & D\&D of PHL 306H Building & TPHL 306H & TPHL 306H \\
\hline & D\&D of PHL 314 Building & TPNL 314 & TPNL_314 \\
\hline & D\&D of PHL 326 Building & TPHL_326 & TPHL 326 \\
\hline & D\&D of PHL 327 Building & TPHL 327 & TPHL 327 \\
\hline & D\&D of PHL 331 Building & TPNL 331 & TPHL 331 \\
\hline & D\&D of PHL 324 Building & TPHL 324 & TPHL 324 \\
\hline & D\&D Of PUREX & TUHC PUREX & TUHC 202A \\
\hline & D\&D of T Plant & THHC T PLAHT & THHC 221T \\
\hline & Past Practice Remediation & PAST PRAC REM & PAST PRAC REH \\
\hline & PUREX Tunnel Waste & TWHC PUREX TH & THHC 202A_TH \\
\hline & Surplus Facilities & WHC SURPLS FAC & MHC SURPLS FAC \\
\hline & Well Drilling - Envirormental Projects & HHC_MELL_DRL & HHC MELL_DRL \\
\hline
\end{tabular}


Table E-1. (contd)

\begin{tabular}{|c|c|c|c|}
\hline Program Area & Waste Generator Name & 1994 Abbreviation & 1993 Abbreviation \\
\hline \multirow[t]{3}{*}{$\begin{array}{l}\text { Liquid } \\
\text { Effluent }\end{array}$} & $\begin{array}{l}200 \text { Area Effluent Treatment .Facility (Project } \\
\text { C018H) }\end{array}$ & HHC_200_ETF & WHC_PX_CO18H \\
\hline & $\begin{array}{l}300 \text { Area Treated Effluent Disposal Facility } \\
\text { (Project L045H) }\end{array}$ & HHC_300_TEDF & WHC_LO45H \\
\hline & $\begin{array}{l}\text { Waste Neutralization Facility (340/307 } \\
\text { Buildings) }\end{array}$ & WHC_HASTE_MEUT & WHC_340 \\
\hline \multirow{25}{*}{$\begin{array}{l}\text { Hon- } \\
\text { Programmatic } \\
\qquad\end{array}$} & 105DR Large Sodiun Fire Facility & HHC_SOOIUH_FAC & HHC_105DR \\
\hline & 1154 Radio Maintenance-3000 Area & HHC RADIO HAIN & WHC 11543000 \\
\hline & 1163 Procurement \& Material Management - & WHC PROCURMENT & WHC 1163 \\
\hline & 1171 FSS Fleet Maintenance & WHC FLEET MAIN & WHC 1171M \\
\hline & 1171 FSS Transportation & WHC TRANSPORT & WHC $1171 T$ \\
\hline & 2101M FSS Electric Utilities & WHC ELEC UTIL & HHC $2101 \mathrm{M}$ \\
\hline & 2703E Chemical Engineering Laboratory & UHC CHEH E LAB & HHC $2703 E$ \\
\hline & 2715EA General Area Services North & WHC GAS NORTH & WHC 2715EA \\
\hline & 2724W Laundry/Equipment Decontamination & WHC LAUHDRY & MHC $2724 \mathrm{H}$ \\
\hline & 284E/284W East \& West Powerhouses & WHC 200 PUR & UHC 284E \\
\hline & 305 Engineering Testing Facility & HHC EHG TEST & HHC 305 \\
\hline & 306 Development/Fabrication/Test Lab. & WHC ENG DEV & WHC 306 \\
\hline & 308 Fuels Development Laboratory & HHC FUEL DEV & WHC 308 \\
\hline & 335 Sodium Test Facility & HHC SOOIUH TST & WHC 335 \\
\hline & 3707C Safeguards/Security Maintenance & WHC SS MAIN & WHC $3707 \mathrm{C}$ \\
\hline & 3717B Standards Laboratiory & HHC STAND LAB & WHC $3717 \mathrm{~B}$ \\
\hline & 377 Geotechnical Engineering Laboratory & WHC GEOTEC LAB & WHC 377 \\
\hline & 384 Power House, 300 Area & UHC 300 PLR & HHC 384 \\
\hline & BCSR Computer Service Federal Bldg. & WHC FED & HHC_FED \\
\hline & Buried Equipment (OSS/TFS) 1992 & WHC BQUIP & WHC BQUIP \\
\hline & FFTF/FHEF/MASF Maintenance & WHC FFTF MAIN & WHC FFTF MAINT \\
\hline & Fuel \& Materials Examination & WHC FHEF & WHC FMEF \\
\hline & General Area Services South & WHC GAS SOUTH & WHC $\mathbf{S 3 0 0}$ \\
\hline & Hanford Environmental Health Foundation & HEHF & HEHF \\
\hline & Kaiser Construction Services- 100 Area & KEH 100 & KEH 100 \\
\hline \multirow[t]{7}{*}{ Offsite } & Ames Laboratory-Ames, Iova & AMES & AMES \\
\hline & Argonne National Laboratory-East & ANL $E$ & ANL E \\
\hline & Bates Accelerator-Massachusetts & MITT BATES & MIT BATES \\
\hline & Battelle Columbus Laboratories & BAT CLBS LAB & BAT CLBS LAB \\
\hline & Bettis Atonic Power-Laboratory & BAPL & BAPL \\
\hline & Bettis Atomic Pouer-Shipyards & BAPL SHIPYDS & BAPL SHIPYOS \\
\hline & Brookhaven National Laboratory & BRKHVN & BRKHVH \\
\hline
\end{tabular}


Table E-1. (contd)

\begin{tabular}{|c|c|c|c|}
\hline Program Area & Haste Generator Hame & 1994 Abbreviation & 1993 Abbreviation \\
\hline \multirow{16}{*}{$\begin{array}{l}\text { Offsite } \\
\text { (contd) }\end{array}$} & EG\&G Rocky flats Plant & EG\&G & EG\&G \\
\hline & FERHI National Accelerator Laboratory & FERHI & FERHI \\
\hline & $\begin{array}{l}\text { Formerly Utilized Sites Remedial Action } \\
\text { Program }\end{array}$ & FUSRAP & FUSRAP \\
\hline & General Atomics & GEH ATOY & GEN ATOM \\
\hline & Knolls Atonic Power-Laboratory & KAPL & KAPL \\
\hline & Knolls Atomic Power-Shipjards & KAPL SHIPYDS & KAPL SHIPYDS \\
\hline & Lawrence Berkeley Laboratory & LBL & LBL \\
\hline & Paducah Energy Systems & PADUCAH_ES & PADUCAH ES \\
\hline & Paducah Utility Services & PADUCAH US & PADUCAH US \\
\hline & Princeton Plasma Physics Laboratory & PRIMCETOH & PRINCETOH \\
\hline & Portsmouth Energy Systems & PORTSHOUTH ES & PORTSMOUTH ES \\
\hline & Portsmouth Utility Services & PORTSMOUTH US & PORTSMOUTH US \\
\hline & Rockwell-Canoga Park & RKW CANOGA & RKU CANOGA \\
\hline & Stänford Linear Accelerator Center & STANFORD & STAMFORD \\
\hline & University of California-Davis & B LEHR DAV & B LEHR DAV \\
\hline & University of Utah & $\underline{u} \mathbf{u}$ & $\mathbf{u} \mathbf{u}$ \\
\hline PHL & Pacific Northwest Laboratory & PNL & PNL \\
\hline $\begin{array}{l}\text { Spent Nuclear } \\
\text { Fuel }\end{array}$ & 100K K-Basin Operations & WHC_K_BASIN & HHC_100K \\
\hline \multirow[t]{4}{*}{ Solid Waste } & 218E/W Low Level Burial Grounds & UHC LLBG & WHC LLBG \\
\hline & 221T/2706T T-Plant Operations & UHC T PLANT & WHC $221 T$ \\
\hline & 224T TRU Storage and Assay Facility (TRUSAF) & WHC TRUSAF & WHC TRUSAF \\
\hline & Mixed Waste Storage Facility & WHC EA H112 & WHC EA H112 \\
\hline \multirow{5}{*}{$\begin{array}{l}\text { Transition } \\
\text { Facilities }\end{array}$} & Purex (202A) Operations & WHC_PUREX & HHC_202A \\
\hline & Purex (202A) Surveillance \& Maintenance & HHC PUREX SM & Hot Applicable \\
\hline & B-Plant & WHC B PLANT & WHC 225B \\
\hline & Plutoniun Finishing Plant & WHC PFP & UHC 23452 \\
\hline & 300 Area Fuel Supply & WHC FUEL TRAHS & UHC $303 \mathrm{~K}$ \\
\hline \multirow[t]{8}{*}{ THRS } & Aging Waste Transfer Lines & HHC TLP W028 & HHC THP NOZB \\
\hline & Cross-Site Transfer System & HHC TUP MO58 & WHC TWP N058 \\
\hline & DST 101AZ Retrieval (1 tank) & HHC TWP H151 & WHC TUP H151 \\
\hline & DST Retrieval Systens (10 tanks) & WHC TUP U211 & WHC TLP W211 \\
\hline & Tank Farm Restoration & WHC TUP U314 & WHC TUP U314 \\
\hline & High Level Vitrification Project & WHC HLVP & WHC HLVP \\
\hline & Low Level Vitrification Project & WHC LLVP & HHC LLVP \\
\hline & SST Long Length Equipment & WHC_SST LLE & WHC SSTLLE \\
\hline
\end{tabular}


Table E-1. (contd)

\begin{tabular}{|c|c|c|c|}
\hline Program Area & Waste Generator Name & 1994 Abbreviation & 1993 Abbreviation \\
\hline \multirow[t]{8}{*}{ TURS (contd) } & SST Retrieval (149 tanks) & WHC SST RET & WHC SSTRET \\
\hline & SST Tank 106C Manipulator & WHC TUP 1340 & UHC TUP U340 \\
\hline & SST Tank 106C sluicing & WHC TUP W320 & HHC TUP W320 \\
\hline & SST/DST Tank Farm Operations & WHC TF OPER & WHC 2ETF \\
\hline & Tank Farm Ventilation Upgrade & WHC TWP W030 & WHC TUP N030 \\
\hline & Tank Waste Pretreatment Facility & WHC PRETRT TH & UHC PRETRT TH \\
\hline & U315 Pilot Scale Retrieval & WHC DST PSRET & WHC DSTPSRET \\
\hline & U343 DST Retrieval (17 tanks). & HHC ST RET & HHC ST_RET \\
\hline
\end{tabular}


HHC-EP-0888

\section{DISTRIBUTION}

Number of Copies

ONSITE

$9 \quad$ U.S. Department of Energy,

Richland Operations Office

R. F. Guercia (5)

R3-81

Public Reading Room

A1-65

Central Files

OSTI (2)

A3-88

E6-44

41

Pacific Northwest National Laboratory

T. J. DeForest (20)

K7-97

D. B. Elliott

$\mathrm{K} 8-17$

J. T. Fulton

K9-26

J. T. Hauth (5)

K7-97

H. S. Konynenbelt

K8-18

J. E. Marler

K8-07

G. W. McNair

P7-75

W. A. Ross

H. J. Roye

R. W. Schultz

J. V. Stangland

K. D. Syverstad

K. J. Templeton (5)

K7-94

K7-10

$\mathrm{K} 8-17$

K9-26

K7-10

Technical Files

K7-94

K1-11

3 ICF Kaiser Hanford Company

M. R. Carver

G2-02

A. R. Gadeken

G3-05

E. H. Koellermeier

G3-09

2

Bechtel Hanford Incorporated

W. S. Stufin

H4-79

M. A. Woll in

H4-79

Westinghouse Hanford Company

R. R. Ames

T4-02

J. D. Anderson

T3-04

F. L. Ashworth

H6-29

W. S. Ayers

T3-28

T. B. Bergman

R3-86

R. R. Borisch

R3-56 
DISTRIBUTION (continued)

Number of Copies

ONSITE

Westinghouse Hanford Company (continued)

H. C. Boynton

T3-04

P. A. Callender

HO-57

A. B. Carlson

T4-02

D. B. Cartme11

R3-50

F. M. Coony

T4-02

G. Cox

J. L. Deichman

H8-64

A. J. Diliberto

T6-03

D. B. Encke

J. I. Forsberg

H6-10

$\times 5-53$

L. A. Fort

J. C. Fulton

B. J. Fuquay

A. D. Gadeken

N2-13

H5-36

R3-11

R3-11

G3-05

K. A. Gasper

C. J. Geier

G3-21

R. J. Giroir

R2-36

C. K. Girres

T4-05

J. H. Hales

T3-04

D. G. Hamrick

G3-21

P. L. Hapke

S6-15

J. A. Harris

T4-05

T6-12

R. E. Heineman, Jr.

S6-65

N. O. Hinojosa

X3-67

J. E. Holmes

B3-53

J. 0. Honeyman

S7-81

S. C. Howald

H6-30

K. L. H7adek

T4-02

R. K. Hulvey

N2-33

P. H. Jacobsen

T3-01

H. G. Jasen

S6-17

R. D. Jensen

G3-21

J. R. Kirkendal1

B3-28

E. F. Loika

N2-51

S. S. Lowe

H6-29

T. L. Main

S6-65

D. Marshall

S7-40

D. E. McKenney

T3-01

L. C. Mercado

D. S. Merry

R2-54

G. A. Meyer

B3-28

D. A. Moore

S2-48

H6-21 
DISTRIBUTION (continued)

Number of Copies

ONSITE

Hestinghouse Hanford Company (continued)

R. J. Murkowski

H5-03

D. E. Nester

F. J. Orsag

S. M. O'Toole

T3-01

D. B. Pabst

H6-35

R. D. Pierce

B. G. Place

D. B. Powel1, Jr.

H6-33

R. H. Powe 17

H7-03

T3-04

T3-01

T4-03

R. J. Puigh

G3-21

K. M. Quigley

HO-31

J. G. Riddelle

T3-01

S. B. Roakes

T3-01

H. A. Ross

H6-32

J. C. Sabin

N. A. Samuels

F. D. Sargent

R. M. Schwenk

K7-94

T4-02

H6-30

S6-31

R. R. Slaybaugh

H8-64

T3-01

D. J. Sommer

R. A. Spohr

H6-33

J. M. Steffen

T6-04

N1-47

J. A. Swenson

H5-61

R. R. Thompson

H6-32

O. J. Valero (20)

T4-02

A. 0. Vance

T4-02

P. J. Vandervert

HO-57

E. C. Vogt

J. L. Westcott

T5-50

J. H. Wicks

T3-04

R2-50

J. P. Hitlis

R. D. Hojtasek

T3-04

J. C. Homack

$57-80$

M. S. Wright

R3-86

M. T. York

T3-28

H6-32 
This page intentionally left blank. 